UNIVERSIDAD POLITÉCNICA DE MADRID

ESCUELA TÉCNICA SUPERIOR DE INGENIEROS DE CAMINOS, CANALES Y PUERTOS
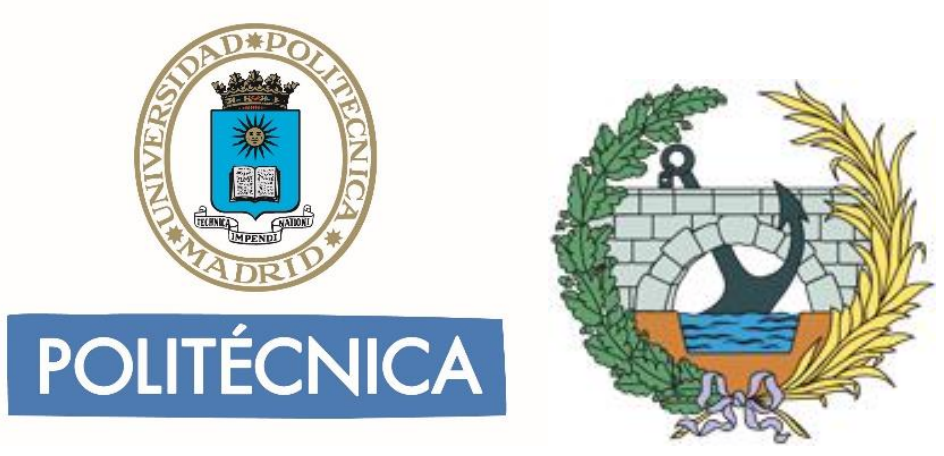

\title{
MODELOS DE COMPORTAMIENTO PARA EL CONTROL DE PANTALLAS ANCLADAS EN TERRENOS CON FLUENCIA
}

TESIS DOCTORAL

NATALIA SALI MONTERO CUBILLO

Ingeniera Geóloga

Madrid, 2020 

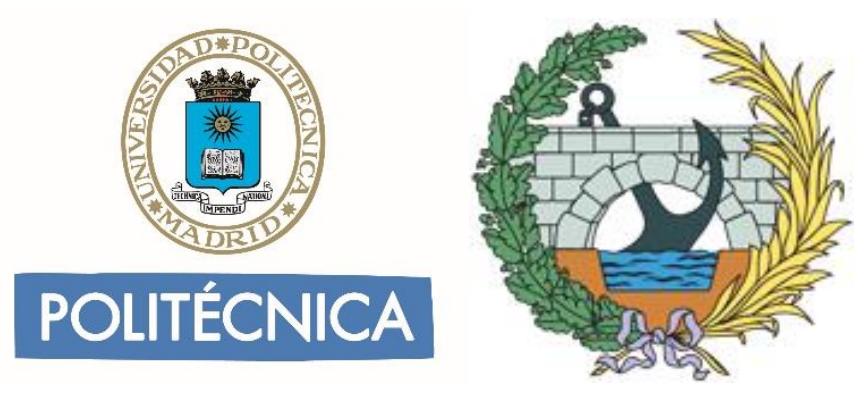

MODELOS DE COMPORTAMIENTO PARA EL CONTROL DE PANTALLAS ANCLADAS EN TERRENOS CON FLUENCIA

\author{
NATALIA SALI MONTERO CUBILLO \\ Ingeniera Geóloga
}

Directores de tesis:

CLAUDIO OLALLA MARAÑÓN Doctor Ingeniero de Caminos, Canales y Puertos

RUBÉN ÁNGEL GALINDO AIRES Doctor Ingeniero de Caminos, Canales y Puertos Licenciado en Ciencias Matemáticas 


\section{RESUMEN}

En algunas de las normativas y guías existentes sobre anclajes al terreno se indican una serie de recomendaciones para el control a largo plazo de éstos, estableciendo un mínimo de células de carga a instalar y controlar periódicamente, según las dimensiones de la obra. Sin embargo, no existe un conocimiento real ni una solución teórica analítica sobre el problema de fluencia en estas condiciones de contorno y, por tanto, sobre la evolución de la carga del anclaje con el tiempo, en las condiciones de trabajo. Por esta razón, se suele introducir un coeficiente de seguridad respecto a la fluencia del terreno. Además, se impide la construcción de anclajes en ciertos terrenos por restricciones mediante límites del índice de plasticidad o del contenido en materia orgánica del suelo o del coeficiente de fluencia obtenido en ensayos de investigación in situ.

Por ello, se propone un nuevo modelo analítico de interacción suelo-estructura para terrenos con fluencia que permite obtener la evolución de la carga en el anclaje con el tiempo, así como las deformaciones por fluencia asociadas. La solución completa del problema de contorno utiliza el modelo de Burgers, o cualquier modelo mecánico o empírico, la hipótesis de asiento de Borowicka y el movimiento producido por una fuerza en el interior de un semiespacio elástico. La singularidad que aparece cuando se aplica una fuerza puntual al comienzo de la longitud del bulbo se resuelve mediante integración de la ecuación de Mindlin para una carga constante repartida a una profundidad determinada actuando en una superficie anular.

En primer lugar, el problema teórico se ha resuelto para una losa anclada verticalmente al terreno $\mathrm{y}$, a continuación, para una pantalla anclada, incorporando al conjunto la rigidez de este nuevo elemento, factor clave en la pérdida de carga en el anclaje. Asímismo, el modelo analítico queda implementado en una hoja de cálculo. Con la finalidad de mostrar su utilidad práctica, la solución desarrollada se aplica a un caso real de estructura flexible anclada, situada en Escocia.

También, se ha llevado a cabo la simulación numérica de ambos modelos analíticos en el programa de análisis geotécnico en diferencias finitas FLAC 3D. 
De tal manera que se dispone de un modelo general parametrizado para el cálculo de pantallas ancladas en terrenos con fluencia. La validación de los modelos análiticos de pantalla anclada y losa anclada se realiza comparando estos con modelos representativos en diferencias finitas. Esto es, incorporando las mismas leyes constitutivas de fluencia y condiciones de contorno. Las predicciones realizadas a partir de los respectivos modelos numéricos y analíticos muestran un buen ajuste.

Los resultados obtenidos revelan la importancia de la necesidad de disponer de ensayos de fluencia específicos, de un mínimo de duración (superior a los 60 o 120 minutos, de los ensayos de investigación en obra) y al nivel de carga de trabajo, para realizar una estimación adecuada de los parámetros y obtener con ellos una predicción lo más ajustada posible.

En esta línea y con un conocimiento previo de los factores que afectan al fenómeno, se presenta un nuevo ensayo de fluencia en laboratorio que permite medir los desplazamientos bajo carga constante durante largos periodos de tiempo, en un anclaje embebido en una roca blanda de grandes dimensiones. Se detallan los fundamentos teóricos que han servido para el diseño del equipo, así como sus características mecánicas y geométricas, definiendo el procedimiento de ensayo. En particular, se ha ensayado un geomaterial, compuesto por bentonita, cemento y agua, que ha sido caracterizado geotécnicamente mediante ensayos de identificación, resistencia y deformabilidad. Se define el proceso específico de fabricación de los bloques sobre los que se efectúan ensayos de fluencia con duraciones de hasta dos meses. Los resultados obtenidos revelan la necesidad de tener en cuenta dos factores para la adecuada interpretación de los desplazamientos instantáneos que se obtienen con el ensayo: la mayor rigidez en pequeñas deformaciones y el efecto escala. 


\begin{abstract}
Some of the current standards and guides about ground anchors indicate recommendations for long-term control of these, establishing a minimum of load cells to install and control periodically depending on the size of the work. However, there is no real knowledge or an analytical theoretical solution to the creep problem in these boundary conditions. The anchor load evolution over time in working conditions is therefore unknown. A safety coefficient considering the soil creep is introduced for this reason. Anchors are not installed in certain soils due to restrictions employing in situ investigation tests, limits of the plasticity index or the content of organic matter.
\end{abstract}

Thus, a new analytical model of soil-structure interaction for creep grounds is proposed, which can be used to calculate the load evolution on an anchored structure over time and the associated creep strains. This complete boundary problem solution employs the Burgers model or any mechanical or empirical model, the Borowicka hypothesis of settlement and movement produced by a force into an elastic semi space. The singularity that appears when a point load is applied at the beginning of the anchor depth was solved with the integration of the Mindlin equation for a distributed constant load at particular depth acting on an annular surface.

Firstly, the theoretical problem has been solved for a slab anchored vertically to the ground, and then for an anchored wall, incorporating the stiffness of this new element, a key factor in the anchor load loss. Likewise, the analytical model is implemented in a spreadsheet. To show its practical utility, the developed solution is applied to an actual case of an instrumented anchored wall, located in Scotland.

The analytical models previously presented are also simulated through finitedifference models fitted with the geotechnical software FLAC 3D. In such a way that a general parameterized model is available for the calculation of anchored walls in creep soils. The validation of the analytical models of the anchored wall and the anchored slab was performed by comparing it with representative finitedifferences models. That is, by incorporating the same constitutive creep law 
and the same boundary conditions. The numerical and analytical prediction models show good agreement.

The results demonstrate the relevance of having specific creep tests, for at least a minimum duration (greater than 60 or 120 minutes, of the on-site investigation tests) and at the workload level, to make an adequate estimation of the parameters and to obtain an accurate prediction.

In this way and with previous knowledge of the factors that affect the phenomenon, this thesis introduces a laboratory creep test for measuring displacements under constant load for long periods in anchors embedded in large-scale soft rocks. The preliminary theoretical fundamentals that have served for the design of the equipment are detailed, as well as its mechanical and geometrical characteristics, defining the test procedure. A rock-like material composed of bentonite, cement and water has been tested, which has been geotechnically characterized by identification, strength and deformability tests. The specific manufacturing process of the test samples is also defined. Three creep tests are carried out with durations between 46 and 75 days each. Two effects that must be considered to make an adequate interpretation of the instantaneous deformations achieved in the test have been shown with the obtained results: the greater stiffness in small deformations and the scale effect. 
A mi sobrina y a mi hermana,

Por hacerme despertar.

A mi madre Isabel,

Por su apoyo incondicional.

A Curro,

Mi ejemplo. Qué bien lo hiciste y lo sigues haciendo. 


\section{AGRADECIMIENTOS}

En primer lugar, me gustaría mostrar mi más sincero agradecimiento a mis dos directores de tesis. A Claudio Olalla, por la confianza depositada en mí al concederme la beca de investigación, siempre mostrando una actitud positiva, cercana y alentadora en todas las propuestas y en el trabajo que iba realizando, la cual valoro y agradezco enormemente. Le quedo muy agradecida por su capacidad de integrarme en el grupo de trabajo del laboratorio y por la búsqueda de recursos. A Rubén Galindo, por su infinita paciencia a la hora de desarrollar el modelo matemático, compartiendo pensamientos e ideas, y explicándome nuevos conceptos que en un principio veía lejanos de comprender. Me llevo un grato recuerdo de esta fase de la tesis, horas de desgranar el modelo, durante las cuales he podido disfrutar de sus conocimientos y brillantez. Le agradezco su inmensa dedicación y su orientación durante todo el doctorado. Me parece primordial agradecer y apreciar la contribución de las ideas originales que han servido de base para el desarrollo del modelo matemático a D. Alcibíades Serrano.

No puedo dejar de mencionar a José Estaire, pues gracias a sus enseñanzas nació mi interés por el mundo de la geotecnia. Posteriormente, me animó a participar en mi primer congreso y realicé mis primeros trabajos en laboratorio, iniciándome en el mundo de la investigación. Su rigor y su forma de trabajar y de comunicarse son para mí un gran ejemplo a seguir. Le agradezco la confianza depositada en mí en tantas ocasiones.

Me parece fundamental el papel que han tenido mis compañeros de la ETSICCP (Daniel, Ana, Nacho, José Gregorio, Salvador), por nuestros ratos de desahogo y por sus consejos profesionales y personales. Especialmente a Ramiro, por su apoyo y amistad, que es una de las recompensas de esta etapa.

Darles las gracias muy especialmente, a Mauro Muñiz y a Felipe García, por las horas dedicadas al nuevo equipo y por su participación en la engorrosa tarea de fabricación y traslado de las muestras. Quiero mencionar también a Jesús Page por su constante colaboración y dedicación en relación a la 
fabricación de las muestras y mejoras del nuevo equipo. Pero principalmente por su sonrisa diaria, sus detalles y su buen humor que facilitan enormemente el trabajo.

Me gustaría agradecer particularmente a Montse Senís y Pedro Velasco, de Itasca Consultores S.L., la asistencia técnica recibida con los modelos numéricos y su acogida durante mi estancia en Asturias.

Los trabajos de investigación en los que está basada la tesis doctoral han sido financiados por el Programa Estatal de Investigación, Desarrollo e Inovacción Orientada a los Retos de la Sociedad (Ministerio de Economía y Competitividad), en el cual ha participado como organismo principal encargado de la investigación Ferrovial Agroman S.A. Agradezco la información proporcionada y, en particular la colaboración durante todo el desarrollo del proyecto, a Davor Simic y a Juan José Sánchez. En este mismo proyecto, la supervisión administrativa por parte de la Fundación Agustín de Betancourt (ETSICCP, UPM), en particular, de Alberto Bornstein, han sido esenciales.

Darles mis más sinceras gracias a mis amigos. En estos años, los momentos con ellos han hecho que pudiera superar cualquier circunstancia. A Rober, por ayudarme a tomar las decisiones más importantes y saberme escuchar como nadie. A Alberto, por estar siempre ahí, pase lo que pase, y poniéndole su sentido del humor. A Paloma, por habernos recuperado. A Soraya, por su psicología y amistad. A Bea, por tranquilizarme solo con existir. A Curro, por su ejemplo.

Me gustaría expresar mi eterno agradecimiento a mi madre, Isabel, pues su apoyo incondicional, es el pilar esencial que ha hecho posible la finalización de esta tesis doctoral y el aguante en los momentos más difíciles. Gracias por entenderme y ayudarme durante esta etapa que tantos cambios ha conllevado. Agradecer también a mis abuelos, Isabel y Antonio, su cariño y ayuda en tantos sentidos, y a mi tío Enrique, por darme ejemplo en la responsabilidad y constancia. 


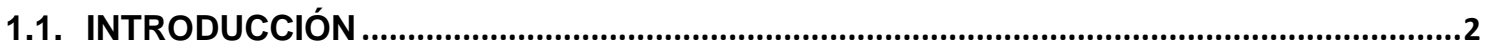

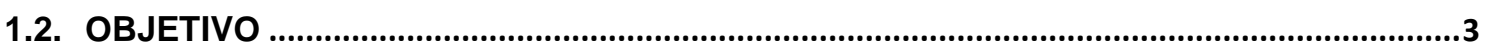

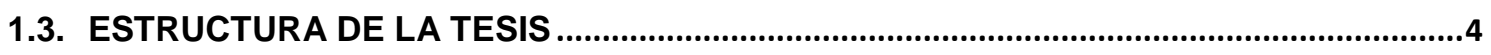

CAPÍTULO 2: ESTADO DEL CONOCIMIENTO. CARACTERIZACIÓN DE LA FLUENCIA DEL TERRENO.

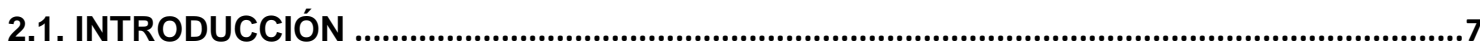

2.2. COMPRENSIÓN DEL FENÓMENO DE FLUENCIA ...............................................................

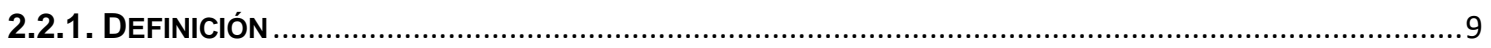

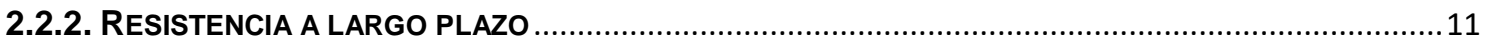

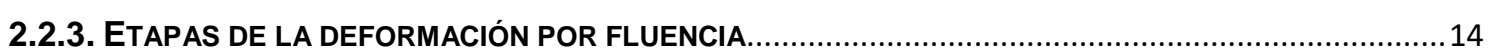

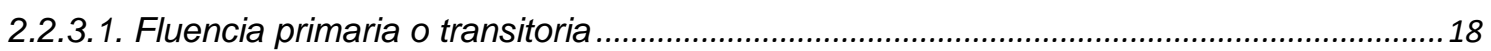

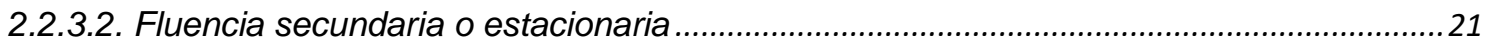

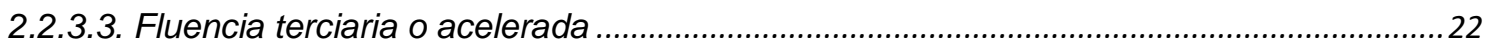

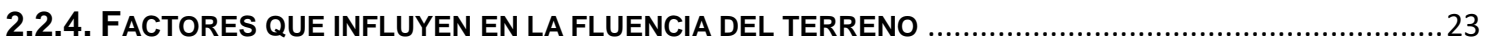

2.2.4.1. Naturaleza, tipo de enlace y otras propiedades del suelo ...............................................23

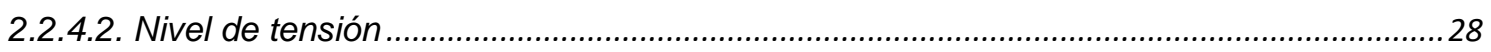

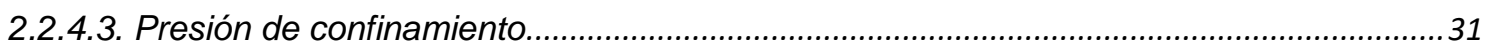

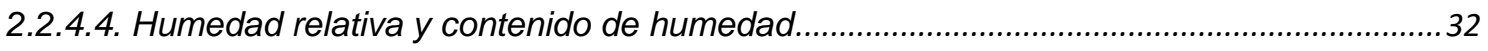

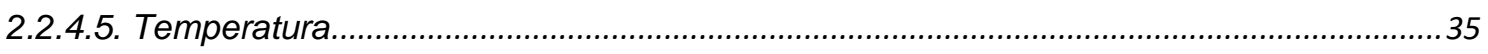

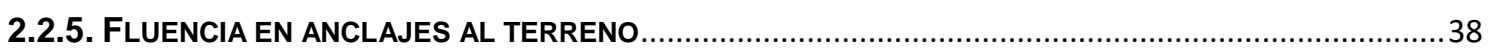

2.3. MODELOS REOLÓGICOS CONSTITUTIVOS …................................................................4

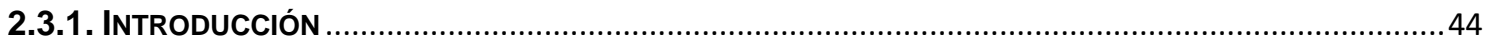

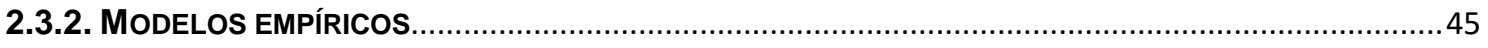

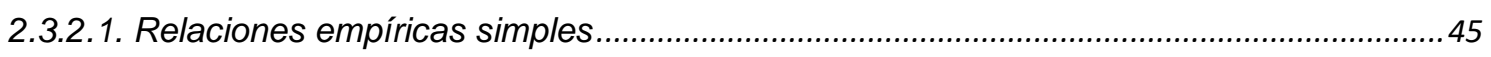

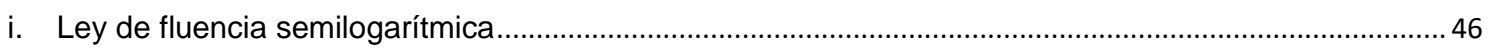

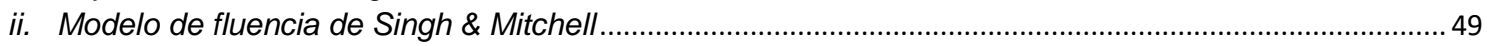

iii. Modelo de relajación de Lacerda \& Houston ............................................................................................. 51

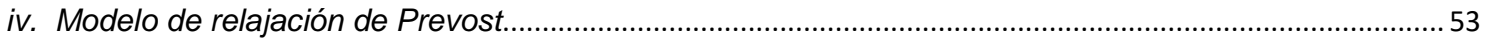

v. Enfoque de la velocidad de deformación ("strain rate approach") ..............................................................5 54

vi. Comentarios sobre los modelos empíricos simples................................................................................5

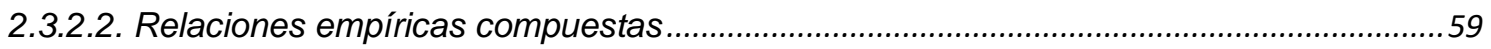

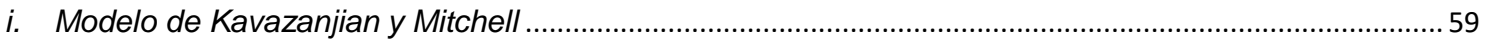

iii. Otros modelos unidimensionales para la consolidación secundaria de arcillas .......................................62

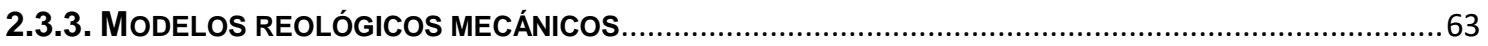

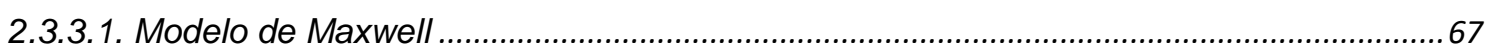

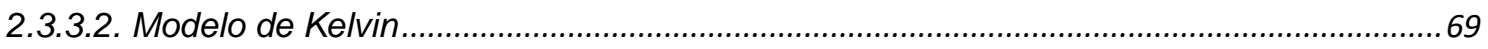

2.3.3.3. Modelo de Zener o sólido lineal estándar........................................................................... 71

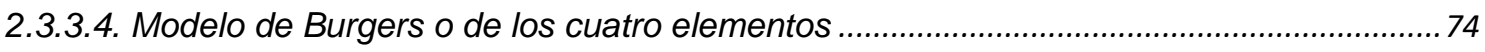

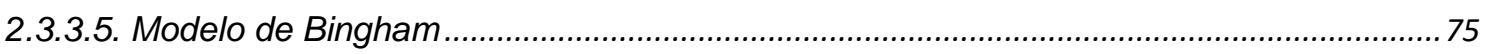


2.3.3.6. Comentarios sobre los modelos reológicos mecánicos ................................................77

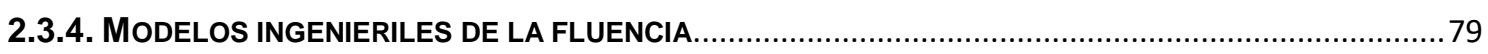

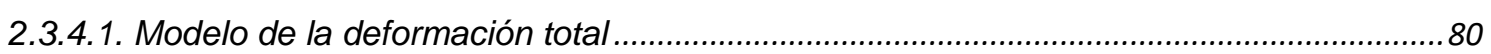

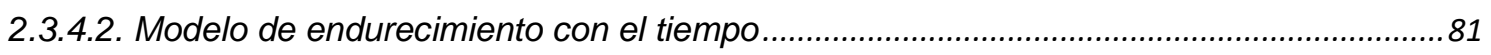

2.3.4.3. Modelo de endurecimiento con la deformación .................................................................81

2.3.4.4. Comentarios sobre los modelos ingenieriles ...............................................................8 82

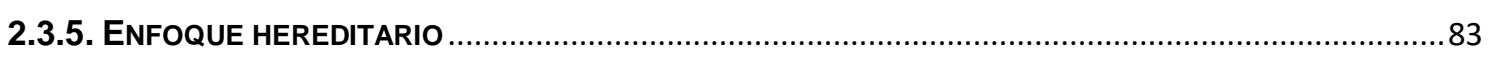

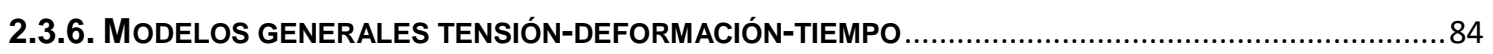

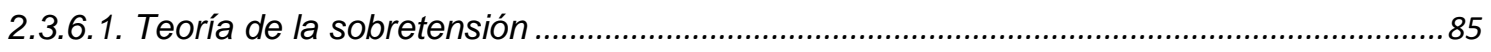

2.3.6.2. Teoría de la superficie de flujo no estacionaria .............................................................89

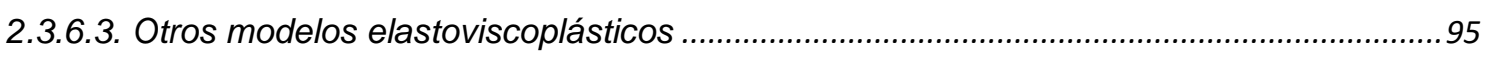

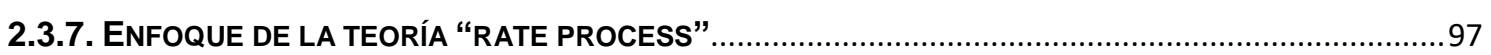

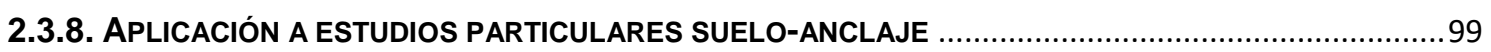

CAPÍTULO 3: MODELO ANALÍTICO DE INTERACCIÓN DE PANTALLA ANCLADA..........102

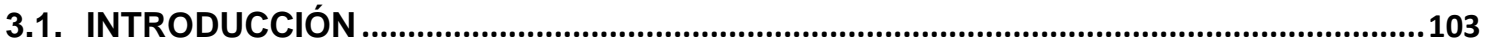

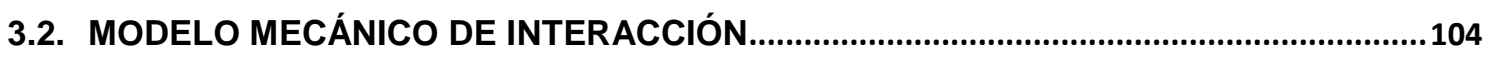

3.2.1. Planteamiento mecánico del PRoblema de CONTORNo.................................................104

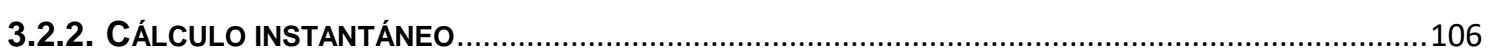

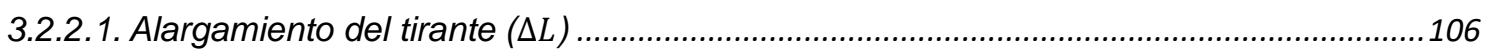

3.2.2.2. Desplazamiento de la losa por compresión del terreno por la carga del gato (upc) .....107

3.2.2.3. Desplazamiento de la losa por la tracción desde el bulbo del anclaje (upt)..................107

3.2.2.4. Desplazamiento del tirante producido por la carga en cabeza del bulbo (uA)...............109

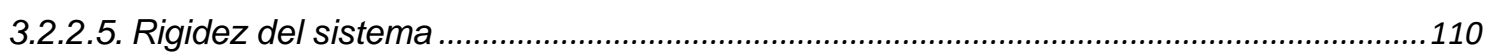

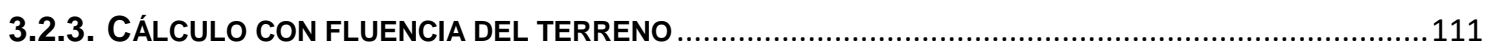

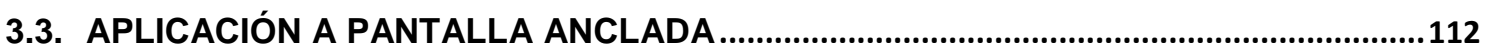

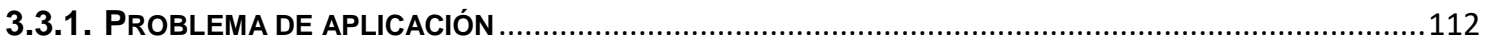

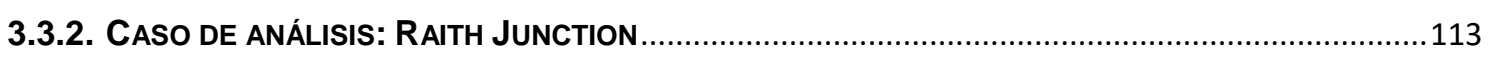

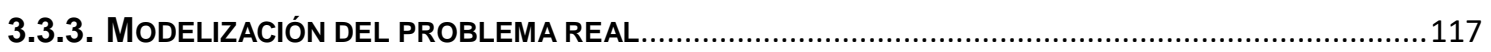

3.3.4. OBTENCIÓN DE LOS PARÁMETROS DEL MODELO DE FLUENCIA DEL TERRENO.........................119

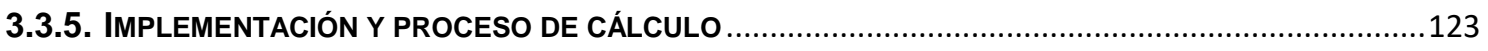

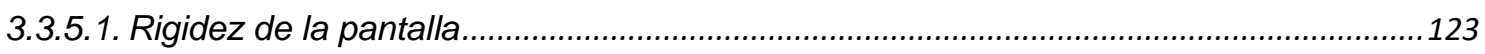

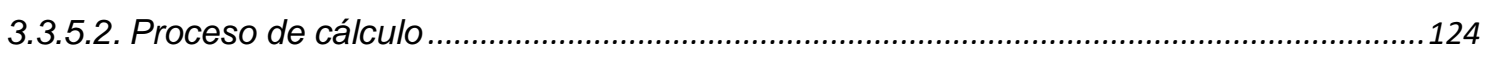

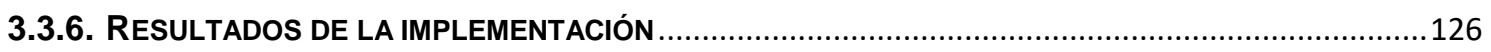

3.3.6.1. Influencia del área de reparto de la carga en cabeza del bulbo....................................126

3.3.6.2. Simulación del modelo teórico................................................................................... 128

CAPÍTULO 4: IMPLEMENTACIÓN NUMÉRICA ..................................................................... 131

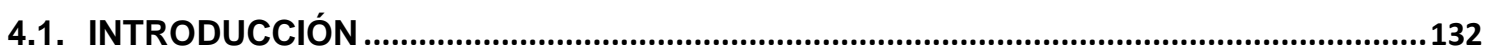


4.2. APLICACIÓN NUMÉRICA AL MODELO TEÓRICO ORIGINAL ......................................134

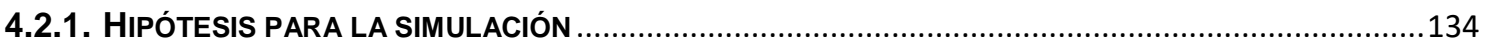

4.2.2. ConTRASTE CON EL MODELO ANALÍtICO DE LOSA ANCLADA....................................................137

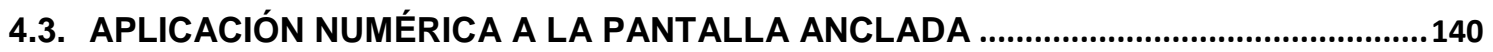

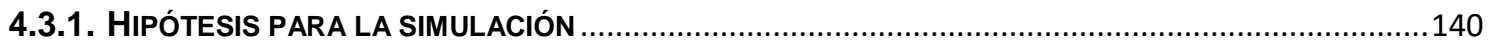

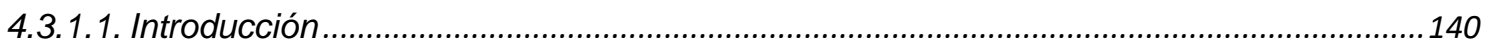

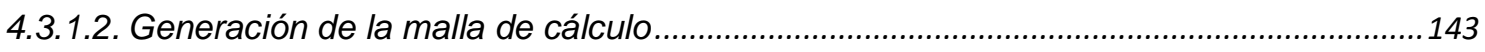

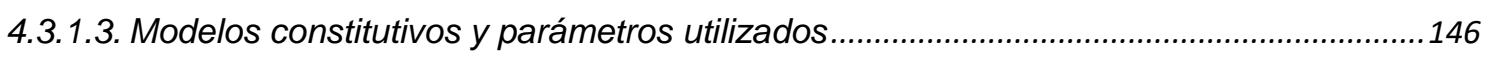

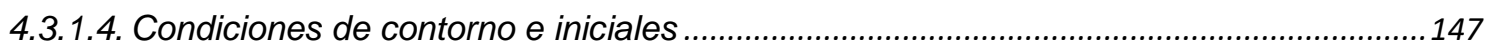

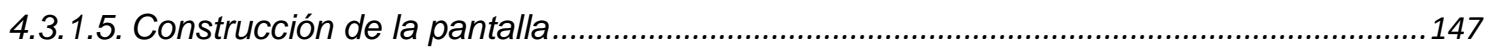

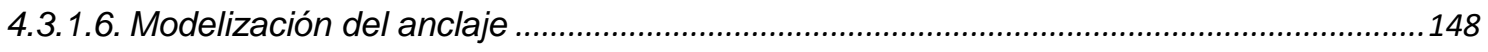

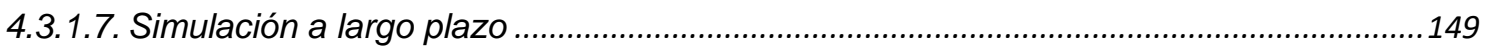

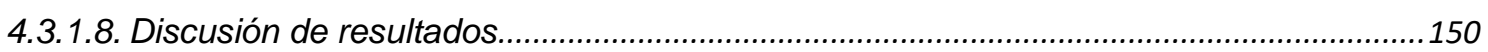

4.3.2. ContRASTE CON EL MOdELO ANALítiCo DE PANTALLA ANCLADA........................................158

CAPÍTULO 5: DESARROLLO DE UN NUEVO ENSAYO DE FLUENCIA EN LABORATORIO 161

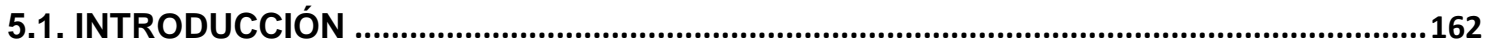

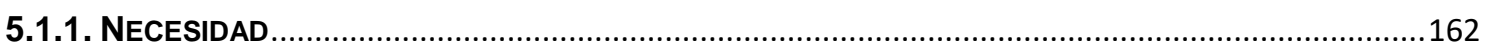

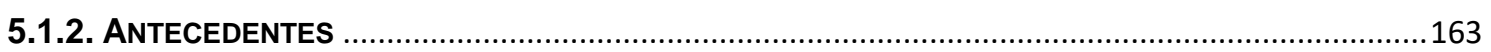

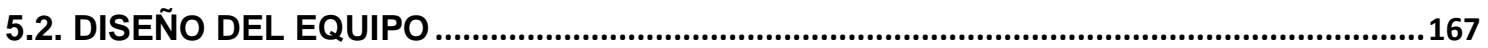

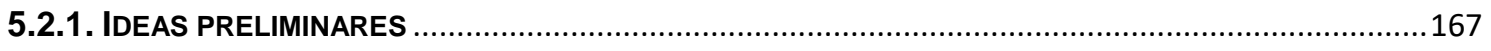

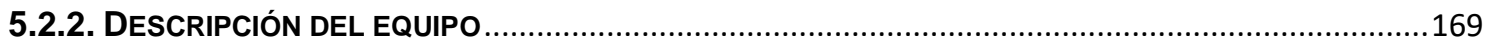

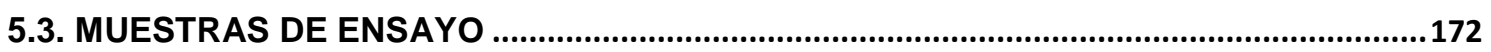

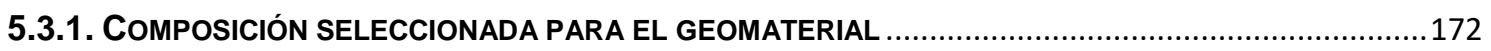

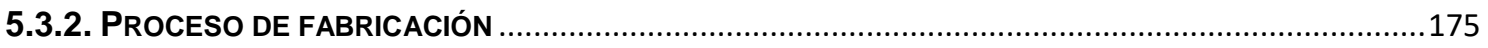

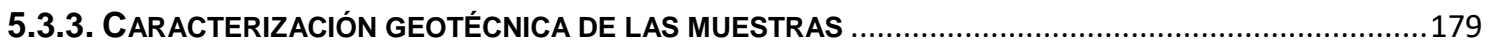

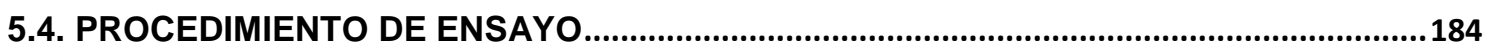

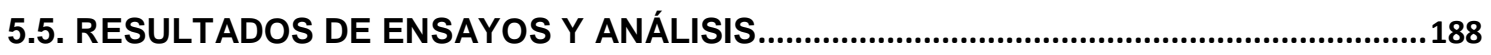

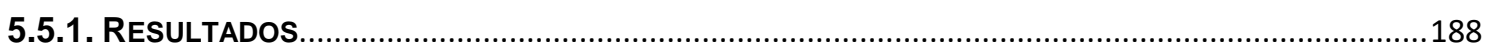

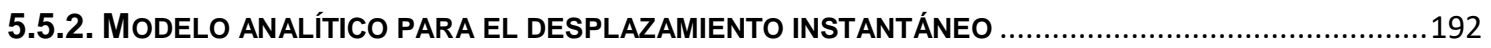

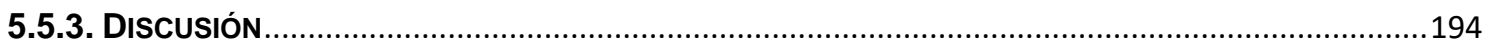

5.6. PRESCRIPCIONES RESPECTO A LA MONITORIZACIÓN.............................................195

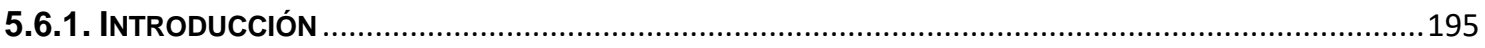

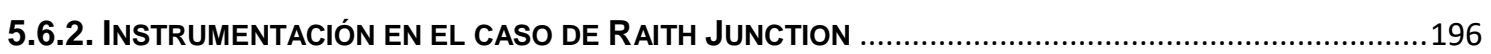

CAPÍTULO 6: CONCLUSIONES Y FUTURAS LÍNEAS DE INVESTIGACIÓN .........................203

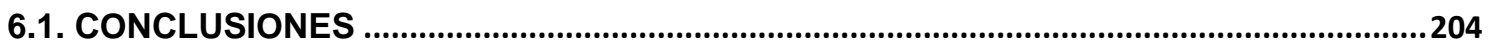




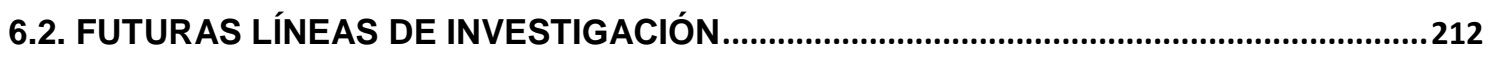

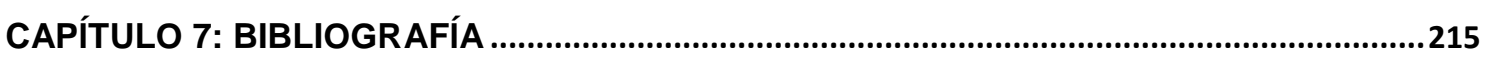

ANEXO I: PROGRAMACIÓN EN FLAC3D

I-A: MODELO NUMÉRICO DE LOSA ANCLADA EN TERRENO CON FLUENCIA

I-B: MODELO NUMÉRICO DE PANTALLA ANCLADA EN TERRENO CON FLUENCIA 


\section{ÍNDICE DE TABLAS}

Tabla 1. Máxima carga de trabajo para anclajes permanentes. (Ostermayer, 1975) ... 41

Tabla 2. Relación entre los modelos empíricos y las teorías ingenieriles para determinar la fluencia. (Liingaard et al., 2004).

Tabla 3. Comportamiento supuesto de los elementos del sistema de la Figura 47 ... 105

Tabla 4. Rigidez de cada componente del desplazamiento del modelo planteado..... 111

Tabla 5. Valores de los parámetros del modelo analítico utilizados en el caso real de aplicación.

Tabla 6. Valores de los parámetros del terreno utilizados en el caso real de aplicación.

Tabla 7. Influencia del área circular de reparto para el cálculo del desplazamiento del tirante producido por la carga en cabeza del bulbo

Tabla 8. Parámetros empleados para el contraste (losa anclada)

Tabla 9. Resultados de desplazamiento por componentes obtenidos con ambos modelos...

Tabla 10. Parámetros del terreno constantes en el análisis de sensibilidad. 151

Tabla 11. Propiedades geotécnicas empleadas en los cuatro casos de cálculo.

Tabla 12. Parámetros adicionales para la simulación de la pantalla en FLAC.

Tabla 13. Resistencia media al arrancamiento aparente para el contacto terreno/lechada a lo largo de la zona del bulbo (posterior a FHWA, 1999).

Tabla 14. Resultados de ensayos a compresión simple en muestras artificiales de prueba a diferentes edades.

Tabla 15. Resultados de los ensayos de caracterización geotécnica de las muestras. 


\section{ÍNDICE DE FIGURAS}

Figura 1. Problema de estudio. 2

Figura 2. Desplazamiento de un muro de contención en Kensal Green (Reino Unido) debido a la fluencia progresiva a largo plazo de la arcilla de Londres. (Skempton y Hutchinson, 1969)

Figura 3. Curva tensión - deformación completa del ensayo de compresión triaxial a corto plazo. (Modificada de Goodman; 1980)

Figura 4. (a) Curvas teóricas de fluencia; (b) Curva teórica de resistencia a largo plazo del suelo. (Vyalov, 1986).

Figura 5. Desarrollo de la deformación con el tiempo en un cuerpo. 1: flujo viscoso; 2: fluencia no atenuada; 3 : fluencia atenuada.

Figura 6. Curva teórica "Deformación - Tiempo" debido a la fluencia. (Lama y Vutukuri, 1978)

Figura 7. Representación esquemática de los cambios en la microestructura del suelo debidos a la fluencia. (a) estructura inicial; (b) estructura en la etapa I; (c) estructura en la etapa II; (d) estructura en la etapa III. 1 = microagregados de partículas de arcilla; 2 = cavidades y huecos; 3 = arcilla cementada; 4 = micro y macro-fracturas. Vyalov (1986)

Figura 8. Ensayos de fluencia a compresión simple con roca de sal de la misma muestra y con tres escalones de carga sucesivos (a temperatura del ambiente). a) Deformación - tiempo; b) velocidad de deformación - tiempo, $\boldsymbol{\varepsilon} \boldsymbol{s}$ velocidad en el estado estacionario; c) velocidad de deformación - deformación de uno de los ensayos. (Cristescu \& Hunsche, 1998)

Figura 9. Curvas de fluencia de una muestra de mármol. Los valores sobre la curva de $\varepsilon_{1}$ son las $\sigma_{1}$ en MPa, $\sigma_{3}=1.176 \mathrm{MPa}$. (Cristescu, 1993).....

Figura 10. Deformación por fluencia en función del tiempo, según ensayos en diferentes suelos arcillosos (1 PCF =0,016 g/ $\mathrm{cm} 3$ ). (Mitchell \& Soga, 2005). .............. 25

Figura 11. Curvas de fluencia del alabastro. Todos los especímenes fueron sumergidos en agua destilada y ensayados a la misma temperatura, pero con diferente tensión desviadora. (Griggs, 1940) 29

Figura 12. Ensayos triaxiales drenados de fluencia en arcillas de Londres fisuradas. (Bishop \& Lovenbury, 1969)

Figura 13. Curva de fluencia de un ensayo triaxial en una muestra de roca de sal realizado a $45{ }^{\circ} \mathrm{C}$ de temperatura y $15 \mathrm{MPa}$ de confinamiento. Sobre la curva se muestran los incrementos de tensión desviadora. Nótese la fluencia transitoria inversa en la etapa con reducción de la tensión. (Cristescu \& Hunsche; 1998)

Figura 14. Curvas "Tiempo - Deformación" de dos ensayos triaxiales de fluencia a 50ㄷ con variaciones escalonadas de la presión de confinamiento. La curva del ensayo (2) está ligeramente afectada debido a que el espécimen se impregnó con aceite mineral 
en el punto indicado con (+), el espécimen fue limpiado y el ensayo fue reiniciado. (Cristescu \& Hunsche, 1998) 32

Figura 15. Curvas tiempo - deformación de dos ensayos de fluencia en compresión simple. El espécimen (1) fue ensayado variando escalonadamente la humedad relativa del aire. El espécimen (2) fue ensayado en el mismo equipo, pero con la humedad relativa constante. (Cristescu \& Hunsche, 1998) 33

Figura 16. Descenso de la resistencia no confinada a largo plazo con el incremento de la humedad de muestras de argilitas del Cretácico Superior. (Houska, 1981) . 34

Figura 17. Curvas de fluencia de la formación "Osaka clay". Ensayos triaxiales no drenados a diferentes temperaturas. (Murayama, 1969) 35

Figura 18. Influencia de la temperatura en la tensión inicial y final en ensayos de relajación tensional en Osaka clay (compresión triaxial no drenada). (Murayama, 1969)

Figura 19. Ensayos de fluencia en compresión simple en roca de sal, con cuatro incrementos sucesivos de temperatura (BGR); a) deformación vs tiempo; b) velocidad de deformación vs tiempo, $\varepsilon s$ velocidad de fluencia secundaria. (Cristescu \& Hunsche, 1998)

Figura 20. Curvas desplazamiento - tiempo para distintas cargas en una arena uniforme. (Ostermayer, 1975)

Figura 21. Coeficientes de fluencia en relación con la capacidad de carga movilizada (resultados de 56 ensayos). (Ostermayer, 1975) 40

Figura 22. Evaluación de la tensión crítica de fluencia. (FHWA, 1999) 43

Figura 23: Curvas de fluencia deducidas según el modelo de Singh y Mitchell (1968). (a) Deformación-tiempo, (b) Deformación-logaritmo del tiempo. (Liingaard et al., 2004).

Figura 24: (a) Curvas tensión-deformación para diferentes velocidades de deformación. La relajación comienza en los puntos A, B y C. (b) Curvas de relajamiento tensional para varias velocidades de deformación antes de la relajación. (Lacerda \& Houston, 1973)

Figura 25: Nivel de tensión desviadora frente a logaritmo de tiempo durante el relajamiento tensional. La ecuación (10) se ha representado para diferentes valores del parámetro b. (Prevost, 1976) 54

Figura 26: Relación tensión-deformación-velocidad de deformación del strain rate approach: (a) Variación normalizada de la presión de preconsolidación con la velocidad de deformación, Ec. (11); (b) Relación entre la tensión efectiva normalizada y la deformación, Ec. (12); y (c) Curvas experimentales obtenidas a distintas velocidades de deformación. (Leroueil et al., 1985) 56

Figura 27. Curvas de deformación de varios cuerpos. (a) elástico; (b) elastoplástico; (c) elástico no lineal; (d) viscoso; (e) viscoplástico; (f) viscoso no lineal. (Vyalov, 1986)... 64 
Figura 28. Desarrollo de la deformación con el tiempo al aplicar una carga constante y una posterior descarga en: (a) cuerpo elástico ideal; (b) cuerpo viscoso ideal; (c) cuerpo viscoelástico ideal; (d) cuerpo viscoelastoplástico ideal. (Vyalov, 1986).....

Figura 29. Esquema simplificado de elementos mecánicos: (a) resorte o cuerpo elástico Hookeano; (b) amortiguador o cuerpo viscoso Newtoniano; (c) amortiguador de fricción seca o cuerpo plástico de Saint-Venant. (Liingaard et al., 2004).................. 66

Figura 30. Modelo de Maxwell.. .67

Figura 31. Comportamiento reológico (fluencia, recuperación de fluencia y relajación de la tensión) de un cuerpo según el modelo de Maxwell ................................................... 68

Figura 32. Modelo de Kelvin-Voigt... 69

Figura 33. Comportamiento reológico (fluencia, recuperación de fluencia y relajación de la tensión) de un cuerpo según el modelo de Kelvin-Voigt. ......................................... 71

Figura 34. Modelo de Zener o del sólido lineal estándar. Modelo H-K............................. 72

Figura 35. Comportamiento reológico (fluencia, recuperación de fluencia y relajación de la tensión) de un cuerpo según el modelo del sólido lineal estándar .......................... 74

Figura 36. Modelo de Burgers o de los cuatro elementos. (Kränkel et al, 2015) ............ 74

Figura 37. Comportamiento por fluencia y recuperación de la fluencia según el modelo

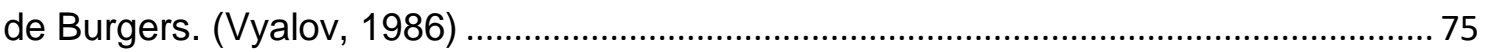

Figura 38. Modelo de Bingham. Cuerpo elasto-viscoplástico. (Liingaard et al., 2004). 76

Figura 39. Respuesta del modelo de Bingham ante (a) fluencia; (b) relajación; y (c) distintas velocidades de deformación constantes. (Liingaard et al., 2004)..................... 77

Figura 40. El estado de tensión $P$ es parte de la superficie dinámica de fluencia $f d$ y la sobretensión $F$ se define como la distancia entre $P$ y la superficie estática de fluencia $f s$. Además, el vector velocidad de deformación viscoplástica es perpendicular a la

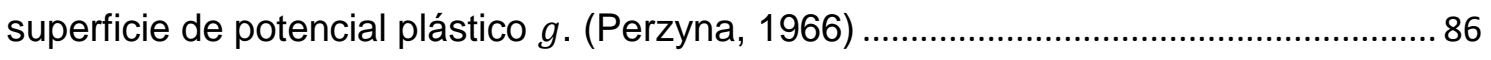

Figura 41. (a) Proceso de fluencia para un material sin endurecimiento, y (b) Proceso de fluencia para un material con endurecimiento. (Perzyna, 1966) ................................. 88

Figura 42. Trayectorias de carga y superficies de fluencia.(Liingaard et al., 2004)...... 90

Figura 43. Criterio completo de carga para un material elastoviscoplástico basado en el concepto de la teoría de la superficie de flujo no estacionaria. (Liingaard et al., 2004) 93

Figura 44. Proceso de fluencia según la teoría NSFS. (Liingaard et al., 2004) .............. 94

Figura 45. Barreras energéticas en la teoría "rate process"(Feda, 1989)

Figura 46. (a) Ensayos triaxiales drenados reales en Toyoura Sand (Murayama et al., 1984), (b) 14 simulaciones mediante el modelo MED de 1002 discos circulares (Kuhn y Mitchell, 1993). 98

Figura 47. Esquema mecánico del problema planteado. 104

Figura 48. Esquema de desplazamiento de los diferentes elementos del modelo de cálculo. 105 
Figura 49. Hipótesis para el asiento según Borowicka. 107

Figura 50. Esquema para la aplicación de las ecuaciones de Mindlin en superficie... 108 Figura 51. Esquema de la aplicación de ecuaciones de Mindlin en el bulbo y en el eje $r=0$. 109

Figura 52. Esquema mecánico del modelo reológico de Burgers. 111

Figura 53. Esquema del problema de aplicación. 113

Figura 54. Vista en planta y ubicación del tramo de autopista estudiado. (Google Earth, 2019)

Figura 55. Vista general de la obra seleccionada para la aplicación del modelo.

Figura 56. Mapa geotécnico del área estudiada. (Cortesía de Ferrovial Agromán y elaboración propia)

Figura 57. Fotografía de la pantalla de pilotes secantes real y esquemas del modelo de cálculo geotécnico de pantalla y del modelo matemático planteado.

Figura 58. Localización de la sección de aplicación del modelo.

Figura 59. Situación del perfil calculado y esquema para la utilización de los parámetros de la Tabla 5 .

Figura 60. Datos de deformación con el tiempo del ensayo de fluencia en campo y ajuste según la ecuación de Burgers.

Figura 61. Ensayo de corte directo de fluencia en argilita de Strahov inalterada. (Feda, 1992).

Figura 62. Datos de deformación con el tiempo del ensayo de fluencia en campo modificado a 20 años y ajuste según la ecuación de Burgers.

Figura 63. Esquema de cálculo del método del pie fijo o europeo y parámetros utilizados.

Figura 64. Esquema del proceso de pérdida de carga en el anclaje debido a la fluencia del terreno

Figura 65. Evolución de la fuerza en el anclaje (durante 1 año) según el modelo analítico con los parámetros de los casos 1 (trazo continuo) y 2 (trazo discontinuo). 128

Figura 66. Fuerza en el tirante al cabo de 20 años, geometría del modelo y parámetros.

Figura 67. Asiento uniforme de la losa circular y geometría del modelo..... 136

Figura 68. Desplazamiento instantáneo obtenido con ambos modelos. 139

Figura 69. Desplazamiento diferido obtenido con el modelo analítico y con FLAC 3D.

Figura 70. Sección geotécnica empleada para la puesta a punto del modelo. 141

Figura 71. Esquema de los diferentes parámetros que intervienen en una sección tipo para el modelo numérico. 
Figura 72. Vista del modelo de cálculo. Detalle de los pilotes........................................ 144

Figura 73. Vista del modelo de cálculo. Situación del anclaje...................................... 145

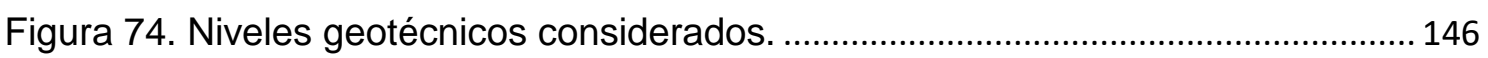

Figura 75. Desplazamientos horizontales (y) en el terreno tras la construcción........... 153

Figura 76. Desplazamientos en el terreno al cabo de 20 años (solo de fluencia)....... 154

Figura 77. Desplazamientos (a) horizontales y (b) verticales en el terreno................... 155

Figura 78. Desplazamientos horizontales en los pilotes................................................ 155

Figura 79. Evolución de desplazamientos en puntos de control en el fuste del pilote.156

Figura 80. Evolución de la tensión en el anclaje transcurridos 20 años para el Caso D.

Figura 81. Evolución de la deformación en el anclaje $(\mathrm{m} / \mathrm{m})$....................................... 157

Figura 82. Pérdida de carga obtenida con el modelo analítico y con FLAC 3D........... 160

Figura 83. Tipos de rotura frecuentes en anclajes en roca. .......................................... 168

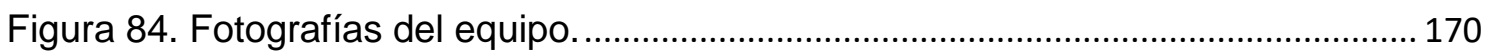

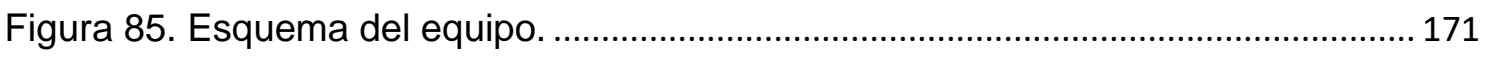

Figura 86. Aspecto de las muestras transcurridas $24 \mathrm{~h}$ de curado................................. 173

Figura 87. Ensayo edométrico de muestras de geomaterial con bentonita elegido.... 174

Figura 88. Algunos de los materiales empleados para la fabricación de las muestras.

Figura 89. Materiales auxiliares empleados para la fabricación de las muestras......... 176

Figura 90. Mezclado de las partes sólidas y proceso óptimo de mezclado..................... 176

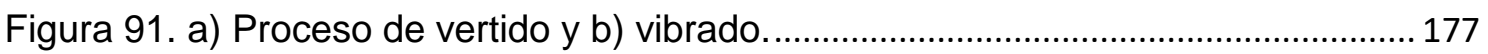

Figura 92. Aspecto final y nivelado de la muestra antes del proceso de curado. ......... 177

Figura 93. Aspecto final tras llenado y vibrado de los moldes para probetas. .............. 178

Figura 94. Aspecto final tras 72 horas de fraguado en cámara húmeda....................... 178

Figura 95. Extracción de testigos para caracterización del bloque 3 (Tabla 15)......... 179

Figura 96. Diagramas de valores geotécnicos representativos del geo-material fabricado: a) Resistencia a compresión simple; b) Velocidad de ondas p; c) Densidad

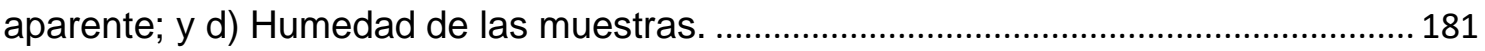

Figura 97. Aspecto interno y de la superficie de rotura de las muestras fabricadas... 182

Figura 98. Aspecto de las probetas tras roturas en compresión simple y en triaxial. . 182

Figura 99. Ejemplo de curvas de rotura obtenidas en ensayos RCS y TCU................ 183

Figura 100. Detalle de la plomada y de la capa para nivelación. .................................... 184

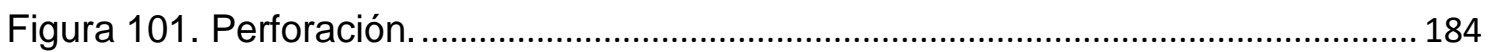




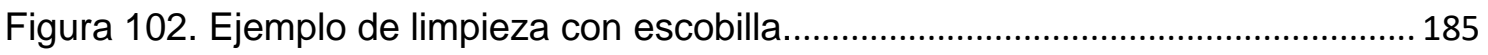

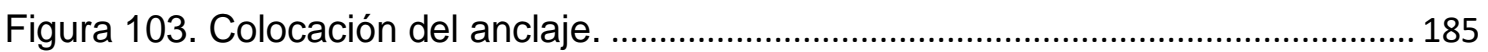

Figura 104. Detalle de mejora de la adherencia del material base con diferentes resinas 186

Figura 105. Detalle de comparadores y nivel de agua. 186

Figura 106. Ejemplo de ensayo de fluencia en funcionamiento. 187

Figura 107. Resultados de los tres ensayos de fluencia efectuados: a) Ensayo en Bloque 2; b) Ensayo en Bloque 4; c) Ensayo en Bloque 5; d) Deformación vertical en superficie (con fluencia); e) Deformación vertical instantánea en superficie................. 190

Figura 108. Ejemplos de tipos de roturas obtenidas. 191

Figura 109. Variación del desplazamiento instantáneo en función del módulo de corte.

Figura 110. Vista en planta del tramo de estudio y secciones instrumentadas. (Cortesía de Ferrovial Agromán y elaboración propia)

Figura 111. Fotografías de las células de carga en la obra. (Cortesía de FerrovialAgromán y elaboración propia)

Figura 112. Fotografías del la instalación y aspecto de los conductos de SAA instalados. (Solvver y Ferrovial-Agroman) 198

Figura 113. Valores de las células de carga en las secciones de estudio (Figura 110) en marzo de 2019.

Figura 114. Desplazamiento a lo largo de la longitud libre obtenido mediante SAA (sección W11, Figura 109). 200

Figura 115. Desplazamiento a lo largo de la longitud libre obtenido mediante SAA (sección E3, equivalente a la W4, Figura 110). 200

Figura 116. Curva de desplazamiento-tiempo de la sección W4 obtenida con el modelo analítico..... 201 


\subsection{INTRODUCCIÓN}

Existen multitud de situaciones en las obras de ingeniería civil en las que participa de manera activa el fenómeno de la fluencia en el terreno (p.e. consolidación secundaria en presas y vertederos o cualquier tipo de terraplén, convergencia o fluencia en túneles). Es decir, las deformaciones que ocurren al aplicar una carga efectiva constante al suelo o roca durante largos periodos de tiempo. Particularmente, resulta interesante el estudio del comportamiento de los anclajes permanentes en estructuras ancladas al terreno. Este tipo de elementos someten al entorno en que se encuentran instalados a un estado tensional prácticamente constante a largo plazo, durante la vida útil de la obra. Aparecen entonces, en el terreno de alrededor del bulbo, tensiones (Figura 1), que irán provocando desplazamientos con el tiempo cuya magnitud variará principalmente en función de la carga aplicada por el anclaje y del tipo de suelo.

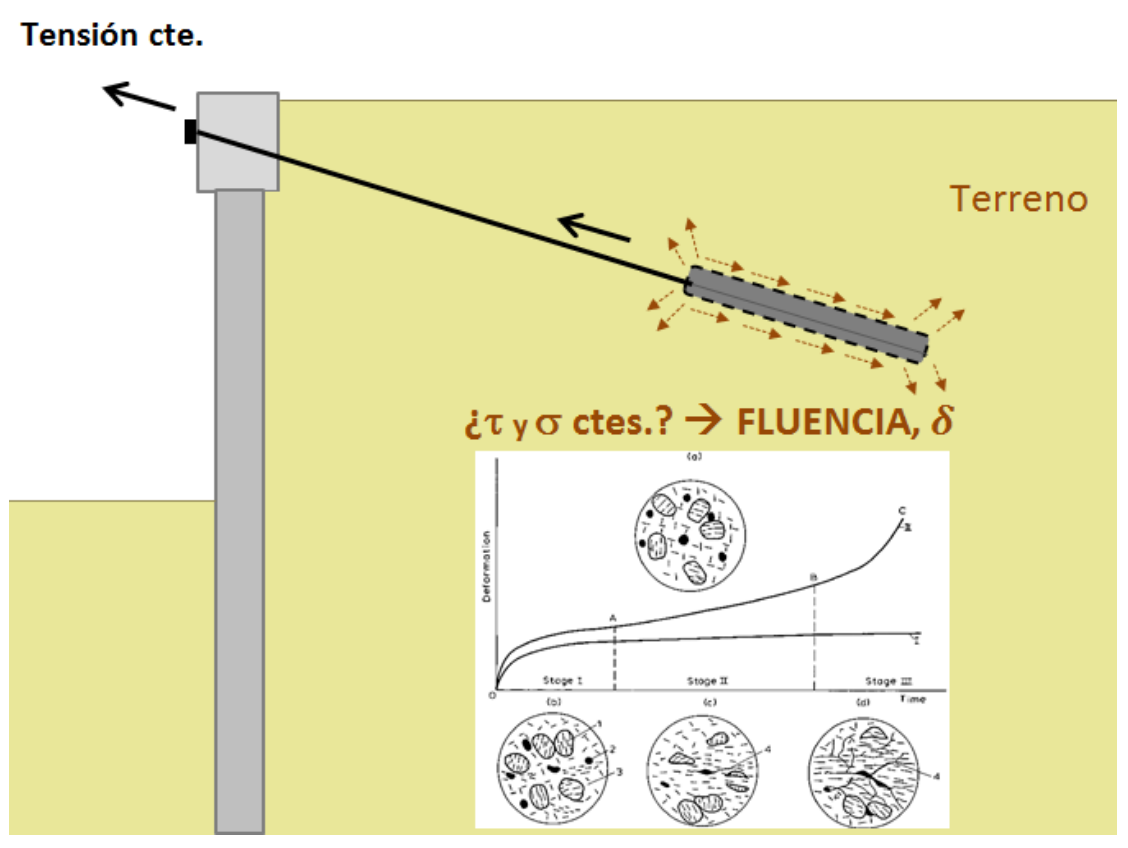

Figura 1. Problema de estudio.

Cuando el anclaje comienza a trabajar, la parte de la cuña activa del terreno que sostiene la pantalla también tendrá cierto desplazamiento con el tiempo. Esta combinación de movimientos es la causante de las pérdidas de carga en el anclaje con el tiempo. 
En este tipo de obras se introduce un coeficiente de seguridad respecto a la fluencia del terreno por cierto desconocimiento del fenómeno, o directamente, no se construye en algunos terrenos por restricciones de actividad, plasticidad o contenido en materia orgánica. Además, en las normativas existentes se indican una serie de recomendaciones para el control a largo plazo, estableciendo un mínimo de células de carga a instalar y controlar periódicamente, según las dimensiones de la obra. Sin embargo, no existe un conocimiento real sobre el problema de fluencia en estas condiciones de contorno y sobre la evolución de la carga con el tiempo, siendo este el motivo principal de la tesis.

\subsection{OBJETIVO}

El principal objetivo de la tesis es introducir un nuevo modelo analítico de interacción suelo-estructura para terrenos con fluencia que permite obtener la evolución de la carga en un anclaje con el tiempo, así como las deformaciones por fluencia asociadas, tanto del terreno como de la estructura anclada. Este objetivo se alcanza, para las condiciones de contorno definidas en el problema, mediante la combinación de las ecuaciones de movimiento de los distintos componentes del sistema (elemento estructural, anclaje y terreno), junto con un modelo constitutivo de fluencia del suelo o roca. Por un lado, se estudia el problema de contorno específico y, por otro, se incorpora una variación de los parámetros del suelo convirtiéndolos en parámetros dependientes del tiempo.

El modelo se implementa con datos reales de ensayos de campo y laboratorio, que permiten obtener los parámetros del terreno correspondientes al modelo reológico elegido. A este respecto se desarrolla un nuevo equipo de fluencia en laboratorio a gran escala, con la finalidad de determinar adecuadamente los parámetros de fluencia del suelo o roca.

Para demostrar su aplicabilidad en casos reales el modelo de interacción desarrollado se resuelve para un caso concreto de una pantalla anclada, que además cuenta con monitorización en tiempo real. 
Por último, el modelo desarrollado matemáticamente se contrasta con un modelo numérico, con ley constitutiva semejante, implementado en un programa de cálculo en diferencias finitas.

\subsection{ESTRUCTURA DE LA TESIS}

A continuación, se describen de manera somera las fases que se han seguido con el fin de alcanzar los objetivos mencionados:

- Elaboración de un estado del conocimiento sobre modelos constitutivos dependientes del tiempo que puedan ser aplicables al sistema terreno-anclaje (capítulo 2). La recopilación bibliográfica está enfocada, más concretamente, hacia la búsqueda de modelos de comportamiento deformación-tiempo (modelos de fluencia) empleados en mecánica de suelos y rocas.

- Desarrollo de un nuevo modelo analítico que gobierne el comportamiento de un sistema compuesto por un elemento estructural anclado al terreno (capítulo 3). Se tienen en cuenta unas condiciones de contorno específicas y se incorpora uno de los modelos recopilados en el estado del conocimiento para simular la respuesta del suelo o roca. De este modo se resuelve el principal objetivo de la tesis que es conocer la evolución dependiente del tiempo de un sistema de estas características, de tal manera que pueda ser aplicable en casos reales.

- Aplicación del modelo analítico a un caso real de estructura de contención, en concreto, una pantalla anclada a un suelo con esperables problemas de fluencia (capítulo 3).

- Simulación numérica en diferencias finitas del modelo teórico original, estableciendo las mismas condiciones de contorno y ley constitutiva, y contraste de la solución analítica y numérica de un caso particular (capítulo 4). De igual modo, simulación numérica en diferencias finitas de un modelo general para pantallas ancladas en terrenos con 
fluencia, y resolución del caso real de pantalla anclada mediante el modelo numérico y contraste con su solución analítica (capítulo 4).

- Desarrollo de un nuevo ensayo de laboratorio de fluencia a gran escala, gracias al cual se obtiene directamente un modelo de comportamiento deformación-tiempo, en este caso el de una roca artificial fabricada específicamente para esta investigación (capítulo $5)$. 


\section{CAPÍTULO 2: ESTADO DEL CONOCIMIENTO. CARACTERIZACIÓN DE LA FLUENCIA DEL TERRENO}




\subsection{INTRODUCCIÓN}

Una de las principales incógnitas asociadas al cálculo y definición de taludes y estructuras de contención se encuentra relacionada con el comportamiento de los anclajes a lo largo de toda su vida útil. Los fenómenos de fluencia (presentes en mayor o menor medida en cualquier tipo de terreno) asociados, por un lado, al macizo rocoso/suelo y por otro, a la inyección y los elementos que materializan dicho anclaje en el propio terreno, son los responsables de una disminución de las propiedades resistentes del anclaje en el tiempo.

La reología de los materiales del terreno, provoca una evolución de las características geomecánicas del medio que puede producir un incremento de las deformaciones con el tiempo de las masas del trasdós de una estructura de contención y consecuentemente una menor capacidad resistente. Dichas deformaciones pueden superar los límites admisibles, por lo que deben ser controladas durante toda la vida útil de la obra.

Para ilustrar la problemática mencionada y explicar el motivo principal del estudio se muestra la Figura 2, debida a Skempton y Hutchinson (1969). En 1912 se construyó un muro de contención en Kensal Green (Reino Unido) excavado en las arcillas de Londres, rígidas y fisuradas, $\left(\mathrm{w}=33 \%, \mathrm{~W}_{\mathrm{L}}=83 \%\right.$, $\mathrm{W}_{\mathrm{P}}=30 \%$ ), que falló en dos ocasiones, en 1929 y en 1941. El muro tiene una altura libre aproximada de $4 \mathrm{~m}$. La velocidad de desplazamiento de la ladera, registrada a lo largo de unos pocos años después de la reconstrucción del muro en 1929, era de $0.6 \mathrm{~cm} /$ año. Eventualmente, la velocidad de desplazamiento se incrementó gradualmente $y$, justo antes de la segunda rotura, el desplazamiento total registrado fue de $46 \mathrm{~cm}$.

Nótese que el fallo del muro en Kensal Green es debido, probablemente, al deslizamiento a lo largo de un plano formado en las arcillas marrones de Londres. Sin embargo, en el planteamiento de esta tesis el fallo o rotura vendría dado por la superación de los límites admisibles de deformación y la pérdida excesiva de la resistencia de los anclajes permanentes instalados. Es decir, la rotura en este caso podría ocurrir por un movimiento conjunto de toda 
la masa del terreno. Los desplazamientos en el contacto anclaje-terreno, debidos principalmente al fenómeno de la fluencia del terreno, pueden provocar la pérdida progresiva de la tensión de diseño en los anclajes, hecho que puede llevar a la rotura de la pantalla, si no se realizan los retesados oportunos, a lo largo de la vida útil de la obra.

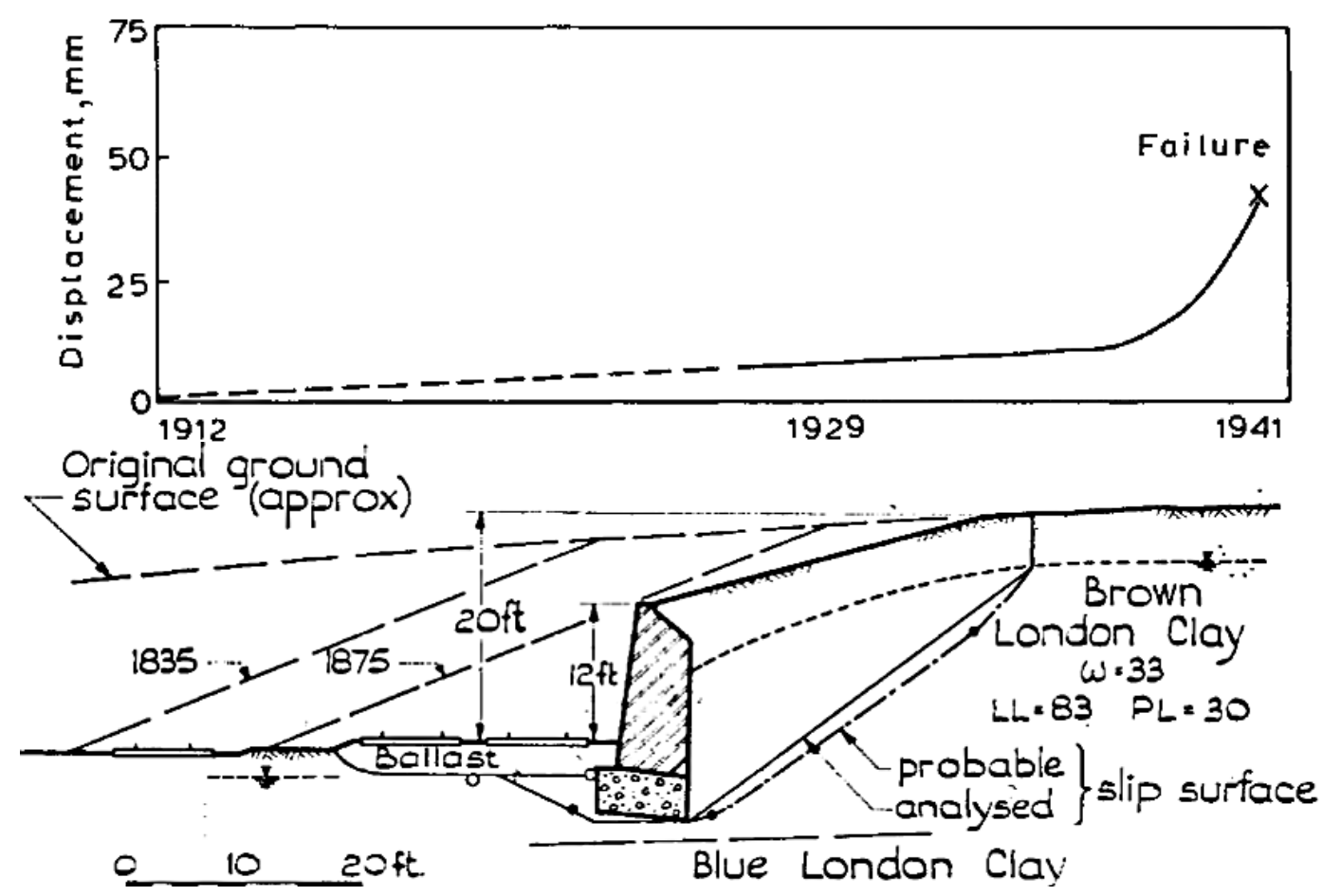

Figura 2. Desplazamiento de un muro de contención en Kensal Green (Reino Unido) debido a la fluencia progresiva a largo plazo de la arcilla de Londres. (Skempton y Hutchinson, 1969)

Skempton (1964) trata este caso de rotura con el tiempo como un ejemplo de deslizamiento a largo plazo en arcillas, en el cual la resistencia media de la arcilla se va reduciendo y acercando al valor de la tensión de corte existente, con mayores movimientos asociados, hasta que se alcanza su resistencia residual. Más tarde, Feda (1992), incorpora este mismo incidente como un ejemplo de fallo por fluencia progresiva del terreno.

La implementación de un adecuado modelo reológico para el estudio de las pantallas ancladas es uno de los principales objetivos de la tesis y debe comenzar por el estudio bibliográfico y documentado de los trabajos previos relacionados con el estudio del comportamiento reológico de suelos y rocas. $\mathrm{A}$ 
partir de dichos análisis previos, se puede establecer un estado del conocimiento que permite identificar los factores y variables que influyen significativamente en el fenómeno de estudio. Dichos factores permiten definir las variables del modelo constitutivo que se pretende emplear.

De este modo, la estructura del capítulo 2 queda dividida de la siguiente manera:

- Una primera parte (apartado 2.2.) sobre conceptos generales del fenómeno objeto de estudio: la fluencia del terreno. En este apartado se define el concepto de fluencia (2.2.1.) y de resistencia a largo plazo (2.2.2.), se describen las distintas etapas de deformación que aparecen durante el fenómeno (2.2.3.), se destacan los factores que influyen en su evolución (2.2.4.), y por último, se incluyen algunas ideas sobre fluencia en anclajes al terreno (2.2.5.).

- Una segunda parte (apartado 2.3.) en la cual se lleva a cabo una recopilación de los modelos actuales de comportamiento dependiente del tiempo que pueden ser aplicables al sistema anclaje-terreno. Se ha supuesto que la mayor parte de las deformaciones se deben al terreno, aunque también existe una ínfima proporción de fluencia y relajación del acero del tirante de los anclajes (su pérdida de tensión se produce en los primeros días de puesta en carga). Así, la búsqueda bibliográfica se ha centrado en la reología, concretamente en el fenómeno de fluencia de suelos y rocas, con el objetivo de estudiar cómo se modelizan las deformaciones a largo plazo producidas en el terreno, más que tratar la pérdida de resistencia en este mismo.

\subsection{COMPRENSIÓN DEL FENÓMENO DE FLUENCIA}

\subsubsection{Definición}

El concepto de reología fue originalmente planteado en abril de 1929, en el Tercer Simposio sobre Plasticidad celebrado en Columbus (Ohio), tras una propuesta de E.C. Bingham y M. Reiner. Estos autores sugirieron la definición de la reología como el estudio de la deformación y el fluir de la materia en 
términos de tensión, deformación, temperatura y tiempo. De este modo se abarcaba la descripción del comportamiento fluyente de todo tipo de materiales.

Con el objetivo de centrarse únicamente en el estudio de la fluencia y para diferenciar esta del término de reología, se establecen las siguientes distinciones. La reología se refiere al comportamiento dependiente del tiempo de los materiales (fluencia, relajamiento tensional o relajación, resistencia a largo plazo, etc.), mientras que el concepto de fluencia atiende únicamente a la evolución de la deformación con el tiempo.

Los procesos químicos, geomicrobiológicos y mecánicos dependientes del tiempo pueden resultar en cambios composicionales y estructurales que generan reblandecimiento, rigidización, pérdida de la resistencia, aumento de la resistencia o alteración de las propiedades conductivas. La fluencia y la relajación son dos consecuencias del mismo fenómeno, los cambios dependientes del tiempo en la estructura del suelo. La velocidad y la magnitud de las deformaciones dependientes del tiempo están determinadas por esos cambios estructurales (Mitchell y Soga, 2005).

Feda (1992) describe más rigurosamente la fluencia como el desarrollo o progreso de la deformación con el tiempo bajo carga efectiva constante. Es decir, excluye procesos como la consolidación primaria (durante la cual se producen deformaciones o asientos con el tiempo según la presión de agua disminuye pasando a ser adoptada por la tensión efectiva del suelo, bajo carga total constante) de los comportamientos asociados a la fluencia, como la consolidación secundaria (proceso de deformación cuando el suelo se encuentra en condiciones drenadas y sometido a tensión efectiva constante). Sin embargo, durante la consolidación primaria, se pueden llegar a producir deformaciones viscosas del esqueleto del suelo.

En una definición muy general del concepto de fluencia (Feda, 1992) se acepta que cualquier material sujeto a una carga constante en el tiempo, se deformará, y la magnitud de esa deformación difiere en función de la resistencia de la estructura del material. 
En el campo de la mecánica de rocas, la fluencia es la deformación irreversible en el tiempo, sin fracturación, y que es observada en la mayoría de las rocas blandas como la sal y el carbón y de algún modo, en mayor o menor medida, en todos los tipos de rocas (Cristescu \& Hunsche, 1998).

La fluencia de los suelos y de las rocas se expresa normalmente en términos de la velocidad de deformación $\dot{\varepsilon}$ a condiciones constantes de tensión desviadora, temperatura y humedad relativa alrededor del suelo o roca, a partir del tiempo transcurrido desde la aplicación de la carga inicial que produce la fluencia (Cristescu \& Hunsche, 1998).

\subsubsection{Resistencia a largo plazo}

La resistencia a largo plazo es la tensión máxima que puede soportar la roca (o el suelo) sin que se produzca su rotura, independientemente del tiempo que transcurra soportando esta tensión (Ladanyi, 1993).

De acuerdo con Lama y Vutukuri (1978) esta resistencia ha sido denominada de diferentes formas, por diversos autores, como "resistencia residual", "tensión segura en el tiempo", "resistencia a largo plazo" y "resistencia con carga sostenida".

En la Figura 3 de Goodman (1980) se ha representado la curva tensión - deformación de un ensayo de compresión triaxial incluyendo a la fase de post pico, para un proceso de carga rápida o lenta. De acuerdo con este autor, se pueden distinguir dos niveles críticos en la tensión de compresión, indicados por los puntos $\mathrm{T}$ y $\mathrm{U}$ de esta figura.

Para una tensión inferior a la marcada por el punto $\mathrm{T}$ el comportamiento de la roca es elástico e independiente del tiempo que se mantenga la carga. Sin embargo, para tensiones comprendidas entre los niveles marcados por los puntos $\mathrm{T}$ y $U$ se produce de forma inmediata una deformación, definida por la curva de la figura para ese nivel de tensión, pero si se mantiene la tensión durante un tiempo prolongado, la deformación 
aumenta constantemente, tendiendo a un valor final situado sobre la recta definida por los puntos $\mathrm{T}$ y $U$, siguiendo la trayectoria EF.

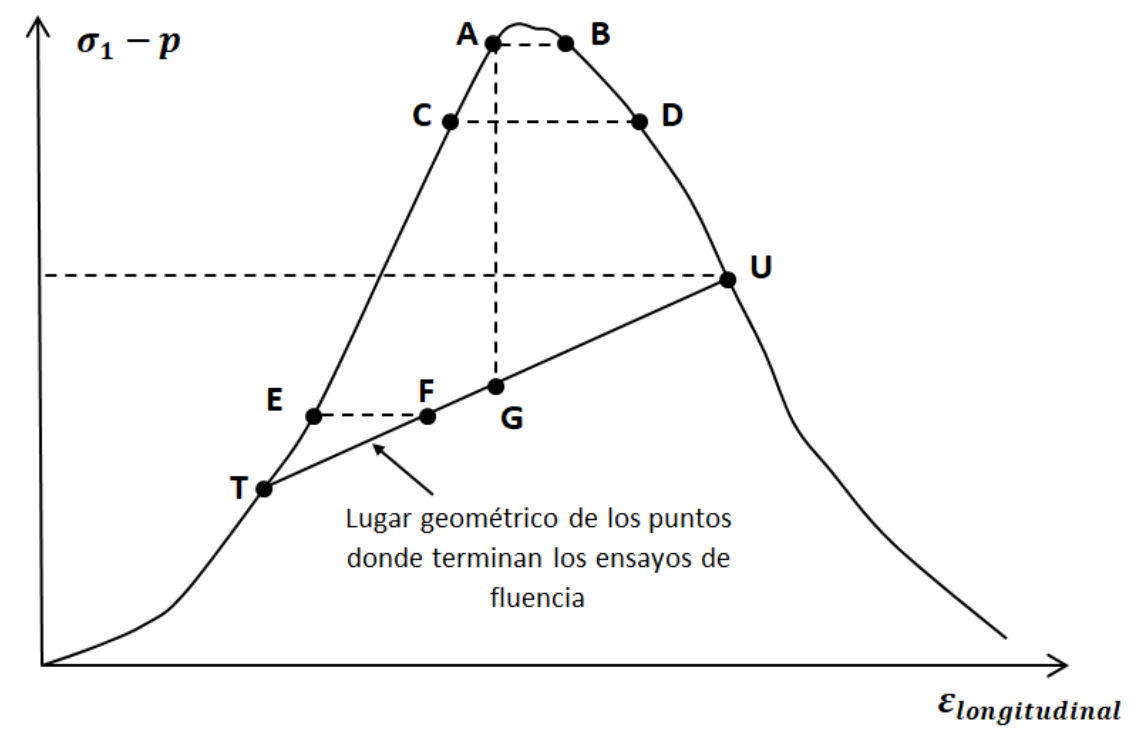

Figura 3. Curva tensión - deformación completa del ensayo de compresión triaxial a corto plazo. (Modificada de Goodman; 1980)

Si la tensión es superior al nivel marcado por el punto $U$ y se mantiene un tiempo suficiente la deformación aumenta constantemente hasta alcanzar la zona de postpico de la curva, momento en el que se producirá la rotura de la roca o suelo (trayectoria $C D$ y $A B$ de la figura). Es decir, para estos niveles de tensión se alcanza la rotura para una carga inferior a la que produce la rotura a corto plazo, si dicha carga se mantiene el tiempo suficiente. Por tanto, en las rocas que presentan fluencia se puede hablar de una resistencia a largo plazo (la tensión marcada por el punto $U$ en la figura) distinta de la que se obtiene en un ensayo rápido.

La definición de la resistencia a largo plazo de los suelos es similar a la introducida anteriormente para rocas, en el sentido en que la rotura de un suelo bajo carga constante se produce a una tensión de una magnitud menor que cuando la rotura se produce en condiciones de carga rápida o a corto plazo. Cuanto menor es la tensión aplicada, mayor es el tiempo necesario para alcanzar la rotura por fluencia. 
La Figura 4 muestra que la disminución de la tensión necesaria para alcanzar la rotura por fluencia va asociada al aumento del tiempo transcurrido hasta la rotura, lo que resulta una clara evidencia de la degradación de la resistencia del suelo (relajación tensional del suelo). En esta Figura se deben distinguir varios valores de la resistencia:

a) $\tau_{0}$, resistencia instantánea (o a corto plazo) supuesta,

b) $\tau(t)$, resistencia a largo plazo; definida por la tensión que produce la rotura del material a un determinado intervalo de tiempo, ese valor está representado por la posición actual en la curva de resistencia a largo plazo del suelo (Figura 4 b, donde se representan los valores decrecientes de $\tau$, desde el mayor, $\tau_{1}$, al mayor, $\tau_{7}$ ),

c) $\tau_{\infty}$, resistencia a largo plazo última, corresponde a una tensión a la cual, y por debajo de la cual, la deformación posee naturaleza atenuada y no tiene lugar la rotura bajo prácticamente ningún periodo de tiempo observable de carga aplicada.
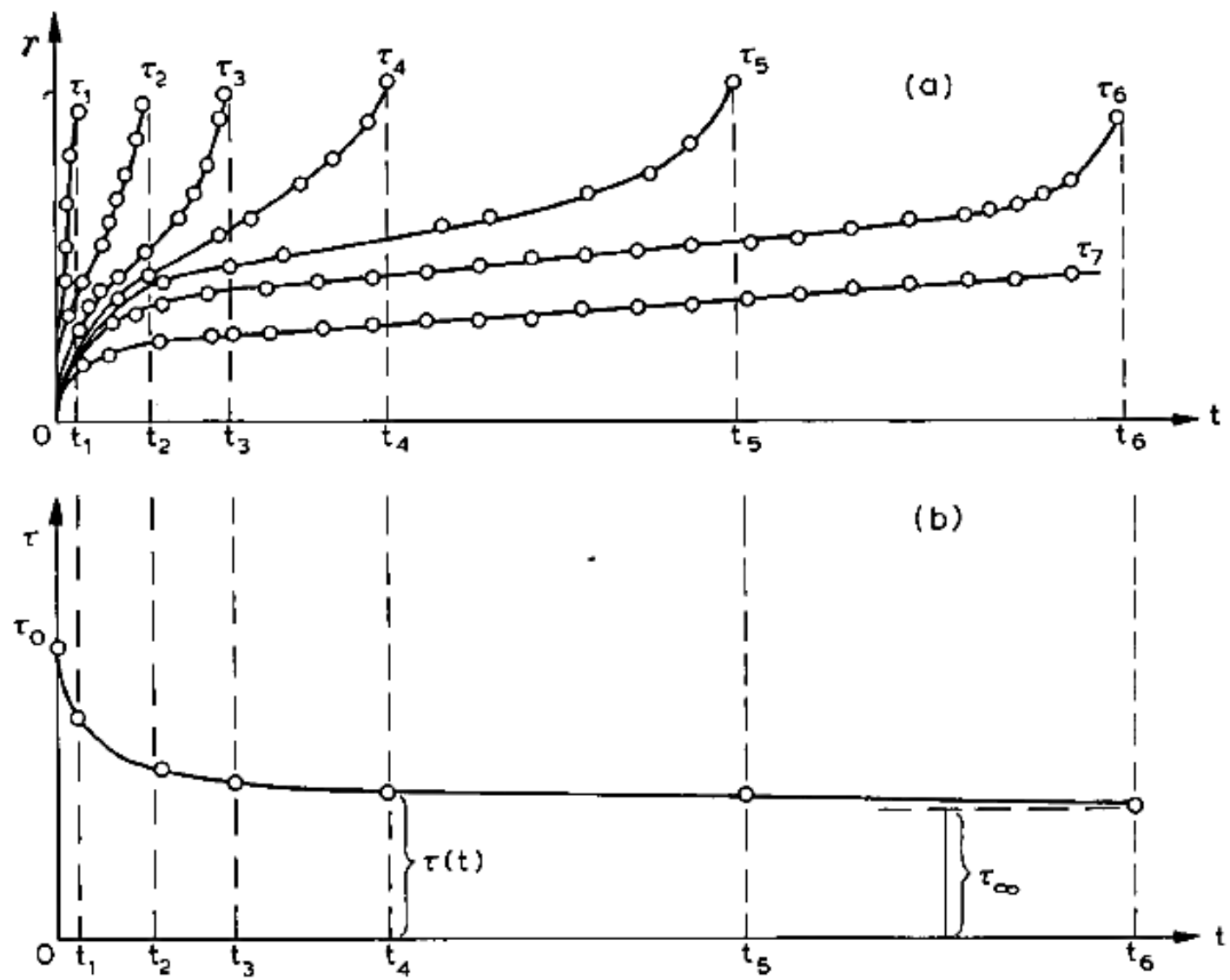

Figura 4. (a) Curvas teóricas de fluencia; (b) Curva teórica de resistencia a largo plazo del suelo. (Vyalov, 1986) 
En algunos terrenos la fluencia no atenuada es inducida por cualquier tensión diferente de cero. Para dichos terrenos, $\tau_{\infty}=0$, y la curva de resistencia a largo plazo se aproxima de manera asintótica al eje de abscisas.

\subsubsection{Etapas de la deformación por fluencia}

En la teoría de la fluencia, el flujo plástico es comprendido como un flujo viscoso inducido por una carga que excede cierto límite (el conocido límite de Bingham de plasticidad) y que frecuentemente se denomina como flujo viscoplástico. En la curva 1 de la Figura 5 se aprecia que el flujo viscoso ocurre a una velocidad de deformación constante. En este sentido el término "flujo", aplicado a sólidos, denota un cambio en la forma de manera incesante y no confinada. Conviene destacar que un cambio en la forma significa por lo general un cambio de deformación de corte y, por lo tanto, el flujo se puede expresar como un proceso de deformación de corte en el tiempo a velocidad constante.

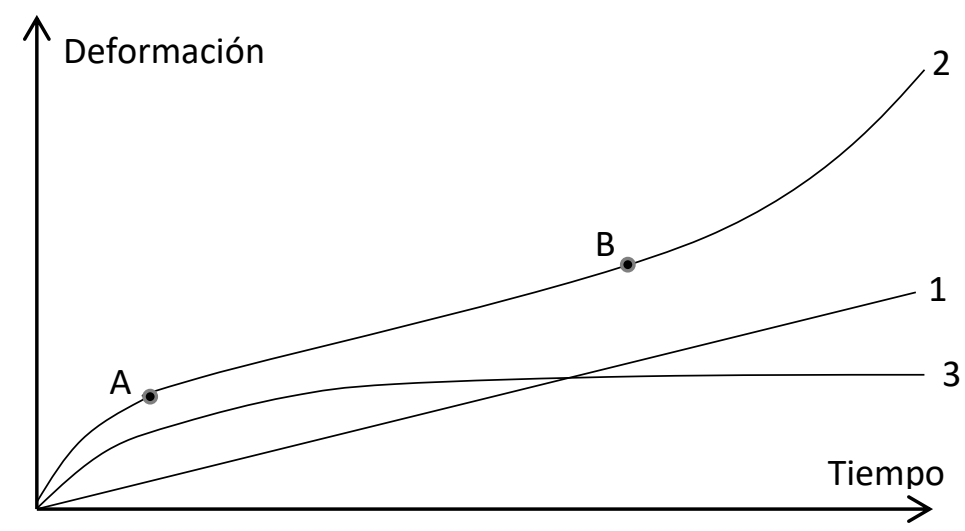

Figura 5. Desarrollo de la deformación con el tiempo en un cuerpo. 1: flujo viscoso; 2 : fluencia no atenuada; 3 : fluencia atenuada.

Vyalov (1986) diferencia dos tipos de fluencia; la fluencia atenuada (curva 3 de la Figura 5), que presenta una velocidad de deformación decreciente; y la fluencia no atenuada (curva 2 de la Figura 5), en la cual se diferencian tres etapas de fluencia con velocidades de deformación decreciente, constante, y creciente, con el avance del tiempo. El segmento $A B$, 
el cual corresponde a la fluencia estacionaria, o de velocidad de deformación constante, se puede asemejar a una etapa en la cual el cuerpo se comporta como un flujo de naturaleza viscoplástica, debido a su parecido con la curva 1.

Kuhn y Mitchell (1993), centrando su estudio en suelos, mencionan que para tensiones de fluencia lo suficientemente elevadas como para llevar al material a la rotura (a carga constante), la deformación por fluencia ocurre en las tres etapas principales de la fluencia no atenuada (Figura 6): primaria o transitoria, durante la cual la velocidad de deformación decrece; secundaria 0 estacionaria, cuando la velocidad de deformación se vuelve constante y; terciaria o acelerada, en la que finalmente la velocidad de deformación aumenta, pudiendo alcanzarse la rotura por fluencia del material. Lo mismo ocurre en el estudio experimental aplicado a rocas, según Lama y Vutukuri (1978).

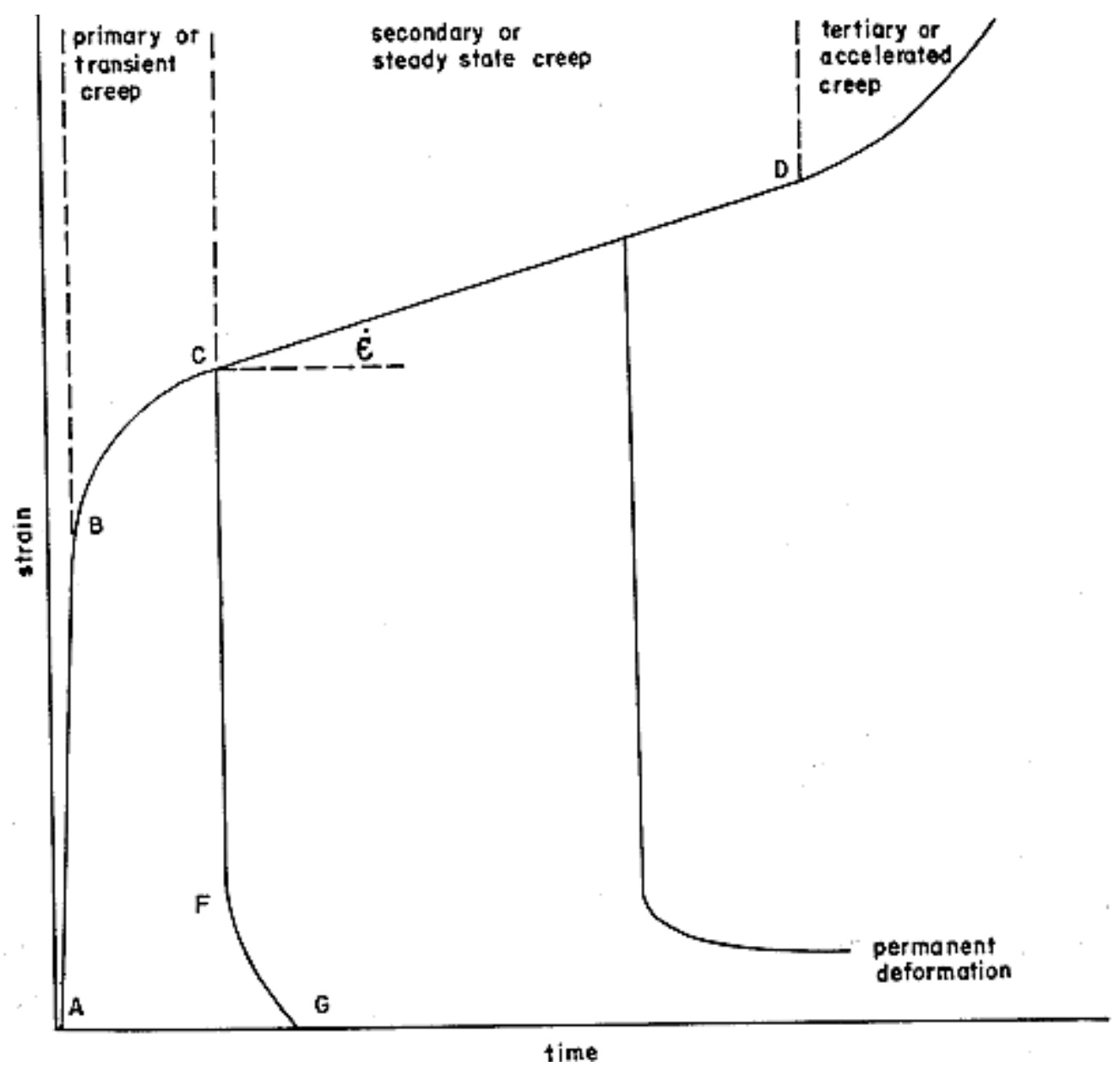

Figura 6. Curva teórica "Deformación - Tiempo" debido a la fluencia. (Lama y Vutukuri, 1978) 
En el núcleo de la deformación por fluencia de un suelo, existen dos fenómenos mutuamente excluyentes, el endurecimiento y el reblandecimiento del suelo (Vyalov, 1986). Si predomina el primer fenómeno, la deformación se detiene, y no se produce la rotura. En cambio, si prevalece el reblandecimiento se induce en el suelo una fluencia no atenuada, que terminará en la rotura de este.

El fenómeno de endurecimiento y reblandecimiento del suelo resulta de los cambios estructurales que son causados por la carga aplicada. Estos cambios se pueden representar gráficamente mediante un diagrama como el presentado en la Figura 7. Cuando se aplica una carga externa a un suelo, las concentraciones de tensiones producen deformaciones y la rotura de algunos enlaces. La rotura ocurre comúnmente en los puntos más débiles de la estructura, y como resultado, las partículas con enlaces dañados tienden a desplazarse a otras posiciones más estables.

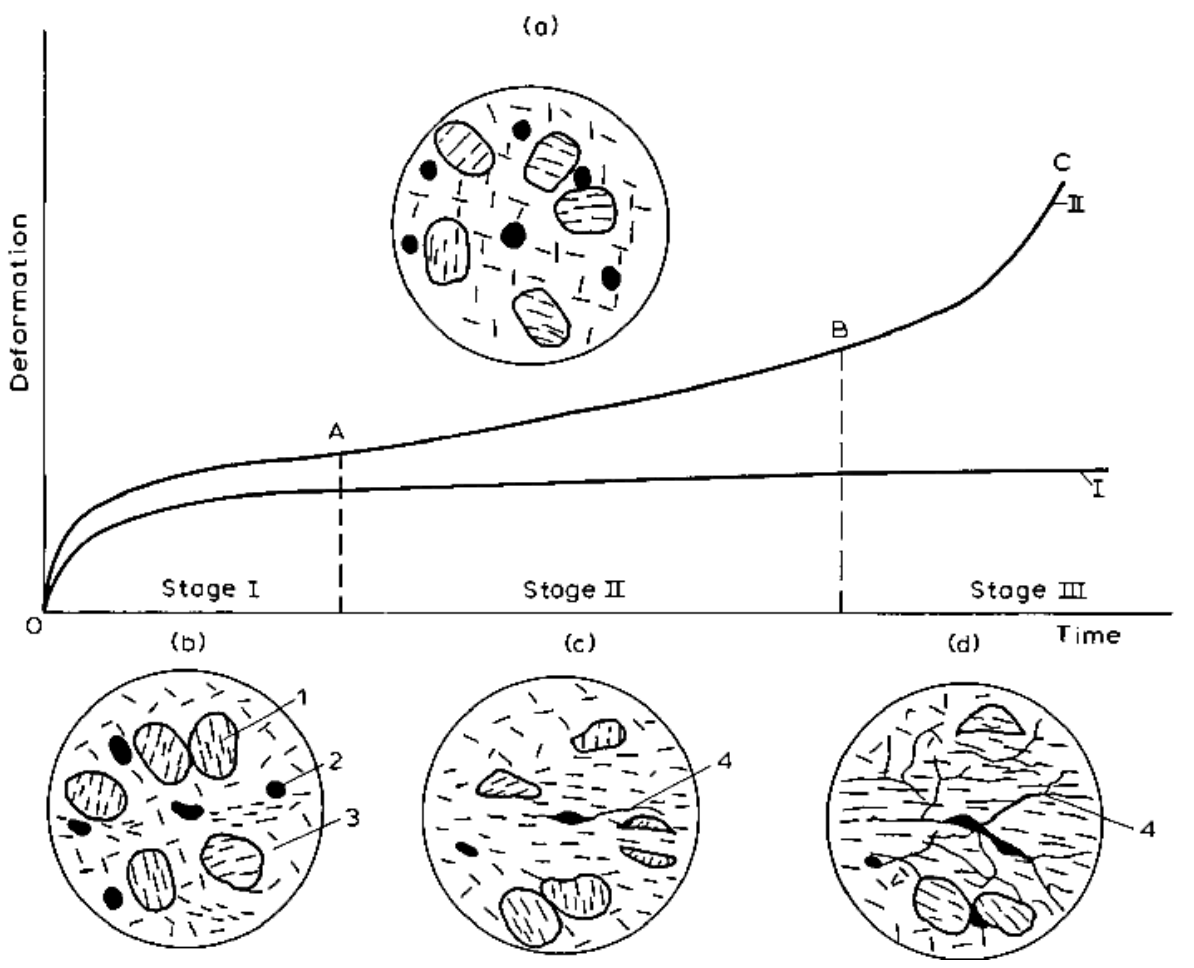

Figura 7. Representación esquemática de los cambios en la microestructura del suelo debidos a la fluencia. (a) estructura inicial; (b) estructura en la etapa I; (c) estructura en la etapa II; (d) estructura en la etapa III. 1 = microagregados de partículas de arcilla; 2 = cavidades y huecos; 3 = arcilla cementada; 4 = micro y macro-fracturas. Vyalov 
En una etapa temprana, las partículas desplazadas generan una disposición más compacta, y simultáneamente se forman nuevos enlaces, mientras que los defectos estructurales (microfracturas, fisuras, huecos, cavidades, etcétera) se contraen (encogen) y decrecen en número. La disminución de los defectos viene acompañada de la reducción del volumen del suelo, es decir, de la compactación de este último. Consecuentemente, el suelo endurece. Todo esto puede ocurrir, incluso, bajo condiciones de cizallamiento puro; pero la aplicación de una presión de confinamiento suele obviamente incrementar la intensidad del proceso.

La reducción de los defectos estructurales puede venir acompañada de la creación de nuevas alteraciones en el suelo, las cuales debilitan los enlaces estructurales. Sin embargo, si la carga aplicada es baja, los nuevos enlaces formados y la disminución de los defectos estructurales preexistentes, superan en número a los posibles defectos más recientes. Por lo tanto, el fenómeno de endurecimiento del suelo prevalece y da explicación al carácter atenuante de la deformación del mismo.

Bajo una carga lo suficientemente elevada, el endurecimiento del suelo se produce durante la etapa de fluencia transitoria o primaria (etapa I de la Figura 7), mientras la debilidad de los enlaces está compensada por el fortalecimiento de la estructura. Con el progreso de la fluencia, se producen cada vez más roturas de enlaces, con desintegración de los agregados, reorientación de partículas y el daño estructural cada vez mayor causa un rápido deterioro de la resistencia del suelo frente a la carga, es decir, su reblandecimiento. Sin embargo, en alguna etapa, este reblandecimiento se compensa por el endurecimiento, de tal manera que la fluencia toma la forma de un flujo estacionario con velocidad de deformación constante (etapa II de la Figura 7).

Los cambios en la estructura provocados por la deformación del suelo (bajo cargas elevadas) y la acumulación de daños estructurales hacen que el reblandecimiento prevalezca sobre el endurecimiento. Como consecuencia, la velocidad de deformación aumenta y el proceso de deformación del suelo entra en la etapa de fluencia acelerada (etapa III de la Figura 7) o etapa de flujo 
progresivo. En esta fase los defectos en el suelo se encuentran en su intensidad máxima y, tan pronto como el daño estructural alcance un valor crítico, se producirá la rotura por fluencia.

\subsubsection{Fluencia primaria o transitoria}

En base a la curva clásica de deformación por fluencia presentada en la Figura 7, la etapa I corresponde a la primera etapa de la deformación por fluencia, también denominada fluencia primaria, transitoria o atenuada.

La Figura 8, debida a Cristescu \& Hunsche (1998), muestra los resultados típicos de los ensayos de fluencia realizados en una roca con propiedades reológicas. Cuando la roca o el suelo se somete a una tensión desviadora, se puede producir solo una deformación instantánea o bien una deformación instantánea más otra diferida en el tiempo, dependiendo del valor de la tensión desviadora aplicada en relación a la resistencia a largo plazo del terreno, como se ha visto en el apartado anterior.

La parte (b) de la Figura 8, muestra cómo evoluciona la velocidad de la deformación con el transcurso del tiempo. Se puede observar que inmediatamente después de la aplicación de cada escalón de carga, la deformación se produce a una velocidad alta, que disminuye continuamente con el tiempo hasta que llega a un valor constante. A la primera etapa, en la que la velocidad de la deformación disminuye continuamente, se le suele denominar fluencia primaria o transitoria. 

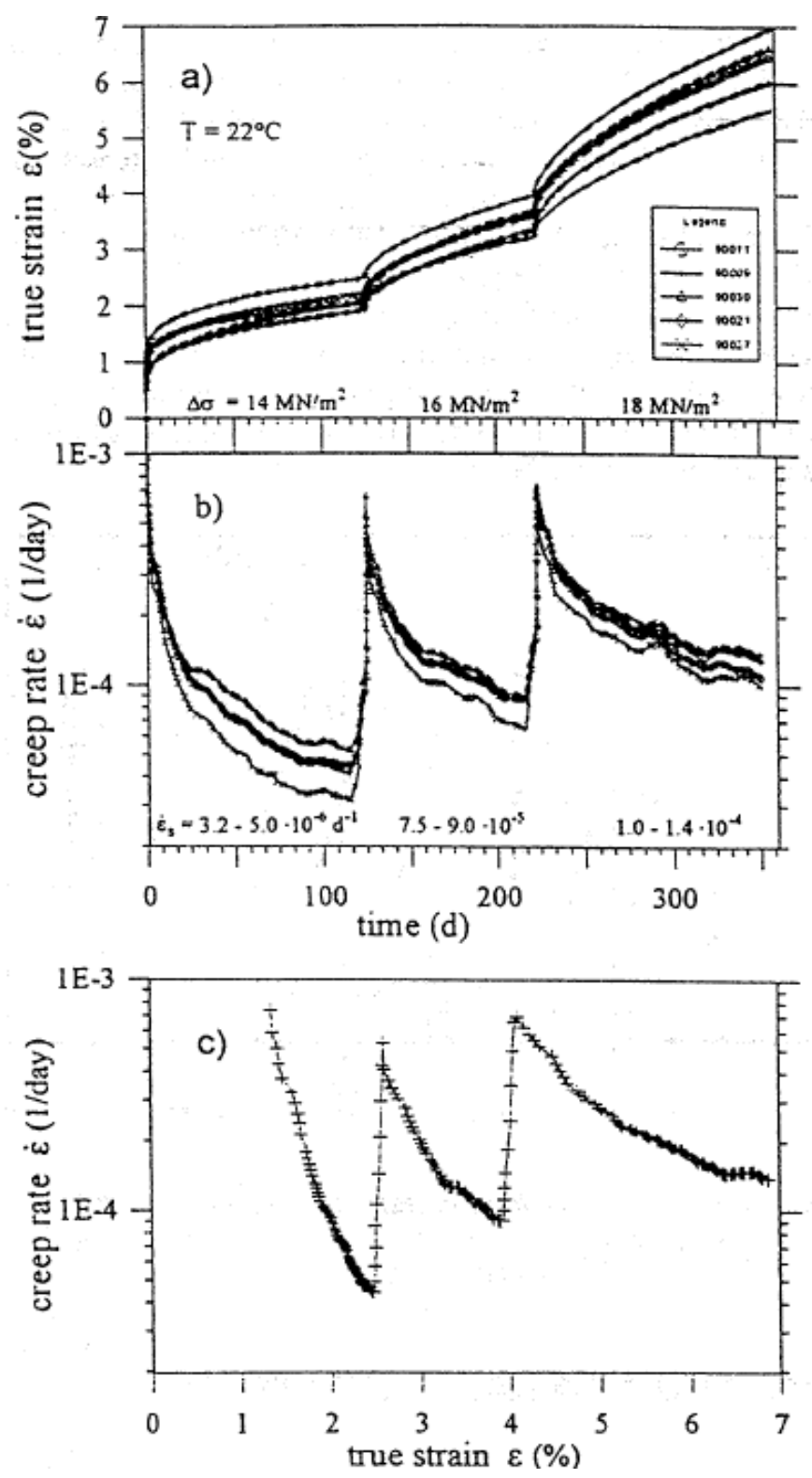

Figura 8. Ensayos de fluencia a compresión simple con roca de sal de la misma muestra y con tres escalones de carga sucesivos (a temperatura del ambiente). a) Deformación - tiempo; b) velocidad de deformación - tiempo, $\boldsymbol{\varepsilon}_{\boldsymbol{s}}$ velocidad en el estado estacionario; c) velocidad de deformación - deformación de uno de los ensayos.

(Cristescu \& Hunsche, 1998)

Cuando la tensión desviadora que actúa sobre la roca es menor que su resistencia a largo plazo, solo se presentará esta etapa de la fluencia. La velocidad de la deformación disminuirá prácticamente a cero y, por lo tanto, se detendrá la evolución de la misma con el transcurso del tiempo. De lo 
contrario, a la fluencia primaria le sucederá la etapa de fluencia secundaria tratada a continuación.

Según Cristescu \& Hunsche (1998) la velocidad de deformación de la fluencia, Figura 8(b), disminuye constantemente debido al endurecimiento por deformación de la roca. Este autor opina que el endurecimiento por deformación se debe a que la cantidad de las dislocaciones aumenta rápidamente en esta etapa, por lo que la interferencia entre ellas se incrementa. Del mismo modo, Feda (1989) asocia la disminución progresiva de la velocidad durante la fluencia primaria, al endurecimiento por deformación y al aumento del número de contactos entre partículas de suelo.

Además, la energía producida por el desviador en esta etapa no es suficiente para mantener la velocidad de deformación constante conforme aumenta la cantidad de dislocaciones, con la consecuente disminución rápida de la velocidad de la deformación cuando se mantiene constante la carga a la que está sometida.

La Figura 9 (Cristescu, 1993) muestra otra característica de esta etapa de la fluencia, que está relacionada con el cambio de volumen de una roca. La figura muestra la deformación que se produce a lo largo del tiempo en una muestra de mármol sometida a una tensión desviadora $\sigma_{1}-\sigma_{3}$ aplicada en escalones crecientes. Se han representado las deformaciones; axial $\left(\varepsilon_{1}\right)$, circunferencial $\left(\varepsilon_{2}\right)$, como la deformación volumétrica, $\varepsilon_{v}$, que se producen en la muestra. Como puede observarse, hasta el desviador de 4.23 $\mathrm{MPa}$, el cambio volumétrico es compresible, es decir, se produce una disminución de volumen. El agrietamiento que ocurre en esta etapa es estable y no produce la rotura de la roca. Incluso en las rocas duras o poco porosas el cambio volumétrico producido en esta etapa es compresible, como es de esperar de acuerdo con la teoría de la elasticidad. Las ecuaciones constitutivas se representan bien en esta etapa mediante los modelos viscoelásticos. 


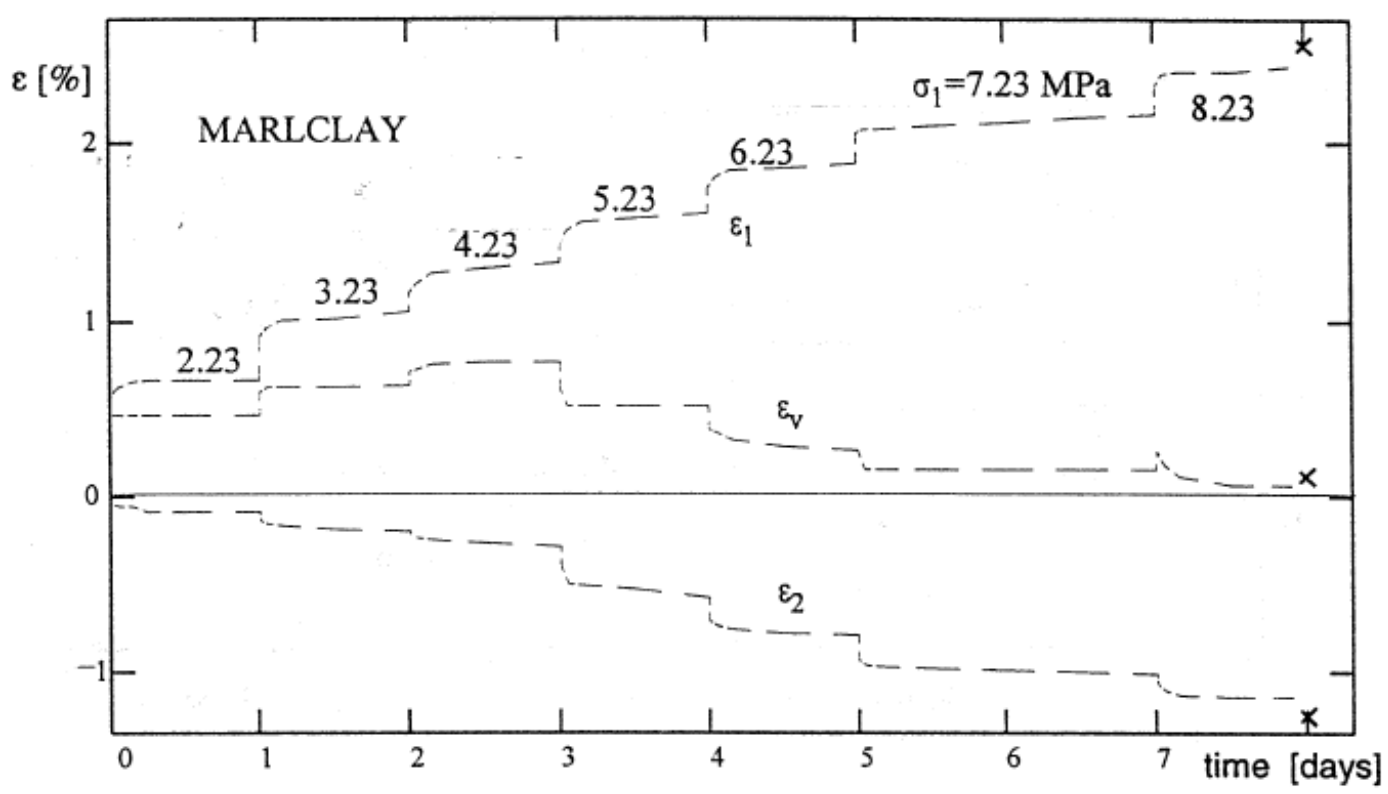

Figura 9. Curvas de fluencia de una muestra de mármol. Los valores sobre la curva de $\varepsilon_{1}$ son las $\sigma_{1}$ en MPa, $\sigma_{3}=1.176 \mathrm{MPa}$. (Cristescu, 1993)

\subsubsection{Fluencia secundaria o estacionaria}

Como se ha mencionado anteriormente, si la tensión desviadora a la que está sometida la roca es superior a su resistencia a largo plazo, después de la etapa de la fluencia primaria se produce una segunda etapa de fluencia, denominada secundaria o estacionaria. En esta etapa la velocidad del proceso se hace constante, como se muestra en la Figura 6.

La deformación transitoria necesaria para llegar a esta condición de fluencia estacionaria depende de la tensión, de la temperatura, así como de la historia de tensiones.

A diferencia de la mayoría de materiales, los suelos no suelen presentar un periodo de fluencia secundaria extendido, y consecuentemente la velocidad de deformación, o bien disminuye continuamente tras la aplicación de la primera carga, o bien eventualmente aumenta al comienzo de la rotura por fluencia (Kuhn \& Mitchell, 1993). La deformación por fluencia es un resultado complejo de cambios estructurales que dependen del tiempo y de la deformación, tendiendo cualquiera de los dos al endurecimiento o reblandecimiento estructural. En su balance podría encontrarse la fluencia 
secundaria, que es, como regla general, muy breve o incluso no llega a aparecer. Solo la falta o la reducción radical de los cambios estructurales, como ocurre en arcillas reconstituidas de consistencia blanda a media, pueden producir condiciones favorables para darse un proceso de fluencia secundaria lo suficientemente pronunciado.

En la Figura 8 (b) se muestra claramente el proceso indicado: después de la aplicación de cada escalón de carga, la velocidad de la deformación disminuye continuamente hasta un valor prácticamente constante. Como puede verse, el valor de esta velocidad depende del nivel de la tensión desviadora actuante. Para una tensión desviadora de $14 \mathrm{MPa}$, el rango de la velocidad de la fluencia secundaria es $3,2 \cdot 10^{-6}<\dot{\varepsilon}_{S}<5,0 \cdot 10^{-6}$; para 16 MPa es $7,5 \cdot 10^{-5}<\dot{\varepsilon}_{S}<9,0 \cdot 10^{-5} \mathrm{y}$, para $18 \mathrm{MPa}$ es $1,0 \cdot 10^{-4}<\dot{\varepsilon}_{s}<1,4$. $10^{-4}$. Las velocidades de deformación anteriores se expresan en 1(\%)/días.

Se suele aceptar que en esta etapa de la fluencia las deformaciones son irreversibles y que únicamente dependen del transcurso del tiempo para que se produzca la rotura. El tiempo necesario para que se produzca la rotura dependerá del nivel de la tensión desviadora actuante y será tanto menor cuanto mayor sea esta.

Debido a que el cambio volumétrico en esta etapa es producido por la plastificación de la roca, los modelos mecánicos viscoelásticos, que se definirán más adelante, no son adecuados para simularla (Cristescu, 1993). Por ejemplo, Gurinsky (2002) utiliza un modelo viscoplástico para simular la etapa secundaria en base a la clara predominancia de ésta en los ensayos de laboratorio y de campo que realizó con arcillas sensitivas blandas.

\subsubsection{Fluencia terciaria o acelerada}

Si la muestra no se descarga, el punto (D) de la Figura 6, la velocidad de la fluencia crece continuamente y se alcanza la rotura. Esta fase (DE) es llamada fluencia terciaria. 
La velocidad de deformación crece tan rápidamente que una vez iniciada no se puede evitar la rotura. No representa un proceso de deformación pura sino también de daño progresivo rápido. Es fundamentalmente diferente de las dos etapas anteriores. Se presenta únicamente con niveles de tensión relativamente altos.

La mayor parte de las publicaciones están dedicadas a la investigación de la fluencia transitoria y de la fluencia estacionaria y no se ha investigado con detalle esta etapa del proceso; probablemente debido a que no es importante, desde el punto de vista de la elaboración de los cálculos del proyecto (Lama y Vutukuri, 1978).

Cristescu \& Hunsche (1998) afirman que esta etapa de la fluencia, que finaliza en la rotura, se produce por un incremento continuo del daño sufrido por la roca. Así mismo indican que las rocas poco porosas y resistentes en donde la rotura se produce por agrietamiento, (no por el colapso de la estructura como el caso de las rocas porosas), esta etapa de la fluencia se produce con cambio volumétrico dilatante. Por lo tanto, en esta etapa, la fluencia y la dilatancia positiva se producen conjuntamente. Dependiendo del nivel de tensión aplicado, esta etapa se puede producir después de la fluencia secundaria o directamente después de la fluencia transitoria, como muestra la Figura 11.

\subsubsection{Factores que influyen en la fluencia del terreno}

\subsubsection{Naturaleza, tipo de enlace y otras propiedades del suelo}

En general las deformaciones por fluencia son más pronunciadas en suelos cohesivos o arcillosos que en suelos arenosos. En consecuencia, existen mayor cantidad de estudios centrados en el comportamiento fluyente de las arcillas.

El fenómeno de la fluencia de los suelos ha sido discutido con detalle por muchos autores; Murayama \& Shibata (1958), Budin (1969), Lacerda 
(1976), Pusch \& Feltham (1981), Vyalov (1986) y otros. Más recientemente por Feda (1992); Kuhn y Mitchell (1993) y Gurinsky (1986).

Pekarskaya (1976) sostiene que existe una dependencia de las propiedades reológicas de los suelos con la naturaleza de los enlaces estructurales. En base a lo anterior, llevó a cabo un análisis de los patrones de comportamiento del suelo en el ámbito de los enlaces estructurales, llegando a la conclusión de que la naturaleza del proceso de deformación está definida fundamentalmente por el tipo de enlace. Por lo tanto, las arcillas blandas que poseen predominantemente enlaces coloidales presentan todas las fases de la deformación por fluencia. Cuanto más blando es el suelo antes comienza la etapa de fluencia estacionaria y más significativo es su efecto en el proceso de deformación. En suelos blandos con este tipo de enlaces, la fluencia acelerada (terciaria) se induce bajo cargas muy variables (entre el 40 al $75 \%$ de la resistencia a corto plazo, según datos presentados por Vyalov, 1986), y se desarrollan durante largos períodos de tiempo, con un tipo de rotura de carácter más viscoso, como regla general.

En general, la relación entre la deformación por fluencia y el logaritmo del tiempo puede ser lineal, creciente o decreciente, como se puede apreciar en la Figura 10. A pesar de que se suele aceptarse una relación lineal, como aproximación ingenieril, para los intervalos de tiempo de interés, no hay disponible una ley fundamental de comportamiento que dicte que la forma es de un tipo u otro. 


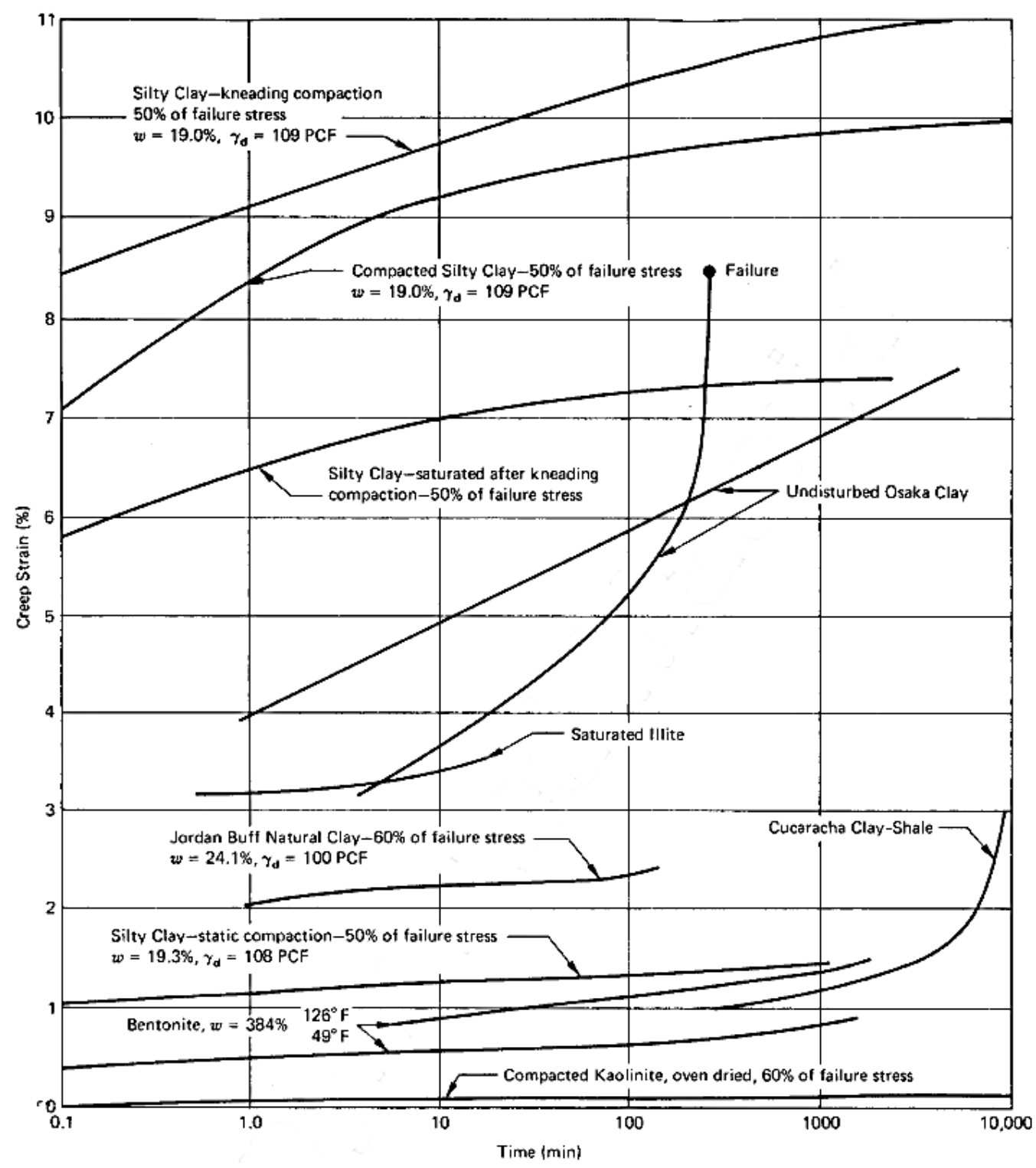

Figura 10. Deformación por fluencia en función del tiempo, según ensayos en diferentes suelos arcillosos (1 PCF $\left.=0,016 \mathrm{~g} / \mathrm{cm}^{3}\right)$. (Mitchell \& Soga, 2005).

En arcillas rígidas caracterizadas por presentar fuertes enlaces y fenómenos de cristalización, la etapa dominante es la primaria. La fluencia acelerada se produce para tensiones aplicadas del orden del 70-80 \% de la resistencia a corto plazo del material, y la transición desde la deformación primaria a la terciaria suele producirse sin que se presente la etapa de flujo viscoso, en la mayoría de los casos. La etapa acelerada es corta, en comparación con la de las arcillas blandas, y su rotura es predominantemente frágil. 
Existen patrones de comportamiento similares en rocas blandas y en algunas rocas duras con enlaces de cristalización. Sin embargo, el comportamiento de estos materiales está regido principalmente por la naturaleza de las sustancias que condicionan la cementación. Una roca con un material de cementación que posee propiedades reológicas provocará un comportamiento similar al conjunto.

Ejemplos de este tipo de rocas podrían ser un suelo congelado cementado por hielo o una arenisca cementada por arcilla. En el primero de los ejemplos, la etapa de fluencia estacionaria será más prolongada que en el segundo ejemplo y la carga necesaria para inducir la etapa terciaria es bastante menor en el primer caso que en el segundo.

Existen dos procesos que contribuyen a la deformación viscosa y que pueden tener lugar simultáneamente (Kurz, et al., 2016): en uno de ellos, el reordenamiento geométrico de las partículas del suelo implica una redistribución estadística de los contactos entre partículas más estresados y menos estresados. Como los primeros buscan la manera de relajar su estado tensional, las partículas se mueven para acomodar la transferencia tensional y los últimos aceptan la carga adicional. La redistribución puede tener lugar bajo largos períodos de tiempo a presión intersticial esencialmente constante, no solo en suelos de grano fino, sino también en materiales granulares como zahorras, escombros o escolleras. En el otro proceso, los cationes hidratados de la doble capa difusa que rodean estrechamente las partículas de arcilla poseen agua adsorbida con una viscosidad superior a la del agua libre 0 gravitacional. La capa doble difusa, de tan solo unos pocos angstroms de espesor, varía con la mineralogía de las partículas, la constante dieléctrica, la temperatura, etc., y todos estos factores pueden cambiar debido a la intervención de las obras de ingeniería (Mitchel y Soga, 2005).

Según se ha comentado al comienzo del capítulo, existen numerosos estudios sobre la fluencia de las arcillas, pero también, es ampliamente conocido que los materiales granulares fluyen bajo cargas efectivas constantes (Lade \& Liu, 1998; Leung, et al., 1996). Murayama, et al. (1984) proponen un modelo reológico para la fluencia de las arenas, y Kuhn \& 
Mitchell (1993) han empleado la teoría del "rate process" para ilustrar que el deslizamiento viscoso y friccional entre partículas podría ser la razón del tipo de comportamiento por fluencia que presentan los suelos granulares. Sin embargo, existen evidencias (Leung et al., 1996; Lade and Liu, 1998) que muestran que la fluencia de los materiales granulares viene acompañada de la trituración de partículas. Bowman y Soga (2003) llevaron a cabo una serie de ensayos de fluencia en la arena "Leighton Buzzard" y en la arena "Montpellier" a bajos niveles de tensión (50 y $500 \mathrm{kPa}$ ). Se pudo observar que la fluencia de los suelos arenosos a bajos niveles tensionales es debida a la reorganización de los granos a lolargo del tiempo. Lade (2013) considera que la trituración de granos debida a la fatiga estática de estos elementos, junto con la fricción entre partículas y el reordenamiento podrían ser los mecanismos presentes en el proceso de deformación por fluencia de las arenas.

En la mayoría de las arcillas naturales, la fluencia es una propiedad inherente de las relaciones entre partículas, particularmente si estas contienen bentonita, montmorillonita y materia orgánica; y de manera menos significativa, las cloritas-illitas (Mitchell y Soga, 2005). La magnitud de la deformación por fluencia aumenta con la plasticidad, la actividad, y la humedad del suelo. Las arcillas más activas presentan normalmente los mayores comportamientos dependientes del tiempo (esmectita>illita>caolinita). Esto es debido a que, para un menor tamaño de partícula, presentan una mayor superficie específica y, por lo tanto, una mayor capacidad de adsorción de agua. De tal manera que, para una tensión de consolidación o tensión desviadora dada, las arcillas más activas y plásticas (esmectitas) tendrán un mayor contenido en agua y menor densidad que las arcillas inactivas (caolinitas). Como cabe esperar, los suelos normalmente consolidados presentan mayor fluencia que los suelos sobreconsolidados. Sin embargo, la forma básica de comportamiento es la misma para todos los tipos de suelos; arcilla alterada y remoldeada, arcilla húmeda, arcilla seca, suelo normalmente y sobreconsolidado, y arena seca y húmeda (Mitchell \& Soga, 2005). 
Otros materiales característicos por presentar problemas de fluencia y que han sido ampliamente estudiados son: las rocas salinas, los suelos congelados y los suelos con elevado contenido en materia orgánica.

\subsubsection{Nivel de tensión}

En el apartado 2.2.2., se ha visto que la rotura por fluencia del terreno sucede cuando se aplica una carga constante de valor inferior al de la carga que produce la rotura a corto plazo del material. La magnitud de la resistencia a largo plazo, expresada habitualmente como un porcentaje respecto a la resistencia a corto plazo, dependerá de la estructura de cada tipo de terreno, y del tiempo transcurrido en esas condiciones de carga constante, a humedad y temperatura constantes.

La velocidad y la magnitud de la deformación debidas a la fluencia, en cualquier momento dek proceso, dependen del nivel de carga relativo al límite de la tensión de plastificación del terreno.

Los suelos presentan un comportamiento similar en cuanto a la aparición de las distintas etapas de fluencia, conforme la tensión desviadora aplicada se aproxima a la resistencia a corto plazo del suelo. Un incremento del nivel de tensión desviadora produce un incremento de la velocidad de deformación por fluencia.

Algunos suelos pueden romper bajo tensiones constantes significativamente menores que las tensiones de pico, medidas en un ensayo de corte, en el cual la muestra se carga hasta la rotura en apenas unos minutos u horas (tan bajas como el $50 \%$ de la tensión en un ensayo rápido). Según Mitchel \& Soga (2005), un ejemplo de la importancia de la ruptura por fluencia fue el desarrollo de los deslizamientos en la formación "Cucaracha clay shale", los cuales comenzaron algunos años después de la excavación del Canal de Panamá (Casagrande \& Wilson, 1951).

Lama \& Vutukuri (1978) han publicado la Figura 11, correspondiente a los trabajos realizados por Griggs (Griggs, 1939; 1940), para mostrar que la 
respuesta viscosa de las rocas depende del nivel de tensión. Dependiendo de este nivel puede suceder que: únicamente ocurra fluencia transitoria (curva con $10 \mathrm{MPa}$ ); se den las tres etapas de la fluencia (curvas desde $12.5 \mathrm{MPa}$ hasta $25 \mathrm{MPa}$ ); u ocurra la fluencia terciaria directamente después de la fluencia transitoria (curva con $30 \mathrm{MPa}$ ). Los valores límite de tensión desviadora citados son específicos para el caso presentado por Griggs (1940), en la Figura 2.6., es decir, las distintas etapas se presentan para esos valores de tensión aplicada en muestras de alabastro a la misma humedad y temperatura.

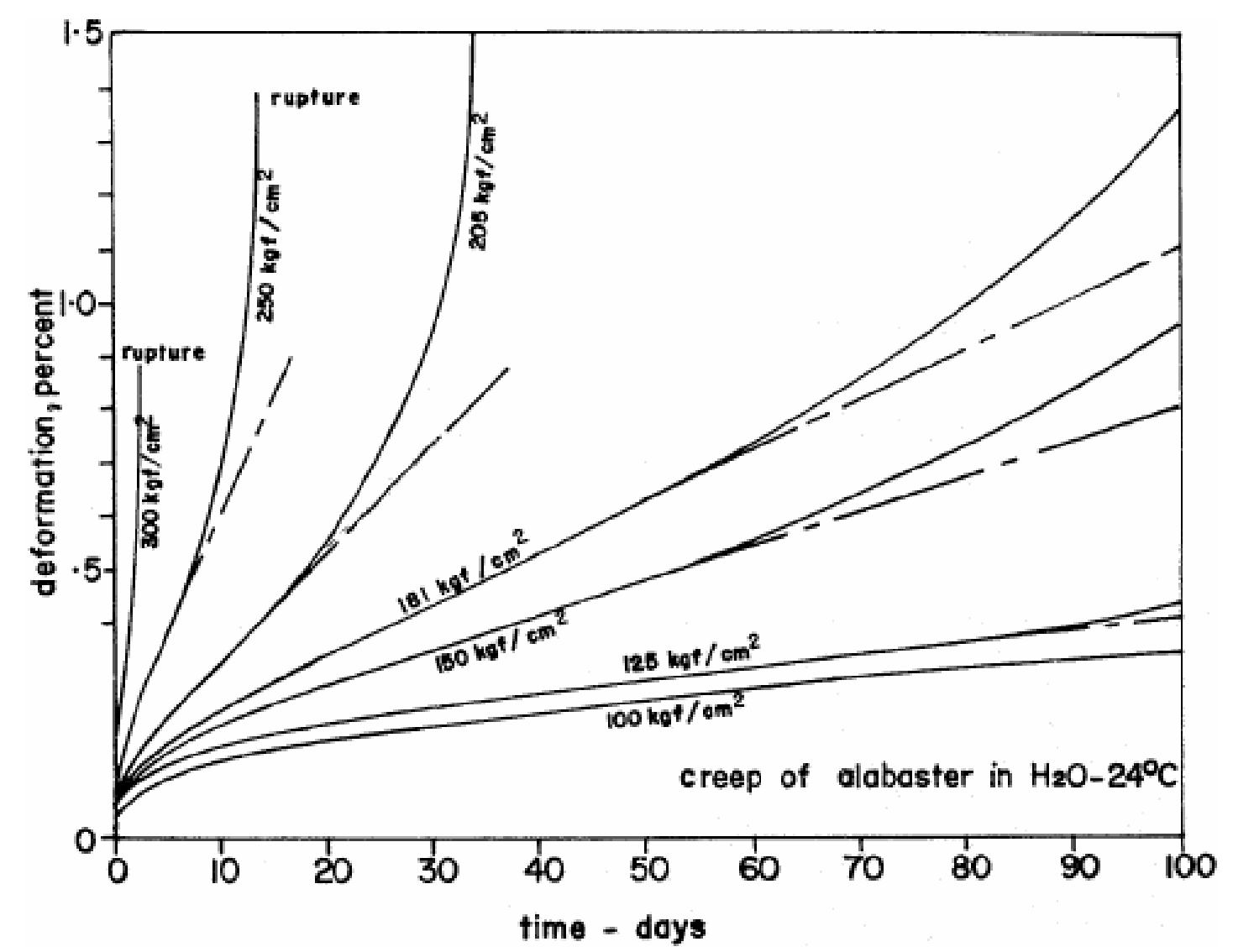

Figura 11. Curvas de fluencia del alabastro. Todos los especímenes fueron sumergidos en agua destilada y ensayados a la misma temperatura, pero con diferente tensión desviadora. (Griggs, 1940)

Bishop \& Lovenbury (1969) llevaron a cabo ensayos triaxiales drenados (véase la Figura 12) de hasta tres años y medio de duración, con muestras inalteradas de arcillas de Londres fisuradas (\% arcillas = $58 \%$, humedad 
natural $w=29.3 \%$, límite plástico $w_{P}=29 \%$, y límite líquido $w_{L}=76 \%$ ). Los distintos desviadores aplicados durante los ensayos de fluencia son un porcentaje de la máxima tensión desviadora registrada en ensayos convencionales a corto plazo. En la Figura 12 se aprecia como para las cargas que varían entre el 90 y $106 \%$ y el 80 y 94\%, del desviador máximo a corto plazo, la rotura por fluencia del terreno se puede producir al cabo de dos días, en el primer caso, o bien al cabo de 1250 días, en el segundo caso.

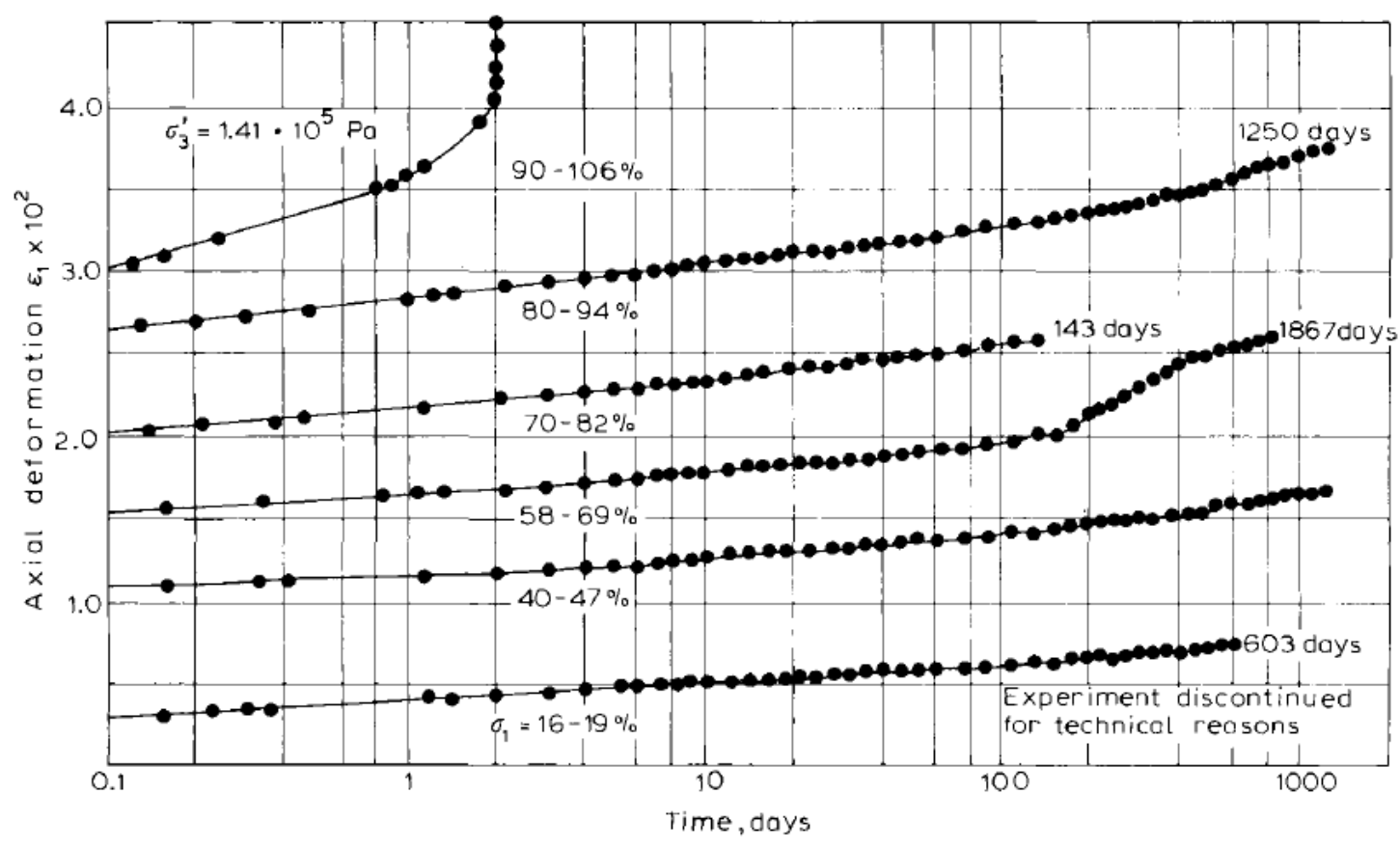

Figura 12. Ensayos triaxiales drenados de fluencia en arcillas de Londres fisuradas. (Bishop \& Lovenbury, 1969)

Cristescu \& Hunsche (1998) ha mostrado que la reacción de una muestra de roca de sal a la reducción de la tensión desviadora depende del grado de reducción de la tensión y del nivel de deformación por el endurecimiento sufrido previamente. En la Figura 13 este autor muestra la curva de fluencia de un ensayo triaxial realizado en una probeta de sal a temperatura $\left(45^{\circ} \mathrm{C}\right)$ y presión de confinamiento constante (15 MPa), para diferentes escalones de carga y de descarga. Sobre la curva se muestran los incrementos de tensión desviadora. Como puede observarse, al reducir la tensión de $20 \mathrm{MPa}$ a $17 \mathrm{MPa}$ se presenta lo que Cristescu \& Hunsche (1998) denomina fluencia transitoria inversa. 


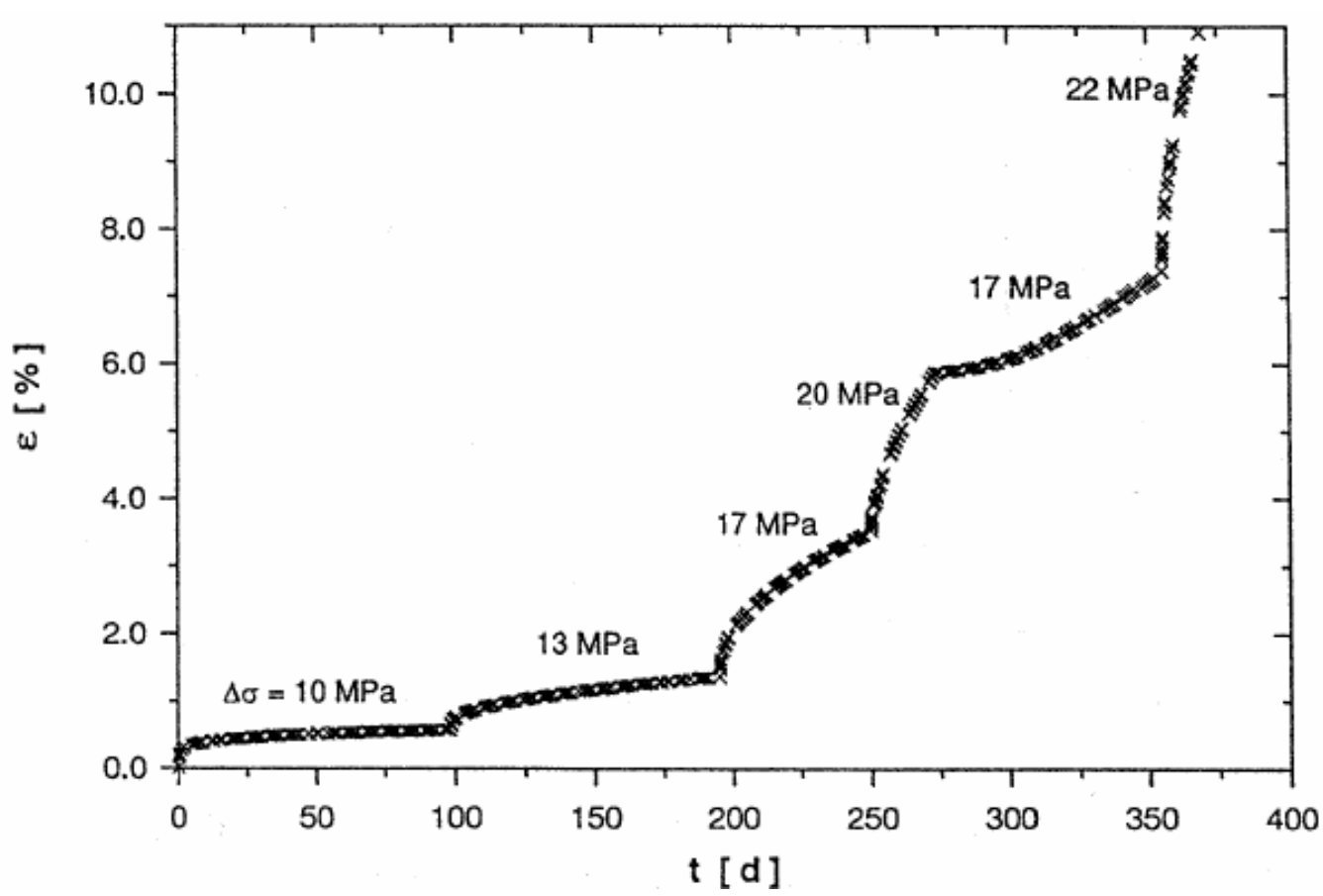

Figura 13. Curva de fluencia de un ensayo triaxial en una muestra de roca de sal realizado a $45{ }^{\circ} \mathrm{C}$ de temperatura y $15 \mathrm{MPa}$ de confinamiento. Sobre la curva se muestran los incrementos de tensión desviadora. Nótese la fluencia transitoria inversa en la etapa con reducción de la tensión. (Cristescu \& Hunsche; 1998)

\subsubsection{Presión de confinamiento}

La Figura 14 muestra otro ensayo de fluencia en compresión triaxial a $50^{\circ} \mathrm{C}$ con cambios escalonados de la presión de confinamiento a lo largo del tiempo (Cristescu \& Hunsche, 1998). La curva del ensayo (2) está ligeramente afectada debido a que el espécimen se impregnó con aceite mineral en el punto indicado con (+), el espécimen fue limpiado y el ensayo fue reiniciado. Esta y otras evidencias experimentales muestran que hay una relación inversamente proporcional entre la velocidad de la fluencia secundaria y la presión de confinamiento. Es decir, que al disminuir la presión de confinamiento la velocidad de la fluencia aumenta. Además, la figura revela que después de cada cambio en la presión de confinamiento se produce la fluencia transitoria y luego la fluencia secundaria.

Según Lama y Vutukuri (1978) la cantidad de deformación desviadora y volumétrica debida a la fluencia es menor cuanto mayor es la presión de 
confinamiento. También manifiestan que la presión de confinamiento retrasa la rotura de la roca, dado que, para la misma tensión desviadora, el tiempo transcurrido antes de la rotura será mayor cuanto mayor sea la presión de confinamiento.

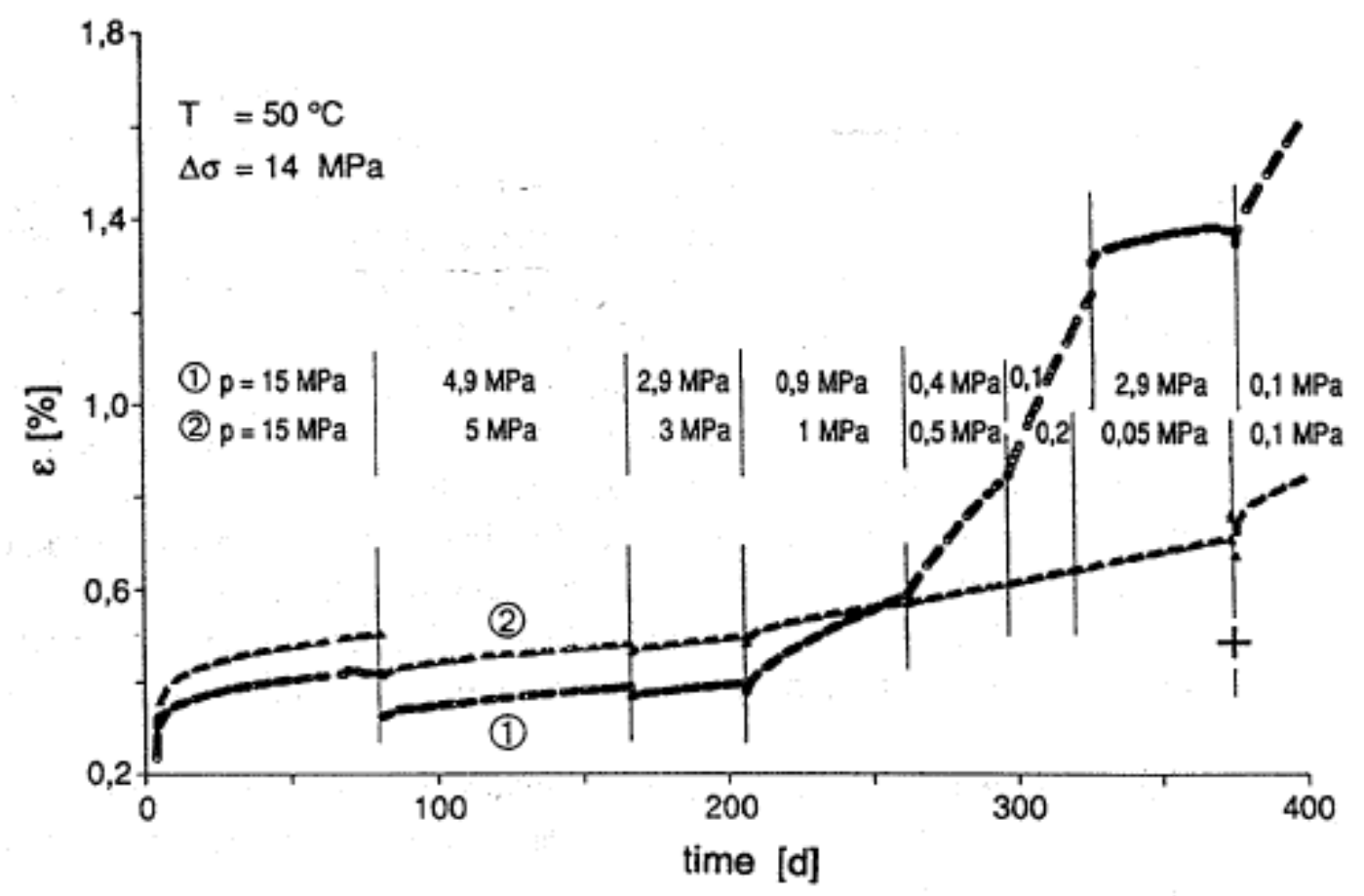

Figura 14. Curvas "Tiempo - Deformación" de dos ensayos triaxiales de fluencia a 50드 con variaciones escalonadas de la presión de confinamiento. La curva del ensayo (2) está ligeramente afectada debido a que el espécimen se impregnó con aceite mineral en el punto indicado con (+), el espécimen fue limpiado y el ensayo fue reiniciado.

(Cristescu \& Hunsche, 1998)

\subsubsection{Humedad relativa y contenido de humedad}

La Figura 15 (Cristescu \& Hunsche, 1998) muestra que la humedad relativa tiene un gran efecto en la velocidad de la fluencia de una muestra de roca de sal. En ella se observan las curvas de fluencia correspondientes a dos ensayos de compresión simple realizados a presión y temperatura constante, con diferente humedad relativa del aire ambiente. En la curva 1 dicha humedad relativa se varió a lo largo del ensayo, desde $0 \%$ a $65 \%$ disminuyéndola nuevamente a $0 \%$ y aumentándola de nuevo al $65 \%$. La curva 
2 corresponde a un ensayo en la misma roca en el que la humedad relativa se mantuvo todo el tiempo constante e igual al $43 \%$.

Como puede observarse, en el ensayo 1, el incremento escalonado de la humedad relativa desde $0 \%$ a $65 \%$ produjo el incremento de la velocidad de la fluencia secundaria en quince veces. La misma figura muestra que el efecto es reversible, es decir que, así como el incremento de la humedad relativa aumenta la velocidad de la fluencia, la posterior disminución de la humedad relativa disminuye la velocidad. La variación de esta condición además produce siempre la fluencia transitoria. Los resultados muestran que la reacción de la roca es casi inmediata.

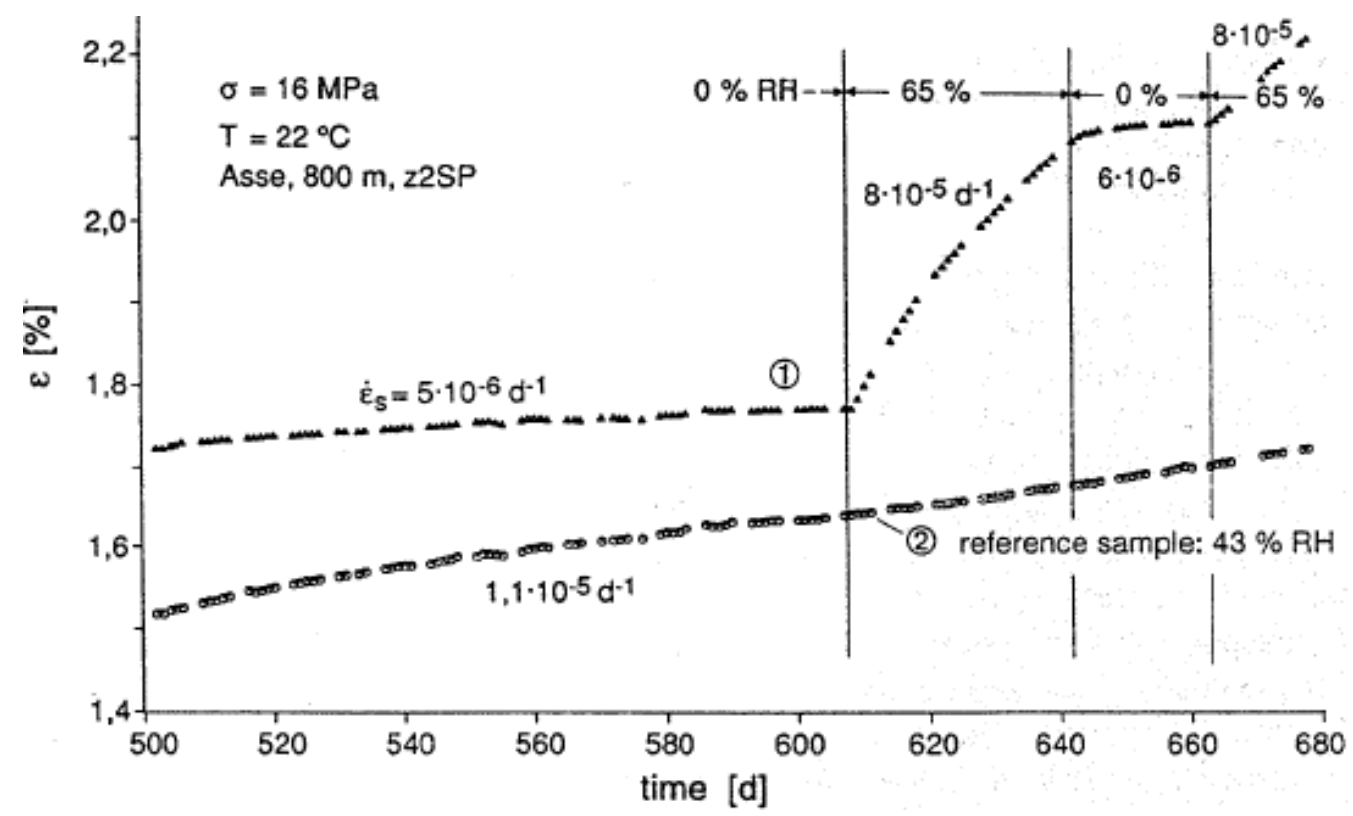

Figura 15. Curvas tiempo - deformación de dos ensayos de fluencia en compresión simple. El espécimen (1) fue ensayado variando escalonadamente la humedad relativa del aire. El espécimen (2) fue ensayado en el mismo equipo, pero con la humedad relativa constante. (Cristescu \& Hunsche, 1998)

El efecto de la humedad se produce siempre que el agua pueda penetrar hacia el interior de la roca. Por lo tanto, puede ser observado únicamente bajo condiciones tensionales que producen la dilatancia de la roca (Cristescu \& Hunsche, 1998). 
Los trabajos publicados por Wawersik \& Brown (1973) indican que la deformación por fluencia en granito y arenisca aumenta con el contenido de humedad de la muestra. En los ensayos de fluencia en compresión simple, la velocidad de la fluencia secundaria de las muestras saturadas con agua fue de alrededor de dos órdenes de magnitud mayor que la correspondiente a las muestras secadas al aire. Afrouz \& Harvey (1974) hallaron que en rocas saturadas blandas la velocidad de fluencia se incrementaba tres veces en el caso del carbón y ocho veces en el caso del esquisto.

En relación al contenido en humedad del terreno, según la Figura 16, presentada por Houska (1981), un cambio en el contenido en agua (de las muestras de laboratorio) del $1 \%$ no tiene las mismas consecuencias en el rango de las humedades bajas que en el de las altas. Además, la condición de humedad constante no es tan importante para determinados suelos. Por ejemplo, no es relevante en suelos limpios sin cohesión (en el proceso de deformación sin fracturación de los granos). Sin embargo, en relación a los suelos cohesivos, los más sensibles son los que se encuentran con contenidos de agua bajos donde el suelo no está saturado (p.e. la colapsabilidad del loess está habitualmente asociada a un grado de saturación de alrededor del $60 \%)$.

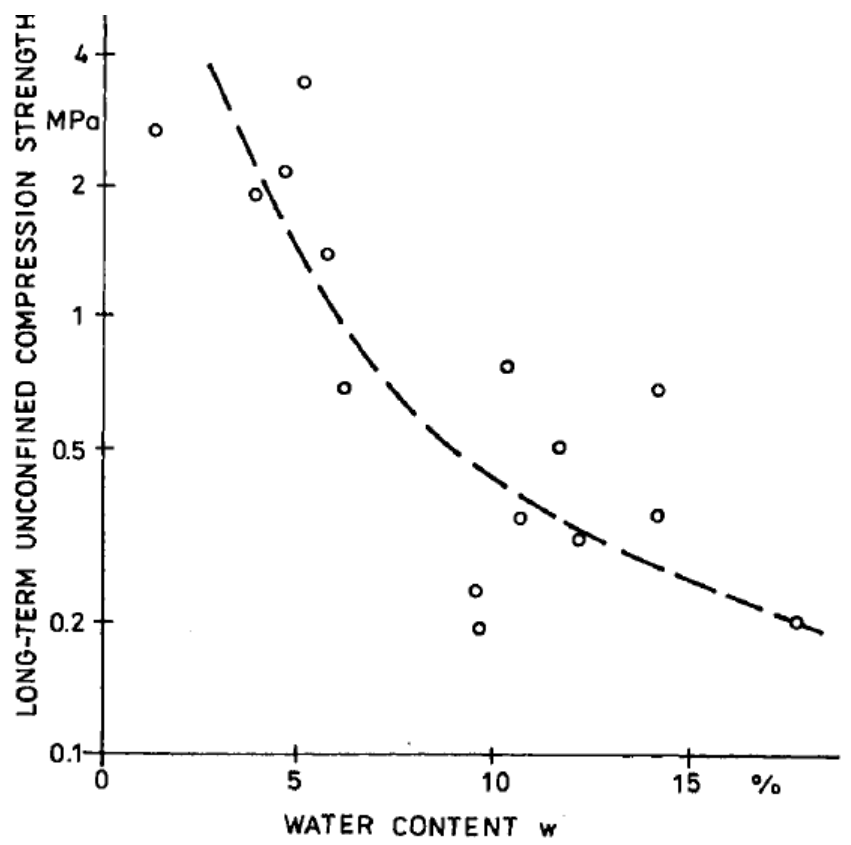

Figura 16. Descenso de la resistencia no confinada a largo plazo con el incremento de la humedad de muestras de argilitas del Cretácico Superior. (Houska, 1981) 


\subsubsection{Temperatura}

Respecto del comportamiento de suelos, se ha estudiado experimentalmente la influencia de la temperatura ambiente en el laboratorio, observándose que la velocidad de deformación aumenta y que las tensiones relajadas correspondientes a valores específicos de deformación decrecen a temperaturas elevadas (Figura 17 y en la Figura 18).

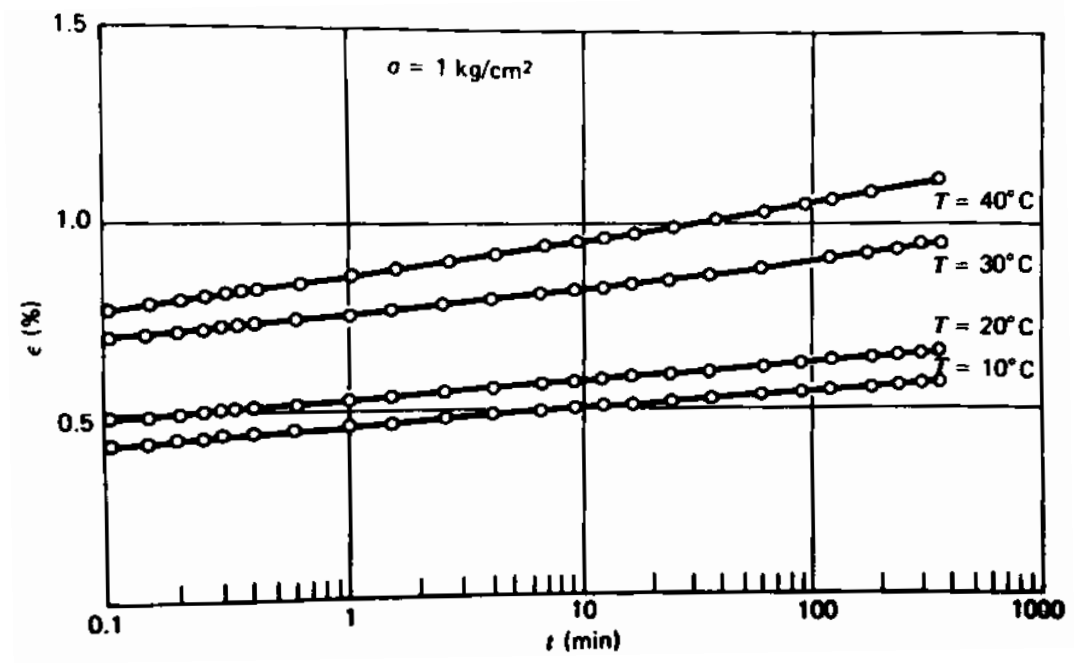

Figura 17. Curvas de fluencia de la formación "Osaka clay". Ensayos triaxiales no drenados a diferentes temperaturas. (Murayama, 1969)
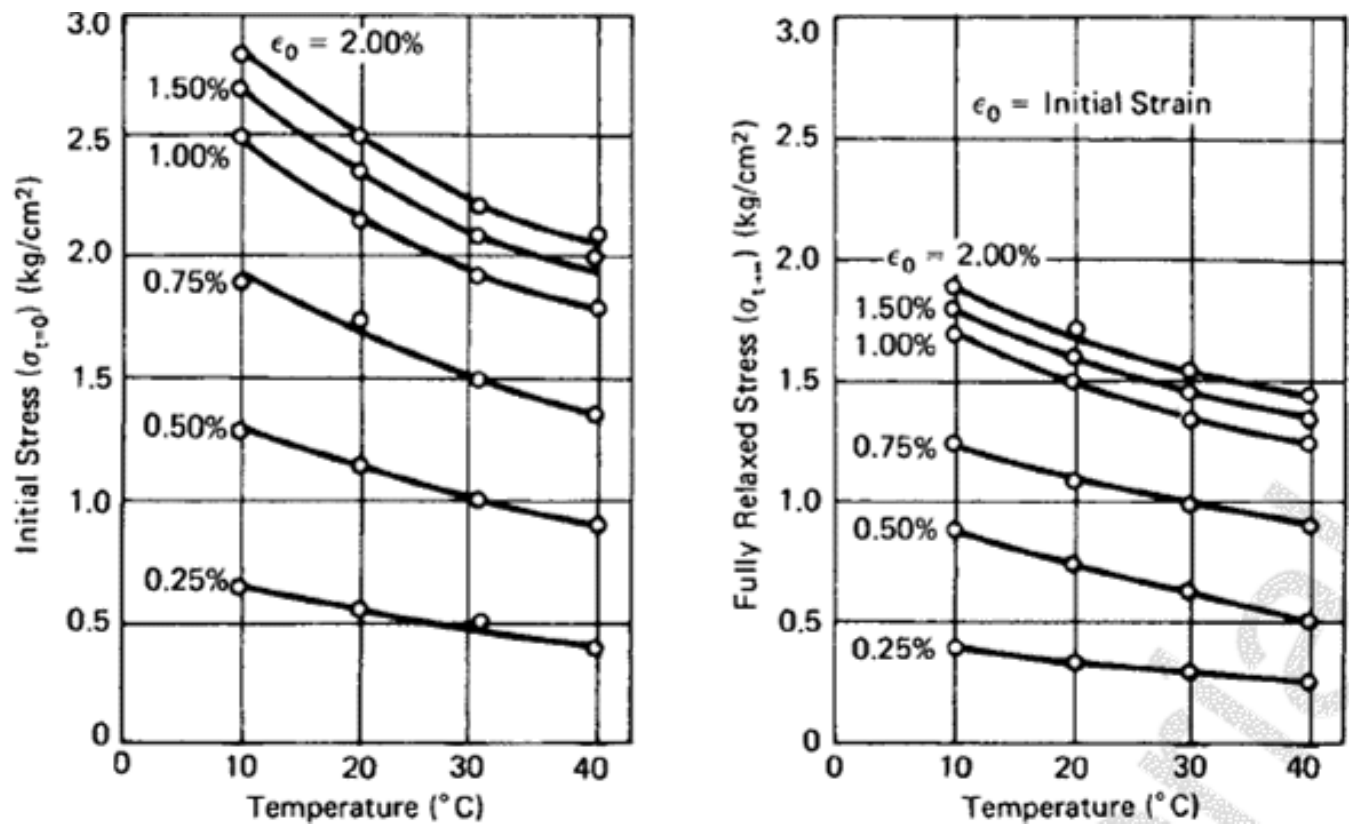

Figura 18. Influencia de la temperatura en la tensión inicial y final en ensayos de relajación tensional en Osaka clay (compresión triaxial no drenada). (Murayama, 1969) 
Un incremento en la temperatura aumenta la presión intersticial, disminuye la tensión efectiva y consecuentemente debilita la estructura del suelo (Mitchel y Soga, 2005).

El efecto de la temperatura en la velocidad de deformación se puede evaluar teóricamente usando la teoría del proceso de activación térmica, la cual se detalla en capítulos posteriores.

La mayoría de los ensayos de fluencia en laboratorio se llevan a cabo a la temperatura ambiente de la sala, que suele ser próxima a los $20 \stackrel{\circ}{\circ}$. La temperatura media del suelo susceptible de cimentación es de alrededor de $10^{\circ}$ en regiones de climas templados. Por ejemplo, a este respecto, Murayama (1969) no encontró cambios en la velocidad de deformación en muestras de la "Osaka clay" cuando incrementó la temperatura desde $10 \stackrel{\circ}{ } \mathrm{C}$ hasta $20 \stackrel{\circ}{\circ}$.

De manera general las opiniones de numerosos investigadores (los cuales se citan en Feda, 1992) llevan a establecer que los rangos permitidos para las oscilaciones de temperatura cuando se está midiendo fluencia son de entre $\pm 0.1{ }^{\circ} \mathrm{C}$ y $\pm 2.5^{\circ} \mathrm{C}$.

Cabe destacar que la variedad de opiniones a este respecto es debida a que los efectos de la temperatura dependen de la estructura de suelo y del nivel tensional. En general los suelos con estructuras más débiles y que están sometidos a niveles tensionales elevados serán más susceptibles a las variaciones de temperatura; especialmente, en los ensayos no drenados donde la temperatura afecta principalmente a la presión intersticial.

Por otro lado, la temperatura tiene un efecto pronunciado en la fluencia de una roca, incluso para temperaturas muy por debajo de la de fusión de la misma, el aumento de la temperatura incrementa considerablemente la velocidad de la deformación debido a la activación térmica de mecanismos micromecánicos (Cristescu \& Hunsche, 1998).

Este hecho se observa claramente en la Figura 19 (Cristescu \& Hunsche, 1998). En dicha figura se muestra la evolución de la deformación en el tiempo en una probeta de sal sometida a compresión simple, cuando se 
mantiene constante la carga y se aumenta la temperatura. Se pueden destacar los dos aspectos siguientes: el aumento de la temperatura produce un aumento notable de la velocidad de deformación, asimismo, la velocidad de deformación para una determinada temperatura tiende a un valor constante, que es tanto mayor cuanto mayor es esta.

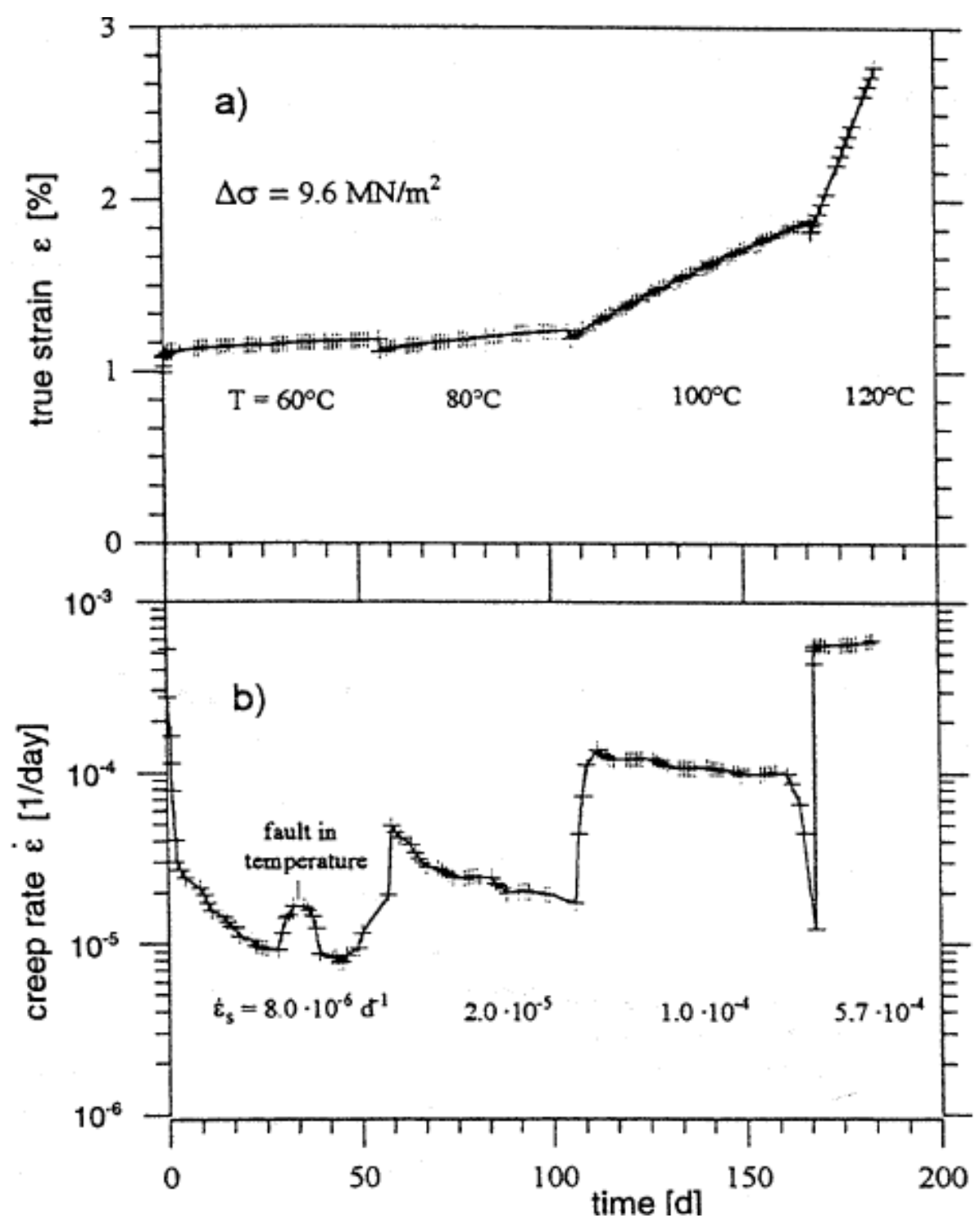

Figura 19. Ensayos de fluencia en compresión simple en roca de sal, con cuatro incrementos sucesivos de temperatura (BGR); a) deformación vs tiempo; b) velocidad de deformación vs tiempo, $\varepsilon$ s velocidad de fluencia secundaria. (Cristescu \& Hunsche, 1998) 


\subsubsection{Fluencia en anclajes al terreno}

Llevando a cabo una revisión de los artículos, normativas y guías relativas a anclajes al terreno más empleadas, se incluyen a continuación algunas consideraciones en relación a: terrenos más susceptibles (no recomendados para la instalación de anclajes) y limitaciones del coeficiente de fluencia, entre otros.

Según cita Ostermayer (1975), tanto en suelos cohesivos como en suelos uniformes granulares, pueden producirse, antes de alcanzar la rotura, desplazamientos por fluencia elevados. Para el diseño de anclajes permanentes es imprescindible conocer el desplazamiento por fluencia en función de tiempo.

Observando que la relación entre el desplazamiento por fluencia y el tiempo era una función exponencial (es decir una línea recta cuando se traza en escala semi-logarítmica), como se puede apreciar en la Figura 20; Ostermayer (1975) definió el coeficiente de fluencia, $k_{s}$, como la pendiente de esa línea recta, de tal manera que resulta:

$$
k_{s}=\frac{s_{2}-s_{1}}{\log \frac{t_{2}}{t_{1}}}
$$

La pendiente de cada una de las curvas aumenta con la carga constante aplicada, hasta que se alcanza la carga de rotura (p.e. $580 \mathrm{kN}$ para el anclaje Wh 7) y el desplazamiento alcanza un valor "infinito". 


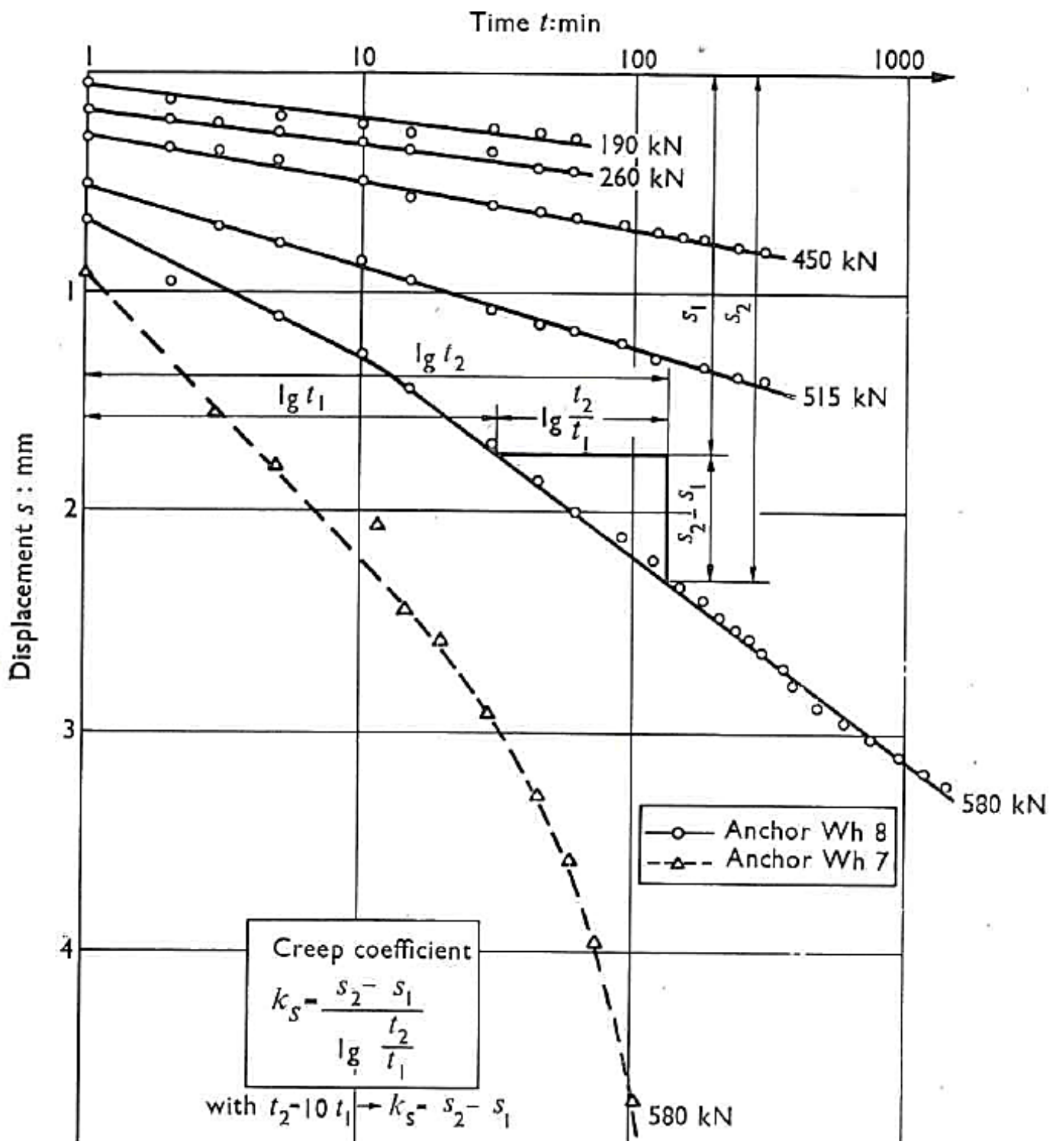

Figura 20. Curvas desplazamiento - tiempo para distintas cargas en una arena uniforme. (Ostermayer, 1975)

La pendiente de cada una de las curvas aumenta con la carga constante aplicada, hasta que se alcanza la carga de rotura (p.e. $580 \mathrm{kN}$ para el anclaje Wh 7) y el desplazamiento se vuelve de nuevo "infinito".

En la Figura 21 se puede observar cómo, al principio, los valores del coeficiente de fluencia son pequeños pero se incrementan rápidamente: para arcillas de consistencia rígida con plasticidad de media a alta, el aumento rápido del coeficiente de fluencia se produce en el entorno del $40 \%$ de la carga de rotura, para arcillas de consistencia rígida a muy rígida con 
plasticidad de media a alta alrededor del $55 \%$ de la carga de rotura, para arcillas de consistencia muy rígida a dura con plasticidad de media a alta y para arenas uniformes alrededor del $80 \%$ de la carga de rotura.

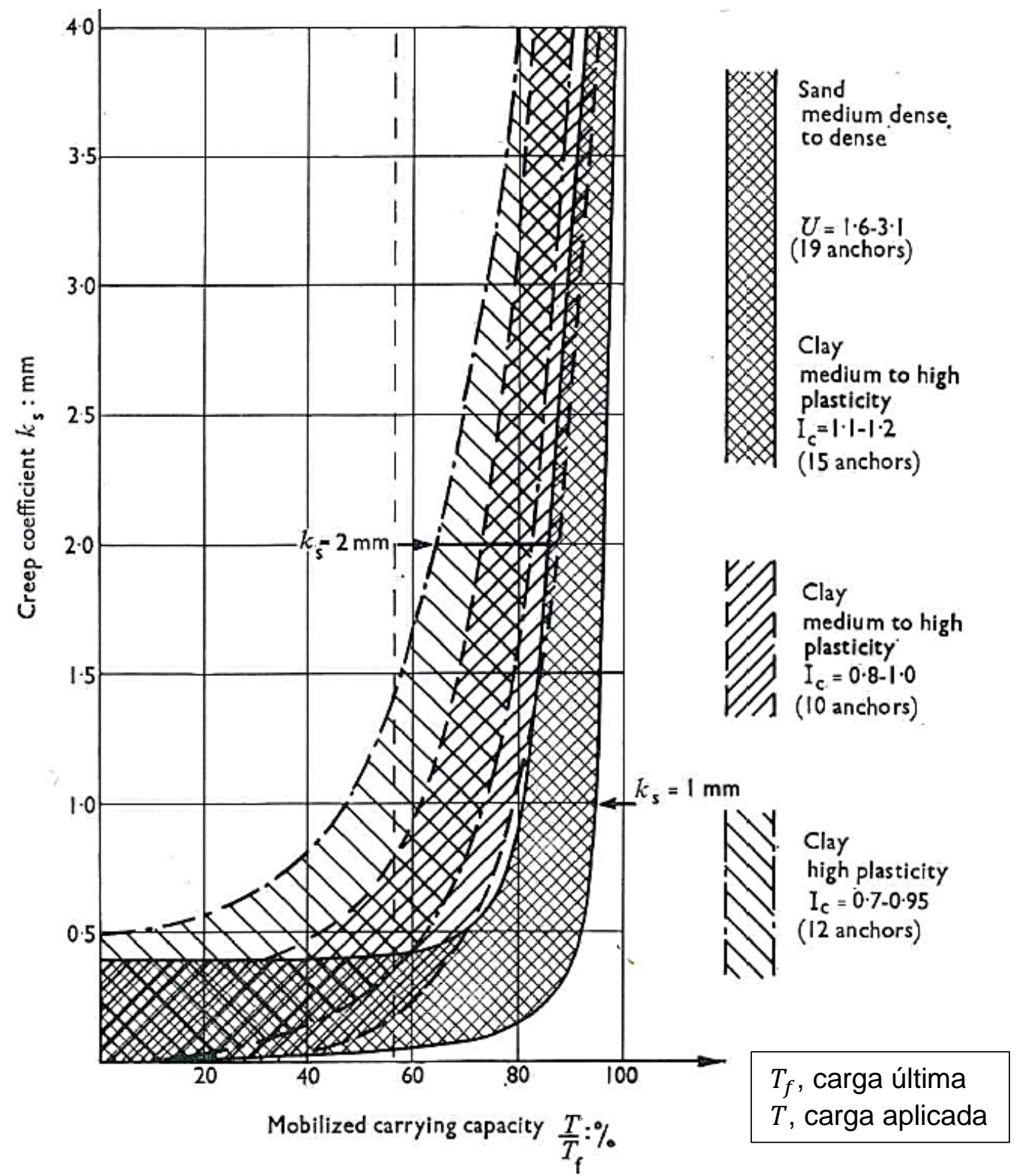

Figura 21. Coeficientes de fluencia en relación con la capacidad de carga movilizada (resultados de 56 ensayos). (Ostermayer, 1975)

Se puede aceptar que el comienzo del flujo plástico del suelo alrededor de la zona cementada, más en el caso de anclajes permanentes, no debe llegar a tener lugar (Ostermayer, 1975). Por eso, además de introducir el factor de seguridad ante rotura (1.75), se debe introducir un factor de 
seguridad frente a desplazamientos por fluencia (1.5), como se puede apreciar en la Tabla 1.

Tabla 1. Máxima carga de trabajo para anclajes permanentes. (Ostermayer, 1975)

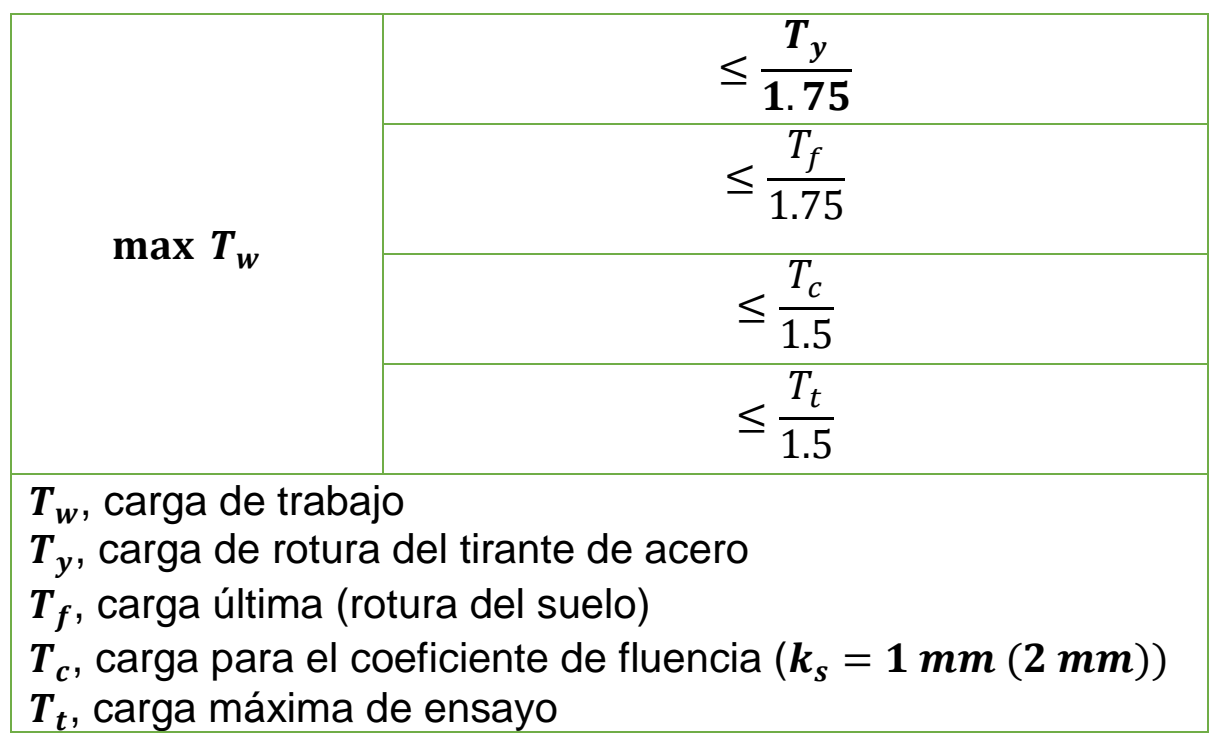

Según Ostermayer (1975) la parte de los desplazamientos por fluencia es debida al desprendimiento parcial del contacto acero/cemento, a la fluencia de la lechada de cemento y a la relajación del tirante de acero, se corresponde generalmente a un coeficiente de fluencia de alrededor de 0,4 $\mathrm{mm}$. Un incremento del coeficiente más allá de 0,4 es el resultado de la fluencia en el contacto cemento/suelo. Este mismo autor indica que los anclajes cementados de eje recto ("straight shaft anchors") corresponden a las líneas rectas de la Figura 20, mientras que en el caso de anclajes reinyectados ("multi-underreamed") se esperan desplazamientos por fluencia mayores debidos a la concentraciones locales de tensión que causan consolidación y deformaciones plásticas.

Debido a la fluencia bajo carga constante y al comportamiento bajo cargas repetidas, en el año 1973, el Institut Für Bautechnik, no permitía la construcción de anclajes permanentes cuando el cambio en la carga retesada del tirante para los distintos escalones de carga es mayor del $20 \%$ de la carga de trabajo o cuando el suelo tiene un elevado contenido en materia orgánica, bien afecta a una arena suelta, o bien es un suelo cohesivo con índice de consistencia $I_{c}<0.9$ o límite líquido $w_{L}>50 \%$. 
El criterio para los ensayos de aceptación e idoneidad (Osterrmayer, 1975) es que el coeficiente de fluencia debe ser menor de $2 \mathrm{~mm}$ bajo cargas de 1.5 veces la carga permitida de trabajo (Figura 21). A pesar de que los coeficientes de fluencia para la carga de trabajo caen por debajo del punto crítico de rotura de las curvas (a partir de que comienza el flujo) no se alcanza el factor de seguridad deseado de 1.75 frente a rotura para anclajes permanentes en gravas y arenas y arcillas de rígidas a muy rígidas. Por lo tanto, se recomienda que el coeficiente de fluencia no debe ser superior a 1 $\mathrm{mm}$ para 1.5 veces la carga de trabajo. Ese valor de $1 \mathrm{~mm}$ para $k_{S}$ corresponde a una diferencia de desplazamientos de $6 \mathrm{~mm}$ en un intervalo de tiempo comprendido entre 30 minutos y 50 años.

Teóricamente es posible calcular el desplazamiento por fluencia de la zona cementada a partir del coeficiente de fluencia obtenido del ensayo de aceptación. A partir de ese desplazamiento se puede establecer una idea sobre la pérdida de las tensiones iniciales en los anclajes con el tiempo (Ostermayer, 1975).

Sin embargo, los cambios en las cargas de los anclajes instalados pueden ser debidos también a movimientos y asientos de la estructura anclada, además de a los desplazamientos por fluencia. Para separar estos dos factores, las medidas en la cabeza del anclaje deben ser muy precisas. Con estas medidas se puede concluir que para longitudes de anclaje de 20-25 $\mathrm{m}$ (diámetros de perforación de $10-15 \mathrm{~cm}$ ) una pérdida tensional del $6 \%$ en arcillas de muy rígidas a duras y de un $12 \%$ en arcillas de rígidas a muy rígidas se considera que es debida a la fluencia. Resulta interesante señalar que en general no suelen tener lugar pérdidas notables de tensión después de un periodo de entre 2-4 meses. La pérdida tensional medida ("real") en ese periodo es inferior a la calculada a partir del coeficiente de fluencia, por lo que los valores calculados quedan del lado de la seguridad.

Según el informe "Ground anchors and anchored systems" de la Federal Highway Administration (FHWA) los suelos potencialmente susceptibles de sufrir elevadas deformaciones a largo plazo bajo carga 
constante son: suelos orgánicos; suelos arcillosos con un índice de líquidez ${ }^{1}$ $\left(I_{L}\right)$ medio superior a 0.2 ; suelos arcillosos con un límite líquido $\left(w_{L}\right)$ medio superior al $50 \%$; y suelos arcillosos con un índice de plasticidad (IP) medio superior al $20 \%$.

Como se puede apreciar en la Figura 22a, el coeficiente de fluencia ( $\alpha$ ) está directamente relacionado con la carga aplicada. Siguiendo la línea de lo mencionado en el apartado anterior (Ostermayer, 1975), en las recomendaciones de la FHWA se menciona que gracias a numerosos ensayos de fluencia en anclajes, se ha visto que cuando la velocidad de fluencia excede los $2 \mathrm{~mm}$ por cada ciclo del logaritmo del tiempo, cualquier carga adicional aplicada al tirante resulta en movimientos del cuerpo cementado inaceptables. En la Figura 21b, se ilustra la definición de carga crítica de fluencia que corresponde a la carga a la cual el coeficiente de fluencia ( $k_{s}$ o $\alpha$ en esta figura) exhibe un aumento brusco.

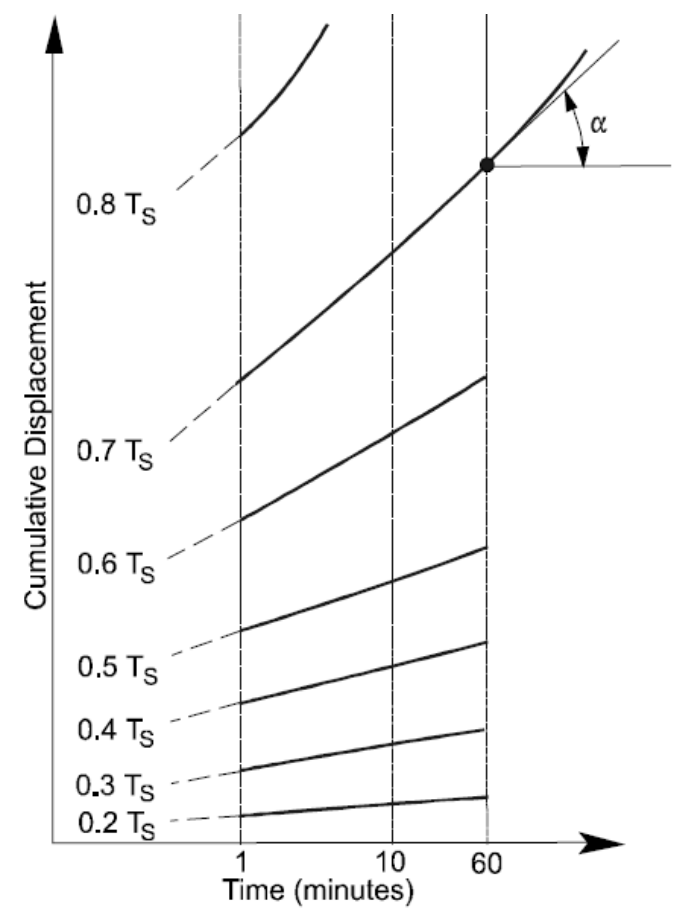

Note: $T_{S}=$ Ultimate strength of the tendon

(a) Creep curves

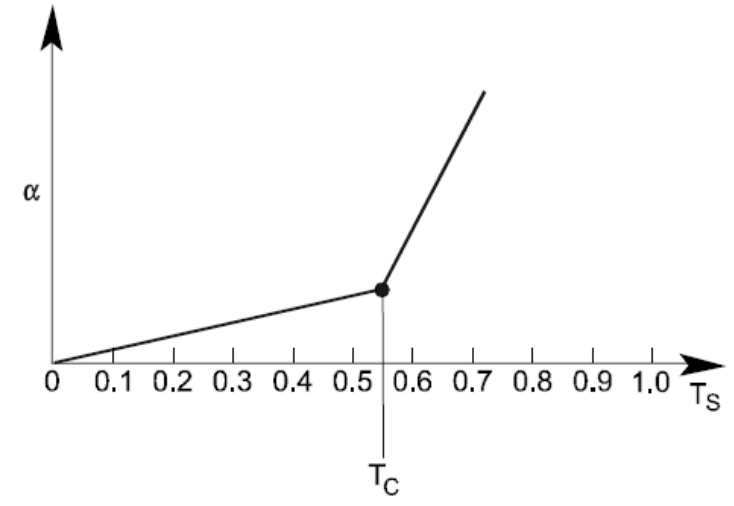

(b) Critical creep tension

Figura 22. Evaluación de la tensión crítica de fluencia. (FHWA, 1999)

\footnotetext{
${ }^{1} I_{L}=\frac{W_{n}-W_{P}}{W_{L}-W_{P}}$, cuanto más elevado sea $I_{L}$ más blando será el suelo arcilloso (su humedad natural, $w_{n}$, está muy próxima a la humedad del $\left.w_{L}\right)$.
} 


\subsection{MODELOS REOLÓGICOS CONSTITUTIVOS}

\subsubsection{Introducción}

En este capítulo se presenta una recopilación bibliográfica de los modelos empleados para caracterizar el comportamiento evolutivo de los suelos, es decir, la variación de sus propiedades geomecánicas con el tiempo. Los siguientes apartados están centrados en la formulación de los fenómenos de fluencia y relajamiento tensional (o relajación), aplicados al comportamiento de suelos granulares y cohesivos. Si bien, es cierto que la mayoría de los modelos que se describen a continuación son aplicables a suelos y a macizos rocosos. En este documento se empleará el término "suelo", en su sentido más amplio y general, pudiendo representar también a medios rocosos, si bien se es, obviamente consciente, de la diferencia entre estos tipos de materiales naturales.

La recopilación llevada a cabo en este documento divide los modelos de comportamiento dependientes del tiempo o modelos reológicos de los suelos, en seis ramas principales:

- Modelos empíricos

- Modelos mecánicos o analógicos

- Modelos ingenieriles

- Enfoque hereditario

- Leyes constitutivas o modelos generales tensión-deformacióntiempo

- Enfoque de la teoría "rate process"

A continuación, se detallan cada una de las anteriores posibilidades. 


\subsubsection{Modelos empíricos}

De manera general, las relaciones empíricas son solo aplicables a problemas con condiciones de contorno y de carga específicas, y que además se desarrollan en tiempos naturales. Esto quiere decir que sus relaciones no son generales (por ejemplo, se genera un modelo específico de fluencia y otro de relajamiento tensional).

La mayoría de los modelos empíricos se obtienen de ajustar resultados experimentales de ensayos de fluencia, con relajamiento tensional y velocidad de deformación constante. Una de sus principales ventajas es que reflejan el comportamiento real de los suelos. A pesar de tener limitada aplicación y, en ocasiones, cierta inconsistencia teórica, se emplean como base para el desarrollo de relaciones constitutivas más sofisticadas.

A su vez las relaciones empíricas que simulan el comportamiento dependiente del tiempo de los suelos, se dividen en: relaciones empíricas simples, obtenidas directamente del ajuste de los datos observados en los ensayos con funciones matemáticas simples; y modelos empíricos compuestos, que provienen de combinar dos o más relaciones empíricas simples.

\subsubsection{Relaciones empíricas simples}

Las relaciones experimentales simples, también denominadas primarias (Liingaard et al., 2004), que reflejan el comportamiento reológico del suelo ensayado se pueden dividir a su vez en: la ley de fluencia semilogarítmica, el modelo de fluencia de Singh y Mitchell, el modelo de relajación de Lacerda y Houston, el modelo de relajación de Prevost, y el enfoque de la velocidad de deformación. 


\section{i. Ley de fluencia semilogarítmica}

Esta ley viene asociada al concepto de consolidación o compresión secundaria entendida como un fenómeno de deformación bajo carga efectiva constante, tras finalizar la etapa de consolidación primaria. Numerosas investigaciones han demostrado que resulta muy práctico dibujar la compresión secundaria observada en un ensayo edométrico frente al logaritmo del tiempo. Para describir la magnitud de las deformaciones por fluencia se emplea el coeficiente de compresión secundaria descrito a continuación:

ó

$$
C_{\alpha e}=\frac{\Delta e}{\Delta \log t}
$$

$$
C_{\alpha \varepsilon}=\frac{\Delta e}{\left(1+e_{i}\right) \cdot \Delta \log t}=\frac{\varepsilon_{z}}{\Delta \log t}=\frac{C_{\alpha e}}{1+e_{i}}
$$

donde: $e$, índice de huecos; $\varepsilon_{z}$, deformación vertical; $e_{i}$, índice de huecos inicial; $t$, tiempo; $C_{\alpha e}$, coeficiente de compresión secundaria con respecto a $e ; C_{\alpha \varepsilon}$, coeficiente de compresión secundaria con respecto a $\varepsilon$.

Dentro del marco de referencia de la ley logarítmica existen tres interpretaciones que afectan al coeficiente de consolidación secundaria $C_{\alpha}$ $\left(C_{\alpha e} \circ C_{\alpha \varepsilon}\right)$.

- $C_{\alpha}$ constante

El enfoque más sencillo de interpretación de la ley semilogarítmica es considerar que el coeficiente $C_{\alpha}$ es constante para cada tipo de suelo. Esto es una simplificación de la fluencia volumétrica confinada de cualquier suelo, ya que muchos estudios sobre los factores que influyen en $C_{\alpha}$ han demostrado que la tensión efectiva vertical de los ensayos edométricos $\left(\sigma_{z}^{\prime}\right)$, el tiempo $t, \mathrm{y}$, otros factores, afectan a la compresión secundaria. 
Si se evalua en términos de deformación vertical por fluencia, el incremento de la compresión secundaria para un suelo dado se puede escribir como:

$$
\varepsilon_{z}=C_{\alpha \varepsilon} \cdot \log \left(1+\frac{t}{t_{i}}\right)
$$

donde: $\varepsilon_{z}$, deformación vertical; $t$, tiempo; $t_{i}$, tiempo de referencia. Una de las mayores dificultades que aparecen al utilizar la ecuación (4), es decidir cuando comienza la deformación por fluencia, es decir, determinar el tiempo de referencia $t_{i}$ (Liingaard et al., 2004).

La suposición de que $C_{\alpha \varepsilon}$ es constante es aceptable cuando se trabaja en el rango normalmente consolidado. La variación de $C_{\alpha \varepsilon}$ con la tensión efectiva decrece considerablemente cuando el estado de tensiones efectivas se encuentra por debajo de la presión de preconsolidación (Mesri, 1973).

- $C_{\alpha} / C_{c}$ constante

Walker \& Raymond (1968) muestran que la velocidad de consolidación secundaria en ensayos de laboratorio en muestras de la "Leda Clay" parece ser linealmente dependiente al índice de compresión $C_{c}$, para todo el rango de tensiones efectivas, con una media de $C_{\alpha} / C_{c}$ de aproximadamente 0,025 . El índice de compresión basado en esta interpretación se define como:

$$
C_{c e}=\frac{\Delta e}{\Delta \log \left(\sigma_{z}^{\prime}\right)} \quad 0 \quad C_{c \varepsilon}=\frac{C_{c e}}{1+e_{i}}
$$

donde $e_{i}$, índice de huecos inicial; $\sigma_{z}^{\prime}$, tensión vertical efectiva; y $C_{c e} \mathrm{y}$ $C_{c \varepsilon}=$ índices de compresión con respecto a e y $\varepsilon$, respectivamente.

Mesri (1973) concluye que los suelos que son muy compresibles en la primera fase (consolidación primaria) también tendrán este comportamiento en la segunda fase. Esto concuerda con los numerosos estudios existentes que analizan la relación entre $C_{\alpha}$ y $C_{c}$. 
Mesri y Godlewski (1977) encontraron que $C_{\alpha}$ depende de la tensión efectiva vertical aplicada y está relacionado con la presión de preconsolidación. Se ha demostrado que ambos $C_{\alpha}$ y $C_{c}$ aumentan a medida que la tensión efectiva se aproxima a la presión de preconsolidación, luego alcanza un máximo sobre, o muy próximo a, la preconsolidación. Dspués permanece relativamente constante. Durante estos cambios tensionales la relación se mantiene aproximadamente constante. Por lo que, combinando la ley logarítmica con el concepto de $C_{\alpha} / C_{c}$ constante, la ecuación (4) se puede reescribir como:

$$
\varepsilon_{z}=\frac{1}{m^{\prime}} \cdot C_{c \varepsilon} \cdot \log \left(1+\frac{t}{t_{i}}\right), m^{\prime}=\frac{C_{c \varepsilon}}{C_{\alpha \varepsilon}}
$$

donde $C_{c \varepsilon}$, índice de compresión con respecto a $\varepsilon_{z}$ que corresponde al estado tensional para el cual se ha determinado el valor de $C_{\alpha \varepsilon}$, y $\mathrm{m}^{\prime}$ es un parámetro que define la relación única entre la compresión $\left(C_{c}\right)$ y la compresión secundaria $\left(C_{\alpha}\right)$.

- $C_{\alpha}$ variable con el tiempo

Muchos autores, incluidos Mesri y Godlewski (1977), cuestionan el concepto de un valor de $C_{\alpha} / C_{c}$ único y constante, en el cual se acepta que $C_{\alpha}$ y $C_{c}$ son independientes del tiempo. En general, $C_{\alpha}$ no permanece constante con el tiempo. Yin (1999) fue el primero en proponer el concepto de que este parámetro representativo de la fluencia variaba con el tiempo. Su motivación fue el observar que la ley logarítmica podría causar un error considerable en la estimación del asiento a largo plazo, debido a que cuando el tiempo tiende a infinito, también lo hace la deformación. De este modo, la ley logarítmica puede sobrestimar el asiento por fluencia a largo plazo.

Yin (1999) presenta una nueva función de fluencia que es capaz de describir el comportamiento no lineal por fluencia como una función del tiempo, todo ello dentro del marco de la ley logarítmica: 


$$
\varepsilon_{z}=\frac{\psi}{v} \cdot \ln \left(\frac{t+t_{0}}{t_{0}}\right)
$$

donde $\psi / v$ se define como

$$
\frac{\psi}{v}=\frac{\psi_{0}^{\prime}}{1+\left(\psi_{0}^{\prime} / \varepsilon_{\infty}\right) \cdot \ln \left[\left(t+t_{0}\right) / t_{0}\right]}
$$

$Y$ donde $v=1+e$, es el volumen específico. Si la relación $\psi / v$ es constante (igual a $C_{\alpha \varepsilon} / \ln (10)$ ), la ecuación (4) se simplifica a la ley logarítmica descrita en la ecuación (7). Según muestra la ecuación (8) $\psi / v$ no es una constante, sino que decrece con el tiempo. Cuando el tiempo es infinito, la deformación por fluencia es $\varepsilon_{z}=\varepsilon_{\infty}$. En consecuencia, $\varepsilon_{\infty}$ es la deformación límite y es otro parámetro del modelo, así como $\psi_{0}^{\prime} \circ t_{0}$.

Se puede concluir que la limitación de la ley logarítmica se encuentra principalmente en que ésta es estrictamente válida para condiciones unidimensionales y simplificadas. En las expresiones presentadas, la deformación varía con el logaritmo del tiempo, esto significa que el suelo endurece con el tiempo. Implica que cualquier aplicación de estas expresiones requiere la definición de un origen para la escala temporal. Para solucionar las dificultades de definir el origen de las deformaciones por fluencia, Suklje (1957) introdujo el enfoque de la velocidad de deformación ("strain rate approach"), el cual será expuesto más adelante.

\section{ii. Modelo de fluencia de Singh \& Mitchell}

Singh \& Mitchell (1968) proponen una ecuación experimental basada en tres parámetros y formulada a partir de los resultados de ensayos triaxiales drenados y no drenados en varios tipos de arcillas. Esta ecuación muestra buena correspondencia con las velocidades de deformación desviadora observadas en muchos suelos, para deformaciones anteriores a la rotura por fluencia.

El modelo describe el comportamiento fluyente de los suelos arcillosos bajo rangos de tensiones de alrededor que varían del 30\% hasta un máximo 
de $90 \%$ o más de la resistencia inicial. En este dominio, los autores observaron una relación general entre el logaritmo de la velocidad de deformación axial y el logaritmo del tiempo, independientemente de que esa relación de la deformación frente al logaritmo del tiempo sea lineal o no lineal:

$$
\dot{\varepsilon}_{1}=A e^{\overline{\alpha q}} \cdot\left(\frac{t_{i}}{t}\right)^{m}
$$

donde $\bar{\alpha}=\alpha q_{\max }$ y $\bar{q}=q / q_{\max }$; el parámetro $A$ se interpreta como una propiedad del suelo que refleja su composición, estructura, e historia tensional; $\alpha$ indica la intensidad del efecto de la tensión en la velocidad de deformación y el parámetro $m$ controla el gradiene al cual la velocidad de deformación axial decrece con el tiempo. Los tres parámetros $A, \alpha$ y $m$ se pueden determinar mediante ensayos de fluencia ordinarios para cualquier tipo de suelo (Singh \& Mitchell, 1968). $\bar{q}$ es el nivel de tensión desviadora expresado como el cociente entre la tensión de fluencia q y la resistencia $q_{\max }$ al inicio del proceso de fluencia. La integración de la ecuación (9) produce una relación general en función del tiempo, obteniéndose la deformación axial. Se producen dos soluciones, una para $m=1$, para la cual la deformación axial varía linealmente con el logaritmo del tiempo, y otra para $m \neq 1$ (valor más frecuente), dando lugar a una relación no lineal entre la deformación axial y el logaritmo del tiempo (ver Figura 23 b).

De este modo, se esclarece que el modelo de Singh y Mitchell es capaz de reproducir la fluencia atenuada (cuando la deformación alcanza un valor asintótico) y la fluencia no atenuada, dependiendo del valor que tome el parámetro del suelo, $m$.

En cambio, presenta las siguientes desventajas (Liingaard et al, 2004): el modelo describe el comportamiento fluyente para un nivel tensional constante en condiciones unidimensionales; además, el modelo solo es válido para la primera carga del terreno; por otra parte, para un suelo en concreto, $m$ se asume constante; otras curvas de fluencia a diferentes niveles tensionales pueden involucrar diferentes valores de $m$ para el mismo suelo. 


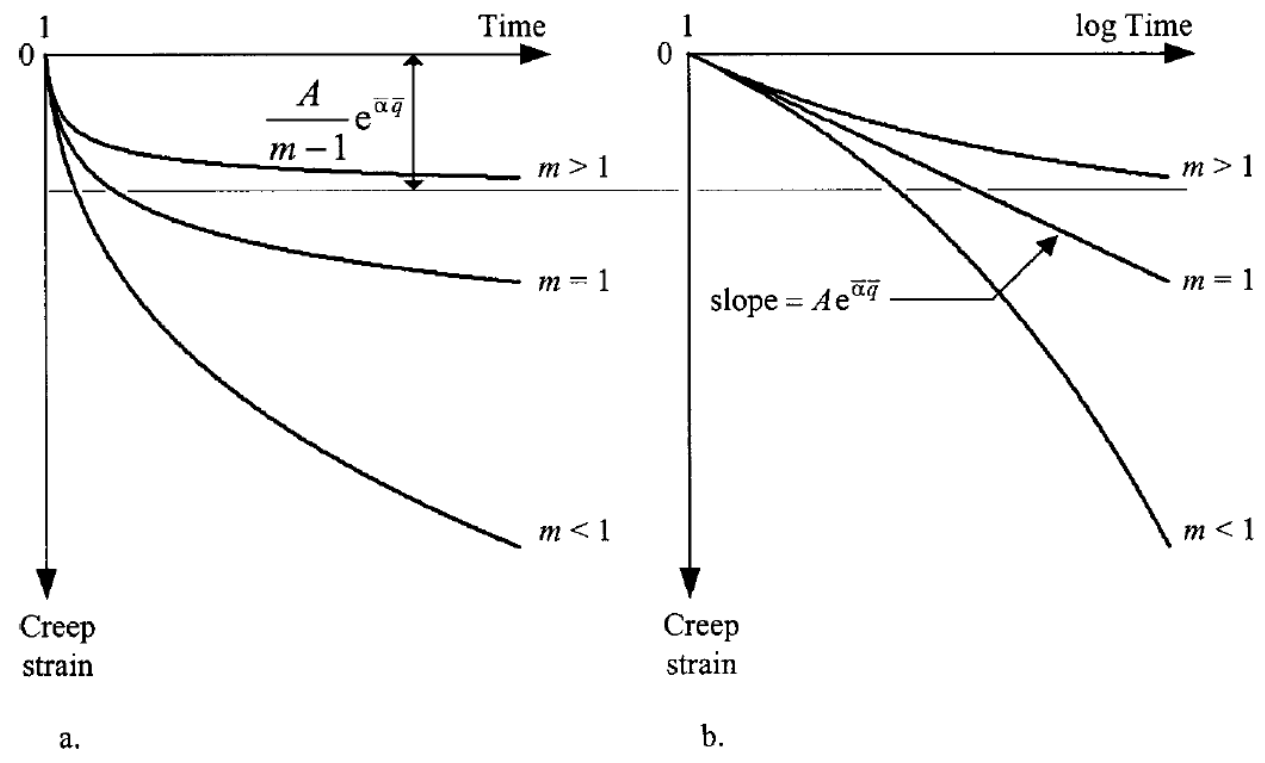

Figura 23: Curvas de fluencia deducidas según el modelo de Singh y Mitchell (1968).

(a) Deformación-tiempo, (b) Deformación-logaritmo del tiempo. (Liingaard et al., 2004).

iii. Modelo de relajación de Lacerda \& Houston

El propósito del estudio de Lacerda \& Houston (1973) fue correlacionar los parámetros que describen el comportamiento de relajamiento tensional con los parámetros de fluencia, obtenidos en los ensayos de fluencia tradicionales realizados con arcillas y arenas. Para describir la relación entre los parámetros de relajamiento tensional y los de fluencia aplican la ecuación (9) de Singh y Mitchell (1968). Lacerda y Houston (1973) observaron que cuando se inicia un ensayo de relajación después de deformar un suelo a velocidad de deformación constante hasta un desviador $q_{0}$, se produce una disminución de la tensión $q$ con el tiempo. Esta disminución varía linealmente con el logaritmo del tiempo después de sobrepasar un tiempo inicial $t_{0}$ :

$$
\frac{q}{q_{0}}=\frac{\bar{q}}{\overline{q_{0}}}=1-s \cdot \log \left(\frac{t}{t_{0}}\right), \text { para } t>t_{0}
$$

donde $\bar{q}$, es el nivel de tensión desviadora; $\overline{q_{0}}$, nivel de tensión desviadora en el instante $t_{0} ; t_{0}$, periodo de tiempo transcurrido hasta el inicio de la relajación; $t$, tiempo transcurrido desde el inicio de la relajación; y $s$, pendiente de la curva de relajación en un diagrama $\frac{q}{q_{0}}-\log (t)$, análogo al 
de la Figura 24 b. La pendiente $s$ está relacionada con los parámetros $\bar{\alpha}$ y $m$ de la ecuación (9) del siguiente modo:

$$
s=\frac{\Phi}{\overline{q_{0}}}, \text { donde } \Phi=\frac{2.3(1-m)}{\bar{\alpha}}
$$

Se debe tener en cuenta que la ecuación (11) está establecida para el caso $m<1$, que corresponde al caso de fluencia no atenuada (es decir, se pueden dar las tres etapas de la fluencia). Las ecuaciones (10) y (11) se derivan invirtiendo la ecuación (9), como indican Lacerda y Houston (1973).

La ecuación (10) es una aproximación del comportamiento real de relajamiento tensional de un suelo. Describe el fenómeno mediante una línea recta en un diagrama $q / q_{0}-\log (t)$ y dos parámetros $s$ y $t_{0}$. El tiempo $t_{0}$ también se denomina tiempo de retardo, debido a que las tensiones no comienzan a relajarse inmediatamente después de que la velocidad de deformación $\dot{\varepsilon}_{1}$ se vuelva nula. El tiempo $t_{0}$ es una variable que depende del tipo de suelo y de la velocidad de deformación como muestra la Figura 24.

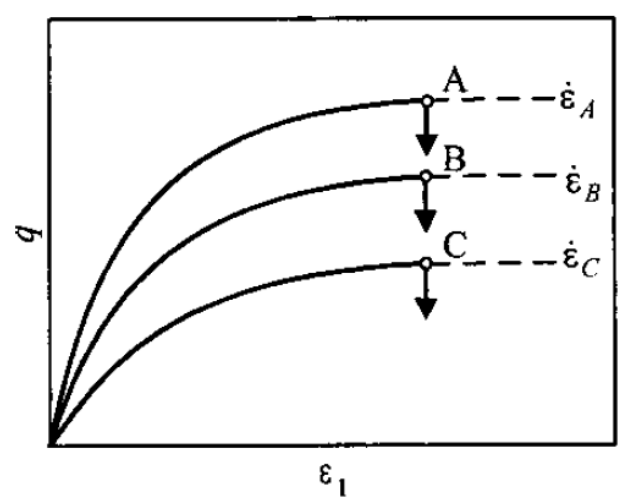

a.

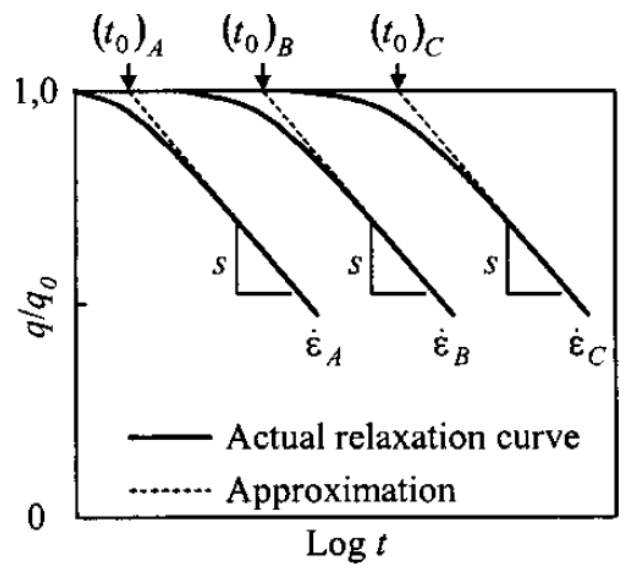

b.

$$
\dot{\varepsilon}_{A}>\dot{\varepsilon}_{B}>\dot{\varepsilon}_{C}=\text { Strain rate prior to relaxation }
$$

Figura 24: (a) Curvas tensión-deformación para diferentes velocidades de deformación. La relajación comienza en los puntos A, B y C. (b) Curvas de relajamiento tensional para varias velocidades de deformación antes de la relajación.

(Lacerda \& Houston, 1973) 
De nuevo, este modelo está limitado a aplicarse en condiciones unidimensionales, debido a que la expresión (8) proviene del modelo también unidimensional de Singh y Mitchell. Se debe resaltar que el trabajo de Lacerda y Houston (1973) está basado en resultados de ensayos de relajamiento tensional no drenados. Además, estos autores y Murayama \& Shibata (1961), observaron en arcillas que la variación de la sobrepresión durante los ensayos no drenados era prácticamente igual a cero.

La ecuación (10) predice un comportamiento no atenuado de relajamiento tensional porque la función logarítmica tiende a infinito junto con el tiempo. Esto significa que el modelo está limitado para aplicarse solo a un valor de tiempo concreto

\section{iv. Modelo de relajación de Prevost}

Prevost (1976) desarrolló un modelo experimental para describir el comportamiento de arcillas saturadas bajo condiciones triaxiales no drenadas. En el caso del relajamiento tensional donde el estado inicial anterior al relajamiento se alcanza aplicando una tensión cortante a velocidad de deformación constante, la relación propuesta es:

$$
q\left(\varepsilon_{1,0}, t\right)=q\left(\varepsilon_{1,0}, t_{0}\right)-\left[q\left(\varepsilon_{1,0}, t_{0}\right)-q\left(\varepsilon_{1,0}, 0\right)\right] \cdot \tanh \left[b \cdot \ln \left(\frac{t}{t_{0}}\right)\right]
$$

donde $q\left(\varepsilon_{1,0}, t\right)$, tensión desviadora actuando para una deformación axial fija $\varepsilon_{1,0}$ y un tiempo $t>t_{0} ; q\left(\varepsilon_{1,0}, t_{0}\right)$, tensión desviadora actuando al inicio del relajamiento tensional y alcanzada llevando a cabo un ensayo de corte a velocidad de deformación constante $\dot{\varepsilon}_{1}=a$ ( $a$ es una constante) hasta un nivel de deformación $\varepsilon_{1,0}$ en un tiempo $t_{0}$, es decir, $\varepsilon_{1,0}=a \cdot t_{0}$. $q\left(\varepsilon_{1,0}, t_{0}\right) ; q\left(\varepsilon_{1,0}, 0\right)$, tensión desviadora a una deformación $\varepsilon_{1,0}$ en un ensayo "estático" no drenado ( $\dot{\varepsilon}_{1}$ aproximadamente 0$) ; b$, es una constante experimental; y $t_{0}$, instante de tiempo en el que comienza el relajamiento. De este modo, los parámetros de entrada son $a, t_{0}, b, q\left(\varepsilon_{1,0}, 0\right)$ y $q\left(\varepsilon_{1,0}, t_{0}\right)$ y los contornos de la ecuación (12) son los que se presentan en la Figura 25. 


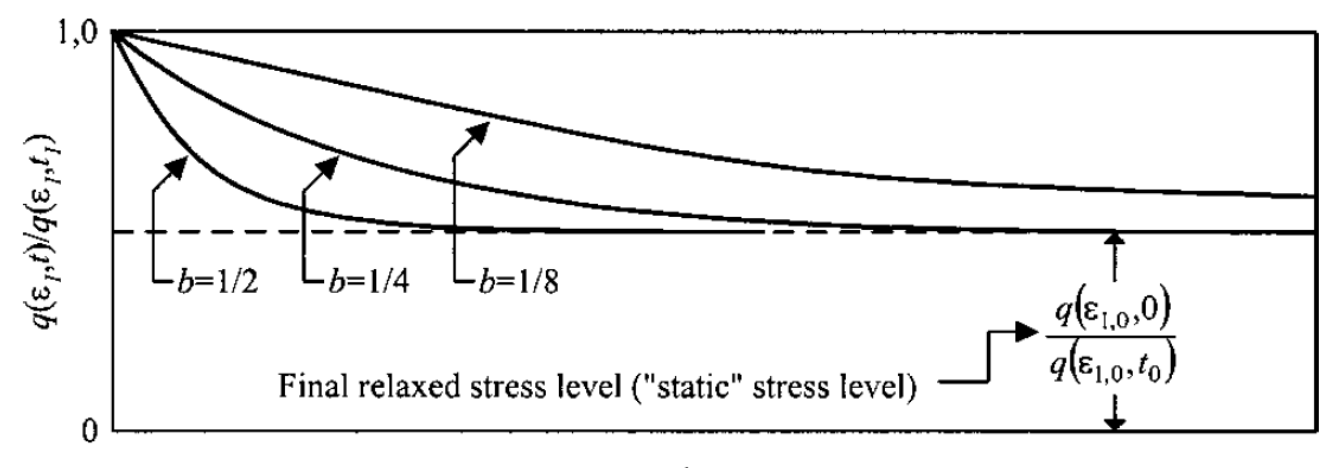

$\log t$

Figura 25: Nivel de tensión desviadora frente a logaritmo de tiempo durante el relajamiento tensional. La ecuación (10) se ha representado para diferentes valores del parámetro b. (Prevost, 1976)

El modelo de Prevost es capaz de describir relaciones de relajación no lineales en espacios $q-\log (t)$, en contraste con el modelo de Lacerda y Houston (1973) cuya relación presentada en el mismo espacio es lineal. Otra distinción es que el modelo de Prevost tiende a un estado final relajado de tensión desviadora (o estado estático de tensión) cuando el tiempo tiende a infinito, mientras que el modelo de Lacerda y Houston nunca alcanza un nivel final "estático".

La expresión (12) fue aplicada satisfactoriamente por Silvestri, et al. (1988) para interpretar los resultados de ensayos de relajamiento tensional no drenados en arcillas sensitivas blandas.

v. Enfoque de la velocidad de deformación ("strain rate approach")

Esta propuesta está basada en la existencia de una relación única entre el estado actual de tensiones verticales efectivas $\sigma_{z}^{\prime}$ y la deformación $\left(\varepsilon_{z} \circ e\right)$ para una velocidad de deformación constante $\left(\begin{array}{lll}\dot{\varepsilon}_{z} & \circ & \dot{e}\end{array}\right)$ dada, independientemente de la historia tensión-deformación-tiempo previa. Este comportamiento está de acuerdo con las investigaciones desarrolladas por Vaid \& Campanella (1977). 
El concepto original fue propuesto por Sukjle (1957) y ha sido investigado en detalle por Leroueil, et al. (1985), quienes lo confirman con ensayos de escalones múltiples de carga, ensayos de velocidad de deformación constante, ensayos de gradiente hidráulico controlado y ensayos de fluencia a largo plazo. Basándose en investigaciones experimentales con varios tipos de arcillas, los autores informaron que la variación de la tensión efectiva con el tiempo $\sigma_{z}^{\prime}$ no tiene una influencia significativa en el comportamiento reológico de las arcillas. Por esta razón ${\sigma_{z}^{\prime}}_{z}$ se elimina de la relación propuesta. Proponen que la relación entre $\sigma_{z}^{\prime}, \varepsilon_{z}$ y $\dot{\varepsilon}_{z}$ se puede describir completamente mediante dos ecuaciones, una proporciona la variación de la presión de preconsolidación con la velocidad de deformación:

$$
\sigma_{z, p c}^{\prime}=f\left(\dot{\varepsilon}_{z}\right)
$$

y la otra presenta la relación entre la tensión efectiva normalizada y la deformación:

$$
\frac{\sigma_{z}^{\prime}}{\sigma_{z, p c}^{\prime}}=g\left(\varepsilon_{z}\right)
$$

Una vez que las dos relaciones anteriores son conocidas para un suelo determinado, se puede reconstruir cualquier relación tensión-deformaciónvelocidad de deformación para ese terreno.

Las expresiones (13) y (14) se han representado en la Figura 26. La curva tensión normalizada-deformación suele variar de una arcilla a otra. Mediante la curva de la Figura 26 (a) se ilustra la capacidad de la arcilla para fluir a distintas velocidades de deformación; se puede apreciar como para tensiones verticales reducidas, la magnitud de la velocidad de deformación impuesta apenas afecta a la magnitud de la deformación desarrollada. 


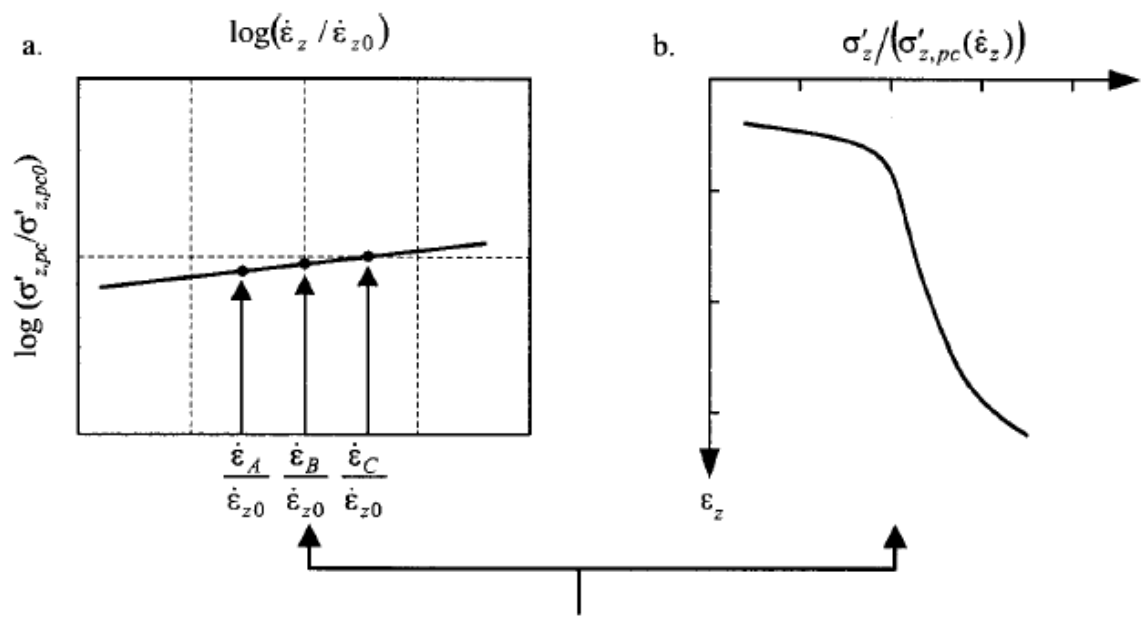

c.

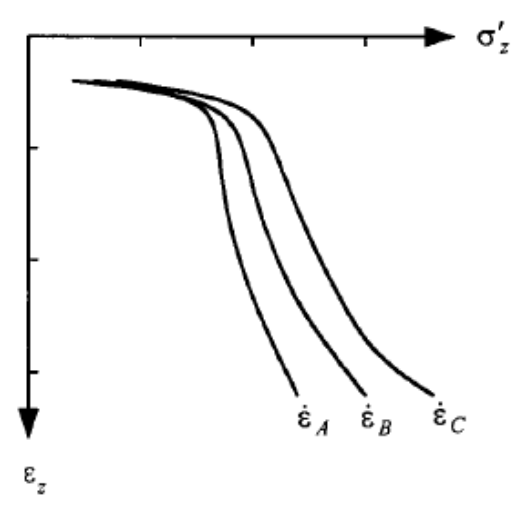

$$
\dot{\varepsilon}_{A}>\dot{\varepsilon}_{B}>\dot{\varepsilon}_{C}
$$

Figura 26: Relación tensión-deformación-velocidad de deformación del strain rate approach: (a) Variación normalizada de la presión de preconsolidación con la velocidad de deformación, Ec. (11); (b) Relación entre la tensión efectiva normalizada y la deformación, Ec. (12); y (c) Curvas experimentales obtenidas a distintas velocidades de deformación. (Leroueil et al., 1985)

Leroueil et al. (1985) no recomiendan usar ninguna solución analítica para $f$ y $g$ en las ecuaciones (13) y (14), pero al combinarlas la forma general de cualquier solución debe ser dada mediante la siguiente expresión:

$$
\dot{\varepsilon}_{z}=f^{-1}\left(\frac{\sigma_{z}^{\prime}}{g\left(\varepsilon_{z}\right)}\right)
$$

El modelo propuesto por Leroueil et al. (1985), dado por las ecuaciones (13) y (14), se basa en ensayos en los cuales las deformaciones son siempre crecientes, y solo ha sido verificado bajo ciertas condiciones. No debería ser 
usado cuando la arcilla sufre relajamiento bajo deformación constante. En los ensayos de relajación, la deformación es constante, la velocidad de deformación es cercana a cero y, para esas condiciones, la singularidad de una relación tensión efectiva-deformación-velocidad de deformación implica una tensión efectiva constante. Sin embargo, es bien conocido que la tensión efectiva disminuye durante la relajación. Este error es debido al hecho de que el concepto fue desarrollado respecto a deformaciones totales. Para reproducir el fenómeno de la relajación, el modelo debe ser descompuesto en sus componentes elástica y plástica, respectivamente. Otra limitación es que el concepto está desarrollado principalmente a partir de observaciones en el rango normalmente consolidado, el cual proporciona prediciones pobres en el rango fuermente sobreconsolidado, donde las deformaciones elásticas son relativamente significantes.

Una de las características del modelo de Leroueil et al. (1985) es que las propiedades viscosas de la arcilla están directamente relacionadas con las propiedades de la deformación primaria. Esto se refleja cuando la ecuación (13) se aproxima a una relación lineal en el diagrama log-log de Leroueil y Marques (1996):

$$
\log \frac{\sigma_{z, p c}^{\prime}}{\sigma_{z, p c 0}^{\prime}}=A+\frac{1}{m^{\prime}} \cdot \log \frac{\dot{\varepsilon_{z}}}{\varepsilon_{z 0}^{\cdot}}
$$

donde $A$ y $m^{\prime}$ son constantes, y $\sigma_{z, p c 0}^{\prime}$ y $\varepsilon_{z 0}^{\prime}$ son valores de referencia.

Leroueil y Marques (1996) demostraron que la ecuación (16) es aplicable a numerosas arcillas inorgánicas y que el valor correspondiente a $m$ está relacionado con $C_{\alpha} / C_{c}$ como sigue:

$$
\frac{1}{m^{\prime}}=\frac{C_{c}}{C_{\alpha}}
$$

Esto implica que el concepto de $C_{\alpha} / C_{c}=c t e$. y el enfoque de la velocidad de deformación son equivalentes cuando se describe el comportamiento viscoso durante la consolidación secundaria. 


\section{vi. Comentarios sobre los modelos empíricos simples}

Los modelos empíricos para suelos están fuertemente influenciados por los conceptos clásicos de la reología de los materiales. Esto es, que la fluencia, la relajación y la dependencia con la velocidad están consideradas como debidas al mismo mecanismo básico, y, de acuerdo a Sheahan \& Kaliakin (1999) esta idea se denomina, "el principio de correspondencia". principio enuncia que uno de los fenómenos, por ejemplo, la relajación, puede deducirse a partir del comportamiento fluyente observado, y viceversa. Los siguientes enunciados confirman la validez del principio de correspondencia: (a) No hay modelos únicos para la relajación. El modelo de Lacerda y Houston (1973) está basado en una ley de fluencia primeramente adoptada, y (b) el parámetro $\boldsymbol{m}^{\prime}$ obtenido de ensayos a velocidad constante de deformación es aceptado como equivalente al cociente $C_{\alpha} / C_{c}$ donde $C_{\alpha}$ se obtiene a partir de ensayos de fluencia.

La diferencia fundamental entre los modelos primarios se encuentra en si estos son de endurecimiento por deformación o de endurecimiento con el tiempo. Los primeros están caracterizados por ecuaciones en la cuales la deformación funciona como el parámetro de endurecimiento, mientras que los segundos están caracterizados por ecuaciones en la cuales el tiempo funciona como el parámetro de endurecimiento. Las relaciones de endurecimiento debido al tiempo se pueden escribir como:

$$
\varepsilon^{c}=f(\sigma) g(t) o \dot{\varepsilon}^{c}=f(\sigma) g(t)
$$

donde $\varepsilon^{c}$, deformación por fluencia; $\dot{\varepsilon}^{c}$, velocidad de deformación por fluencia; y $f$ y $g$ funciones de la tensión y del tiempo, respectivamente. Las dos relaciones que se presentan en la ecuación (18) se refieren normalmente al modelo de endurecimiento con el tiempo, mientras que la ecuación de la izquierda se denomina modelo de deformación total.

De acuerdo a la ecuación (18), la ley logarítmica y el modelo de Singh y Mitchell (1968) se pueden categorizar como modelos de endurecimiento con el tiempo. 
La relación funcional de un modelo de endurecimiento por deformación se puede escribir como:

$$
\dot{\varepsilon}^{c}=f(\sigma) g\left(\varepsilon^{c}\right)
$$

donde $\varepsilon^{c}$, deformación por fluencia; $\dot{\varepsilon}^{c}$, velocidad de deformación por fluencia; y $f$ y $g$ funciones de la tensión y de la deformación, respectivamente. Está reconocido que la forma general del enfoque de la velocidad de deformación, que proporciona una relación única entre la tensión, la deformación, y la velocidad de deformación, es equivalente a la expresión funcional de un modelo de tipo endurecimiento por deformación.

\subsubsection{Relaciones empíricas compuestas}

Como se ha mencionado, los modelos semiempíricos secundarios están clasificados como modelos obtenidos a partir de la combinación de dos o más modelos primarios. Estas relaciones pueden, en cierto modo, ser empleadas como modelos tensión-deformación-tiempo o tensión-deformaciónvelocidad de deformación, que generan soluciones para la fluencia y la relajación dentro de un modelo particular. Se entienden como soluciones cerradas de diferentes fenómenos, de manera diferente a los modelos viscoplásticos/elásticos revisados en este documento, los cuales se presentan como formulaciones de la velocidad en forma incremental.

\section{i. Modelo de Kavazanjian y Mitchell}

Uno de los primeros intentos para desarrollar una relación constitutiva tensión-deformación-tiempo multiaxial fue propuesto por Kavazanjian \& Mitchell (1977). Estos autores presentaron un modelo de comportamiento general tensión-deformación-tiempo para suelos de grano fino, y fue formulado separando las componentes volumétrica y desviadora. Se acepta que los modelos volumétricos y desviadores tienen una componente instantánea y una componente retardada de la deformación. 
La parte volumétrica está basada en la ley logarítmica para la compresión secundaria con la hipótesis de que $C_{\alpha \varepsilon}$ es aproximadamente constante bajo condiciones normalmente consolidadas. Por lo tanto, la componente volumétrica retardada se puede escribir como:

$$
\dot{\varepsilon_{v}}=\frac{C_{\alpha \varepsilon}}{\ln (10)} \frac{1}{t}
$$

donde $\dot{\varepsilon_{v}}$, velocidad de deformación volumétrica retardada; y $C_{\alpha \varepsilon}$, coeficiente de consolidación secundaria. El modelo desviador esta basado en el modelo de Singh y Mitchell (1968) anteriormente descrito. Cuando la velocidad de deformación axial en la ecuación (9) se toma como la velocidad de deformación a lo largo del eje principal primario, la ecuación (9) se puede describir como:

$$
\dot{\varepsilon}_{1}=A e^{\overline{\alpha q}} \cdot\left(\frac{t_{i}}{t}\right)^{m}
$$

La velocidad de deformación desviadora puede ser calculada de manera indirecta a partir de las deformaciones axiales en condiciones triaxiales según Tavenas, et al. (1978):

$$
\dot{\varepsilon_{q}}=\dot{\varepsilon_{1}}-\dot{\varepsilon_{v}} / 3
$$

donde $\dot{\varepsilon}_{v}$ y $\dot{\varepsilon}_{1}$ se obtienen a partir de las ecuaciones (20) y (21) respectivamente.

La combinación de velocidades de fluencia de corte y volumétrica también fue propuesta por Poulos et al. (1976). Conviene señalar que el trabajo de Kavazanjian y Mitchell (1977) se ha desarrollado dentro de un modelo viscoplástico general, como se verá en la sección 2.3.6. Modelos generales tensión-deformación-tiempo. 


\section{ii. Modelo de Tavenas}

Tavenas et al. (1978) también dividen las deformaciones por fluencia en sus componentes volumétrica y desviadora. Basándose en resultados de ensayos de fluencia en arcillas ligeramente sobreconsolidadas, concluyeron que el desarrollo con el tiempo de ambas deformaciones, volumétrica y de corte, pueden representarse mediante la ecuación (9) del siguiente modo:

$$
\begin{aligned}
& \dot{\varepsilon}_{v}=B f\left(\sigma^{\prime}{ }_{i j}\right) \cdot\left(\frac{t_{i}}{t}\right)^{m} \\
& \dot{\varepsilon}_{q}=A g\left(\sigma^{\prime}{ }_{i j}\right) \cdot\left(\frac{t_{i}}{t}\right)^{m}
\end{aligned}
$$

donde $f\left(\sigma_{i j}^{\prime}\right)$ y $g\left(\sigma_{i j}^{\prime}\right)$ son funciones del estado actual de tensiones efectivas $\sigma_{i j}^{\prime}$; los parámetros $A$ y $B$, reflejan propiedades del suelo como la composición, la estructura y la historia tensional; y $m$, parámetro que controla la velocidad, la cual disminuye con el tiempo. Basándose en las formas de las líneas de contorno de velocidades de deformación iguales, para $t=100 \mathrm{~min}$, Tavenas et al. (1978) sugirieron que las funciones tensionales $f\left(\sigma^{\prime}{ }_{i j}\right)$ y $g\left(\sigma_{i j}{ }_{i j}\right)$ deben ser expresadas en términos de la superficie del estado límite, también denominada superficie de fluencia.

Sekiguchi (1985) indicó que el cociente entre la velocidad por fluencia volumétrica y por fluencia desviadora puede ser una función, únicamente, del estado actual de tensiones $\sigma_{i j}^{\prime}$. Atendiendo a las ecuaciones (23) y (24) lo anterior se puede escribir como:

$$
\frac{\dot{\varepsilon}_{v}}{\dot{\varepsilon}_{q}}=\frac{f\left(\sigma^{\prime}{ }_{i j}\right)}{g\left(\sigma^{\prime}{ }_{i j}\right)}=h\left({\sigma^{\prime}}_{i j}\right)
$$

donde $h\left(\sigma^{\prime}{ }_{i j}\right)$ es una función material. La ecuación (25) es actualmente una regla de flujo para la deformación por fluencia. De acuerdo a la ecuación (25), las deformaciones volumétricas representadas frente a las deformaciones desviadoras durante un ensayo de fluencia deben formar una línea recta, independientemente del tiempo transcurrido. Sin embargo, la ecuación (25) solo es válida si el valor de $\boldsymbol{m}$ para la fluencia desviadora coincide con el valor de $\boldsymbol{m}$ para la fluencia volumétrica, lo cual no suele ser el 
patrón general de comportamiento. Esto ha sido estudiado en numerosas investigaciones (Feda, 1992; Lade \& Liu, 1998; Tatsuoka, et al., 2000). No obstante, la regla de flujo de la ecuación (25) ha sido empleada muy a menudo en modelos constitutivos viscoplásticos.

iii. Otros modelos unidimensionales para la consolidación secundaria de arcillas

Como el principal objetivo de esta investigación no es un caso exclusivo de consolidación secundaria en suelos arcillosos, solo se presentan esquemáticamente, añadiéndolos a los anteriores, dos de los modelos más conocidos: el modelo de Bjerrum, y el modelo de Yin y Graham. Una explicación más detallada ha sido presentada por Liingaard et al. (2004).

El modelo de Bjerrum (1967) presenta una teoría para el análisis del asiento de arcillas normal o ligeramente sobreconsolidadas, que incluye la ley logarítmica. Introduce el concepto de las líneas de tiempo, ya sugeridas por Buisman (1936) y Taylor (1942), como curvas que corresponden a diferentes duraciones de la carga aplicada en un ensayo edométrico. La magnitud de la presión de preconsolidación es diferente para cada una de esas líneas. Otra de las claves del modelo de Bjerrum es que se tiene en cuenta la sobreconsolidación observada en depósitos envejecidos de arcillas normalmente consolidadas se tiene, mediante el concepto de la dependencia con la edad del depósito.

Yin \& Graham (1989a, 1989b, 1994) desarrollaron una serie de modelos para describir el comportamiento unidimensional dependiente del tiempo de los suelos arcillosos, y mediante estudios adicionales (Yin, et al., 1994; Yin \& Graham, 1996) se acoplan ecuaciones para la disipación de las presiones intersticiales. El modelo de Yin y Graham conlleva avances sustanciales con respecto al modelo de Bjerrum y el enfoque de la velocidad de deformación, vistos anteriormente, como la capacidad de modelizar la diferencia entre las arcillas normalmente consolidadas y las arcillas sobreconsolidadas y el fenómeno de la relajación. 
El concepto más novedoso del modelo de Yin y Graham es el del tiempo equivalente, que emplean para reproducir el comportamiento fluyente de arcillas normalmente consolidadas y sobreconsolidadas como una función de la tensión efectiva vertical y su velocidad de aplicación, y la deformación vertical y su velocidad. Esto permite predecir el comportamiento de las arcillas en varias condiciones de ensayo, por ejemplo, ensayos de relajación, de velocidad de deformación constante, y de velocidad de carga constante.

\subsubsection{Modelos reológicos mecánicos}

El enfoque diferencial, mejor conocido como el método de los modelos reológicos mecánicos o analógicos, está basado en relaciones constitutivas que se construyen combinando diferentes modelos de materiales elementales, como son los materiales Hookeanos, de Saint-Vernant, y Newtonianos. Se puede definir un modelo analógico como aquel que se basa en estructuras materiales (p.e. muelles) que funcionan de manera semejante al sistema en consideración.

En los siguientes apartados se exponen los principios básicos del enfoque diferencial, habiéndose simplificado la formulación a condiciones uniaxiales.

Como referencias generales sobre el empleo del método diferencial en la mecánica de suelos se pueden consultar textos clásicos como Murayama \& Shibata (1961); Barden (1965); y Murayama, et al. (1984), entre otros.

La mayoría de los materiales idealizados poseen propiedades elásticas, plásticas y viscosas y se representan en la Figura 27. Las curvas a, b, y c corresponden a cuerpos sólidos, y las curvas $d$, e, y f corresponden a cuerpos viscosos. El estudio del comportamiento de los cuerpos sólidos sometidos a una carga se puede llevar a cabo mediante su relación tensión-deformación $(\tau-\gamma)$, mientras que, en el caso de cuerpos viscosos, es necesario conocer la relación de la deformación $(\gamma)$ con el tiempo $(t)$, (Figura 28), es decir, su velocidad de deformación $(\dot{\gamma})$. Por lo tanto, el comportamiento de estos últimos 
bajo una carga aplicada se define mediante la relación entre la tensión y la velocidad de deformación.

(a)

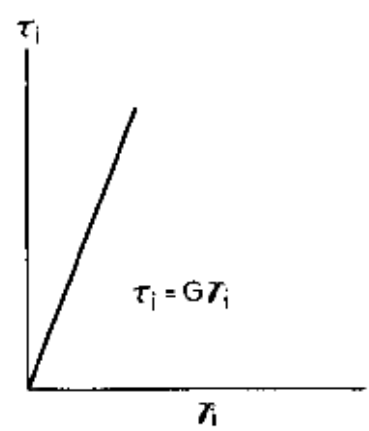

(d)

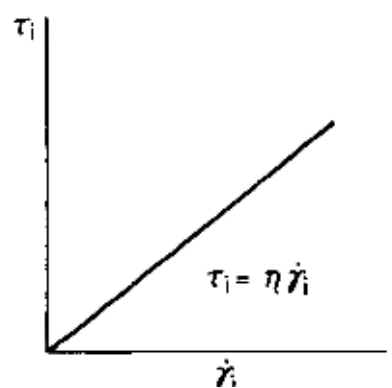

(b)

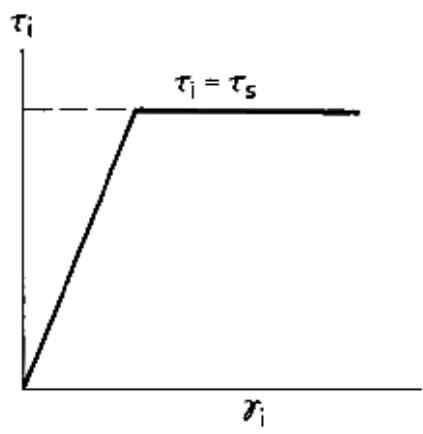

(e)

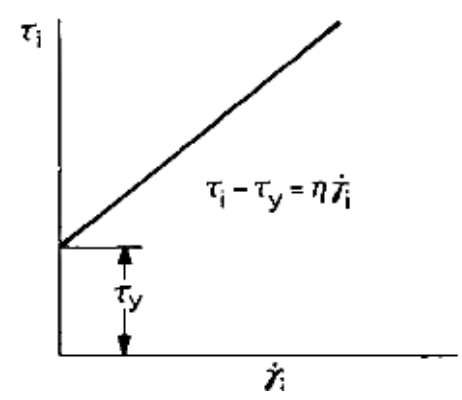

(c)

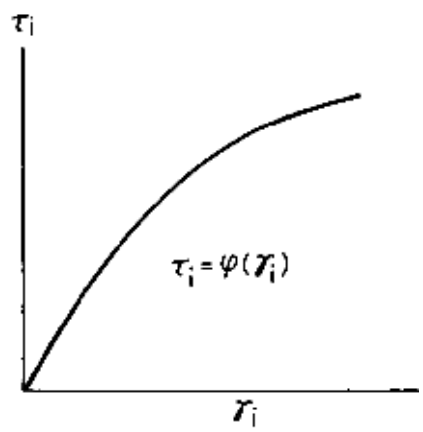

(f)

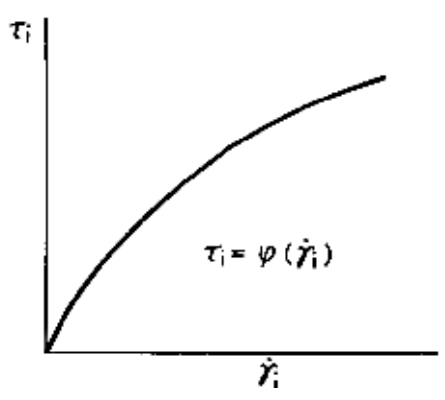

Figura 27. Curvas de deformación de varios cuerpos. (a) elástico; (b) elastoplástico; (c) elástico no lineal; (d) viscoso; (e) viscoplástico; (f) viscoso no lineal. (Vyalov, 1986)

(a)

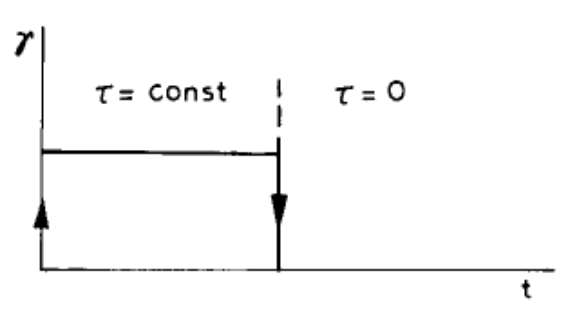

(c)

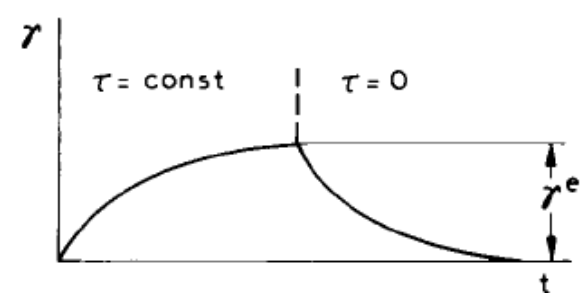

(b)

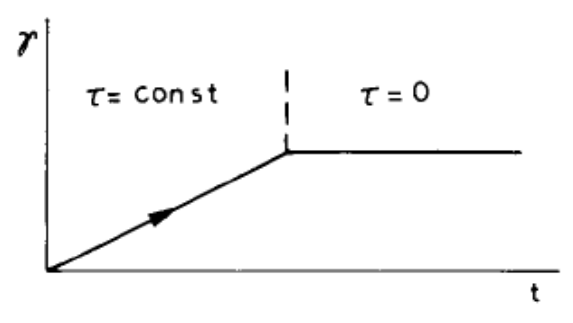

(d)

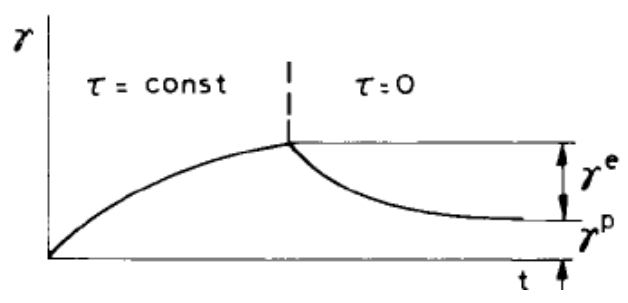

Figura 28. Desarrollo de la deformación con el tiempo al aplicar una carga constante y una posterior descarga en: (a) cuerpo elástico ideal; (b) cuerpo viscoso ideal; (c) cuerpo viscoelástico ideal; (d) cuerpo viscoelastoplástico ideal. (Vyalov, 1986) 
Por ejemplo, la viscoelasticidad supone una elaboración de la respuesta elástica que incorpora los efectos de dependencia de la velocidad de deformación e historia del suelo. Estos efectos son imprescindibles para poder modelar sólidos que poseen fluencia y relajación, dos comportamientos fundamentales que se dan en los polímeros, los suelos e incluso el hormigón. Como se verá más adelante, la forma más sencilla de abordar la viscoelasticidad es mediante modelos reológicos.

Dentro del comportamiento reológico de los sólidos existen varios fenómenos: la fluencia, la recuperación de la fluencia y la relajación de la tensión; han de modelarse con leyes constitutivas distintas de las elásticas. Los modelos reológicos se formularon con dicho objetivo. Estos modelos son sistemas mecánicos elementales que reproducen de forma sencilla los distintos tipos de comportamientos, a partir de una combinación de elementos mecánicos que simulan cuerpos elásticos, viscosos y plásticos (Figura 29).

En la mayoría de los textos referenciados y en el presente documento, se hace uso de las deformaciones longitudinales, $\varepsilon$ (y tensiones normales, $\sigma$ ) $\mathrm{y}$ de las deformaciones de corte, $\gamma$ (y tensiones tangenciales, $\tau$ ), indistintamente, a la hora de plantear la formulación de los modelos reológicos mecánicos básicos. Esta simplificación se hace con el objeto de presentar una explicación simplificada y visual de los modelos mecánicos más conocidos.

Por otro lado, en elasticidad, es bien sabido que las respuestas volumétrica y desviadora están desacopladas, es decir, que incrementos de la tensión principal no generan deformaciones desviadoras, e incrementos de la tensión desviadora no producen deformaciones volumétricas. Se observa experimentalmente, al igual que en el caso elástico, que en el caso viscoelástico, el comportamiento volumétrico y desviador están desacoplados. Además, los modelos viscoelásticos suelen suponer que la respuesta volumétrica es totalmente elástica. 


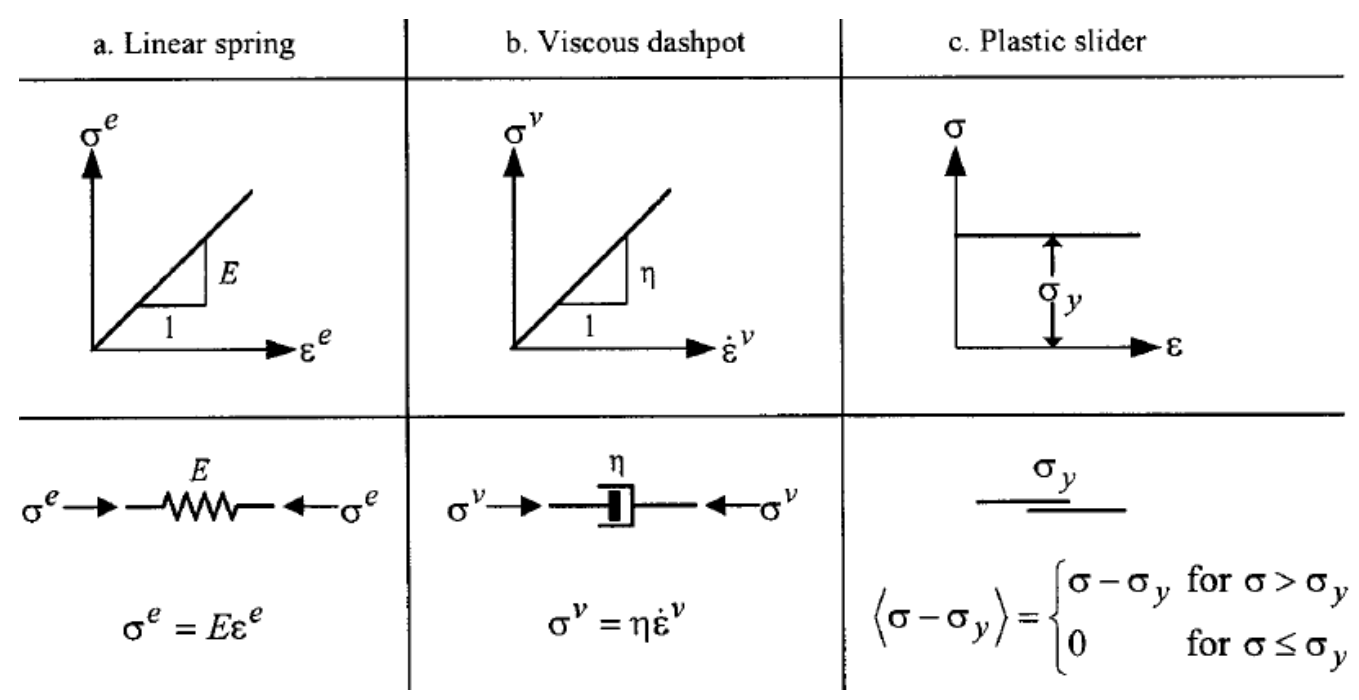

Figura 29. Esquema simplificado de elementos mecánicos: (a) resorte o cuerpo elástico Hookeano; (b) amortiguador o cuerpo viscoso Newtoniano; (c) amortiguador de fricción seca o cuerpo plástico de Saint-Venant. (Liingaard et al., 2004).

El muelle o resorte, se identifica mediante la letra $\mathrm{H}, \mathrm{y}$ es el elemento elástico básico que sigue la ley de Hooke, cuya tensión $\sigma$ es proporcional a la deformación $\varepsilon$, siendo $E$ el módulo elástico del resorte. El amortiguador 0 émbolo ( $N$ ) es el elemento viscoso que se comporta según la ley de Newton, cuya tensión es proporcional a la velocidad de deformación $\dot{\varepsilon}$, donde $\eta$ es el coeficiente de viscosidad. Por último, el amortiguador de fricción seca (SV) obedece la ley de Saint-Venant, donde $\sigma_{y}$ es la tensión por debajo de la cual no se producen deformaciones plásticas o tensión de fluencia, según Bingham. Las distintas expresiones de respuesta de los tres elementos mecánicos se aprecian en la Figura 29.

Cuando se estudia un modelo con conexiones en serie de los tres elementos citados, se simboliza mediante un guión (p.e. H-N); mientras que la conexión en paralelo se distingue con una línea vertical (p.e. $H \mid N)$ (Vyalov, 1986).

En los siguientes apartados se presentan algunos de los modelos clásicos más conocidos, pero existen otras numerosas combinaciones posibles, su descripción se puede consultar en Vyalov (1986), Feda (1992), entre otros. De este modo se estudia la respuesta de los distintos modelos 
ante los fenómenos reológicos de interés, observando cuál de ellos se puede ajustar mejor al caso de estudio y que parámetros del terreno es necesario determinar.

\subsubsection{Modelo de Maxwell}

El modelo reológico de Maxwell, representado mediante la letra $\mathrm{M}=\mathrm{H}-\mathrm{N}$, se basa en la conexión de un muelle y un amortiguador en serie, tal y como se observa en la Figura 30.

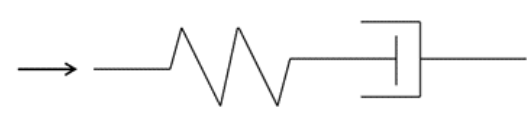

Figura 30. Modelo de Maxwell

Según la disposición del modelo de Maxwell la tensión elástica y la viscosa son idénticas $\left(\tau=\tau_{e}=\tau_{v}\right)$, mientras que la deformación total está distribuida entre los dos elementos $\left(\gamma=\gamma_{e}+\gamma_{v}\right)$ sometidos a la tensión total. La ecuación que gobierna el comportamiento del modelo que resulta es:

$$
\dot{\gamma}_{l}=1 /{ }_{G} \cdot \dot{\tau}_{i}+1 / \eta \cdot \tau_{i}
$$

Cuando se aplica una tensión constante $\tau_{0}$ a la ecuación (26), $\dot{\tau}_{i}=0$, para el estudio del fenómeno de la fluencia, la expresión resultante es:

$$
\dot{\gamma}_{\iota}=1 / \eta \cdot \tau_{0}
$$

e integrando, $\gamma=1 / \eta \cdot \tau_{0} \cdot t+$ cte., y dao que para el instante inicial $t=0, \gamma=\tau_{0} / G=\gamma_{e}+\left(\gamma_{v}=0\right)$, resulta finalmente:

$$
\gamma_{i}=\tau_{0} / \eta \cdot t+{ }^{\tau_{0}} / G
$$

La expresión (27) indica que la velocidad de deformación es constante durante la etapa de fluencia, lo que es acorde a un comportamiento exclusivamente viscoso. De todo ellos se deduce que este tipo de modelo es adecuado para la reproducción de la etapa de fluencia secundaria. 
En el momento en el que se deja de aplicar carga, la expresión (26) resulta $\dot{\gamma}_{l}=0$, por lo que la deformación será constante y la recuperación de la fluencia nula, excepto la correspondiente a la deformación elástica inicial.

Cuando se quiere estudiar el relajamiento tensional, $\gamma_{i}=c t e .=\gamma_{0}$, la expresión (26) resulta:

$$
\begin{gathered}
\gamma_{i}={ }^{\tau_{0}} / \eta \cdot t+{ }^{\tau_{0}} / G \\
\dot{\tau}_{i} / \tau_{i}=(-G / \eta) d t
\end{gathered}
$$

que integrada desde el instante inicial de aplicación de la tensión hasta que transcurre un tiempo $\mathrm{t}$, queda $\tau_{i}=\tau_{0} e^{-G t} / \eta$. Por lo que para un tiempo $t=\infty$ el relajamiento tensional es total $\left(\tau_{\infty}=0\right)$, sin existir tensión remanente o residual.

En la Figura 31 se observa como el modelo propuesto por Maxwell es adecuado para modelizar el relajamiento tensional de un material viscoelástico ideal, con una curva de tipo exponencial. Sin embargo, no se conserva una tensión remanente, lo que se aleja bastante del comportamiento real de los suelos.
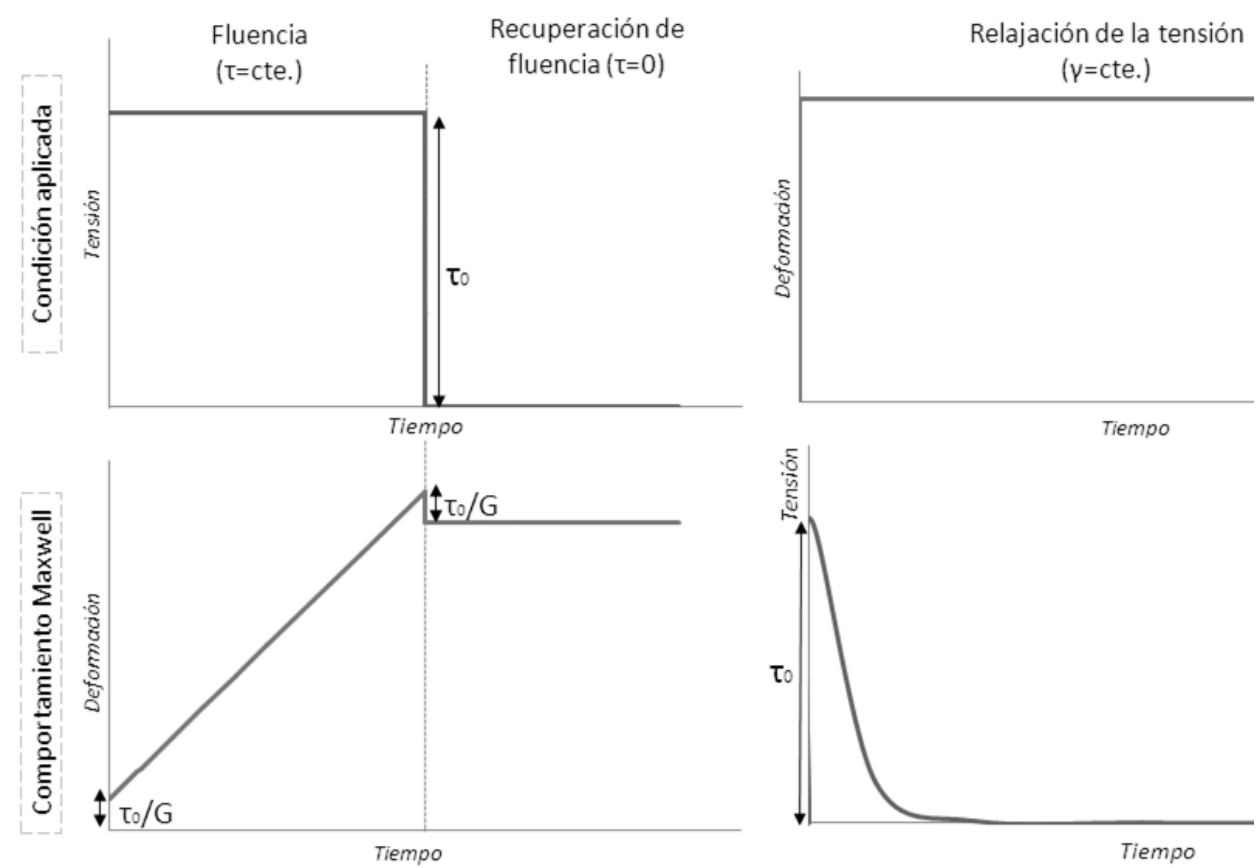

Figura 31. Comportamiento reológico (fluencia, recuperación de fluencia y relajación de la tensión) de un cuerpo según el modelo de Maxwell 
Por otra parte, la velocidad de deformación durante la etapa de fluencia es constante, lo que refleja un comportamiento puramente viscoso; además, la recuperación de la parte elástica ocurre de manera instantánea dejando de reflejar el comportamiento dependiente del tiempo.

\subsubsection{Modelo de Kelvin}

La ecuación de Kelvin-Voigt se puede representar mediante un resorte y un amortiguador colocados en paralelo, como se ilustra en la Figura 32, y se indica con la letra $\mathrm{K}=\mathrm{H} \mid \mathrm{N}$. La tensión aplicada se distribuye entre ambos elementos, $\tau=\tau_{e}+\tau_{v}$, y las deformaciones elástica y viscosa son idénticas, $\gamma=\gamma_{e}=\gamma_{v}$.

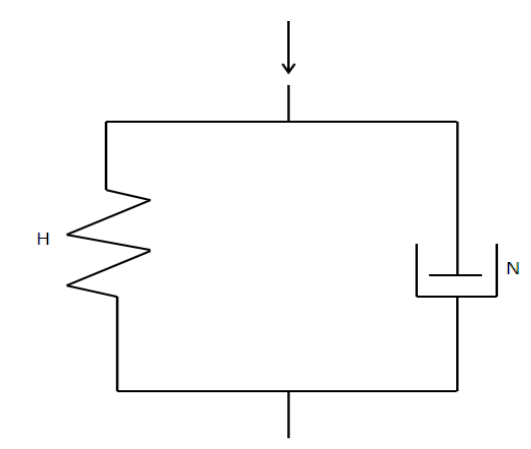

Figura 32. Modelo de Kelvin-Voigt

La ecuación reológica de un medio viscoelástico fue propuesta por Kelvin, en 1980, en su conocido tratado "Elasticity" y, casi simultáneamente por Voigt. La expresión enunciada es la siguiente:

$$
\tau_{i}=G \cdot \gamma_{i}+\eta \cdot \dot{\gamma}_{i}
$$

Resolviendo la ecuación (31) para $\gamma_{i}$, o lo que es lo mismo para las deformaciones en cualquier instante de tiempo $\gamma(t)$, cuando se quiere estudiar la fluencia $\left(\tau_{i}=c t e=\tau\right)$, se obtiene:

$$
\gamma_{i}=\tau / G \cdot\left(1-e^{\left.-t / T_{a}\right)}\right.
$$


La expresión (32) describe el proceso de una deformación elástica retardada (o deformación anelástica), donde $T_{a}={ }^{\eta} /{ }_{G}$ es el tiempo de retardo. Para el instante de tiempo inicial $t=0$ la deformación es nula. En cambio, para un instante $t=\infty$, la deformación toma un valor asintótico $\gamma_{\infty}=\tau /{ }_{G}$; y toda la tensión la soporta el elemento elástico del modelo.

Este fenómeno de deformación retardada, de acuerdo al modelo de Kelvin, es atribuido a las fuerzas de fricción internas (viscosidad). El cuerpo al que se refiere su modelo es un medio poroso elástico cuyos huecos están rellenos con un líquido viscoso.

Cuando se procede a la descarga del cuerpo $\left(\tau_{i}=0\right)$ la ecuación (30), que gobierna el comportamiento del modelo, se transforma en:

$$
0=G \cdot \gamma_{i}+\eta \cdot \dot{\gamma}_{i}
$$

que integrada con la condición inicial $t=0, \gamma=\gamma_{0}$, resulta:

$$
\gamma=\gamma_{0} \cdot e^{-t / T_{a}}
$$

donde $t$ es ahora el tiempo transcurrido desde la eliminación de la tensión y $\gamma_{0}$ es la deformación existente en el instante previo a la descarga. Como se puede observar en la Figura 33, se produce una recuperación total (no existen deformaciones residuales o permanentes en el tiempo) de la deformación de forma exponencial.

Para evaluar el fenómeno reológico de la relajación tensional, hay que aplicar la condición de deformación constante en la ecuación (31), de tal manera que se obtiene:

$$
\tau_{i}=G \cdot \gamma_{i}
$$

que corresponde a la respuesta de un material elástico, por lo que no hay relajación de tensiones bajo deformación constante.

Por lo tanto, observando la Figura 33, se puede concluir que el modelo de Kelvin-Voigt simula adecuadamente el proceso de fluencia y de 
recuperación de fluencia de un cuerpo viscoelástico ideal (según Figura 28c); sin embargo, no es apropiado para el relajamiento tensional.
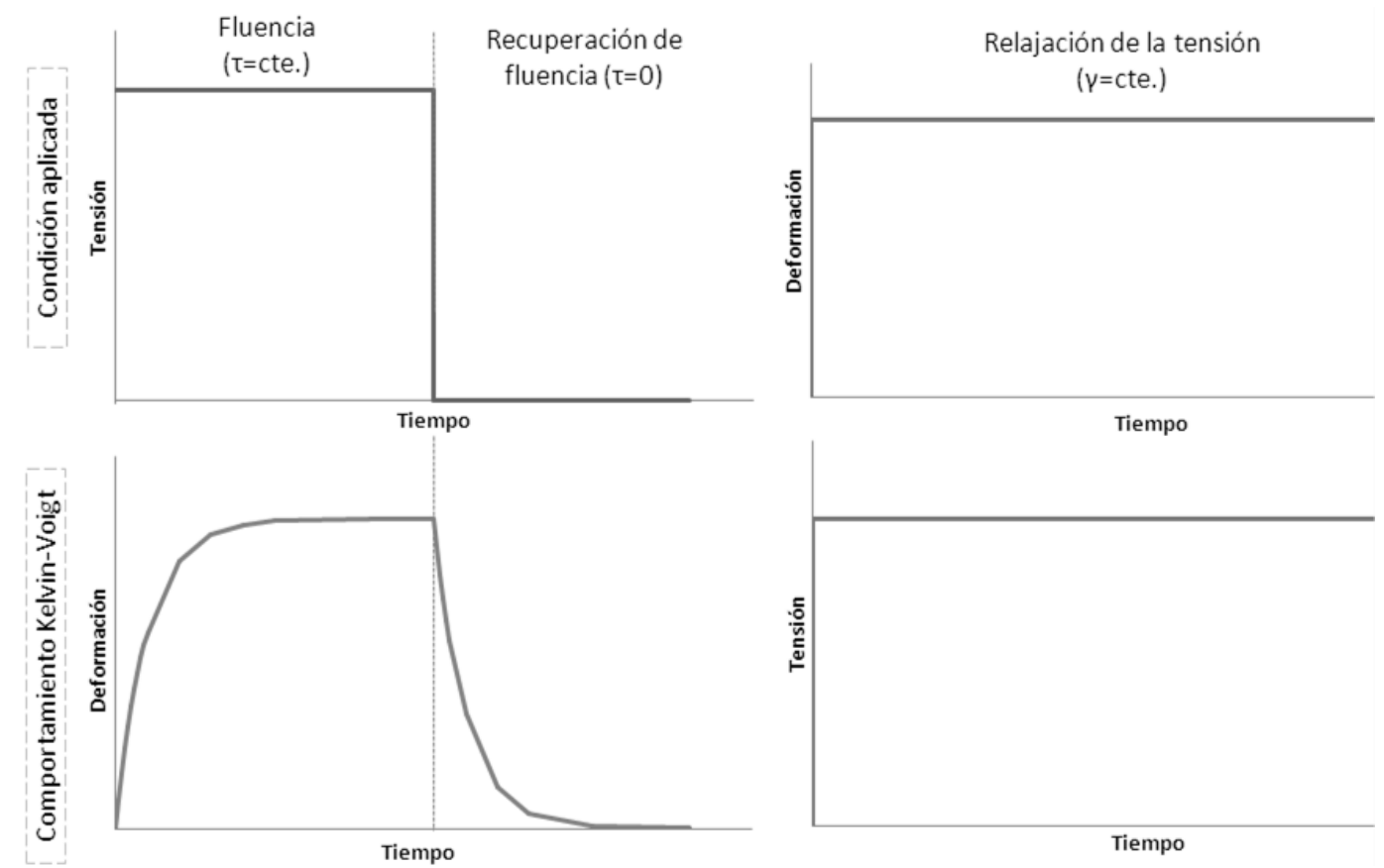

Figura 33. Comportamiento reológico (fluencia, recuperación de fluencia y relajación de la tensión) de un cuerpo según el modelo de Kelvin-Voigt.

\subsubsection{Modelo de Zener o sólido lineal estándar}

Como se acaba de exponer, el modelo de Kelvin experimenta una respuesta al someterlo a una tensión constante que se puede identificar con la fluencia, y por su parte el cuerpo de Maxwell exhibe relajación de tensiones al someterlo a un campo de deformaciones constantes.

Con el objetivo de representar adecuadamente ambos fenómenos reológicos en un único modelo, se han desarrollado propuestas que combinan de manera más compleja los distintos elementos elástico y viscoso. La formulación de algunos de estos modelos viscoelásticos (Jeffreys, J=N|M; Lethersich, L=N-K; Burger, Bu=M-K; y otros) se encuentra desarrollada en Vyalov (1986). 
El modelo del sólido lineal estándar, también denominado cuerpo viscoelástico generalizado emplea un elemento de Maxwell $(\mathrm{M}=\mathrm{H}-\mathrm{N})$ en paralelo con un resorte, o bien un modelo Kelvin-Voigt $(K=H \mid N)$ en serie con un resorte, tal y como se ilustra en la Figura 34 .

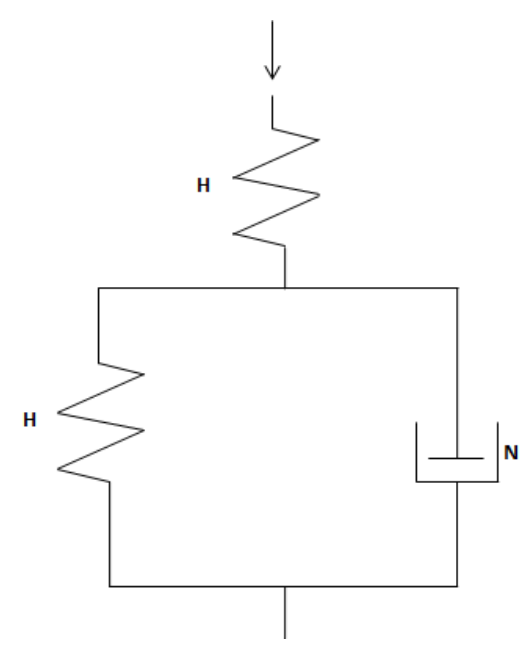

Figura 34. Modelo de Zener o del sólido lineal estándar. Modelo H-K

Refiriendo las ecuaciones a la disposición de la Figura 34 resulta el siguiente sistema:

$$
\begin{gathered}
\tau=\tau_{1}=\tau_{2}+\tau_{3} \\
\left(\gamma_{2}=\gamma_{3}\right) \quad \gamma=\gamma_{1}+\gamma_{2}
\end{gathered}
$$

Tras la aplicación de las ecuaciones que rigen el comportamiento del muelle y del amortiguador, vistas en la Figura 29, al sistema de ecuaciones (36) y (37) del modelo de Zener y una serie de reordenaciones, se obtiene la ecuación que relaciona la tensión aplicada con la deformación total, representando el comportamiento del modelo:

$$
\dot{\gamma}(t)+\left(G_{2} / \eta_{3}\right) \cdot \gamma(t)=1 / G_{1} \cdot \dot{\tau}(t)+\left(G_{1}+G_{2} / G_{1} \eta_{3}\right) \cdot \tau(t)
$$

Analizando el comportamiento del modelo bajo carga constante $\tau=c t e .=\tau_{0}$, la ecuación (38) se transforma tras su integración y reordenación en: 


$$
\gamma(t)=\tau_{0} / G_{1}+\tau_{0} / G_{2}\left(1-e^{\left(-t / T_{r}\right)}\right)
$$

donde $T_{r}=\eta_{3} /_{G_{2}}$ es el tiempo de retardo. La relación (39) expresa la existencia de una deformación instantánea correspondiente al muelle y una respuesta retardada que se deriva de la extensión del émbolo.

Al retirar la carga al modelo de Zener, se tiene la ecuación $\dot{\gamma}(t)+$ $\left(G_{2} / \eta_{3}\right) \cdot \gamma(t)=0$, cuya integral es:

$$
\gamma(t)=\gamma_{c} e^{\left(-t / T_{r}\right)}
$$

donde $\gamma_{c}$ es la deformación de fluencia en el elemento de Kelvin-Voigt debida a la carga previa.

Cuando se somete el modelo a una deformación constante $\gamma_{0}$, la ecuación (38) se transforma tras su integración y reordenación en:

$$
\tau(t)=\left(G_{1} * \gamma_{0} / G_{1}+G_{2}\right) \cdot\left(G_{2}+G_{1} * e^{\left(-t / T_{r e l .}\right.}\right)
$$

donde $T_{\text {rel. }}=\eta_{3} / G_{1}+G_{2}$ es el tiempo de relajación.

Como se aprecia en la Figura 35 el proceso de relajamiento tensional es acorde a lo expuesto teóricamente, conservándose además una tensión residual. La combinación de los modelos de Maxwell o de Kelvin con un resorte provoca que la reproducción del fenómeno de la fluencia y recuperación de la fluencia sea más acorde al comportamiento de un cuerpo viscoelástico. Sin embargo, a diferencia de lo ocurrido en el suelo, no existen deformaciones plásticas remanentes, recuperando el terreno su deformación de manera retardada pero total, según el modelo del sólido lineal estándar o puramente viscoelástico. 

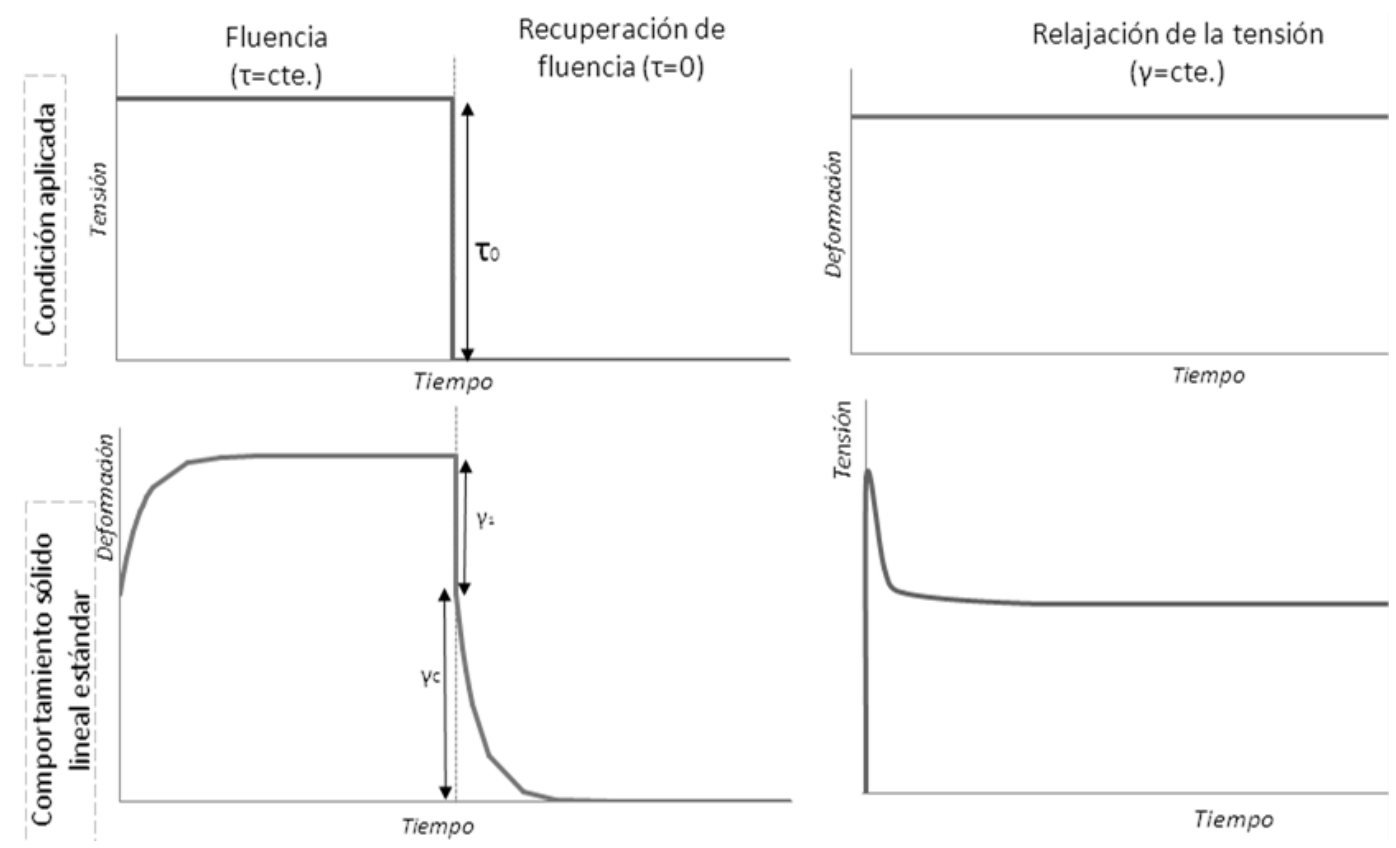

Figura 35. Comportamiento reológico (fluencia, recuperación de fluencia y relajación de la tensión) de un cuerpo según el modelo del sólido lineal estándar

\subsubsection{Modelo de Burgers o de los cuatro elementos}

Con este modelo se sugiere la colocación en serie de un elemento de Maxwell y un elemento de Kelvin-Voigt $(\mathrm{Bu}=\mathrm{M}-\mathrm{K})$, como se aprecia en la Figura 36 de tal manera que se consigue modelizar materiales que presentan componentes de deformación instantánea y viscoelástica.

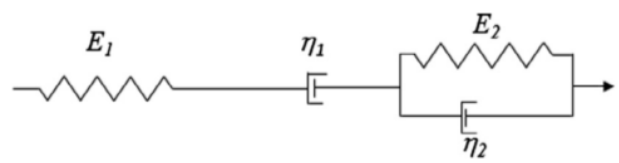

Figura 36. Modelo de Burgers o de los cuatro elementos. (Kränkel et al, 2015)

Es un modelo muy completo, empleado en la simulación numérica del medio continuo isótropo, viscoelástico en distorsión angular y elástico en deformación volumétrica. Como se menciona más adelante, este es uno de los modelos implementados en el programa de diferencias finitas FLAC. Existen numerosos estudios recientes con este modelo, aplicados 
principalmente a rocas y a rocas blandas (Kränkel et al., 2015; Wang, et al., 2015; Ping, et al., 2016; Sun, et al., 2016; Wang, et al., 2016; Zhang, et al., 2016).

La ecuación que gobierna el comportamiento bajo una carga constante $\sigma_{0}$ según el modelo de Burgers es:

$$
\varepsilon(t)=\sigma_{0} / E_{1}+\frac{\sigma_{0}}{\eta_{1}} t+{ }^{\sigma_{0}} / E_{2}\left(1-e^{\left(-E_{2} t / \eta_{2}\right)}\right)
$$

donde $\varepsilon(t)$ es la deformación por fluencia en el tiempo; $E_{1}$ y $E_{2}$ módulos de elasticidad de los respectivos muelles, y $\eta_{1}$ y $\eta_{2}$ los coeficientes de viscosidad de los respectivos amortiguadores.

La representación gráfica de los fenómenos de fluencia y relajamiento tensional según un cuerpo mecánico de tipo Burgers, se ilustra en la Figura 37. Como se aprecia en esta última figura, en relación con la Figura 28, el modelo de Burgers representa razonada y adecuadamente un comportamiento viscoelastoplástico ideal.
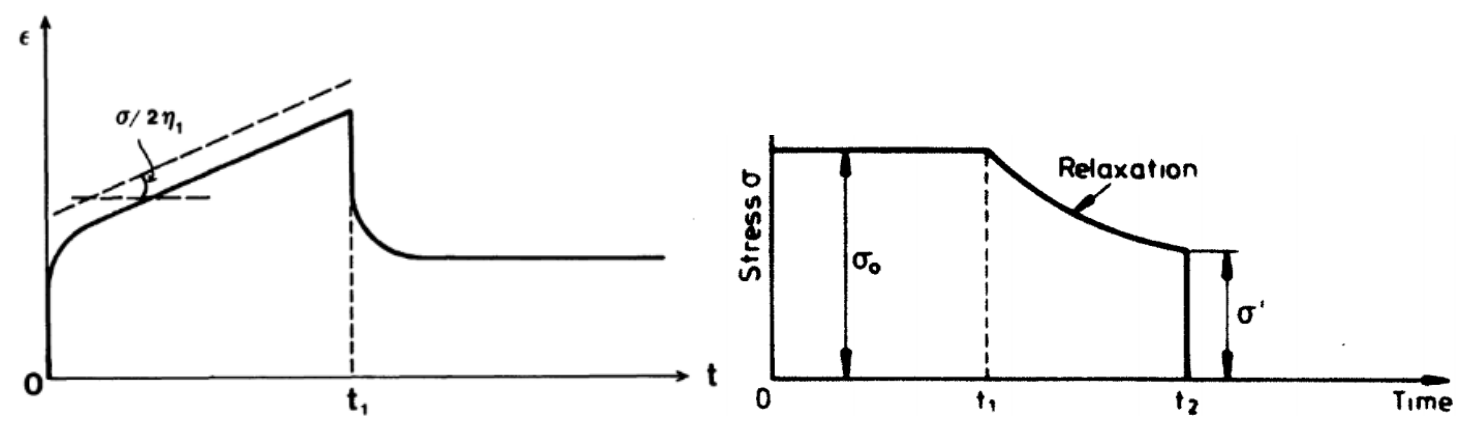

Figura 37. Comportamiento por fluencia y recuperación de la fluencia según el modelo de Burgers. (Vyalov, 1986)

\subsubsection{Modelo de Bingham}

Hasta ahora se han introducido los modelos mecánicos más simples, basados en la viscoelasticidad lineal. Uno de los modelos elastoviscoplásticos (EVP) mecánicos más conocido es el modelo de Bingham. El modelo EVP de Bingham consiste en un elemento de Saint-Venant colocado en paralelo con un elemento de Newton, que a su vez se encuentran en serie con un elemento 
de Hooke, $\mathrm{B}=\mathrm{H}-(\mathrm{N} \mid \mathrm{SV})$, como se puede apreciar en Figura 38. Donde $\boldsymbol{\tau}_{\boldsymbol{y}}$ es la resistencia al corte última del elemento de Saint-Venant, también denominada tensión de fluencia; $\dot{\boldsymbol{\varepsilon}}^{\boldsymbol{e}}$ velocidad de deformación del elemento elástico; y $\dot{\boldsymbol{\varepsilon}}^{\boldsymbol{v} \boldsymbol{p}}$ velocidad de deformación del elemento viscoplástico.

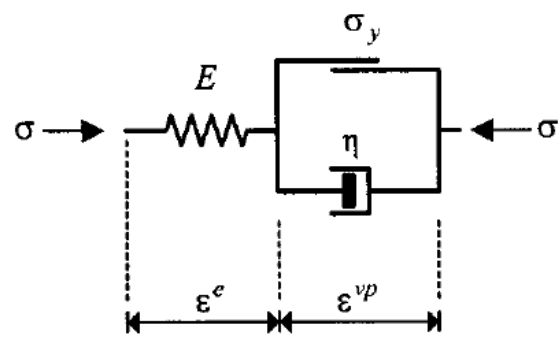

Figura 38. Modelo de Bingham. Cuerpo elasto-viscoplástico. (Liingaard et al., 2004).

Las ecuaciones del sistema en velocidades de deformación se pueden expresar como:

$$
\dot{\varepsilon}= \begin{cases}\dot{\varepsilon}^{e}+\dot{\varepsilon}^{v p}=\dot{\sigma} / E+\left(\sigma-\sigma_{y}\right) / \eta & \text { para } \sigma>\sigma_{y} \\ \dot{\varepsilon}^{e}=\dot{\sigma} / E & \text { para } \sigma \leq \sigma_{y}\end{cases}
$$

donde $\dot{\varepsilon}$ es la velocidad de deformación total del sistema. De tal manera que el modelo muestra un comportamiento puramente elástico por debajo de la tensión de fluencia (el elemento viscoplástico permanece inactivo mientras $\sigma \leq \sigma_{y}$ ), y por encima se comporta como un flujo viscoso de tipo Maxwell (Liingaard et al., 2004).

Se pueden dar dos hipótesis diferentes:

- La tensión límite $\sigma_{y}$ es nula y el material no se endurece durante el proceso de carga ("nonhardening"), o bien,

- $\sigma-\sigma_{y}$ es constante y el material no se endurece; es "nonhardening".

En el primer caso, el modelo de Bingham es idéntico al conocido modelo de Maxwell, que describe un material que se deforma a una velocidad de deformación constante, como en la etapa de fluencia secundaria o 
estacionaria (Meschyan, 1995). En el segundo caso, la ecuación constitutiva del modelo de Maxwell obedece la ley de Bingham si la tensión impuesta $\sigma$ se sustituye por $\sigma-\sigma_{y}$. En este último caso, la velocidad de deformación también es constante.

En la Figura 39 se representa el comportamiento del modelo mecánico de Bingham frente a fluencia, con relajamiento tensional y velocidad de deformación constante, para rangos de tensiones por debajo y por encima de la tensión de fluencia.
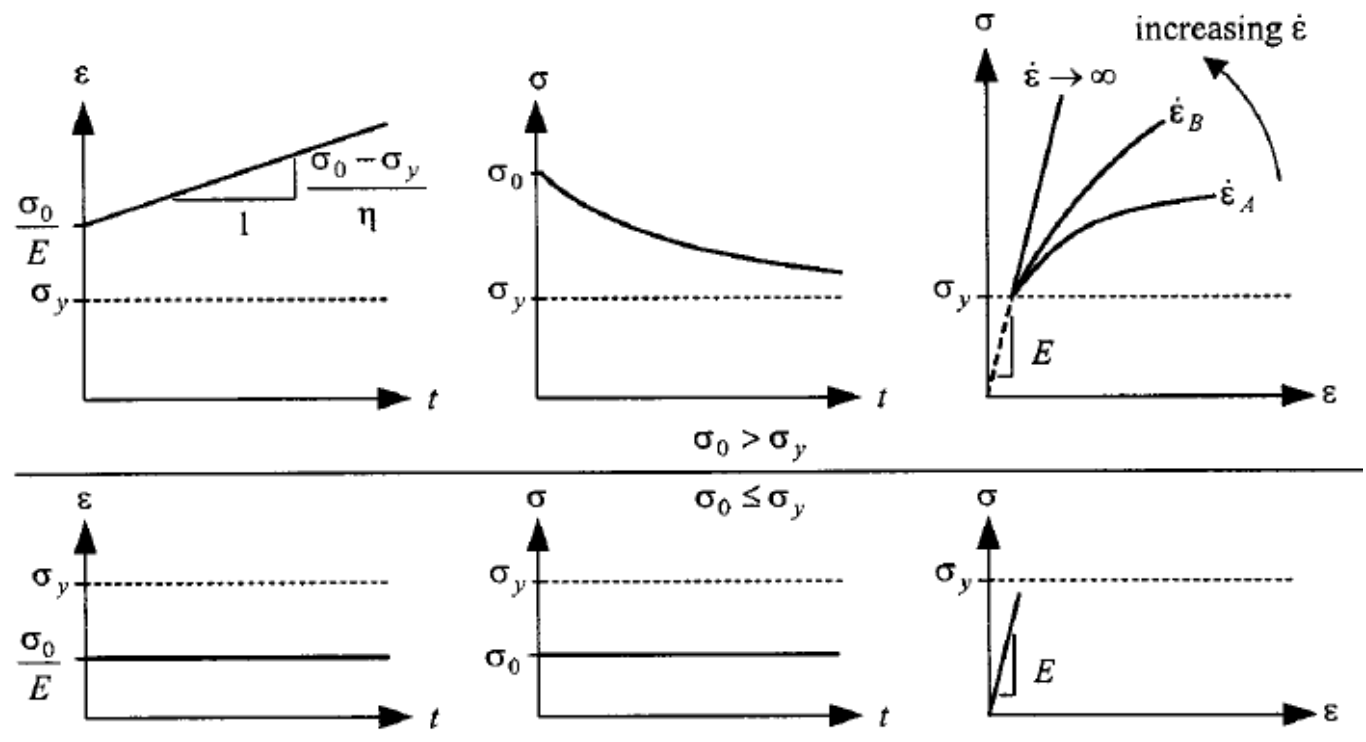

a. Creep

b. Relaxation

c. Constant rate of strain

Figura 39. Respuesta del modelo de Bingham ante (a) fluencia; (b) relajación; y (c) distintas velocidades de deformación constantes. (Liingaard et al., 2004).

\subsubsection{Comentarios sobre los modelos reológicos mecánicos}

El principal inconveniente del enfoque diferencial podría ser el hecho de que las relaciones constitutivas en que se basa son muy simples, en cuanto a que los elementos mecánicos (el muelle, el amortiguador y el cuerpo plástico) describen relaciones constitutivas lineales (Liingaard et al., 2004). Este planteamiento resulta poco adecuado porque el comportamiento elástico y plástico, real, de los suelos es marcadamente no lineal. A este respecto existen combinaciones posibles que introducen la no linealidad combinada 
con algunos de los modelos mecánicos existentes. Algunos casos de aplicación y contraste con ensayos de laboratorio se decriben a continuación.

Kränkel et al. (2015); emplean el modelo de Burgers modificado, suponiendo un comportamiento no lineal viscoelástico de un anclaje de tipo adhesivo en hormigón. En su estudio la no linealidad depende de: (a) el nivel de tensiones aplicado, (b) la duración del periodo de carga y (c) el proceso de degradación del material.

Wang et al. (2016); aplican el modelo de Kelvin-Heard modificado. El primer modelo mecánico se emplea para simular la fluencia primaria y el segundo para simular la fluencia estacionaria, de una thenardita. La modificación de los modelos mecánicos originales se lleva a cabo para tener en cuenta la variación de los parámetros bajo distintas cargas desviadoras.

$\mathrm{Xu}$, et al. (2007); utilizan el modelo de Zener no lineal, para caracterizar la variación de la fuerza de arranque de un anclaje con el tiempo.

Feda (1992); propone el empleo de la teoría del proceso de cambio ("rate process theory"), presentada más adelante, como un método que tiene en cuenta la no linealidad de un material Newtoniano (es decir, del coeficiente de viscosidad).

También al respecto de introducir la no linealidad en los modelos mecánicos clásicos, Feda (1992) sugiere la utilización del modelo hiperbólico (Duncan \& Chang, 1970) para definir un material Hookeano no lineal.

En la actualidad y atendiendo a los párrafos anteriores, parece poco adecuado considerar como un problema la aplicación de relaciones no lineales a los modelos mecánicos.

Según algunos autores (Singh y Mitchell, 1968) la generalización de los modelos mecánicos desde formulaciones unidimensionales a tridimensionales es posible, pero la calibración práctica y su aplicación parece complicada. Sin embargo, existe bibliografía con estas soluciones desarrolladas (Jaeger, 1969; Cristescu \& Hunsche, 1998) y aplicadas a casos reales (Sandoval, 2008). 
Avances relativamente recientes son los propuestos por Xia-Ting, et al. (2006) que, para hallar el modelo reológico más acorde al material estudiado, publicaron un método basado en algoritmos de programación genética y de optimización de elementos dentro de un grupo,.

Como conclusión se puede decir que los modelos mecánicos no representan una visualización de los cambios estructurales a los que está sujeto el material en un proceso de deformación, si no que sirven para hacer una descripción formal de su comportamiento fenomenológico, resultando ser modelos muy prácticos y aplicables, por su fácil comprensión visual en una dimensión.

\subsubsection{Modelos ingenieriles de la fluencia}

En este apartado se describen las hipótesis generales que permiten determinar la respuesta por fluencia inelástica de los sólidos, muy aplicadas en la mecánica del hormigón y de los metales. La mayoría del trabajo realizado dentro de las teorías ingenieriles de la fluencia se concentra en el campo de los metales, el hormigón y el hielo, donde la carga está por debajo de la tensión de fluencia (Liingaard et al., 2004).

Este tipo de teorías no son de aplicación única al fenómeno de la fluencia. Son leyes fenomenológicas basadas en observaciones experimentales y existen obviamente similitudes entre las estructuras de los modelos empíricos vistos y estas. Sin embargo, las teorías ingenieriles de la fluencia difieren conceptualmente de la manera habitual de tratar con la fluencia en suelos, porque son vistas como teorías de fluencia para materiales donde los estados tensionales están por debajo de la tensión de fluencia. Para suelos, el fenómeno de la fluencia fue originalmente desarrollado para arcillas plásticas normalmente consolidadas. Los modelos ingenieriles de la fluencia son, según Skrzypek (1993): el modelo de la deformación total, el modelo del endurecimiento con el tiempo, y el modelo del endurecimiento con la deformación. 
Las definiciones expuestas en los siguientes capítulos están basadas en condiciones uniaxiales. La deformación, la velocidad de deformación y la tensión efectiva uniaxiales, se desginan $\operatorname{con} \varepsilon$, $\dot{\varepsilon}$ y $\sigma$, respectivamente. El tiempo transcurrido desde el comienzo de la fluencia es $t$. Los superíndices $e$ y $c$ corresponden a las componentes elástica y de fluencia de la deformación, respectivamente.

\subsubsection{Modelo de la deformación total}

Con este modelo se asume que la deformación total consiste en una componente elástica instantánea y una componente de fluencia viscosa:

$$
\varepsilon=\varepsilon^{e}+\varepsilon^{c}
$$

Para un ensayo de fluencia estándar donde la tensión $\sigma$ es aplicada instantáneamente, la componente de deformación por fluencia está dada como una función de la tensión aplicada $\sigma$ y de la historia de carga. En la relación funcional del modelo, la historia de carga está únicamente relacionada con el tiempo de carga $t$. Esto proporciona una relación sencilla entre la deformación, la tensión y el tiempo:

$$
\varepsilon^{c}=f(\sigma) g(t)
$$

donde $f$ y $g$ son funciones de la tensión y del tiempo, respectivamente. La relación entre las deformaciones por fluencia y la tensión se modela a menudo mediante una función potencial, es decir, que las deformaciones por fluencia tienen una dependencia no lineal con la tensión. La influencia del tiempo de carga también se formula habitualmente mediante una relación de tipo potencial, siendo posible emplear otras funciones, por ejemplo, exponenciales o hiperbólicas.

En el modelo de la deformación total, la deformación viscosa (componente por fluencia) a tensión constante está directamente relacionada con el tiempo $t$. Esto da lugar a un modelo sencillo que se puede adoptar en las predicciones de fluencia. Rabotnov (1969) recomienda que esta teoría se 
utilice únicamente en condiciones de tensión constante o con variaciones de tensión muy lentas.

\subsubsection{Modelo de endurecimiento con el tiempo}

Para tener en cuenta los cambios de tensión arbitrarios, la relación constitutiva debe ser de naturaleza incremental, es decir, formulada con respecto a la velocidad de deformación por fluencia en vez de a la deformación por fluencia.

$$
\dot{\varepsilon}^{c}=f(\sigma) g(t)
$$

donde $f$ y $g$ son funciones no lineales de la tensión y del tiempo, respectivamente. En la ecuación (46), el tiempo se puede identificar como el parámetro de endurecimiento, de ahí el nombre "endurecimiento con el tiempo". Uno de los inconvenientes de este modelo, al igual que el modelo de la deformación total, es que las ecuaciones que lo gobiernan no son invariantes con respecto al origen del tiempo, porque el tiempo se ha introducido de forma explícita.

\subsubsection{Modelo de endurecimiento con la deformación}

En el modelo de endurecimiento por deformación, el endurecimiento está gobernado por la deformación por fluencia. El modelo está basado en la acumulación de deformaciones plásticas como mecanismo de endurecimiento. La ecuación generalizada del modelo es:

$$
\dot{\varepsilon}^{c}=f(\sigma) g\left(\varepsilon^{c}\right)
$$

donde $f$ y $g$ son funciones no lineales de la tensión y del tiempo, respectivamente. La deformación por fluencia $\varepsilon^{c}$ caracteriza el estado del material y se puede considerar como una variable interna. 


\subsubsection{Comentarios sobre los modelos ingenieriles}

El concepto del endurecimento en las teorías ingenieriles de la fluencia, ya sea endurecimiento por tiempo o por deformación, es análogo a las ideas tradicionales cuando se considera el comportamiento dependiente del tiempo de los suelos. Es decir, se han observado semejanzas entre las estructuras matemáticas de los modelos empíricos vistos y los modelos ingenieriles de fluencia (véase la Tabla 2).

Tabla 2. Relación entre los modelos empíricos y las teorías ingenieriles para determinar la fluencia. (Liingaard et al., 2004).

\begin{tabular}{|c|c|c|}
\hline $\begin{array}{c}\text { Teoría de la } \\
\text { fluencia }\end{array}$ & Relación & Modelo empírico \\
\hline $\begin{array}{c}\text { "Total strain } \\
\text { model" }\end{array}$ & $\varepsilon^{c}=f(\sigma) g(t)$ & $\begin{array}{r}\text { Ley logarítmica. La función tensional } f \text { o } \\
\text { es constante (enfoque } C_{\alpha} \text { ) o varía con la } \\
\left.\text { presión de confinamiento (enfoque } C_{c} / C_{\alpha}\right) . \\
\text { La función temporal } g \text { es la función } \\
\text { logarítmica. }\end{array}$ \\
\hline $\begin{array}{c}\text { "Time- } \\
\text { hardening } \\
\text { model" }\end{array}$ & $\dot{\varepsilon}^{c}=f(\sigma) g(t)$ & $\begin{array}{r}\text { Modelo de Singh y Mitchell. La función } \\
\text { tensional } f \text { es una función exponencial, y } \\
\text { la función temporal } g \text { es una función } \\
\text { potencial de exponente } m .\end{array}$ \\
\hline $\begin{array}{c}\text { "Strain- } \\
\text { hardening } \\
\text { model" }\end{array}$ & $\dot{\varepsilon}^{c}=f(\sigma) g\left(\varepsilon^{c}\right)$ & $\begin{array}{c}\text { Método de la velocidad de deformación. La } \\
\text { forma general del enfoque de la velocidad } \\
\text { de deformación enuncia una relación única } \\
\text { entre la tensión, la deformación y la } \\
\text { velocidad de deformación. }\end{array}$ \\
\hline
\end{tabular}

En la Tabla 2 solo se describen las relaciones entre los modelos empíricos y las teorías ingenieriles de la fluencia, pero estas últimas se pueden relacionar también con otros conceptos. Por ejemplo, los modelos de endurecimiento por deformación y por tiempo, son modelos de Maxwell con componentes de la fluencia no lineales. El elemento viscoso lineal en el modelo originial de Maxwell puede ser remplazado por la ecuación (46) o (47), lo que significa que la componente viscosa endurece con el tiempo o con la deformación, respectivamente. El modelo de Maxwell no lineal se puede formular entonces como: 


$$
\begin{gathered}
\dot{\varepsilon}=\dot{\varepsilon}^{e}+\dot{\varepsilon}^{c}=\frac{\dot{\sigma}}{E}+\frac{\sigma}{\eta(\sigma, t)} \text {, para endurecimiento con el tiempo } \\
\dot{\varepsilon}=\dot{\varepsilon}^{e}+\dot{\varepsilon}^{c}=\frac{\dot{\sigma}}{E}+\frac{\sigma}{\eta\left(\sigma, \varepsilon^{c}\right)}, \text { para endurecimiento por deformación }
\end{gathered}
$$

En las ecuaciones (48) y (49) se observa que el parámetro viscoso $\boldsymbol{\eta}$ ya no es constante, si no que endurece o bien con la tensión y el tiempo, o bien con la tensión y la deformación.

Las soluciones para el modelo del endurecimiento con el tiempo son relativamente sencillas, pero las soluciones del modelo de endurecimiento con la deformación son algo más complejas. Existen también soluciones dadas para el relajamiento tensional basadas en las teorías del endurecimiento con el tiempo y con la deformación (Rabotnov, 1969; Borm \& Haupt, 1988; Huneault, 1992; Ladanyi \& Melouki, 1993; Ladanyi \& Benyamina, 1995).

\subsubsection{Enfoque hereditario}

Este modelo reológico basado en la representación integral establece que la deformación o la tensión dependientes del tiempo se definen mediante una función de fluencia o de relajación, las cuales son a su vez, funciones hereditarias (con memoria) que describen la dependencia histórica de las deformaciones o tensiones.

El principio del método hereditario es que la deformación actual $\boldsymbol{\varepsilon}(\boldsymbol{t})$ se obtiene por integración sobre la historia de cargas total, es decir, la integración sobre todas las variaciones infinitesimales de tensión hasta el tiempo actual.

La teoría se ha desarrollado para dos casos, el primero y más simple está basado en la viscoelasticidad lineal, la cual es una generalización del enfoque diferencial. También es posible adaptar el método hereditario al comportamiento no lineal de un material que corresponde a una generalización de las teorías ingenieriles de la fluencia.

La opinión general es que el enfoque hereditario es demasiado complicado de incorporar al estudio de la mecánica de suelos. Esto es debido 
al hecho de que se necesitan un gran número de ensayos para su calibración. Por ejemplo, Feda (1992) señala que fueron necesarios 28 ensayos para describir un ensayo de tensión uniaxial mediante la teoría hereditaria no lineal, y que para un estado triaxial de tensión se requieren una cantidad de ensayos del orden de seis veces mayor. Es decir, se requiere un volumen de información de partida poco viable.

Para conocer más detalles sobre el enfoque hereditario se puede consultar: Rabotnov (1969), Feda (1992), Ter-Martirosyan (1992), Skrzypek (1993), y Meschyan (1995).

\subsubsection{Modelos generales tensión-deformación-tiempo}

Esta sección está dedicada exclusivamente a las leyes constitutivas generales (tridimensionales) que describen tanto los efectos viscosos como los no viscosos (o independientes de la velocidad de deformación) que gobiernan el comportamiento de los suelos, en principio, bajo cualquier condición de carga.

Se ha puesto una atención especial en los modelos elastoviscoplásticos (EVP), los cuales combinan los comportamientos elástico no viscoso y plástico viscoso o dependiente del tiempo. En este documento no se han incluido los modelos viscoelástico-viscoplásticos. Su descripción se encuentra, por ejemplo, en Naghdi \& Murch (1963); Murayama (1983); y Murayama, et al. (1984).

Los modelos elásticos-viscoplásticos se pueden dividir en tres tipos (Sekiguchi, 1985): modelos elastoviscoplásticos basados en el concepto de la sobretensión; modelos elastoviscoplásticos basados en el concepto de la superficie de flujo no estacionaria; y otros. 


\subsubsection{Teoría de la sobretensión}

El concepto de la teoría de la sobretensión fue introducido y desarrollado por Ludwick (1922), Prandtl (1928), Hohenemser \& Prager (1932), Sokolovsky (1948) y Malvern (1951), como indica Satake (1989). La teoría de la sobretensión de Perzyna es la versión tridimensional del modelo constitutivo unidimensional de Malvern. La descripción de la teoría de la sobretensión de Perzyna, que se realiza a continuación, está basada en Perzyna (1963a, 1963b, 1963c, 1966), Olszak \& Perzyna (1966a, 1970) y Sekiguchi (1985).

Existen numerosos modelos elastoviscoplásticos basados en la teoría de la sobretensión y aplicados a distintos tipos de terrenos. Algunos ejemplos se citan en Liingaard et al. (2004).

Un supuesto clave en la teoría de la sobretensión de Perzyna es que los efectos viscosos son inapreciables en la región elástica, es decir, las deformaciones viscosas no se producen dentro de la superficie de fluencia estática. Esta última corresponde con la superficie de fluencia tradicional, asociada con la plasticidad independiente del tiempo. En otras palabras, las deformaciones elásticas son independientes del tiempo, mientras que las deformaciones inelásticas sí se consideran viscosas o dependientes del tiempo.

La velocidad de deformación total está compuesta por la suma de las velocidades de deformación elástica y la viscoplástica:

$$
\dot{\varepsilon}_{i j}=\dot{\varepsilon}_{i j}{ }^{e}+\dot{\varepsilon}_{i j}{ }^{p p}
$$

donde $\dot{\varepsilon}_{i j}$ es la componente $(i j)$ del tensor de velocidad total de deformación; y los superíndices $e$ y $v p$ atienden a las componentes elástica e inelástica, respectivamente. En la teoría de la elastoviscoplasticidad, la velocidad de deformación inelástica representa efectos plásticos y viscosos combinados. En el caso de la velocidad de deformación elástica $\dot{\varepsilon}_{i j}{ }^{e}$ se acepta que sigue la ley de Hooke generalizada, mientras que en el caso de la 
velocidad de deformación viscoplástica $\dot{\varepsilon}_{i j}{ }^{v p}$ se supone que esta sigue la siguiente regla de flujo no asociada:

$$
\dot{\varepsilon}_{i j}{ }^{v p}=\gamma \phi(F) \frac{\partial g}{\partial \sigma_{i j}^{\prime}}
$$

donde $\gamma$ es el parámetro de fluidez; $\phi$ es el núcleo viscoso; $F$ es la función de sobretensión; $g$ es la función de potencial plástico; y $\sigma_{i j}^{\prime}$ es el estado de tensiones efectivas.

La función de sobretensión se puede expresar como:

$$
F\left(\sigma_{i j}^{\prime}, W^{v p}\right)=\frac{f_{d}\left(\sigma^{\prime}{ }_{i j}, W^{v p}\right)}{\kappa_{S}\left(W^{v p}\right)}-1 \text { donde } \kappa_{s}=\kappa_{S}\left(\int_{0}^{\dot{\varepsilon}_{i j}{ }^{v p}}{\sigma^{\prime}}_{i j} \dot{\varepsilon}_{i j}{ }^{v p}\right)
$$

En la ecuación (52) la función $f_{d}$ depende del estado de tensiones y del trabajo viscoplástico $W^{v p}$. La función $f_{d}$ describe la superficie de carga dinámica (o superficie dinámica de fluencia) en la cual el estado actual de tensiones $(P)$ es localizado, como se ilustra en la Figura $40 . \kappa_{s}$ es el parámetro de endurecimiento, y $F=0$ cuando $f_{d}\left(\sigma^{\prime}{ }_{i j}, W^{v p}\right)=\kappa_{s}\left(W^{v p}\right)$ lo cual implica que $\kappa_{s}$ debe ser una expresión para la superficie estática de fluencia $f_{s}$.

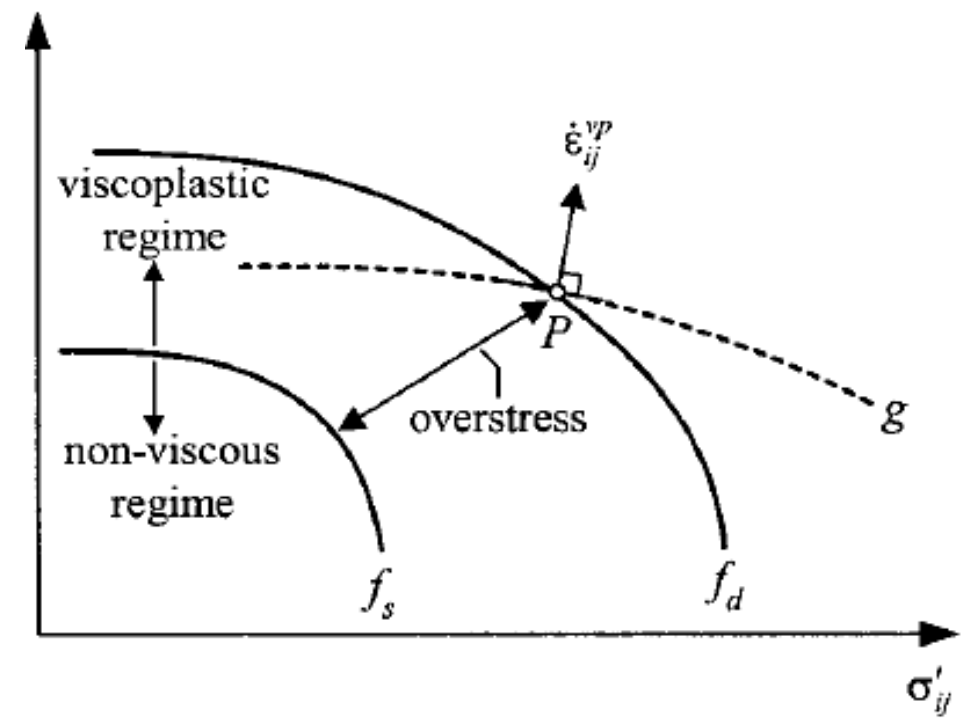

Figura 40. El estado de tensión $P$ es parte de la superficie dinámica de fluencia $f_{d}$ y la sobretensión $F$ se define como la distancia entre $P$ y la superficie estática de fluencia $f_{s}$. Además, el vector velocidad de deformación viscoplástica es perpendicular a la superficie de potencial plástico g. (Perzyna, 1966) 
La teoría de la sobretensión difiere de la elastoplasticidad general en el sentido en que la regla de consistencia no se utiliza en el desarrollo de la misma. Esto implica que las deformaciones inelásticas en el modelo de la sobretensión no están relacionadas con la historia tensional y sí con el punto de tensión actual, mientras que en la elastoplasticidad las deformaciones inelásticas están relacionadas con la razón de tensiones. Además, aceptando la no validez de la regla de consistencia, se permite que el estado de tensión se encuentre sobre, por debajo o fuera de la superficie estática de fluencia. Esto se emplea en la definición de la sobretensión $F$. Cuando $F=0$, el estado de tensiones actual $(P)$, se encuentra sobre la superficie de fluencia estática $f_{S}$ (límite entre régimen no viscoso y régimen viscoplástico); cuando $F>0, P$ se encuentra fuera de $f_{s}$ (en el régimen viscoplástico); y cuando $F<0, P$ se encuentra dentro de $f_{s}$ (en el régimen no viscoso).

De acuerdo a la suposición de régimen no viscoso por debajo de la superficie estática de fluencia y a la regla de flujo, se aplican las siguientes restricciones al núcleo viscoso $\phi$ :

$$
\langle\phi(F)\rangle= \begin{cases}0 & \text { para } F \leq 0 \\ \phi(F) & \text { para } F>0\end{cases}
$$

La ecuación (53) se puede considerar como el criterio de carga para las deformaciones inelásticas. La dirección de $\dot{\varepsilon}_{i j}{ }^{v p}$ en la ecuación (51) es perpendicular a la superficie de potencial plástico $g$ en el estado actual de tensiones $(P)$, como se apreciaba en la Figura 40. La magnitud de $\dot{\varepsilon}_{i j}{ }^{v p}$ está dada por el parámetro de fluidez $\gamma$, y el núcleo viscoso $\langle\phi(F)\rangle$. La determinación de este último se realiza a partir de ensayos (Phillips \& Wu, 1973; Adachi \& Okano, 1974; Adachi \& Oka, 1982a; Oka, et al., 1986; di Prisco \& Imposimato, 1996). La determinación de $\phi(F)$ a partir de ensayos se puede consultar, por ejemplo, en Akai, et al. (1977) y en Adachi y Okano (1974). Se presentan a continuación dos de los procedimientos más empleados:

$$
\phi(F)=a F^{6} \quad \mathrm{y} \quad \phi(F)=c e^{\left(j F^{k}\right)}-1
$$


donde a, c, j, y k son constantes. Como se deduce de la ecuación (54), el núcleo viscoso es una función creciente con la sobretensión $F$.

Mientras que la sobretensión se define como la diferencia entre las superficies estática y dinámica de fluencia, la posición de $f_{s}$ en el espacio de tensiones no es sencilla de determinar.

Considerando un proceso de fluencia iniciado en un punto de tensión que se encuentra fuera de la superficie estática de fluencia, cuando se impone un estado de tensión constante de tal manera que $F>0$, el flujo viscoplástico ocurrirá y continuará desarrollándose a una velocidad constante si $f_{s}$ es una superficie de fluencia que no endurece y perfectamente plástica (ver Figura 41a); es decir, la distancia entre las superficies de fluencia dinámica y estática es constante con el tiempo.

Si $f_{s}$ es una función de fluencia con endurecimiento, el flujo viscoplástico ocurre a una velocidad decreciente, debido a que según la deformación viscoplástica y el consecuente trabajo viscoplástico $W^{v p}$ se acumulan, la superficie estática de fluencia cambia de tal manera que $F \rightarrow 0$, y en consecuencia $\dot{\varepsilon}^{v p} \rightarrow 0$. Es decir, la distancia entre $f_{s}$ y $f_{d}$ decrece con el tiempo, y ésta se reduce conforme disminuye la velocidad de deformación. En otras palabras, la superficie estática de fluencia $f_{s}$ se desplaza hacia afuera con el tiempo y finalmente, después de un tiempo infinito llega a coincidir con la superficie dinámica de fluencia, como se indica en la Figura 41b. En ese momento la nueva superficie estática de fluencia se estabiliza y $\dot{\varepsilon}^{v p}=0$.
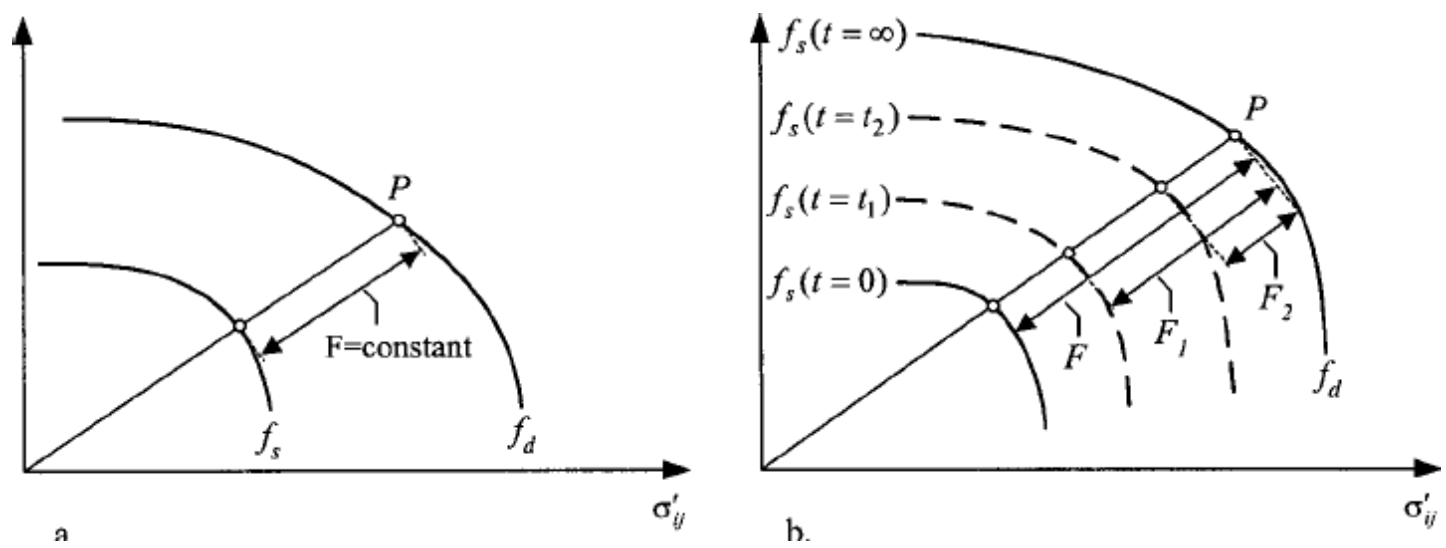

Figura 41. (a) Proceso de fluencia para un material sin endurecimiento, y (b) Proceso de fluencia para un material con endurecimiento. (Perzyna, 1966) 
Si se considera ahora un nuevo proceso de fluencia, iniciado desde un estado de tensiones que se encuentra dentro de la superficie estática de fluencia, en ese caso, la sobretensión es $F<0$. De acuerdo a las ecuaciones (51) y (53), no se producen deformaciones viscoplásticas, y esto es físicamente incorrecto porque la naturaleza de las deformaciones por fluencia es similar a la de las deformaciones plásticas postuladas por Lade \& Liu (1998).

Una desventaja de la teoría de la sobretensión es que no es capaz de modelar la fluencia terciaria o acelerada.

Por otra parte, la teoría de la sobretensión reproduce adecuadamente el fenómeno del relajamiento tensional, aunque solo en el caso en el que el proceso se inicia desde un estado tensión que cae fuera de la superficie estática de fluencia actual. También es posible simular los ensayos de velocidad de deformación constante mediante esta teoría (Liingaard et al, 2004).

Liingaard et al. (2004) presentan la teoría de la sobretensión como una expansión tridimensional del modelo unidimensional de Bingham estudiado anteriormente.

\subsubsection{Teoría de la superficie de flujo no estacionaria}

El concepto de la teoría de la superficie de flujo no estacionario (NSFS) fue introducido y desarrollado por Naghdi y Murch (1963) y Olszak \& Perzyna (1966b, 1970) como indica Matsui \& Abe (1985a, b). La descripción aquí presentada está basada en Olszak y Perzyna (1966b, 1970), Sekiguchi (1985), y Satake (1989).

Esta teoría es el resultado de un desarrollo de la teoría no viscosa de la elastoplasticidad y, por lo tanto, está basada en sus conceptos básicos. Por ese motivo, en las siguientes líneas, se discuten únicamente las diferencias entre ambas. 
La mayor diferencia entre la teoría NSFS y la elastoplasticidad clásica recae en la definición de la condición de fluencia. De acuerdo a esta última, la condición de fluencia para un material isotrópico con endurecimiento está dada por:

$$
f\left(\sigma^{\prime}{ }_{i j}, \varepsilon^{p}{ }_{i j}\right)=0
$$

donde $\sigma^{\prime}{ }_{i j}$ y $\varepsilon^{p}{ }_{i j}$ son el estado tensional y las deformaciones plásticas, respectivamente. De acuerdo a la ecuación (55), la condición de fluencia no cambia con el tiempo cuando las deformaciones plásticas se mantienen constantes. En este sentido, la superficie de fluencia se puede denominar "estática". En cambio, la condición de fluencia asociada con la teoría NSFS depende del tiempo:

$$
f\left(\sigma^{\prime}{ }_{i j}, \varepsilon^{v p}{ }_{i j}, \beta\right)=0
$$

donde $\varepsilon^{v p}{ }_{i j}$ y $\beta$ son, las deformaciones viscoplásticas y la función dependiente del tiempo, respectivamente. De la ecuación (56) se puede deducir que la superficie de fluencia se desplaza continuamente en el espacio mientras las deformaciones viscoplásticas se mantienen constantes, y es por este hecho que se denomina superficie de fluencia no estacionaria.

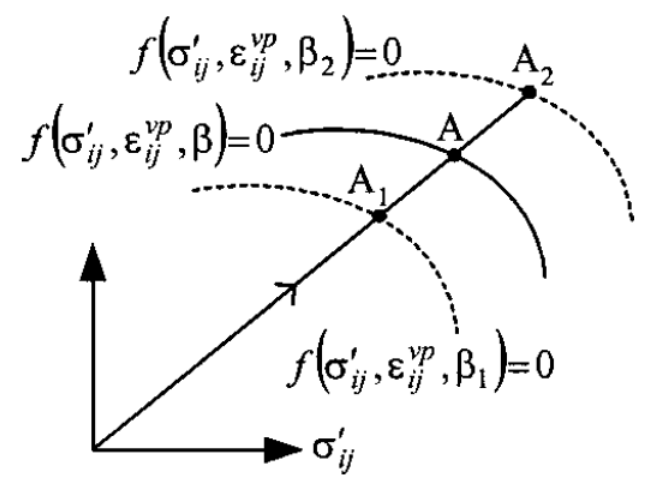

Figura 42. Trayectorias de carga y superficies de fluencia ${ }^{2}$.(Liingaard et al., 2004).

\footnotetext{
2 Para un material elastoviscoplástico, la superficie de fluencia corresponde a una deformación viscoplástica dada que se alcanza en diferentes puntos $A, A_{1}, A_{2}$, dependientes del tiempo. Para un material elastoplástico, la superficie de fluencia corresponde a una deformación viscoplástica dada para una trayectoria de carga alcanzada en el mismo punto (por ejemplo, el punto A) independientemente del tiempo.
} 
La condición de fluencia no estacionaria $f=0$ define una superficie en el espacio de tensiones y todos los estados tensionales posibles están sobre o dentro de la superficie. En el caso $f<0$, el estado tensional actual se encuentra dentro de la superficie de flujo y el suelo está entonces en un estado elástico en el que solo se producen deformaciones elásticas. Cuando $f=0$ y se considera una condición de carga, se sabe que el suelo está en un estado elastoviscoplástico y se producen deformaciones elásticas y viscoplásticas.

Como en la teoría de la sobretensión, la velocidad de deformación total asociada con la teoría NSFS se puede descomponer en una velocidad de deformación elástica $\varepsilon_{l \jmath}^{e}$ y en una velocidad de deformación viscoplástica $\varepsilon_{l j}^{i p}$, siguiendo la ecuación (45). La velocidad de deformación elástica atiende a la ley de Hooke generalizada y, como distinción con la teoría de la sobretensión, la velocidad de deformación viscoplástica está definida de acuerdo a la siguiente regla de flujo:

$$
\dot{\varepsilon}_{i j}^{v p}=\langle\Lambda\rangle \frac{\partial g}{\partial \sigma^{\prime}{ }_{i j}}
$$

donde $\Lambda$ es un producto no negativo, $g$ el potencial viscoplástico, y \langle\rangle los paréntesis de MacCauley. Estos paréntesis aseguran que las deformaciones viscoplásticas ocurran cuando se carga desde un estado plástico y, en el resto de casos, las deformaciones viscoplásticas son nulas. El factor $\Lambda$ se puede determinar empleando la regla de consistencia, que postula que cargar desde un estado tensional que cae en la superficie actual de fluencia debe conllevar de nuevo a un estado tensional que se encuentra en otra superficie de fluencia, la cual constituye la nueva superficie de fluencia actual (Prager, 1949). La expresión de $\Lambda$ es:

$$
\Lambda=-\frac{\frac{\partial f}{\partial{\sigma^{\prime}}_{i j}^{\prime}} \dot{\sigma}^{\prime}{ }_{i j}+\frac{\partial f}{\partial \beta} \dot{\beta}}{\frac{\partial f}{\partial \varepsilon_{k l}^{v p}} \frac{\partial g}{\partial \sigma^{\prime}}}
$$

donde el factor $\Lambda$ definido en la ecuación (58) debe entenderse como la suma de dos contribuciones $\Lambda_{1}$ y $\Lambda_{2}$. 


$$
\Lambda=\Lambda_{1}+\Lambda_{2}, \quad \Lambda_{1}=-\frac{\frac{\partial f}{\partial \sigma^{\prime}{ }_{i j}} \dot{\sigma}^{\prime}{ }_{i j}}{\frac{\partial f}{\partial \varepsilon_{k l}^{v p}} \frac{\partial g}{\partial \sigma^{\prime}{ }_{i j}}} y \Lambda_{2}=-\frac{\frac{\partial f}{\partial \beta} \dot{\beta}}{\frac{\partial f}{\partial \varepsilon_{k l}^{v p}} \frac{\partial g}{\partial \sigma^{\prime}{ }_{i j}}}
$$

El parámetro $\Lambda_{1}$ es idéntico al factor plástico $\lambda$ definido en conexión con la elastoplasticidad clásica. Entonces, se puede concluir de la ecuación (50), que la única diferencia entre el factor plástico $\lambda$ y el factor viscoplástico $\Lambda$ es que el último incluye un término adicional $\dot{\beta}(\partial f / \partial \beta)$ en el numerador. El término adicional implica que las deformaciones elastoviscoplásticas se producen incluso cuando las tensiones se mantienen constantes, como ocurre en un proceso de fluencia.

Las ecuaciones constitutivas en relación con la teoría NSFS, empleando las ecuaciones (58), (59), (51) y la expresión generalizada de la ley de Hooke, se pueden describir como:

$$
\dot{\varepsilon}=\frac{\dot{\sigma}^{\prime}}{E}+\left\langle-\frac{\frac{\partial f}{\partial \sigma_{i j}^{\prime}} \dot{\sigma}^{\prime}{ }_{i j}+\frac{\partial f}{\partial \beta} \dot{\beta}}{\frac{\partial f}{\partial \varepsilon_{k l}^{v p}} \frac{\partial g}{\partial \sigma_{i j}^{\prime}}}\right\rangle \frac{\partial g}{\partial{\sigma^{\prime}}_{i j}}
$$

De acuerdo a Naghdi y Murch (1963) y Perzyna (1963a, b, c), el criterio de descarga, carga neutra y carga se describe como:

$$
\begin{array}{ccc}
f=0 & L\left(\dot{\sigma}^{\prime}{ }_{i j}, \dot{\beta}\right)<0 & (\text { descarga }) \\
f=0 & L\left(\dot{\sigma}^{\prime}{ }_{i j}, \dot{\beta}\right)=0 & (\text { carga neutra }) \\
f=0 & L\left(\dot{\sigma}^{\prime}{ }_{i j}, \dot{\beta}\right)>0 & (\text { carga })
\end{array}
$$

donde el operador $L\left(\dot{\sigma}_{i j}^{\prime}, \beta\right)$ se define como:

$$
L\left(\dot{\sigma}_{i j}^{\prime}, \dot{\beta}\right)=\frac{\partial f}{\partial \dot{\sigma}_{i j}^{\prime}} \dot{\sigma}_{i j}^{\prime}+\frac{\partial f}{\partial \beta} \dot{\beta}
$$

Dado que el tiempo influye en el criterio de carga, cargar a una velocidad puede ser descargar a otra, y en la carga neutra pueden resultar direcciones no tangentes a la superficie de fluencia. Esto se puede ilustrar mediante el uso de la interpretación geométrica de las condiciones de carga, la cual se discute a continuación. La condición de fluencia para un material isótropo con endurecimiento de la ecuación (56) se puede replantear como: 


$$
\begin{gathered}
f\left({\sigma^{\prime}}_{i j}, \varepsilon_{i j}^{v p}, \beta\right)=f^{\prime}\left({\sigma^{\prime}}_{i j}, \varepsilon_{i j}^{v p}\right)-\kappa\left(\varepsilon_{i j}^{v p}, \beta\right)=0 \\
\rightarrow f^{\prime}\left(\sigma^{\prime}{ }_{i j}, \varepsilon_{i j}^{v p}\right)=\kappa\left(\varepsilon_{i j}^{v p}, \beta\right)
\end{gathered}
$$

donde $\kappa$, es la función de endurecimiento. Diferenciando la ecuación (63) e introduciéndola en las ecuaciones (61) y (62), para el caso de carga neutra resulta:

$$
\frac{\partial f}{\partial \sigma_{i j}^{\prime}} \dot{\sigma}_{i j}^{\prime}-\frac{\partial \kappa}{\partial \beta} \frac{\partial \beta}{\partial t}=0 \Leftrightarrow \cos \theta=\left(\frac{\frac{\partial \kappa}{\partial \beta} \frac{\partial \beta}{\partial t}}{\left|\frac{\partial f}{\partial \sigma_{i j}^{\prime}}\right|\left|\dot{\sigma}_{i j}^{\prime}\right|}\right)
$$

donde $\theta$ es el ángulo entre la velocidad de aplicación de la carga $\dot{\sigma}_{i j}$ y la normal a la superficie de fluencia, $\partial f / \partial \sigma^{\prime}{ }_{i j}$, en el caso de la carga neutra. Esto es, la carga neutra se puede simbolizar geométricamente como un cono en el espacio de tensiones con un ángulo de apertura $\theta$ como se ilustra en la Figura 43.

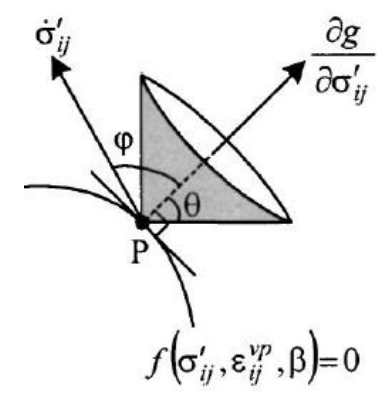

a. Unloading

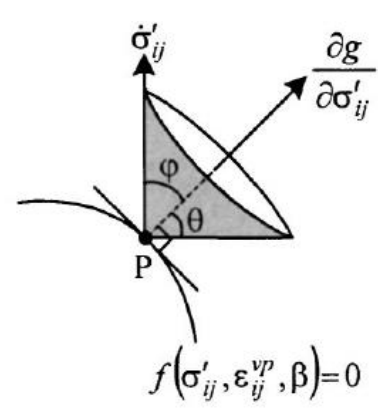

b. Neutral loading

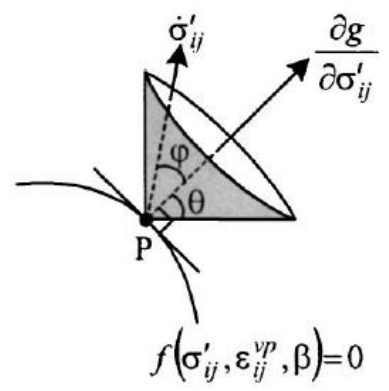

c. Loading

Figura 43. Criterio completo de carga para un material elastoviscoplástico basado en el concepto de la teoría de la superficie de flujo no estacionaria. (Liingaard et al., 2004)

Para cualquier criterio de carga (descarga, carga neutra, y carga), el ángulo formado por el vector velocidad de aplicación de carga y la normal a la superficie de fluencia, se representa mediante $\varphi$. Entonces el criterio completo de carga se define como (Naghdi y Murch, 1963):

$$
\begin{gathered}
\varphi>\theta(\text { descarga }) \\
\varphi=\theta(\text { carga neutra }) \\
\varphi<\theta(\text { carga })
\end{gathered}
$$


En principio (no hay literatura disponible que indique lo contrario) es posible modelizar el proceso de relajamiento tensional en un suelo mediante la teoría de la superficie de flujo no estacionaria, pero solo si ese proceso de relajación se inicia desde un punto sobre la superficie de fluencia y no por debajo de esta. Cuando el proceso de relajamiento tensional se inicia por debajo de la superficie de fluencia, según la teoría NSFS, solo se producen deformaciones elásticas y no puede tener lugar el fenómeno de relajación de la tensión.

Del mismo modo, considerando un proceso de fluencia iniciado en un estado tensional localizado dentro de la superficie de fluencia, la teoría NSFS no es capaz de predecir deformaciones inelásticas, por lo tanto, tampoco es adecuada para predecir deformaciones por fluencia del terreno, satisfactoriamente. Sin embargo, sí permite averiguar las deformaciones por fluencia cuando el proceso empieza desde un estado tensional en la superficie de fluencia actual.

El desarrollo de deformaciones viscoplásticas implica que la superficie de fluencia se expande en el espacio tensional y en un instante de tiempo $t_{1}$ el estado tensional constante impuesto $Q$ estará dentro de la nueva superficie de fluencia actual $f_{1}$ como se ilustra en la Figura 44.

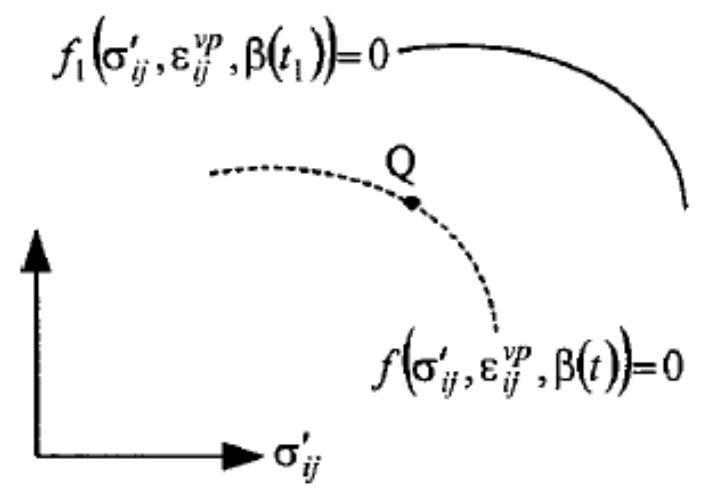

Figura 44. Proceso de fluencia según la teoría NSFS ${ }^{3}$. (Liingaard et al., 2004)

\footnotetext{
${ }^{3}$ Si un proceso de fluencia se inicia en un estado tensional $Q$ que cae sobre la superficie de fluencia actual, se producen deformaciones viscoplásticas, y la superficie de fluencia se extiende con el tiempo mientras el estado tensional $Q$ se mantenga constante.
} 
Se puede concluir que la respuesta del suelo es elástica hasta que el estado tensional alcanza la superficie de fluencia actual $y$, entonces, la respuesta es elastoviscoplástica. Esta idea es consecuente con el modelo de Bjerrum mencionado anteriormente.

Se pueden encontrar en la literatura numerosos modelos elastoviscoplásticos (EVP) basados en la teoría NSFS. Se pueden consultar algunos de ellos en Liingaard et al. (2004).

\subsubsection{Otros modelos elastoviscoplásticos}

Además de la teoría de la sobretensión y la teoría de la superficie de flujo no estacionaria, existen otros modelos EVP. A continuación, se exponen algunos de ellos de manera simplificada. Para una revisión más completa se puede acudir a los documentos orignales:

- Modelo de Borja: Hsieh, et al. (1990) proponen una relación tensióndeformación-tiempo para suelos cohesivos en la región húmeda, y Borja, et al. (1990) verifican la capacidad del modelo para describir los efectos del tiempo. El modelo predice el comportamiento de arcillas de normal a ligeramente sobreconsolidadas.

- Modelo de la superficie límite ("bounding surface model"): Dafalias (1982) y Kaliakin \& Dafalias (1990a, 1991) describen un modelo constitutivo dependiente del tiempo para suelos cohesivos, y Kaliakin \& Dafalias (1990b) verifican su capacidad para reproducir adecuadamente el fenómeno. Al-Shamrani \& Sture (1998) desarrollaron un modelo de la superficie límite, anisotrópico, e incorporan una microestructura basada en la ley del daño con el fin de simular la ruptura por fluencia.

- Modelo endocrónico: propuesto por Valanis (1971), tiene la principal característica de que no se incorpora una superficie de fluencia en la definición. Además, el tiempo se incluye en las ecuaciones por 
medio de una escala de tiempo intrínseca, la cual es una propiedad del material. Cuéllar, et al. (1977) lo aplican posteriormente para reproducir el comportamiento de arenas sometidas a corte cíclico.

- Modelo de superficie débilmente cargada ("subloading surface model"): ha sido desarrollado por Hashiguchi y sus colaboradores: Hashiguchi y Ueno (1977) y Hashiguchi y Okayasu (2000), entre otros. Extendieron este modelo elastoplástico al comportamiento dependiente del tiempo, el cual permite la simulación de una respuesta rápida ante un cambio brusco en la carga.

- Modelo de Tian y sus colaboradores (Tian et al., 1994): proponen un modelo con endurecimiento isótropo como una variación del modelo la superficie límite.

- Adachi, et al. (1990) proponen un modelo que consta de memoria y variables internas, en el sentido de que una medida de la deformación se introduce en las ecuaciones constitutivas en vez del tiempo real (Oka \& Adachi, 1985). Adachi, et al. (1997) introdujeron otra medida del tiempo y una regla de flujo no asociada.

- Cristescu (1991) propone un nuevo modelo con el propósito de describir de manera original el comportamiento de las arenas.

- El modelo de Lade \& Liu (2001) incluye los efectos del tiempo en la respuesta del terreno. El modelo es una extensión del modelo de endurecimiento único ("single harndenig model") (Lade \& Kim, 1988a, 1988b) (Kim \& Lade, 1988). El tiempo se introduce mediante el factor plástico.

- Mac, et al., (2014) sugieren un nuevo modelo EVP para simular el comportamiento dependiente del tiempo de suelos arcillosos. Este modelo propuesto incorpora en sus ecuaciones constitutivas un nuevo parámetro de endurecimiento y una regla de endurecimiento. 


\subsubsection{Enfoque de la teoría "rate process"}

La teoría "rate process" (Eyring, 1936; Joly, 1970) ha sido empleada por numerosos autores para el estudio de la fluencia en suelos (Murayama y Shibata, 1961; Mitchell, 1964; Christensen \& Wu, 1964; Mitchell, et al., 1968; Feda, 1989; Kuhn \& Mitchell, 1993). Cabe destacar que este enfoque ha sido retomado en la última "Rankine Lecture" (2017), impartida por E. Alonso, en la que se tratan los mecanismos de activación del movimiento de laderas. En estos estudios la ecuación fundamental de la teoría de la velocidad de reacción absoluta se desarrolla para su aplicación en el estudio de la deformación dependiente del tiempo en los suelos.

De acuerdo a esta teoría, las unidades fluyentes tienen su movimiento restringido por barreras energéticas virtuales separando sus posiciones de equilibrio adyacentes. Para activar los movimientos es necesaria la adquisición de una energía tal que se suficiente para superar esas barreras energéticas (veáse Figura 45).

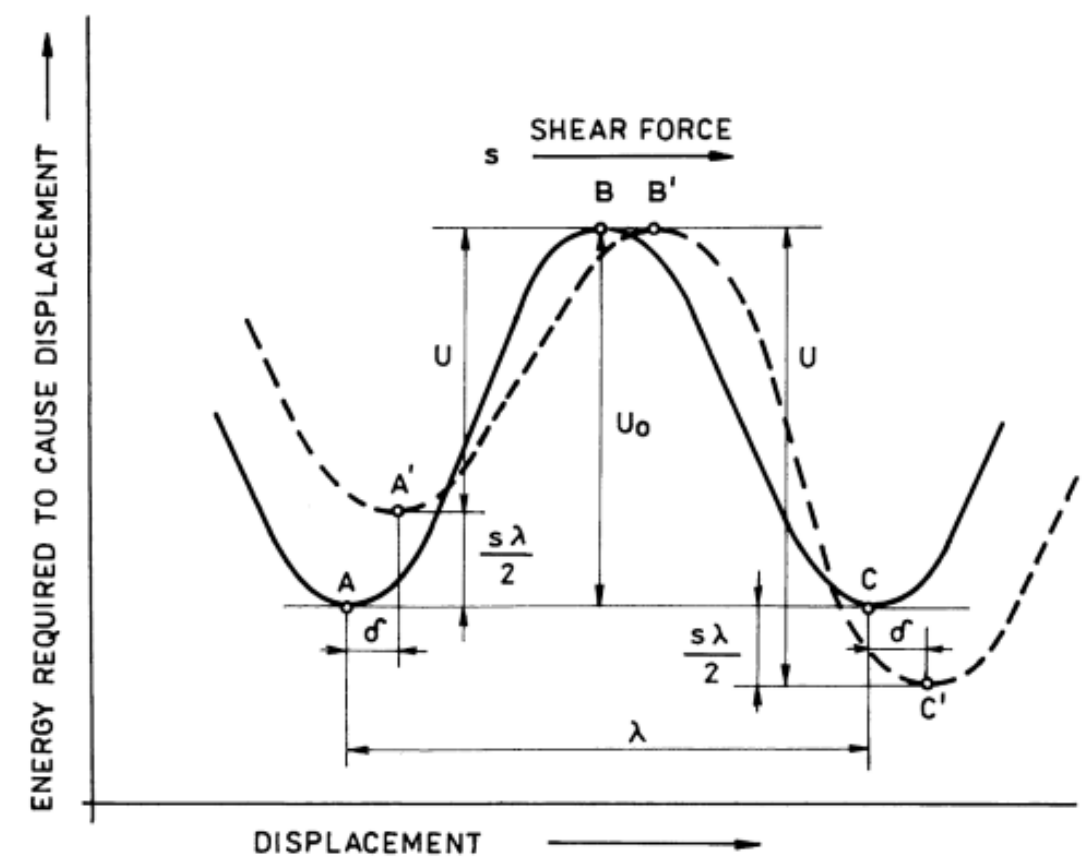

Figura 45. Barreras energéticas en la teoría "rate process" (Feda, 1989).

Mitchel et al. (1968) demuestran la dependencia de la velocidad de deformación por fluencia con la temperatura, por lo que sugieren que la 
fluencia de los suelos puede ser tratada como un proceso de activación térmica. Los autores sugieren que la ecuación (9) del modelo empírico de Singh y Mitchell (1968) es aplicable bajo condiciones de temperatura constante y, a su vez, compatible con el enfoque de la teoría de activación térmica.

El concepto principal subyace en entender, por un lado, el endurecimiento del suelo como un aumento en el número de contactos entre partículas (asociado a la etapa de fluencia primaria y a la reducción de la velocidad de deformación), y por otro, el reblandecimiento del suelo como una disminución de los contactos entre partículas (asociado a la etapa de fluencia terciaria y al incremento de la velocidad de deformación). Según demuestra Feda (1989), en el balance de las dos hipótesis anteriores se encuentra la fluencia estacionaria o secundaria.

Kuhn y Mitchell (1993) sugieren un mecanismo de deslizamiento viscofriccional, basado en esta teoría del proceso de cambio, y lo incorporan a un modelo de elementos discretos (MED) de 1002 discos circulares, proporcionando unos resultados muy acordes a los obtenidos en ensayos de laboratorio (veáse Figura 46).
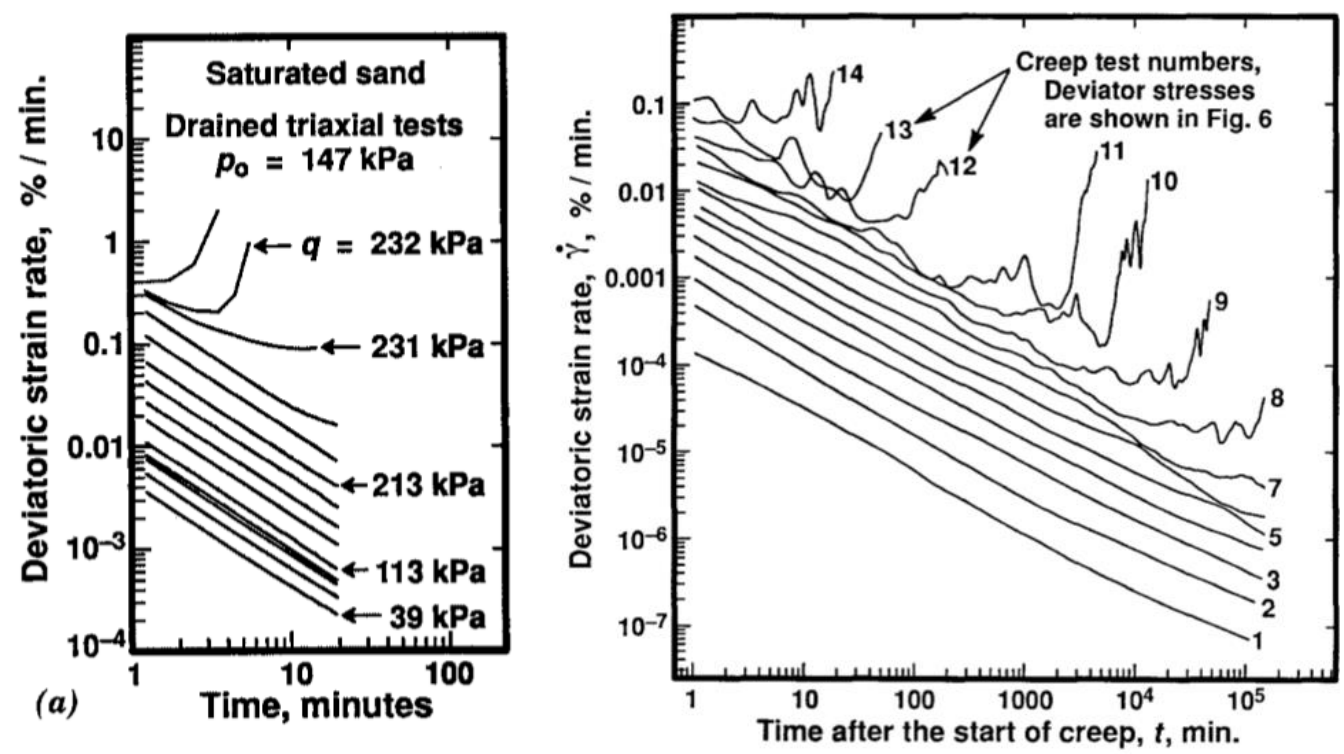

Figura 46. (a) Ensayos triaxiales drenadog reales en Toyoura Sand (Murayama et al., 1984), (b) 14 simulaciones mediante el modelo MED de 1002 discos circulares (Kuhn y Mitchell, 1993). 
Los resultados numéricos obtenidos presentan gran semejanza con la ecuación de Singh y Mitchell, estudiada en anteriores apartados. Estas similitudes sirven de base para defender la teoría del proceso de cambio como una manera de modelizar de manera realista los procesos micromecánicos (a nivel de partícula) que dan lugar a las deformaciones por fluencia.

Posteriormente (Kwok \& Bolton, 2010) reutilizaron el mecanismo en un modelo de 3421 esferas, obteniendo resultados similares en tres dimensiones.

\subsubsection{Aplicación a estudios particulares suelo-anclaje}

En el diseño de anclajes en suelos (especialmente en los permanentes), los efectos del tiempo en la resistencia al arrancamiento de estos deben ser considerados con el objetivo de poder realizar estudios más ajustados, razonables y más seguros. En definitiva, poder pronosticar de la manera más precisa posible las relajaciones que se produzcan a lo largo del tiempo de las tensiones (activas) que representan la interacción suelo-estructura.

Enfocando este documento al objetivo final, que es disponer de un buen modelo de comportamiento en función del tiempo para poderlo aplicar al sistema suelo-anclaje, se han recopilado una serie de estudios que emplean modelos reológicos, orginales o modificados, en condiciones similares.

Como se verá, la bibliografía al respecto es reducida. Según Xu et al. (2007), existen numerosas investigaciones experimentales, analíticas, y numéricas, que tratan el comportamiento de anclajes inyectados en suelos y su resistencia última o fuerza de arrancamiento. Sin embargo, es escaso el conocimiento publicado sobre el comportamiento de los anclajes al terreno dependiente del tiempo.

Se ha encontrado muy poca bibliografía específica sobre investigaciones concretas que apliquen un modelo reológico a un sistema suelo-anclaje, valorando la deformación y pérdida de carga, del terreno y del anclaje, respectivamente, a lo largo del tiempo. Por lo que, con mayor motivo, resulta de especial interés tratar este tema. 
i. Ranjan, et al. (1985) proponen una relación tensión-deformacióntiempo basada en un modelo de Kelvin no lineal, compuesto por un muelle que se reblandece con la deformación ("strain-softening") y un amortiguador que se endurece con el tiempo ("time hardening"). El modelo considerado consta de cuatro parámetros reológicos interdependientes, los cuales se pueden obtener mediante ensayos triaxiales bajo condiciones de desviador constante. Los suelos estudiados por estos autores son arcillas de alta plasticidad. Las tracciones a las que están sometidas los anclajes ensayados están comprendidas entre la mitad y un cuarto de la fuerza de arrancamiento.

ii. Gurinsky (2002) realizó un estudio aplicado a anclajes embebidos en suelos arcillosos sensitivos y susceptibles ante la fluencia. Los ensayos de laboratorio en este tipo de suelos determinaron que la etapa secundaria de la fluencia era la predominante, presentando la etapa transitoria muy corta duración. Junto con este motivo se da el hecho de que las deformaciones dependientes del tiempo aparecen a partir de un cierto umbral de carga, que es asemejable a la tensión de fluencia descrita en la Figura 39. Los gráficos teóricos que representan el modelo de Bingham proporcionan un buen ajuste a los obtenidos experimentalmente por el autor, basándose en ensayos de campo y de laboratorio.

iii. Li, et al. (2002) analizaron la deformación de anclajes en estructuras de contención. En su estudio los terrenos están formados por suelos con abundante materia orgánica. Mediante ensayos "in situ" obtenidos en obra, proporcionan un índice de diseño para la carga de los anclajes en este tipo de suelos. Como aplicación de su investigación, introducen un ejemplo en Wuhan (China).

iv. Xu et al. (2002) aplicaron un modelo mecánico para reproducir la variación de la fuerza de arrancamiento de los anclajes con el tiempo. Posteriormente, $\mathrm{Xu}$ et al. (2007) lo aplican a un anclaje embebido en una arcilla limosa, analizando la dependencia del daño acontecido por fluencia del anclaje. El modelo empleado fue el de 
Zener, obteniéndose resultados acordes de los ensayos de fluencia con el análisis teórico previamente efectuado.

v. Kim (2003) llevó a cabo ensayos de arrancamiento en siete anclajes inyectados a baja presión e instrumentados, en suelos alterados. También realizó ensayos de fluencia y de relajamiento, e investigó y evaluó el mecanismo de transferencia de carga para anclajes en compresión y en tensión, a lo largo del tiempo, mediante un modelo numérico basado en una simple viga con muelles. 


\section{CAPÍTULO 3: MODELO ANALÍTICO DE INTERACCIÓN DE PANTALLA ANCLADA}




\subsection{INTRODUCCIÓN}

El problema que se trata de resolver en esta tesis y concretamente de manera analítica, en este capítulo, es la interacción de los distintos elementos; terreno, elemento estructural y anclaje, en términos de su funcionamiento a largo plazo. Como se ha visto en el capítulo anterior, debido a la fluencia del terreno se desarrollarán movimientos a lo largo de la vida útil de una estructura anclada.

La resolución de este problema de contorno, comienza por analizar en el apartado 3.2. el caso de una losa rígida anclada verticalmente a un terreno con fluencia. La ley de deformación bajo carga constante en el terreno es conocida, estableciendo alguno de los modelos constitutivos de fluencia, presentados en el capítulo anterior, por ejemplo el modelo mecánico de Burgers o cualquier modelo empírico. De este modo, en el apartado 3.2.1.se ilustra el problema de contorno que consta de cuatro partes: el alargamiento del tirante, el levantamiento de la losa debido a la fuerza que actúa en el bulbo, el asiento de la losa por la acción del gato que ejerce la fuerza en el anclaje por medio de la reacción en la losa, y, el desplazamiento del propio anclaje por acción de la fuerza. En el apartado 3.2.2. se lleva a cabo el cálculo instantáneo del desplazamiento del sistema. A continuación, en el apartado 3.2.3. se explica el cálculo a largo plazo, mediante la conversión de los parámetros del terreno en parámetros dependientes del tiempo.

En el apartado 3.3., el sistema matemático de interacción descrito anteriormente en el apartado 3.2. se aplica a una pantalla anclada. Para ello es necesario incorporar la rigidez de un nuevo elemento como la pantalla, en este caso flexible (apartado 3.3.1.). De este modo se alcanza uno de los objetivos finales de la tesis que es obtener un modelo analítico para el cálculo de pantallas ancladas en terrenos con fluencia. Para resolver el modelo se emplean los datos de una obra real situada en Escocia, la cual es presentada en el apartado 3.3.2., y modelizada mediante los datos incluidos en el apartado 3.3.3. Un punto esencial del modelo es el desarrollado en el apartado 3.3.4. en cuanto a la obtención de los parámetros del modelo de fluencia del terreno. El 
proceso de implementación analítica de la rigidez de la pantalla y el procedimiento de cálculo se exponen en el apartado 3.3.5. Por último, se muestra la simulación del modelo analítico para el caso de la pantalla de Escocia, así como la resolución de algunas singularidades matemáticas que han aparecido durante la implementación (apartado 3.3.6.).

\subsection{MODELO MECÁNICO DE INTERACCIÓN}

\subsubsection{Planteamiento mecánico del problema de contorno}

En la Figura 47 se puede apreciar un esquema del problema planteado, donde el desplazamiento en la cabeza de un anclaje (sometido a una carga constante) se produce como suma de los movimientos de los siguientes elementos: tirante (cables o barra), elemento estructural (viga de atado o losa), anclaje (bulbo) y terreno.

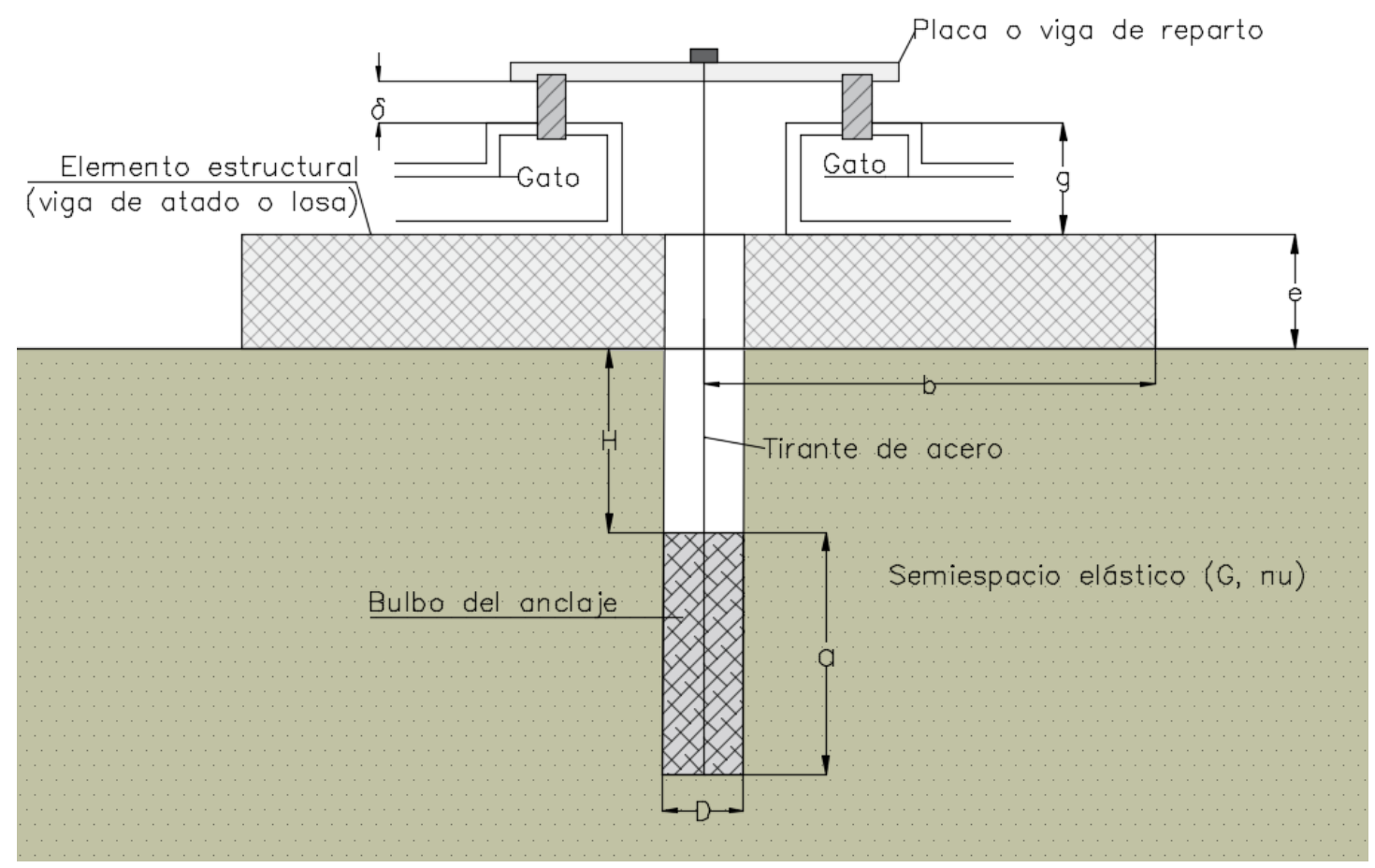

Figura 47. Esquema mecánico del problema planteado. 
En la Tabla 3 se indican los diferentes tipos de comportamiento supuestos para cada elemento del modelo físico planteado.

Tabla 3. Comportamiento supuesto de los elementos del sistema de la Figura 47.

\begin{tabular}{|c|c|}
\hline ELEMENTO & COMPORTAMIENTO \\
\hline Viga de reacción/losa & Rígido \\
\hline Gato & Rígido \\
\hline Tirante & Elástico \\
\hline Terreno & Viscoelástico \\
\hline Anclaje & Elástico \\
\hline
\end{tabular}

Para calcular el desplazamiento en cabeza del anclaje $(\delta)$ producido por una carga constante $\left(P_{0}\right)$ actuando sobre dicho anclaje, es necesario evaluar cada una de las siguientes contribuciones: alargamiento del tirante $(\Delta L)$, compresión del terreno por la losa $\left(u_{p c}\right)$, levantamiento de la losa por la tracción en el anclaje $\left(u_{p t}\right)$ y el movimiento del tirante producido por el bulbo $\left(u_{A}\right)$ debido a la carga $\mathrm{A}$ actuando en la cabeza de dicho bulbo (que es igual a la carga $P_{0}$ ). En la Figura 48 se puede ver un esquema de los diferentes elementos que actúan y su nomenclatura.

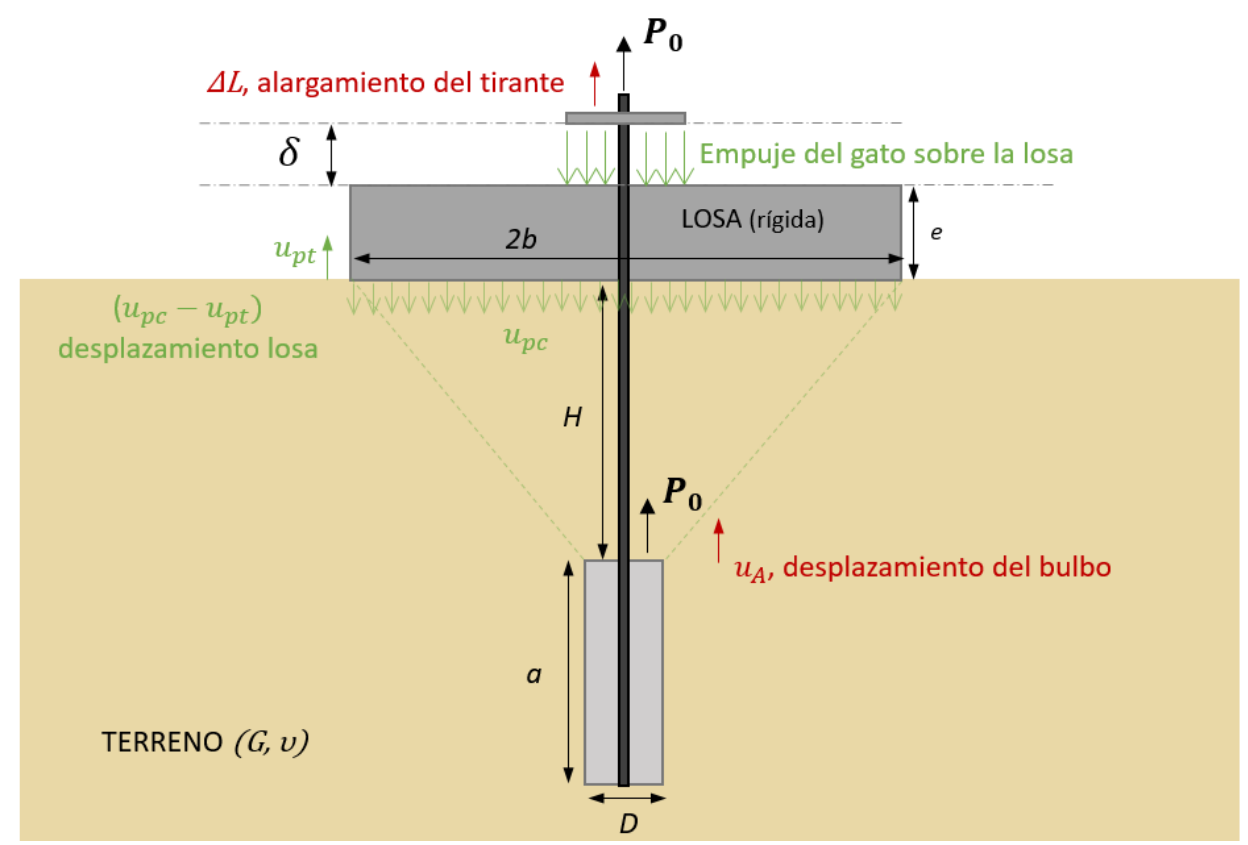

Figura 48. Esquema de desplazamiento de los diferentes elementos del modelo de cálculo. 
De esta manera, se dispone de dos sistemas mecánicos trabajando simultáneamente: por un lado el que influye sobre la losa, cuyo movimiento hacia el terreno (sentido negativo) es igual a $u_{p c}-u_{p t}$; y por otro lado, el que afecta al tirante, cuyo desplazamiento en el sentido de aplicación de la carga $P_{0}$ (sentido positivo) es $\Delta L+u_{A}$. Ambos sistemas tienen, por consiguiente, movimientos de sentido opuesto y por tanto, situando los ejes de referencia en la cara superior de la losa puede obtenerse el movimiento relativo producido entre la misma y el tirante, $\delta$ (Figura 48). Así, la ecuación que permite calcular el desplazamiento de la cabeza del anclaje respecto a la cara superior de la losa, según el sentido definido como positivo, resulta:

$$
\delta=\Delta L+u_{A}+\left(u_{p c}-u_{p t}\right)
$$

\subsubsection{Cálculo instantáneo}

En el modelo planteado la aplicación de la carga constante sobre la cabeza del tirante produce un desplazamiento instantáneo y un movimiento diferido función de la ley constitutiva de fluencia considerada. En este apartado se describen las expresiones de cada una de las componentes que producen el desplazamiento instantáneo expresado por (66).

\subsubsection{Alargamiento del tirante $(\Delta L)$}

Considerando un comportamiento elástico y lineal en el tirante que constituye el anclaje, la ecuación de su alargamiento $(\Delta L)$ es función de las características del mismo (módulo de elasticidad $E_{c}$, sección $\Omega_{c}$ y longitud inicial $\mathrm{L}$ ) y de la carga de tracción $\left(P_{0}\right)$. Tiene la siguiente expresión:

$$
\Delta L=\frac{1}{E_{c}} \frac{L}{\Omega_{c}} P_{0}
$$


3.2.2.2. Desplazamiento de la losa por compresión del terreno por la carga del gato $\left(u_{p c}\right)$

Si se supone una losa rígida circular, de radio $b$, se produce un desplazamiento del terreno constante a lo largo de toda su base (Figura 49), de manera que se puede utilizar la solución de Borowicka (1936) que cuantifica el asiento de una carga sobre un medio seminfinito.

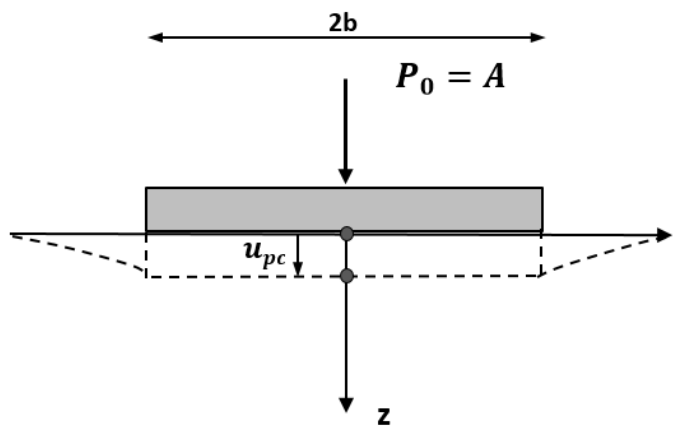

Figura 49. Hipótesis para el asiento según Borowicka.

Por tanto, para un terreno elástico (coeficiente de Poisson $v$ y módulo de corte $\mathrm{G})$, el movimiento de la losa por compresión del terreno $\left(u_{p c}\right)$ viene dado por la ecuación:

$$
u_{p c}=\frac{1-v}{(1+v) G}\left(\frac{P_{0}}{4 b}\right)
$$

3.2.2.3. Desplazamiento de la losa por la tracción desde el bulbo del anclaje $\left(u_{p t}\right)$

Se produce un levantamiento de la losa debido a la tracción A (igual a $P_{0}$ ) que se transmite desde el bulbo del anclaje. Este movimiento se puede calcular con suficiente precisión como el valor medio correspondiente al volumen de levantamiento respecto a la superficie de apoyo de la losa.

El cálculo se puede hacer particularizado las ecuaciones de Mindlin (1936) en superficie $(r, z=0)$. Para un terreno elástico $(v, G)$ y las variables 
geométricas referidas en la Figura 50, la ecuación de Mindlin para el levantamiento vertical de la losa tiene la siguiente expresión:

$$
\begin{gathered}
u_{p t}=P_{0}\left(\frac{1-v}{\pi a b^{2} G} \int_{H}^{H+a} \frac{\tau(z) \varphi_{31}(z)}{\beta} d z+\frac{1}{2 \pi a b^{2} G} \int_{H}^{H+a} \frac{\tau(z) \varphi_{32}(z)}{\beta} d z\right) \\
\varphi_{31}(z)=\sqrt{b^{2}+z^{2}}-z ; \varphi_{32}(z)=z-\frac{z^{2}}{\sqrt{b^{2}+z^{2}}}
\end{gathered}
$$

donde, de forma general:

$\tau(z)$ es la tensión de adherencia (función de la profundidad en el bulbo)

$\beta$ es la tensión de adherencia media $\left(\beta=\int_{H}^{H+a} \tau(z) d z / a\right)$

Si se considera una tensión de adherencia constante en todo el bulbo ( $\beta),(69)$ admite solución analítica cerrada:

$$
\begin{gathered}
u_{p t}=\frac{P_{0}}{\pi a b^{2} G}\left((1-v) \int_{H}^{H+a} \varphi_{31}(z) d z+\frac{1}{2} \int_{H}^{H+a} \varphi_{32}(z) d z\right) \\
\int_{H}^{H+a} \varphi_{31}(z) d z=\left[\frac{z \cdot \sqrt{b^{2}+z^{2}}}{2}+\frac{b^{2} \cdot \ln \left(z+\sqrt{\left.b^{2}+z^{2}\right)}\right.}{2}-\frac{z^{2}}{2}\right]_{z=H}^{z=H+a} \\
\int_{H}^{H+a} \varphi_{32}(z) d z=\left[\frac{b^{2} \cdot \ln \left(z+\sqrt{\left.b^{2}+z^{2}\right)}\right.}{2}-\frac{z \cdot \sqrt{b^{2}+z^{2}}}{2}+\frac{z^{2}}{2}\right]_{z=H}^{z=H+a}
\end{gathered}
$$

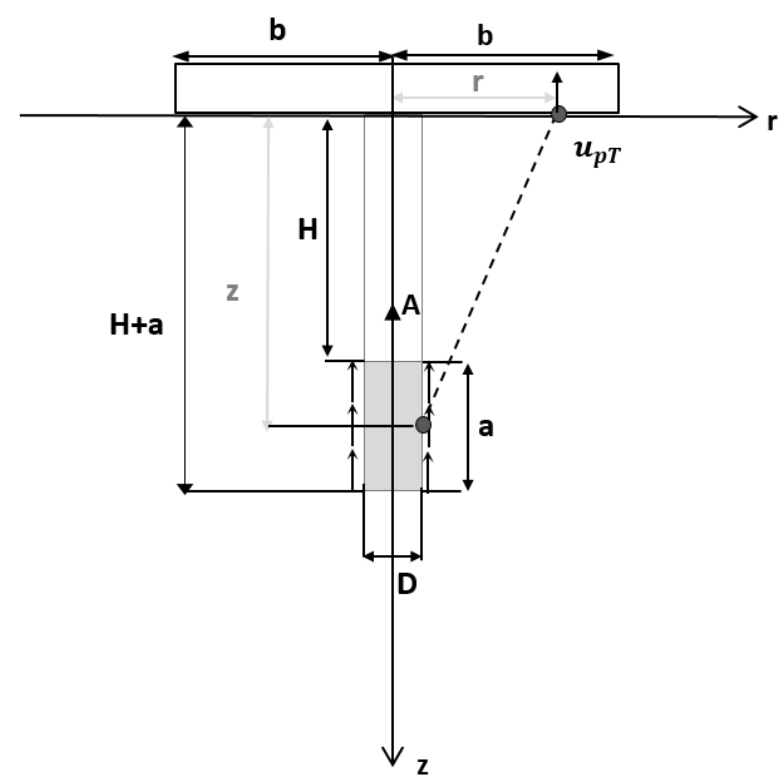

Figura 50. Esquema para la aplicación de las ecuaciones de Mindlin en superficie. 
3.2.2.4. Desplazamiento del tirante producido por la carga en cabeza del bulbo $\left(u_{A}\right)$

El desplazamiento $\left(u_{A}\right)$ en cabeza del bulbo (y por tanto del tirante) debido a la tracción $P_{0}$ transmitida a este, se puede calcular particularizando la solución de Mindlin (1936) a lo largo de la longitud anclada $(r=0)$ en la profundidad del bulbo $(z \in[H, H+a])$, según indica la Figura 51 .

Sin embargo, la aplicación de una carga puntual a la profundidad $z=H$ hace que la solución del movimiento $\left(u_{A}\right)$ obtenido se haga singular.

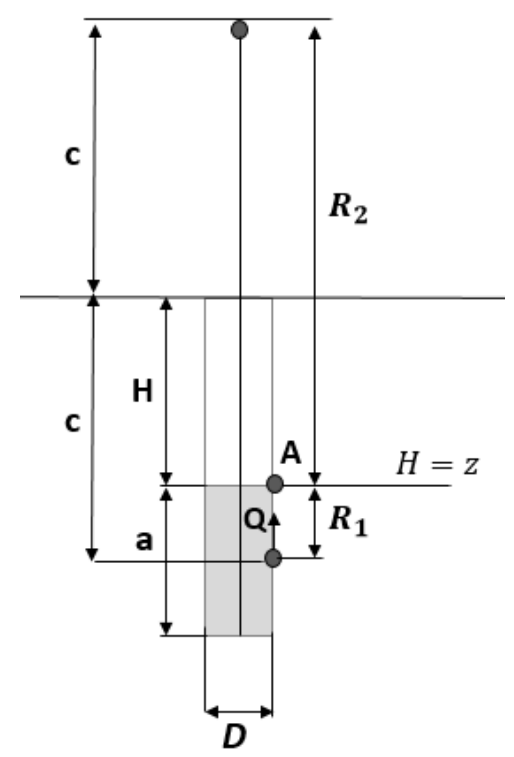

Figura 51. Esquema de la aplicación de ecuaciones de Mindlin en el bulbo y en el eje $r=0$.

Esta singularidad se resuelve mediante la integración de la ecuación de Mindlin para una carga repartida constante a una determinada profundidad actuando sobre una superficie anular (Justo, 1993):

$$
u_{A}=\frac{P_{0}}{2 \pi D^{2} G(1-v)} \int_{H}^{H+a}\left[I^{\prime}\left(r_{e}\right)-I^{\prime}\left(r_{i}\right)\right] d z=\frac{P_{0}}{2 \pi D^{2} G(1-v)} I\left(r_{i}, r_{e}\right)
$$

La expresión de la integral (denominada coeficiente de influencia, $\left.I\left(r_{i}, r_{e}\right)\right)$ correspondiente al área de integración de la corona circular donde se 
reparte la carga $P_{0}$ en la cabeza del bulbo $\left(r=r_{e}\right.$, radio externo y $r=r_{i}$, radio interno) se puede calcular a partir de:

$$
I^{\prime}(r)=B R_{1}-\frac{z_{1}^{2}}{R_{1}}+C R_{2}-\frac{K}{R_{2}}-\frac{M}{R_{2}^{3}}
$$

donde,

$$
\begin{gathered}
z_{1}=z-H \\
R_{1}=\sqrt{(z-H)^{2}+r^{2}} ; R_{2}=\sqrt{(z+H)^{2}+r^{2}} \\
M=2 H z(z+H)^{2} ; K=(3-4 v)(z+H)^{2}-2 H z \\
B=3-4 v ; C=5-12 v+8 v^{2}
\end{gathered}
$$

El caso $r_{i}=0$ equivaldría a considerar la superficie circular del bulbo de diámetro D. La influencia del radio interno de la corona circular supuesta para la aplicación de la carga será discutida en el apartado 3.6.1. en la resolución de una aplicación práctica.

\subsubsection{Rigidez del sistema}

La rigidez se define como la fuerza impuesta $\left(P_{0}\right)$ dividida por el desplazamiento instantáneo provocado $\left(\delta_{0}\right)$. De este modo, (66) se puede expresar como:

$$
\delta_{0}=\left[\frac{1}{k_{L}}+\frac{1}{k_{p c}}-\frac{1}{k_{p t}}+\frac{1}{k_{A}}\right] P_{0}
$$

Y así la rigidez del sistema $\left(k_{S}\right)$ viene dada por:

$$
k_{S}=\frac{1}{\frac{1}{k_{L}}+\frac{1}{k_{p c}}-\frac{1}{k_{p t}}+\frac{1}{k_{A}}}
$$

Las rigideces $k_{L}, k_{p c}, k_{p t}$ y $k_{A}$ de cada una de las componentes del desplazamiento del sistema, vistas anteriormente en las ecuaciones (67) a (70), se pueden expresar según se indica en la Tabla 4. 
Tabla 4. Rigidez de cada componente del desplazamiento del modelo planteado.

\begin{tabular}{|c|c|}
\hline Relación & Rigidez inversa \\
\hline $\boldsymbol{P}_{\mathbf{0}}=\Delta \boldsymbol{L} \cdot \boldsymbol{k}_{\boldsymbol{L}}$ & $\frac{1}{k_{L}}=\frac{L}{\Omega_{c} E_{c}}$ \\
\hline $\boldsymbol{P}_{\mathbf{0}}=\boldsymbol{u}_{\boldsymbol{p} \boldsymbol{c}} \cdot \boldsymbol{k}_{\boldsymbol{p} \boldsymbol{c}}$ & $\frac{1}{k_{p c}}=\frac{1-v}{4 b(1+v) G}$ \\
\hline $\boldsymbol{P}_{\mathbf{0}}=\boldsymbol{u}_{\boldsymbol{p} \boldsymbol{t}} \cdot \boldsymbol{k}_{\boldsymbol{p} \boldsymbol{t}}$ & $\frac{1}{k_{p T}}=\frac{1}{\pi a b^{2} G}\left(1-v \int_{H}^{H+a} \varphi_{31}(z) d z+\frac{1}{2} \int_{H}^{H+a} \varphi_{32}(z) d z\right)$ \\
\hline $\boldsymbol{P}_{\mathbf{0}}=\boldsymbol{u}_{\boldsymbol{A}} \cdot \boldsymbol{k}_{\boldsymbol{A}}$ & $\frac{1}{k_{A}}=\frac{1}{2 \pi D^{2} G(1-v)} \int_{H}^{H+a}\left[I^{\prime}\left(r_{e}\right)-I^{\prime}\left(r_{i}\right)\right] d z$ \\
\hline
\end{tabular}

\subsubsection{Cálculo con fluencia del terreno}

Para conocer las deformaciones con el tiempo $(\delta(t))$ del modelo mecánico planteado se debe poder evaluar el coeficiente de rigidez del sistema en el tiempo $\left(k_{S}(t)\right)$ que dependerá de las correspondientes rigideces de cada componente del movimiento dependientes del tiempo. De esta manera:

$$
\delta(t)=\frac{P_{0}}{k_{S}(t)}
$$

Las rigideces $k_{p c}(t), k_{p t}(t)$ y $k_{A}(t)$ variarán únicamente en la incorporación de los parámetros del terreno $G(t)$ y $v(t)$ en vez de los instantáneos $G$ y $v$. Estos parámetros proceden de la transformación de Laplace de la ecuación constitutiva usada para considerar las deformaciones por fluencia en el suelo.

La reología del terreno se evalúa en este trabajo considerando el modelo mecánico de Burgers para las deformaciones cortantes (Figura 52) y el modelo elástico para las deformaciones volumétricas.

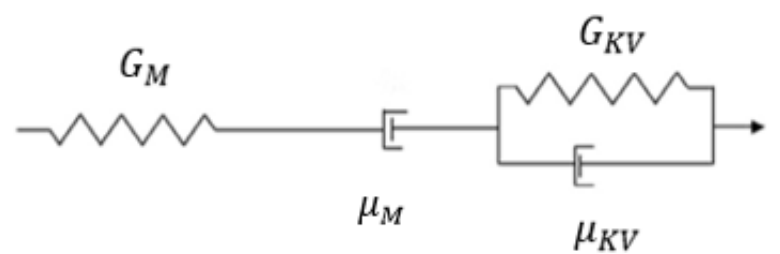

Figura 52. Esquema mecánico del modelo reológico de Burgers. 
En un medio unidimensional la expresión de la deformación en función del tiempo del modelo de Burgers, bajo una carga constante instantánea (que sigue una función de tipo escalón), puede ser obtenida a partir de la transformada inversa de Laplace (Fung, 1965). Así, se establece que el módulo de corte en función del tiempo se puede expresar como:

$$
G(t)=\frac{1}{\frac{1}{G_{M}}+\frac{t}{\mu_{M}}+\frac{1-e^{\left({ }^{-t G_{K V} / \mu_{K V}}\right)}}{G_{K V}}}
$$

donde,

$\mu_{M}$, viscosidad del elemento amortiguador del modelo de Maxwell

$G_{M}$, módulo de corte del elemento elástico (muelle) del modelo de Maxwell

$\mu_{K V}$, viscosidad del elemento amortiguador del modelo de Kelvin-Voigt

$G_{K V}$, módulo de corte del elemento elástico (muelle) del modelo de Kelvin-Voigt

Por otra parte, al considerar un modelo elástico en cambio volumétrico, el módulo de compresibilidad es constante, es decir: $K(t)=K$, y por tanto $v(t)$ se obtiene a partir de la siguiente expresión:

$$
v(t)=\frac{3 K-2 G(t)}{2(3 K+G(t))}
$$

\subsection{APLICACIÓN A PANTALLA ANCLADA}

\subsubsection{Problema de aplicación}

El sistema matemático de interacción descrito anteriormente en el apartado 3.2. se aplica a una pantalla anclada. Para ello es necesario incorporar la rigidez de un nuevo elemento (Figura 53), en este caso una pantalla flexible. Así, la ecuación para la rigidez del sistema (73) debe ser modificada e incluir la rigidez de la pantalla. 


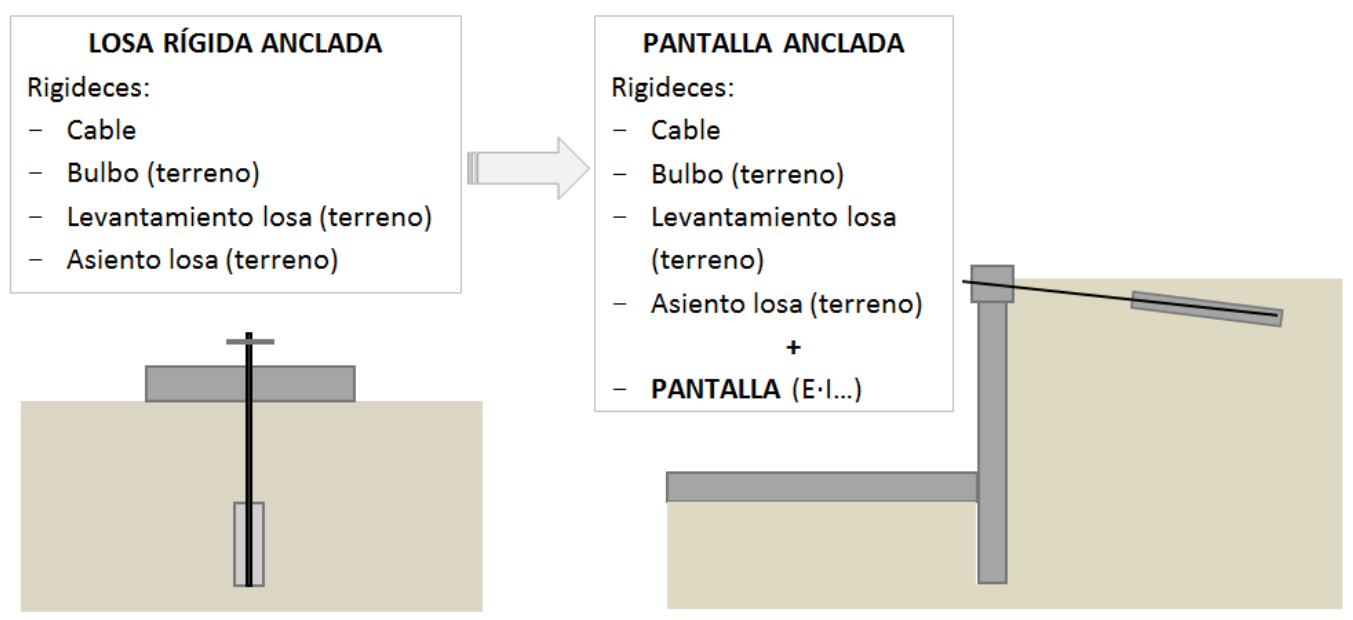

Figura 53. Esquema del problema de aplicación.

\subsubsection{Caso de análisis: Raith Junction}

Para la implementación del modelo analítico se estudia un caso real. Proviene de una autopista ya ejecutada por la empresa FERROVIAL situada en Escocia. En concreto se trata del nudo de Raith (Figura 54 y Figura 55), que enlaza la autopista M74 (carretera principal que comunica Glasgow con el Suroeste de Escocia) con la A725, mediante un paso inferior.

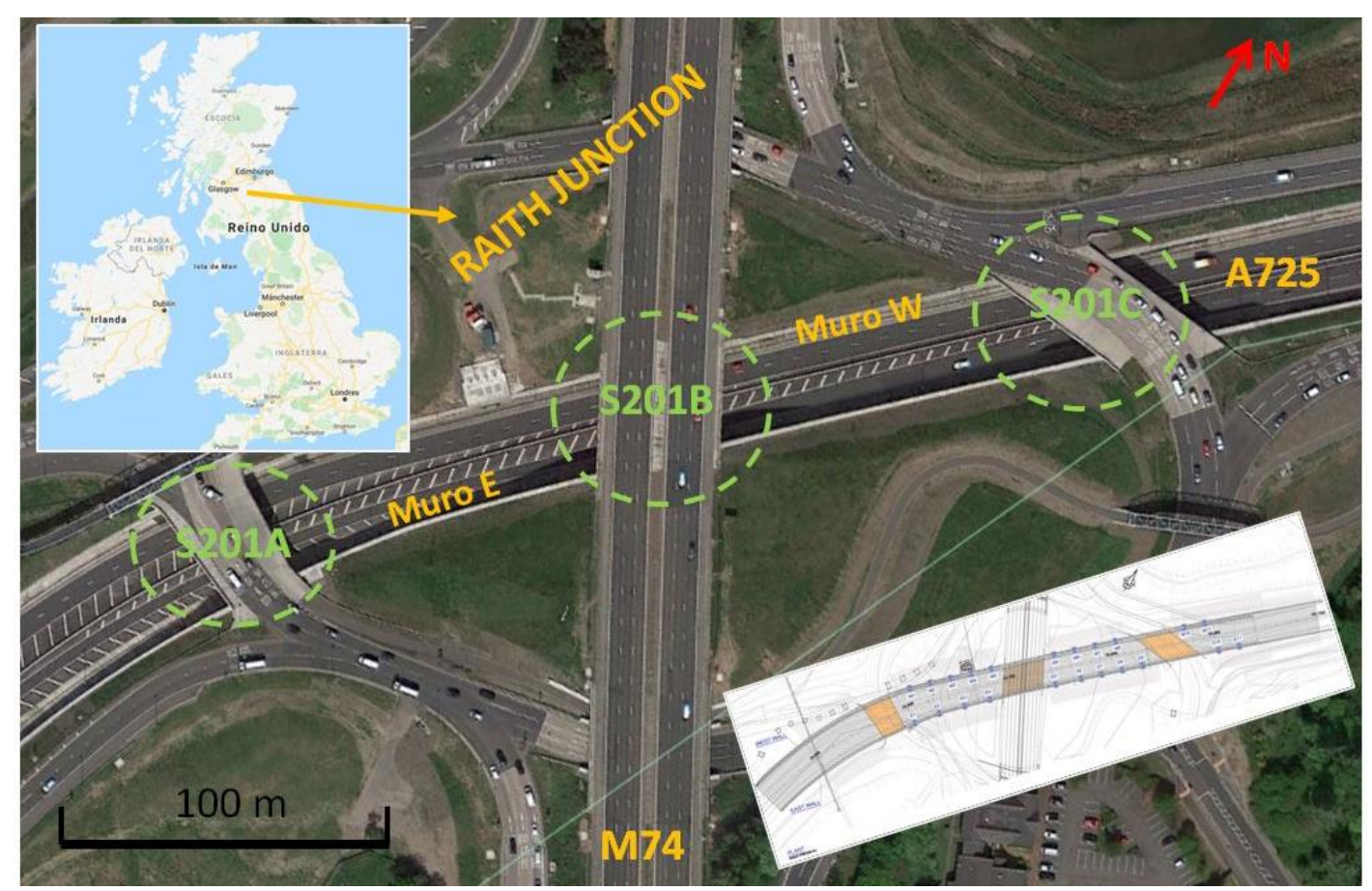

Figura 54. Vista en planta y ubicación del tramo de autopista estudiado. (Google Earth, 2019) 
El tramo de aproximadamente $500 \mathrm{~m}$ de la estructura de contención elegida está formado por una pantalla de pilotes secantes de 600, 900 y 1200 $\mathrm{mm}$ de diámetro y anclajes activos permanentes en cabeza de aproximadamente $20 \mathrm{~m}$ de longitud total.

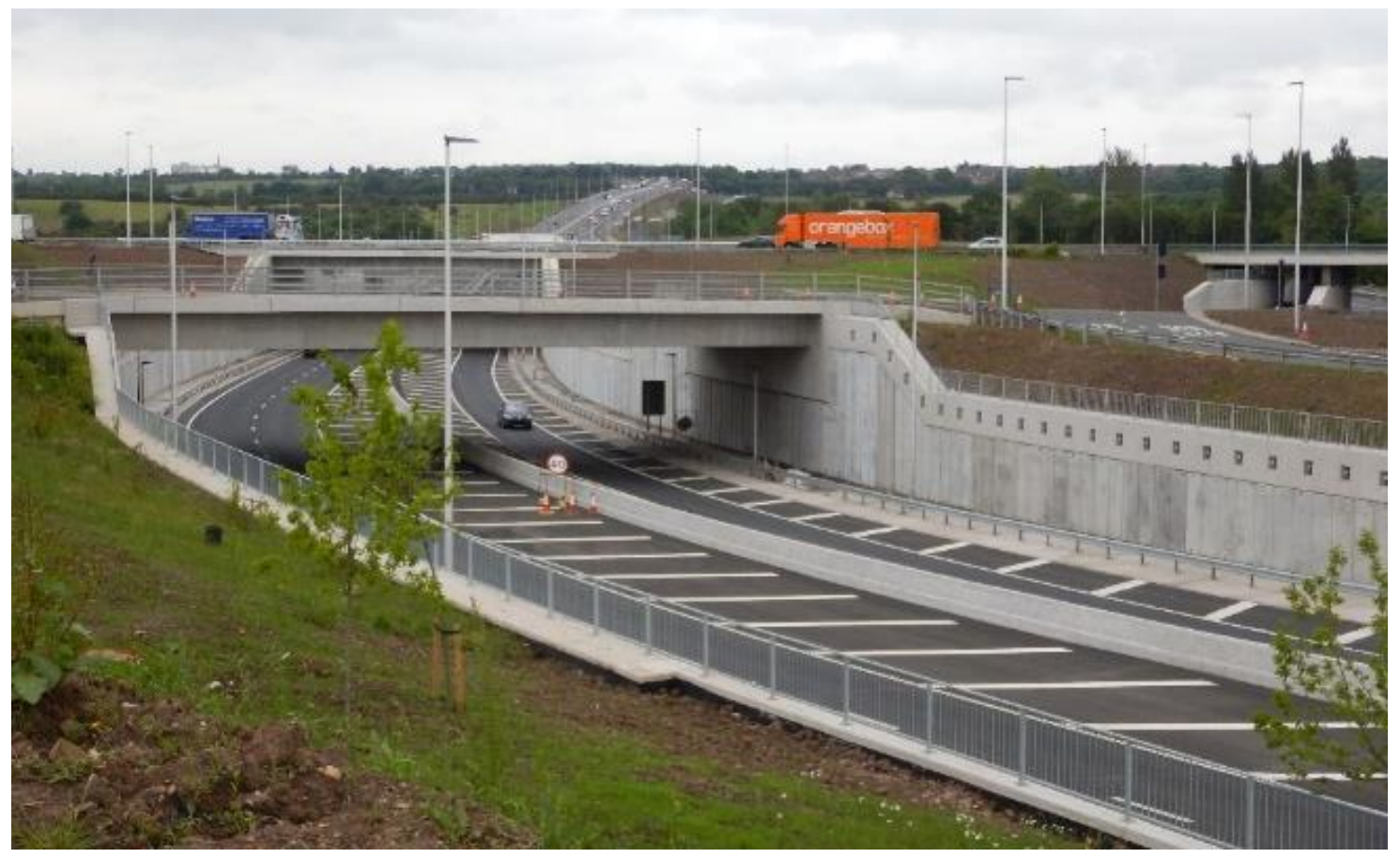

Figura 55. Vista general de la obra seleccionada para la aplicación del modelo.

La geología del área de estudio está formada por depósitos marinos de litología variable como gravas, arenas, limos y arcillas, los cuales presentan frecuentemente elevados contenidos en materia orgánica. En la Figura 56 se pueden apreciar las unidades geotécnicas consideradas, en un perfil simplificado y longitudinal, con motivo de la obra ejecutada. 


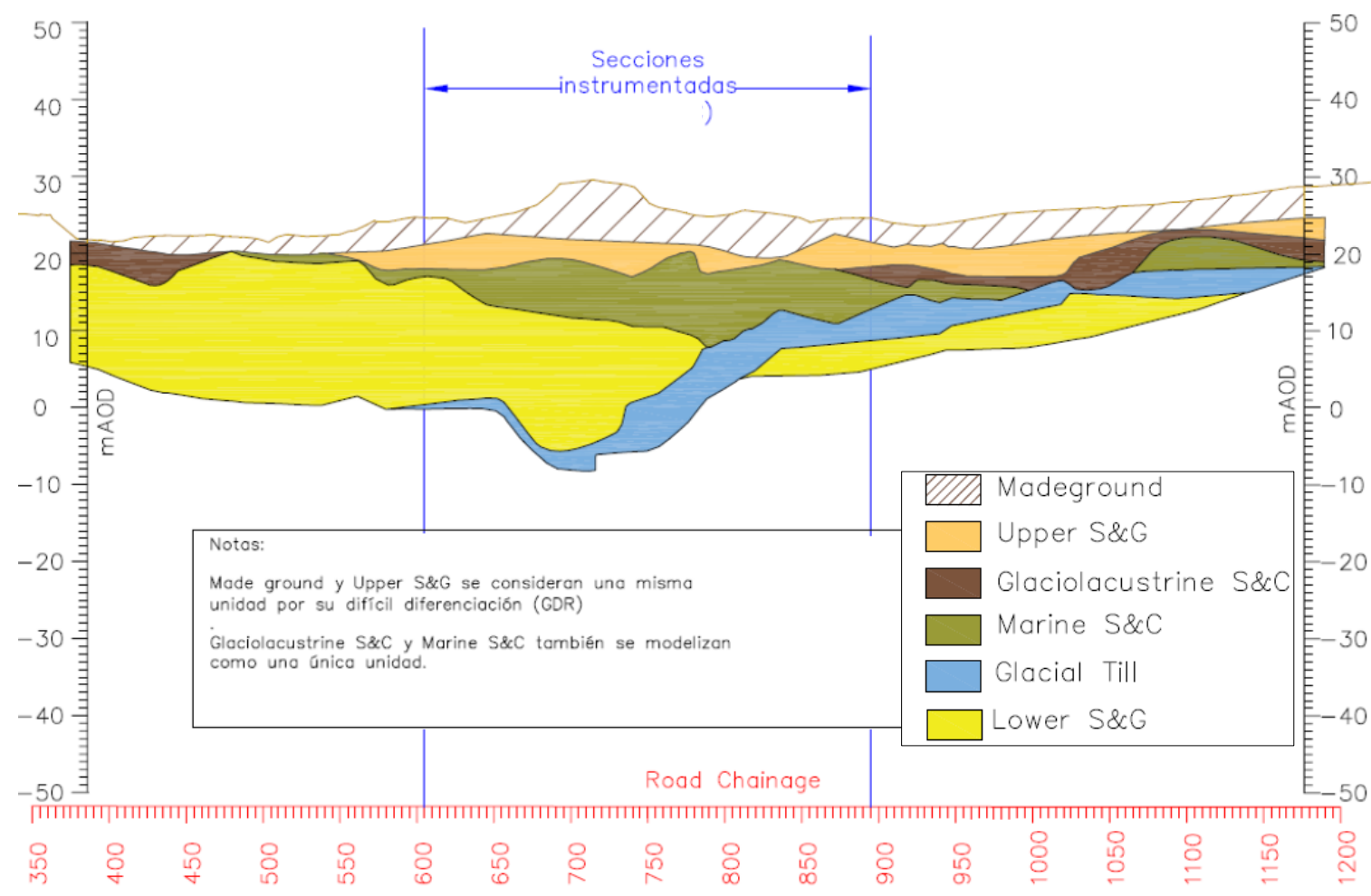

Figura 56. Mapa geotécnico del área estudiada. (Cortesía de Ferrovial Agromán y elaboración propia)

Las unidades que están presentes en el área son seis y se describen a continuación, de techo a muro, como:

- Relleno (Made Ground): está formado frecuentemente tanto por material cohesivo como granular, en un estado medio y flojo de densidad. El relleno granular es no plástico, se trata de unas gravas y arenas arcillosas de color marrón rojizo y medio densas, con inclusiones de cenizas, pizarras, escorias y guijarros. El relleno cohesivo comprende frecuentemente arcillas de plasticidad baja a media, descritas como arcillas areno gravosas, firmes de color marrón rojizo y con fragmentos de cenizas y pizarras.

- Gravas y Arenas Superiores (Upper Sands and Gravels): esta unidad está formada por una mezcla de materiales granulares que incluyen arena, arena y gravas y arena limosa, con finas capas de 
limo y arcilla intercaladas. Se encuentra habitualmente en un estado de compactación de medio denso a denso. Los datos de los ensayos y de las testificaciones indican que se trata de gravas y arenas arcillosas de color marrón grisáceo, de medio densas a densas y no plásticas. Dentro de la unidad aparecen algunos suelos cohesivos definidos como arcillas limo o gravo arenosas de color marrón.

- Limos y Arcillas Glacio-lacustres (Glacio-Lacustrine Silts and Clays): esta unidad difiere de los "Limos y Arcillas Marinos" en que es generalmente más débil, siendo de consistencia blanda a muy blanda. Los limos tienden a ser cohesivos y aparecen menos frecuentemente. Los datos de ensayos y testificaciones indican que se trata de una arcilla arenosa laminada de consistencia muy blanda a blanda, de color marrón y con plasticidad de intermedia a alta, así como con una compresibilidad de media a alta.

- Limos y Arcillas Marinos (Marine Silts and Clays): este depósito se presenta frecuentemente como una capa superior de arcilla superpuesta a unos limos. En algunos casos la arcilla puede estar ausente, mientras que la ausencia del horizonte de limos es rara. La arcilla se encuentra normalmente en un estado firme, aunque localmente puede ser blanda o rígida. El limo se describe como compacto y arenoso. El limo está en un estado medio denso y ocasionalmente suelto. En las arcillas aparecen frecuentemente niveles de limos o arenas, mientras que en los limos aparecen niveles arcillosos. Las testificaciones indican que se trata de un limo laminado, ligeramente arenoso de color marrón grisáceo, de baja a media compresibilidad, y baja a media plasticidad, con inclusiones frecuentes de arcilla limo arenosa firme.

- Gravas y Arenas Inferiores (Lower Sands and Gravels): este nivel geotécnico deriva de dos formaciones geológicas. La formación superior, compuesta principalmente por arena, con proporciones menores de limo o grava. Ocasionalmente los depósitos están formados por arena y grava o grava únicamente. Generalmente 
se encuentra en estado de medio denso a denso. Pueden aparecer niveles aislados de arcilla y lentejones cohesivos, aunque no son muy comunes. Los depósitos de la formación inferior son generalmente arenas y gravas en estado denso a muy denso.

- Sedimentos Glaciales (Glacial Till): esta unidad está formada por una arcilla areno gravosa rígida o muy rígida de color marrón rojizo. Presentan baja plasticidad y muy baja compresibilidad.

\subsubsection{Modelización del problema real}

La finalidad del caso de análisis es realizar una aplicación práctica del modelo matemático presentado en el apartado 3.2. De esta manera, la Figura 57 muestra como este modelo puede incorporarse en el cálculo a largo plazo de desplazamientos y pérdidas de carga en los anclajes situados en pantallas y otros elementos estructurales anclados.

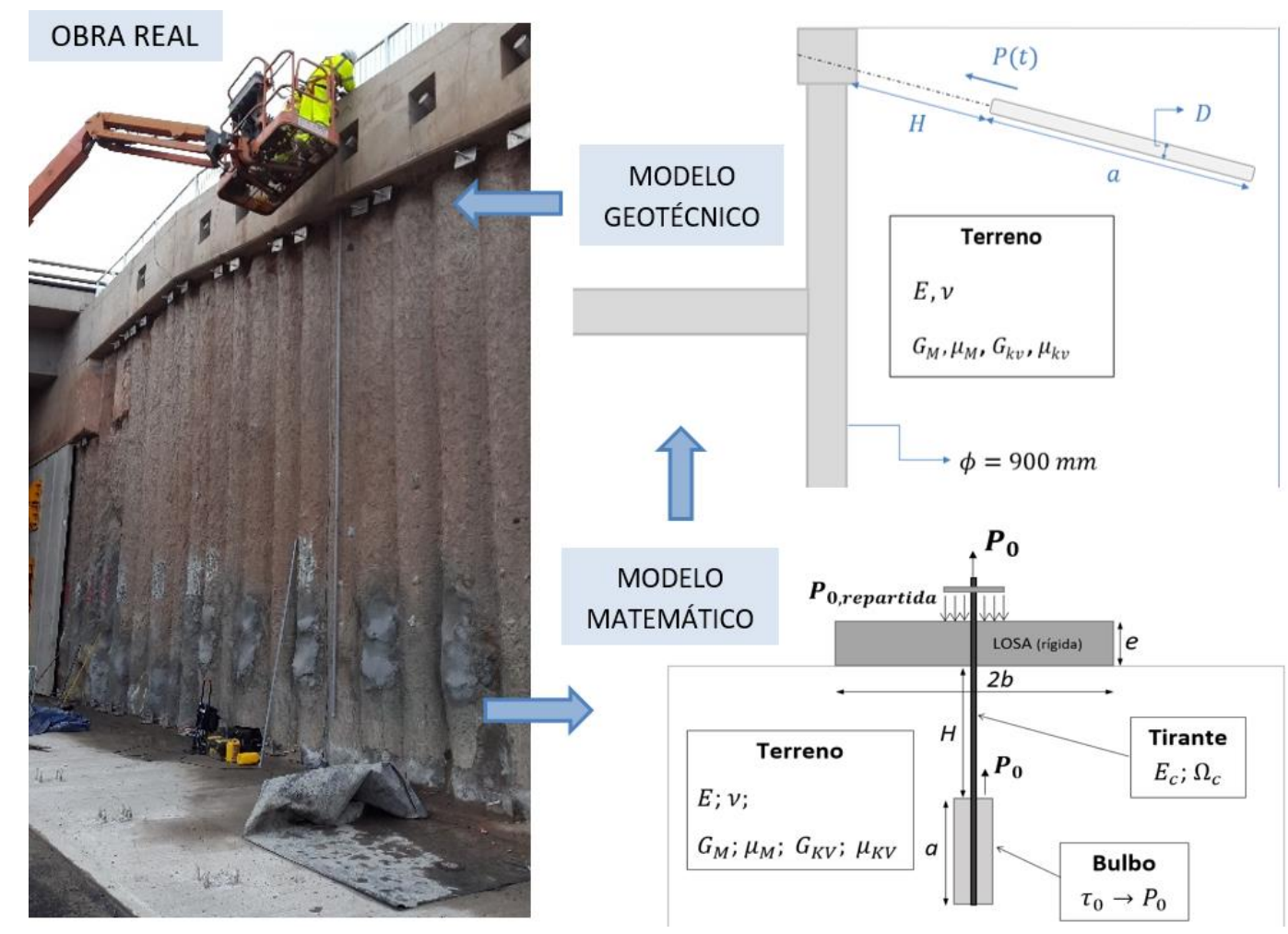

Figura 57. Fotografía de la pantalla de pilotes secantes real y esquemas del modelo de cálculo geotécnico de pantalla y del modelo matemático planteado. 
Como ejemplo se va a desarrollar el cálculo analítico de la sección de la pantalla situada en el P.K 645 del lado Oeste, que tiene los datos de construcción indicados en la Tabla 5.

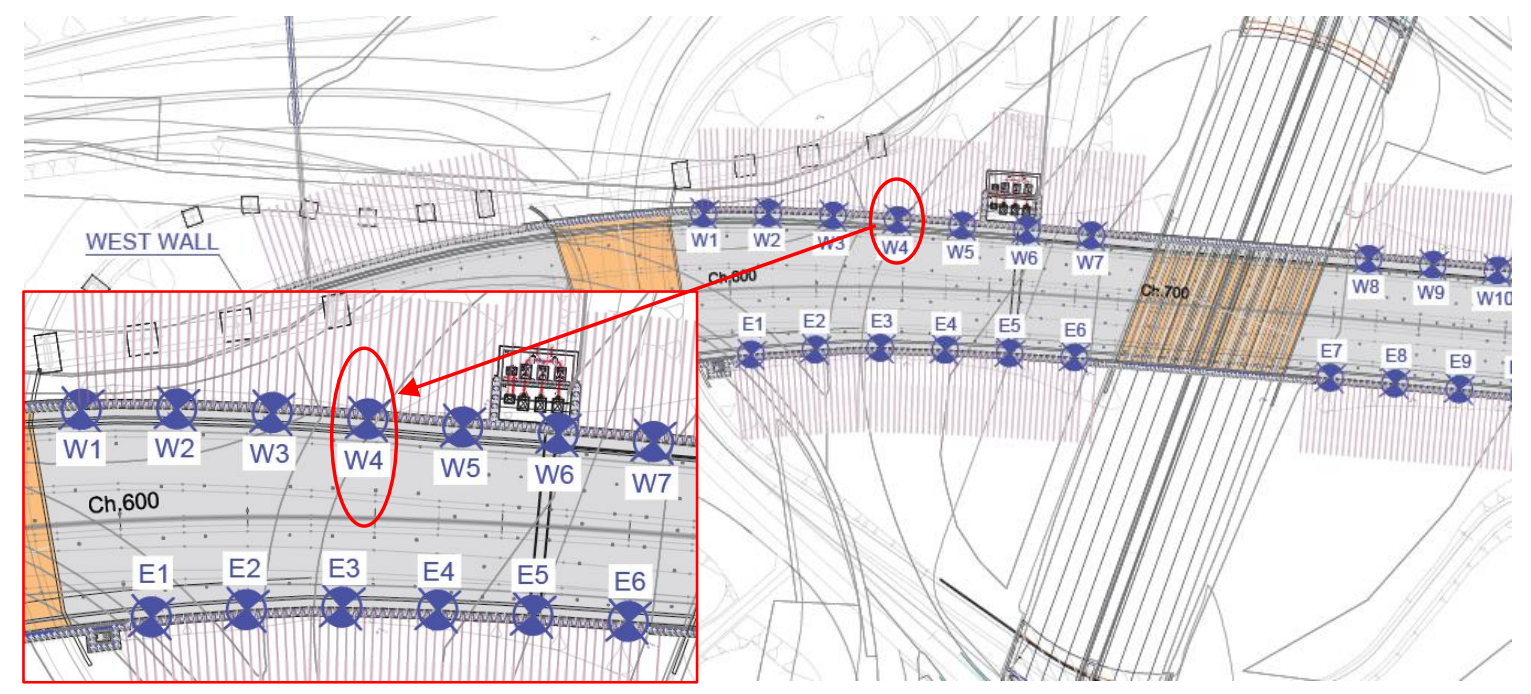

Figura 58. Localización de la sección de aplicación del modelo.

Tabla 5. Valores de los parámetros del modelo analítico utilizados en el caso real de aplicación.

\begin{tabular}{|l|c|c|}
\hline Parámetros de la pantalla & Valor & Unidades \\
\hline e (ancho de viga de atado) & 1.40 & $\mathrm{~m}$ \\
\hline $\boldsymbol{H}_{\boldsymbol{L}}$ (altura libre) & 4.83 & $\mathrm{~m}$ \\
\hline$\Phi$ (diámetro pilotes pantalla) & 0.90 & $\mathrm{~m}$ \\
\hline 2 b (separación entre pilotes) & 1.40 & $\mathrm{~m}$ \\
\hline $\boldsymbol{H}_{\boldsymbol{T}}$ (empotramiento real) & 9.92 & $\mathrm{~m}$ \\
\hline $\boldsymbol{E}_{\text {str } \text { (módulo elasticidad hormigón) }}$ & 29 & $\mathrm{GPa}$ \\
\hline Parámetros del anclaje & Valor & Unidades \\
\hline $\boldsymbol{H}$ (longitud libre en el terreno) & 7.10 & $\mathrm{~m}$ \\
\hline $\boldsymbol{a}$ (longitud del bulbo) & 10 & $\mathrm{~m}$ \\
\hline $\boldsymbol{D}$ (diámetro del bulbo) & 0.18 & $\mathrm{~m}$ \\
\hline $\boldsymbol{P}_{\mathbf{0}}$ (Carga inicial del anclaje) & 200 & $\mathrm{kN}$ \\
\hline $\boldsymbol{E}_{\boldsymbol{c}}$ (modulo elasticidad del tirante) & 195 & $\mathrm{GPa}$ \\
\hline $\boldsymbol{\Omega}_{\boldsymbol{c}}$ (área del tirante) & 660 & $\mathrm{~mm}^{2}$ \\
\hline
\end{tabular}

Un esquema con representación de los parámetros, geométricos y geotécnicos, necesarios para el cálculo analítico, se incluye en la Figura 59. 


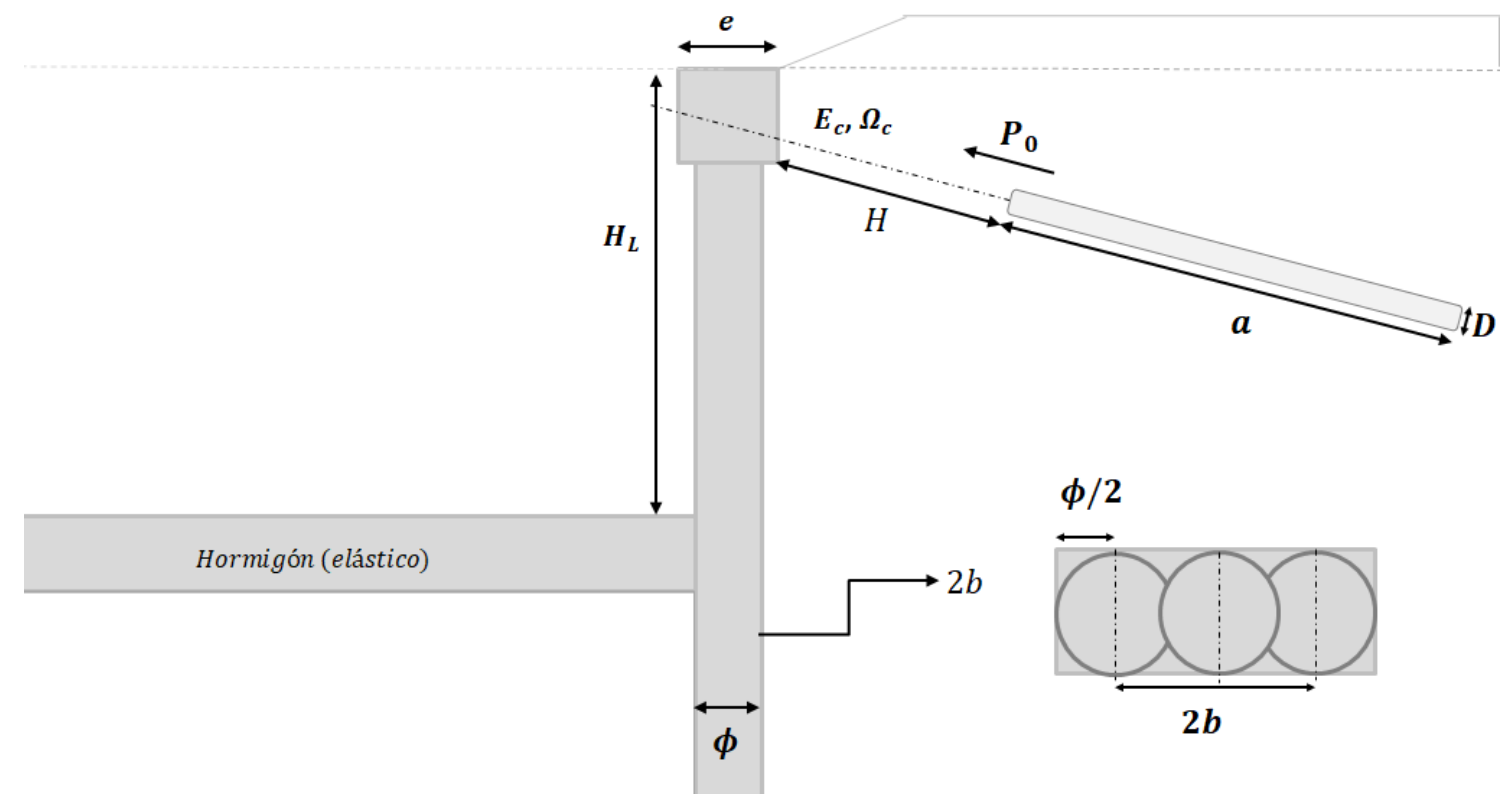

Figura 59. Esquema para la utilización de los parámetros de la Tabla 5.

\subsubsection{Obtención de los parámetros del modelo de fluencia del terreno}

El modelo de fluencia de Burgers, representado en la Figura 52 requiere de cuatro parámetros $\left(\mu_{M}, \mu_{K V}, G_{M}\right.$ y $\left.G_{K V}\right)$, que pueden obtenerse a partir de un modelo deformación-tiempo bajo carga constante en el terreno.

Se dispone de algunos ensayos de investigación de fluencia realizados en campo sometiendo a un anclaje a distintos escalones de carga crecientes y consecutivos, para después realizar la descarga y continuar con un nuevo ciclo. La máxima carga aplicada en cada ciclo, se mantiene durante 60 min y se miden los desplazamientos en cabeza a ciertos instantes de tiempo. Se debe notar que la normativa (BS-EN 1537:2000) recomienda que el desplazamiento consecutivo entre dos instantes de tiempo no debe ser superior a $2 \mathrm{~mm}$. Este límite se ha establecido para las cargas de prueba, en el ensayo de investigación, de entre 0,8 veces respecto a la carga característica del tirante y 0,95 veces la carga característica para la cual hay una deformación permanente del $0,1 \%$. 
En la Figura 60 se pueden apreciar los puntos de medida del ensayo de investigación en campo más representativo de la zona de análisis y los parámetros que resultan del ajuste de regresión no lineal al modelo de Burgers realizado con el programa estadístico SPSS (IBM SPSS Statistics), conservando en la extrapolación la misma pendiente de 60 min a 20 años.

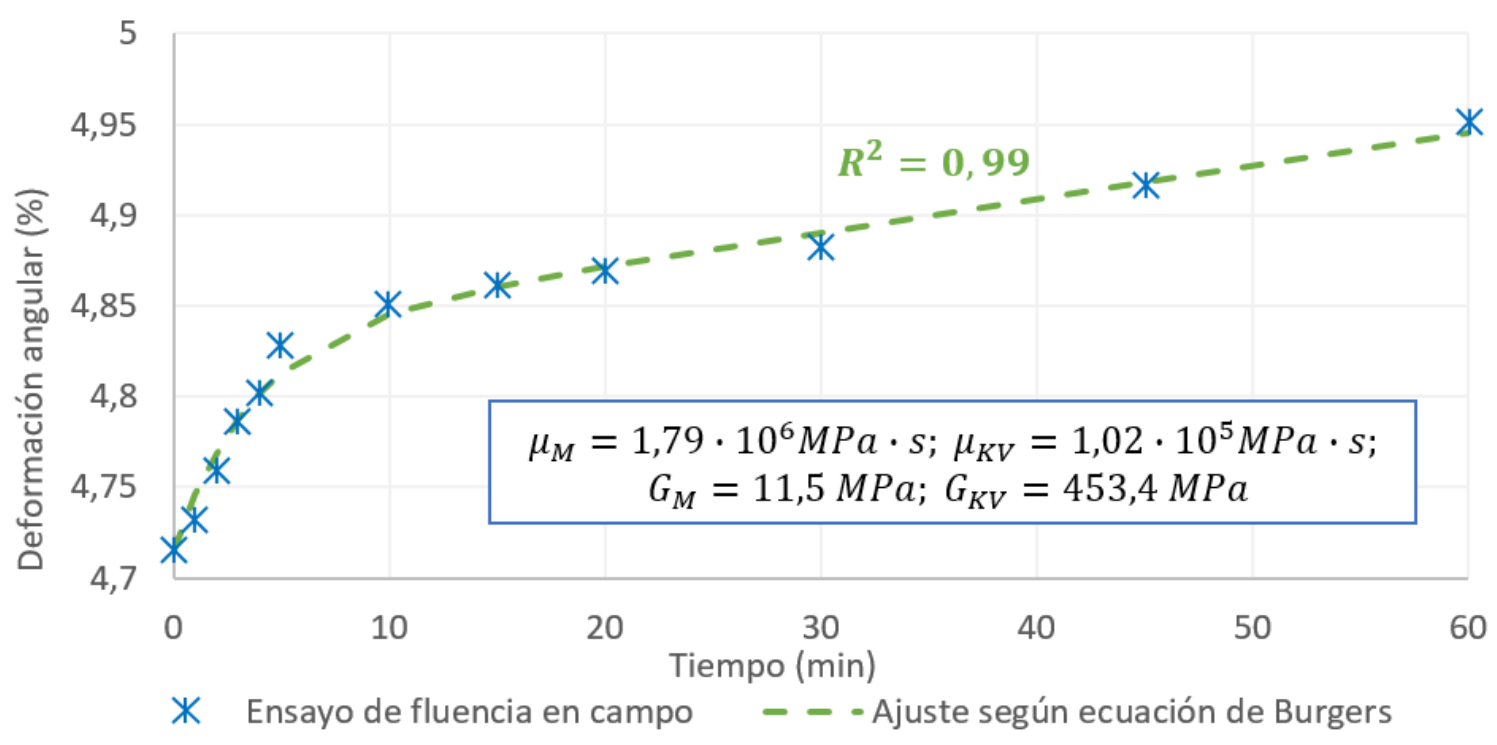

Figura 60. Datos de deformación con el tiempo del ensayo de fluencia en campo y ajuste según la ecuación de Burgers.

Si bien, en terrenos poco evolutivos, estos ensayos de investigación permiten su validación frente a deformaciones por fluencia excesivas o fuera de los límites habituales, resulta en general insuficiente emplear un intervalo de tiempo tan reducido (60 minutos) cuando quiere analizarse toda la vida útil de la estructura (en este caso 20 años). A este respecto, es ilustrativo fijar la atención en terrenos reales referenciados como, por ejemplo, la argilita de Strahov (Feda, 1992) cuyo ensayo de corte directo bajo carga constante durante 35 días se representa en la Figura 61. Sobre esta figura se indica la zona que correspondería a un ensayo de investigación de 60 min y se puede observar la evolución de la deformación diferida, cuyo incremento con el tiempo va disminuyendo, pero con valores aún significativos de 0,024 \%/día a los 35 días. Obsérvese también en este caso como la deformación acumulada de los 60 min a los 35 días es del 2,4\%. 


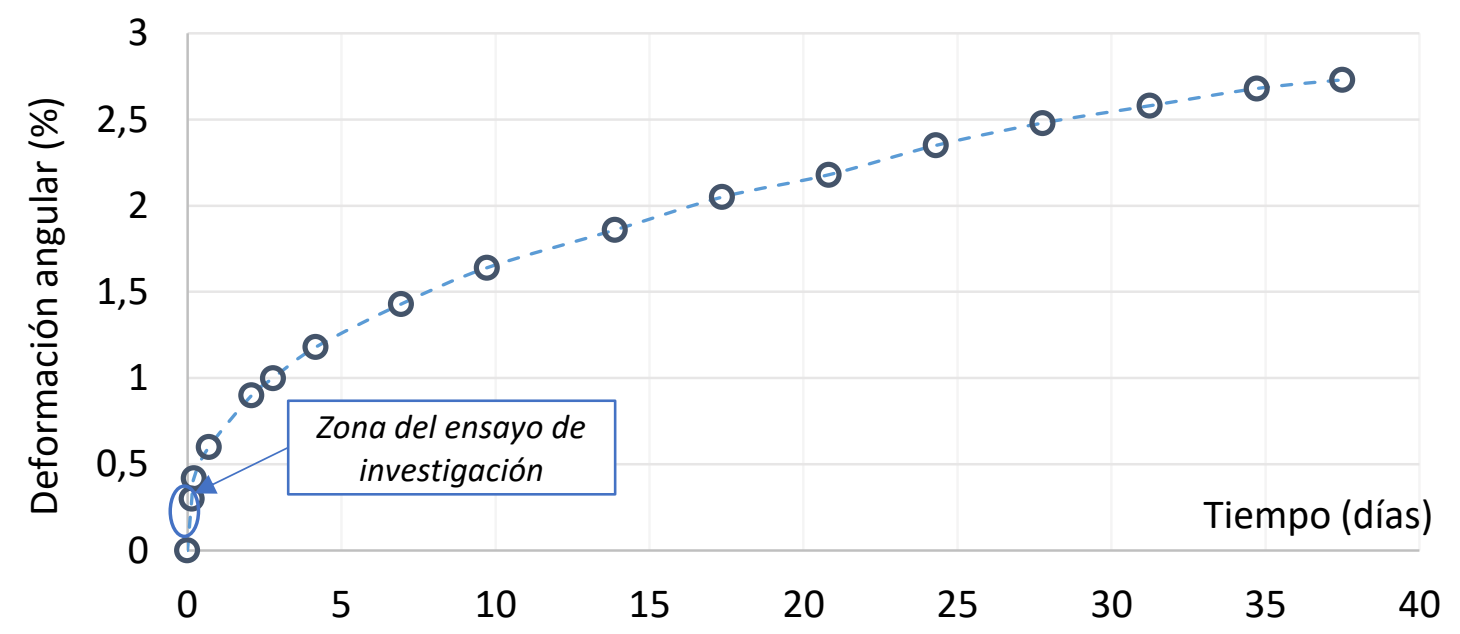

Figura 61. Ensayo de corte directo de fluencia en argilita de Strahov inalterada. (Feda, 1992).

Si, a partir del valor incremental obtenido a los 60 min en el ensayo real de investigación de la Figura 60, se supone una evolución con el tiempo similar, por ejemplo, a la del ensayo de referencia de la Figura 61, se puede mostrar la necesidad e importancia disponer de unos tiempos de ejecución más elevados, para los ensayos de fluencia. En la Figura 62 se muestra la representación de esta supuesta evolución a 20 años frente al mejor ajuste que se puede obtener con los datos reales disponibles.

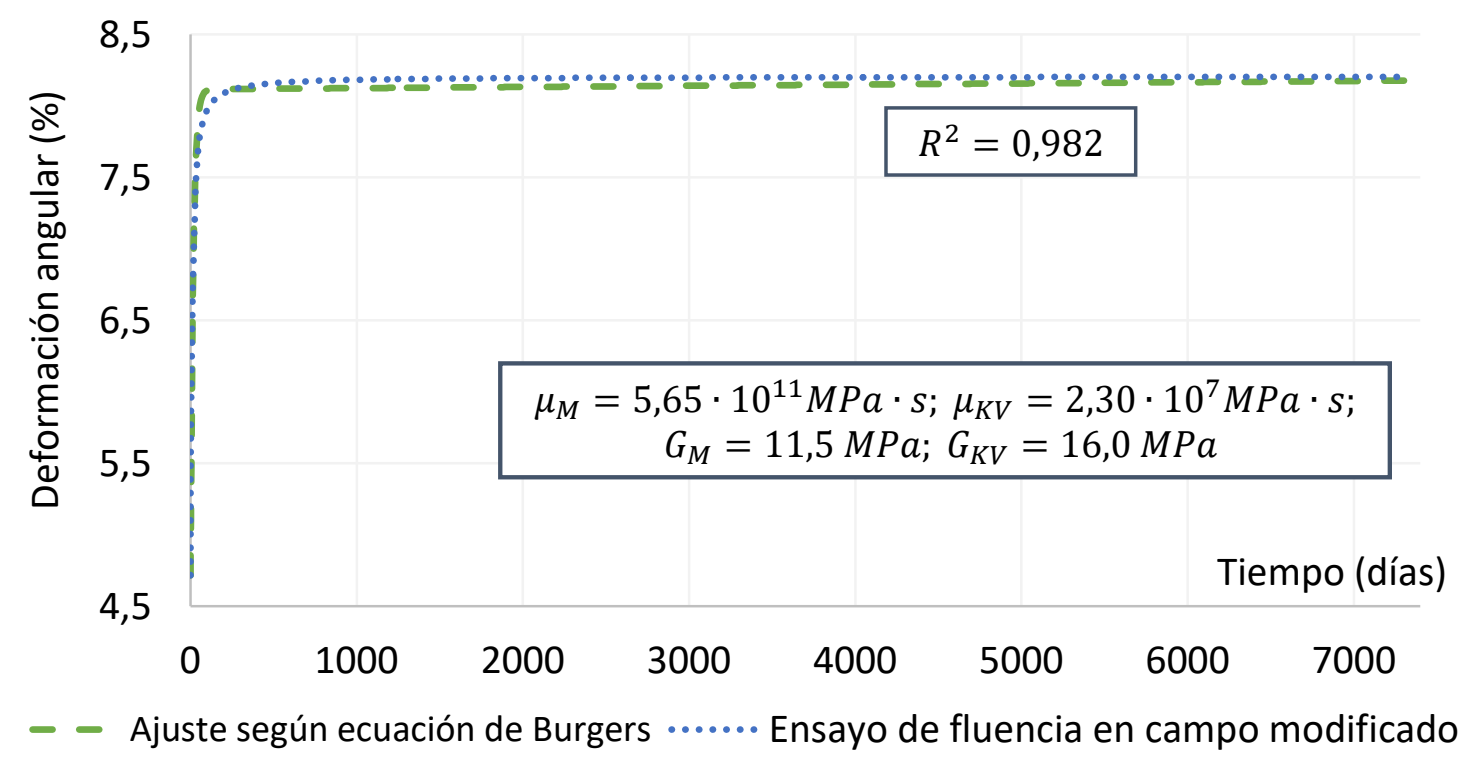

Figura 62. Datos de deformación con el tiempo del ensayo de fluencia en campo modificado a 20 años y ajuste según la ecuación de Burgers. 
Se necesita disponer de ensayos específicos, que se lleven a cabo en los terrenos donde se instalan los bulbos y en condiciones de carga constante, para lograr un adecuado análisis. De manera que el tiempo ensayado debe asegurar que el suelo se encuentra en una etapa de fluencia secundaria con pendiente constante. De este modo, generalmente se alcanza un valor incremental de la deformación con el tiempo reducido, que permite establecer que el terreno va a desarrollar una deformación por fluencia de tipo atenuado y que la pérdida de carga en el anclaje asociada a esa segunda etapa es mínima, si se compara con la perdida inicialmente, durante la fluencia transitoria.

En la Tabla 6 se incluyen los parámetros del terreno que resultan necesarios para resolver el modelo analítico propuesto. Los parámetros elegidos, o que se consideran más realistas con la información disponible, para el terreno de implantación de la pantalla anclada analizada son los correspondientes a la evolución supuesta en la Figura 60 (Caso 1) y en la Figura 62 (Caso 2).

Tabla 6. Valores de los parámetros del terreno utilizados en el caso real de aplicación.

\begin{tabular}{|l|c|c|}
\hline Parámetros del terreno & Valor & Unidades \\
\hline $\boldsymbol{\varphi}$ (ángulo de rozamiento) & 25 & $\underline{0}$ \\
\hline $\boldsymbol{V}$ & 0.3 & - \\
\hline $\boldsymbol{G}$ & 11.5 & $\mathrm{MPa}$ \\
\hline Parámetros del modelo (Burgers) & Valor (Caso 1/ Caso 2) & Unidades \\
\hline $\boldsymbol{G}_{\boldsymbol{M}}$ & $11.5 / 11.5$ & $\mathrm{MPa}$ \\
\hline $\boldsymbol{\mu}_{\boldsymbol{M}}$ & $1.79 \cdot 10^{6} / 5.65 \cdot 10^{11}$ & $\mathrm{MPa} \cdot \mathrm{S}$ \\
\hline $\boldsymbol{G}_{\boldsymbol{K} V}$ & $453.4 / 16$ & $\mathrm{MPa}$ \\
\hline $\boldsymbol{\mu}_{\boldsymbol{K} V}$ & $1.02 \cdot 10^{5} / 2.30 \cdot 10^{7}$ & $\mathrm{MPa} \cdot \mathrm{S}$ \\
\hline
\end{tabular}

La simulación del modelo analítico de pantalla anclada se llevará a cabo con los parámetros de fluencia de los Casos 1 y 2. De esta manera, se ilustra la importancia de considerar unos parámetros u otros en base a ensayos de fluencia adecuados. 


\subsubsection{Implementación y proceso de cálculo}

\subsubsection{Rigidez de la pantalla}

En el apartado 3.2. se presentó el modelo teórico para reproducir el sistema anclaje - losa rígida - terreno. Como se ha venido mencionando, es necesario incorporar la rigidez del nuevo elemento flexible para poder aplicar el nuevo modelo analítico al caso real de una pantalla anclada y, de manera general, a cualquier caso de estructuras flexibles ancladas al terreno.

Para el cálculo de la longitud de empotramiento útil de la pantalla $(t)$, se puede emplear un método de equilibrio límite, concretamente, el método del pie fijo o europeo (Figura 63), cuyas hipótesis de cálculo se deben a Blum (1931). De esta manera, para una longitud libre de pantalla $\left(H_{L}\right)$ y a partir del coeficiente de empuje activo $\left(k_{a}\right)$ y del pasivo $\left(k_{p}\right)$, se obtiene:

$$
t=\frac{H_{L}}{\sqrt{k_{p} / k_{a}}-1}
$$

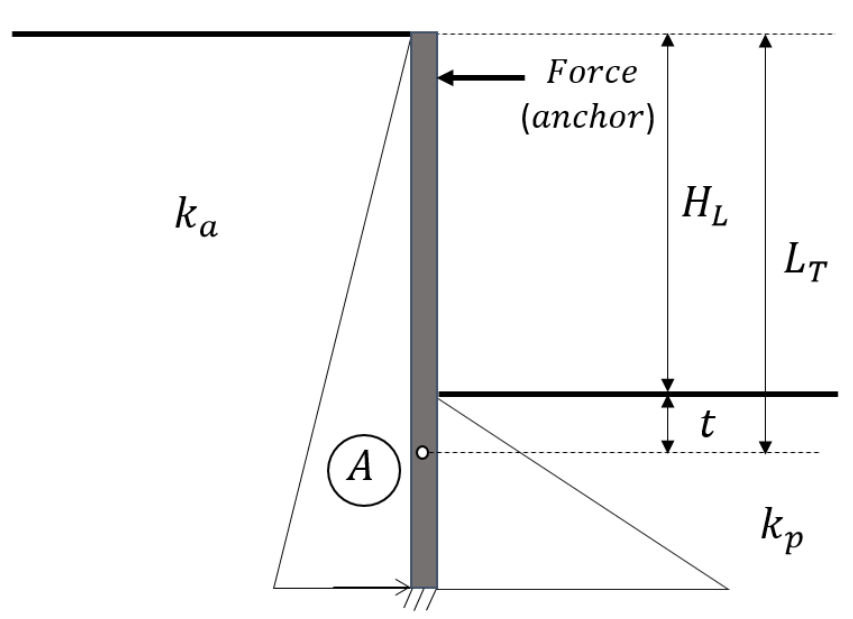

Figura 63. Esquema de cálculo del método del pie fijo o europeo y parámetros utilizados.

Por tanto, dicha longitud de empotramiento de cálculo no depende de los parámetros elásticos y por tanto permanece constante en el tiempo. 
La rigidez de la pantalla de inercia $\left(I_{s t r}\right.$, inercia de un pilote circular repartida por metro de cálculo) y módulo de elasticidad $\left(E_{s t r}\right)$ puede ser calculada como la de una viga empotrada con un apoyo en el extremo. De tal manera que, la rigidez buscada se puede expresar mediante la siguiente ecuación:

$$
k_{s t r}=\frac{3 E_{s t r} I_{s t r}}{L_{T}{ }^{3}}
$$

Siendo $L_{T}=H_{L}+t$, la longitud de giro correspondiente al punto de momento nulo (punto A, en la Figura 63).

\subsubsection{Proceso de cálculo}

Al producirse el tesado inicial del anclaje en cabeza de la pantalla, tiene lugar una deformación instantánea y en particular se produce el alargamiento elástico del tirante sometido a tracción. La fluencia del terreno provoca deformaciones diferidas, de manera que el alargamiento elástico inicial del tirante se va recuperando con el tiempo y consecuentemente se va reduciendo la carga en él.

El proceso de cálculo puede hacerse de manera iterativa, en el tiempo, según el siguiente esquema:

0 . Se calcula la deformación instantánea $\left(\delta_{0}\right)$ en cabeza del anclaje para $t=t_{0}$ a partir de (72) y la Tabla 4 . En el tirante se habrá producido un alargamiento elástico debido a la carga de tesado $P_{0}$.

1. Se elige un incremento de tiempo $(\Delta t)$ para discretizar el tiempo de la vida útil de la estructura (20 años en este caso de aplicación) y calcular las propiedades elásticas del terreno en cada instante de tiempo $t_{i}=t_{i-1}+\Delta t$, mediante (75) y (76).

2. Se calcula inicialmente el desplazamiento diferido $\Delta \delta_{1}=\delta_{1}-\delta_{0}$ para un valor de la carga constante igual al inicial $P_{0}$, entre el instante $t_{0}$ y $t_{1}=t_{0}+$ 
$\Delta t$. Para ello se utiliza (74) empleando las propiedades elásticas calculadas en el paso $1 \mathrm{y}$ considerando $t_{0}$ como origen de tiempos (es decir, $t_{0}=0$ ). Como las propiedades elásticas hacen el terreno más deformable se producirá un $\Delta \delta_{1}$ positivo de la cabeza del anclaje.

3. Para garantizar la compatibilidad de deformaciones entre la cabeza de la pantalla y el anclaje, en el instante $t_{1}$, debiera producirse un desplazamiento diferido $\left(\Delta u_{1}\right)$ en la parte superior de la misma de valor $\Delta u_{1}=\Delta \delta_{1}-\Delta L_{1}$ (siendo $\Delta L_{1}$ el incremento de longitud del anclaje entre el instante $t_{0}$ y $t_{1}$ ). Sin embargo, como los empujes no dependen de las características elásticas resultaría un desplazamiento diferido nulo de la pantalla en cabeza. Para cumplir la compatibilidad es necesario una variación de la carga de tesado del anclaje $\left(\Delta P_{1}\right)$, que se puede obtener de la condición de compatibilidad:

$$
\Delta u_{1}=\Delta \delta_{1}-\Delta L_{1} \rightarrow \frac{\Delta P_{1}}{k_{s t r}}=\Delta \delta_{1}-\frac{\Delta P_{1}}{k_{L}} \rightarrow \Delta P_{1}=\frac{\Delta \delta_{1}}{\frac{1}{k_{s t r}}+\frac{1}{k_{L}}}
$$

Por tanto, el valor $\Delta \delta_{1}$ positivo de la cabeza del anclaje se corresponde a una menor carga del tirante en el instante $t_{1}$, de valor $P_{1}=P_{0}-\Delta P_{1}$ (es decir, el tirante se destesa).

Mediante el esquema de la Figura 64 se pretende ilustrar el modo en el que se produce la pérdida de carga, empleando expresiones generales.

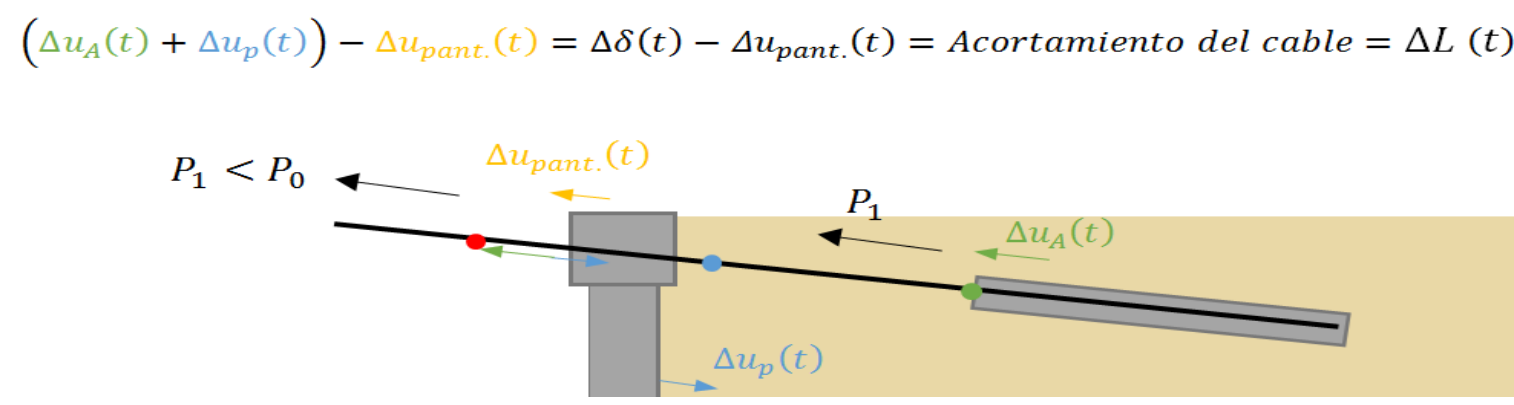

Figura 64. Esquema del proceso de pérdida de carga en el anclaje debido a la fluencia del terreno. 
4. Se calcula el desplazamiento diferido $\Delta \delta_{2}=\delta_{2}-\delta_{1}$ en la cabeza del anclaje para un valor de la carga constante igual a $P_{1}$, entre el instante $t_{1} \mathrm{y}$ $t_{2}=t_{1}+\Delta t$. Para ello se utiliza (74) empleando las propiedades elásticas calculadas en el paso 1 para $t=t_{2}$.

5. Se realiza nuevamente el paso 3, entrando en (79) con el valor de $\Delta \boldsymbol{\delta}_{2}$, para calcular $\Delta P_{2}$ en el instante $t_{2}$ y obtener, por tanto, el valor de la carga de tesado $P_{2}$.

6. Se repiten iterativamente los pasos 2 y 3 , que permiten calcular secuencialmente los valores $\Delta \delta_{i}, \Delta P_{i}$ y $P_{i}$ para cada $t_{i}=t_{i-1}+\Delta t$ hasta alcanzar el tiempo total de fluencia. De manera que el desplazamiento diferido acumulado en cada instante de tiempo $\left(t_{i}\right)$ es $\delta_{i}=\sum_{1}^{i} \Delta \delta_{i}$ y $u_{i}=\sum_{1}^{i} \Delta u_{i}$ para la cabeza del anclaje y la pantalla, respectivamente.

En este proceso de cálculo iterativo se va obteniendo progresivamente con el tiempo la evolución del movimiento diferido acumulado de la cabeza del anclaje $\left(\delta_{i}\right)$, de la carga actuante sobre el anclaje $\left(P_{i}\right)$ y del desplazamiento total de la cabeza de la pantalla $\left(u_{i}\right)$.

Como consecuencia del proceso de fluencia se va destesando el tirante y deformándose la pantalla. En terrenos con una apreciable fluencia, el destesado del tirante evoluciona hasta anularse, momento en el cual se ha recuperado completamente el alargamiento elástico instantáneo producido.

Por último, cabe indicar que debe comprobarse que el intervalo de tiempo elegido $(\Delta t)$ es lo suficientemente pequeño como para garantizar un adecuado funcionamiento del esquema numérico de resolución.

\subsubsection{Resultados de la implementación}

3.3.6.1. Influencia del área de reparto de la carga en cabeza del bulbo 
Según se indicó en el apartado 3.2.2.4, para obtener el desplazamiento del tirante producido por la carga en la cabeza del bulbo $\left(u_{A}\right)$ fue necesario repartir la carga en una corona circular, dado que la carga puntual producía una singularidad matemática en las ecuaciones analíticas. De esta manera, dicho desplazamiento $u_{A}$ depende del área de reparto considerada.

El coeficiente $\alpha$ entre el desplazamiento $\left(u_{A}\left(0, r_{e}\right)\right)$ debido a una carga repartida en una superficie circular de radio $r_{i}$ y el desplazamiento $\left(u_{A}\left(r_{i}, r_{e}\right)\right)$ debido a una carga repartida en una superficie anular de radio interior $r_{i}$ y radio externo $r_{e}$, se puede expresar como:

$$
\alpha=\frac{u_{A}\left(0, r_{e}\right)}{u_{A}\left(r_{i}, r_{e}\right)}=\left[1-\left(\frac{r_{i}}{r_{e}}\right)^{2}\right] \frac{I\left(0, r_{e}\right)}{I\left(r_{i}, r_{e}\right)}
$$

Siendo $I\left(r_{i}, r_{e}\right)$ el coeficiente de influencia definido en (71).

Para la aplicación de (80) cuando $r_{i} \rightarrow r_{e}$ es necesario calcular un límite aplicando la regla de L'Hopital para resolver la indeterminación. En la Tabla 7 se presenta una comparativa de resultados considerando diferentes valores del radio interior del área circular de la carga en la cabeza del bulbo de anclaje en el momento inicial del cálculo.

Tabla 7. Influencia del área circular de reparto para el cálculo del desplazamiento del tirante producido por la carga en cabeza del bulbo.

\begin{tabular}{|c|c|c|c|c|c|c|c|}
\hline $\begin{array}{c}\boldsymbol{G} \\
(\boldsymbol{M P a})\end{array}$ & $v$ & $r_{e}(\boldsymbol{m})$ & $r_{i}(\boldsymbol{m})$ & $I\left(r_{i}, r_{e}\right)$ & $\begin{array}{c}\boldsymbol{u}_{\boldsymbol{A}}\left(\boldsymbol{r}_{\boldsymbol{i}}, \boldsymbol{r}_{\boldsymbol{e}}\right) \\
(\boldsymbol{m} \boldsymbol{m})\end{array}$ & $\begin{array}{c}P_{0} / \text { área } \\
\left(\boldsymbol{M N} / \boldsymbol{m}^{2}\right)\end{array}$ & $\alpha$ \\
\hline \multirow{4}{*}{11.5} & \multirow{4}{*}{0.3} & \multirow{4}{*}{0.09} & 0.00 & 0.0735 & 8.94 & 7.86 & 1.000 \\
\hline & & & 0.05 & 0.0479 & 8.55 & 11.37 & 1.046 \\
\hline & & & 0.08 & 0.0144 & 8.37 & 37.45 & 1.069 \\
\hline & & & 0.09 & 0.0000 & 8.24 & $\infty$ & $1.086^{\star}$ \\
\hline
\end{tabular}

*Nota: calculado con un límite, aplicando la regla de L'Hopital

Se pone de manifiesto que, aunque una reducción del área de aplicación produce una mayor presión aplicada al bulbo, el coeficiente de influencia $I\left(r_{i}, r_{e}\right)$, es cada vez menor y la variación de resultados varía aproximadamente un $8 \%$ para los casos extremos de cálculo. De este modo, se comprueba que 
suponer la carga repartida en un círculo completo, de diámetro igual al del bulbo del anclaje, es una hipótesis razonable, que produce, de entre todas las posibles áreas de reparto, el mayor desplazamiento por fluencia en cabeza del bulbo.

\subsubsection{Simulación del modelo teórico}

A partir de la descripción del caso de aplicación y de los datos disponibles (Tabla 5 y Tabla 6), se realiza una simulación siguiendo el proceso de cálculo indicado en el apartado 3.3.5.2. bajo los dos supuestos de ajustes de los parámetros de Burgers comentados (Caso 1 y Caso 2 de la Tabla 6).

Si se consideran los parámetros del modelo de Burgers del Caso 1, obtenidos con los valores disponibles de deformación angular con el tiempo del ensayo de investigación de tan solo 60 min (Figura 60), la simulación del modelo analítico predice una pérdida de carga en el anclaje completa a los 120 días (Figura 65).

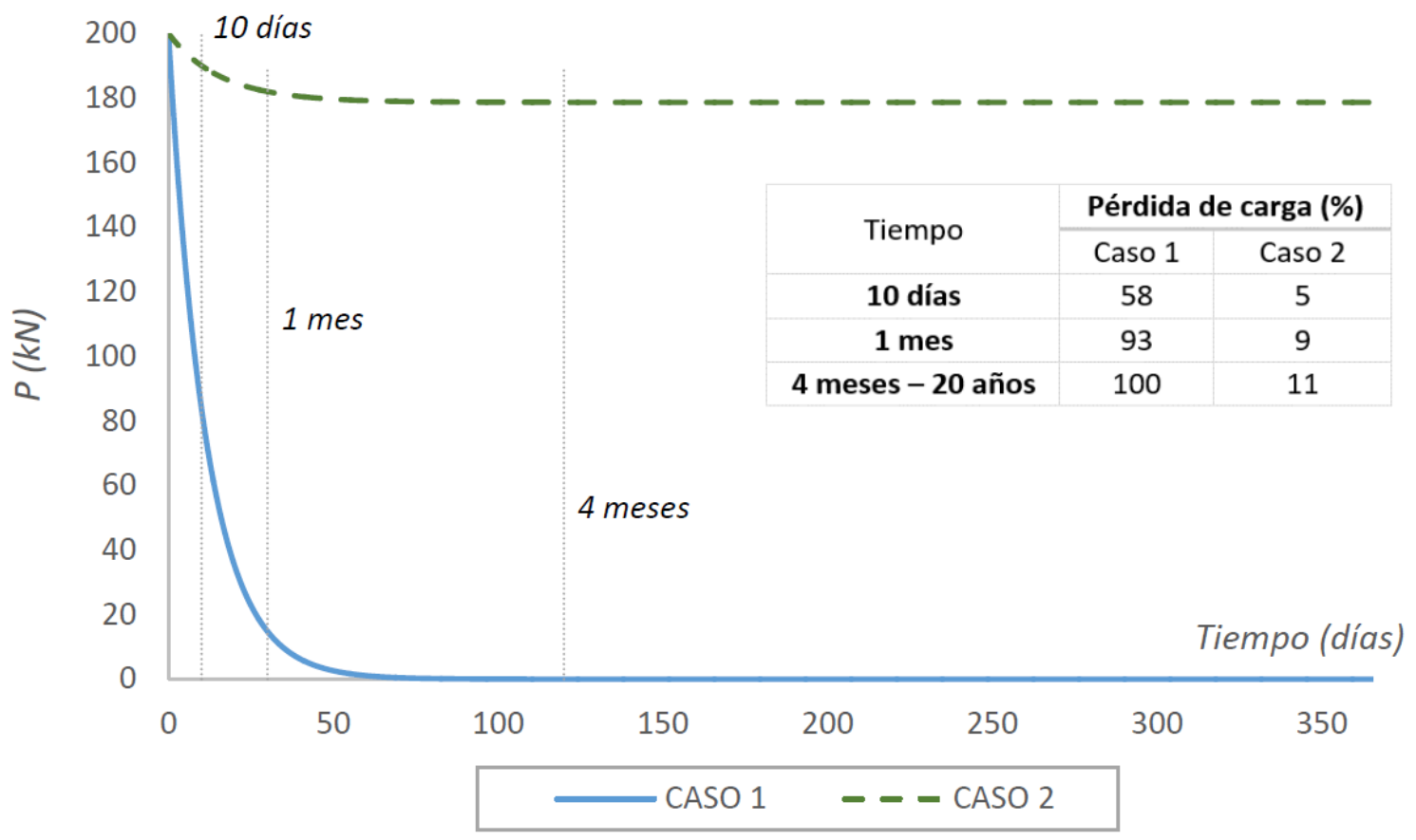

Figura 65. Evolución de la fuerza en el anclaje (durante 1 año) según el modelo analítico con los parámetros de los casos 1 (trazo continuo) y 2 (trazo discontinuo). 
En el primer supuesto (Caso 1), el desplazamiento diferido acumulado de la cabeza del anclaje se eleva a los $6,68 \mathrm{~cm}$ a los 120 días, lo cual era de esperar, pues supone recuperar el alargamiento inicial del tirante. Por su parte, el desplazamiento de la cabeza de la pantalla esperable a 120 días es igual a $5,36 \mathrm{~cm}$ según predice el modelo, siendo de $4,96 \mathrm{~cm}$ cuando han transcurrido 30 días.

Partiendo de los valores proporcionados por el ensayo de investigación supuesto en el Caso 2 (Figura 62), la evolución de la carga de tesado con el tiempo que se obtiene siguiendo el proceso de cálculo del modelo teórico, se representa también en la Figura 65. Así, la pérdida de carga al cabo de un mes sería del $9 \%$ respecto a la carga inicial de tesado y tras un periodo de 1 año del $11 \%$. Además, se obtiene un desplazamiento diferido acumulado de 7,1 $\mathrm{mm}$ de la cabeza del anclaje, que alcanza un valor aproximadamente constante a los 90 días. En ese mismo instante de tiempo el desplazamiento de la cabeza de la pantalla esperable es de $5,7 \mathrm{~mm}$.

Trasladando ambos supuestos a la práctica real en obra cabría destacar que el primer supuesto (modelo analítico resuelto con los parámetros del terreno del Caso 1) predice una pérdida de carga total en el anclaje. Sin embargo, para el cálculo de la longitud de empotramiento útil de la pantalla se ha empleado el método europeo (equilibrio límite) y la consideración de que los empujes del terreno sobre la pantalla son constantes a lo largo del tiempo, por lo que se puede asegurar la estabilidad de la misma para este caso, aunque la fuerza remanente en el anclaje sea nula. Durante el proceso de construcción de la pantalla la presencia del anclaje es útil, debido a que sin este elemento los movimientos en la pantalla tras la excavación podrían llegar a alcanzar valores elevados del orden de $9,5 \mathrm{~cm}$. Si se lleva a cabo el cálculo de la flecha para el caso en el que el anclaje ha perdido toda la carga, es decir, solo por el empuje del terreno sobre una estructura con esa rigidez se obtienen $1,8 \mathrm{~cm}$ de desplazamiento en la cabeza de la pantalla. Es decir, aunque se produjera una pérdida de carga total tras 20 años, el movimiento asociado sería únicamente 
del orden del $20 \%$ del movimiento máximo que se produciría durante la construcción si no hubiera anclaje.

Por otro lado, el Caso 2 permite diseñar la pantalla sabiendo que quedará una carga remanente considerable del $89 \%$ respecto a la carga inicial. Si fuera necesario, con el modelo propuesto se podría predecir cuánto y cuando hay que retesar los anclajes o bien, simplemente, conocer cuándo (en qué momento de la curva deformación-tiempo) hay que llevar cierto control. Adicionalmente, el factor de seguridad que se añade por fluencia se podría ver ajustado, conocida la ley de comportamiento de la carga durante la vida útil del anclaje. Es decir, este coeficiente se añade por falta de conocimiento sobre el comportamiento por fluencia del suelo y si se logra conocer cómo se comportarán los anclajes durante la vida útil de la obra gracias a este modelo, la magnitud del factor de seguridad podría verse reducida considerablemente, permitiendo diseños más realistas y ajustados y, consecuentemente, más económicos.

Por lo tanto, cabe destacar principalmente la notable diferencia de pérdida de carga entre ambos supuestos, en función de que se empleen para resolver el modelo analítico los parámetros del modelo de fluencia estimados a partir de un caso u otro (Caso 1 o Caso 2 de la Tabla 6).

Con la aplicación del modelo propuesto a un caso real se constata la validez de la solución matemática desarrollada y, por otro lado, queda ilustrada la necesidad de disponer de ensayos de fluencia a largo plazo, bien sean en campo o en laboratorio, y en los terrenos en los que se instalan los anclajes, todo ello para llevar a cabo una predicción representativa. 


\section{CAPÍTULO 4: IMPLEMENTACIÓN NUMÉRICA}




\subsection{INTRODUCCIÓN}

En los capítulos anteriores se presentó un modelo analítico desarrollado para el cálculo de pantallas ancladas en suelos con fluencia. En primer lugar, se obtuvo la solución teórica original de una losa rígida anclada verticalmente y, a continuación, la solución para la aplicación a pantallas ancladas. En este capítulo se presenta la simulación numérica en diferencias finitas de sendos modelos resueltos anteriormente de manera analítica. De tal manera que, en el apartado 4.2. se incluyen las hipótesis empleadas (apartado 4.2.1.) para generar el modelo numérico de la losa anclada a un terreno con fluencia (modelo teórico original) y su contraste con la solución analítica (apartado 4.2.2.). Asimismo, en el apartado 4.3. se lleva a cabo la modelización de la pantalla anclada a un terreno con fluencia, cuyas hipótesis (apartado 4.3.1.) y contraste con la solución analítica correspondiente (apartado 4.3.2.), también se incluyen.

Para realizar los modelos numéricos se ha empleado el software FLAC ("Fast Lagrangian Analysis of Continua"). Se trata de un código informático que realiza cálculos geotécnicos avanzados, utilizando una formulación explícita en diferencias finitas que es capaz de modelizar comportamientos complejos, como problemas que presentan numerosas etapas, desplazamientos y deformaciones muy elevadas, comportamiento no lineal del material, o sistemas inestables (incluso en el caso del colapso total). Está diseñado para acomodarse a cualquier tipo de proyecto de ingeniería que requiera un análisis del medio continuo. Entre sus principales ventajas, cuenta con un lenguaje de escritura abierta (FISH) que permite, por ejemplo, definir un modelo constitutivo propio.

Con la finalidad de presentar los modelos más utilizados actual e históricamente, resulta adecuado incorporar aquí a la recopilación del capítulo 2, los modelos constitutivos dependientes del tiempo que están implementados en un programa como el código FLAC, en una de sus versiones más recientes (7.0). Existen seis modelos implementados en FLAC 7.0 que sirven para la 
simulación de distintos materiales que presentan comportamiento dependiente del tiempo:

i. Modelo viscoelástico clásico: se refiere a la formulación original (en componentes desviadora y volumétrica) del modelo de Maxwell. Las propiedades del suelo necesarias para definir el modelo son el módulo de corte $G$, el módulo volumétrico $K$ y la viscosidad $\eta$. Supone que bajo tensiones de corte el comportamiento es puramente viscoso (el material fluye continuamente), mientras que bajo tensiones isótropas el comportamiento es elástico.

ii. Ley potencial de dos componentes: se recomienda su uso, por ejemplo, en medios rocosos propios de aplicaciones mineras (minería de la sal). Utiliza la ley potencial de Norton (Norton, 1929) comúnmente empleada para reproducir el comportamiento fluyente de formaciones salinas.

iii. Formulación de referencia para estudios de aislamiento de residuos nucleares (Modelo WIPP). Es una ley de fluencia empírica desarrollada para describir la dependencia con el tiempo y con la temperatura de una roca de sal. El modelo es formulado por Herrmann, et al. (1980a, b) y Senseny (1985) y proporciona una expresión propia pero diferente de la misma ley de fluencia.

iv. El modelo viscoplástico de Burgers; es una combinación del modelo viscoelástico original de Burgers, tratado anteriormente, y el modelo de plasticidad basado en el criterio de rotura de Mohr-Coulomb. Se caracteriza por un comportamiento desviador viscoelastoplástico y un comportamiento volumétrico elastoplástico. Incorppora la hipótesis de que las componentes viscoelástica y plástica de la velocidad de deformación actúan en serie.

v. Modelo viscoplástico WIPP: este modelo se implementa combinando el modelo viscoelástico WIPP con el modelo de plasticidad de Drucker-Prager. Esta combinación es más manejable, debido a que ambos modelos se formulan en términos del segundo invariante del tensor desviador. 
vi. Modelo constitutivo para sal triturada ("crushed-salt constitutive model"): se emplea para simular los comportamientos de compactación volumétrica y desviadora. Es una variación de la ley WIPP y está basado en la ley descrita por Sjaardema \& Krieg (1987), añadiendo una componente desviadora como la propuesta por Callahan \& DeVries (1991).

\subsection{APLICACIÓN NUMÉRICA AL MODELO TEÓRICO ORIGINAL}

\subsubsection{Hipótesis para la simulación}

El modelo analítico para una losa rígida anclada a un terreno con fluencia se valida mediante un esquema en diferencias finitas realizado con el software geotécnico FLAC 3D (Itasca, 2012).

Como primer paso se ha realizado un cálculo previo para reproducir la distribución de tensiones verticales bajo una carga uniforme aplicada en una superficie circular y optimizar así el tamaño de elemento bajo la losa. Con este modelo se ha determinado que 8 elementos bajo el radio de aplicación de la carga produce errores $<5 \%$ en la distribución de tensiones verticales. Como se puede apreciar en la Figura 66 y en la Figura 67 se ha creado un modelo axilsimétrico discretizado con 8 elementos bajo el radio de la losa circular y con elementos mayoritariamente cúbicos. 
FLAC3D 5.01

@2018 Itasca Consulting Group, Inc.

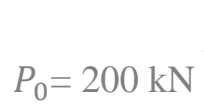

$P_{0}=200 \mathrm{kN}$

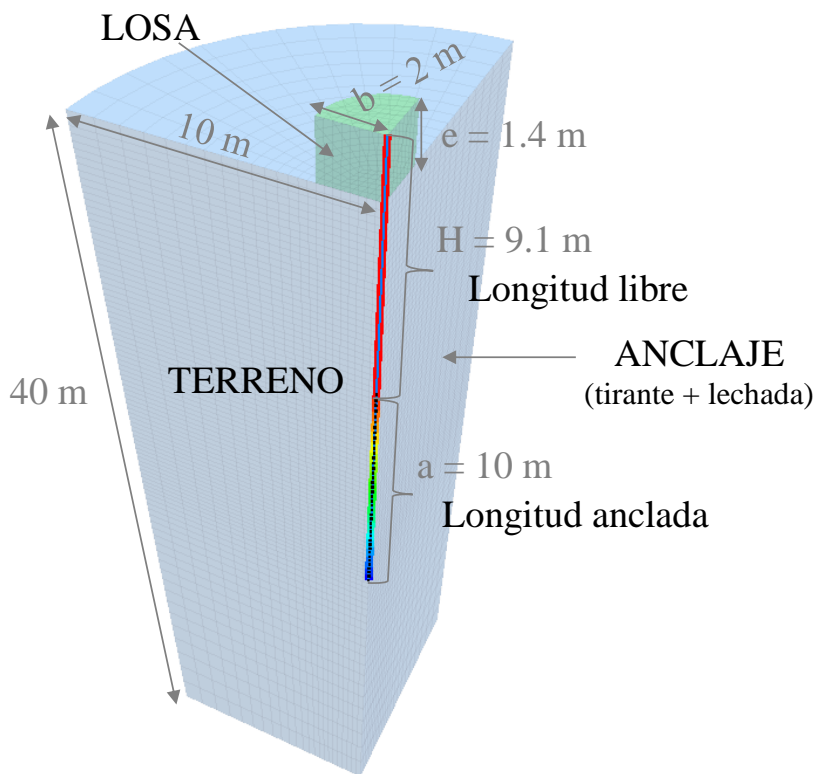

Figura 66. Fuerza en el tirante al cabo de 20 años, geometría del modelo y parámetros.

El mallado del modelo se ha llevado a cabo mediante formas primitivas ${ }^{4}$. Las formas empleadas en este caso son de tipo prisma y de tipo cuña. Para crear la geometría circular se emplea una rutina de deformación de las formas originarias que convierte las aristas de los hexaedros en arcos, de una manera más precisa que si se emplea directamente la primitiva "cilindro".

\footnotetext{
${ }^{4}$ Formas geométricas originales del programa que permiten la generación de la malla y sirven como "celdas unidad" (p.e. cuadrados en dos dimensiones o tetraedros en tres dimensiones).
} 


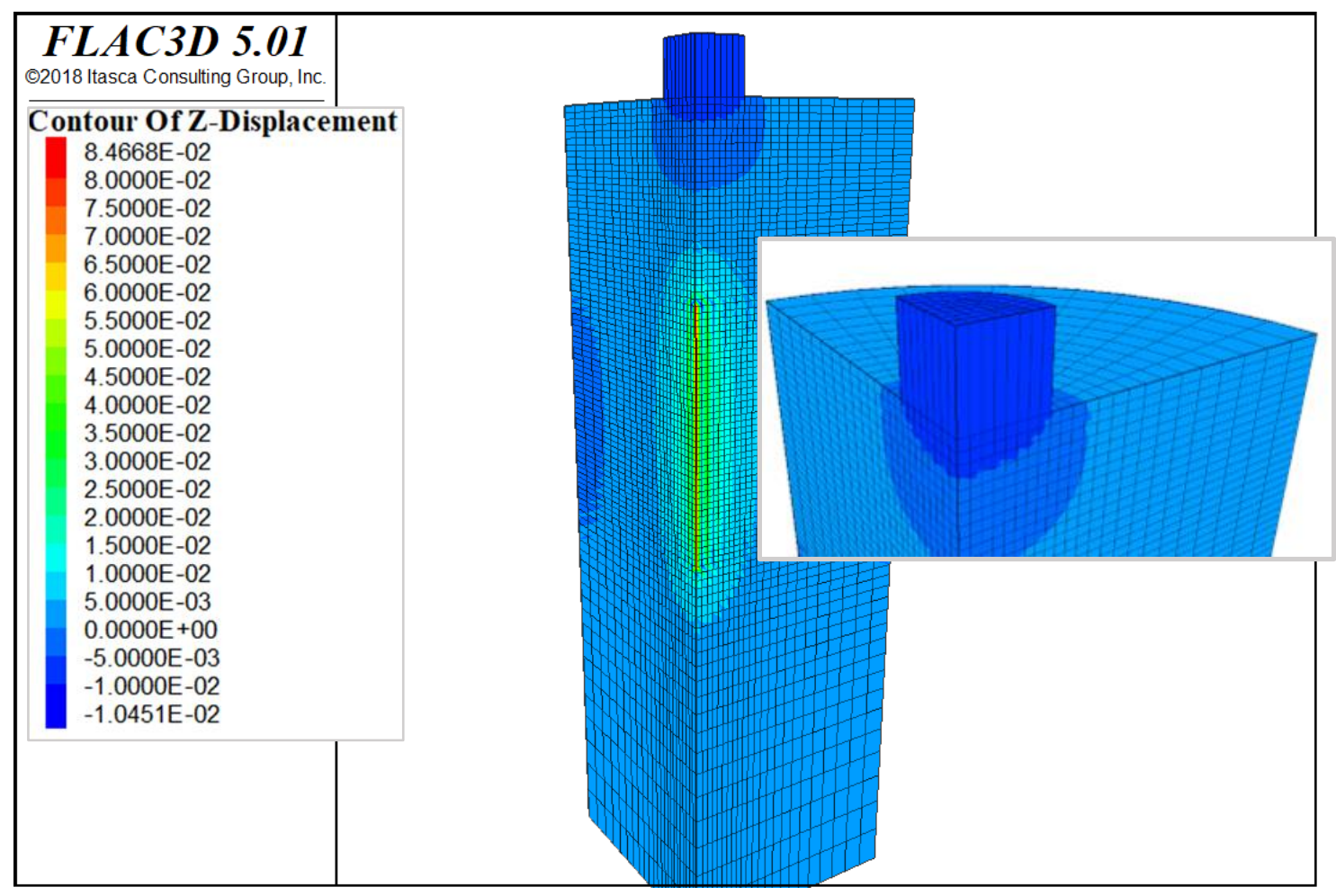

Figura 67. Asiento uniforme de la losa circular y geometría del modelo.

Los contornos laterales del modelo se encuentran a cinco radios de la losa circular desde el centro. El contorno inferior se sitúa a una profundidad de dos veces la longitud total del anclaje más el espesor de la losa.

Se han creado únicamente dos nodos en los extremos del ancho de la losa, para procurar que su comportamiento sea rígido, como se pretende ilustrar con la Figura 67.

El cable está modelizado mediante un elemento estructural unidimensional, que trasfiere las cargas al mallado del terreno mediante muelles. La parte libre está formada únicamente por dos nodos, uno en cabeza y otro al inicio del bulbo, ya que esta zona no interfiere con el terreno.

Sin embargo, la zona adherida al terreno presenta la misma discretización que el modelo, para estudiar adecuadamente la interacción con el suelo. 
La carga de tesado se aplica al anclaje de forma incremental para evitar inestabilidades numéricas, del mismo modo que la reacción ejercida por el gato en la losa. En la Figura 65 se puede apreciar que se mantiene la hipótesis de carga constante con el tiempo en el anclaje, para estudiar estrictamente el problema de fluencia en el terreno.

Los modelos de comportamiento son: elástico para la losa de hormigón y viscoelástico de Burgers para el terreno.

\subsubsection{Contraste con el modelo analítico de losa anclada}

En la Tabla 8 se muestran los parámetros de cálculo empleados en el modelo numérico, de los cuales se han tomado los necesarios para resolver el modelo analítico expuesto en el apartado 3.2. del capítulo 3 y realizar un contraste entre ambas soluciones.

Cada uno de los parámetros geométricos está indicado, a su vez, en la Figura 66. 
Tabla 8. Parámetros empleados para el contraste (losa anclada).

PARÁMETROS GEOMÉTRICOS

\begin{tabular}{|c|c|c|}
\hline $\boldsymbol{e}$ & $m$ & 2 \\
\hline$H$ & $m$ & 7.1 \\
\hline $\boldsymbol{a}$ & $m$ & 10 \\
\hline $\boldsymbol{b}$ & $m$ & 2 \\
\hline \multicolumn{3}{|l|}{ PARÁMETROS DEL TERRENO } \\
\hline $\boldsymbol{E}$ & $\mathrm{MPa}$ & 30 \\
\hline $\boldsymbol{v}$ & - & 0.3 \\
\hline$\gamma_{d}$ & $\mathrm{~N} / \mathrm{m}^{2}$ & 1800 \\
\hline$K$ & $\mathrm{MPa}$ & (elástico) 25 \\
\hline$G_{M}$ & $\mathrm{MPa}$ & (elástico) 1.54 \\
\hline$\mu_{M}$ & $\mathrm{MN} \cdot \mathrm{s} / \mathrm{m}$ & $2.1 \cdot 10^{10}$ \\
\hline$G_{K}$ & $\mathrm{MPa}$ & 1.22 \\
\hline$\mu_{K}$ & $\mathrm{MN} \cdot \mathrm{s} / \mathrm{m}$ & $5.28 \cdot 10^{6}$ \\
\hline \multicolumn{3}{|l|}{ PARÁMETROS DEL HORMIGÓN } \\
\hline Densidad $^{\mathrm{a}}$ & $\mathrm{N} / \mathrm{m}^{2}$ & 2500 \\
\hline Módulo de Young $^{\mathrm{a}}$ & $\mathrm{GPa}$ & 6 \\
\hline Coeficiente de Poisson ${ }^{\mathrm{a}}$ & - & 0.2 \\
\hline \multicolumn{3}{|l|}{ PARÁMETROS DEL ANCLAJE } \\
\hline Carga de tesado, $\boldsymbol{P}_{\mathbf{0}}$ & $\mathrm{kN}$ & 200 \\
\hline Sección del cable, $\boldsymbol{\Omega}_{c}$ & $\mathrm{~mm}^{2}$ & 660 \\
\hline Límite elástico del cable $^{\mathrm{a}}$ & $\mathrm{N}$ & $1 \cdot 10^{20}$ \\
\hline Adherencia de la lechada $^{\mathrm{b}}$ & $\mathrm{kN} / \mathrm{m}$ & 400 \\
\hline Módulo de Young del cable, $\boldsymbol{E}_{\boldsymbol{c}}$ & $\mathrm{GPa}$ & 195 \\
\hline
\end{tabular}

Con los datos de partida presentads en la Tabla 8 , se obtiene un desplazamiento relativo entre la cabeza del tirante (que sale) y la placa de apoyo (que entra), de $2.32 \mathrm{~cm}$ con el modelo de FLAC 3D; mientras que el modelo analítico proporciona un valor de $2.40 \mathrm{~cm}$. Por lo tanto y como se ilustra en la Figura 68, se considera que se alcanza un buen ajuste para el caso instantáneo, lo cual permite validar el modelo analítico desarrollado. 


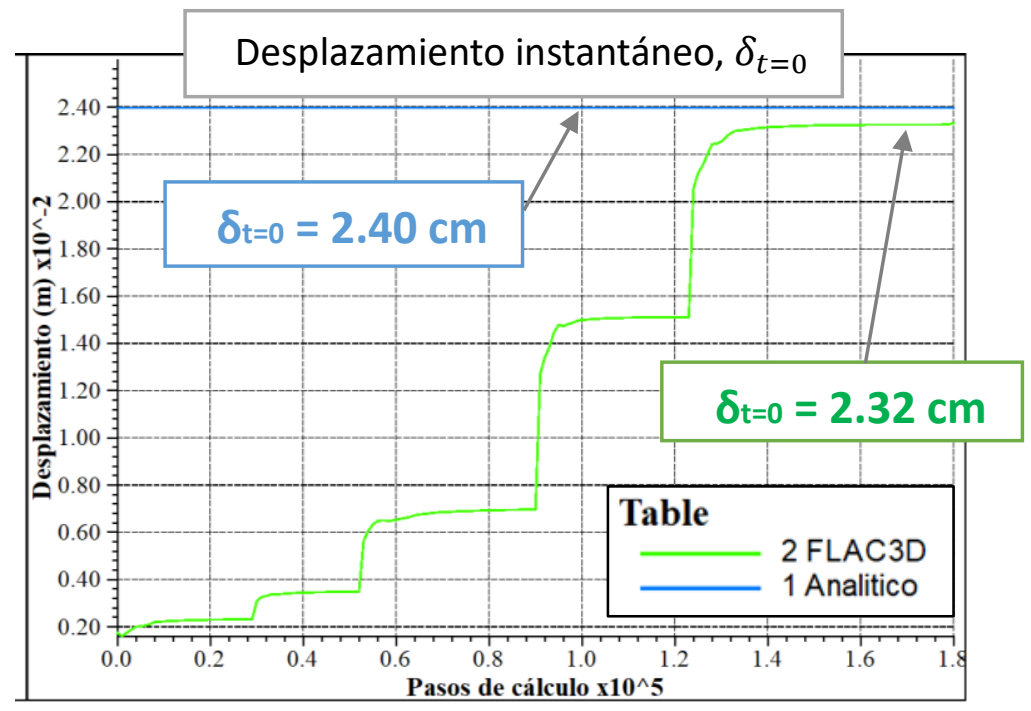

Figura 68. Desplazamiento instantáneo obtenido con ambos modelos.

En el caso del desplazamiento por fluencia del sistema $(\delta(t))$ al cabo de 20 años se obtienen $1.04 \mathrm{~cm}$ de diferencia entre ambos modelos. La predicción de desplazamiento por fluencia en el tiempo se puede apreciar en la Figura 69, alcanzando un valor de aproximadamente $8 \mathrm{~cm}$ con el modelo de FLAC 3D y de $9 \mathrm{~cm}$ con el modelo analítico, al cabo de 20 años. Del mismo modo que para el caso puramente elástico, se puede aceptar que las discrepancias numéricas observadas al cabo de 20 años, entre ambos modelos, son poco relevantes, del orden del $12.5 \%$.

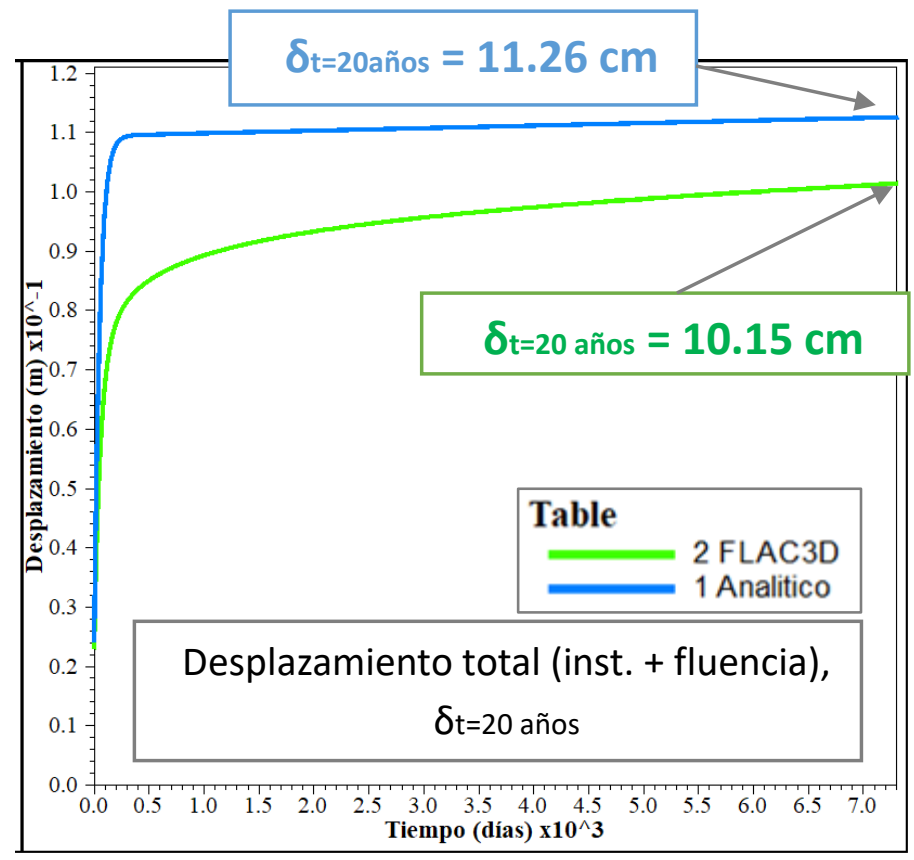

Figura 69. Desplazamiento diferido obtenido con el modelo analítico y con FLAC 3D. 
Además, como se puede ver en la Tabla 9, los desplazamientos instantáneos por componentes obtenidos mediante ambos métodos son idénticos, para el alargamiento del cable $(\Delta L)$ y el movimiento del bulbo $\left(u_{A}\right)$.

Tabla 9. Resultados de desplazamiento por componentes obtenidos con ambos modelos.

\begin{tabular}{|c|c|c|c|c|c|}
\hline \multirow{2}{*}{ Modelo } & \multirow{2}{*}{ Tiempo } & \multicolumn{4}{|c|}{ Desplazamientos por componentes (cm) } \\
\hline & & $\Delta L$ & $u_{p}=\left(u_{p c}-u_{p t}\right)$ & $u_{A}$ & $\delta$ \\
\hline \multirow{3}{*}{ ANALÍTICO } & 0 & 1.41 & 0.09 & 0.89 & 2.40 \\
\hline & \multirow{2}{*}{20 años } & 1.41 & 0.56 & 9.28 & 11.26 \\
\hline & & \multicolumn{3}{|c|}{ Solo fluencia, $\delta(t)$} & 8.86 \\
\hline \multirow{3}{*}{ FLAC 3D } & 0 & 1.41 & 0.03 & 0.89 & 2.32 \\
\hline & \multirow{2}{*}{20 años } & 1.41 & 0.58 & 8.46 & 10.15 \\
\hline & & \multicolumn{3}{|c|}{ Solo fluencia, $\delta(t)$} & 7.82 \\
\hline
\end{tabular}

\subsection{APLICACIÓN NUMÉRICA A LA PANTALLA ANCLADA}

\subsubsection{Hipótesis para la simulación}

\subsubsection{Introducción}

El objetivo de esta segunda parte de aplicación del software geotécnico consiste en poner a punto un modelo de cálculo para el estudio del comportamiento deformacional con el tiempo de una pantalla anclada real. En el presente caso se aplicará al tramo de pantalla de pilotes anclados, situada en el nudo de Raith (Escocia) y descrita en el apartado 3.3.2. del capítulo 3.

Los archivos principales del cálculo, en buena parte suministrados por la empresa constructora, se pueden consultar en el ANEXO I, y son los siguientes:

- pantalla_datos.f3dat: archivo de datos en el que se introducen los datos para el cálculo

- pantalla_malla.f3dat: archivo de datos para la generación de la malla 
- pantalla_calculo.f3dat: archivo de datos con las fases de construcción y cálculo

Como se verá posteriormente, los resultados numéricos presentados en esta memoria corresponden a la sección situada en el pp.kk. 645 del muro W, cuyo perfil geotécnico se ha representado en la Figura 70. Esta sección ya fue calculada de manera analítica en el apartado 3.3.6.2. del capítulo 3. La sección tipo evaluada tiene un solo anclaje y está instrumentada mediante una célula de carga en la cabeza del anclaje y un conducto flexible con acelerómetros a lo largo de la longitud libre de la pantalla. Existen otras secciones, igualmente instrumentadas, con dos y tres anclajes. Sin embargo, se ha considerado que la influencia de un único anclaje es la situación más sencilla para un primer ajuste del modelo.

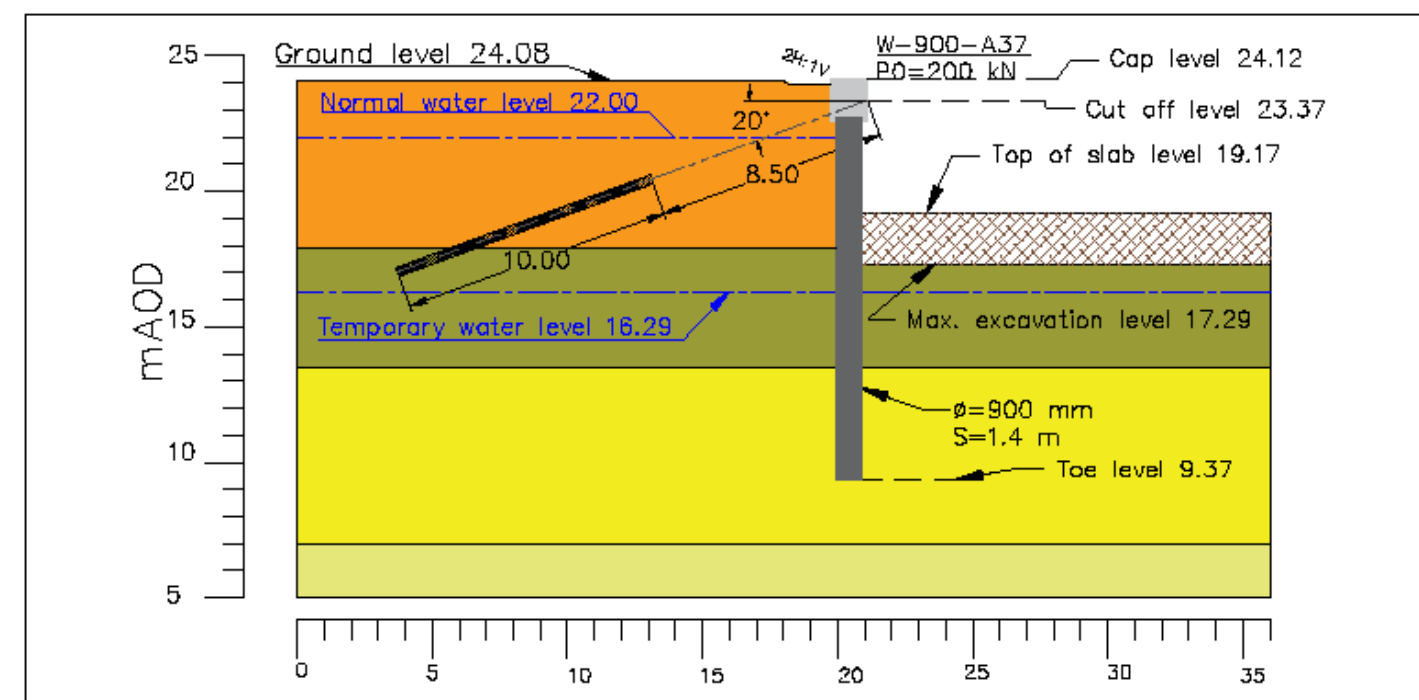

up Fill $\left(\gamma=19 \mathrm{kN} / \mathrm{m} 3, \phi^{\prime}=35^{\circ}\right)$
Mode ground $\left(\gamma=18 \mathrm{kN} / \mathrm{m} 3, \phi^{\prime}=25^{\circ}, C u=50 \mathrm{kPa}\right)$
$\square$ Soft clay $\left(\gamma=18 \mathrm{kN} / \mathrm{m} 3, \phi^{\prime}=20^{\circ}, \mathrm{Cu}=25 \mathrm{kPa}\right)$
$\square$ Loose dense S\&G $\left(\gamma=18 \mathrm{kN} / \mathrm{m} 3, \phi^{\prime}=28^{\circ}\right)$
$\square$ Medium dense $5 \& \mathrm{G}\left(\gamma=18 \mathrm{kN} / \mathrm{m} 3, \phi^{\prime}=32^{\circ}\right)$

Figura 70. Sección geotécnica empleada para la puesta a punto del modelo.

Se cuenta con nueve secciones más que se corresponden con el perfil de la Figura 70, en cuanto a que poseen la misma cota de comienzo de la viga 
de atado, misma carga inicial o de bloqueo, un solo anclaje con iguales dimensiones, y otras características idénticas (p.e. posición del nivel freático, dimensiones de la viga y de los pilotes, espesor de la losa y del relleno inferior).

Dadas las diferentes configuraciones (propiedades geomecánicas de los terrenos, geometría de la pantalla, del anclaje y de los terrenos, y propiedades elásticas de los elementos estructurales) que se pueden dar en cualquier obra de este tipo, se ha desarrollado un modelo parametrizado de cálculo, de tal manera que se facilita el estudio de secciones variables con variación de los parámetros implicados e indicados en la Figura 71.
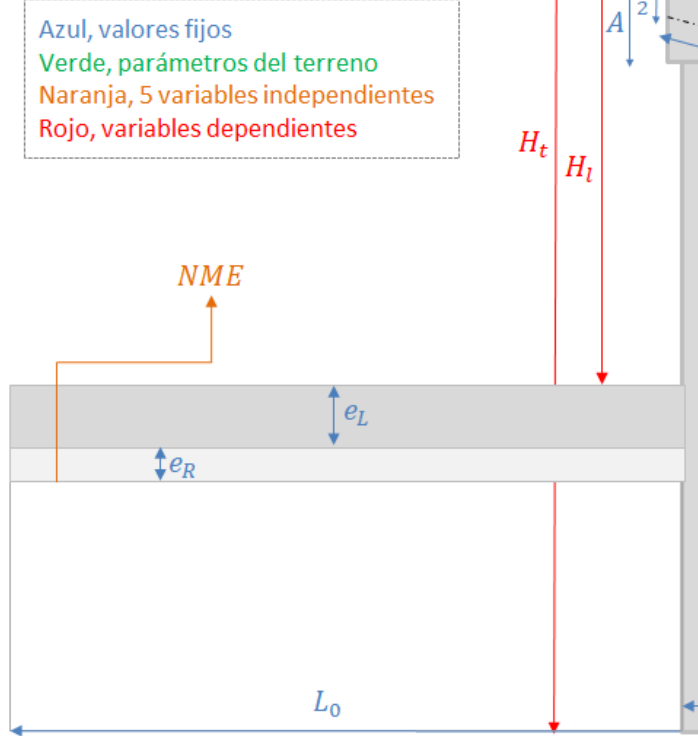

Figura 71. Esquema de los diferentes parámetros que intervienen en una sección tipo para el modelo numérico.

En el caso de estudio los datos variables entre secciones, fundamentalmente son: el espesor de los tres terrenos que aparecen en la longitud de la pantalla, la cota del nivel máximo de excavación y la posición del nivel freático temporal. 


\subsubsection{Generación de la malla de cálculo}

Dada la clara condición tridimensional del problema (pilotes circulares con anclajes), se planteó la necesidad de llevar a cabo una modelización numérica tridimensional. Ahora bien, a efectos de optimizar el modelo de cálculo (y por tanto los tiempos de respuesta), se han considerado las simetrías suficientes para reproducir el problema de forma eficaz, por lo que el modelo representa una rebanada de un espesor igual a la separación entre ejes de anclajes.

La creación de la malla de cálculo se ha llevado a cabo mediante la definición de puntos clave y primitivas, así como rutinas $\mathrm{FISH}^{5}$ de modificación de la malla, desarrolladas específicamente para este trabajo. Se ha seleccionado esta técnica más "artesana" porque se facilita la parametrización de ciertas variables, y que consecuentemente serán muy útiles a la hora de calcular otras secciones a lo largo de la pantalla.

Los pilotes se han modelizado con elementos volumétricos, a los que se asigna un modelo constitutivo elástico. El contacto pilote-terreno se simula mediante interfaces (de tipo Mohr Coulomb con ángulo de rozamiento tierraspantalla de 2/3 del ángulo de rozamiento del terreno).

Este esquema reproduce los pilotes circulares y tiene en cuenta las simetrías en función de la separación entre anclajes.

Dado que los anclajes irán en pilotes alternos, se reproduce el pilote central (en el que va instalado el anclaje) así como la mitad de cada uno de los pilotes laterales (sin anclaje). Al aplicar la condición de contorno correcta (fijando los desplazamientos en la dirección transversal a los contornos) se reproduce una pantalla de pilotes "infinita" con anclajes instalados cada dos unidades.

\footnotetext{
${ }^{5}$ FISH, es un lenguaje de programación propio de FLAC, integrado para la personalización del modelo, la automatización y parametrización.
} 
La Figura 72 muestra un detalle de la malla de cálculo generada, que incluye:

- Pilotes anclados y sin anclar

- Viga de atado

- Losa de fondo

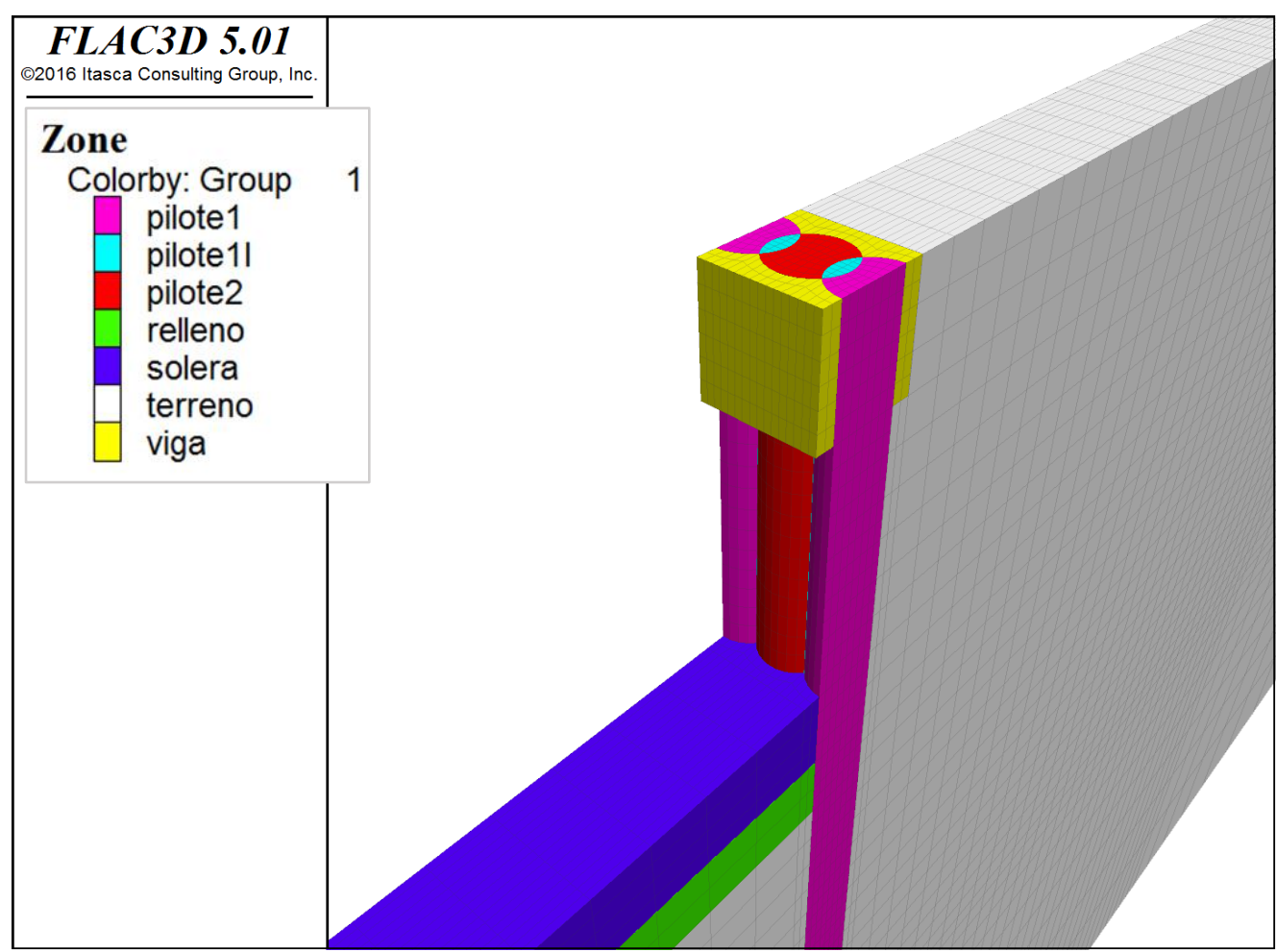

Figura 72. Vista del modelo de cálculo. Detalle de los pilotes.

Al ser pilotes secantes, se ha considerado la construcción de pilotes primarios y secundarios, en la que el pilote secundario "morderá" al pilote primario cuando se construya. Se distinguen por tanto en el modelo los pilotes armados (definitivos) de los pilotes en masa (que serán excavados cuando se construyan los secundarios).

El modelo reproduce la mitad de la calzada, es decir la mitad de la longitud entre muros. El contorno izquierdo se fija en su dirección para tener en cuenta la simetría existente. 
El cable está anclado al pilote central desde la viga de atado y consta de dos partes: la longitud libre y la longitud anclada, tal y como se muestra en la Figura 73.

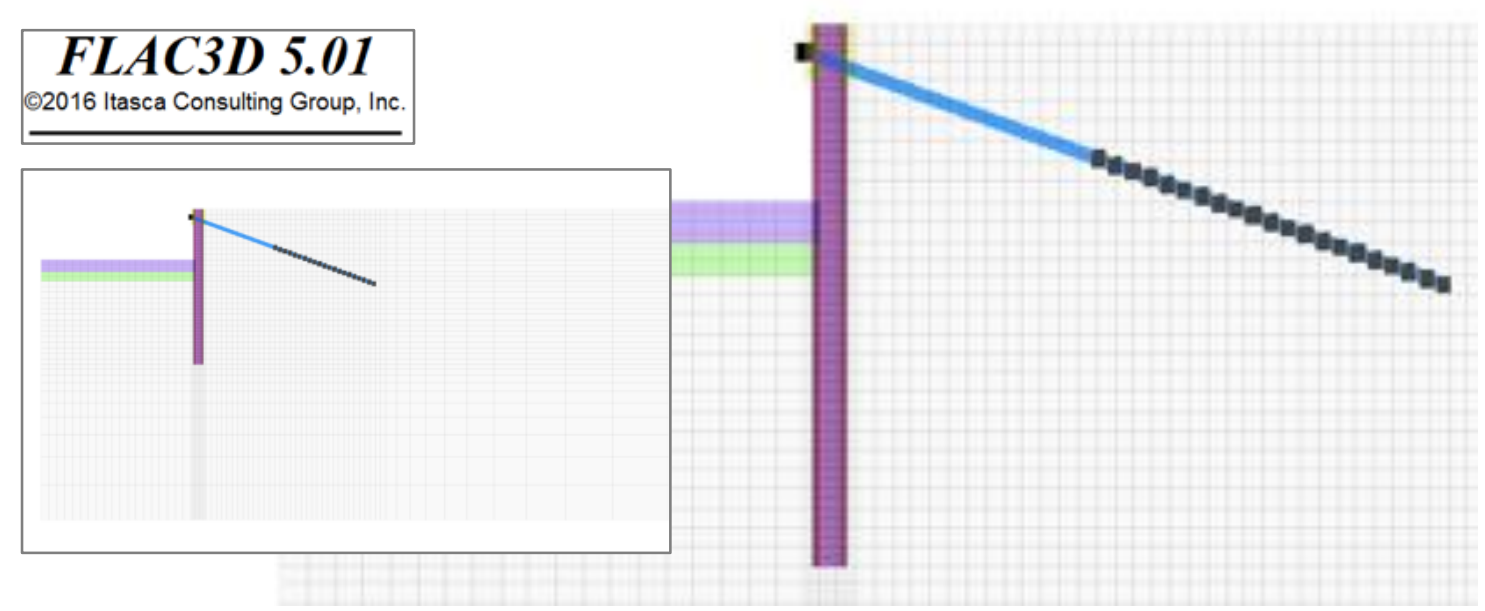

Figura 73. Vista del modelo de cálculo. Situación del anclaje.

En cuanto a los materiales implicados, en el modelo se han incluido 3 niveles de terreno, tal y como se ilustra en la Figura 74.

Se han parametrizado sus contactos, de forma que pueden variar en altura, modificando las variables correspondientes en la introducción de datos.

Aunque en el diseño geotécnico de la obra se han definido más niveles geotécnicos solo se han tenido en cuenta los tres materiales que figuran en la Figura 74 , debido a que el terreno subyacente al denominado "terreno 3" comienza siempre (en los perfiles que se van a estudiar) por debajo del pie de la pantalla o pilote, por lo que se considera que se puede despreciar la afección al desplazamiento de la zona libre de la pantalla y del anclaje. 


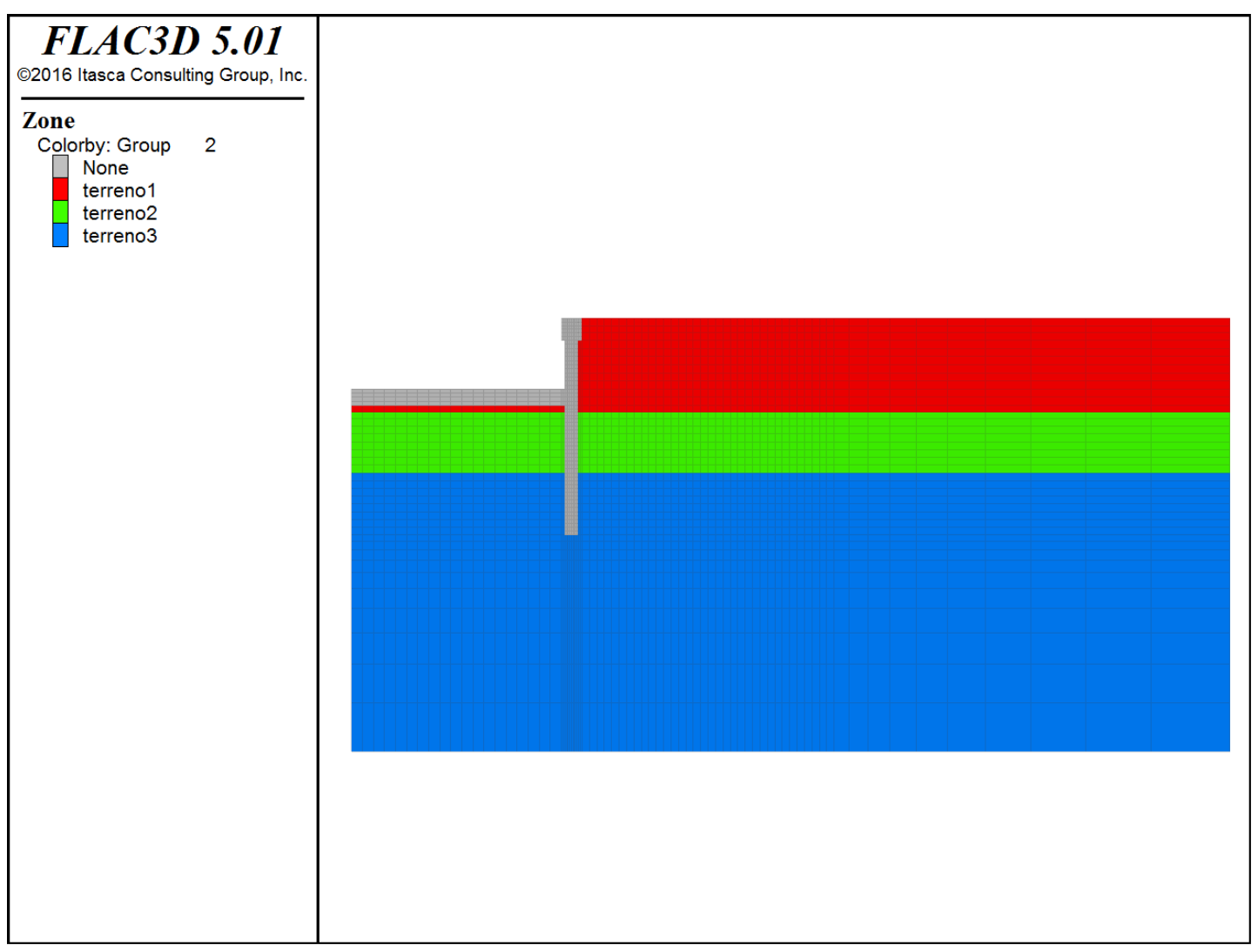

Figura 74. Niveles geotécnicos considerados.

\subsubsection{Modelos constitutivos y parámetros utilizados}

El modelo constitutivo asignado es el elasto-plástico de Mohr-Coulomb en la parte mecánica y el viscoelastoplástico de Burgers con un criterio de rotura tipo Mohr-Coulomb en la parte de fluencia. El modelo de fluencia solo se ha asignado en un principio a los dos terrenos superiores, considerando que el "terreno 3" no presenta fluencia. Tampoco se dispone de ensayos a esta profundidad que permitan estimar unos parámetros representativos.

En función del origen de los datos reales empleados, los parámetros geotécnicos de diseño de proyecto varían en gran medida. Por este motivo, se ha incluido, en el último capítulo, un estudio de sensibilidad para el modelo desarrollado, en el que se han variado sus magnitudes, dentro de unos rangos de variación realistas. 
Las propiedades geotécnicas y características resistentes de los pilotes de hormigón armado y de hormigón en masa, del relleno granular bajo la losa base, y del hormigón de la losa base son las mismas que las indicadas en el proyecto y han sido proporcionadas por la empresa FERROVIAL.

\subsubsection{Condiciones de contorno e iniciales}

Como condiciones de contorno se fijan los desplazamientos en los bordes del modelo (en la dirección transversal a cada borde) y en la base del modelo.

Se introduce además el nivel freático y la distribución de presiones intersticiales de partida.

Es preciso inicializar el estado tensional de partida para lo que se parte de la profundidad a la que se encuentra cada material y su densidad. Para las tensiones horizontales se considera un coeficiente de empuje al reposo $k_{0}=1$, como primera aproximación razonable.

Se permite una primera fase de equilibrio mecánico del modelo para que se redistribuyan las tensiones de acuerdo con la disposición del terreno y el agua. En dicha fase no se permite la fluencia, por lo que debe ser convenientemente desactivada.

En principio, si todo es correcto y está bien inicializado, el modelo calculará una primera fase y estará en equilibrio. Este paso es recomendable hacerlo para comprobar que los datos de partida están bien introducidos.

\subsubsection{Construcción de la pantalla}

Finalizado el equilibrio inicial del modelo se procede a la construcción de la pantalla y excavación. 
Se trata de reproducir una secuencia progresiva, previa a la activación de la fluencia, de cara a tener una situación lo más parecida a la realidad posible. Estas fases se realizan considerando sólo la parte de deformación instantánea. No se activa en ningún momento la fluencia.

Las fases de cálculo que se han considerado son:

1) Excavación hasta la plataforma de trabajo. Esta fase se hará solo si hay terreno preexistente por encima de la viga de atado. Si no lo hay el programa pasa automáticamente a la fase 2.

2) Instalación de los pilotes primarios. En este caso, la parte definitiva de estos pilotes será de hormigón armado. La parte que será "mordida" por los secundarios, será de mortero.

3) Instalación de los pilotes secundarios, que serán todos de hormigón en masa. Antes de la instalación, se excavarán las partes de mortero de los primarios.

4) Excavación hasta la viga de atado. Se rebaja el nivel freático ${ }^{6}$ en la parte interna de la excavación, si corresponde.

5) Instalación de la viga de atado y del anclaje (ver el punto siguiente para detalles).

6) Excavación hasta el nivel máximo de excavación (NME) y rebaje del nivel freático hasta el nivel temporal (1 $\mathrm{m}$ por debajo del NME).

7) Instalación del relleno y solera.

8) Recuperación del nivel freático hasta la posición original.

\subsubsection{Modelización del anclaje}

En la fase 5 del apartado anterior se instalan tanto la viga de atado como el anclaje. Los anclajes se modelizan como elementos estructurales tipo "cable". Los anclajes constan de una longitud libre y un bulbo. Para simular esta geometría, el programa permite crear diferentes tramos conectados entre

\footnotetext{
${ }^{6}$ En los cálculos no se ha considerado red de flujo. Se ha definido una rutina manual que cambia las tensiones efectivas teniendo en cuenta las sobrepresiones.
} 
sí a los que se asignan diferentes propiedades geomecánicas a la interfase cable-terreno, sin adherencia en la parte libre y con ella en la parte anclada.

La cabeza de anclaje se simula fijando los desplazamientos y rotaciones en el nodo de cabeza.

La instalación de los anclajes se realiza mediante una rutina desarrollada específicamente, mediante el lenguaje FISH (pone_cable), en la que se calculan los puntos de origen y fin del cable, según su longitud y ángulo:

- Se calculan las coordenadas de inicio, fin y longitud libre del anclaje en función de su posición en la pared y de su longitud.

- Se instala el cable discretizado en elementos de $0.5 \mathrm{~m}$ de longitud, desde la posición inicial a la posición final en dos tramos: tramo libre y tramo anclado.

- Se fijan las condiciones de la cabeza de anclaje (fijándolas a la viga de atado).

- Se asignan las propiedades de cada tramo.

Para simular el tesado, además de asignar la carga correspondiente, se emplea una alternativa que se ha utilizado frecuentemente y de acuerdo a la propia experiencia funciona de forma bastante ajustada. Consiste en asignar un módulo de deformación muy bajo a la longitud libre y se deja equilibrar el modelo con esta condición. De esta forma, el cable empezará a trabajar a la carga de tesado, pero no cogerá más carga (o la que asimilará será muy pequeña, aunque se deforme). Una vez equilibrado el modelo, se reasigna el módulo de deformación a la parte libre, dándole el valor real del módulo del cable de acero, previamente a continuar con las siguientes fases de excavación.

\subsubsection{Simulación a largo plazo}

Antes de comenzar con el proceso de fluencia se inicializan los desplazamientos producidos durante la fase de excavación, de forma que se 
puedan evaluar directamente las deformaciones inducidas por el "largo plazo". En cualquier caso, estos desplazamientos previos se almacenan en variables de nodo para que puedan ser recuperadas en cualquier momento posterior del cálculo, si se desease.

A continuación, se incluyen las rutinas y comandos para simular esta fase, así como el seguimiento de la instrumentación.

Para la etapa de estudios predictivos a largo plazo, se activa la fluencia en el modelo.

Se activa el cálculo durante varios años, en períodos de 1 año, lo que se incorpora mediante la rutina FISH (calcula_LP). Se ha escogido inicialmente un período total de 20 años.

El número de pasos de cálculo viene definido por el tiempo de fluencia.

Se definen unas rutinas con la instrumentación, también en lenguaje FISH:

- Desplazamientos en los pilotes y losa: lee_disp_pil. Con esta rutina se seleccionan puntos en la losa y a lo largo del fuste de los pilotes y se reproducen las variaciones de los desplazamientos horizontales y verticales con el tiempo.

- Control de deformación en los anclajes:_cab_strL. Con esta rutina se conoce la deformación del anclaje, calculada de la forma siguiente: se lee la longitud original de los tramos del cable, y la posición tras el desplazamiento, con lo que se calcula la deformación producida, tanto en el tramo libre como en toda la longitud del cable.

\subsubsection{Discusión de resultados}

Se ha venido mencionando que las propiedades geotécnicas de diseño del proyecto tienen un rango de variación muy alto, en función del origen de los datos reales empleados, y por tanto, se ha tenido que realizar un estudio de sensibilidad con valores realistas de dichos parámetros para el modelo 
numérico desarrollado. Considerando las diferentes hipótesis, así como los parámetros estructurales y geométricos del proyecto, representativos del terreno,estimados a partir de ensayos de laboratorio o campo, que deben considerarse suficientemente precisos. Los valores del coeficiente de viscosidad provienen de valores medios estimados a partir de ensayos de investigación y aceptados como válidos en anclajes instalados en terrenos similares. Estos parámetros constantes para los cuatro casos calculados se presentan en la Tabla 10.

Tabla 10. Parámetros del terreno constantes en el análisis de sensibilidad.

\begin{tabular}{|c|c|c|c|}
\hline TERRENO & 1 & 2 & 3 \\
\hline$\gamma\left(k N / m^{3}\right)$ & 18 & 18 & 18 \\
\hline$S_{u}(k P a)$ & 50 & 25 & - \\
\hline Design $\boldsymbol{N}_{S P T}$ & 10 & 14 & 10 \\
\hline$k_{h}(k N / m 3)$ & 21839 & 9588 & 14274 \\
\hline$v$ & 0.30 & 0.35 & 0.30 \\
\hline$\mu(\boldsymbol{M N} * \boldsymbol{s} / \boldsymbol{m})$ & $2.00 \mathrm{E}+08$ & $1.00 \mathrm{E}+08$ & NO \\
\hline \multicolumn{4}{|c|}{$\begin{array}{l}\text { Resistencia al corte sin drenaje, } \boldsymbol{S}_{\boldsymbol{u}} \\
\text { Número de golpes en el ensayo SPT, } \boldsymbol{N}_{S P T} \\
\text { Densidad aparente, } \gamma \\
\text { Coef. de balasto horizontal, } \boldsymbol{k}_{\boldsymbol{h}}\end{array}$} \\
\hline
\end{tabular}

De este modo, se han adoptado distintos rangos de valores para ciertos parámetros de resistencia y deformabilidad $\left(\varphi^{\prime}, c^{\prime}, E\right)$, realizando así un análisis de sensibilidad. Con ello se han ido obteniendo unos valores representativos del terreno aproximados, que generan unos desplazamientos del mismo y un aumento de la carga en el anclaje que, en caso de análisis retrospectivos, pueden ser comparados con medidas de monitorización. Los casos calculados para llevar a cabo la puesta a punto del modelo han sido los siguientes:

- Caso A: parámetros resistentes más conservadores de proyecto (FERROVIAL - AGROMÁN). Los valores del módulo de Young provienen de la estimación más conservadora deducida a partir del número de golpes obtenido en el ensayo SPT y de la resistencia al corte sin drenaje. 
- Caso B: en el segundo caso de cálculo se aumentan los módulos de Young de los terrenos que intervienen respecto al Caso A. En este caso los módulos se han estimado a partir del coeficiente de balasto horizontal proporcionado, seleccionando el valor medio proporcionado por algunas de las correlaciones más conocidas (Jiménez Salas et al, 1976; Terzaghi, 1955 y Vesic, 1961).

- Caso C: se aumentan los módulos de Young de los tres terrenos que intervienen hasta lograr desplazamientos aceptables.

- Caso D: parámetros resistentes conservadores aunque menos que los del Caso A. Con el objetivo de reducir los módulos de Young del paso anterior se realiza un nuevo cálculo aumentando la cohesión de los primeros terrenos y estableciendo el ángulo de rozamiento del terreno en $28^{\circ}$, valor sugerido en el informe geotécnico de diseño de la obra (Geotechnical Design Report. M8, M73, M74 Motorway improvements. Structure S201. Raith Underpass, 2015, CIRIA 580).

Tabla 11. Propiedades geotécnicas empleadas en los cuatro casos de cálculo.

\begin{tabular}{|c|c|c|c|c|c|c|c|c|c|c|c|c|c|}
\hline \multirow{2}{*}{\multicolumn{2}{|c|}{$\begin{array}{c}\text { Parámetro } \\
\text { Caso }\end{array}$}} & \multicolumn{4}{|c|}{$\varphi^{\prime}(\underline{o})$} & \multicolumn{4}{|c|}{$c^{\prime}(k P a)$} & \multicolumn{4}{|c|}{$E(k P a)$} \\
\hline & & $A$ & $B$ & $C$ & $D$ & $A$ & $B$ & $C$ & $D$ & $A$ & $B$ & $C$ & $D$ \\
\hline \multirow{3}{*}{ Terreno } & 1 & 25 & 25 & 25 & 28 & 0 & 0 & 0 & 60 & 8 & 30 & 150 & 60 \\
\hline & 2 & 20 & 20 & 20 & 20 & 0 & 0 & 0 & 60 & 5 & 15 & 120 & 30 \\
\hline & 3 & 28 & 28 & 28 & 28 & 0 & 0 & 500 & 500 & 7 & 20 & 250 & 40 \\
\hline
\end{tabular}

En el Caso A los desplazamientos instantáneos en condiciones drenadas alcanzan un valor máximo de $14 \mathrm{~cm}$ en cabeza (ver Figura 75), considerándose movimientos inadmisibles en una pantalla. Consecuentemente, la carga en el anclaje alcanza los 391 kN tras la fase final de excavación (200 $\mathrm{kN}$ de carga de bloqueo), incremento excesivo en contraste con lo ocurrido en la realidad de la obra. En el Caso $B$ se obtienen desplazamientos en el terreno inferiores a los del primer caso. Sin embargo, desplazamientos instantáneos máximos de $5 \mathrm{~cm}$ en cabeza continúan siendo inaceptables. Así como que la carga en el anclaje alcance un valor de $343 \mathrm{kN}$ tras la fase final de construcción de la pantalla. Un aumento del $72 \%$ respecto a la inicial de bloqueo. 
CASO A

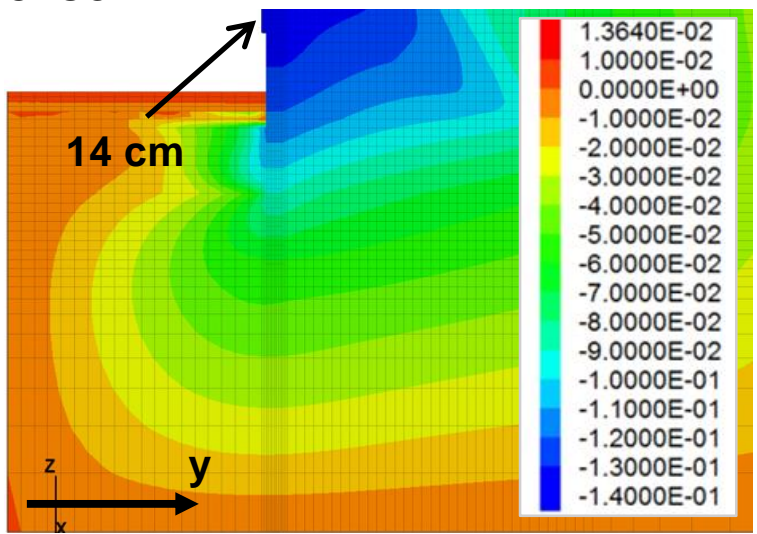

CASO C

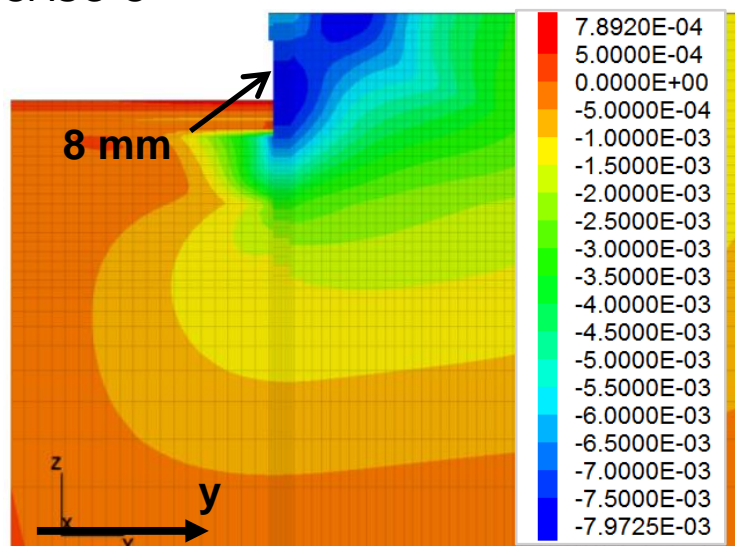

CASO B

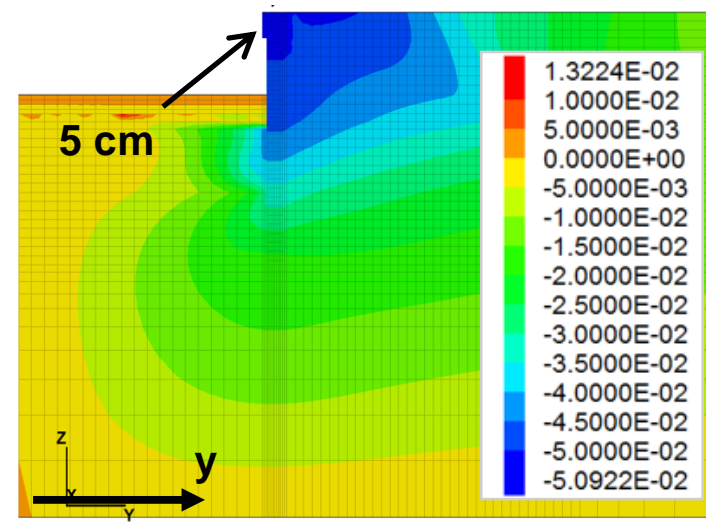

CASO D

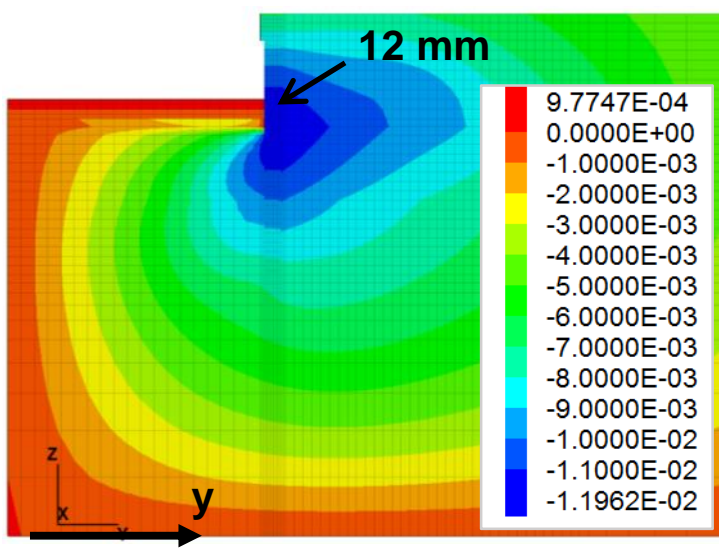

Figura 75. Desplazamientos horizontales (y) en el terreno tras la construcción.

En el Caso $C$ se obtienen desplazamientos muy aceptables, con valores de 6.5 $\mathrm{mm}$ en cabeza y máximos de $8 \mathrm{~mm}$ en la zona media donde no hay anclaje (Figura 75). La carga que queda en el anclaje tras el final de la construcción alcanza un valor de $228 \mathrm{kN}$. Los resultados de desplazamiento y carga en anclaje serían aceptables, sin embargo, los módulos del terreno son demasiado poco realistas (véase Tabla 11) con los terrenos presentes.

Los desplazamientos instantáneos obtenidos en el Caso D son de $7 \mathrm{~mm}$ en cabeza y $12 \mathrm{~mm}$ en la zona media de la pantalla (Figura 75). La carga en el anclaje alcanza los $243 \mathrm{kN}$ tras la fase total de excavación, valor que parece más razonable que los aumentos de carga tan marcados en casos anteriores (asociados a mayores desplazamientos).

En cuanto a los resultados de los cálculos incorporando el proceso de fluencia del terreno, debe tenerse en cuenta que los desplazamientos se 
inicializan a cero tras el final de excavación por lo que los resultados que se muestran en las siguientes figuras son los inducidos únicamente por dicho fenómeno de fluencia durante 20 años. En la Figura 76 se presentan los desplazamientos horizontales y verticales en el terreno transcurridos 20 años.

CASO A

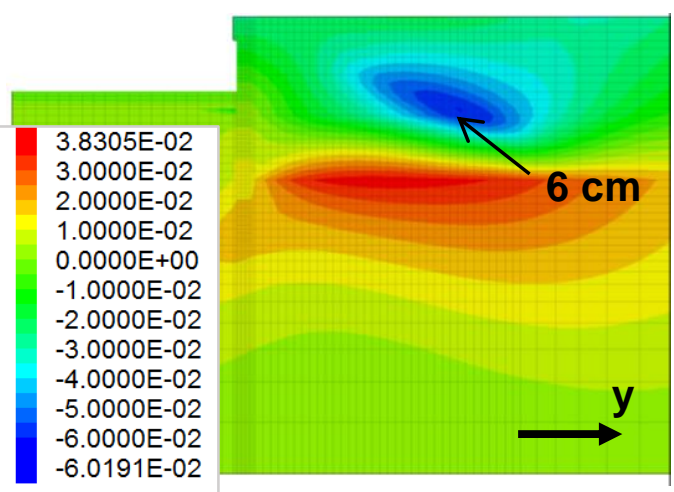

a. Desplazamientos horizontales

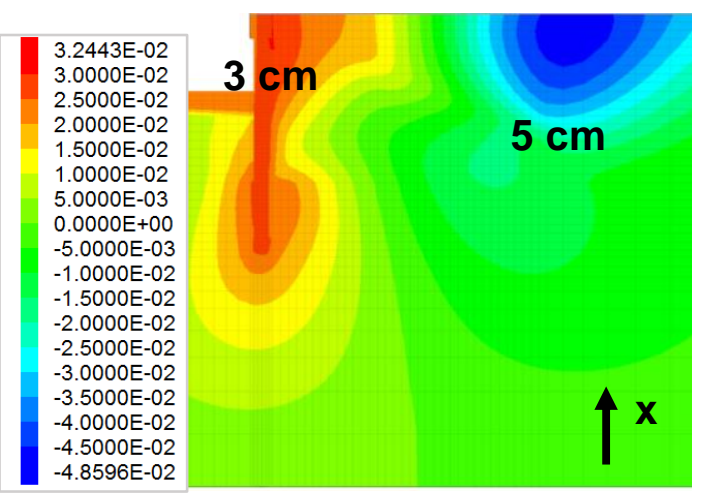

b. Desplazamientos verticales
CASO B
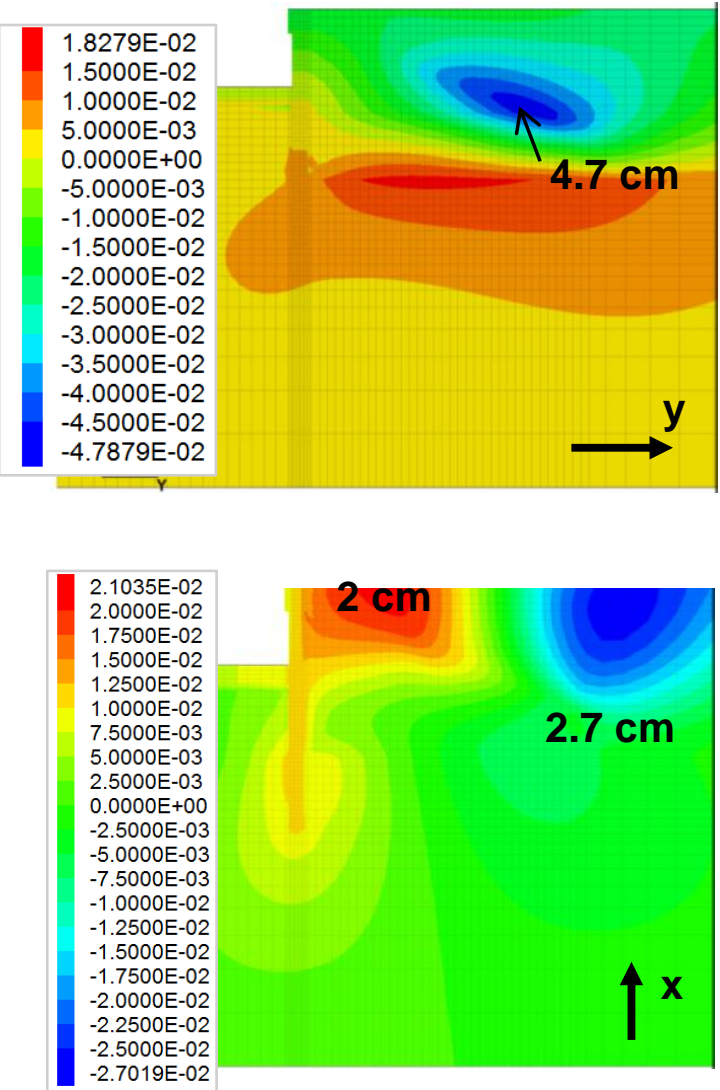

Figura 76. Desplazamientos en el terreno al cabo de 20 años (solo de fluencia).

En el primer supuesto (Caso A) también se alcanzarían a largo plazo desplazamientos horizontales demasiado elevados, de $6 \mathrm{~cm}$ alrededor del anclaje. Así como desplazamientos verticales en zonas colindantes de $5 \mathrm{~cm}$ de descenso y de aproximadamente $3 \mathrm{~cm}$ hacia la excavación en cabeza.

En el Caso B se alcanzan desplazamientos de $4.7 \mathrm{~cm}$ en el entorno del anclaje, un ascenso de $2 \mathrm{~cm}$ por detrás de la pantalla y un descenso en superficie en zonas colindantes de casi $3 \mathrm{~cm}$. 
Los cálculos del Caso C solo se han llevado a cabo para simular el corto plazo, con el fin de tener un orden de magnitud de la disminución de los desplazamientos en relación con el módulo de Young de los tres terrenos. Como se ha comentado anteriormente, a partir del Caso C se plantea el Caso D, con módulos más creíbles y cercanos a la realidad. Los resultados de este último son desplazamientos horizontales de $2.6 \mathrm{~cm}$ en el entorno del anclaje, un ascenso de $1 \mathrm{~cm}$ por detrás de la pantalla y un descenso en superficie de $1.2 \mathrm{~cm}$, como se aprecia en la Figura 77 .
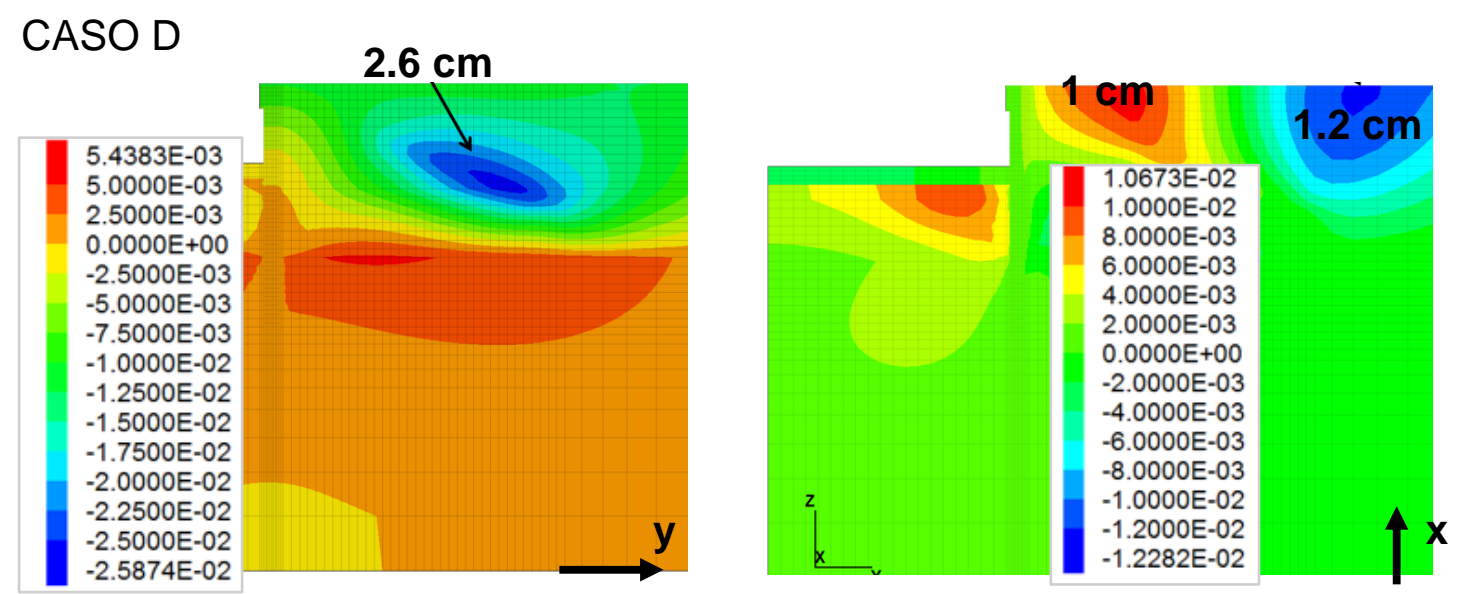

Figura 77. Desplazamientos (a) horizontales y (b) verticales en el terreno.

Los desplazamientos horizontales máximos al cabo de 20 años se producen en todos los casos en la coronación de la pantalla y hacia el intradós. Concretamente, en el Caso D, el valor alcanzado debido a los efectos de la fluencia del terreno es de tan solo $1 \mathrm{~cm}$, como se aprecia en la Figura 78.
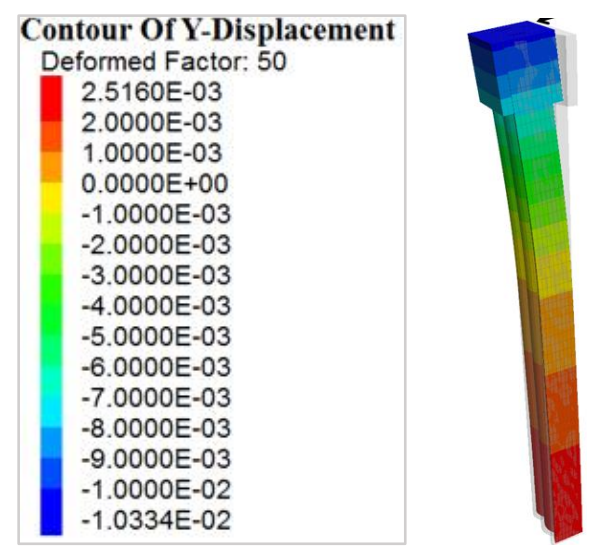

Figura 78. Desplazamientos horizontales en los pilotes. 
Con la finalidad de realizar una posterior comparación y ajuste con los valores registrados con la monitorización de la obra (cap. 3 ap. 3.2.) se han establecido ciertos puntos de control de desplazamiento (véase Figura 79), allí donde existen mediciones de desplazamientos reales en las pantallas.

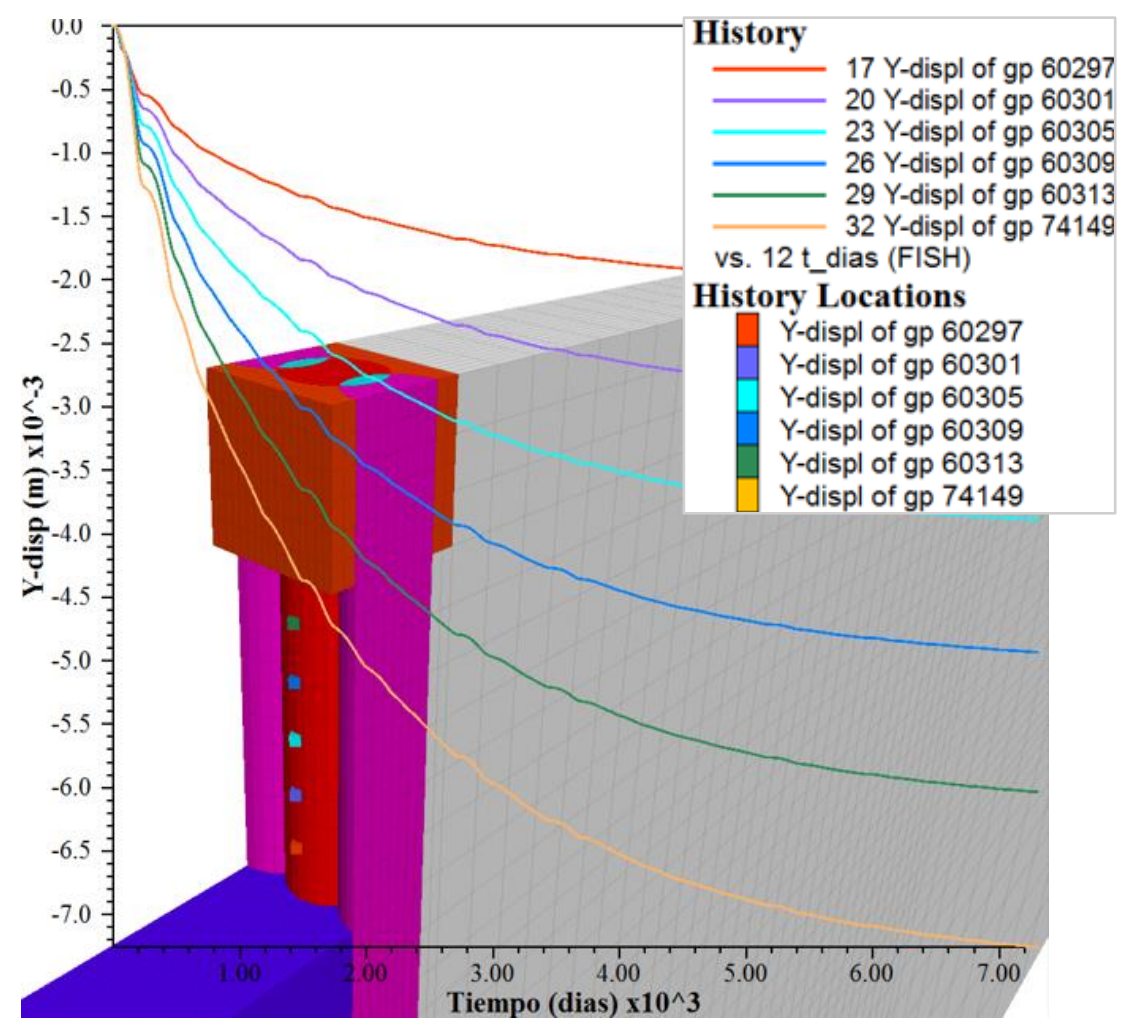

Figura 79. Evolución de desplazamientos en puntos de control en el fuste del pilote.

En cuanto a la carga remanente que quedaría transcurridos 20 años de servicio de la pantalla anclada, ha resultado ser del $17 \%, 6 \%$ y $3 \%$ respecto al valor inicial alcanzado tras la construcción, para los casos A, B y D, respectivamente. En la Figura 80 se ha incluido la evolución de la carga para el caso $\mathrm{D}$ también con la finalidad de mostrar las salidas gráficas del modelo para el cálculo a largo plazo. 


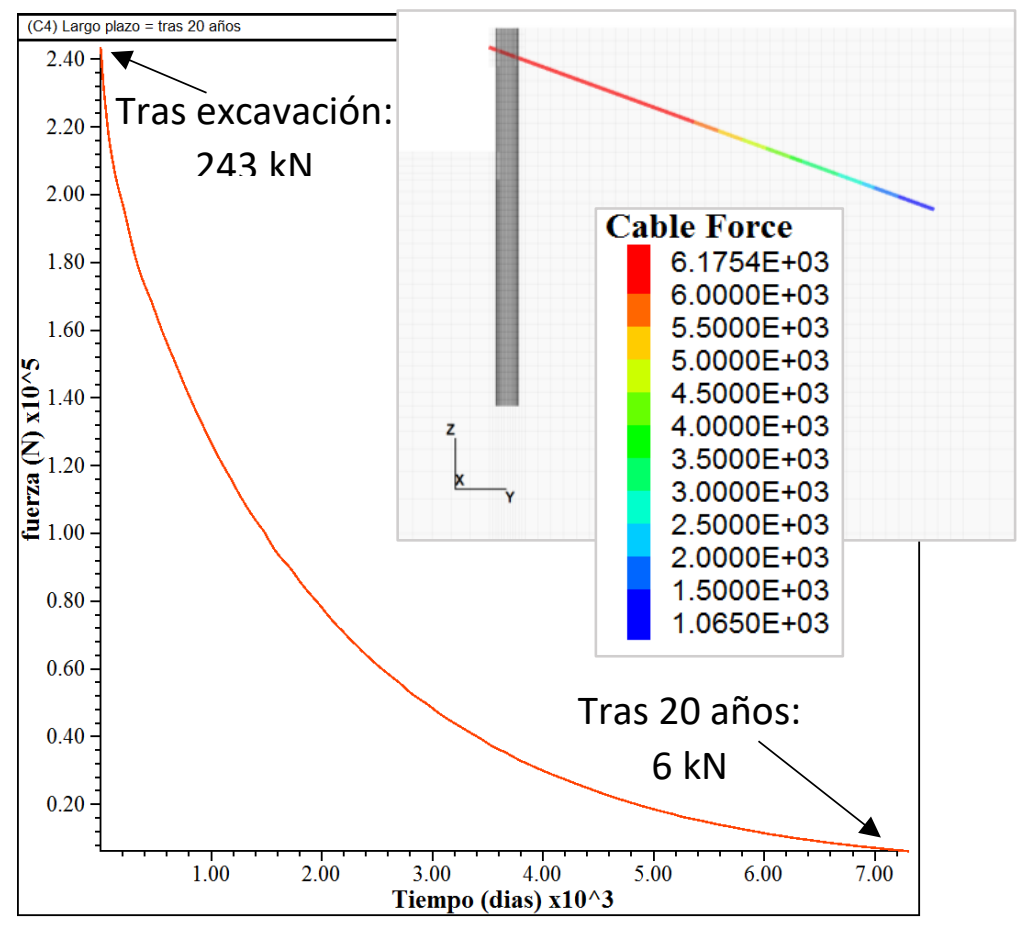

Figura 80. Evolución de la tensión en el anclaje transcurridos 20 años para el Caso D.

La evolución de la carga en el anclaje es equivalente a la deformación negativa registrada, aspecto que quiere decir que ha habido un acortamiento de la longitud libre del anclaje, como se puede apreciar en la Figura 81.

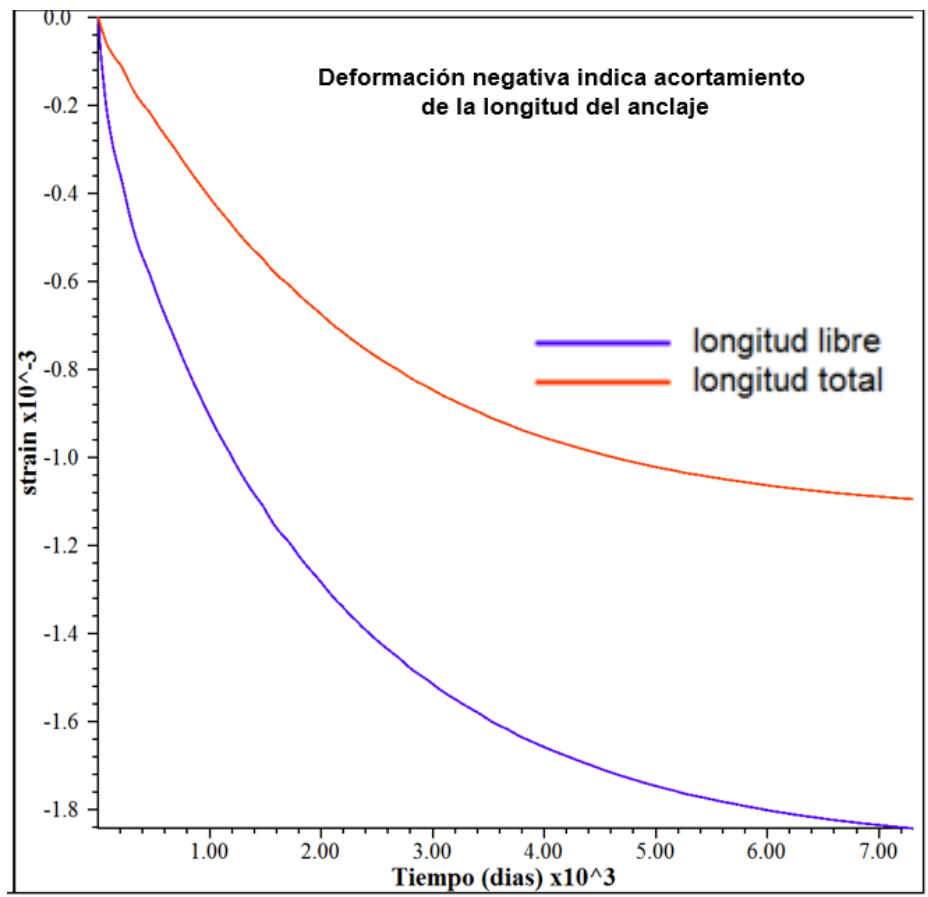

Figura 81. Evolución de la deformación en el anclaje $(\mathrm{m} / \mathrm{m})$. 
En un principio, el cuarto caso de cálculo se considera válido como una primera solución del comportamiento deformacional de la sección de estudio, tanto a corto como a largo plazo.

No obstante, los resultados y valores propuestos forman parte de una primera puesta a punto del modelo de cálculo, que queda disponible para su aplicación en otros casos de pantallas ancladas en terrenos con fluencia. La clave estará en que los parámetros de fluencia que se incorporen al modelo sean lo más representativos posible del comportamiento durante la vida útil de la obra. Estos parámetros han de ser estimados a partir de los resultados de ensayos adecuados, realizados con los terrenos presentes alrededor del bulbo y en contacto, principalmente, con la altura libre de la pantalla.

\subsubsection{Contraste con el modelo analítico de pantalla anclada}

En el apartado 4.2.2. del presente capítulo se llevaba a cabo la validación del modelo de losa anclada mediante un modelo numérico en diferencias finitas, aportando credibilidad al modelo analítico. De igual modo se realiza la validación del modelo analítico de pantalla anclada mediante su contraste con un modelo representativo en diferencias finitas. Las hipótesis para la simulación vistas en el apartado anterior son las mismas para este caso de contraste, aunque han tratado de precisarse: las propiedades geotécnicas del terreno, ciertas condiciones de contorno, y un $k_{0}$ recalculado y ajustado a los terrenos presentes, según se indica a continuación.

Las propiedades geotécnicas y características resistentes de los pilotes de hormigón armado y de hormigón en masa, del relleno granular bajo la losa base, y del hormigón de la losa base son las indicadas en la Tabla 12. A estos elementos se les asigna un modelo constitutivo elástico. 
Tabla 12. Parámetros adicionales para la simulación de la pantalla en FLAC.

\begin{tabular}{|c|c|c|}
\hline \multicolumn{1}{|c|}{ Parámetro } & Valor & Unidades \\
\hline Espesor, $\boldsymbol{e}_{\boldsymbol{R}}$ & RELLENO GRANULAR LOSA & $\mathrm{m}$ \\
\hline $\boldsymbol{\varphi}$ & 0.9 & $\underline{-}$ \\
\hline $\boldsymbol{c}$ & 35 & - \\
\hline $\boldsymbol{\gamma}$ & 0 & $\mathrm{~N} / \mathrm{m}^{2}$ \\
\hline $\boldsymbol{E}$ & 1900 & $\mathrm{MPa}$ \\
\hline $\boldsymbol{v}$ & 100 & - \\
\hline PILOTES (HA Y MORTERO), VIGA ATADO Y SOLERA & \\
\hline Espesor solera, $\boldsymbol{e}_{\boldsymbol{L}}$ & 0.3 & $\mathrm{~m}$ \\
\hline Altura viga atado, $\boldsymbol{A}$ & 1.1 & $\mathrm{~m}$ \\
\hline $\boldsymbol{\gamma}$ & 2500 & $\mathrm{~N} / \mathrm{m}^{2}$ \\
\hline $\boldsymbol{E}$ & 24.5 (pilote mortero), 29 (pilote HA y viga de & $\mathrm{GPa}$ \\
\hline $\boldsymbol{v}$ & atado), 30 (solera) & - \\
\hline
\end{tabular}

Al igual que en el caso de puesta a punto del apartado anterior, se han considerado tres niveles geotécnicos diferentes para reproducir la pantalla real. De los cuales, los dos primeros, que afectan a la longitud de giro y en los cuales se instalan los anclajes, tienen las propiedades incluidas en la Tabla 6 , con los parámetros viscoelásticos del Caso 2. El modelo constitutivo asignado es el elasto-plástico de Mohr-Coulomb en la parte mecánica y viscoelastoplástico de Burgers con un criterio de rotura tipo Mohr-Coulomb en la parte de fluencia. Sin embargo, se toman cohesiones consideradas como infinitas $\left(\mathrm{c}=1 \cdot 10^{20} \mathrm{~Pa}\right)$ para no alcanzar la plasticidad y simular un problema viscoelástico.

Por otro lado, al considerarse un modelo viscoelástico en el terreno, el empuje aumenta por verse afectados los parámetros de deformación del suelo. Para simular la hipótesis de empuje del terreno constante con el tiempo e igualar el modelo numérico al analítico, se fijan los desplazamientos en la dirección "y" y en la longitud total de giro $\left(\mathrm{L}_{\mathrm{T}}\right)$ de la pantalla.

El resto de parámetros (geométricos y del anclaje) necesarios para el modelo de pantalla creado en FLAC 3D son los presentados en la Tabla 5. Llevando a cabo la simulación del modelo en FLAC 3D con los parámetros, 
bajo las condiciones vistas, se obtiene la curva de pérdida de carga en el anclaje representada en la Figura 82.

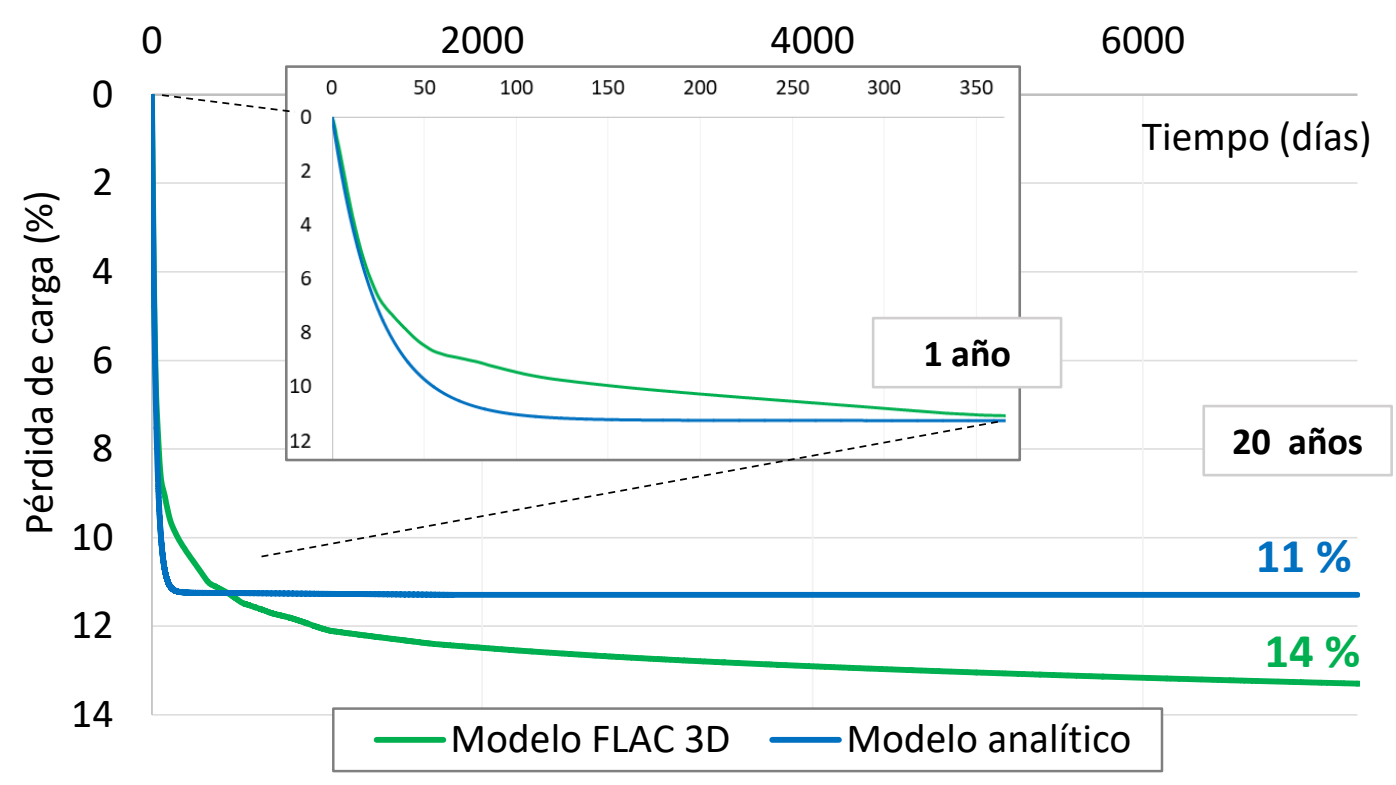

Figura 82. Pérdida de carga obtenida con el modelo analítico y con FLAC 3D.

Se puede apreciar una diferencia en la predicción de pérdida de carga a un año entre ambos modelos de tan solo un 1\%. Al cabo de 20 años la diferencia en la predicción entre ambos modelos es únicamente de un $3 \%$.

Por lo tanto, se puede afirmar que los resultados obtenidos mediante el modelo numérico confirman la validez del modelo analítico desarrollado para el caso de pantalla anclada. 
CAPÍTULO 5: DESARROLLO DE UN NUEVO ENSAYO DE FLUENCIA EN LABORATORIO 


\subsection{INTRODUCCIÓN}

\subsubsection{Necesidad}

En apartados anteriores (3.3.4. y 3.3.6.2.) quedó reflejada la necesidad de disponer de ensayos de fluencia de mayores duraciones. Es decir, ensayos con los cuales sea posible estimar parámetros de fluencia del modelo constitutivo que sean representativos de la vida útil de la obra y del comportamiento por fluencia bajo las condiciones impuestas. De manera que, el tiempo ensayado debe asegurar que el suelo se encuentra en una etapa de fluencia secundaria con pendiente aproximadamente constante, tras haber transcurrido la etapa de fluencia primaria. Durante esta última se produce la mayor pérdida de carga en el anclaje por las deformaciones asociadas y suele presentarse en la mayor parte de los suelos durante los primeros días de su puesta en carga. La etapa secundaria se alcanza habitualmente en el primer mes. La pérdida de carga en el anclaje asociada a esa segunda etapa es mínima, si se compara con la perdida inicialmente, durante la fluencia primaria o transitoria. Por tanto, de manera general, se puede establecer que un tiempo de ensayo del orden de 30 días sería el mínimo adecuado para estimar los parámetros representativos de la fluencia del terreno objeto de estudio.

Adicionalmente, ya se realicen en campo o en laboratorio, estos ensayos también deben estar sometidos a niveles tensionales similares a los de la carga de trabajo o de servicio. Es conocido (apartado 2.2.4.2.) que, según el nivel de tensión al que se encuentre sometida la estructura del suelo o de la roca, las distintas etapas de la fluencia pueden aparecer en mayor o menor magnitud y antes o después en el tiempo.

Los ensayos de fluencia en campo (normalmente ensayos de investigación) se realizan para evaluar la fluencia del terreno bajo ciertos niveles de carga muy elevados (respecto a la resistencia última del tirante) y con duraciones muy breves (60 o $120 \mathrm{~min}$ ). Por un lado, son muy útiles, ya que se realizan in situ en los terrenos completamente inalterados y sirven para tener una primera orientación del comportamiento por fluencia del terreno en condiciones extremas de carga. Sin embargo, se someten a cargas mayores 
de las de trabajo, induciendo en el terreno un nivel tensional que, en principio, no es al que se verá sometido durante su vida útil $\mathrm{y}$, por lo tanto, no es representativo en cuanto al fenómeno de fluencia en sí, que se va a desarrollar en ese terreno en condiciones de trabajo normales.

Por lo tanto, para llevar a cabo predicciones mediante los modelos analítico o numérico propuestos en la tesis, resultará clave estimar los valores de los parámetros del modelo de fluencia elegido, a partir de otro tipo de ensayos o con niveles de carga y duraciones más adecuadas que las de los ensayos de investigación.

Commo consecuencia de lo expuesto en los párrafos anteriores, se ha visto la necesidad de realizar ensayos nuevos y específicos en laboratorio que cuenten con:

- duraciones mínimas de un mes,

- unas condiciones de contorno particulares del problema objeto de estudio,

- unas determinadas dimensiones que superen las correspondientes de los ensayos más convencionales empleados para el estudio de la fluencia secundaria, como por ejemplo, un edómetro a largo plazo,

- y la posibilidad de emplear los niveles tensionales reales (respecto a la resistencia última del terreno en esas condiciones) de aplicación y su mantenimiento en el tiempo.

\subsubsection{Antecedentes}

Jamiolkowski et al., (1985) mencionan equipos como: el edómetro, el triaxial en deformación plana (deformación plana o corte simple), el triaxial convencional, el corte directo, el triaxial verdadero y el corte a torsión, como 
los más apropiados para la investigación de los efectos del tiempo en laboratorio.

El equipo ideal para el ensayo de la fluencia debe permitir la medida de las deformaciones a largo plazo de las muestras con la posibilidad de aplicar un tensor de tensiones efectivas constante, con la anisotropía tensional seleccionada, que varíe desde tensiones isotrópicas hasta tensiones de rotura. El entorno del ensayo debería preservar una humedad y temperatura constantes en la muestra y evitar la exposición ante cualquier posible impacto.

El denominado triaxial verdadero emplea probetas cúbicas o prismáticas con un estado general de tensiones conforme a las condiciones que se han indicado más arriba. Sin embargo, la complejidad intrínseca de este equipo, a menudo combinada con el delicado sistema electrónico de registro y control de las medidas (que en el caso de la fluencia no es necesario que sean tan frecuentes), complica el empleo del triaxial verdadero en pruebas de duración semanal o incluso mensual, como es el caso de ensayos para evaluar el fenómeno de la fluencia.

Otra opción posible es emplear el equipo triaxial convencional. A pesar de que el estado de tensiones de la muestra es simétricamente axial y no tan general como en el caso del triaxial verdadero, el equipo es más simple y también lo es la forma de la probeta. En el transcurso de los ensayos de fluencia, la sección transversal de la probeta varía y la carga axial debe ser reajustada para mantener la condición de tensión efectiva constante (la versión habitual de velocidad de deformación constante se debe remplazar por un sistema de carga permanente). La fricción de las bases de las probetas no se puede reducir de la manera convencional (láminas de goma lubricadas con silicona) ya que este sistema no es duradero, y la membrana de plástico alrededor de las probetas puede, bajo ciertas condiciones, ser permeable 0 rigidizable y se necesita tomar ciertas medidas como, por ejemplo, rodear la probeta de mercurio.

En el ensayo edométrico la condición de carga axial efectiva constante es fácil de mantener debido a que la sección transversal de la probeta no 
varía. Sin embargo, por las condiciones del ensayo edométrico $\left(\varepsilon_{H}=0\right)$, el coeficiente de empuje al resposo $\left(k_{0}\right)$ es constante y por lo tanto también lo es la tensión horizontal efectiva. Dicho de otro modo, mediante el ensayo edométrico la anisotropía tensional no se puede controlar, limitando la generalidad del procedimiento experimental. Según Feda (1992), el edómetro solo se debe aceptar como herramienta suplementaria en la investigación de la fluencia.

En el equipo de corte directo, se puede medir la resistencia al corte en el plano de corte prestablecido. Sin embargo, el estado tensional no está definido con claridad y las deformaciones de las probetas no son homogéneas. Aun así, las resistencias y las velocidades de tensión de rotura medidas con equipos de corte directo y triaxiales coinciden en las arenas estudiadas por Feda (1992).

La mayoría de los ensayos con argilitas y arcillas llevados a cabo por Feda (1992) se realizaron en un equipo de corte anular a torsión, tipo Hvorslev. Las ventajas y desventajas del edómetro y del triaxial se ven combinadas en este ensayo a cambio de sacrificar la forma sencilla de la probeta. Esta posee forma de cilindro hueco, lo que complica la utilización de muestras inalteradas. Como principal ventaja se encuentra la posibilidad de desplazamientos tangenciales ilimitados que permiten la medida de resistencias residuales. Además, la deformación uniaxial de las muestras en el equipo de corte anular a torsión (con deformación radial nula) permite obtener deformaciones volumétricas fiables y la sección transversal de la muestra se mantiene constante, por lo que no es necesario reajustar ni la carga vertical ni la de corte.

Sin embargo, ninguno de ellos caracteriza directamente el estado tensional producido por el anclaje y las dimensiones reducidas de los equipos no permiten el estudio del efecto escala de los procesos con el tiempo.

Existen trabajos recientes que han llevado a cabo ensayos de fluencia y de arrancamiento en anclajes con hormigón como material base. Kränkel et al. (2015) presentan un estudio bajo carga constante de anclajes en hormigón, 
mediante la realización de tres tipos de ensayos, que estiman: la dependencia no lineal del coeficiente de viscosidad en el tiempo, la dependencia no lineal del módulo de deformación con la tensión, y la degradación causada por la reducción del módulo de deformación en relación al desplazamiento del anclaje. Nilforoush et al. (2016) realizaron ensayos de fluencia, en interior y en exterior, mediante un nuevo equipo para someter anclajes a carga constante en muestras de hormigón. Utilizaron diferentes escalones de carga respecto a la resistencia última a corto plazo, llevando a cabo ensayos de hasta 28 años en colaboración con varios laboratorios. Además, los autores estudiaron el efecto de la temperatura, la humedad ambiente, la adición de sales, y la humedad de las muestras en la resistencia a largo plazo de los anclajes, llevando a cabo los ensayos en diferentes condiciones.

No obstante, las investigaciones son mucho más limitadas en cuanto a ensayos de fluencia en laboratorio en anclajes con suelos y rocas como materiales base. Gurinsky (2002) empleó un nuevo equipo de laboratorio con el que medía el desplazamiento de un anclaje embebido en arcillas sensitivas durante aproximadamente 40 días bajo diferentes escalones de carga constante. El diseño del equipo permitía únicamente medir el desplazamiento en el anclaje, siendo además la humedad en la muestra variable. Fan et al. (2011) llevaron a cabo ensayos de fluencia en anclajes cementados en muestras de lutitas inalteradas. Con duraciones de ensayos de hasta 8000 min (5 días aproximadamente) pudieron establecer una serie de conclusiones en cuanto al desarrollo de las distintas etapas de deformación por fluencia conforme se va aumentando la carga aplicada respecto a la resistencia última a corto plazo. La resistencia al arrancamiento a largo plazo para la roca blanda arcillosa estudiada resultó ser de entre un 26 y un $39 \%$ del valor instantáneo. Zhang et al. (2015) desarrollaron un equipo que les permitió el estudio del mecanismo de interacción tipo "soil-nailing" (GFRP-soil nail), preconsolidando la arena de estudio mediante un sistema incorporado en el propio equipo. El procedimiento de sus ensayos consiste en la aplicación de siete escalones de carga consecutivos, manteniendo dicha carga constante tan solo durante una hora. 
En este capítulo se presenta un ensayo de laboratorio de grandes dimensiones que permite medir los desplazamientos por fluencia durante largos periodos de tiempo en un anclaje embebido en un terreno. La descripción del equipo desarrollado, así como las bases teóricas que han servido para su propuesta se detallan en el capítulo 5.2. En particular, se ha ensayado una roca artificial fabricada con bentonita (arcilla comercial con propiedades reológicas), que ha sido caracterizada geotécnicamente mediante ensayos de identificación, resistencia y deformabilidad (capítulo 5.3.). En el capítulo 5.4. se puede consultar el procedimiento de ensayo finalmente establecido. A continuación, además de los resultados de los tres ensayos de fluencia llevados a cabo, se presenta una interpretación de los resultados obtenidos con los ensayos con carga instantánea considerando la rigidez en pequeñas deformaciones y el efecto escala (capítulo 5.5.). Finalmente se incluyen una serie de conceptos que relacionan la monitorización en obra, el control y la predicción a largo plazo, y la estimación de parámetros mediante un equipo de estas características (capítulo 5.6.).

\subsection{DISEÑO DEL EQUIPO}

\subsubsection{Ideas preliminares}

Es conocido que cuando se realiza un ensayo de arrancamiento los tipos de rotura que pueden tener lugar son los referenciados en Hilti (2002): arrancamiento de un cono de roca, deslizamiento parcial del anclaje, rotura del anclaje (metal), deslizamiento del anclaje en el contacto resina-roca, y desprendimiento lateral.

En la Figura 83 se muestran los modos de rotura más frecuentes obtenidos experimentalmente en muestras de mortero por García-Wolfrum (2005), con motivo de la realización de una tesis doctoral. 


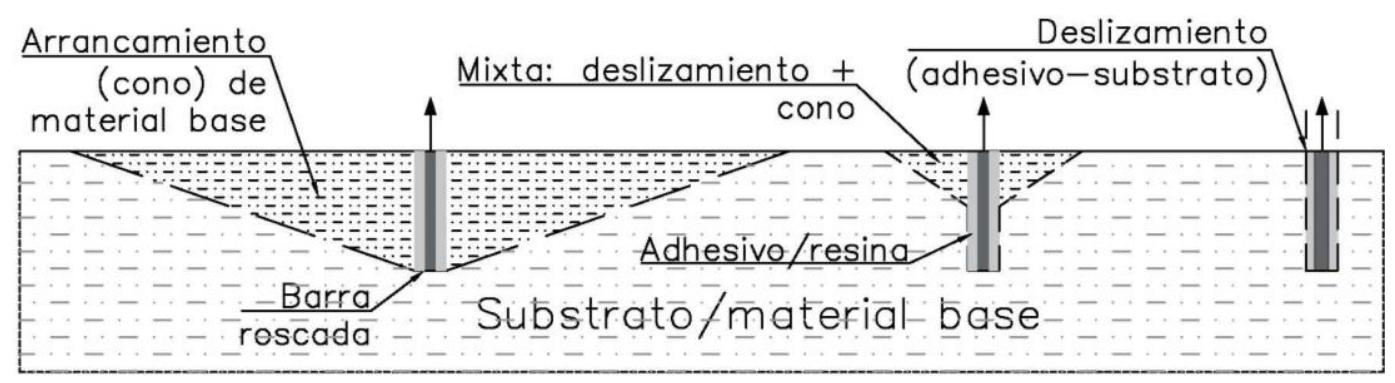

Figura 83. Tipos de rotura frecuentes en anclajes en roca.

Los resultados de 15 ensayos de arrancamiento en muestras de mortero (García-Wolfrum, 2005) establecen unas dimensiones máximas de los conos de rotura de entre 70 y $240 \mathrm{~mm}$ de radio, para longitudes de anclaje $\left(L_{a}\right)$ comprendidas entre 46 y $114 \mathrm{~mm}$ y diámetros de perforación $\left(D_{h}\right)$ entre 15 y 18 $\mathrm{mm}$. La relación geométrica $\left(L_{a} / D_{h}\right)$ empleada por la autora variaba entre $2.6 \mathrm{y}$ 7.2, ensayando morteros de resistencias a compresión simple $\left(\sigma_{c}\right)$ comprendidas entre 2.3 y 7.3 MPa. A partir de estas ideas preliminares, se propone un equipo con las siguientes características geométricas:

- Muestras cúbicas de roca de 745 × 550 × $310 \mathrm{~mm}$.

- Longitud anclada: variable entre 70 y $80 \mathrm{~mm}$.

- Diámetro del bulbo: perforación de $12 \mathrm{~mm}$ con barra roscada de 10 $\mathrm{mm}$ adherida a la muestra mediante resina epoxi (Sika AnchorFix3001 , resistencia a compresión $=85 \mathrm{GPa}$, módulo elástico $=5000$ GPa).

Además, se considera un sistema mecánico (que evita la dependencia externa de un sistema electrónico de registro en largos periodos de tiempo) en el cual la carga se transmite al anclaje a través de la colocación directa de pesas.

Los valores habituales de resistencia media al arrancamiento $\left(\tau_{m}\right)$ para distintos tipos de materiales se pueden consultar en la Tabla 13. 
Tabla 13. Resistencia media al arrancamiento aparente para el contacto terreno/lechada a lo largo de la zona del bulbo (posterior a FHWA, 1999).

\begin{tabular}{|l|c|}
\hline Tipo de roca & $\boldsymbol{\tau}_{\boldsymbol{m}}(\mathbf{M P a})$ \\
\hline Granito y basalto & $1.7-3.1$ \\
\hline Dolomía & $1.4-2.1$ \\
\hline Caliza blanda & $1.0-1.4$ \\
\hline Slates y pizarras duras & $0.8-1.4$ \\
\hline Pizarras blandas & $0.2-0.8$ \\
\hline Areniscas & $0.8-1.7$ \\
\hline Areniscas alteradas & $0.7-0.8$ \\
\hline Creta & $0.2-1.1$ \\
\hline Marga alterada & $0.15-0.25$ \\
\hline Hormigón & $1.4-2.8$ \\
\hline Nota: Para rocas duras la adherencia última corresponde al menor valor de \\
resistencia última entre la roca y el contacto terreno/lechada. \\
\hline
\end{tabular}

Según estos valores (Tabla 13) se puede deducir que dicho equipo puede ser empleado para ensayar la mayoría de terrenos que habitualmente se encuentran en la naturaleza y en las obras de ingeniería. En particular, para ensayar rocas blandas, con resistencias al arrancamiento entre 150 y $1400 \mathrm{kPa}$ (Tabla 13), se necesitarían pesas en el sistema mecánico de 40 kg - 400 kg de masa, respectivamente.

\subsubsection{Descripción del equipo}

Se trata de un equipo mecánico, debido a que la transmisión de la carga se realiza directamente mediante la colocación de pesas. De este modo, se pueden lograr tiempos de ensayo muy prolongados, manteniendo la carga aplicada constante, sin necesidad de depender de sistemas eléctricos que pudieran verse interrumpidos o con los cuales es más difícil generar esta condición. En la Figura 84 se pueden apreciar varias fotografías del equipo desarrollado. 


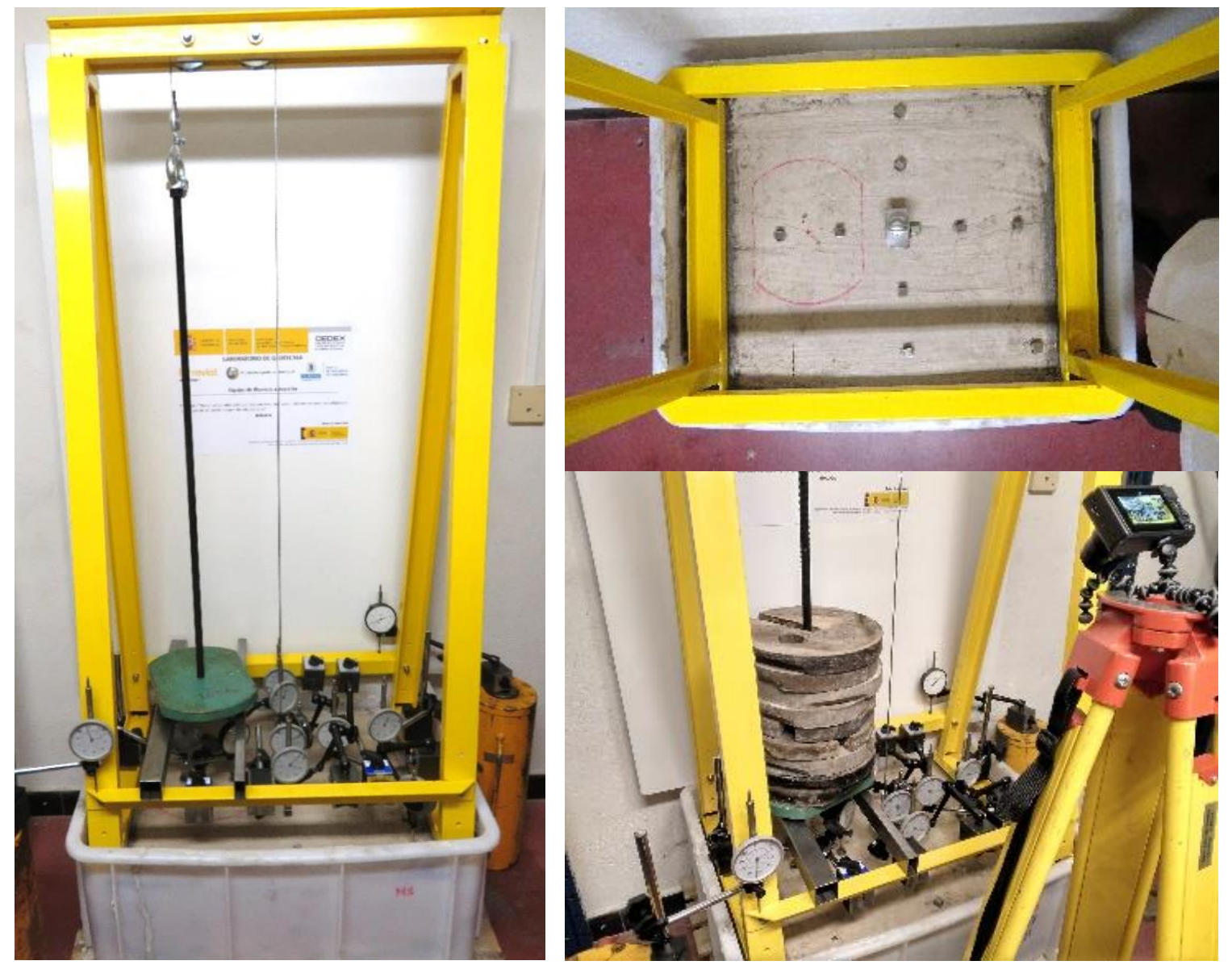

Figura 84. Fotografías del equipo.

El esquema del equipo desarrollado se puede apreciar en la Figura 85 y sus principales componentes son:

- cubeta de plástico endurecido contenedora de la muestra fabricada,

- estructura resistente formada con perfiles metálicos de acero estructural (perfiles UPN de $60 \mathrm{~mm}$ ) con base de apoyo sobre la muestra de $745 \mathrm{x}$ $550 \mathrm{~mm}$. En esta estructura se coloca el cableado (cable de acero galvanizado de $3 \mathrm{~mm}$ de diámetro y resistencia a tracción de $1770 \mathrm{GPa}$ ), apoyado sobre dos poleas, que une el anclaje (en el eje central) con el sistema o barra para colgar las pesas (en un lateral) que ejercerán la tracción constante en el tiempo,

- y nueve comparadores de centésima de milímetro (puntos 1 a 9 en Figura 85) que miden el desplazamiento con el tiempo de la muestra y del anclaje (punto 6, Figura 85). 
Por otro lado, la altura de la cubeta está pensada para poder sumergir la muestra en agua durante el ensayo y mantener su humedad de saturación, factor clave en el estudio de la fluencia del terreno. Además, el ensayo se realiza en una sala con temperatura controlada, manteniendo aproximadamente constante la temperatura ambiente en la muestra, y lo más relevante, la temperatura del agua que se encuentra en sus poros.

Se trata de una sala restringida al paso del personal o maquinaria para preservar la sensibilidad del equipo y protegerlo de cualquier golpe. Se han establecido distintos puntos de medición según el esquema en planta de la Figura 85.

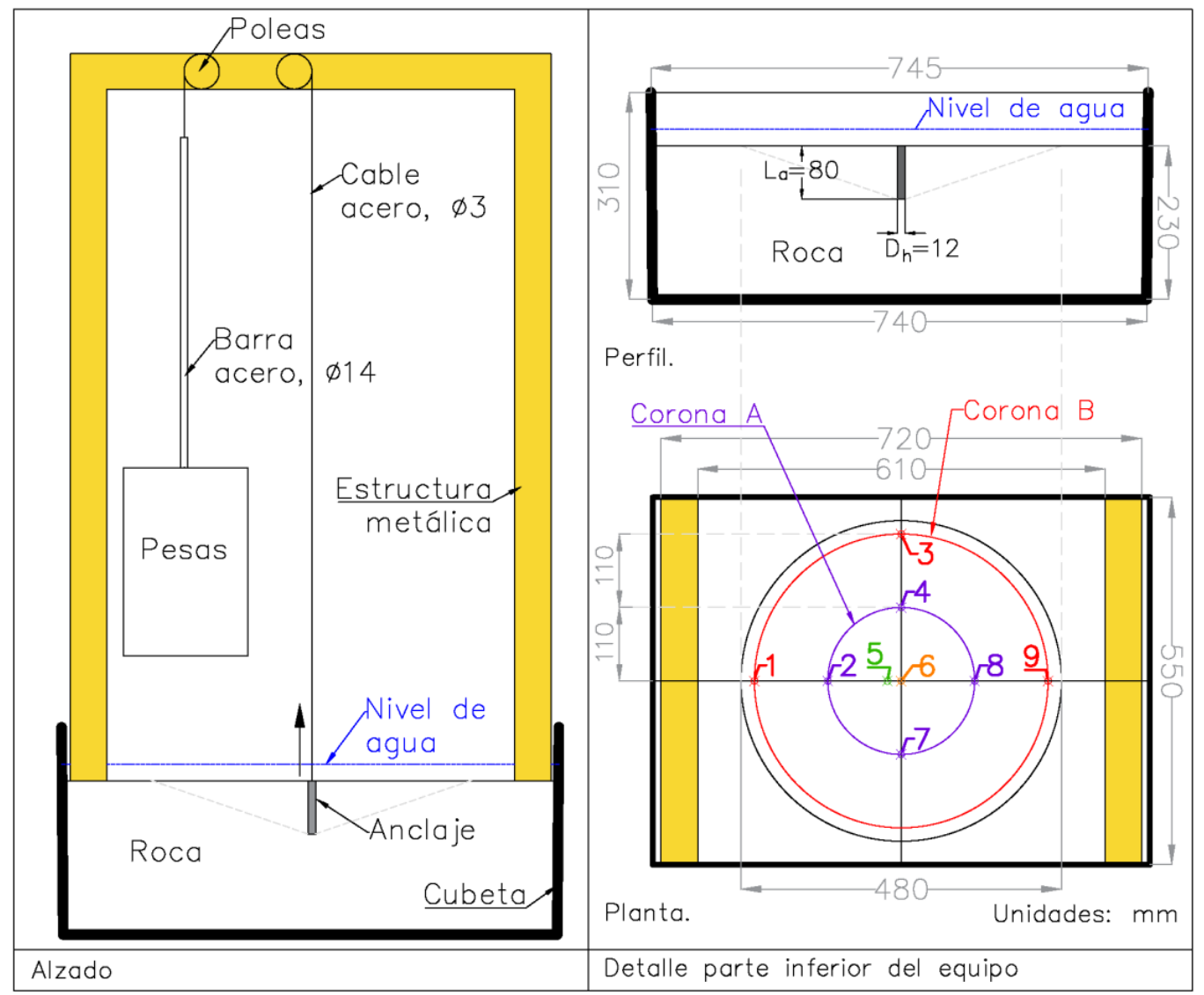

Figura 85. Esquema del equipo.

Los puntos seleccionados son simétricos respecto a dos ejes perpendiculares que pasan por el centro y cubren el área de movimiento esperada, para estudiar la evolución con el tiempo del cono de roca. 


\subsection{MUESTRAS DE ENSAYO}

\subsubsection{Composición seleccionada para el geomaterial}

En la mayoría de las arcillas naturales, la fluencia es una propiedad inherente de las relaciones entre partículas, particularmente si estas contienen bentonita, montmorillonita y materia orgánica; y de manera menos significativa, las cloritas-illitas (Mitchell y Soga, 2005).

De esta manera, se planteó la búsqueda de mezclas artificiales que pudieran presentar fluencia, estudiando distintas composiciones (bentonita, cemento y agua; y yeso, arena, cemento y agua), y que, a su vez, estuvieran en el rango de resistencia establecido para las rocas blandas (Del Cañizo et al., 1976, Leung y Ko, 1993 y Nishimura, 2016). Se realizaron probetas cilíndricas de $70 \mathrm{~mm}$ de diámetro con varias dosificaciones y se ensayaron a compresión uniaxial a diferentes edades (ver Tabla 14) para conocer su resistencia y trabajabilidad.

Tabla 14. Resultados de ensayos a compresión simple en muestras artificiales de prueba a diferentes edades.

\begin{tabular}{|c|c|c|c|c|}
\hline Muestra & Composición (\% p/p) & Edad (días) & $\sigma_{c}(\mathrm{Mpa})$ & Humedad (\%) \\
\hline \multirow{3}{*}{ macsia } & \multirow{3}{*}{$\begin{array}{l}\text { Bentonita: } 20 \\
\text { Cemento: } 30 \\
\text { Agua: } 50\end{array}$} & 10 & 0.6 & 104 \\
\hline & & 42 & 1.0 & 64 \\
\hline & & 91 & 1.3 & 70 \\
\hline \multirow{3}{*}{ B2 } & \multirow{3}{*}{$\begin{array}{l}\text { Bentonita: } 15 \\
\text { Cemento: } 25 \\
\text { Agua: } 60\end{array}$} & 10 & 0.1 & 151 \\
\hline & & 41 & 0.3 & 79 \\
\hline & & 92 & 0.3 & 109 \\
\hline \multirow{2}{*}{ Y1 } & \multirow{2}{*}{$\begin{array}{l}\text { Yeso: } 13 \\
\text { Cemento: } 25 \\
\text { Arena: } 32 \\
\text { Agua: } 30\end{array}$} & 8 & 0.7 & 29 \\
\hline & & 47 & 1.2 & 30 \\
\hline \multirow{2}{*}{ Y2 } & \multirow{2}{*}{$\begin{array}{l}\text { Yeso: } 18 \\
\text { Cemento: } 20 \\
\text { Arena: } 26 \\
\text { Aqua: } 36\end{array}$} & 11 & 0.3 & 57 \\
\hline & & 42 & 0.6 & 46 \\
\hline
\end{tabular}


La trabajabilidad observada con las mezclas que contenían yeso resultó muy compleja debido a la formación de agregados sólidos de manera casi inmediata, dificultando la tarea de mezclado manual para el operador. Al no disponer de una homogeneidad adecuada tras el mezclado, se imposibilita la obtención de una masa uniforme lo suficientemente fluida para ser vertida a continuación, en las probetas para el vibrado. Por esta razón se optó por fabricar las muestras de grandes dimensiones con bentonita. El aspecto heterogéneo (muestras con yeso y arena) y más homogéneo (muestras con bentonita) de las probetas tras 24 horas de curado, se puede apreciar en la Figura 86 a) y 84 b), respectivamente.
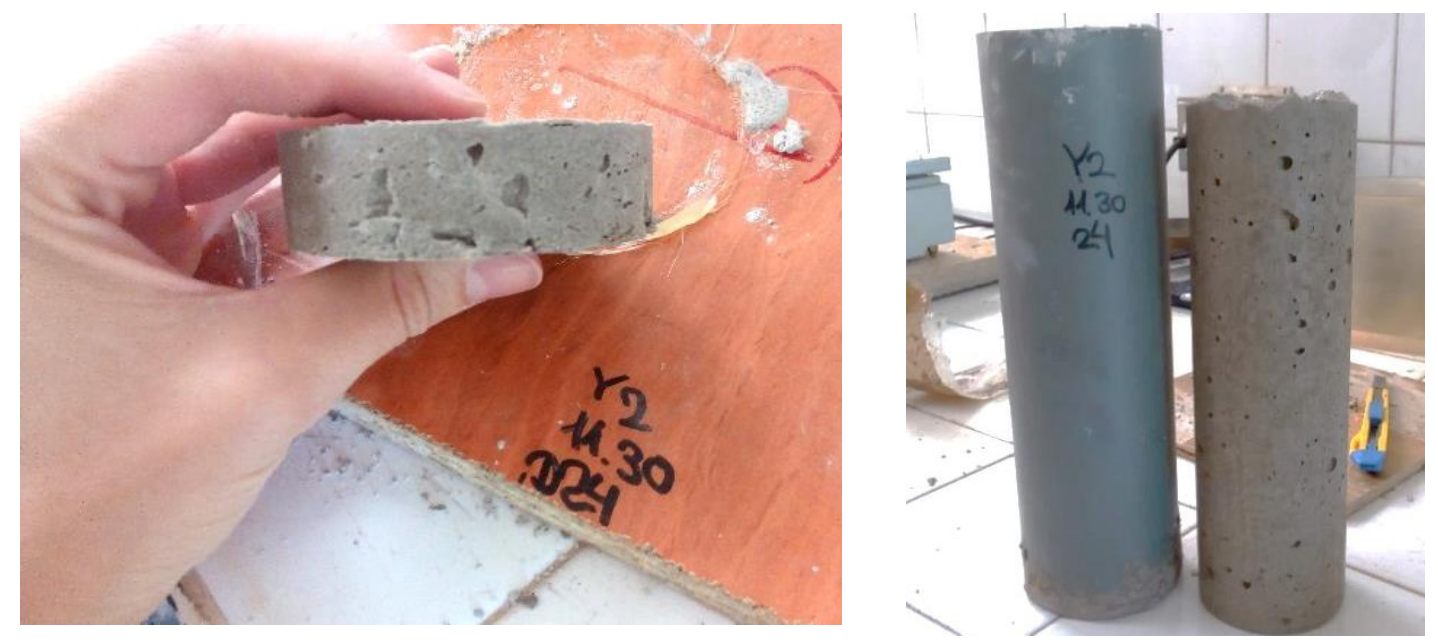

a) Muestras con yeso
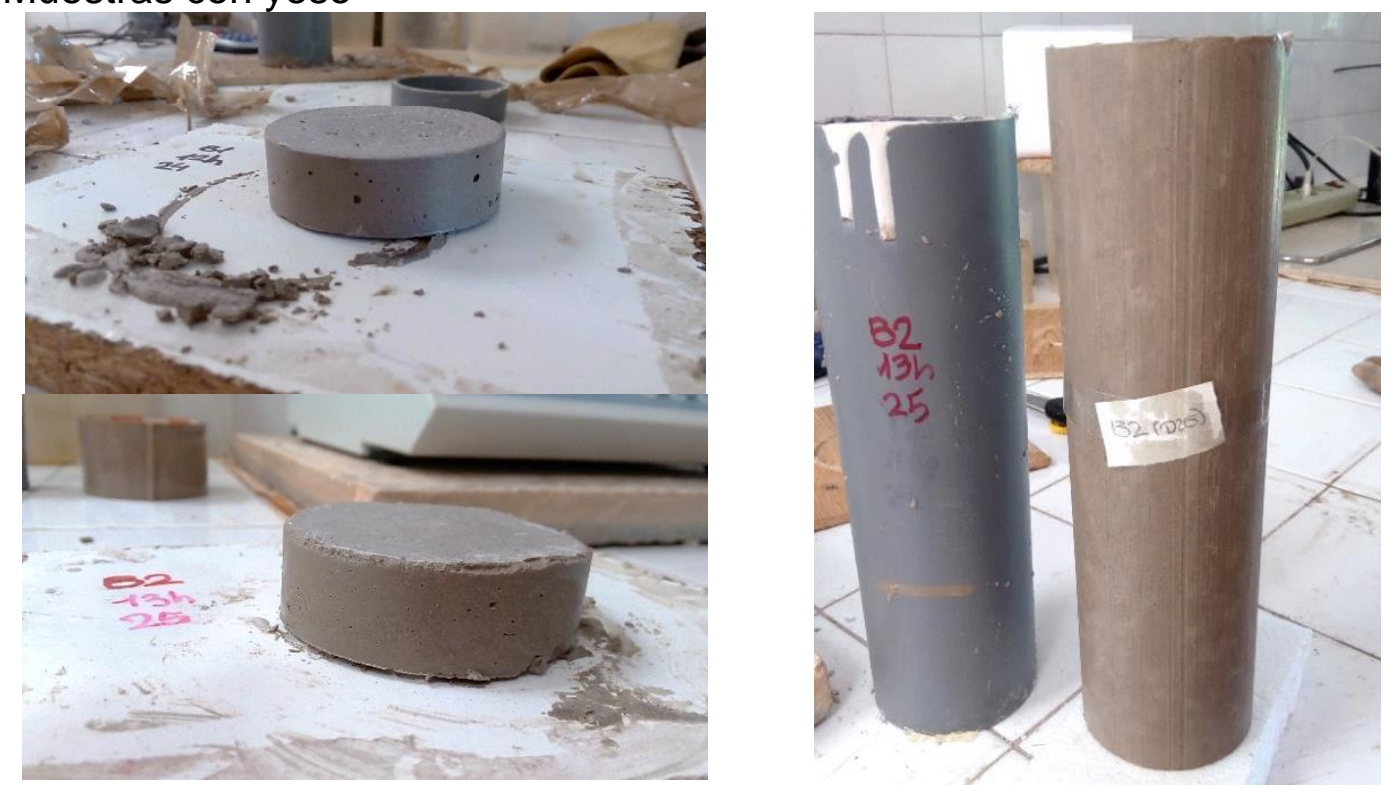

b) Muestras con bentonita

Figura 86. Aspecto de las muestras transcurridas $24 \mathrm{~h}$ de curado. 
Debido al contenido en bentonita, la cual posee un elevado poder retardante en la cementación, se preveía una evolución de las propiedades resistentes de los bloques con el tiempo. Por esta razón, los ensayos de fluencia comienzan cuando las muestras han estado curando en cámara húmeda, necesitándose en este caso al menos una duración de 90 días. Se utilizó una bentonita sódica por ser una arcilla comercial con propiedades reológicas y por proporcionar una buena trabajabilidad para fabricar los bloques de grandes dimensiones.

Se eligió una dosificación de la muestra B1, con un $50 \%$ de agua, $30 \%$ de cemento (CEM II/B-L 32,5 N) y un $20 \%$ de bentonita sódica, que permitían alcanzar resistencias finales de aproximadamente $1 \mathrm{MPa}$ y asegurar una mayor adherencia con la resina que en el caso de dosificaciones B2.

Finalmente, se verificó la fluencia del geomaterial creado con bentonita. Para ello se realizaron pruebas edométricas con las muestras secas, donde se manifestó claramente una rama de deformación con el tiempo (Figura 87) y por tanto indicativa de propiedades reológicas, convirtiéndolo en un material adecuado para su uso en el ensayo de fluencia diseñado.

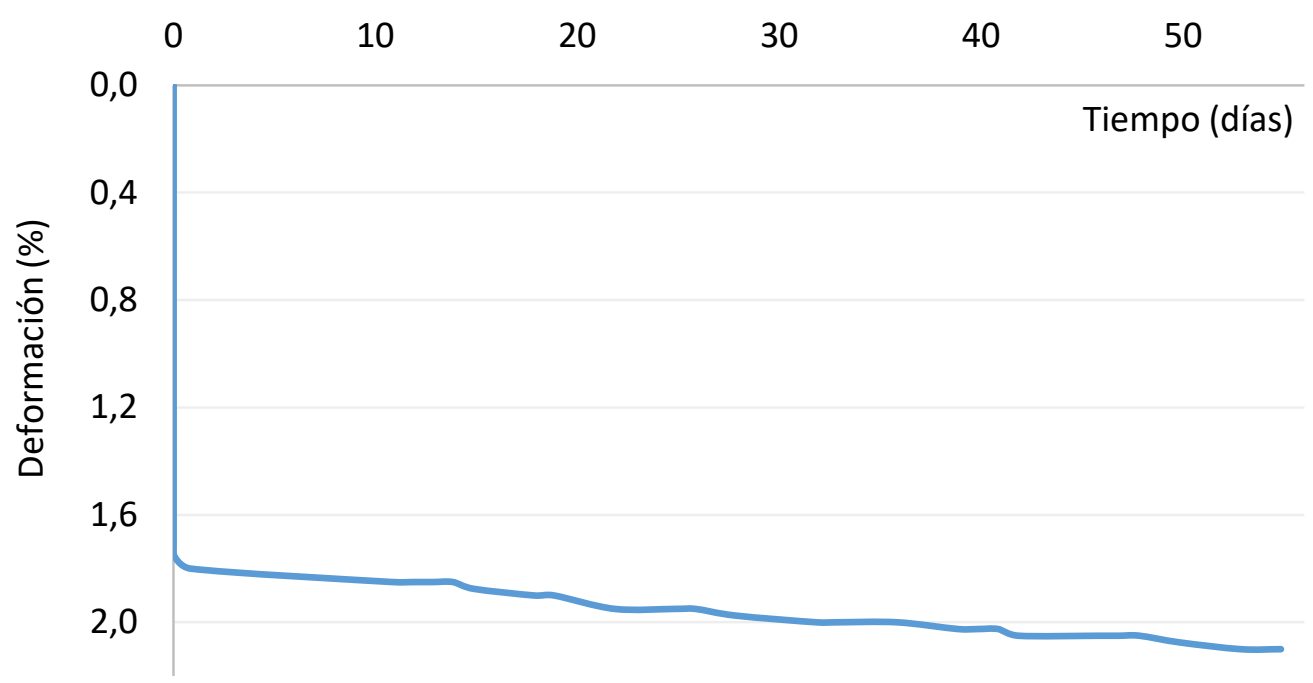

Figura 87. Ensayo edométrico de muestras de geomaterial con bentonita elegido. 


\subsubsection{Proceso de fabricación}

Para la caracterización geotécnica del geo-material se han fabricado, de manera simultánea a los bloques de grandes dimensiones, probetas de $70 \mathrm{~mm}$ de diámetro y $300 \mathrm{~mm}$ de altura.

El proceso de elaboración de las muestras de grandes dimensiones (745 x $550 \times 310 \mathrm{~mm}$ ) y de las probetas se puede resumir en los siguientes pasos:

1) Obtención de los materiales necesarios: hormigonera de 100 litros de capacidad máxima, cubetas, aguja vibradora eléctrica, moldes de PVC para probetas de $70 \mathrm{~mm}$ de diámetro y $300 \mathrm{~mm}$ de altura, varilla metálica para vibrado manual, cantidades para la mezcla (bentonita sódica, cemento y agua), recipientes para pesar, palas para mezclado y ayuda con el vertido y balanza (Figura 88).
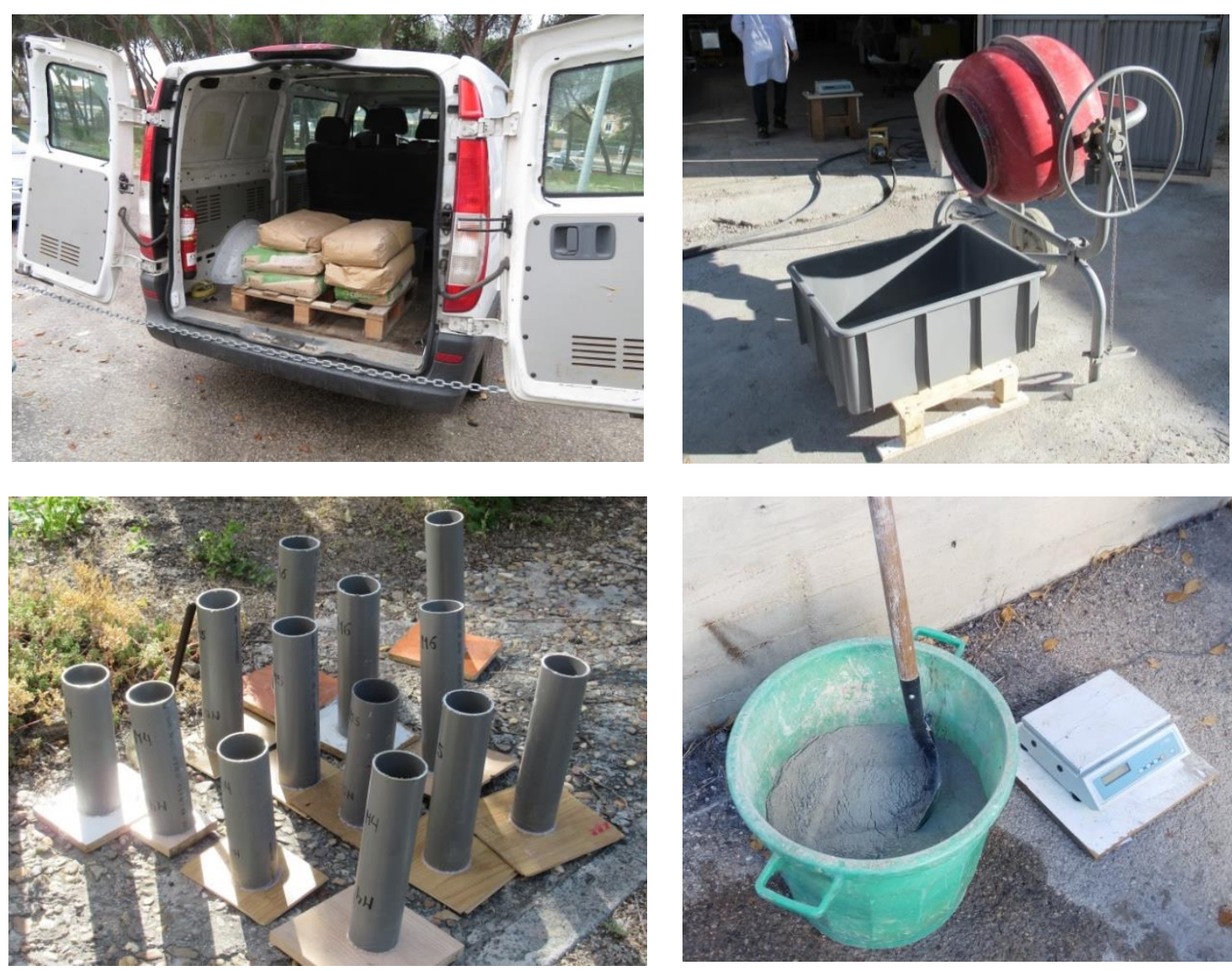

Figura 88. Algunos de los materiales empleados para la fabricación de las muestras. 
2) Engrasado de los moldes de PVC con aceite para facilitar la extracción de las probetas, tras el curado, sin alterarlas signiificativamente (Figura 89).
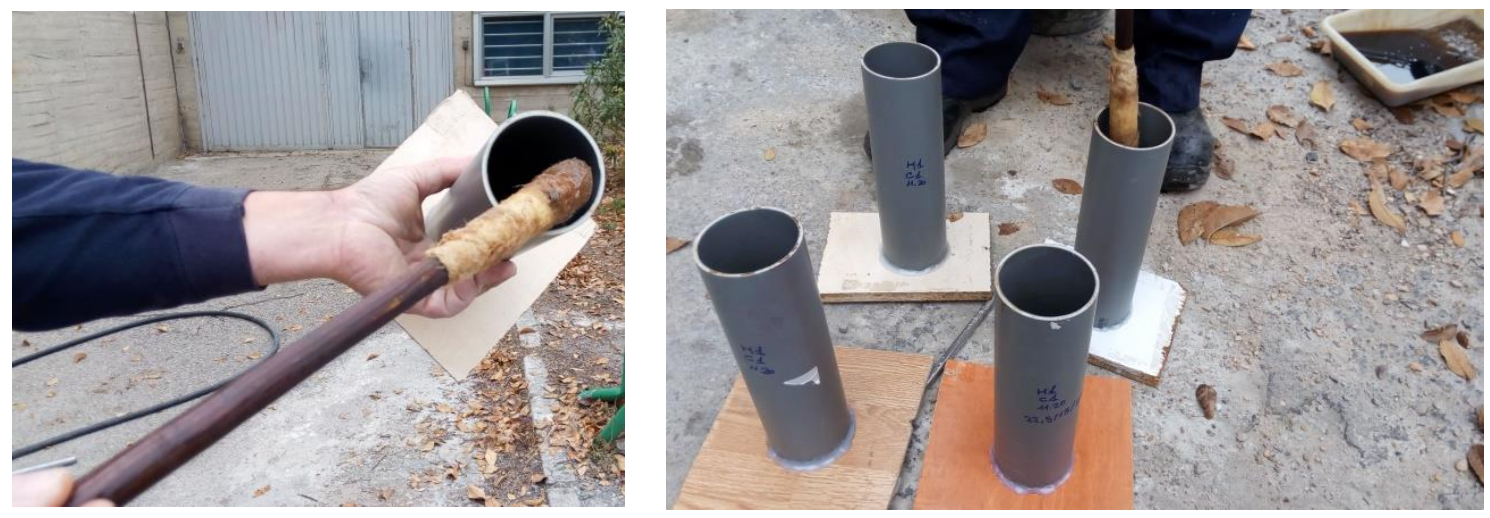

Figura 89. Materiales auxiliares empleados para la fabricación de las muestras.

3) Elección del espesor de cada capa para la fabricación del bloque por condicionantes de la Norma UNE-EN 12390-2. También, con el objetivo de lograr la mayor homogeneidad posible en la profundidad abarcada por el anclaje.

4) Mezclado previo de la mezcla sólida (bentonita y cemento) (Figura 90) depositándola, lentamente y en cantidades inferiores a $200 \mathrm{~g}$, dentro de la hormigonera en funcionamiento con el agua (Figura 90). Este proceso ha sido seleccionado como el óptimo, tras varias pruebas de vertido en las cuales el resultado no fue el deseado en cuanto a la homogeneidad de la mezcla conseguida.
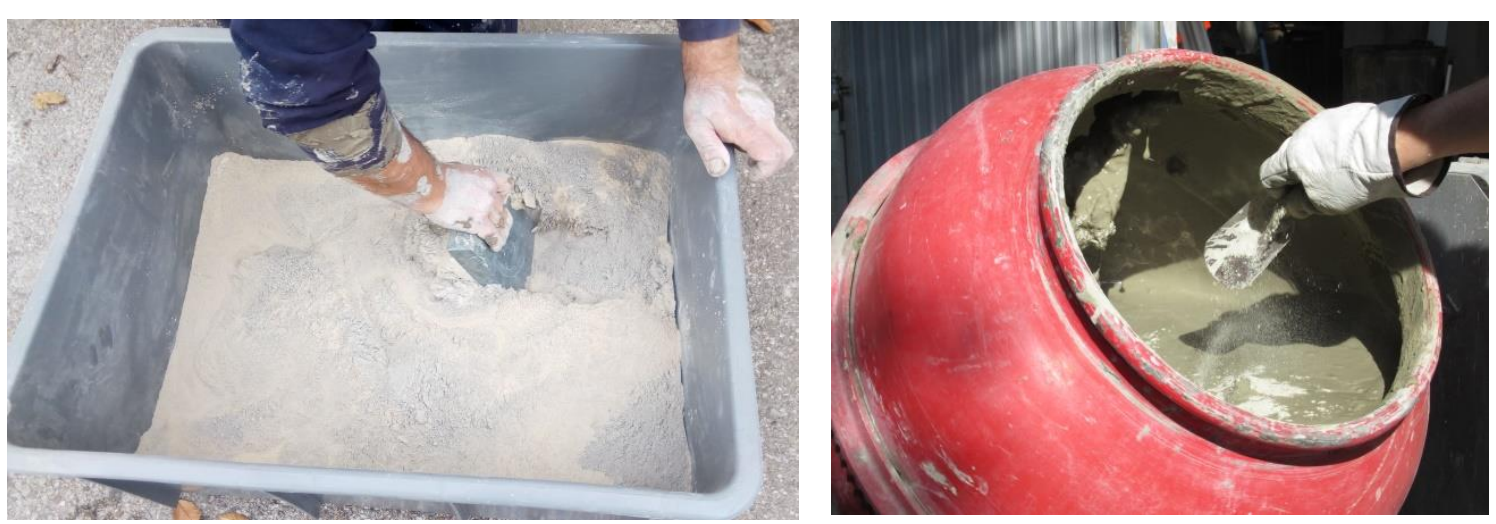

Figura 90. Mezclado de las partes sólidas y proceso óptimo de mezclado. 
5) Vertido sobre la cubeta y vibrado continuo mediante aguja, siguiendo las recomendaciones de la Norma UNE-EN 12390-2 (Figura 91). De este modo, la muestra queda adecuadamente compactada (Figura 92). En función del acabado final de la superficie, puede ser necesaria una ligera nivelación de esta mediante una llana de acero, antes de iniciarse el proceso de curado en cámara húmeda.

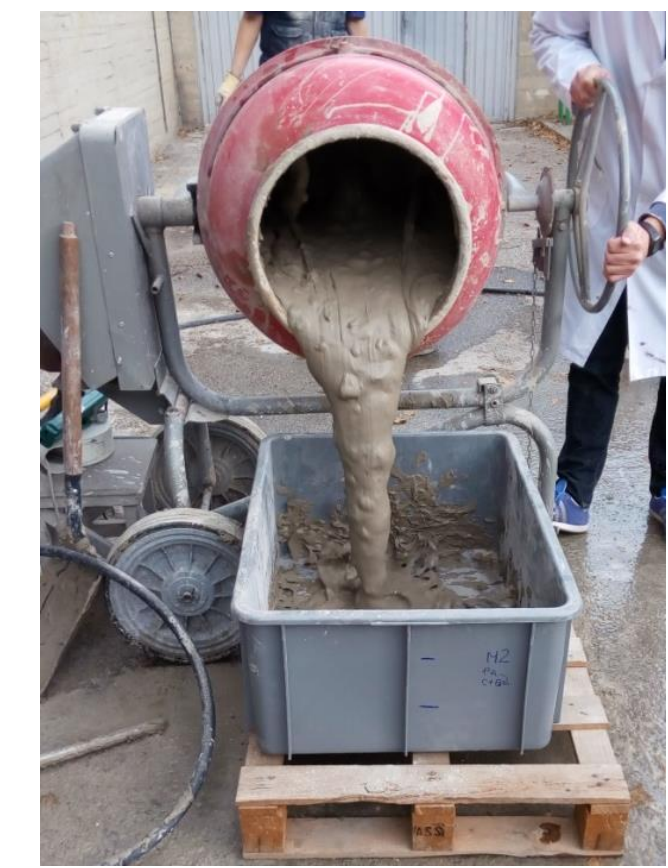

a)

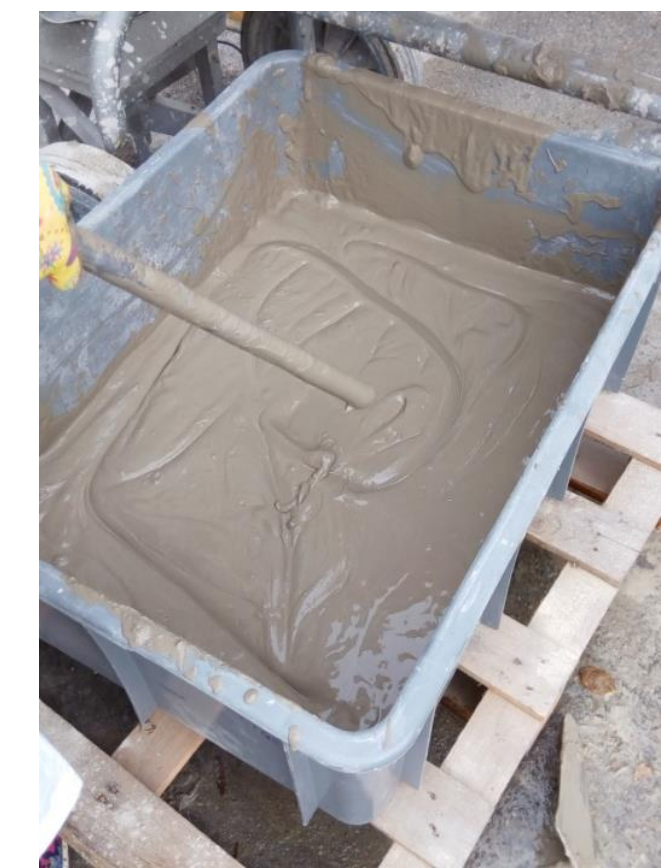

b)

Figura 91. a) Proceso de vertido y b) vibrado.

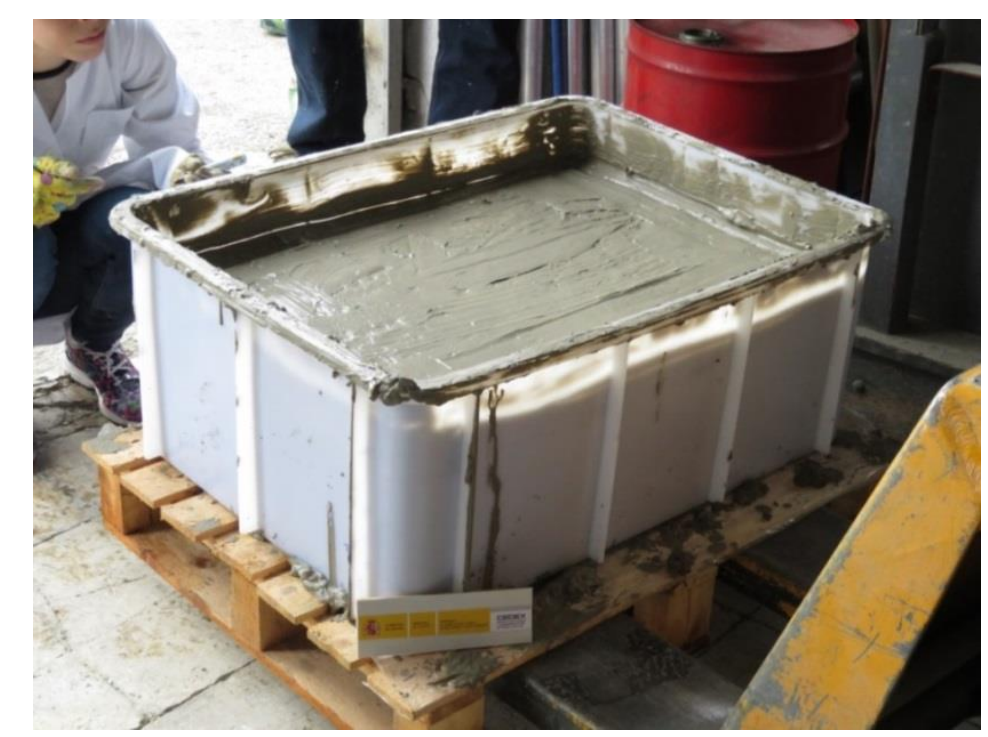

Figura 92. Aspecto final y nivelado de la muestra antes del proceso de curado. 
6) Se rellenan cuatro moldes de PVC con la mezcla final y se vibran manualmente mediante varilla metálica (Figura 93).
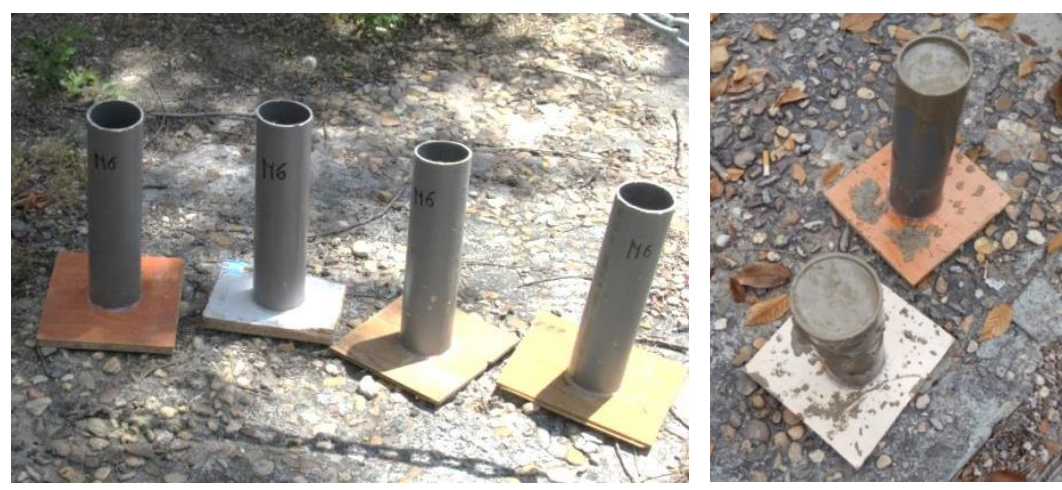

Figura 93. Aspecto final tras llenado y vibrado de los moldes para probetas.

7) Fraguado y curado en cámara húmeda de los bloques y de las probetas fabricadas.Durante las primeras horas de fraguado se coloca un plástico por encima de los bloques para evitar la caída de agua de las boquillas de nebulización de la cámara. A continuación, se retira el plástico, dejando el bloque destapado y en cámara húmeda durante 72 horas (Figura 94). Después de este periodo los bloques presentan gran consistencia y la superficie está completamente seca. En ese momento se inundan las cubetas con agua y las probetas también se sumergen en agua (sin extraer de los moldes, pero quitando las tapas, por similitud con el proceso de curado del bloque).
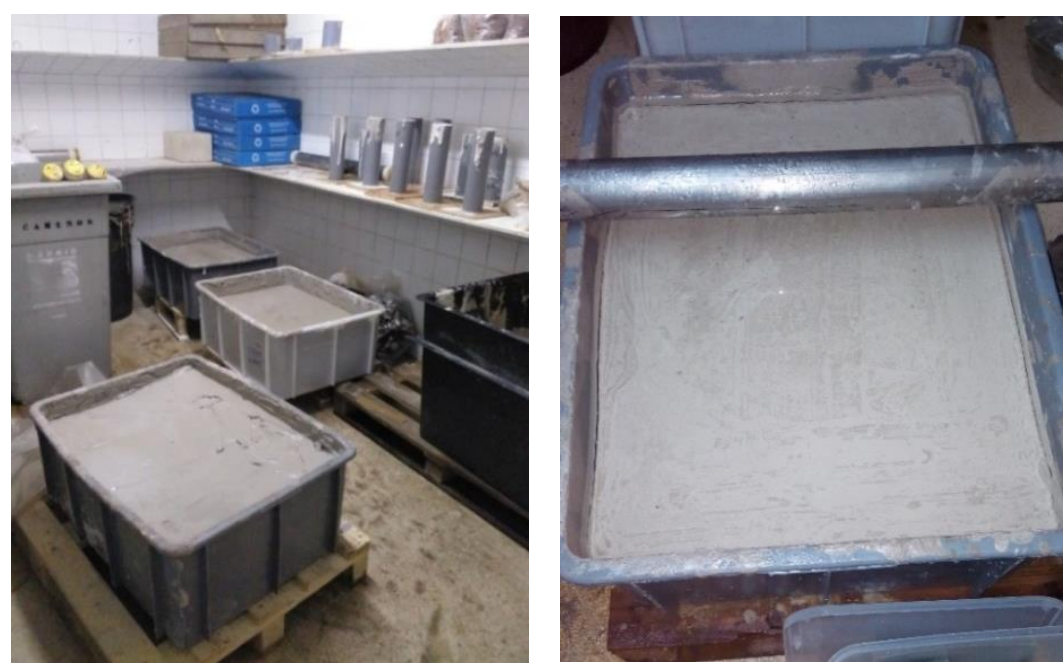

Figura 94. Aspecto final tras 72 horas de fraguado en cámara húmeda. 


\subsubsection{Caracterización geotécnica de las muestras}

Con el objetivo de identificar y conocer el comportamiento resistente y de deformación de los geomateriales ensayados a fluencia, se han llevado a cabo los siguientes ensayos: compresión simple (RCS), triaxiales consolidados no drenados (TCU) y velocidad de ondas longitudinales (Vp).

Como se indica en la Tabla 15 los ensayos se realizan tanto en probetas fabricadas al mismo tiempo que los bloques como en testigos extraídos de dichos bloques (véase Figura 95). Este procedimiento se seleccionó para asegurar la representatividad de las muestras dada su condición inalterada y por si pudieran existir diferencias en la fabricación en pequeñas y grandes dimensiones.
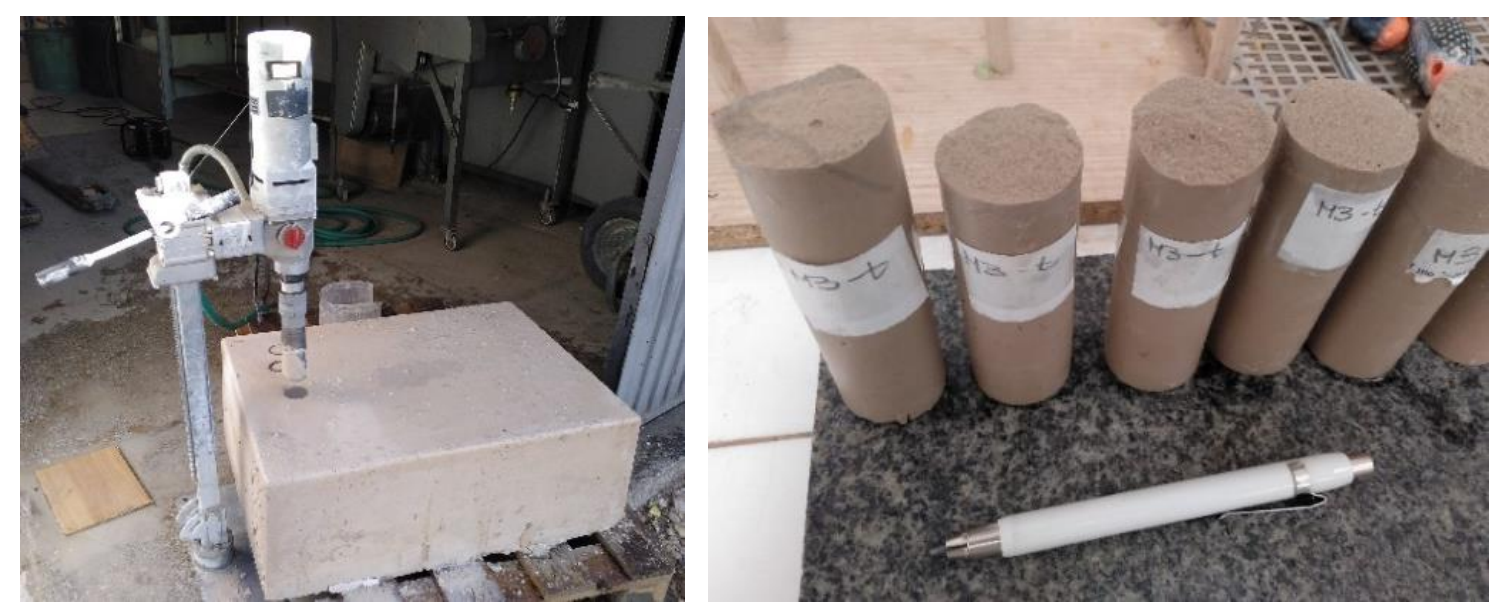

Figura 95. Extracción de testigos para caracterización del bloque 3 (Tabla 15).

Se han elaborado cinco muestras de grandes dimensiones, sin embargo, únicamente los bloques 2, 4 y 6 (Tabla 15) han sido ensayados en el nuevo equipo. El bloque 1 fue un bloque de prueba que se utilizó para establecer la metodología de fabricación, pero fue descartado dada su apreciable heterogeneidad. Por su parte, el bloque 3 se ha utilizado exclusivamente para extraer testigos en condiciones inalteradas (Figura 93). 
Tabla 15. Resultados de los ensayos de caracterización geotécnica de las muestras.

\begin{tabular}{|c|c|c|c|c|c|c|c|c|c|c|c|}
\hline \multirow{3}{*}{$\begin{array}{l}\frac{0}{2} \\
\frac{0}{0}\end{array}$} & \multirow{3}{*}{ 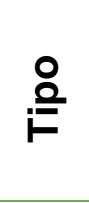 } & \multirow{3}{*}{ № } & \multirow{2}{*}{ Edad } & \multirow{2}{*}{$\rho$} & \multirow{2}{*}{$\mathbf{w}$} & \multicolumn{2}{|c|}{ RCS } & \multicolumn{2}{|c|}{ Triaxial CU } & \multicolumn{2}{|c|}{$V_{p}$} \\
\hline & & & & & & $\sigma_{c}$ & $\mathbf{G}_{\mathbf{u}}$ & $\sigma_{1 f}$ & $\mathbf{G}_{\mathbf{u}}$ & $V_{p}$ & $\mathbf{G}_{\mathbf{u}}$ \\
\hline & & & días & $\begin{array}{c}\mathrm{Mg} / \\
\mathrm{m}^{3}\end{array}$ & $\%$ & $\mathrm{MPa}$ & $\mathrm{MPa}$ & $\mathrm{kPa}$ & $\mathrm{MPa}$ & $\mathrm{m} / \mathrm{s}$ & $\mathrm{MPa}$ \\
\hline \multirow{2}{*}{1} & $\mathbf{S}$ & 2 & 130 & $\begin{array}{l}1.38- \\
1.51\end{array}$ & $88-90$ & 1.3-1.8 & & - & - & 1966 & 1778 \\
\hline & C & 4 & & & $86-90$ & $0.7-1.6$ & - & - & 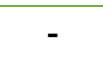 & - & - \\
\hline \multirow{3}{*}{2} & $\mathbf{S}$ & 2 & 145 & 1.68 & $87-91$ & $1.7-2.4$ & $\begin{array}{l}130- \\
243\end{array}$ & - & - & 1927 & 2079 \\
\hline & C & 2 & & 1.50 & $87-91$ & $1.4-1.8$ & $\begin{array}{l}67- \\
100\end{array}$ & - & - & 1911 & 1826 \\
\hline & $C^{*}$ & 3 & $256-468$ & $\begin{array}{l}1.48- \\
1.50\end{array}$ & $87-88$ & - & - & $\begin{array}{l}1795- \\
2063\end{array}$ & $\begin{array}{l}33- \\
57\end{array}$ & - & - \\
\hline \multirow{3}{*}{3} & $\mathbf{S}$ & 1 & & 1.55 & & 20 & 233 & - & - & 1942 & 1949 \\
\hline & C & 5 & & $\begin{array}{l}1.53- \\
1.54\end{array}$ & $91-92$ & $1.4-2.1$ & $\begin{array}{l}77- \\
103\end{array}$ & - & - & $\begin{array}{c}1852- \\
2089\end{array}$ & $\begin{array}{l}1749- \\
2226\end{array}$ \\
\hline & $C^{\star *}$ & 9 & & $\begin{array}{l}1.45- \\
1.56\end{array}$ & $91-93$ & - & - & $\begin{array}{l}1649- \\
2018\end{array}$ & $\begin{array}{l}33- \\
57\end{array}$ & $\begin{array}{c}1805- \\
1947\end{array}$ & - \\
\hline 4 & $\mathrm{~S}$ & 4 & $49-173$ & $\begin{array}{l}1.44- \\
1.54\end{array}$ & $92-99$ & $1.8-2.9$ & $\begin{array}{l}140- \\
213\end{array}$ & - & - & $\begin{array}{c}1980- \\
1985\end{array}$ & $\begin{array}{l}1904- \\
2012\end{array}$ \\
\hline 5 & $\mathbf{S}$ & 4 & $49-263$ & $\begin{array}{l}1.43- \\
1.55\end{array}$ & $91-92$ & $1.7-2.5$ & $\begin{array}{l}167- \\
420\end{array}$ & - & - & $\begin{array}{l}1990- \\
2115\end{array}$ & $\begin{array}{l}2046- \\
2192\end{array}$ \\
\hline
\end{tabular}

$\mathrm{S}$, probetas fabricadas al mismo tiempo que los bloques;

$\mathrm{C}$, testigos extraídos de los bloques (diámetro $=53.4 \mathrm{~mm}$ y relación altura/diámetro > 2);

$\mathrm{C}^{\star}$, testigos (diámetro $=36.6 \mathrm{~mm}$ y altura $=76.0 \mathrm{~mm}$ );

$\mathrm{C}^{* *}$, testigos (diámetro $=36.6 \mathrm{~mm}$ y altura $=81.8 \mathrm{~mm}$ ).

$\rho$, densidad aparente

w, humedad (saturada)

$\sigma_{3}$, presión de confinamiento aplicada (oscila desde 49 a $294 \mathrm{kPa}$ );

$\sigma_{1 f}$, tensión total vertical en rotura

$\mathrm{G}_{\mathrm{u}}$ (Triaxial $\left.\mathrm{CU}\right)$ : considerando un $10 \%$ de $\sigma_{1 \mathrm{ff}}$ en la curva de rotura del ensayo.

$\mathrm{G}_{u}\left(V_{\mathrm{p}}\right)$ : para muy pequeñas deformaciones, módulo dinámico.

La Figura 96 representa, en un diagrama de "cajas", valores medios y dispersión de los resultados incluidos en la Tabla 15. Los límites horizontales superior, intermedio e inferior de las cajas representan los cuartiles tercero (75\% de los datos), segundo (50\% de los datos o mediana) y primero ( $25 \%$ de los datos), respectivamente. Por lo que el $50 \%$ de los datos queda dentro de la "caja". Las líneas horizontales superior e inferior, más cortas, representan el valor máximo y mínimo de los datos, respectivamente. 
Los resultados de los ensayos de resistencia a compresión simple tienen un valor medio de $1.8 \mathrm{MPa}$, con el $50 \%$ de los datos entre 1.4 y $2.1 \mathrm{MPa}$ (Figura 96a). Así, los valores revelan un comportamiento esperable de una roca blanda (p.e. caliza o pizarra blanda de la Tabla 13). Por otro lado, los valores de velocidad de propagación de ondas de compresión son bastante homogéneos ( $\mathrm{CV}=4 \%$, Figura $96 \mathrm{~b}$ ) y coherentes con el tipo de roca blanda fabricada (Coates, 1970). Se puede establecer un valor representativo de 1970 $\mathrm{m} / \mathrm{s}$, quedando el $50 \%$ de los valores concentrados entre 1910 y $2040 \mathrm{~m} / \mathrm{s}$. A su vez, los valores de densidad y humedad de las muestras saturadas presentan gran homogeneidad (Figura 96c y Figura 96d) con valores representativos del geo-material de $1.50 \mathrm{Mg} / \mathrm{m} 3$ de densidad y $91 \%$ de humedad aparente.

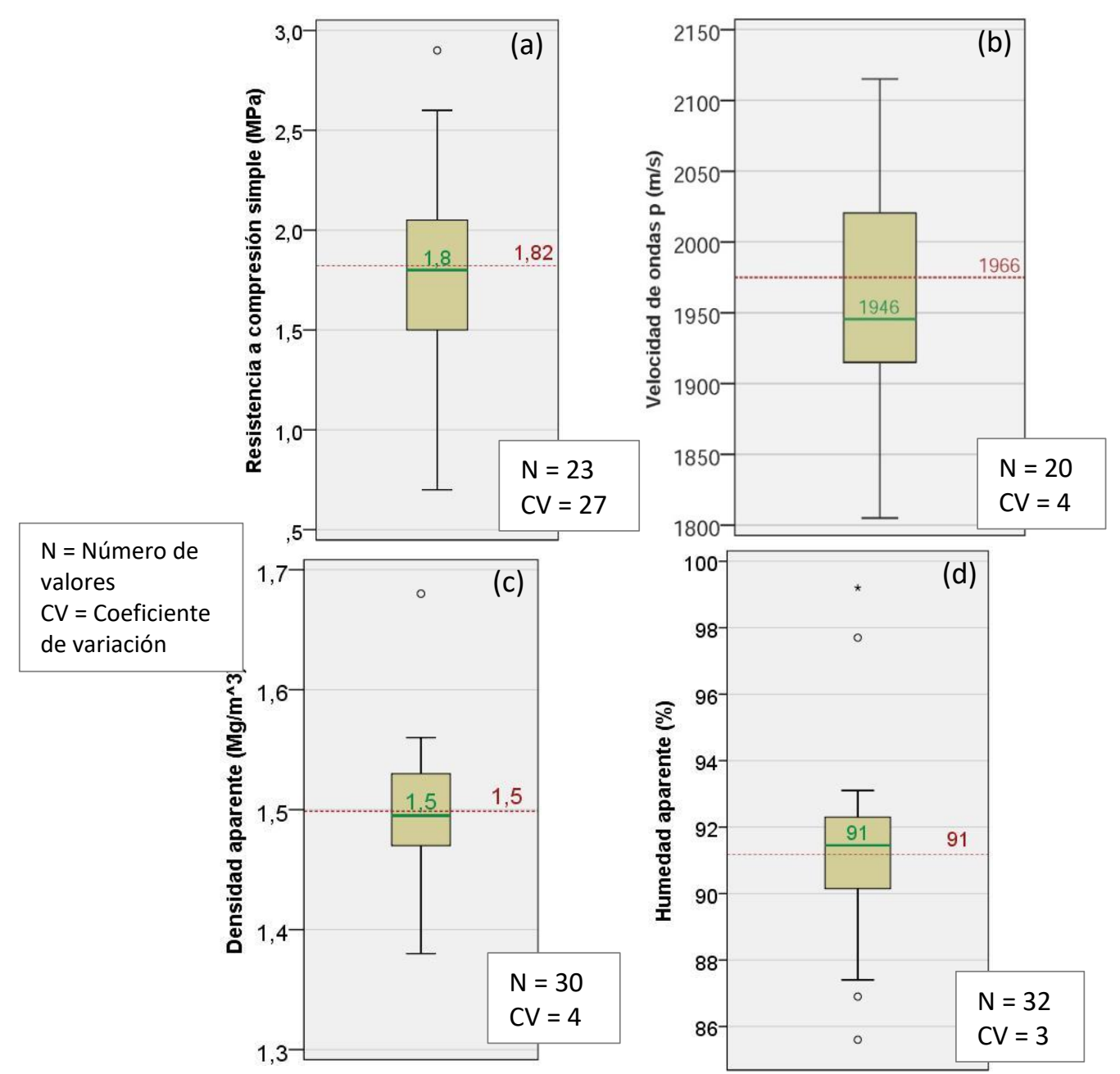

Figura 96. Diagramas de valores geotécnicos representativos del geo-material fabricado: a) Resistencia a compresión simple; b) Velocidad de ondas p; c) Densidad aparente; y d) Humedad de las muestras. 
La superficie de rotura de la Figura 97 permite apreciar muy claramente la homogeneidad y continuidad lograda en las muestras fabricadas.
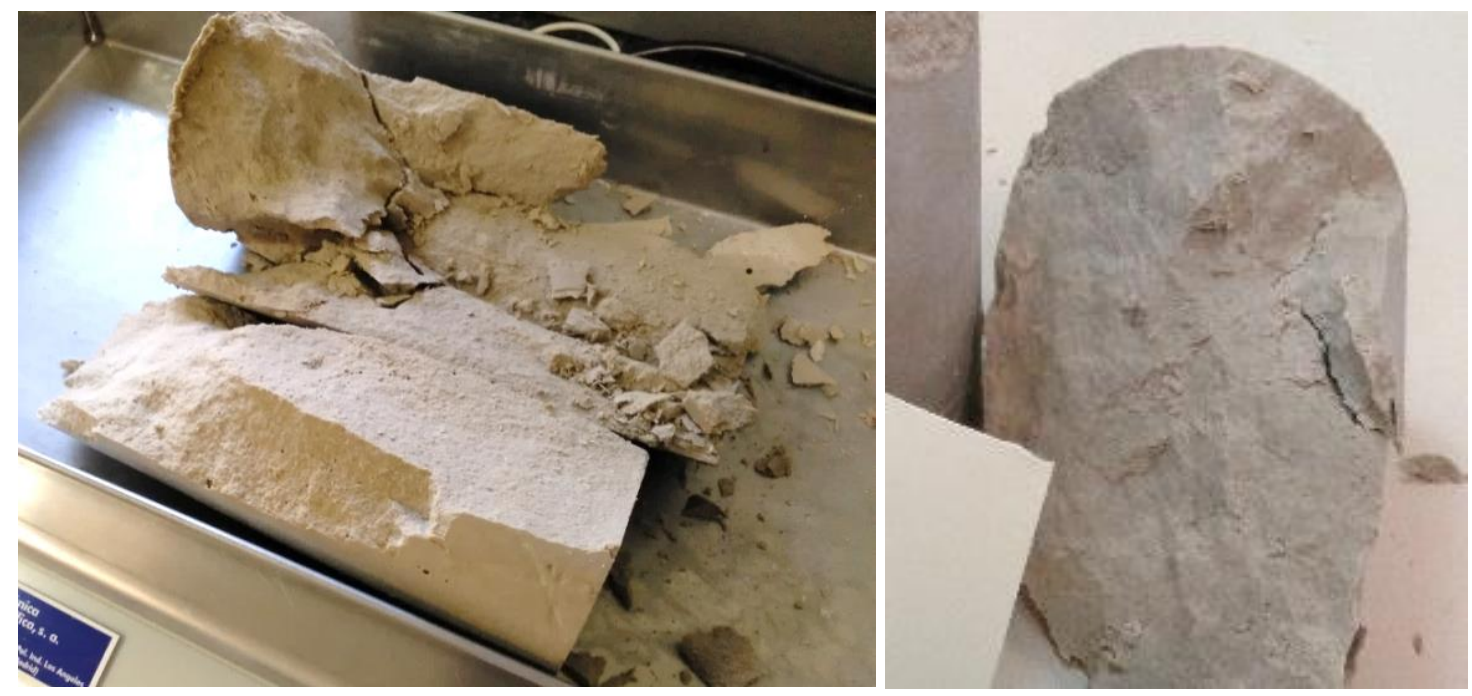

Figura 97. Aspecto interno y de la superficie de rotura de las muestras fabricadas.

También resulta interesante apreciar la forma de rotura experimentada en las probetas al finalizar los ensayos de compresión simple y triaxiales (Figura 98).
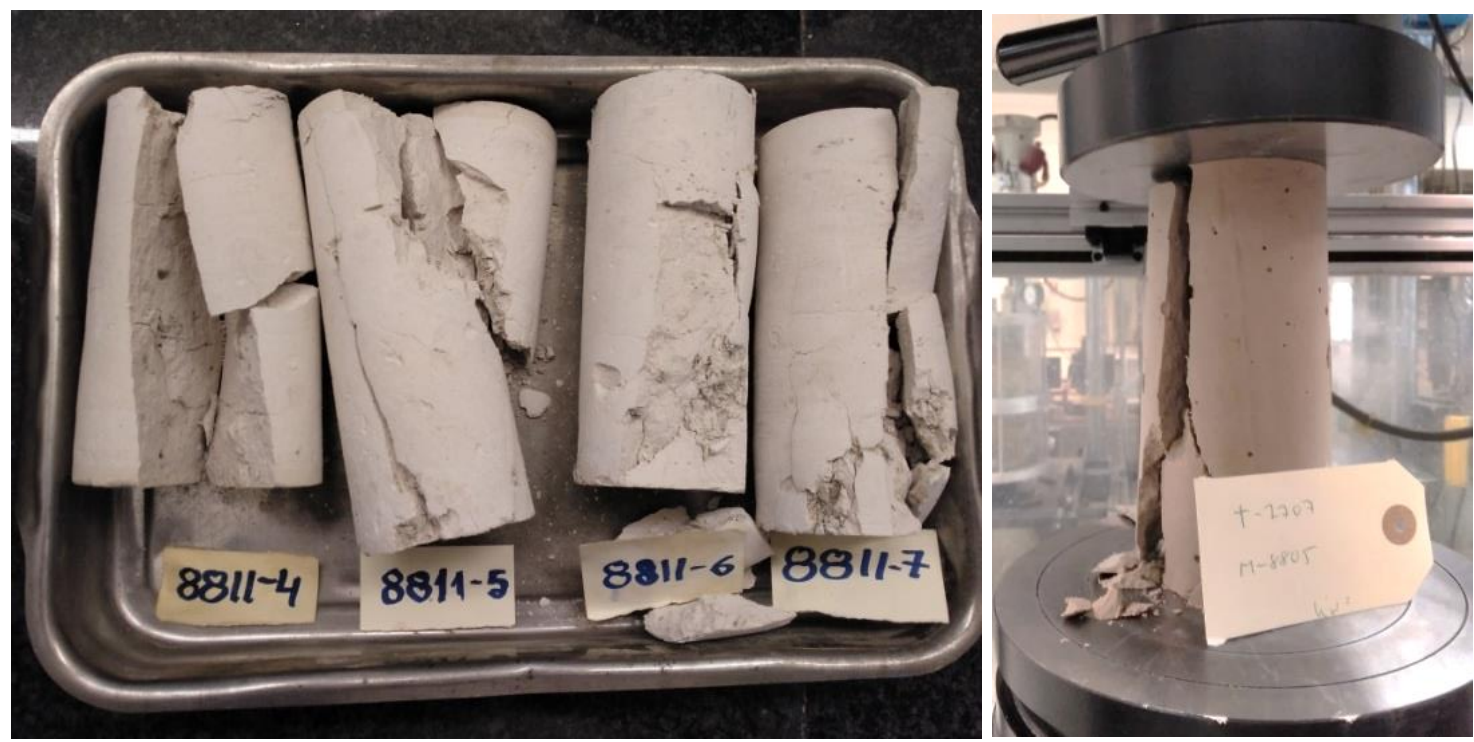

Figura 98. Aspecto de las probetas tras roturas en compresión simple y en triaxial. 
Se incluyen ejemplos de las curvas de rotura de los ensayos de compresión simple y triaxiales no drenados (Figura 99), que señalan un comportamiento en el límite dúctil-frágil, acorde al límite de resistencia entre un suelo duro y una roca blanda en que se encuentra el material creado.
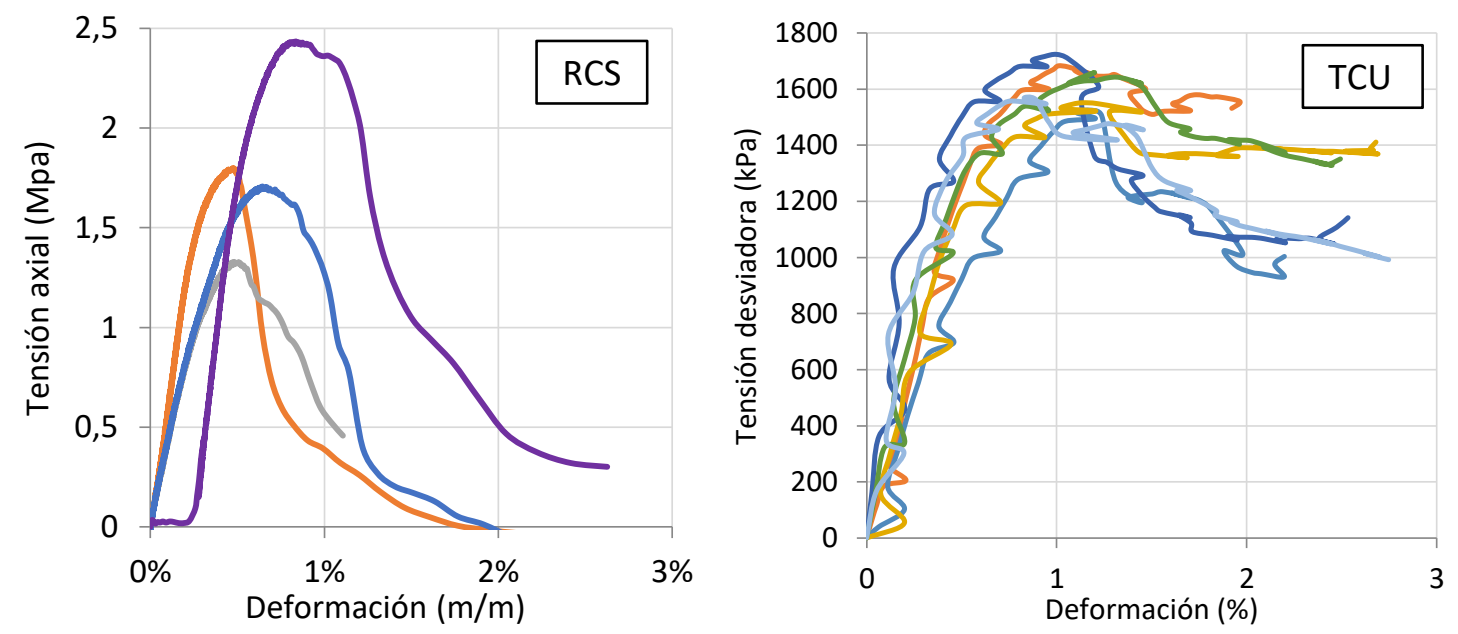

Figura 99. Ejemplo de curvas de rotura obtenidas en ensayos RCS y TCU.

Los módulos de corte no drenados $\left(G_{u}\right)$ obtenidos para los ensayos $\mathrm{TCU}$, tienen un rango bastante cerrado, al variar entre 0.03 y $0.06 \mathrm{GPa}$; siendo más disperso para los ensayos de resistencia a compresión simple, con valores entre 0.07 y $0.4 \mathrm{GPa}$, manifestando el efecto de otros factores al desaparecer el confinamiento. Estos valores pueden considerarse como próximos entre sí y, como cabría esperar, son inferiores a los que proporcionan los ensayos de velocidad de ondas longitudinales, variables entre 1.7 y $2.2 \mathrm{GPa}$.

La caracterización geotécnica llevada a cabo permite clasificar al material como una roca muy blanda, pudiendo verse representada en rocas presentes en las obras de ingeniería (sal, lutita, limolita, marga, yeso, arenisca, toba o carbón), donde se presentan valores habituales de UCS entre 1 y $5 \mathrm{MPa}$ (ISRM, 1981), y de velocidad de propagación entre 1400 y 3200 m/s (González de Vallejo et al., 2002). 


\subsection{PROCEDIMIENTO DE ENSAYO}

Las fases de procedimiento del ensayo son:

1) Retirar el agua hasta aproximadamente la profundidad del anclaje, durante al menos $48 \mathrm{~h}$, para facilitar el proceso de curado de la resina que se inyectará más adelante. Colocación de la estructura sobre la muestra fijando los laterales con una capa de cemento y arena de aproximadamente $2 \mathrm{~cm}$ de espesor. Se emplea una plomada para verificar el centro aproximado de la muestra y se nivela la estructura en el eje X e Y (Figura 100).
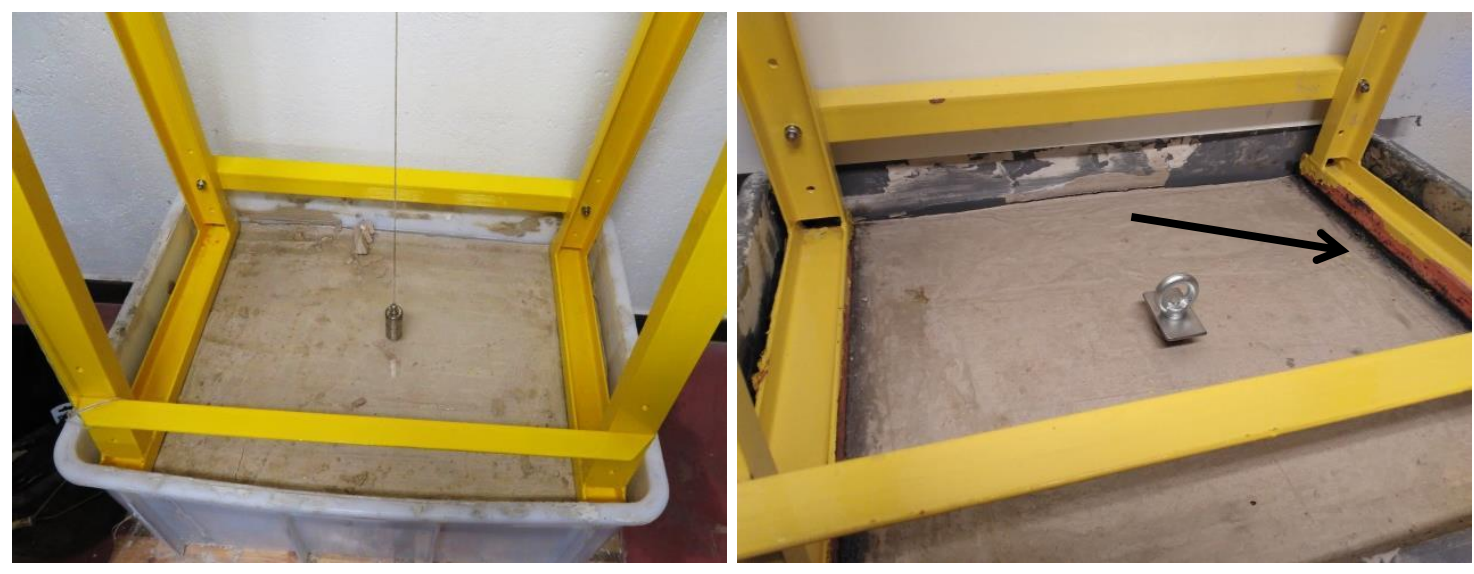

Figura 100. Detalle de la plomada y de la capa para nivelación.

2) Realizar la perforación de $12 \mathrm{~mm}$ de diámetro con taladro de rotación hasta la profundidad deseada (Figura 101).

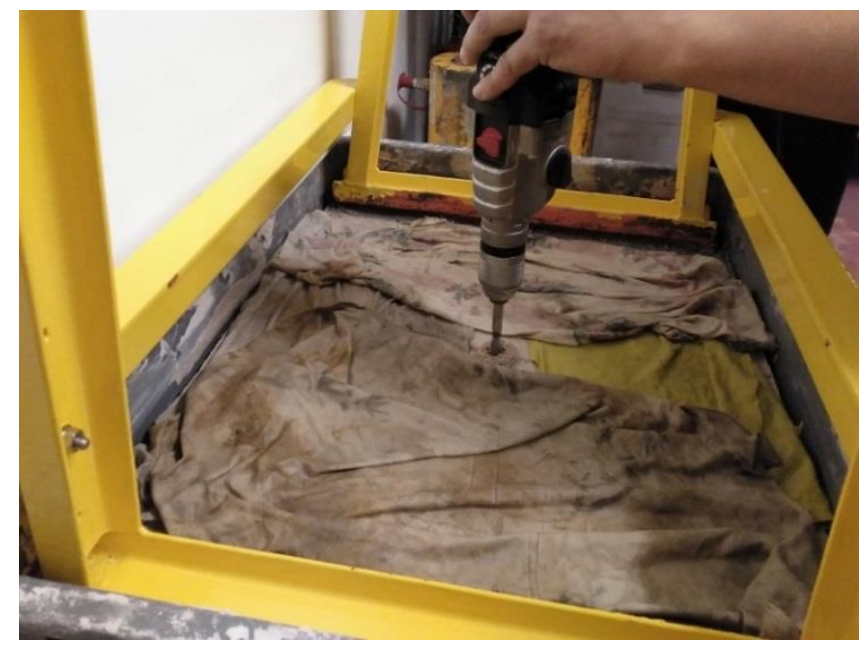

Figura 101. Perforación. 
Después, se seca y se limpia la perforación con aire a presión mediante una boquilla y con una escobilla específica (Figura 102).

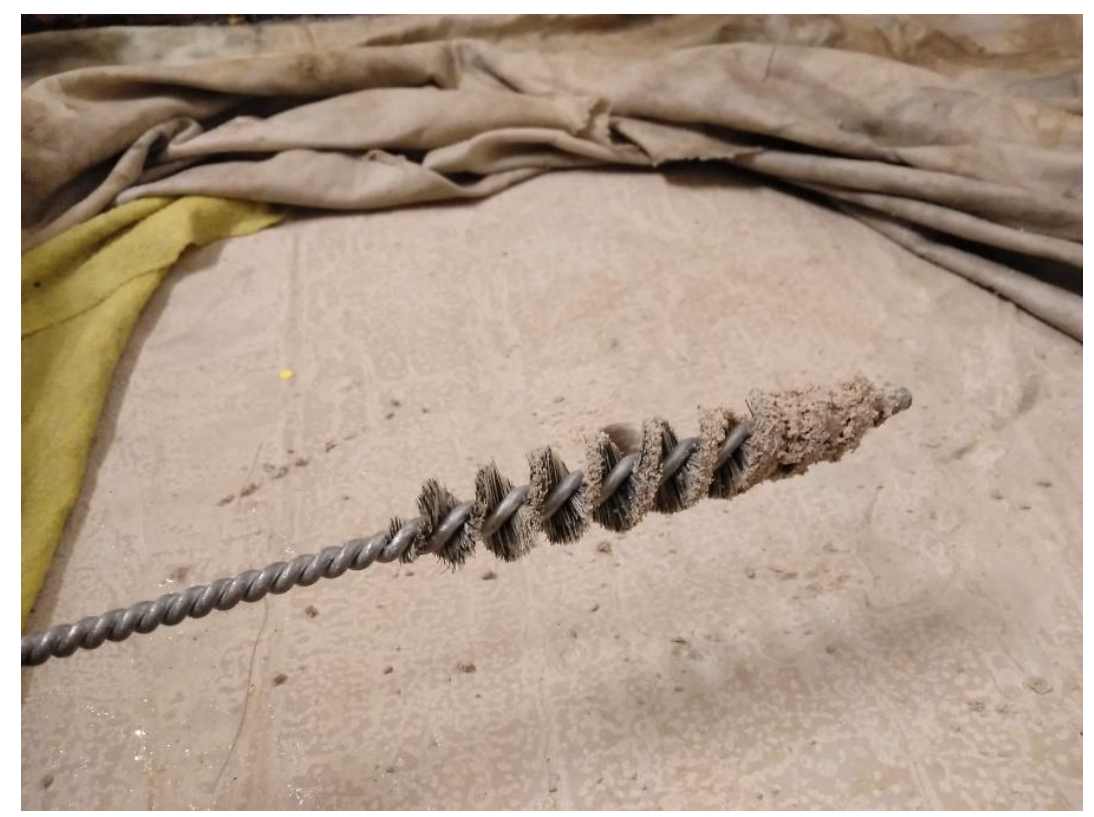

Figura 102. Ejemplo de limpieza con escobilla.

Posterior inyección de la resina de alta resistencia. Se introduce la varilla roscada de acero inoxidable de métrica $10 \mathrm{~mm}$, girándola para una mejor adherencia con la resina (Figura 103) y dejando que endurezca durante un tiempo mínimo de $48 \mathrm{~h}$.

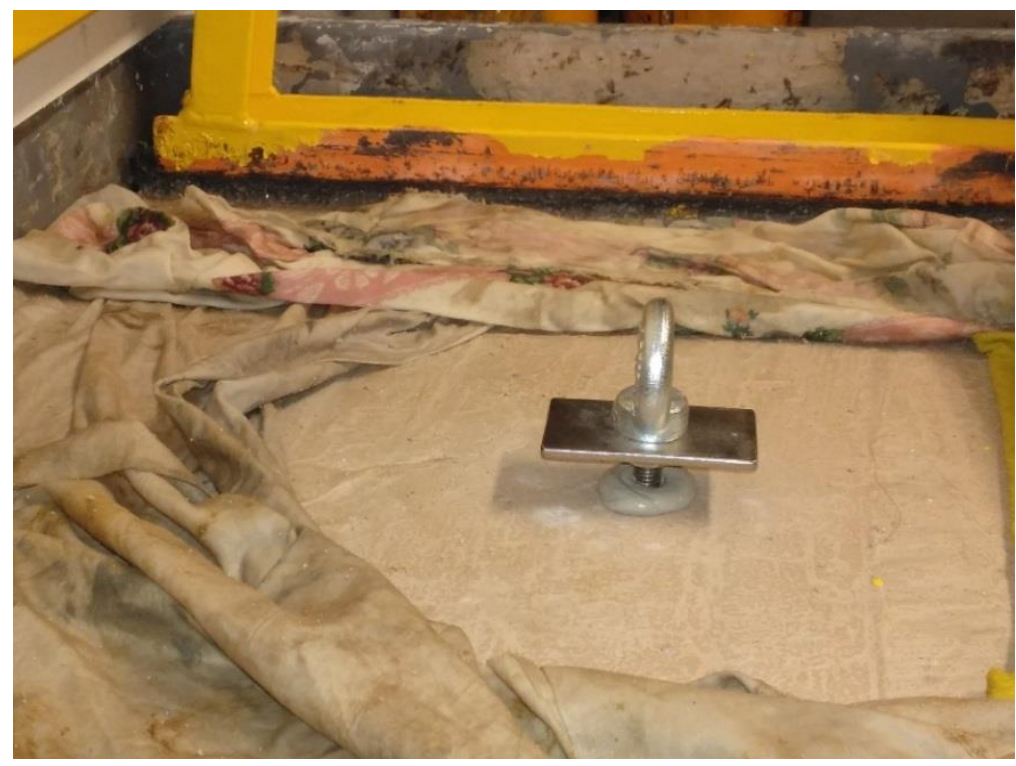

Figura 103. Colocación del anclaje. 
Cabe destacar a este respecto, que al inicio se han realizado pruebas con distintas resinas, observándose una adherencia completa del material base a la resina en la finalmente utilizada (véase Figura 104).

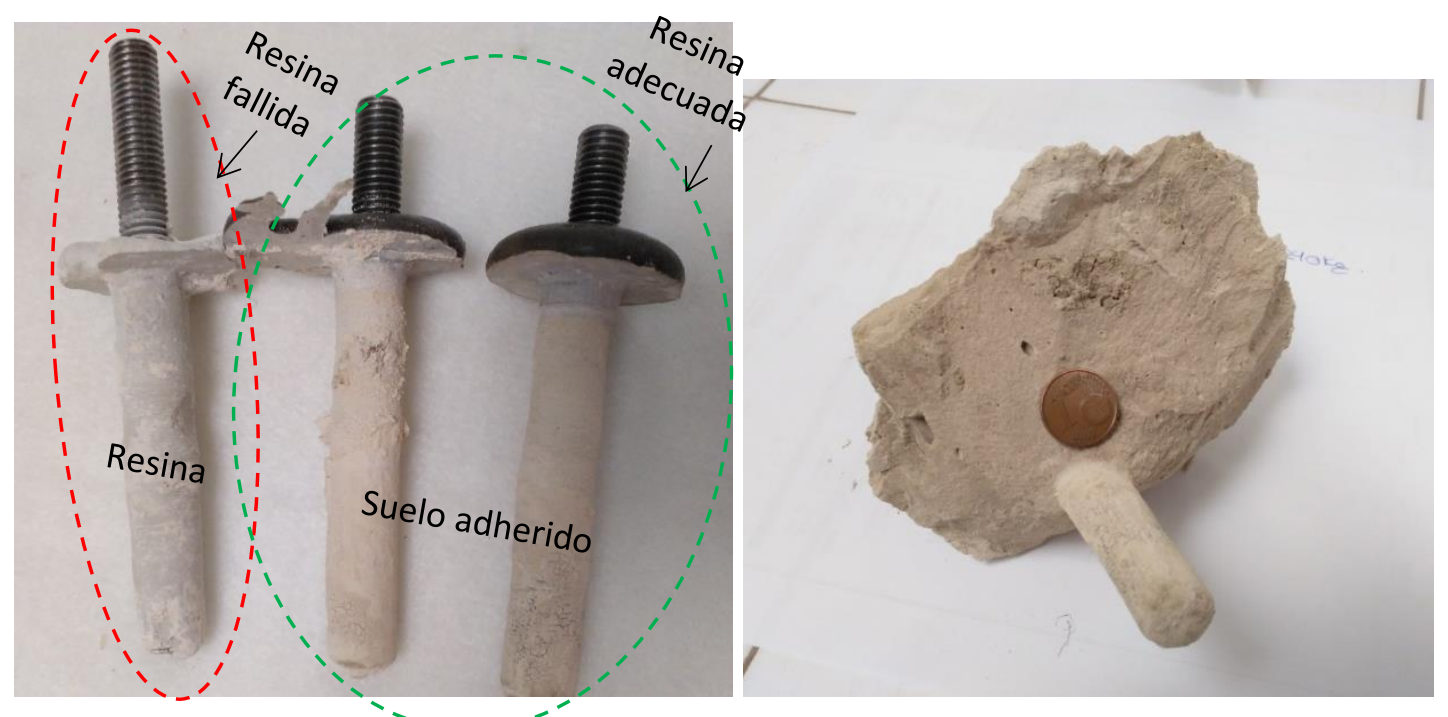

Figura 104. Detalle dè mēejóra de la adherencia del material base con diferentes resinas.

3) Se añade agua hasta cubrir ligeramente la muestra sin sobrepasar los perfiles metálicos colocados y fijados anteriormente con la misma resina del anclaje, en los puntos de registro de desplazamientos (Figura 105). Se coloca el cableado, la barra para las pesas y los comparadores, según el esquema de la Figura 85.

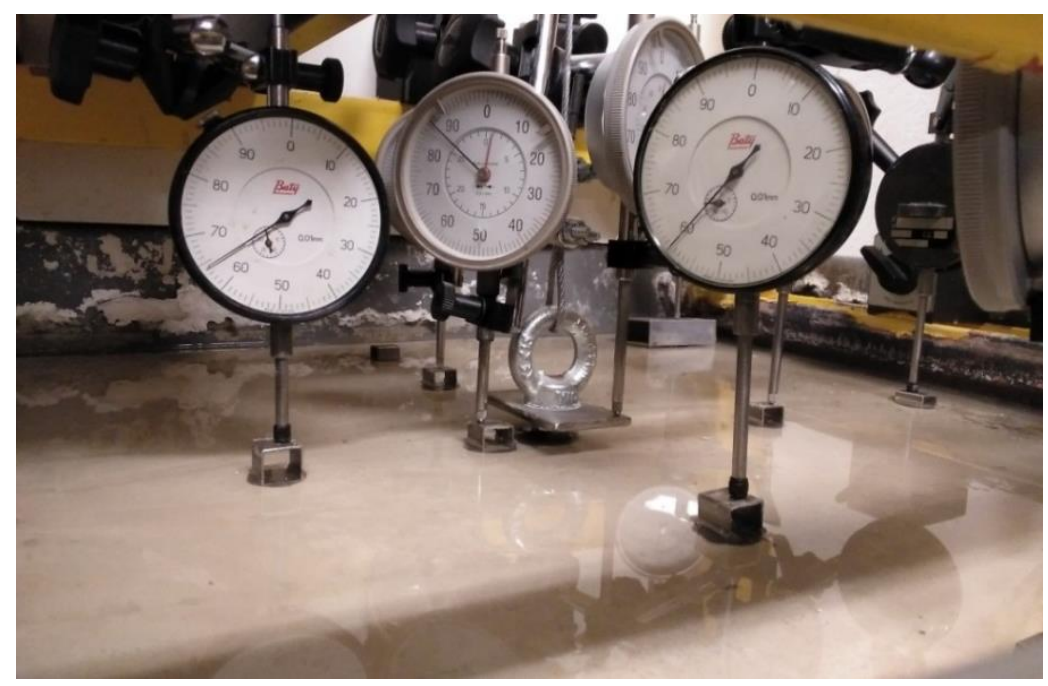

Figura 105. Detalle de comparadores y nivel de agua. 
4) Ensayo de fluencia. Antes de proceder a la primera puesta en carga del anclaje, se fija la lectura cero de los comparadores. Durante los ensayos se han aplicado al menos tres escalones de carga consecutivos, que generan una tensión de adherencia $\left(\tau_{a d h}\right)$ en el anclaje según un porcentaje estimado de la resistencia a compresión simple (15, 20 y 30 \%), previamente estimada en probetas. Cada escalón de carga se lleva a cabo añadiendo pesas adcuadamente hasta llegar a la carga total del escalón. Durante los escalones se mantiene la carga constante en periodos de tiempo variables en función de la evolución de los movimientos, incrementando la carga cuando se aprecia una estabilización de dichos desplazamientos (Figura 106). Por último, se carga el anclaje hasta la rotura.

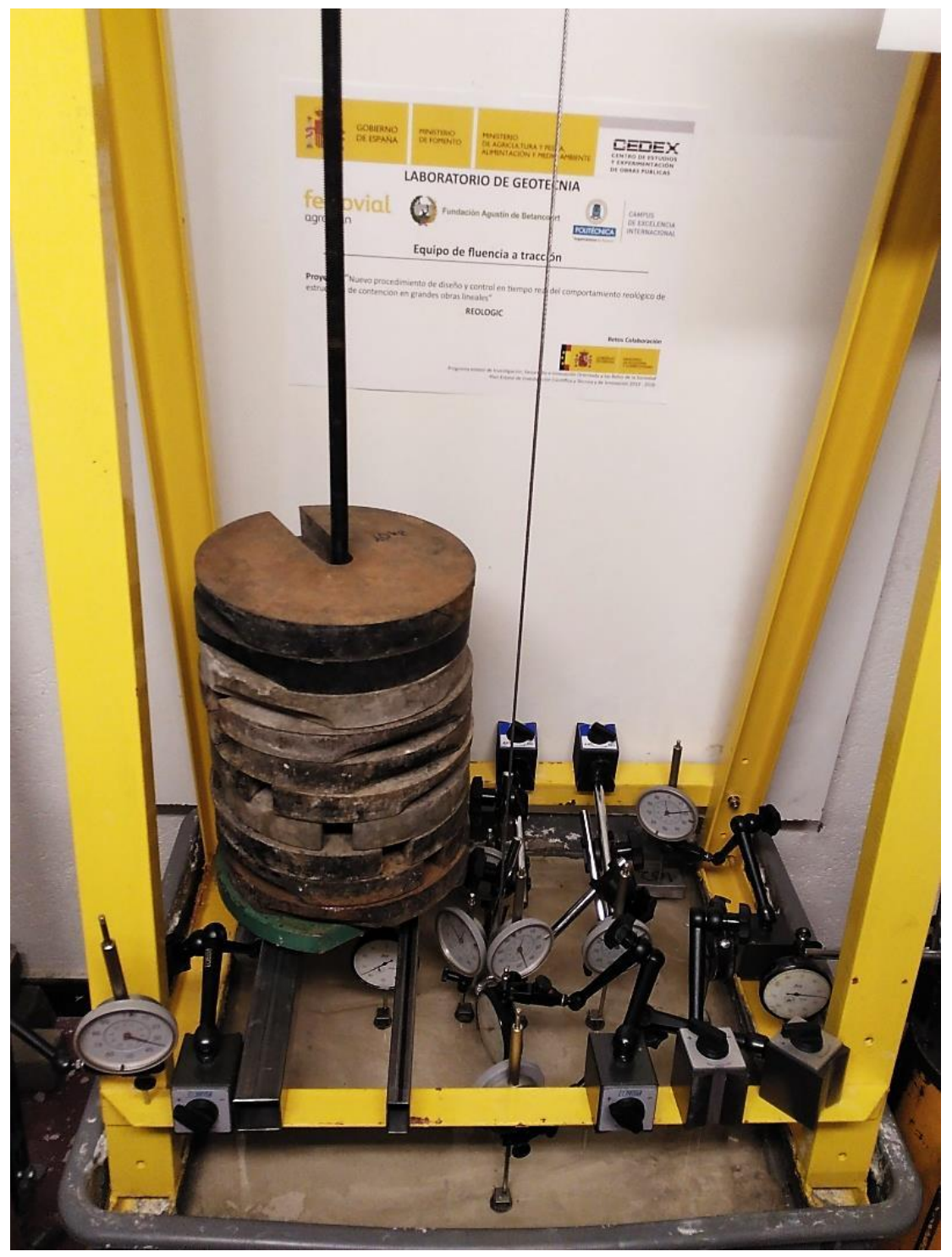

Figura 106. Ejemplo de ensayo de fluencia en funcionamiento. 
Aprovechando las características de movilidad del equipo y las grandes dimensiones de la muestra se han hecho ensayos de arrancamiento instantáneo, en puntos distanciados del centro o en la otra cara de los bloques, al finalizar los ensayos de fluencia.

\subsection{RESULTADOS DE ENSAYOS Y ANÁLISIS}

\subsubsection{Resultados}

Siguiendo el procedimiento descrito en el apartado anterior, se han llevado a cabo los ensayos de fluencia. Con el objetivo de sintetizar la cantidad de datos registrados, se han representado los bloques 2, 4 y 5 (Tabla 15) en la Figura 107. Se presentan los desplazamientos con el tiempo de los puntos de las coronas circulares indicadas en el esquema de la Figura 85 , donde se obtienen movimientos similares, así como el desplazamiento de los puntos 5 (central) y 6 (anclaje) de manera independiente.

Durante el ensayo, se fueron elevando los escalones de carga con pesas en todos los bloques ensayados, para generar una tensión de corte $\left(\tau_{a d h}\right)$ creciente en el contorno del anclaje $(75 \mathrm{~mm}$ aproximadamente de longitud anclada y $12 \mathrm{~mm}$ de diámetro de perforación). El primer escalón de carga (carga instantánea) empezó generando una $\tau_{\text {adh }}$ del $15 \%$ de la resistencia a compresión simple $\left(\sigma_{c}\right)$ obtenida en las probetas (equivalentes a $70 \mathrm{~kg}$ de pesas). Los siguientes escalones subieron, aproximadamente, al 20\% y $30 \%$ de $\sigma_{c}$, es decir 110 y $150 \mathrm{~kg}$ de pesas; y fueron aplicados cuando se observó la estabilización con el tiempo de la deformación medida en los comparadores. En el bloque 5, se añadieron además dos escalones de carga más (de 190 y $230 \mathrm{~kg}$ ) hasta una $\tau_{a d h}$ próxima al $50 \%$ de la resistencia del ensayo de compresión simple.

En los resultados mostrados en la Figura 107, se observa que la deformación crece con el tiempo de aplicación de la carga, de manera que: 
- En el primer escalón de carga, la respuesta instantánea de la deformación vertical en superficie del bloque sigue, en todos los casos, una tendencia decreciente a medida que nos alejamos del anclaje (ver esquema de la Figura 107e).

- La respuesta deformacional vertical en superficie con el tiempo, en cada bloque, se ajusta a la mostrada en la Figura 107d. Se observa que en el contorno inmediato del anclaje la deformación esta contenida, creciendo con la distancia hasta alcanzar un máximo de deformación, a partir del cual decae la zona de influencia y vuelve a decrecer el desplazamiento. Este fenómeno, observable en fluencia, es previsiblemente debido a la condición de contorno impermeable generada por la zona de inyección de la resina del anclaje, que genera un flujo de agua con salida a la superficie.

En todos los casos de ensayo, la corona B de medición está lo suficientemente lejana para correspóndele los puntos de menor deformación ante carga instantánea y durante la fluencia. Por su parte, en correspondencia con la discusión de la Figura 107e y Figura 107d, el punto central medido en cada bloque es, en todos los casos, el de mayor deformación instantánea; mientras que a los puntos de la corona $A$, les corresponde la máxima deformación al final de la fluencia, en todos los bloques ensayados.

Debe mencionarse también que se aprecian movimientos diferidos entre el punto central y el del anclaje en todos los ensayos, que además evoluciona de diferente manera a medida que se producen los efectos de la fluencia. Se deduce que, cuando el desplazamiento del anclaje supera el del centro del bloque ensayado, se están produciendo deslizamientos en el contacto resinaroca. Este hecho sucede claramente en el bloque 4 ensayado, por tanto es esperable que el modo de rotura (cuando se produzca el arrancamiento) sea de tipo mixto. En contraste, para el ensayo de fluencia del bloque 2, en el último escalón de carga se alcanza el mismo valor de desplazamiento entre el punto central y el del anclaje, y mantiene la misma evolución hasta el final del ensayo; por tanto, cuando se igualan los movimientos, es el anclaje el que arrastra 
completamente bloque sin deslizamientos relativos (esperablemente se producirá una rotura completa tipo “rock break-out"). Por último, en el bloque 5, durante el ensayo de fluencia la deformación del anclaje se mantiene por debajo de la del punto central del bloque y por tanto, no será hasta la fase de la rotura (a partir de cuando se igualen dichos desplazamientos), cuando se verá si el anclaje desliza (rotura mixta) o arrastra al bloque en su movimiento ("rock break-out"). En nuestro caso, para el bloque 5 se obtuvo en arrancamiento una rotura de tipo mixto.
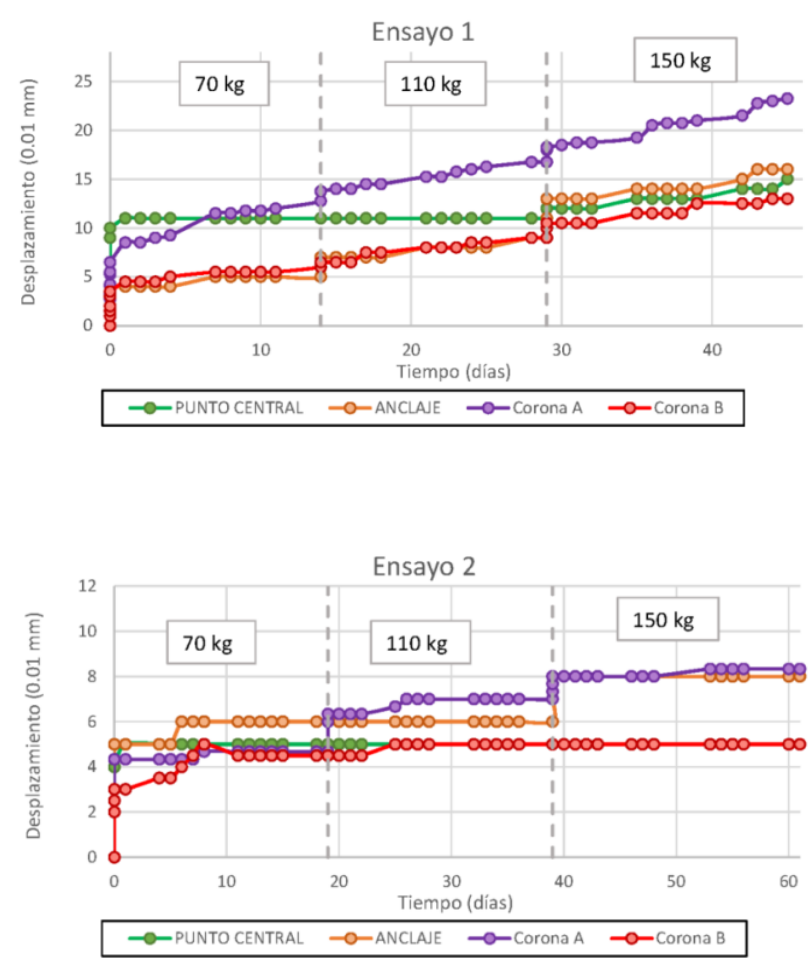

d)
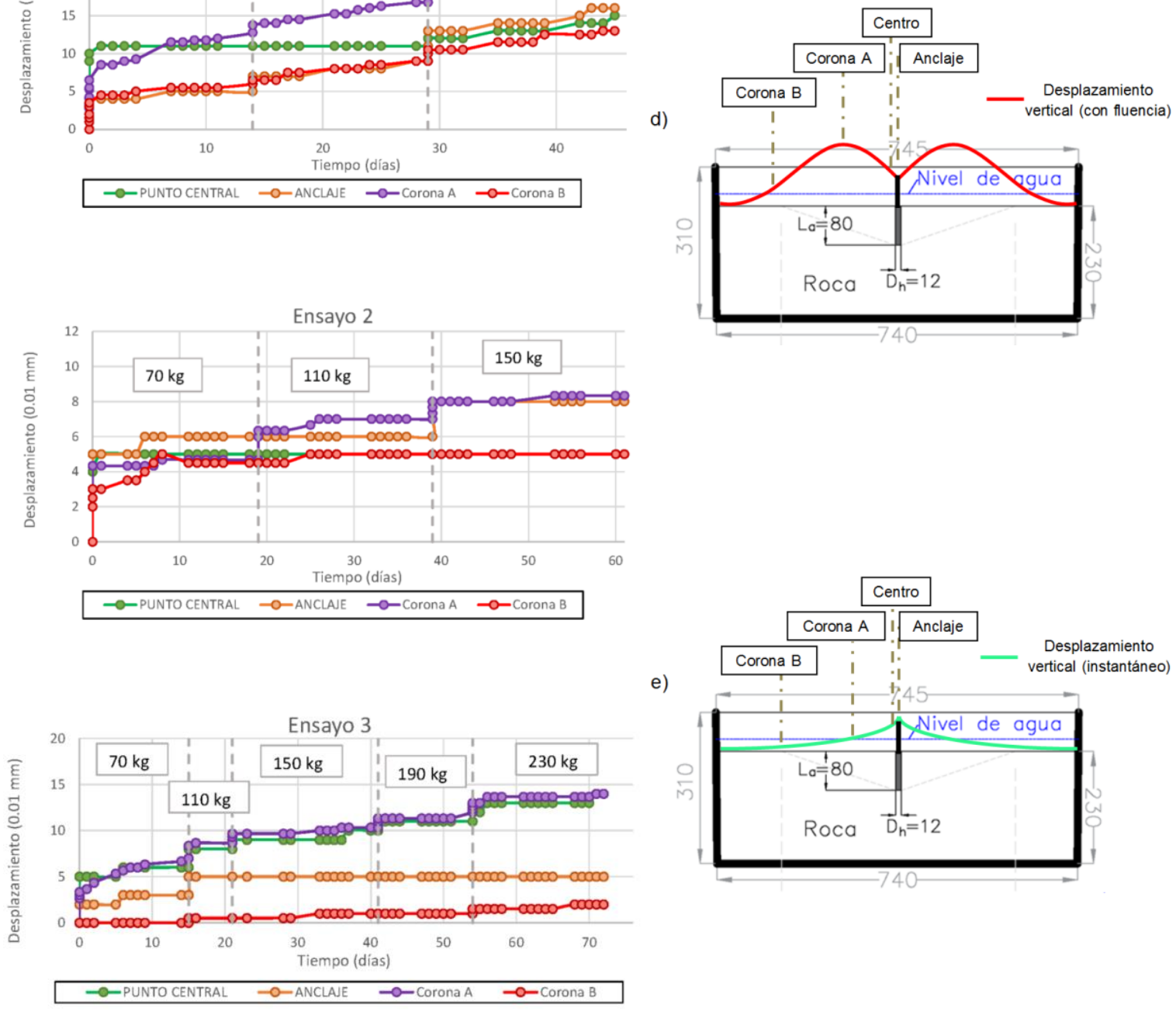

e)

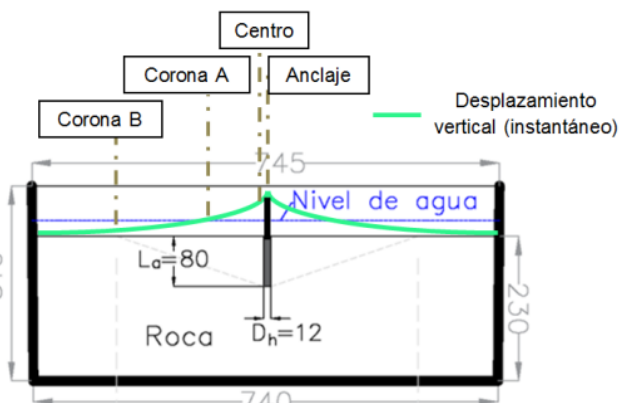

Figura 107. Resultados de los tres ensayos de fluencia efectuados: a) Ensayo en Bloque 2; b) Ensayo en Bloque 4; c) Ensayo en Bloque 5; d) Deformación vertical en superficie (con fluencia); e) Deformación vertical instantánea en superficie. 
Las resistencias al arrancamiento obtenidas al final de los ensayos y en ensayos de arrancamiento adicionales (realizados sobre la superficie inferior del bloque, al darle la vuelta dentro de la cubeta) han variado entre 980 y 1115 $\mathrm{kPa}$ (correspondientes a pesas de entre 280 y $320 \mathrm{~kg}$ ), valores acordes a los límites esperables entre una arcilla de consistencia rígida y una roca blanda, como refleja la Tabla 13. Estos resultados permiten validar el ensayo a resistencia última, ya que dichos valores deben compararse con los que corresponderían a la resistencia al corte para un ensayo no drenado. En este caso, los resultados de arrancamiento del ensayo coinciden bien con los mostrados (Figura 96a) para los ensayos de resistencia a compresión simple $\left(\sigma_{c}=1400-2100 \mathrm{kPa}\right)$, desde los que se puede deducir un valor de resistencia al corte sin drenaje $\left(S_{u}=\sigma_{c} / 2\right)$ entre 700 y $1050 \mathrm{kPa}$.

En la Figura 108 se incluyen fotografías de los dos tipos de rotura principales obtenidos en los experimentos.
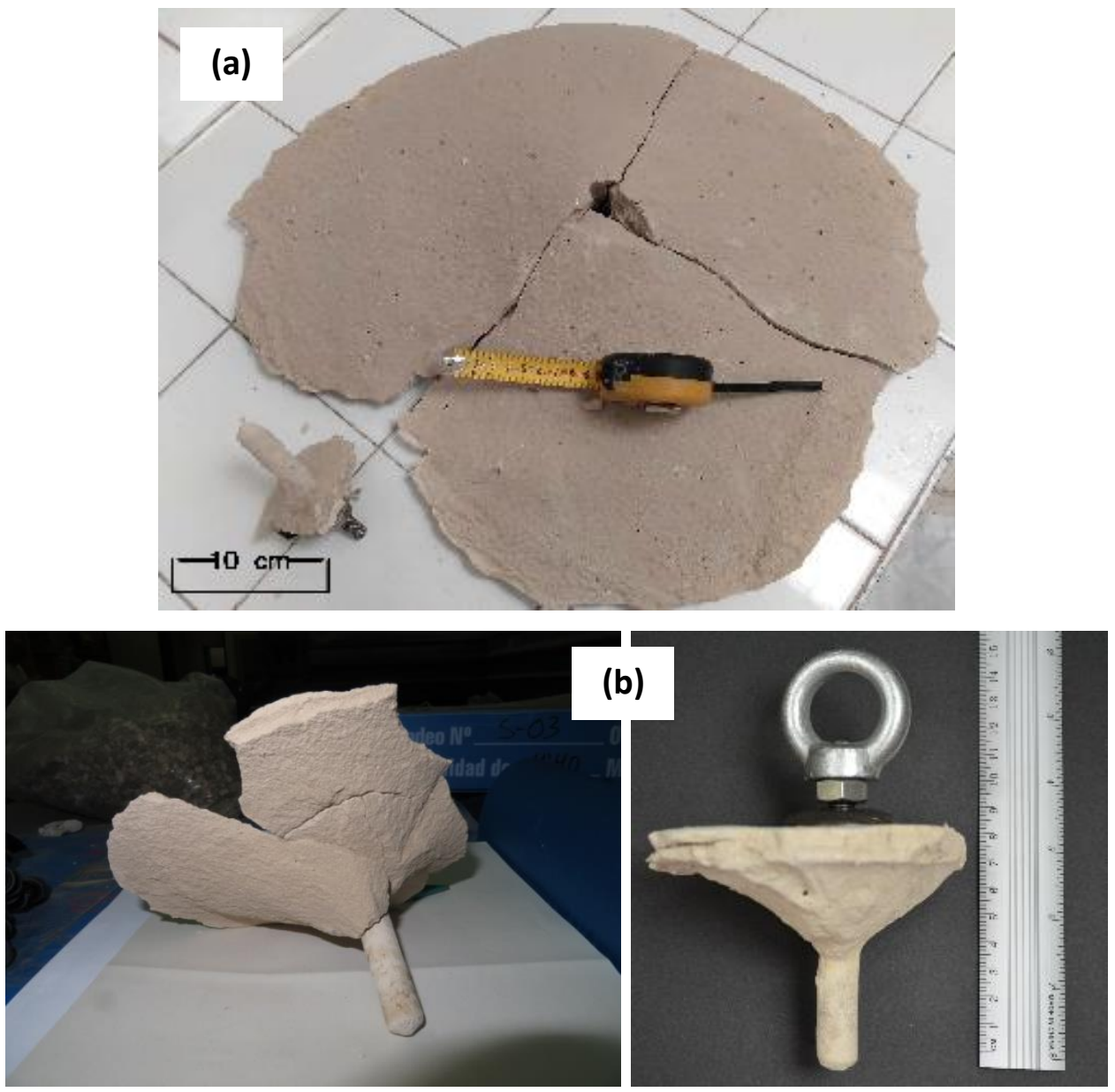

Figura 108. Ejemplos de tipos de roturas obtenidas. 
La rotura mostrada en la Figura 108a muestra el tipo de rotura "rock break-out", que desarrolla completamente el cono de rotura desde la base del vástago de anclaje (Bloque 2); por su parte, en la Figura 108b se aprecia el tipo de rotura mixta (partial adhesive-rock bond failure of the anchor) donde hay un deslizamiento parcial del anclaje con el terreno y un cono superior de rotura (Bloques 4 y 5 ).

\subsubsection{Modelo analítico para el desplazamiento instantáneo}

Los resultados del ensayo permiten estudiar la evolución del anclaje y de los puntos de la superificie del terreno con el tiempo, de manera que puedan obtenerse parámetros de ajuste de los modelos reológicos empíricos y matemáticos necesarios para evaluar problemas aplicados. No obstante, con el objetivo de interpretar adecuadamente el ensayo, se deben remarcar dos aspectos bien conocidos en este tipo de ensayos: la aplicación de la carga por etapas durante el ensayo y por tanto reducidas respecto a la carga última (que implica, por tanto, módulos instantáneos en pequeñas deformaciones) y el efecto escala asociado al tamaño de terreno involucrado (que viene definido principalmente por la longitud de anclaje).

A este respecto y para ayudar a la correcta interpretación del ensayo desarrollado, se plantea la utilización de la formulación analítica de Mindlin (1936) para hallar el desplazamiento instantáneo debido a una fuerza $(T)$ en el interior de un medio semi-infinito. Para estas condiciones de contorno, Justo (1993) propuso la solución analítica producida por una presión constante sobre un área circular, pudiendo obtenerse el movimiento instantáneo del bulbo $\left(u_{a}\right)$ dependiendo del diámetro del anclaje $\left(D_{h}\right)$, de un coeficiente de influencia $\left(I_{j}\right)$ y de las propiedades elásticas $(v, G)$, de la siguiente manera:

$$
u_{a}=\frac{T}{2 \pi D_{h}^{2} G(1-v)} I_{j}
$$

El coeficiente de influencia $\left(I_{j}\right)$ depende de la longitud anclada, del diámetro del anclaje y del módulo de Poisson. De esta manera, a partir de los 
resultados monitorizados en el ensayo para el anclaje, se puede obtener los valores del módulo de elasticidad transversal a corto plazo $\left(G_{u}\right)$, correspondientes a un módulo de Poisson $v_{u}=0,5$.

La Figura 109 muestra los resultados obtenidos aplicando la formulación analítica al equipo desarrollado. En esta figura también se incluyen los módulos de corte no drenados obtenidos a partir de los diferentes ensayos efectuados (RCS, TCU o Vp). La variabilidad del módulo de corte observada, dependiendo del ensayo considerado, es una verificación empírica de los dos efectos indicados para la interpretación adecuada de las deformaciones instantáneas obtenidas en el ensayo: mayor rigidez en pequeñas deformaciones y efecto escala. Por un lado, para las cargas de $70 \mathrm{~kg}$ (correspondientes a un $15 \%$ de la resistencia a compresión simple), se manifiesta una deformabilidad en pequeñas magnitudes más comparables con los ensayos de velocidad de ondas (Vp) que con los de resistencia (RCS, TCU). Por otro lado, la manifestación del efecto escala debido a que se han empleado probetas de 13 a $20 \mathrm{~cm}$ de largo para los ensayos de velocidad de ondas, mientras que en el ensayo propuesto la longitud del anclaje es solo de $7,5 \mathrm{~cm}$.

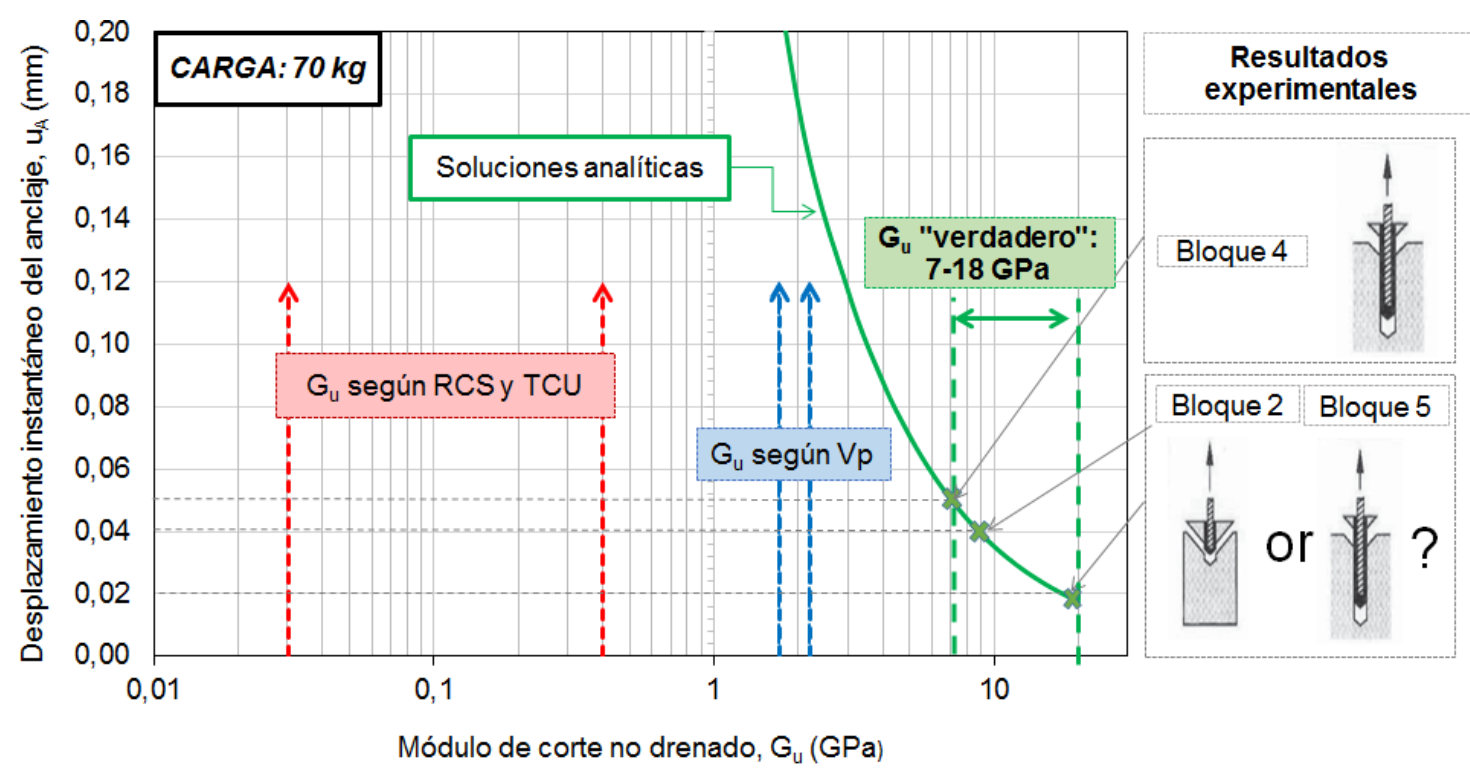

Figura 109. Variación del desplazamiento instantáneo en función del módulo de corte. 
Por último, se muestra que para este primer escalón de carga de $70 \mathrm{~kg}$, el bloque 4 ya muestra un mayor desplazamiento del anclaje y por tanto es de prever una rotura tipo mixta, mientras que los bloques 2 y 5 aún no se han igualado los desplazamientos del anclaje con los del punto central y no es posible predecir el tipo de rotura.

\subsubsection{Discusión}

Gracias a la experiencia adquirida durante la puesta en marcha del equipo propuesto y el ensayo de las tres muestras, se puede establecer lo siguiente:

- El ensayo permite medir los desplazamientos bajo carga constante durante largos periodos de tiempo (46, 61 y 75 días en este caso, respectivamente) en un anclaje embebido en una roca blanda.

- Se ha diseñado un equipo sencillo, al ser exclusivamente mecánico, por lo tanto, muy adecuado para el estudio de deformaciones a largo plazo bajo carga constante. De este modo, se permite el registro de datos sin necesidad de manipulación durante el propio ensayo, y con el periodo de tiempo que se desee, además de mantener la humedad constante en las muestras al quedar estas sumergidas. Las características mecánicas y geométricas del equipo están basadas en investigaciones teóricas y experimentales previas (GarcíaWolfrum, 2005). Se ha definido el procedimiento de ensayo a seguir y se ha constatado su funcionamiento mediante las tres pruebas realizadas.

- Los resultados de los ensayos garantizan su reproducibilidad, salvando las posibles diferencias en cuanto a resistencia y homogeneidad se refiere, en bloques de grandes dimensiones fabricados por el usuario, así como, garantizándose una adecuada precisión de registro de los desplazamientos. 
- Los resultados obtenidos permiten interpretar adecuadamente la diferente respuesta deformacional del terreno ante carga instantánea y en fluencia. De manera que los desplazamientos en superficie tienen una tendencia decreciente con la distancia ante carga instantánea; y obteniendo una evolución con la misma, en forma de campana con el tiempo.

- Se deduce que, cuando el desplazamiento del anclaje supera el del centro del bloque ensayado, se estan produciendo deslizamientos en el contacto resina-roca. En este caso es esperable que el modo de rotura, en arrancamiento, sea de tipo mixto.

- Los tipos de rotura observados durante los ensayos de arrancamiento (rock break-out y mixed) concuerdan con las formas experimentales y teóricas obtenidas por otros autores, además de proporcionar valores de resistencia al arrancamiento $\left(\tau_{\text {adh }}=980\right.$ y $1115 \mathrm{kPa}$ ) que concuerdan con los resultados obtenidos en ensayos de resistencia a compresión simple a corto plazo $\left(S_{u}=700\right.$ y 1050 $\mathrm{kPa})$.

- Los resultados obtenidos ponen de manifiesto los dos efectos que deben considerarse para la interpretación adecuada de las deformaciones instantáneas obtenidas en el ensayo: la mayor rigidez en pequeñas deformaciones y el efecto escala.

\subsection{PRESCRIPCIONES RESPECTO A LA MONITORIZACIÓN}

\subsubsection{Introducción}

El empleo de ensayos como el presentado en los capítulos previos puede resultar muy útil a la hora de estimar la influencia del fenómeno de fluencia en el terreno, con las condiciones de contorno que intervienen. Gracias a la incorporación de unos parámetros adecuados en los modelos de predicción, tanto analítico como numérico, se puede disponer de un modelo de 
pronóstico de las deformaciones en la pantalla y en el anclaje, así como, de la pérdida de carga en el mismo durante la vída útil de la obra.

Disponer de este tipo de modelos resulta muy útil durante la fase de proyecto, permitiendo, por ejemplo, ajustar los coeficientes de fluencia que habitualmente resultan muy elevados. No obstante, el control en obra durante la vida útil de la pantalla resulta muy aconsejable. La retroalimentación entre el control o monitorización en obra y los modelos predictivos, que tienen su origen en soluciones teóricas y ensayos en laboratorio, es muy recomendable. Ello permitirá un ajuste en tiempo real del modelo de deformaciones y pérdidas de carga, aportando nuevas conclusiones que sirvan para crear un modelo de predicción más representativo de los condicionantes propios de la realidad de la obra.

\subsubsection{Instrumentación en el caso de Raith Junction}

La obra objeto de estudio presentada en el apartado 3.3.2. y empleada para la simulación teórica y numérica de los modelos cuenta además con una monitorización en tiempo real. La instrumentación llevada a cabo por FERROVIAL - AGROMÁN consiste en sensores para control de la deformación de las pantallas (arrays) y dispositivos para controlar la carga de tesado de los anclajes (células de carga). Los perfiles instrumentados dentro del tramo de pantalla estudiado, junto con un esquema del estado definitivo de la monitorización en obra, se pueden consultar en la Figura 110. Se cuenta con las siguientes unidades: seis células de carga instaladas en las cabezas de los anclajes permanentes, de las cuales tres han quedado midiendo en tiempo real; $y$ treinta sensores de tipo SAA (Shape Accelerometer Array) instalados, de los cuales se encuentran operativos únicamente catorce. Cabe destacar que en el presente caso no se ha podido disponer de algunas de estas medidas hasta más de dos años después de la instalación de los anclajes. Este hecho permitirá resaltar algunas ideas generales a tener en cuenta para instalaciones futuras, pero dificulta el contraste entre los resultados estimados por los modelos y los obtenidos con la monitorización, en este caso. 


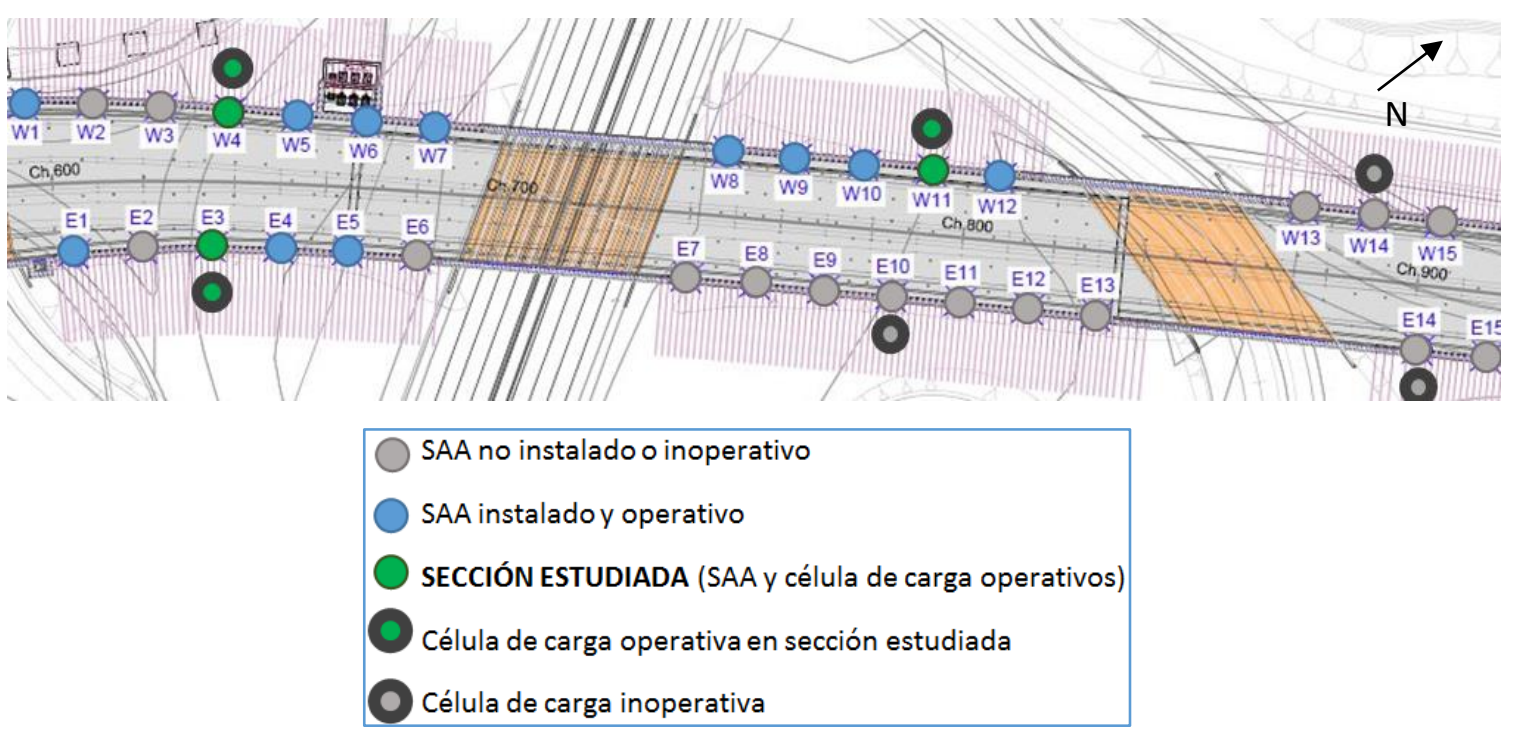

Figura 110. Vista en planta del tramo de estudio y secciones instrumentadas. (Cortesía de Ferrovial Agromán y elaboración propia)

Las células de carga están basadas en la tecnología de cuerda vibrante, cuya transmisión de frecuencias frente al voltaje elimina el ruido y los problemas de interferencias, aumentando la fiabilidad de las lecturas, en mayor medida cuando se trata de monitorización a largo plazo. Deben ser instaladas durante el proceso de puesta en carga, donde la presión aplicada debe ser comprobada con un lector manual. A partir de ese momento las células mandarán las lecturas automáticamente a un registrador de datos. En la Figura 111 se pueden apreciar las células de carga; durante su instalación y en su estado definitivo en la obra.
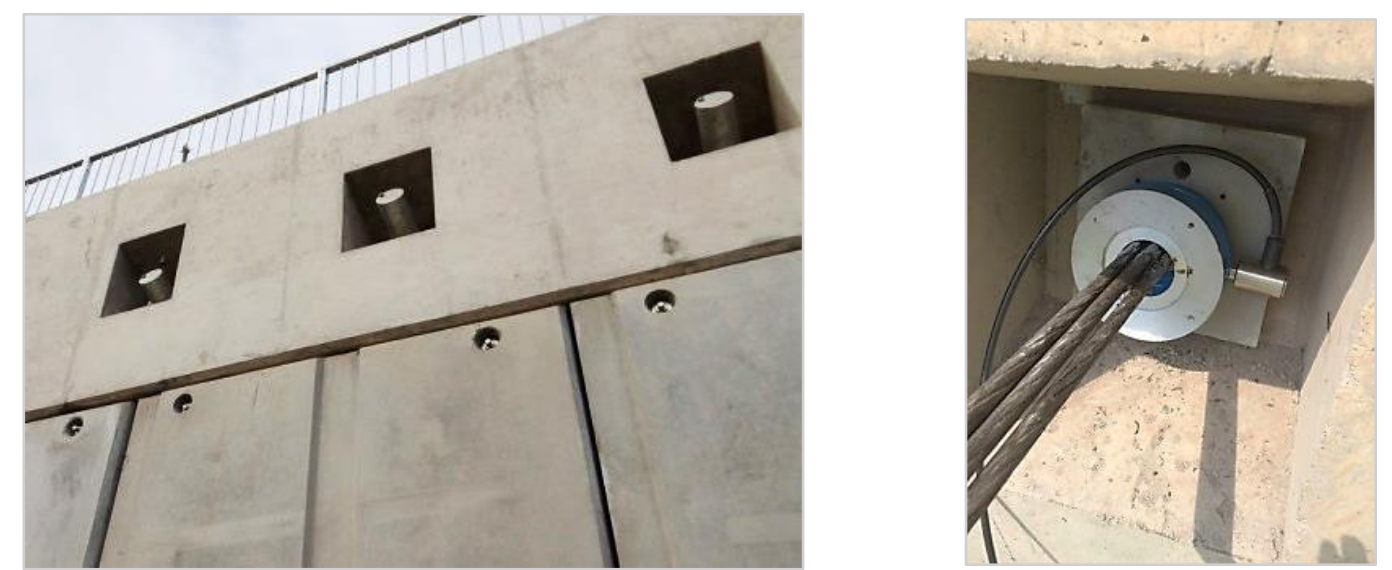

Figura 111. Fotografías de las células de carga en la obra. (Cortesía de Ferrovial-

Agromán y elaboración propia) 
En cuanto al control de las deformaciones, un SAA consiste en un conjunto de segmentos que registran desplazamientos a lo largo de un perfil vertical u horizontal. Cada segmento contiene un acelerómetro de tres ejes con tecnología SMEM (Sistema Microelectromecánico). Los conjuntos de acelerómetros se instalan dentro de un tubo flexible de PVC a lo largo de la longitud libre de la pantalla (ver Figura 112). En este caso, como la pantalla ya estaba construida, los conductos se instalaron anclados a la cara externa de esta. Los puntos de lectura de desplazamientos, son puntos en los que este sistema está fijado a la pantalla y se encuentran cada $0.5 \mathrm{~m}$ desde la losa de fondo. El punto fijado en la base (losa) se considera de desplazamiento nulo y es a partir del cual se integran las aceleraciones medidas. De esta manera, se puede obtener un perfil vertical de la deformada de la pantalla que recorre los puntos de medida desde la base hasta la viga de atado. Como en el caso de las células de carga las lecturas de los SAA se envían automáticamente a un sistema de adquisición de datos.
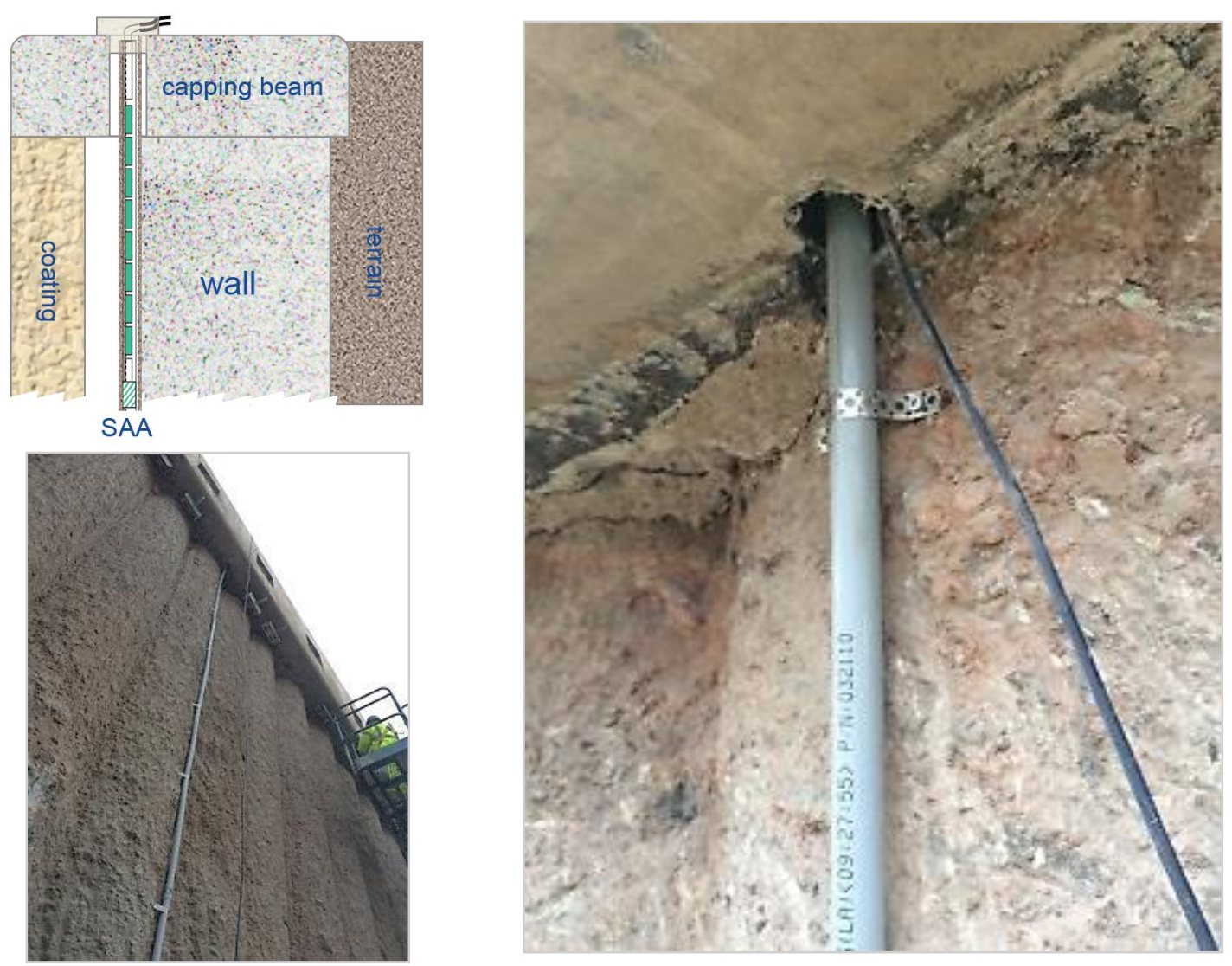

Figura 112. Fotografías del la instalación y aspecto de los conductos de SAA instalados. (Solvver y Ferrovial-Agroman) 
La información proporcionada por la monitorización, que finalmente ha quedado instalada para este proyecto, permite corrobar de manera aproximada algunos hechos esperables. En primer lugar, el registro de las células de carga del que se ha podido disponer para la tesis, permite deducir que se produce un aumento de la carga en el anclaje considerable tras la fase de excavación total, al igual que ocurría con el modelo numérico (Figura 80). Como se aprecia en la Figura 113 (obtenida a partir de los datos de monitorización de la obra), se producen aumentos de hasta el $30 \%$ respecto a la carga de bloqueo $(200 \mathrm{kN})$. En esta figura se aprecia la fuerza en los anclajes transcurridos 2 años y 10 meses desde su puesta en carga, por lo que se supone un aumento inicial real de ese orden que apenas se ha visto reducido por los fenómenos de fluencia del terreno. Conocer el valor de la fuerza real en los anclajes al terminar la excavación total de las pantallas aportaría un dato fundamental, para establecer conclusiones más precisas acerca de la fluencia de los suelos presentes en la obra.

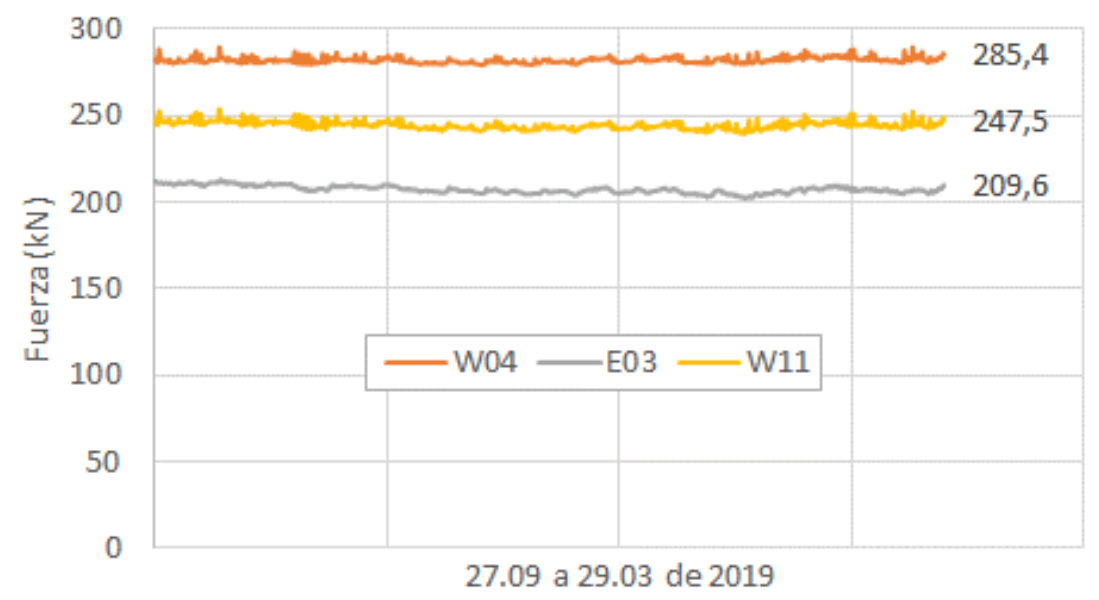

Figura 113. Valores de las células de carga en las secciones de estudio (Figura 110) en marzo de 2019.

Con los datos de monitorización disponibles, en cuanto a deformaciones, se puede apreciar que el perfil de desplazamiento a lo largo de la longitud libre de la pantalla (Figura 114) es similar al estimado con el modelo numérico en el caso de la sección $\mathrm{W} 11$, con desplazamientos cualitativamente similares en cabeza hacia la excavación. 
De igual modo, la monitorización de la sección E3 (equivalente a la sección W4, de la cual no se encuentra disponible el sensor SAA) precide desplazamientos cualitativamente similares en la parte alta de la pantalla hacia el trasdós de ésta (Figura 115), de igual modo que ocurre con el modelo numérico obtenido con FLAC 3D.

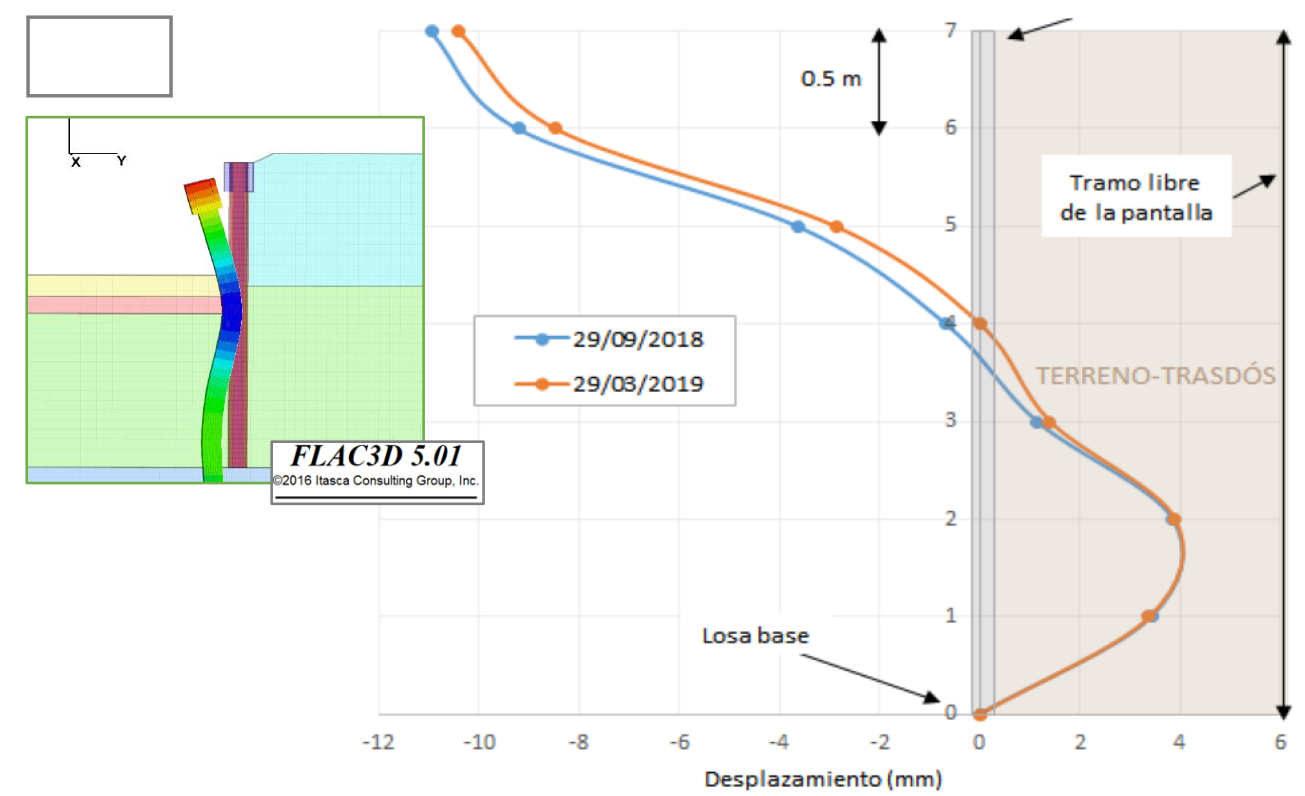

Figura 114. Desplazamiento a lo largo de la longitud libre obtenido mediante SAA (sección W11, Figura 109).

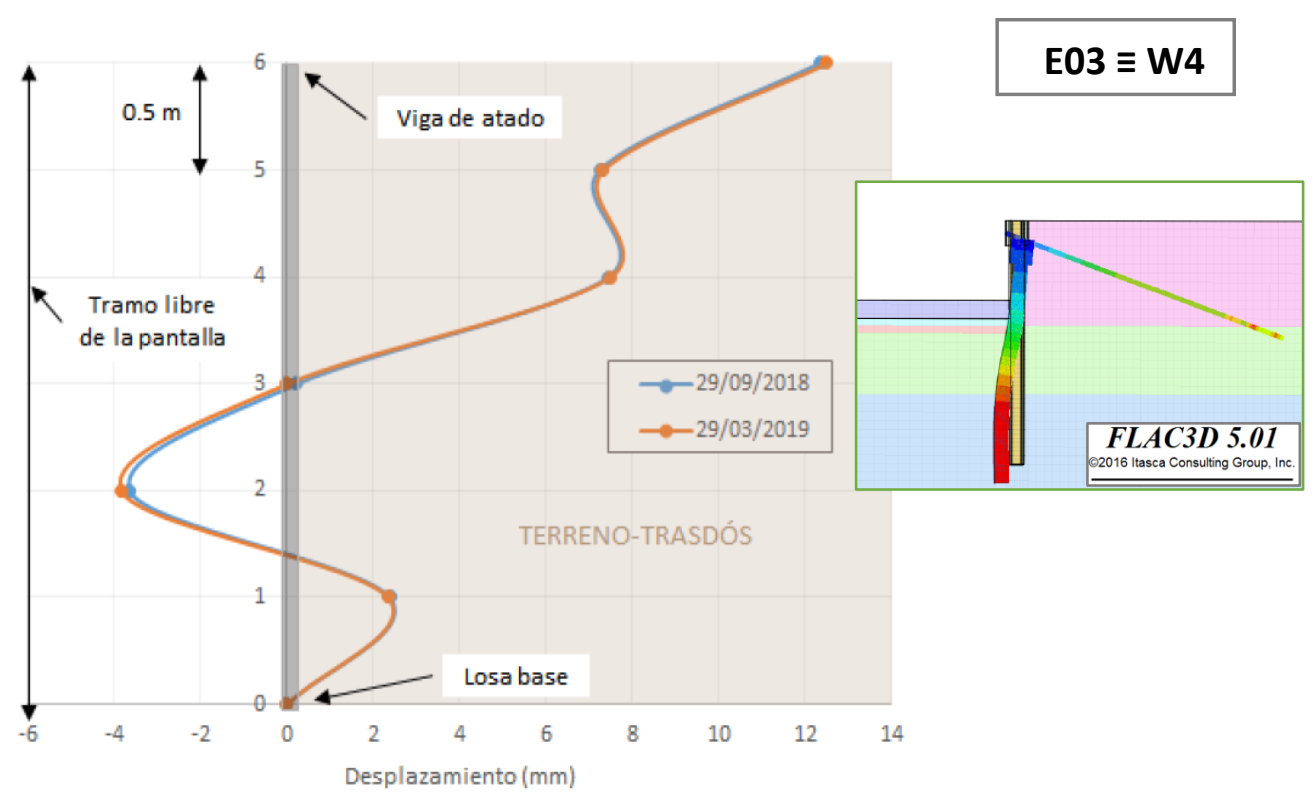

Figura 115. Desplazamiento a lo largo de la longitud libre obtenido mediante SAA (sección E3, equivalente a la W4, Figura 110). 
Según los datos de monitorización, el desplazamiento máximo producido en la cabeza del anclaje, durante el periodo de 6 meses indicado (Figura 113 y Figura 114) es de 0,5 y 0,1 mm, en el caso de la sección W11 y E03 (W4), respectivamente. Sin embargo, al buscar un periodo de tiempo de 6 meses en el que se hayan producido esos desplazamientos en las correspondientes secciones, mediante los modelos analítico y numérico, se encuentra que estos ocurren durante la etapa de fluencia primaria concentrada en ambas secciones en los primeros meses desde la excavación completa de la pantalla. Por ejemplo, veáse la Figura 116, en la cual se representa la curva desplazamiento tiempo de la sección W4, obtenida con el modelo analítico. Un desplazamiento de $0,1 \mathrm{~mm}$ ocurre entre los 2 y 8 meses de tiempo de fluencia desde la excavación total de la pantalla, que es el tiempo cero para el inicio de los desplazamientos en el modelo analítico.

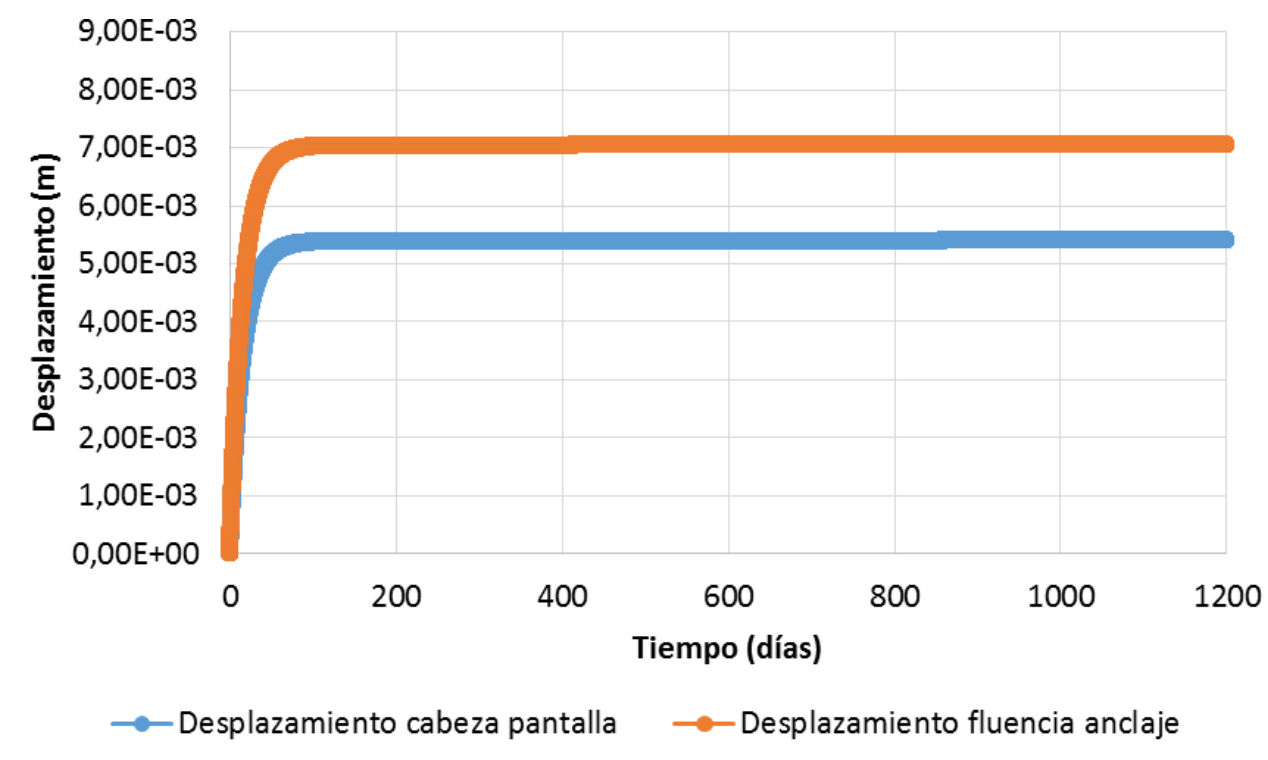

Figura 116. Curva de desplazamiento-tiempo de la sección W4 obtenida con el modelo analítico.

El origen de tiempo de registro de desplazamientos para cada sección es desconocido (aunque sí se estima que hayan pasado más de dos años desde la puesta en carga, como en el caso de las células de carga). Además, cada uno de los perfiles intrumentados se puso en marcha en distintos momentos (cuando los trabajos de ejecución de la obra lineal en sí o el propio 
factor climático, lo permitían), que no se conocen con precisión, aspecto que hace suponer que las pantallas vayan desfasadas temporalmente.

Por tanto, gracias a la experiencia obtenida, se ha observado la importancia de registrar las medidas en los momentos clave de la curva deformación tiempo del terreno y conocer el origen de tiempos para cada una de las secciones. De esta manera, se conoce en qué momento exacto de la curva se encuentra la medida que ofrece la instrumentación, y aunque estas medidas fueran puntuales, discontinuas, permitirían realizar hipótesis de contraste más fiables. Por tanto, para un primer ajuste del modelo propuesto más que disponer de una gran cantidad de puntos instrumentados, sería imprescindible conocer estos factores. Además, se podría plantear un registro mucho más continuado durante la primera etapa de fluencia (primeros 2-3 meses) y un registro más puntual y distanciado durante la segunda etapa (a partir del tercer mes hasta completar la vida útil de la obra). 
CAPÍTULO 6: CONCLUSIONES Y FUTURAS LÍNEAS DE INVESTIGACIÓN 


\subsection{CONCLUSIONES}

Esta tesis propone un modelo que permite conocer la evolución de la carga en anclajes instalados en pantallas construidas en terrenos con fluencia. Para abordar el problema, el documento introduce en primer lugar un estado del conocimiento sobre los modelos reológicos existentes que se puedan incorporar al nuevo modelo propuesto. A continuación, se explican y se desarrollan los dos modelos que resuelven el problema planteado: uno analítico, basado en formulaciones clásicas para el asiento en superficie y en profundidad en un semiespacio elástico, que ha quedado implementado en una hoja de cálculo; y otro numérico, desarrollado en un programa de cálculo geotécnico en diferencias finitas. Por último, se propone un nuevo ensayo de fluencia bajo las mismas condiciones de contorno del problema, que proporcione unos parámetros representativos para introducir en el modelo.

Llevando a cabo un repaso a los distintos tipos de modelos reológicos expuestos (empíricos, mecánicos, teorías ingenieriles, enfoque hereditario, modelos generales tensión-deformación-tiempo y el enfoque de la teoría "rate proccess) se pueden plantear una serie de conclusiones acerca de la conveniencia de un modelo $u$ otro, para aplicarlo en el caso particular que trata esta investigación:

- En primer lugar, se ha visto que los modelos empíricos, tanto los simples como los compuestos, están basados en el principio de correspondencia, es decir que los distintos fenómenos de relajación o de fluencia, están debidos al mismo mecanismo básico, y se pueden deducir uno a partir de la observación del comportamiento del otro. Esta idea es acorde con el hecho de que no exista un modelo empírico único para reproducir la relajación, y que los que se han presentado están basados en leyes de fluencia previamente adoptadas. Una de sus principales ventajas es que reflejan el comportamiento real de los suelos, sin embargo, es un comportamiento "real" en laboratorio, con las numerosas precauciones que hay que tomar para su adecuada y semejante reproducción y escala. Teniendo en cuenta, además, que los 
ensayos no siempre pueden simular las condiciones "in situ" en su totalidad. En principio las desventajas de las relaciones empíricas es que son solo aplicables a problemas con condiciones de contorno y de carga muy específicas (la mayoría de ellas se han desarrollado para estudiar la consolidación secundaria).

- Los resultados de las investigaciones expuestas anteriormente, prueban la posibilidad de introducir en los modelos mecánicos comportamientos no lineales, así como la generalización desde formulaciones unidimensionales a tridimensionales, ya sea considerando condiciones de isotropía u otras, más complejas o reales. La escasa bibliografía encontrada acerca de modelos de comportamiento dependientes del tiempo, aplicados a un sistema suelo-anclaje, revela una amplia preferencia por el empleo de los modelos analógicos, así como un vacío científico e investigador en este campo. Las aportaciones encontradas muestran buenas correlaciones, tanto entre las formulaciones analíticas del modelo mecánico seleccionado y los datos de ensayos de laboratorio y/o de campo, como con las mediciones obtenidas "in situ".

- Además, los modelos mecánicos permiten hacer una descripción formal del comportamiento fenomenológico del material, resultando ser modelos muy prácticos y aplicados, por su fácil comprensión en una dimensión. Otra de las ventajas del enfoque analógico es que se pueden plantear numerosas combinaciones y disposiciones, tanto de los elementos básicos, como de los distintos modelos planteados. Se pueden crear nuevos prototipos que se adecúen a las condiciones de carga que se quieran estudiar; por ejemplo, un caso de consolidación secundaria, o bien, uno de fluencia bajo tensiones de corte, o las diversas combinaciones que se producen en la realidad de las obras de ingeniería. Este hecho representa una gran ventaja frente a las relaciones empíricas vistas, que se establecen para unas condiciones de contorno y de carga en general muy específicas.

- La opinión general acerca de que es necesario un volumen de información de partida muy elevado (gran cantidad de ensayos) hacen que el enfoque hereditario sea descartarlo frente a otros modelos. 
- En la teoría del modelo de sobretensión las deformaciones elásticas son independientes del tiempo, mientras que las deformaciones inelásticas sí se consideran viscosas o dependientes del tiempo. Además, otra desventaja de esta teoría es que no es capaz de reproducir la fluencia acelerada.

- Del mismo modo, considerando un proceso de fluencia iniciado en un estado tensional localizado dentro de la superficie de fluencia, la teoría NSFS no es capaz de predecir deformaciones inelásticas, por lo tanto, tampoco es adecuada para predecir deformaciones por fluencia del terreno en el tiempo.

- El empleo de otros modelos más sofisticados ("rate process theory") están todavía lejos de su aplicabilidad a problemas de ingeniería práctica, si bien sí constituyen una valiosa herramienta para la comprensión conceptual de los procesos micromecánicos.

Como principal objetivo de la tesis, se propone una solución analítica para evaluar el desplazamiento con el tiempo, en la cabeza de anclajes embebidos en terrenos con fluencia. En primer lugar se resuelve el problema de una losa anclada verticalmente a un terreno con fluencia para, a continuación, resolver el problema de un elemento estructural flexible anclado a un terreno con fluencia, incorporando la rigidez del nuevo elemento. Con ello se logra obtener la pérdida de carga en el anclaje a largo plazo. A partir del desarrollo matemático de estas soluciones, se tienen las siguientes conclusiones principales:

- Se desarrolla un modelo teórico de fluencia de interacción anclaje estructura - suelo, resolviendo una serie de singularidades numéricas e indeterminaciones que aparecen al aplicar las formulaciones elásticas clásicas consideradas, al caso de estudio.

- La originalidad del nuevo modelo reside en que la evolución de la deformación en cabeza del anclaje calculada es un conjunto de los movimientos de los distintos elementos de este sistema, que interactúan entre sí, y no únicamente la evolución de un ensayo experimental desplazamiento-tiempo en el terreno involucrado. Es decir, el 
desplazamiento en cabeza es la combinación de las distintas partes del movimiento originado al someter el anclaje a tracción, por un lado, asociadas al cable, y por otro al terreno: todo ello debido a la carga en la losa y a la tracción del bulbo del anclaje.

- El modelo matemático presentado ha sido resuelto para un terreno con comportamiento viscoelástico (Burgers).

- Para obtener el desplazamiento del tirante producido por la carga en la cabeza del bulbo fue necesario repartir la carga en una corona circular, dado que la carga puntual producía una singularidad matemática en la resolución de las ecuaciones analíticas. De esta manera, dicho desplazamiento depende del área de reparto considerada. Estudiando la influencia del área de reparto considerada, se comprueba que suponer la carga repartida en un círculo completo, de diámetro igual al del bulbo del anclaje, es una hipótesis razonable, que produce, de entre todas las posibles áreas de reparto, el mayor desplazamiento por fluencia en cabeza del bulbo.

- Con el objetivo de estudiar su utilidad en la ingeniería práctica, el modelo teórico se aplica a un caso concreto de pantalla anclada, donde es necesario implementar la rigidez del elemento estructural de contención e integrar su efecto en el resultado total del equilibrio en el problema completo de fluencia en el tiempo.

- Se muestra el esquema iterativo del problema de análisis de la pantalla anclada y la implementación del cálculo según un esquema convergente y estable. La solución analítica se ha podido implementar adecuadamente en una hoja de cálculo, lo que facilita su integración en el diseño a largo plazo de estructuras ancladas. En este proceso de cálculo iterativo se va obteniendo progresivamente con el tiempo la evolución del movimiento diferido acumulado de la cabeza del anclaje, de la carga actuante sobre el anclaje y del desplazamiento total de la cabeza de la pantalla. Debe comprobarse que el intervalo de tiempo elegido es lo suficientemente pequeño como para garantizar un adecuado funcionamiento del esquema numérico de resolución. Al estar implementado en una hoja de cálculo se pueden efectuar estudios 
paramétricos de manera muy general en un intervalo de tiempo muy reducido.

- Se muestran los resultados obtenidos al aplicarlo a un caso real, consiguiendo una interpretación física clara y coherente entre la predicción del modelo implementado y los resultados esperables.

- Para la determinación de los parámetros del modelo reológico utilizado (Burgers), se han considerado dos curvas de fluencia diferentes (en cuanto a duración total de las medidas) del suelo presente en la obra. El contraste de resultados del modelo analítico, obtenidos en ambos casos, ha permitido apreciar la importancia de la necesidad de disponer de ensayos de fluencia específicos, de un mínimo de duración, con los materiales presentes en la obra (en los bulbos del anclaje, principalmente) para realizar una estimación adecuada de los parámetros y obtener con ellos una predicción lo más ajustada posible. De este modo se puede señalar que los ensayos de investigación en campo en anclajes con duraciones de 60 o 120 minutos no proporcionan datos suficientes para realizar predicciones de deformaciones a muy largo plazo, mientras que, sí son útiles para poder restringir en primera medida en qué tipo de suelos no es admisible la instalación de anclajes por deformaciones diferidas excesivas.

- Si fuera necesario, con el modelo propuesto, se podría predecir cuánto y cuando hay que retesar los anclajes o bien, simplemente, conocer cuándo (en qué momento de la curva deformación-tiempo) hay que llevar cierto control. Adicionalmente, el factor de seguridad que se añade por fluencia se podría replantear, conocida la ley de comportamiento de la carga durante la vida útil del anclaje. Es decir, este coeficiente se añade por falta de conocimiento sobre el comportamiento por fluencia del suelo y si se logra conocer cómo se comportarán los anclajes durante la vida útil de la obra gracias a este modelo, la magnitud del factor de seguridad podría verse reducida considerablemente, permitiendo diseños más ajustados y más económicos.

- Con la aplicación del modelo propuesto a un caso real se constata que la solución matemática desarrollada es razonablemente acertada. Por 
otro lado, queda ilustrada la necesidad de disponer de ensayos de fluencia a largo plazo, bien sean en campo o en laboratorio, y en los terrenos en los que se instalan los anclajes, para llevar a cabo una predicción ajustada y representativa.

Por otro lado, en la tesis se describe la simulación llevada a cabo con el programa de análisis geotécnico en diferencias finitas FLAC 3D. Para ello ha sido necesario implementar en el código nuevas subrutinas que reproducen las innovaciones desarrolladas. Como principal objetivo, se cuenta con la simulación de un modelo de pantalla anclada con evaluación de la fluencia del terreno y pérdida de carga en el anclaje. Se indican a continuación las conclusiones referentes a la simulación en diferencias finitas:

- El modelo de cálculo se ha parametrizado de tal manera que queda disponible para su aplicación en otros casos de pantallas ancladas en terrenos con fluencia.

- Tras realizar el cálculo instantáneo en la fase final de construcción de la pantalla, se incorpora en el terreno un modelo constitutivo dependiente del tiempo, de tipo viscoelastoplástico, compuesto a su vez por el modelo mecanicista viscoelástico de Burgers (empleado en el modelo analítico) y el conocido criterio de rotura de Mohr-Coulomb cuando se alcanza la plasticidad.

- Los movimientos por fluencia del terreno, así como las pérdidas de carga en el anclaje obtenidas mediante el cálculo en diferencias finitas, con el modelo implementado, han resultado bastante coherentes. La clave está en que los parámetros de fluencia incorporados en el modelo sean lo suficientemente representativos del comportamiento del terreno durante la vida útil de la obra.

- La solución teórica presentada para una losa rígida circular anclada verticalmente a un terreno con fluencia también ha sido validada mediante análisis numérico por diferencias finitas, creando un modelo semejante. Se pone de manifiesto la bondad del modelo teórico de análisis con el buen ajuste obtenido entre ambas soluciones. 
- Del mismo modo, la solución teórica presentada para una pantalla anclada a un terreno fluencia también ha sido validada mediante análisis numérico por diferencias finitas, creando un modelo semejante. Ambas soluciones se ajustan muy adecuadamente, proporcionando valores de pérdida de carga con el tiempo con diferencias despreciables.

En conexión con la necesidad de disponer de ensayos específicos de fluencia para obtener unos parámetros del modelo predictivo a largo plazo, lo suficientemente representativos, se ha desarrollado un nuevo equipo de laboratorio para esta tesis. Se pretenden conocer los movimientos por fluencia en el bulbo bajo las condiciones de contorno del problema de estudio y durante los tiempos en los cuales el fenómeno de fluencia desarrolla por completo su etapa primaria, entrando en su etapa secundaria, en cualquier tipo de terreno. El ensayo permite conocer la deformación del terreno en el tiempo, debido a una tracción impuesta a través de una barra embebida en este y adherida mediante resina. Se trata de un ensayo a escala de grandes dimensiones, para el cual se han fabricado muestras artificiales que simulan un suelo duro/roca blanda. A partir del desarrollo y puesta en marcha del equipo y de la ejecución de los ensayos en laboratorio, se destacan las siguientes evidencias experimentales:

- El ensayo permite medir los desplazamientos bajo carga constante durante largos periodos de tiempo (46, 61 y 75 días en este caso, respectivamente) en un anclaje embebido en una roca blanda.

- Se ha diseñado un equipo sencillo, al ser exclusivamente mecánico, por lo tanto, muy adecuado para el estudio de deformaciones a largo plazo bajo carga constante. De este modo, se permite el registro de datos sin necesidad de manipulación durante el ensayo, y con el periodo de tiempo que se desee, además de mantener la humedad constante en las muestras al quedar estas sumergidas. Las características mecánicas y geométricas del equipo están basadas en investigaciones teóricas y experimentales previas. Se ha definido el procedimiento de ensayo a seguir y se ha constatado su funcionamiento mediante las tres pruebas realizadas. 
- Los resultados de los ensayos garantizan su reproducibilidad, salvando las posibles diferencias en cuanto a resistencia y homogeneidad en bloques de grandes dimensiones fabricados por el usuario, así como, garantizándose una adecuada precisión de registro de desplazamientos.

- Los resultados obtenidos permiten interpretar adecuadamente la diferente respuesta deformacional del terreno ante carga instantánea y en fluencia. De manera que los desplazamientos en superficie tienen una tendencia decreciente con la distancia ante carga instantánea; y obteniendo una evolución con la distancia en forma de campana con el tiempo.

- Se deduce que, cuando el desplazamiento del anclaje supera el del centro del bloque ensayado, se estan produciendo deslizamientos en el contacto resina-roca. En este caso es esperable que el modo de rotura, en arrancamiento, sea de tipo mixto.

- Los tipos de rotura observados durante los ensayos de arrancamiento (rock break-out and mixed) concuerdan con las formas experimentales y teóricas obtenidas por otros autores, además de proporcionar valores de resistencia al arrancamiento $\left(\tau_{a d h}=980\right.$ y $\left.1115 \mathrm{kPa}\right)$ que concuerdan con los resultados obtenidos en ensayos de resistencia a compresión simple a corto plazo ( $S_{u}=700$ y $\left.1050 \mathrm{kPa}\right)$.

- Los resultados obtenidos ponen de manifiesto los dos efectos, que deben considerarse para la interpretación adecuada de las deformaciones instantáneas obtenidas en el ensayo: la mayor rigidez en pequeñas deformaciones y el efecto escala.

Por último, de los trabajos derivados del contraste entre los resultados de los modelos analítico y numérico y los de la monitorización en obra, se tienen las siguientes conclusiones:

- El modelo numérico y los datos reales parecen predecir aumentos de carga considerables en el anclaje tras la fase de excavación total, con valores de hasta un $45 \%$ respecto a la carga de bloqueo. El valor real de la carga en el anclaje tras la excavación resulta un dato fundamental. Este valor permitiría ajustar de manera más precisa los parámetros 
elásticos ( $E$ y $v$ ) reales del terreno, al caso estudiado, y a partir de los cuales se realizan los supuestos de variabilidad en el tiempo, influyendo notablemente en las predicciones a largo plazo.

- Cabe destacar la importancia de la primera fase de deformación por fluencia primaria, en la cual se producen las mayores pérdidas de carga en el anclaje y las mayores deformaciones asociadas. Esta etapa se suele presentar en la mayoría de terrenos durantes el primer mes de su puesta en carga. Sin embargo, las medidas disponibles en tiempo real corresponden a una etapa de fluencia secundaria posterior (se estima que a los 2 años y medio de la puesta en carga), permitiendo, únicamente, un ajuste discontinuo de los datos.

- A partir de la experiencia tenida se ha observado que es más importante tomar las medidas imprescindibles en los momentos clave (al principio) de la curva deformación - tiempo del terreno (o carga - tiempo) que disponer de una gran cantidad de puntos instrumentados. Realmente es necesario obtener un registro continuado durante la primera etapa de fluencia (primeros 2 - 3 meses), pudiéndose pasar a un registro más puntual y distanciado durante la etapa secundaria (a partir del tercer mes hasta completar la vida útil de la obra).

\subsection{FUTURAS LÍNEAS DE INVESTIGACIÓN}

Durante el desarrollo de la tesis se han alcanzado los resultados deseados en cuanto a la puesta en marcha y aplicación al caso concreto de los modelos analítico y numérico. Del mismo modo, se cuenta con un nuevo equipo de laboratorio con el que estudiar las deformaciones con el tiempo del terreno bajo las condiciones de contorno presentes. Adicionalmente, se han puesto de manifiesto la necesidad de desarrollar una serie de futuras líneas de investigación, abordables razonablemente, que se sintetizan a continuación:

- Una de las principales ventajas del modelo analítico propuesto es que permite la incorporación de otros modelos de fluencia en las ecuaciones constitutivas del terreno. En el caso desarrollado se ha empleado el 
modelo de Burgers, siendo posible la incorporación de otros de los modelos vistos en el capítulo de estado del conocimiento, en función de que estos se ajusten mejor cuando se presente un tipo de terreno $u$ otro en la obra. Por ejemplo, también permite la incorporación directa de un modelo empírico, como el que nos ofrece el nuevo ensayo propuesto, para simular los parámetros deformacionales del terreno con el tiempo.

- Otro aspecto a desarrollar en futuras investigaciones sería tener en cuenta la variación de la adherencia a lo largo de la longitud del bulbo, en las ecuaciones del modelo analítico. Este aspecto resultará más relevante cuando se trate del estudio de anclajes en roca $y$, teóricamente, también es posible incorporar a las ecuaciones propuestas, una función para tenerlo en cuenta.

- Sería muy interesante conocer la curva deformación-tiempo bajo las condiciones de contorno de estudio, de múltiples tipos de terrenos, empleando el nuevo equipo de fluencia desarrollado, en otro tipo de suelos y rocas. Se tiene la capacidad de utilizar otros rangos de carga y distintos materiales ya sea en condiciones sumergidas, o completamente secas, para preservar la condición de humedad constante.

- Contar con datos de pérdida de carga en anclajes, desde su puesta en carga y durante los primeros días y meses, para el contraste de estos datos reales con los modelos numérico y analítico, de otras obras con pantallas ancladas. Este hecho completaría el retroanálisis inicialmente buscado, aportando al modelo analítico propuesto una validación extra. Así mismo, resulta clave contar con parámetros deducidos de ensayos con los terrenos realmente presentes en la obra, de mayores duraciones y bajo niveles tensionales similares a los que tendrán los bulbos en servicio.

- Proponer mejoras de las restrictivas limitaciones, para anclajes en terrenos potencialmente reológicos, según la normativa actual (que limita mediante ensayos de investigación, límites del índice de plasticidad o del contenido en materia orgánica). Esta valoración se podría llevar a cabo realizando un primer análisis con el modelo propuesto y un ensayo de fluencia representativo del cual provengan los parámetros a aignar en el 
estudio de fluencia del modelo constitutivo del terreno. Es decir, el ensayo de fluencia debe realizarse con el material que afectará al bulbo, al nivel de cargas de trabajo y con una duración mínima representativa del largo plazo o etapa atenuada (fluencia secundaria). 
CAPÍTULO 7: BIBLIOGRAFÍA 
Adachi, T., Oka, F., \& Poorooshasb, H. B. (1990). A constitutive model for frozen sand. Trans. ASME, 112, 208 -212.

Adachi, T., Oka, F., \& Zhang, F. (1997). An elasto-viscoplastic constitutive model with strain softening. Numerical models in geomechanics, $\mathrm{S}$. Pietruszczak and G. Pande, eds., Balkema, Rotterdam, The Netherlands, 8186.Numerical models in geomechanics, S. Pietruszczak and G. Pande, eds., Balkema, Rotterdam, The Netherlands, 81-86.

Adachi, T., \& Okano, M. (1974). A constitutive equation for normally consolidated clay. Soils Found., 14(4), 55-73.

Adachi, T., \& Oka, F. (1982a). Constitutive equations for normally consolidated clay based on elasto-viscoplasticity. Soils and Foundations, 22(4), 57-70.

Afrouz, A., \& Harvey, J. M. (1974). Rheology of rocks within the soft to medium strength range. International Journal of Rock Mechanics and Mining Sciences \& Geomechanics, 11(7), 281-290.

Akai, K., Adachi, T., \& Nishi, K. (1977). Mechanical properties of soft rocks. Proc., 9th ICSMFE, Tokyo, 1, 7-10.

Al-Shamrani, M. A., \& Sture, S. (1998). A time-dependent bounding surface model for anisotropic cohesive soils. Soils and Found., 38(1), 61-76.

AMEY-RPS: M8, M73, M74 Motorway improvements. Structure S201. Raith Underpass. Geotechnical Design Report (2015).

Barden, L. (1965). Consolidation of clay with nonlinear viscosity. Geotechnique, 15(4), 345-362.

Bishop, A. W., \& Lovenbury, C. O. (1969). Creep characteristics of two undisturbed clays. Proc. 7th ICSMFE, Mexico, 1, 29-37.

Bjerrum, L. (1967). Engineering geology of Norwegian normallyconsolidated marine clays as related to the settlement of buildings. Geotechnique, 17(2), 83-119.

Blum, H. (1931). Einspannungsverhaltnisse Bei Bohlwerken [Restraint Conditions in Cantilevered Retaining Walls]. Berlin: Wilh. Ernstund Sohn.

Borm, G., \& Haupt, M. (1988). Constitutive behavior of rock salt: Power law or hyperbolic sine creep? 6th Int. Conf. on Numerical Methods in Geomechanics, 1883-1893. 
Borja, R. I., Kavazanjian, E., Jr., \& Hsieh, H. S. (1990). Double-yieldsurface cam-clay plasticity Model: II. Implementation and verification.'. J. Geotech. Eng., 116(9), 1402-1421.

Borowicka, H. (1936). "The influence of rigidity of a circular foundation slab on the distribution of pressures over contact surface." Proc. 1st Int. Conf. on Soil Mechanics and Foundation Engineering, Vol. 2, No. 1, 144-149.

Bowman, E. \& Soga, K. (2003). "Creep, ageing and microstructural change in dense granular materials". Soils and Foundations, vol. 43, nº, pp. 107-117.

BS-EN 1537 (British Standard-European Standard). (2000). "Execution of special geotechnical works: ground anchors". Brussels.

Budin, A. (1969). Behavior of sheet pile retaining walls in soils susceptible to creep. Soil Mechanics and Foundation Engineering, 6 .

Buisman, A. S. (1936). Results of long duration settlement tests. Proc.1st Int. Conf.on Soil Mechanics and Foundation Engineering, Cambridge, Mass, 1, 103-107.

Callahan, G. D., \& DeVries, K. L. (1991). Analysis of Backfilled Transuranic Waste Storage Rooms. RE/SPEC, Inc., report to Sandia National Laboratories SAND91-7052.

Casagrande, A., \& Wilson, S. D. (1951). Effect of rate of loading on strength of clays and shales at constant water content. Geotechnique, 2(3), 251-263.

Christensen, R. W., \& Wu, T. H. (1964). Analysis of clay deformation as a rate process. Journal of Soil. Mech. and Found. Eng. Div., ASCE, 125-157.

Cristescu, N. (1991). Nonassociated elasto/viscoplastic constitutive equations for sand. Int. J. Plast., 6, 41-64.

Cristescu, N. (1993). Rock Rheology. En: Hudson John, Comprehensive Rock Engineering. London, New York, Seoul, Yushima. Pergamon Press Itd, 1993. Vol. I, Pp. 523 - 544.

Cristescu, N.; Hunsche, U. (1998). Time effects in rock mechanics. England: John Wiley \& Sons Ltd.1998. Pp 342.

Coates, D. F. (1970). Rock mechanics principles. Mines Branch, Department of Energy, Mines and Resources. 
Cuéllar, V., Baiant, Z. P., Asce, J. M., \& Raymond, J. K. (1977). Densification and hysteresis of sand under cyclic shear. Proc. Amer. Soc. Civ. Eng., J. Geotech. Eng. Div., 103(GT5), 399-416.

Dafalias, Y. F. (1982). Bounding surface elastoplasticity-viscoplasticity for particulate cohesive media. Int. Union of Theoretical and Applied Mechanics Conf. on Deformation and Failure of Granular Materials, P. A. Vermeer and H. J. Luger, eds., 97-107.

Del Cañizo, L., Eraso, A. \& Aguado, J. (1976). La bentonita-cemento y sus aplicaciones. Revista de Obras Públicas, 3130: 67-76 (in Spanish).

Di Prisco, C., \& Imposimato, S. (1996). Time dependent mechanical behaviour of loose sands. Mech. Cohesive-Frict. Mater., 1(1), 45-73.

Duncan, J. M., \& Chang, C. (1970). Nonlinear analysis of stress and strain in soils. ASCE Soil Mechanics and Foundation Division Journal, 96(5), 1629-1653.

European Standard EN 12390-2:2009. Testing hardened concrete - Part 2: Making and curing specimens for strength tests.

Eyring, H. (1936). Viscosity, plasticity and diffusion as examples of absolute reaction rates. J. Chem. Phys., 4(4), 283-291.

Fan, Q., Liu, C., Yang, K. and Wang, M. (2011). Test Study on Creep Character of Anchor Rod in Argillaceous Soft Rock. Advanced Materials Research, Vols. 396-398: 565-570.

Feda, J. (1989). Interpretation of creep of soils as a rate process. Géotechnique, 39(4), 667-677.

Feda, J. (1992). Creep of soils and related phenomena ( $2^{\text {nd }}$ ed.). Amsterdam: Elsevier Science.

FHWA. (1999). GEOTECHNICAL ENGINEERING CIRCULAR NO. 4. Ground anchors and anchored systems. Washington, DC: US Department of Transportation.

Fung, Y. C. (1965). Foundations of solid mechanics ( $1^{\text {st }}$ ed.). Englewood Cliffs, New Jersey: Prentice-Hall.

García Wolfrum, S. (2005). "Anclajes en roca". España: Tesis de Doctorado, Departamento de Ingeniería y Morfología del Terreno (ETSICCP), Universidad Politécnica de Madrid. 
Gonzalez de Vallejo, L. I (2002). “Ingeniería Geológica”. Ed. Pearson.

Goodman, R. (1980). Introduction to Rock Mechanics (2nd ed.). New York: John wiley \& Sons

Griggs, D. T. (1939). Creep of rocks. The Journal of Geology, 47(3), 225251.

Griggs, D. T. (1940). Experimental flow of rocks under conditions favoring recrystalization. Geological Society of America Bulletin, 51(7), 10011022.

Gurinsky M. (1986). "Investigation of long-term strength of ground anchors in creep-sensitive soils". Ph. D. Thesis, St. Petersburg Polytechnic University.

Gurinsky, M. A. (2002). Long-term strength of prestressed ground anchors in creep-sensitive soils. International Deep Foundations Congress, Orlando, Florida, 37-52.

Hashiguchi, K., \& Ueno, M. (1977). Elastoplastic constitutive laws of granular materials. Proc. 9th ICFSME, Spec. Session 9, JSSMFE, Tokyo,, 7382.

Hashiguchi, K., \& Okayasu, T. (2000). Time-dependent elastoplastic constitutive equation based on the subloading surface model and its application to soils. Soils Found., 40(4), 19-36.

Herrmann, W., Wawersik, W. R., \& S., L. H. (1980a). Analysis of Steady State Creep of Southeastern New Mexico Bedded Salt. Sandia National Laboratories, SAND80-0558.

Herrmann, W., Wawersik, W. R., \& Lauson., H. S. (1980b). Creep Curves and Fitting Parameters for Southeastern New Mexico Rock Salt. Sandia National Laboratories, SAND80-0087.

Hilti S.A., 2002. Manual técnico de anclajes. Editado por Hilti Española, S.A.

Hohenemser, K., \& Prager, W. (1932). "Uber die ansatze der mechanik isotroper kontinua". Z. Angew. Math. Mech., 12, 216 -226.

Houska, J. (1981). Rheological tests of claystones from Strahov tunnel in Prague. Res. rep. UGG CSAV, 6. 
Hsieh, H. S., Kavazanjian, E., \& Borja, R. I. (1990). Double yield surface cam-clay plasticity model. I: Theory. J. Geotech. Eng., 116(9), 1381-1401.

Huneault, P. A. (1992). Strain-hardening relaxation. J. Appl. Mech., 59, 217-219.

IBM SPSS Statistics. IBM Corp. Released 2015. IBM SPSS Statistics for Windows, Version 23.0. Armonk, NY: IBM Corp.

ISRM, 1981. Suggested methods for rock characterization, testing and monitoring. ISRM. Suggested methods. Ed. E.T. Brown. Pergamon Press.

Itasca (2012) FLAC 3D - Fast Lagrangian Analysis of Continua in 3 Dimensions. Version 5.01.152. Itasca Consulting Group, Inc., Minneapolis.

Jaeger, J. (1969). Elasticity, Fracture and Flow (3rd ed.). New York: John Wiley \& Sons Inc.

Jamiolkowski, M., Ladd, C. C., Germaine, G. T., \& Lancellotta, R. (1985). New developments in field and laboratory testing of soils. . Proc. 11th ICSMFE, San Francisco, 1, 57-153.

Jiménez Salas, J.A., de Justo Alpañés, J. L. y Serrano González, A. (1976). "Geotecnia y Cimientos II". $2^{\mathrm{a}}$ ed. Editorial Rueda.

Joly, M. (1970). Comportement rhéologique des liquides et suspensions. Cah. GRPE. Fr. Rheol. (№ Special "Rhéologie et hydroechnique", Nov., 397405.

Justo, J. L. 1993. La integración de la fórmula de asientos de Mindlin para carga anular. Revista Internacional de Métodos Numéricos para Cálculo y Diseño en Ingeniería. 9 (1): 35-57, (in Spanish).

Kaliakin, V. N., \& Dafalias, Y. F. (1990a). Theoretical aspects of the elastoplastic-viscoplastic bounding surface model for cohesive soils. Soils Found., 30(3), 11-24.

Kaliakin, V. N., \& Dafalias, Y. F. (1990b). Verification of the elastoplasticviscoplastic bounding surface model for cohesive soils. Soils Found., 30(3), 2536.

Kaliakin, V. N., \& Dafalias, Y. F. (1991). Details regarding the elastoviscoplastic bounding surface model for isotropic cohesive soils. Civil Engineering Rep. No. 91-1, Dept. of Civil Engineering, Univ. of Delaware, Newark, Del. 
Kavazanjian, E., \& Mitchell, J. K. (1977). A general stress-strain-time formulation for soils. Proc., 9th ICSMFE, Tokyo, 113-120.

Kim, N. K. (2003). Performance of tension and compression anchors in weathered soil. Journal of Geotechnical and Geoenvironmental Engineering, ASCE, 129(12), 1138-1150.

Kim, M. K., \& Lade, P. V. (1988). Single hardening constitutive model for frictional materials. I: Plastic potential function. Comput. Geotech., 5(4), 307324.

Kränkel, T., Lowke, D., \& Gehlen, C. (2015). Prediction of the creep behaviour of bonded anchors until fracture: a rheological approach. Construction and Building Materials, 75, 458-464.

Kuhn, M. R., \& Mitchell, J. K. (1993). New perspectives on soil creep. J. Geotech. Engrg., 119(3), 507-524.

Kurz, D., Sharma, J., Alfaro, M., \& Graham, J. (2016). A semi-empirical elastic-thermoviscoplastic model for clay. Canadian Geotechnical Journal.

Kwok, C. Y., \& Bolton, M. D. (2010). DEM simulations of thermally activated creep in soils. Geotechnique, 60(6), 425-433.

Lacerda, W. A. (1976). Stress relaxation and creep effects on soil deformation (Ph.D. Thesis). Berkeley: University of California.

Lacerda, W. A., \& Houston, W. N. (1973). Stress relaxation in soils. Proc., 8th ICSMFE, 1/34, 221-227.

Ladanyi, B. (1993). Time dependent response of rock around tunnel. Comprehensive Rock Engineering, 2, 77-112.

Ladanyi, B., \& Melouki, M. (1993). Determination of creep properties of frozen soils by means of borehole stress relaxation tests. Can. Geotech. J., 30, 170-186.

Ladanyi, B., \& Benyamina, M. B. (1995). Triaxial relaxation testing of a frozen sand. Can. Geotech. J., 32, 496-511.

Lade, P. V. (2013). Time Effects Relate to Particle Crushing in Granular Materials. En Z. J. Yang Q. (Ed.), Constitutive Modeling of Geomaterials. Berlin: Springer Series in Geomechanics and Geoengineering. 
Lade, P. V., \& Kim, M. K. (1988a). Single hardening constitutive model for frictional materials. II: Yield criterion and plastic work contours. Comput. Geotech., 6(1), 13-29.

Lade, P. V., \& Kim, M. K. (1988b). Single hardening constitutive model for frictional materials. III: Comparisons with experimental data. Comput. Geotech., 6(1), 31-47.

Lade, P. V., \& Liu, C. T. (1998). Experimental study of drained creep behavior of sand. J. Eng. Mech., 124(8), 912-920.

Lade, P. V., \& Liu, C. T. (2001). Modeling creep behavior of granular materials. Proc., 10th Int. Conf. on Computer Methods and Advances in Geomechanics, Desai et al., eds., Tuscon, Ariz., 277-284.

Lama, R., \& Vutukuti, V. (1978). Handbook on Mechanical properties of rocks (2nd Edition, ed.). Switzerland: Trans Tech Publications.

Leroueil, S., Kabbaj, M., Tavenas, F., \& and Bouchard, R. (1985). Stressstrain rate relation for the compressibility of sensitive natural. Geotechnique, 35(2), 159-180.

Leroueil, S., \& Marques, M. E. (1996). Importance of strain rate and temperature effects in geotechnical engineering. Measuring and Modeling Time Dependent Soil Behavior, Geotechnical Special Publication. Sheahan, T. C. and Kaliakin, V. N., eds., ASCE, Reston, Va.(61), 1-60.

Leung, C.F. \& Ko, H-Y. 1993. Centrifuge model study of piles socketed in soft rock. Soils and Foundations, 33 (3): 80-91.

Leung, C. F., Lee, F. H., \& Yet, N. S. (1996). The role of particle breakage in pile creep in sand . Can. Geotech. J. 33, 888-898.

Li, H., Zhang, F., \& Zhao, Y. (2002). In-site test of anchor bolt in mucky soil and its engineering application. Chinese J. of Rock Mechanics and Eng., 19, 1014-1017.

Liingaard, M., Augustesen, A., \& Lade, P. V. (2004). Characterization of Models for Time Dependent Behavior of Soils. International Journal of Geomechanics, 4(3), 157-177.

Ludwick, P. (1922). Uber den Einfluss der Deformationsgeschwind-igkeit bei bleibenden Deformationen mit besonderer Berucksichtigung der Nachwirkungserscheinungen. Phys. Z., 10(12), 411-417. (in German) 
Mac, N., Shahbodaghkhan, B., \& Khalili, N. (2014). A Constitutive Model for Time-Dependent Behavior of Clay. International Journal of Civil, Environmental, Structural, Construction and Architectural Engineering, 8(6), 596-601.

Malvern, L. E. (1951). The propagation of longitudinal waves of plastic deformation in a bar of metal exhibiting a strain rate effect. J. Appl. Mech., 18, 203-208.

Matsui, T., \& Abe, N. (1985a). Elasto/viscoplastic constitutive equation of normally consolidated clays based on flow surface theory. 5th Int. Conf. on Numerical Methods in Geomechanics, Nagoya, Japan, 407-413.

Matsui, T., \& Abe, N. (1985b). Undrained creep characteristics of normally consolidated clay based on the flow surface model. Proc., 11th ICSMFE, 140-143.

Meschyan, S. R. (1995). Experimental rheology of clayey soils. Geotechnika 13 (Selected Translation of Russian Geotechnical Literature) A. A. Balkema, Rotterdam/Brookfield.

Mesri, G. (1973). Coefficient of secondary compression. J. Soil Mech. Found. Div., 99(1), 123-137.

Mesri, G., \& Godlewski, P. M. (1977). Time and stress compressibility interrelationship. J. Geotech. Eng., 103(5), 417-439.

Mindlin, R. D. (1936). "Force at point in the interior of a semi-infinite solid". Physics, 7, 195-202.

Mitchell, J. K. (1964). Shearing resistance of soils as a rate process. Journal of Soil Mech. and Found. Div., ASCE (Proceedings Paper 3773), 90, $29-61$.

Mitchell, J. K., \& Soga, K. (2005). Fundamentals of soil behavior (3rd Edition ed.). Hoboken, New Jersey: John Wiley \& Sons.

Murayama, S. (1969). Effect of temperature on elasticity of clays. Highway Research Board Special Report 103, 194-202.

Murayama, S. (1983). Formulation of stress-strain-time behavior of soils under deviatoric stress condition. Soils Found., 23(2), 43-57.

Murayama, S., \& Shibata, T. (1958). On the Rheological Characteristics of clays. Kyoto: Part1, Bull. № 26, Disaster Prevention Research Institute. 
Murayama, S., \& Shibata, T. (1961). Rheological properties of clays. Proc., 5th Int. Conf., S.M.F.E., 1, 269-274.

Murayama, S., Michihiro, K., and Sakagami, T. (1984). "Creep characteristics of sands". Soils and Foundations. Japanese Society of Soil Mechanics and Foundation Engineering, 24(2), 1-15.

Naghdi, P. M., \& Murch, S. A. (1963). On the mechanical behavior of viscoelastic/plastic solids. J. Appl. Meteorol., 30, 321-328.

Nilforoush, R., Nilsson, M., Söderlind, G., and Elfgren, L. 2016. LongTerm Performance of Adhesive Bonded Anchors. ACI Structural Journal, 113 (2): 251-261.

Nishimura T. (2016). "Investigation on creep behavior of geo-materials with suction control technique". E-UNSAT 2016.

Norton, F. H. (1929). Creep of Steel at High Temperatures. New York: McGraw-Hill Book Company.

Oka, F., \& Adachi, T. (1985). An elasto-plastic Constitutive Equation of Geologic Materials with Memory. Proc., 5th Int. Conf. on Numerical Methods in Geomechanics, 293-300.

Oka, F., Adachi, T., \& Okano, Y. (1986). Two-dimensional consolidation analysis using an elastoviscoplastic constitutive equation. Int. J. Numer. Analyt. Meth. Geomech., 10, 1-16.

Olszak, W., \& Perzyna, P. (1966a). On elastic-viscoplastic soils, rheology and soil mechanics. Int. Union of Theoretical and Applied Mechanics Symp., Grenoble, Springer, Berlin.

Olszak, W., \& Perzyna, P. (1966b). The constitutive equations of the flow theory for a nonstationary yield condition. Proc., 11th Int. Con- gress of Applied Mechanics, Springer, Berlin, 545-553.

Olszak, W., \& Perzyna, P. (1970). Stationary and nonstationary viscoplasticity. McGraw-Hill, New York [Kanninen, F. (1969). "Inelasticbehavior of solids" Battelle Institute Materials of Science Colloquia, Columbus and Atwood Lake, Ohio, 53-75.].

Ostermayer, H. 1975. Construction, carrying behaviour and creep characteristics of ground anchors. Proc. ICE Conf. "Diaphragm walls and anchorages". London, 141-151. 
Pekarskaya, N. (1976). Creep of soils depending on the character of structural bonds. Proc. 2nd All-Union Symp. Rheology of Soils. Erevan State University.

Perzyna, P. (1963a). The constitutive equations for work-hardening and rate sensitive plastic materials. Proc., Vib. Probl. , 3(4), 281-290.

Perzyna, P. (1963b). The constitutive equations for rate sensitive plastic materials. Q. Appl. Math., 20(4), 321-332.

Perzyna, P. (1963c). The study of the dynamical behaviour of rate sensitive plastic materials. Arch. Mech. Stos., 1(15), 113-130.

Perzyna, P. (1966). Fundamental problems in viscoplasticity. Adv. Appl. Mech., 9, $244-377$.

Phillips, A., \& Wu, H. C. (1973). A theory of viscoplasticity. Int. J. Solids Struct., 9, 15-30.

Ping, C., Wen, Y. D., Wang, Y. X., Yuan, H. P., \& Yuan, B. X. (2016). Study on nonlinear damage creep constitutive model for high-stress soft rock. Environmental Earth Sciences, 75(10), № de artículo: 900.

Poulos, H. G., Davis, E. H., \& and Ambrosis, L. P. (1976). Method of calculating long-term creep settlements. J. Geotech. Eng. Div., Am. Soc. Civ. Eng., 102(7), 787-804.

Prager, W. (1949). Recent developments in the mathematical theory of plasticity. J. Appl. Phys., 20(3), 235-241.

Prandtl, L. (1928). Ein Gedanken model zur kinetischen Theorie der festen Korper. Z. Angew. Math. Mech., 8, 85-106. (in German)

Prevost, J. H. (1976). Undrained stress-strain-time behavior of clays. J. Geotech. Eng., 102(12), 1245-1259.

Pusch, R., \& Feltham, P. (1981). Computer simulations of creep of clay. ASCE Journal of Geotechnical Engineering, 107(1).

Rabotnov, Y. N. (1969). Creep problems in structural members. North Holland, Amsterdam, The Netherlands: North-Holland Series in Applied Mathematics and Mechanics. North Holland Publishing Company.

Ranjan, G., Swami, S., \& Nene, A. S. (1985). Prediction of time dependent displacement of anchors. Proc. 11th Conf. Soil Mech. Found. Eng, San Francisco, 12-16. 
Sandoval, G. J. (2008). Estudio de la convergencia por fluencia de túneles circulares en medios viscoelásticos plásticos (Tesis doctoral). Madrid.

Satake, M. (1989). Mechanics of granular materials. Rep., Int. Society of Soil Mechanics and Foundation Engineering, technical committee on mechanics of granular materials, 12th, ICSMFE, M. Satake, ed., 62-79.

Sekiguchi, H. (1985). Macrometric Approaches-Static-Intrinsically Time Dependent Constitutive Laws of Soils. Rep., ISSMFE Subcommittee on Constitutive Laws of Soils and Proc. of Discussion Session 1A, Int. Society of Soil Mechanics and Foundation Engineering, Subcommittee on Constitutive Laws of Soils, 11th ICFMFE, San Francisco, 66-98.

Senseny, P. E. (1985). Determination of a Constitutive Lawfor Salt at Elevated Temperature and Pressure. American Society for Testing and Materials, Reprint 869.

Serrano, A. (2015). "Propuesta de un modelo de interacción entre un anclaje y el terreno". Manuscrito personal. No publicado.

Sheahan, T. C., \& Kaliakin, V. N. (1999). Microstructural considerations and validity of the correspondence principle for cohesive soils. Proc., 13th Conf., Engineering Mechanics, N. Jones and R. Ghanem, eds., ASCE, Reston, Va.

Silvestri, V., Soulie', M., Touchan, Z., \& Fay, B. (1988). Triaxial relaxation tests on a soft clay. Advanced triaxial testing of soil and rock, ASTM, Philadelphia, 321-337.

Singh, A., \& Mitchell, J. K. (1968). General stress-strain-time function for soils. J. Soil Mech. Found. Div., 94(1), 21-46.

Sjaardema, G. D., \& Krieg, R. D. (1987). A Constitutive Model for the Consolidation of WIPP Crushed Salt and Its Use in Analyses of Backfilled Shaft and Drift Configurations. Sandia National Laboratories, SAND87-1977.

Skempton, A.W., 1964. Long-term stability of clay slopes (4th Rankine lecture). Géotechnique, 14(2).

Skempton, A.W. \& Hutchinson, J., 1969. Stability of Natural Slopes and Embankment Foundations, State-of-the-Art Volume. Proc. 7th ICSMFE, Mexico City.

Skrzypek, J. J. (1993). Plasticity and creep - Theory, examples and problems. London: CRC Press. 
Sokolovsky, V. V. (1948). Propagation of elastic-viscoplastic waves in bar. Dokl. Akad. Nauk SSSR, 60, 775-778.

Suklje, L. (1957). The analysis of the consolidation process by the isotaches method. Proc., 4th ICSMFE, 1, 200-206.

Sun, M. J., Tang, H. M., Wang, M. Y., Shan, Z. G., \& Hu, X. L. (2016). Creep behavior of slip zone of the Majiagou landslide in the Three Gorges area. Environmental Earth Sciences, 75(16), № de artículo: 1199.

Tatsuoka, F., Santucci de Magistris, F., Hayano, K., Momoya, Y., \& J., a. K. (2000). Some new aspects of time effects on the stress-strain behaviour of stiff geomaterials. Proc., 2nd HSSR, Napoli, A. Evangelista and L. Picarelli, eds., Balkema, Rotterdam, The Netherlands, 2, 1285-1371.

Tavenas, F., Leroueil, S., La Rochelle, P., \& and Roy, M. (1978). Creep behavior of an undisturbed lightly overconsolidated clay. Can. Geotech. J., 15(3), 402-423.

Taylor, D. W. (1942). Research on consolidation of clays. Serial 85, Massachusetts Institute of Technology, Dept. of Civil and Sanitary Engineering, Cambridge, Mass.

Ter-Martirosyan, Z. G. (1992). Rheological parameters of soils and design of foundations. Rotterdam, The Netherlands: A A Balkema Publishers.

Terzaghi K. (1955). "Evaluation of Coeffcients of Subgrade Reaction," Géotechnique, Vol. 5, No. 4, 1955, pp. 297-326.

Tian, W. M., Sadd, M. H., Silva, A. J., \& Veyera, G. E. (1994). Modeling creep behavior of anisotropically consolidated marine clays. Computer methods and advances in geomechanics, H. J. Siriwardane and M. M. Zaman, eds., Balkema, Rotterdam, The Netherlands, 701-706.

Vaid, Y. P., \& Campanella, R. G. (1977). Time-dependent behavior of undisturbed clay. J. Geotech. Eng., 103(7), 693-709.

Valanis, K. C. (1971). A theory of viscoplasticity without a yield surface. I. Arch. Mech., 23(4), 517-533.

Vesic A. B. (1961). "Beams on Elastic Subgrade and Winkler's Hypothesis," Proceedings of the 5th International Conference on Soil Mechanics and Foundation Engineering, Paris, pp. 845-850.

Vyalov, S. S. (1986). Rheological fundamentals of soil mechanics. Amsterdam, The Netherlands: Elsevier Science. 
Walker, L. K., \& Raymond, G. P. (1968). The prediction of consolidation rates in a cemented clay. Can. Geotech. J., 5(4), 192-216.

Wang, X. Z., Qu, Z., Dou, Y. H., \& Ma, W. H. (2015). Loads of casing and cement sheath in the compressive viscoelastic salt rock. Journal of petroleum science and engineering, 135, 146-151.

Wang, X. G., Hu, B., Tang, H. M., Hu, X. L., Wang, J. D., \& Huang, L. (2016). A constitutive model of granite shear creep under moisture. Journal of Earth Science, 27(4), 677-685.

Wawersik, W. R., \& Brown, W. S. (1973). Creep Fracture of Rock. UTAH UNIV SALT LAKE CITY DEPT OF MECHANICAL ENGINEERING.

Yin, J. H. (1999). "Nonlinear creep of soils in oedometer tests". Geotechnique, 49(2), 699-707.

Yin, J. H., \& Graham, J. (1989a). Viscous-elastic-plastic modeling of onedimensional time-dependent behaviour of clays. Can. Geotech. J., 26, 199-209.

Yin, J. H., \& Graham, J. (1989b). General elastic viscous plastic constitutive relationships for 1D straining in clays. Proc., 3rd Int. Symp Numerical Models in Geomechanics, 108-117.

Yin, J. H., \& Graham, J. (1994). Equivalent times and onedimensional elastic visco-plastic modelling of time-dependent stress-strain behaviour of clays. Can. Geotech. J., 31, 45-52.

Yin, J.-H., Graham, J., Clark, J. I., \& Gao, L. (1994). Modelling unanticipated pore-water pressures in soft clays. Can. Geotech. J., , 31, 773778.

Yin, J. H., \& Graham, J. (1996). Elastic visco-plastic modelling of onedimensional consolidation. Geotechnique, 46(3), 515-527.

Xanthakos, P. P. (1991). Ground anchors and anchored structures. John Wiley \& Sons, New York.

Xia-Ting, F., Bing-Rui, C., Chengxiang, Y., \& Hui, Z. (2006). Intelligent analysis of rheological characteristics of rock materials. EUROCK 2006 Multiphysics Coupling and Long Term Behavior in Rock Mechanics. London: Taylor \& Francis Group.

Xu, H. F., Wang, F. J., \& Cheng, X. X. (2007). Pullout creep properties of grouted soil anchors. J. Cent. South Univ. Technol., 14, 474-477. 
Xu, H., HB., L., \& Qian, Q. (2002). Creep damage effects of pulling grouting anchor in soil. Chinese Journal of Geotechnical Engineering, 24(1), 6163.

Zhang, C.-C., Zhu, H.-H., Xu, Q., Shi, B and Mei, G.-X. 2015. Timedependent pullout behavior of glass fiber reinforced polymer (GFRP) soil nail in sand. Can. Geotech. J. 52: 671-681.

Zhang, F. R., Jiang, A. N., Yao, X. C., \& Song, Z. P. (2016). Analysis of creep properties of soft soil subgrade using gravel piles based on Modified Burgers Model. Proceedings of the 2016 5th International Conf.on Sustainable Energy and Environment Engineering (ICSEEE, 2016), 63, 82-88. 
ANEXO I: PROGRAMACIÓN EN FLAC 3D 


\section{I-A: MODELO NUMÉRICO DE LOSA}

ANCLADA EN TERRENO CON FLUENCIA 


\section{anclaje_malla.f3dat}

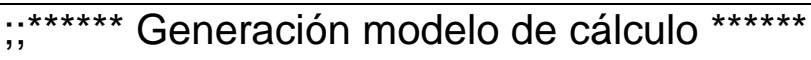

def matriz

array xc(9000) yc(9000) zc(9000)

end

def genera_prim

poi0=poi0

poi1=poi1

poi2=poi2

poi3=poi3

poi4=poi4

poi5=poi5

poi6=poi6

poi7=poi7

$\mathrm{n} 1=\mathrm{n} 1$

$\mathrm{n} 2=\mathrm{n} 2$

n3 $=$ n3

rat1 $=$ rat 1

rat2=rat2

rat3 $=$ rat3

gname $=$ gname

gname2=gname2

if tipo $=0$ then

command

gen zon brick p0 gp poi0 p1 gp poi1 p2 gp poi2 p3 gp poi3 p4 gp poi4 \&

p5 gp poi5 p6 gp poi6 p7 gp poi7 size n1 n2 n3 ratio rat1 rat2 rat3 group gname slot 1

end_command

else

if tipo $=1$ then 
command

gen zon wedge p0 gp poi0 p1 gp poi1 p2 gp poi2 p3 gp poi3 p4 gp poi4 \&

$\mathrm{p} 5 \mathrm{gp}$ poi5 size $\mathrm{n} 1 \mathrm{n} 2 \mathrm{n} 3$ ratio rat1 rat2 rat3 group gname slot 1

end_command

else

end_if

end_if

poi0=poi0 +1

poi1 $=$ poi $1+1$

poi2=poi2 +1

poi3=poi3+1

poi5=poi5+1

poi6=poi6 +1

poi7=poi7+1

end

;,*** Definición keypoints ***

def parm_geom

;; Eje del anclaje

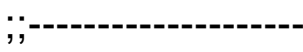

x_cen $=0.0$

y_cen $=0.0$

zz_cen $=0.0$

; Contornos del modelo

$x \_\min =x \_$cen

$x \_\max =x \_c e n+5^{\star} \_b ; 5$ radios de la losa circular_sp

$y \_\min =y \_c e n$ 
y_max $=y \_c e n+5^{*} \_b$

z_top $=z z$ zcen

z_bot=zz_cen - _e - 2* ( h+_a $)$

z_topm $=$ z_top -0.001

z_topp $=$ z_top +0.001

z_botm $=$ z_bot -0.001

z_botp $=$ z_bot +0.001

$x \_\operatorname{minm}=x \_\min -0.001$

$x \_\operatorname{minp}=x \_\min +0.001$

$x \_\operatorname{maxm}=x \_\max -0.001$

$x \_\operatorname{maxp}=x \_\max +0.001$

y_minm $=y \_\min -0.001$

$y \_\operatorname{minp}=y \_\min +0.001$

$y \_\operatorname{maxm}=y \_\max -0.001$

$y \_\operatorname{maxp}=y \_\max +0.001$

z_3 $=$ z_top - _e

z_2 $=$ z_3 - $-h$

z_1 $1=$ z_2 - _a

$\mathrm{nx} 1=6 ; 8$ elementos en la base de la losa circular $(2 \mathrm{~m} / 8=0.25 \mathrm{~m} / \mathrm{elem})$

$\mathrm{n} \times 2=2$

$\mathrm{n} \times 3=15$

$\operatorname{rat} x 1=1.0$

$\operatorname{rat} x 2=1.0$

$\operatorname{rat} \times 3=1.1$ 
$\mathrm{nz} 1=22$

$\mathrm{nz} 2=40 ; 10 \mathrm{~m}$ de long. anclada, a $0.25 \mathrm{~m} / \mathrm{elem}=40$ elem

$\mathrm{nz3}=28 ; 7.1 \mathrm{~m}$ de lomg. lobre, a $0.25 \mathrm{~m} / \mathrm{elem}=28$ elem

$\mathrm{nz} 4=1$

ratz $1=0.92$

ratz2 $=1.00$

ratz3 $=1.00$

ratz4 $=1.00$

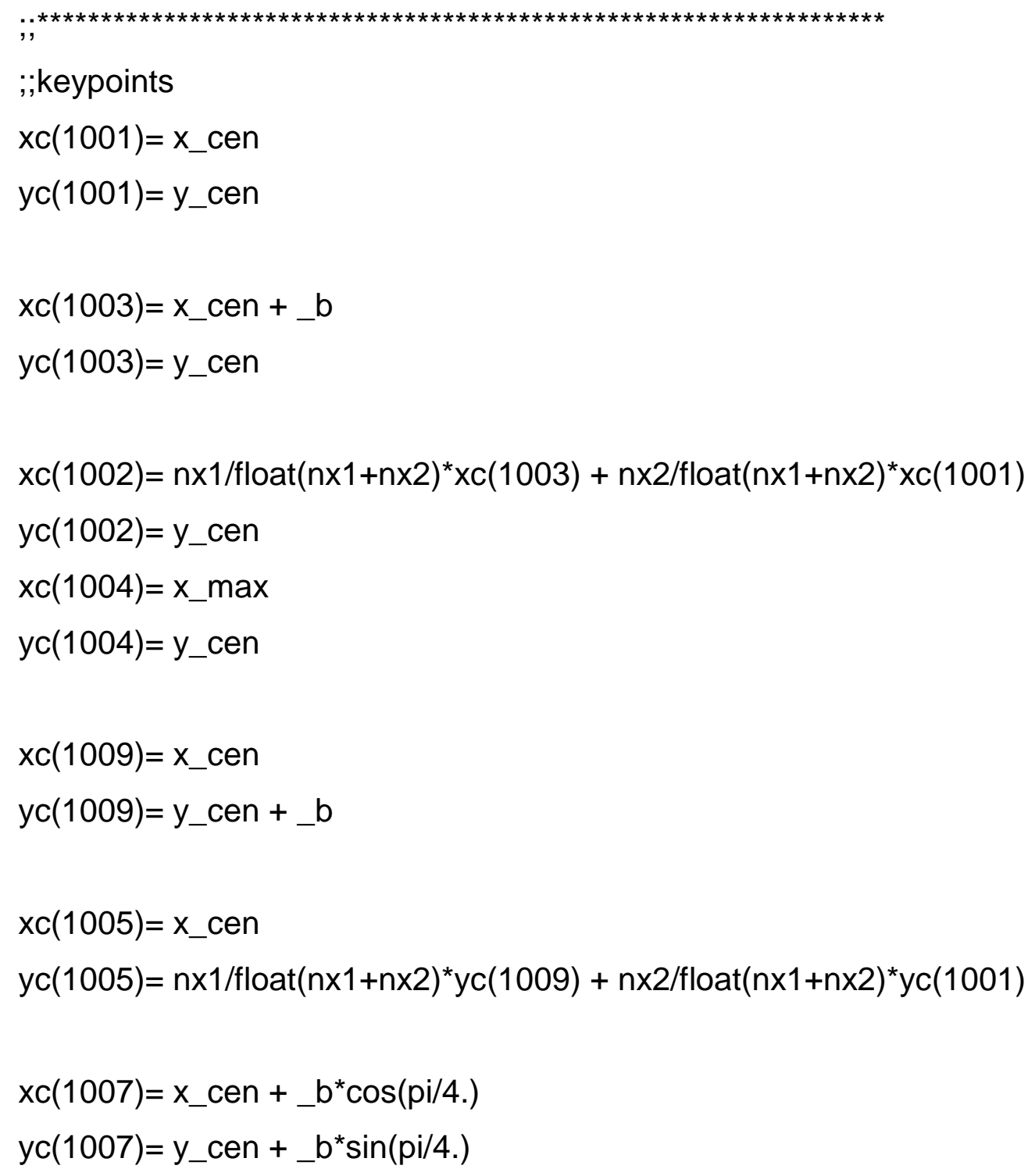


$x c(1006)=x \_c e n+b^{*} \cos (p i / 4 .)^{*}\left(n \times 1^{*} \operatorname{sqrt}(2)\right) / f l o a t\left(n \times 1{ }^{*} \operatorname{sqrt}(2)+n \times 2\right) \quad ; S Q R T$ para tratar de que queden más uniformemente repartidos

$\mathrm{yc}(1006)=y \_c e n+{ }^{*}{ }^{*} \sin (\mathrm{pi} / 4 .)^{*}\left(\mathrm{nx} 1^{*} \operatorname{sqrt}(2)\right) /$ float $\left(\mathrm{n} \times 1{ }^{*} \operatorname{sqrt}(2)+\mathrm{nx} 2\right)$

$x c(1008)=x \_c e n+\left(x \_\max -x \_c e n\right)^{*} \cos (p i / 4$.

$y c(1008)=y \_c e n+\left(x \_m a x-x \_c e n\right)^{*} \sin (p i / 4$.

$x c(1010)=x \_c e n$

$\mathrm{yc}(1010)=y \_\max$

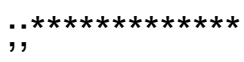

loop j(2,5)

loop $\mathrm{k}(1,10)$

$x c(1000 * j+k)=x c(1000+k)$

$\mathrm{yc}\left(1000^{*} \mathrm{j}+\mathrm{k}\right)=\mathrm{yc}(1000+\mathrm{k})$

end_loop

end_loop

loop $\mathrm{k}(1,10)$

zc $(1000+k)=z \_b o t$

$\mathrm{zc}(2000+\mathrm{k})=\mathrm{z} \_1$

$\mathrm{zc}(3000+\mathrm{k})=\mathrm{z} \_2$

$\mathrm{zc}(4000+\mathrm{k})=\mathrm{z} \_3$

$\mathrm{zc}(5000+\mathrm{k})=\mathrm{z}$ _top

end_loop

loop $\mathrm{h}(1,5)$

loop $\mathrm{i}(1,10)$

$\mathrm{nn}=1000 * \mathrm{~h}+\mathrm{i}$

$\mathrm{XX}=\mathrm{Xc}(\mathrm{nn})$

$\mathrm{yy}=\mathrm{yc}(\mathrm{nn})$

$\mathrm{zz}=\mathrm{zc}(\mathrm{nn})$

command 
gp id nn xx,yy,zz ;;;Flac3D 5.0

end_command

end_loop

end_loop

end

parm_geom

\section{;;GENERACIÓN DE PRIMITIVAS}

;;

;,tipo $=0$ brick, tipo $=1$ wedge

$; ; ; ;$

;; 1000-2000

set tipo=0 poi $=1001$ poi $1=1002$ poi $2=1005$ poi3=2001 poi $4=1006$ poi5=2005 poi6=2002 poi $7=2006$

set gname='terreno' $\mathrm{n} 1=\mathrm{nx} 1 \mathrm{n} 2=\mathrm{nx} 1 \mathrm{n} 3=\mathrm{nz} 1$ rat1=ratx1 rat2=ratx1 rat3=ratz1 genera_prim

set gname='terreno' $n 1=n x 2 \mathrm{n} 2=\mathrm{nx} 1 \mathrm{n} 3=\mathrm{nz} 1$ rat1=ratx2 rat2=ratx1 rat3=ratz1 genera_prim

set gname='terreno' $n 1=n \times 3 n 2=n \times 1 n 3=n z 1$ rat1=ratx3 rat2=ratx1 rat3=ratz1 genera_prim

set tipo $=0$ poi $0=1005$ poi $1=1006$ poi $2=1009$ poi3=2005 poi $4=1007$ poi5=2009 poi6=2006 poi $7=2007$

set gname='terreno' $n 1=n x 1 \mathrm{n} 2=\mathrm{nx} 2 \mathrm{n} 3=\mathrm{nz} 1$ rat1=ratx1 rat2=ratx2 rat3=ratz1 genera_prim

set tipo=0 poi $=1009$ poi $1=1007$ poi $2=1010$ poi $3=2009$ poi $4=1008$ poi $5=2010$ poi6 $=2007$ poi $7=2008$

set gname='terreno' $n 1=n \times 1$ n2=nx3 n3=nz1 rat1=ratx1 rat2=ratx3 rat3=ratz1 genera_prim

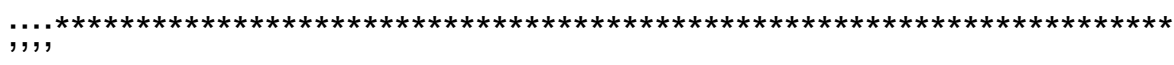

;; 2000-3000 
set tipo $=0$ poi $0=2001$ poi $1=2002$ poi $2=2005$ poi $3=3001$ poi $4=2006$ poi5=3005 poi6=3002 poi7=3006

set gname='terreno' $\mathrm{n} 1=\mathrm{nx} 1 \mathrm{n} 2=\mathrm{nx} 1 \mathrm{n} 3=\mathrm{nz} 2$ rat1=ratx1 rat2=ratx1 rat3=ratz2 genera_prim

set gname='terreno' $n 1=n \times 2 \mathrm{n} 2=\mathrm{nx} 1 \mathrm{n} 3=\mathrm{nz} 2$ rat1=ratx2 rat2=ratx1 rat3=ratz2 genera_prim

set gname='terreno' n1=nx3 n2=nx1 n3=nz2 rat1=ratx3 rat2=ratx1 rat3=ratz2 genera_prim

set tipo $=0$ poi $0=2005$ poi $1=2006$ poi $=2009$ poi3=3005 poi4=2007 poi5=3009 poi6=3006 poi7 $=3007$

set gname='terreno' $\mathrm{n} 1=\mathrm{nx} 1 \mathrm{n} 2=\mathrm{nx} 2 \mathrm{n} 3=\mathrm{nz} 2$ rat1=ratx1 rat2=ratx2 rat3=ratz2 genera_prim

set tipo=0 poi0 $=2009$ poi $1=2007$ poi $2=2010$ poi $3=3009$ poi $4=2008$ poi $5=3010$ poi6=3007 poi $7=3008$

set gname='terreno' n1=nx1 n2=nx3 n3=nz2 rat1=ratx1 rat2=ratx3 rat3=ratz2 genera_prim

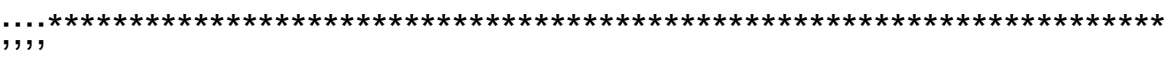

;; 3000-4000

set tipo $=0$ poi $0=3001$ poi $1=3002$ poi $=3005$ poi $3=4001$ poi $4=3006$ poi5=4005 poi6 $=4002$ poi $7=4006$

set gname='terreno' $n 1=n x 1 \mathrm{n} 2=\mathrm{nx} 1 \mathrm{n} 3=\mathrm{nz} 3$ rat1=ratx1 rat2=ratx1 rat3=ratz3 genera_prim

set gname='terreno' $n 1=n \times 2 n 2=n \times 1 n 3=n z 3$ rat $1=$ ratx2 rat2=ratx 1 rat3=ratz3 genera_prim

set gname='terreno' $n 1=n \times 3$ n2=nx1 n3=nz3 rat1=ratx3 rat2=ratx1 rat3=ratz3 genera_prim

set tipo $=0$ poi $0=3005$ poi $1=3006$ poi $2=3009$ poi3 $=4005$ poi $4=3007$ poi5=4009 poi6=4006 poi $7=4007$

set gname='terreno' $\mathrm{n} 1=\mathrm{nx} 1 \mathrm{n} 2=\mathrm{nx} 2 \mathrm{n} 3=\mathrm{nz} 3$ rat1=ratx1 rat2=ratx2 rat3=ratz3 genera_prim

set tipo $=0$ poi $0=3009$ poi $1=3007$ poi $2=3010$ poi $3=4009$ poi $4=3008$ poi $5=4010$ poi6 $=4007$ poi $7=4008$ 
set gname='terreno' $\mathrm{n} 1=\mathrm{nx} 1 \mathrm{n} 2=\mathrm{nx} 3 \mathrm{n} 3=\mathrm{nz} 3$ rat1=ratx1 rat2=ratx3 rat3=ratz3 genera_prim

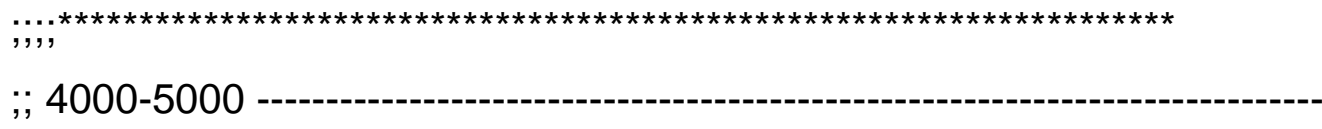

set tipo $=0$ poi $=4001$ poi $1=4002$ poi $=4005$ poi3 $=5001$ poi $4=4006$ poi $5=5005$ poi6 $=5002$ poi $7=5006$

set gname='losa' n1=nx1 n2=nx1 n3=nz4 rat1=ratx1 rat2=ratx1 rat3=ratz4 genera_prim

set gname='losa' $n 1=n \times 2 n 2=n \times 1 n 3=n z 4$ rat1=ratx2 rat2=ratx1 rat3=ratz4 genera_prim

set gname='aire' $n 1=n \times 3 \mathrm{n} 2=\mathrm{nx} 1 \mathrm{n} 3=\mathrm{nz} 4$ rat1=ratx3 rat2=ratx1 rat3=ratz4 genera_prim

set tipo $=0$ poi $0=4005$ poi $1=4006$ poi $2=4009$ poi $3=5005$ poi $4=4007$ poi $5=5009$ poi6 $=5006$ poi $7=5007$

set gname='losa' $n 1=n \times 1 n 2=n \times 2 n 3=n z 4$ rat1=ratx1 rat2=ratx2 rat3=ratz4 genera_prim

set tipo $=0$ poi $=4009$ poi $1=4007$ poi $2=4010$ poi $3=5009$ poi $4=4008$ poi $5=5010$ poi6 $=5007$ poi $7=5008$

set gname='aire' $n 1=n \times 1 \mathrm{n} 2=\mathrm{n} \times 3 \mathrm{n} 3=\mathrm{nz} 4$ rat1=ratx1 rat2=ratx3 rat3=ratz4 genera_prim

def morf1

$\mathrm{xx} 1=\mathrm{xc}(\mathrm{p} 1)$

yy $1=\mathrm{yc}(\mathrm{p} 1)$

$\mathrm{xx} 2=\mathrm{xc}(\mathrm{p} 2)$

yy2=yc $(p 2)$

$x \times 3=x c(p 3)$

yy3 $=y c(p 3)$

$\mathrm{xx} 4=\mathrm{xc}(\mathrm{p} 4)$

yy4=yc $(p 4)$ 


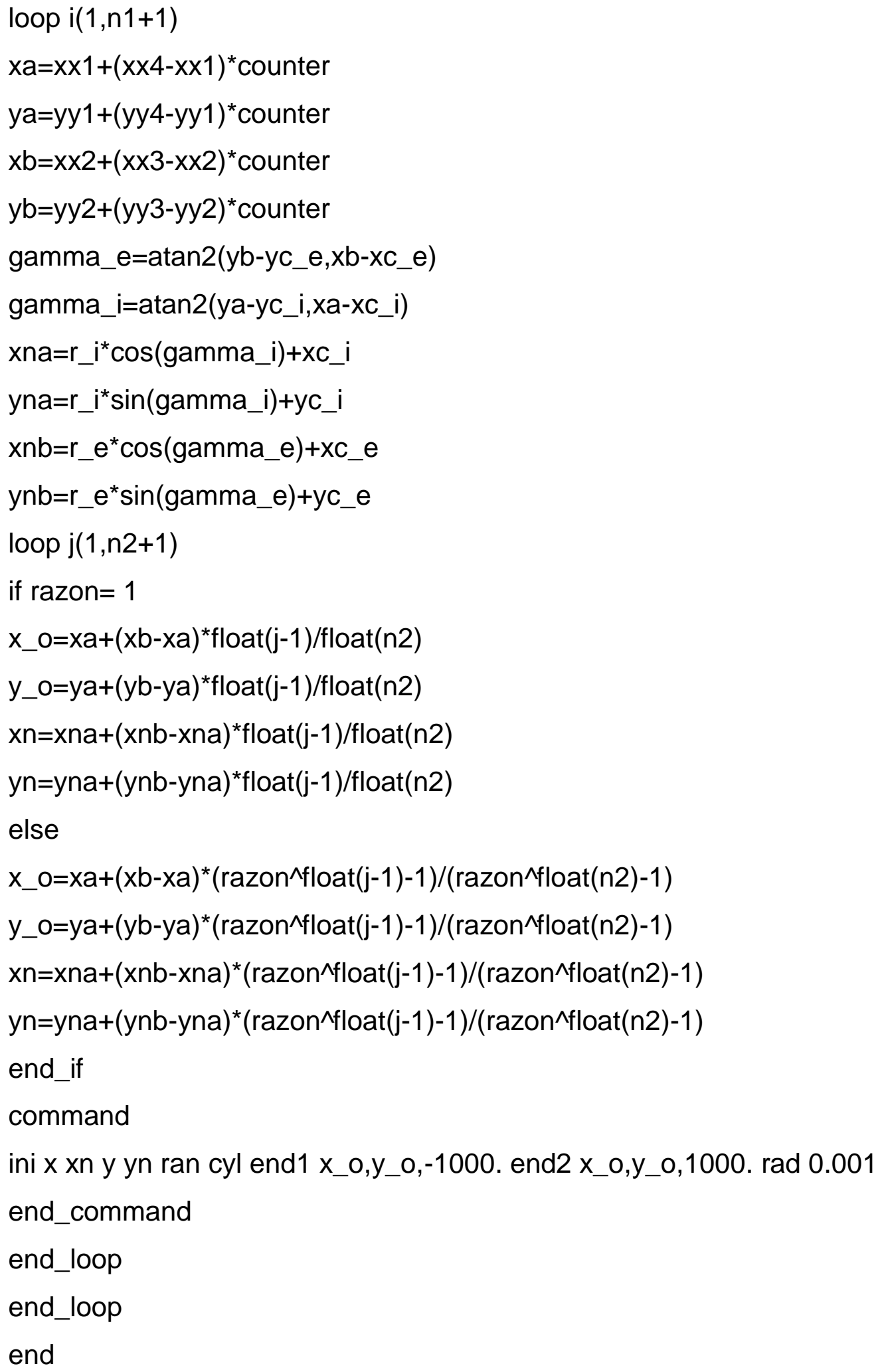


set $x c \_e=x \_c e n \quad y c \_e=y \_c e n \quad x c \_i=x \_c e n \quad y c \_i=y \_c e n \quad r \_e=x \_m a x \quad r \_i=\_b$ razon=ratx 3

morf1

set $\mathrm{p} 1=1007 \mathrm{p} 2=1008 \mathrm{p} 3=1010 \mathrm{p} 4=1009$

set $\mathrm{n} 1=\mathrm{n} \times 1 \mathrm{n} 2=\mathrm{n} \times 3$

set $x c \_e=x \_c e n \quad y c \_e=y \_c e n \quad x c \_i=x \_c e n \quad y c \_i=y \_c e n \quad r \_e=x \_m a x \quad r \_i=\_b$ razon=ratx 3

morf1

def morf3

$x \times 1=x c(p 1)$

$\mathrm{yy} 1=\mathrm{yc}(\mathrm{p} 1)$

$x \times 2=x c(p 2)$

$\mathrm{yy} 2=\mathrm{yc}(\mathrm{p} 2)$

$x \times 3=x c(p 3)$

yy3=yc(p3)

$\mathrm{xx} 4=\mathrm{xc}(\mathrm{p} 4)$

yy4=yc(p4)

loop $\mathrm{i}(1, \mathrm{n} 1+1)$

counter $=$ float $(\mathrm{i}-1) /$ float $(\mathrm{n} 1)$

$x a=x x 1+(x x 4-x x 1)^{*}$ counter

ya $=y y 1+(y y 4-y y 1)^{*}$ counter

$x b=x x 2+(x x 3-x \times 2)^{*}$ counter

yb=yy2+(yy3-yy2)*counter

gamma_e=atan2(yb-yc_e,xb-xc_e)

gamma_i=atan2(ya-yc_i,xa-xc_i)

$x n b=r \_e^{*} \cos ($ gamma_e $)+x c \_e$

$y n b=r \_e^{*} \sin \left(g a m m a \_e\right)+y c \_e$

loop $\mathrm{j}(1, \mathrm{n} 2+1)$

if razon $=1$ then

x_o $=x a+(x b-x a)^{*}$ float $(j-1) / f l o a t(n 2)$ 
y_o $=y a+(y b-y a)^{*}$ float $(j-1) / f l o a t(n 2)$

$\mathrm{xn}=\mathrm{xa}+(\mathrm{xnb}-\mathrm{xa})^{*}$ float $(\mathrm{j}-1) / \mathrm{float}(\mathrm{n} 2)$

$\mathrm{yn}=\mathrm{ya}+(\mathrm{ynb}-\mathrm{ya})^{*}$ float $(\mathrm{j}-1) / \mathrm{float}(\mathrm{n} 2)$

else

$x \_0=x a+(x b-x a)^{*}($ razon^float $(j-1)-1) /($ razon^float $(n 2)-1)$

$y_{-} 0=y a+(y b-y a)^{*}($ razon^float $(j-1)-1) /($ razon^float $(\mathrm{n} 2)-1)$

$x n=x a+(x n b-x a)^{*}($ razon^float $(j-1)-1) /($ razon^float $(n 2)-1)$

yn $=y a+(y n b-y a)^{*}\left(\right.$ razon $^{\wedge}$ float $\left.(j-1)-1\right) /($ razon^float(n2)-1)

end if

command

ini $x$ xn y yn ran cyl end1 $x \_0, y \_0,-999$ end2 $x \_0, y \_0,999$ rad 1e-4

end_command

end_loop

end_loop

end

; Hacia bóveda

set $p 1=1002 p 2=1003$ p3=1007 p4=1006

set $n 1=n \times 1 n 2=n \times 2$ razon $=1$

set $x c \_i=x \_c e n$ yc_e $=y \_c e n ~ r \_e=\_b$

morf3

set $p 1=1006 p 2=1007 p 3=1009 p 4=1005$

set $n 1=n \times 1 n 2=n \times 2$ razon $=1$

set $x c \_i=x \_c e n y c \_e=y \_c e n t r e=\_b$

morf3

def_archivo

fname ='Anclaje_'+string(_caso)+'_gr.sav'

end

_archivo

save @fname 
RET

anclaje_calculo.f3dat

def_archivo

fname ='Anclaje_'+string(_caso)+'_gr.sav'

end

_archivo

rest @fname

config creep

; Contorno izdo.

fix $x$ ran $x$ x_minm, $x \_$minp ;contorno ixdo

fix y ran $x$ x_minm,x_minp y y_maxm,y_maxp

; Contorno anterior

fix y ran y y_minm,y_minp

fix $x$ ran y y_minm,y_minp $x$ x_maxm,x_maxp

; Contorno cilíndrico exterior

apply nvel 0 ran cyl end1 $x \_c e n, y \_c e n, z \_b o t p$ end $2 x \_c e n, y \_c e n, z \_t o p m$ rad

x_maxm,x_maxp \&

$x$ x_minp,x_maxp y y_minp,y_maxp

; Contorno inferior

fix x y z ran z z_botm,z_botp 
; Interfase contacto losa-terreno

gen separate face ran group 'losa' group 'terreno' slot 1

gen separate face ran group 'losa' group 'aire' slot 1

int 1 face ran group 'losa' slot 1 z z_3

int 1 pro kn 1 e10 ks 1 e8

model null ran group 'aire' slot 1

; Modelo de comportamiento

model mech elastic ran group 'losa' slot 1

model mech burgers ran group 'terreno' slot 1

; Propiedades de cálculo

pro dens $=\_$gamma1 bulk=_bulk1 \&

kshear=_kshear1 kvis=_kvisc1 mshear=_mshear1 mvis $=$ mvisc1 ran group 'terreno' slot 1

pro dens_dens $V$ young _young $V$ pois_pois $V$ ran group 'losa' slot 1

; Colocación del cable

def pone_cable

idc $=$ idc

zini=z_top +0.01

yini=y_cen

xini $=x \_$cen

$x 2=x i n i$

y2=yini

z2=zini - _e - _ H 
$x$ fin $=x 2$

yfin=y2

zfin $=z 2$ - _A

nnseg $1=1$

nnseg2=nz2 ; coincide con la discretización del modelo

yinim=yini-0.005

yinip=yini+0.005

xinim $=x 1-0.005$

xinip $=x 1+0.005$

command

sel cable id idc beg xini,yini,zini end x2,y2,z2 nseg nnseg1

sel cable id idc beg x2,y2,z2 end xfin,yfin,zfin nseg nnseg2

sel cable id idc pro e $=$ e_cable $x c a=\_$area gr_k 1e11 gr_c $=\_$grout yten $=\_q$ Lim

end_command

end

set idc $=100$

pone_cable

def carga_cable

_cargaf $=\_$carga*factor

_reac $=-$ _carga ${ }^{*}$ factor $/\left(\mathrm{pi}^{*} \mathrm{~b}^{\wedge} 2\right)$

command

sel node apply remove

sel node apply force 0,0,_cargaf system global ran sph cen xini,yini,zini rad 0.001

apply remove szz

apply szz_reac ran group 'losa' slot 1 z z_top

ini szz_reac ran group 'losa' slot 1

end_command

end 
hist unbal

hist ratio

hist sel node zdis x_cen,y_cen,[z_top + 0.01] ; Cabeza del cable

hist gp zd x_cen,y_cen,z_top ; Cara superior Losa

hist gp zd id 4001 ; Cara inferior losa

hist gp zd x_cen,y_cen,[z_3-0.01] ; Cara superior terreno

hist gp zd x_cen,y_cen,z_2

hist gp zd x_cen,y_cen,z_1

hist gp zd 1.0,y_cen,[z_3-0.01] ; Cara superior terreno

hist gp zd 2.0,y_cen,[z_3-0.01]

hist gp zd 3.0,y_cen,[z_3-0.01]

hist gp zd 4.0,y_cen,[z_3-0.01]

hist gp zd 5.0,y_cen,[z_3-0.01]

def carrera

pntnd=nd_near(x_cen,y_cen, (z_top + 0.01))

pntgp=gp_near(x_cen,y_cen,z_top)

carrera $=$ nd_rdisp(pntnd,1,3)-gp_zdisp(pntgp)

end

hist carrera

;;**Inicialización

set creep off

set factor 0.1

carga_cable

solve ratio $5 \mathrm{e}-5$

save tmp1.sav

set factor 0.15 
carga_cable

solve ratio $5 \mathrm{e}-5$

save tmp2.sav

set factor 0.30

carga_cable

solve ratio $5 \mathrm{e}-5$

save tmp3.sav

set factor 0.65

carga_cable

solve ratio 1 e- 5

save tmp4.sav

set factor 1.0

carga_cable

solve ratio 1 e- 6

def_archivo

fname ='Anclaje_'+string(_caso)+'_0.sav'

end

_archivo

save@fname

rest @fname

;

$;^{* \star * * \star *}$ Largo plazo (creep)

set creep on

set $\mathrm{cr}$ dt auto on

set cr mindt 2 maxdt 5000.

set $\mathrm{cr}$ dt 2

set cr lfob 9e-5 ufob 1e-4 
set cr lat 20

set $\mathrm{cr}$ Imul $1.01 \mathrm{cr}$ umul .1

def t_dias

t_dias=crtime/86400. ;;tiempo en días

t_mes $=$ crtime $/ 86400 . / 30.417$

deltat $=$ crdt

end

hist t_dias

hist t_mes

hist deltat

solve age $\left[30^{\star} 24^{\star} 3600\right] ; 1$ mes

def_archivo

fname ='Anclaje_'+string(_caso)+'_1m.sav'

end

_archivo

save@fname

solve age $\left[60^{\star} 24^{\star} 3600\right] ; 2$ mes

def_archivo

fname ='Anclaje_'+string(_caso)+'_2m.sav'

end

_archivo

save@fname

solve age $[120 * 24 * 3600] ; 6$ mes

def_archivo

fname ='Anclaje_'+string(_caso)+'_6m.sav'

end

_archivo

save@fname 
solve age $\left[1 * 365^{\star} 24^{*} 3600\right] ; 1$ año

def_archivo

fname ='Anclaje_'+string(_caso)+'_1y.sav'

end

_archivo

save@fname

solve age $\left[2^{*} 365^{\star} 24^{\star} 3600\right] ; 2$ año

def_archivo

fname ='Anclaje_'+string(_caso)+'_2y.sav'

end

_archivo

save@fname

solve age $\left[5^{\star} 365^{\star} 24^{\star} 3600\right] ; 5$ año

def_archivo

fname ='Anclaje_'+string(_caso)+'_5y.sav'

end

_archivo

save@fname

solve age $\left[10^{\star} 365^{\star} 24^{\star} 3600\right] ; 10$ año

def_archivo

fname ='Anclaje_'+string(_caso)+'_10y.sav'

end

archivo

save@fname

solve age $\left[20^{\star} 365^{\star} 24^{\star} 3600\right] ; 20$ año

def_archivo

fname ='Anclaje_'+string(_caso)+'_20y.sav'

end

archivo 
save@fname

RET

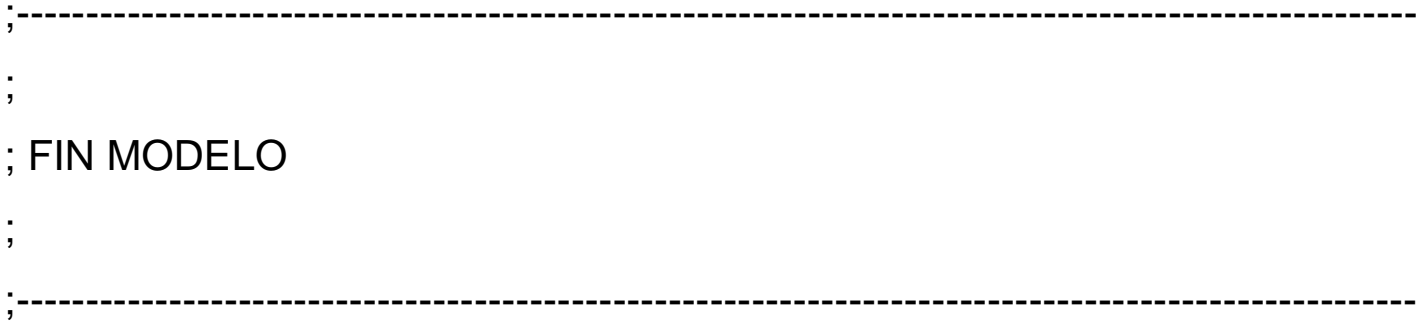

anclaje_datos.f3dat

new

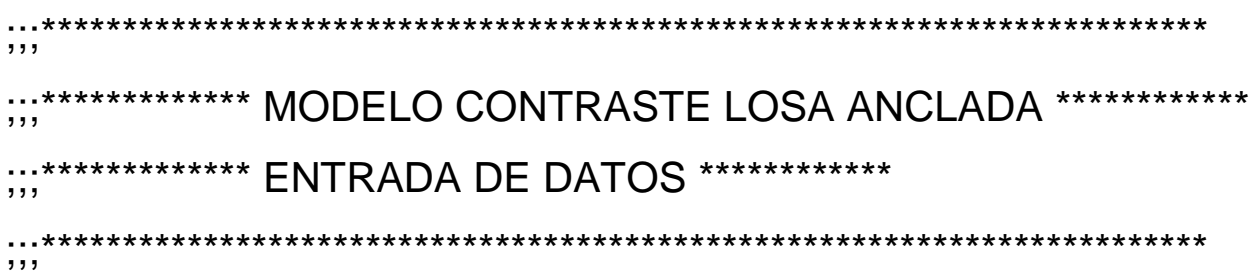

set fish safe off

def nombra_caso ;;número de caso para ir cambiando el nombre a los archivos -caso $=2$

end

nombra_caso

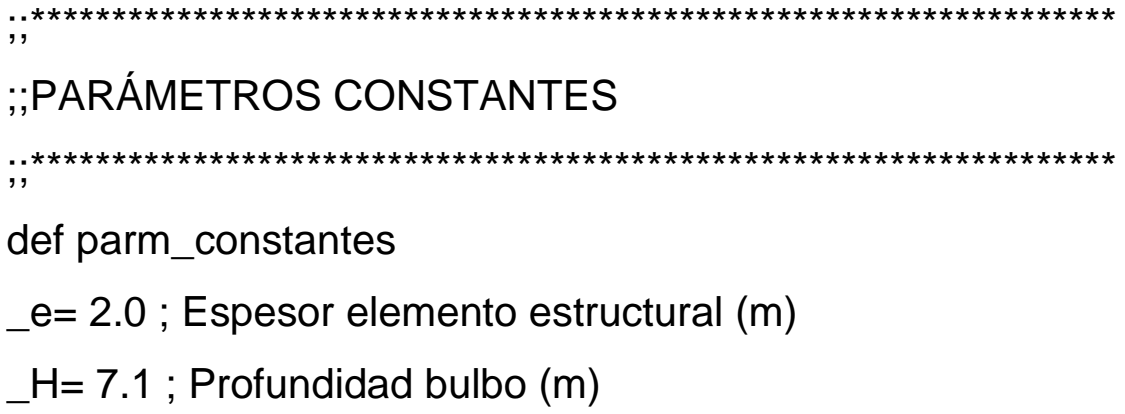


$\_2 b=4.0$; Ancho elemento estructural $(\mathrm{m})$

_a $=10.0$; Longitud anclada (m)

$b=\_2 b / 2$.

end

parm_constantes

;

;;PARÁMETROS TERRENO

;"

def parm_terreno

_ee1=30e6 ; Módulo de Young terreno 1 (Pa)

_pois $1=0.3$; coficiente Poisson terreno 1

_gamma1=1800. ; Densidad terreno 1 (N/m2)

_bulk1=_ee1/(3.*(1.-2.* _pois1))

_mshear1=_ee1/(2.*(1.+_pois1)); Módulo de corte de Maxwell es el elástico

_mvisc1=2.1E10*1e6; Viscosidad Maxwell terreno $1\left(\mathrm{~N}^{*} \mathrm{~s} / \mathrm{m}\right)$

_kshear1=1.22e6 ; Módulo de corte de Kelvin

_kvisc1 $=5.28 \mathrm{e}^{\star} 1 \mathrm{e} 6 ;$ Viscosidad Kelvin terreno $1\left(\mathrm{~N}^{*} \mathrm{~s} / \mathrm{m}\right)$

end

parm_terreno

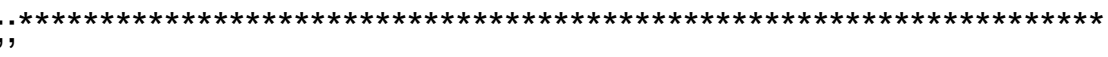

;;PARÁMETROS HORMIGÓN

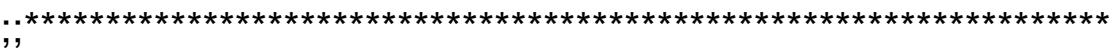

def parm_hormigon

_densV=2500. ; Densidad viga atado (N/m2)

_youngV=6e9 ; Módulo de Young viga atado (Pa)

; OJO minorado a 200 E_suelo para mejorar time-step

_pois $V=0.2$; Coeficiente Poisson viga atado

end

parm_hormigon 
;;

;:PARÁMETROS ANCLAJES

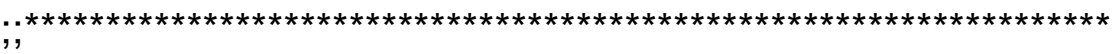

def parm_cables

_carga $=200 \mathrm{e} 3$; Carga tesado

_area $=660$ e- 6 ; Sección cable $(\mathrm{m} 2)$

_qLim=1e20 ; límite elástico cable $(\mathrm{N})$

_grout $=40 \mathrm{e} 4$; adherencia lechada $(\mathrm{N} / \mathrm{m})$

e_cable=195e9; módulo elasticidad del cable (Pa)

end

parm_cable

ret 
I-B: MODELO NUMÉRICO DE PANTALLA ANCLADA EN TERRENO CON FLUENCIA 


\section{Archivo pantalla_datos.f3dat}

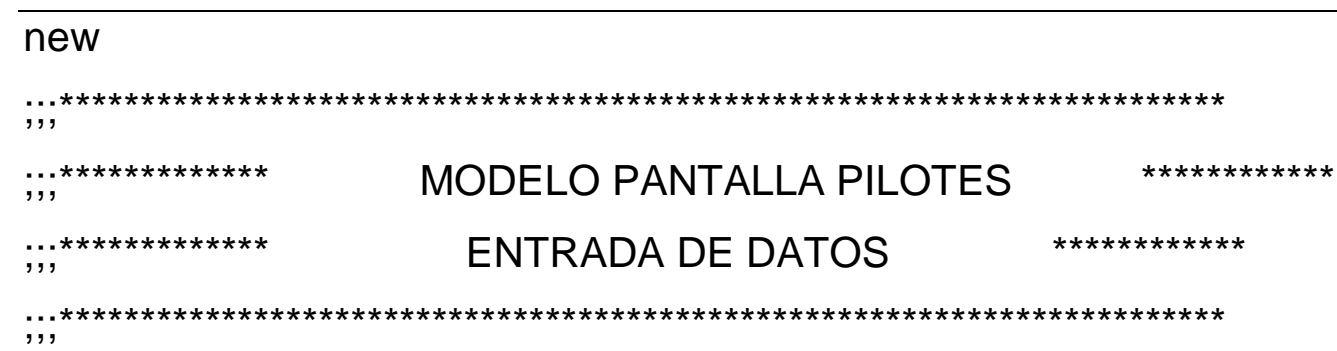

set fish safe off

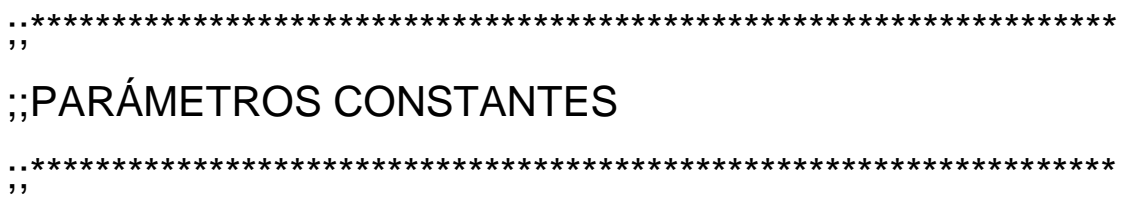

def parm_constantes

z_viga $=24.12$

;;cota superior viga de atado $(\mathrm{m})$

\begin{tabular}{|c|c|}
\hline$A=1.50$ & ;;altura viga de atado $(\mathrm{m})$ \\
\hline $\mathrm{A} 2=0.75$ & ;;distancia cota sup viga atado a inicio anclaje (m) \\
\hline$B=1.40$ & ;;ancho viga de atado $(\mathrm{m})$ \\
\hline$s p=0.70$ & ;;separación pilotes (m) \\
\hline sa $=1.40$ & ;;separación eje anclajes (m) \\
\hline _diam $=0.90$ & ;;diámetro pilote $(\mathrm{m})$ \\
\hline $\mathrm{dnf}=2.12$ & ;;distancia nivel freático a viga atado $(\mathrm{m})$ \\
\hline$F=200.0$ & ;;tracción de bloque $(\mathrm{kN})$ \\
\hline beta $=20.0$ & ;;inclinación anclaje $(\stackrel{\circ}{*})$ \\
\hline LLI $=8.50$ & ;;longitud libre (m) \\
\hline$L a=10.0$ & $; ;$ longitud anclada (m) \\
\hline L $\mathrm{L}=29.0$ & $;$;longitud entre muros (m) \\
\hline el=1.10 & ;;espesor losa $(\mathrm{m})$ \\
\hline eR $=0.90$ & ;;espesor relleno granular $(\mathrm{m})$ \\
\hline alfa=2./1. & ;;pendiente terreno superior \\
\hline
\end{tabular}

end

parm_constantes 
;:PARÁMETROS TERRENO

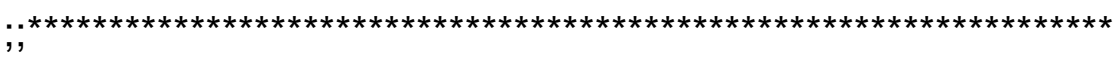

def nombra_caso ;;número de caso para ir cambiando el nombre a los archivos caso $=4$

end

nombra_caso

def parm_terreno

_cc1=6e4 ;;cohesión terreno $1(\mathrm{~Pa})$

_ff1=28.0 ; ;fricción terreno $1(\stackrel{\circ}{*})$

_gamma1=1800. ;;densidad terreno $1(\mathrm{~N} / \mathrm{m} 2)$

_ee1=60.0e6 ;;módulo de Young terreno $1(\mathrm{~Pa})$

_pois $1=0.3 \quad ; ;$ coficiente Poisson terreno 1

_tens $1=\_c c 1 / 10$.

_mvisc1 $=2 \mathrm{e} 8{ }^{*} 1 \mathrm{e} 6 \quad$;;viscosidad Maxwell terreno $1\left(\mathrm{~N}^{*} \mathrm{~s} / \mathrm{m}\right)$

_kvisc1=2e8*1e6 ;;viscosidad Kelvin terreno $1\left(\mathrm{~N}^{*} \mathrm{~s} / \mathrm{m}\right)$

_mshear1=_ee1/(2.*(1.+_pois 1$))$

_kshear1=_ee1/(2.*(1.+_pois 1$))$

_bulk1=_ee1/(3.*(1.-2.. _pois 1$))$

_cc2 $=6 \mathrm{e} 4 \quad$;;cohesión terreno $2(\mathrm{~Pa})$

_ff2 $=20.0 \quad$;;fricción terreno $2(\stackrel{\circ}{\circ})$

_gamma2 $=1800 . \quad$;idensidad terreno $2(\mathrm{~N} / \mathrm{m} 2)$

_ee2=30.0e6 ;;módulo de Young terreno $2(\mathrm{~Pa})$

_pois2 $=0.35 \quad$;;coficiente Poisson terreno 2

_tens $2=\_c c 2 / 10$.

_mvisc2 $=1 \mathrm{e} 8^{*} 1 \mathrm{e} 6 \quad ; ;$ viscosidad Maxwell terreno $1\left(\mathrm{~N}^{*} \mathrm{~s} / \mathrm{m}\right)$

_kvisc2 $=1$ e8*1e6 ;;viscosidad Kelvin terreno $1\left(\mathrm{~N}^{*} \mathrm{~s} / \mathrm{m}\right)$

_mshear2=_ee2/(2.*(1.+_pois2))

_kshear2=_ee2/(2. ${ }^{*}\left(1 .+\_\right.$pois 2$\left.)\right)$ 


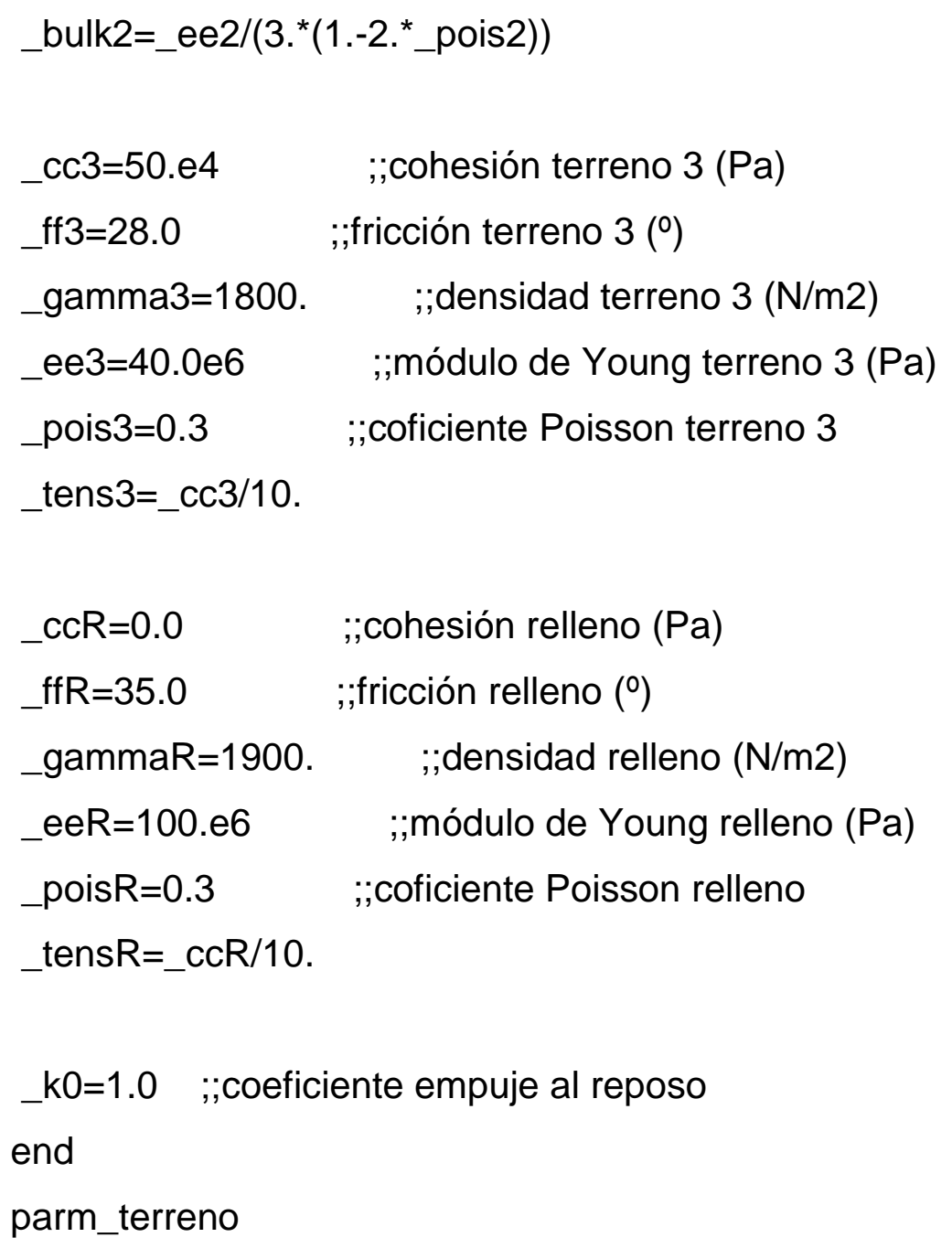




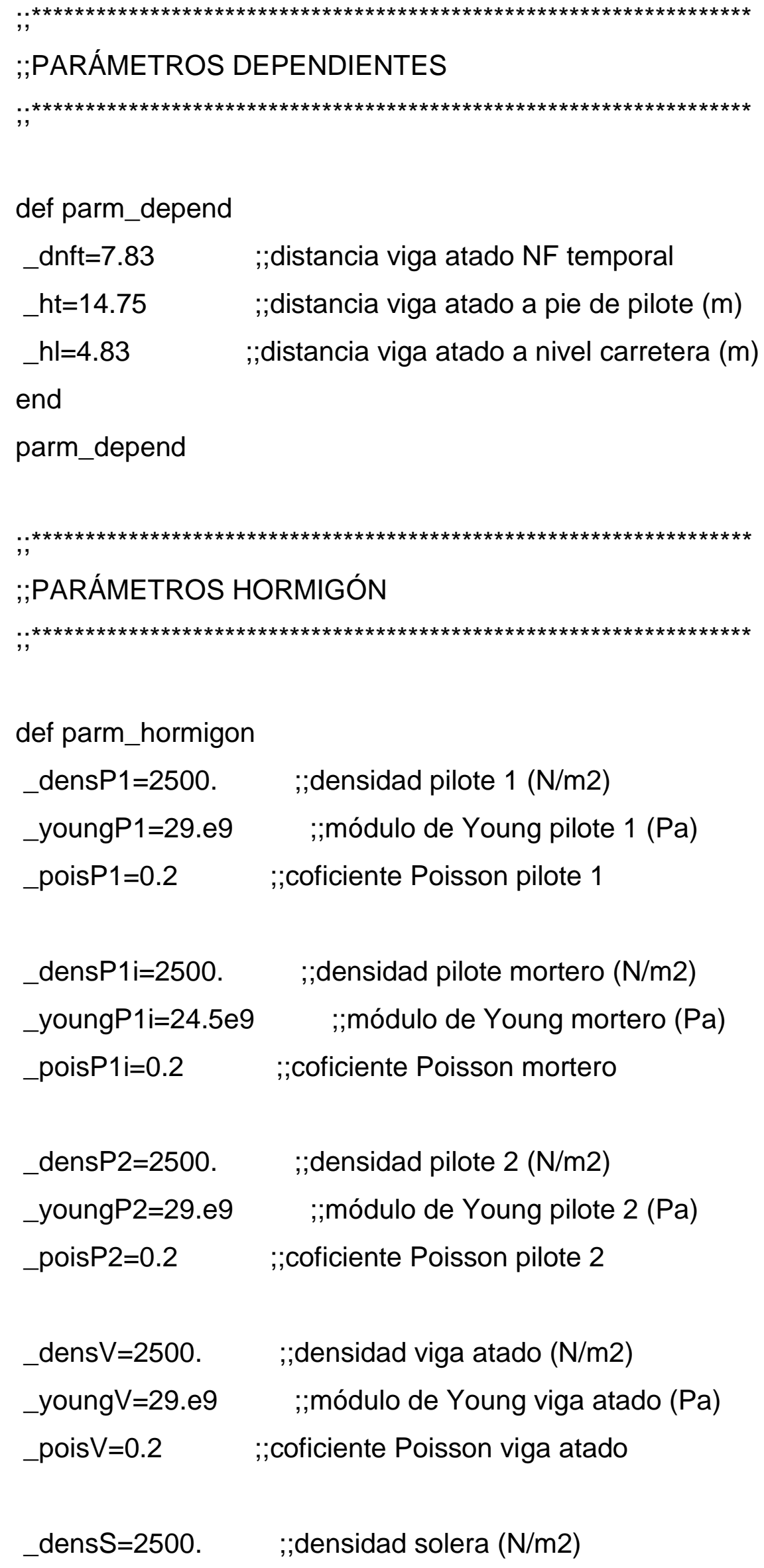


_young $=30.9$ e9 ;;módulo de Young solera $(\mathrm{Pa})$

_poisS $=0.2 \quad$;;coficiente Poisson solera

end

parm_hormigon

;

;;PARÁMETROS ANCLAJES

;

def parm_cables

_area $=660 . / 1 \mathrm{e} 6 \quad$;;area anclaje $(\mathrm{m} 2)$

_carga=1200e3 ;;límite elástico (N)

_grout $=40$ e4 ;;adherencia lechada

e_cable=195e9 ;;módulo elasticidad del cable

end

parm_cables

ca pantalla_malla.f3dat 


\section{Archivo pantalla_malla.f3dat}

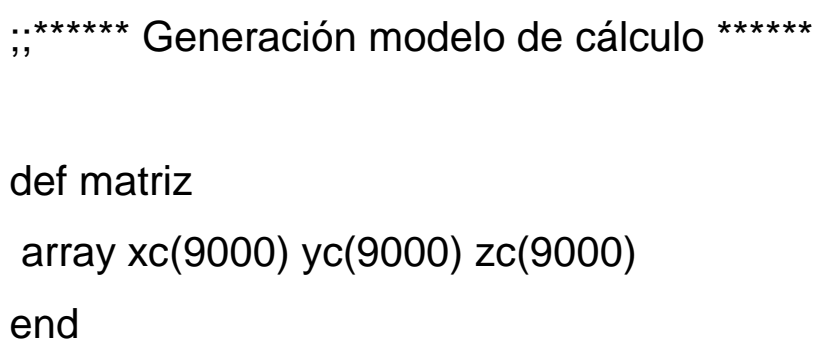




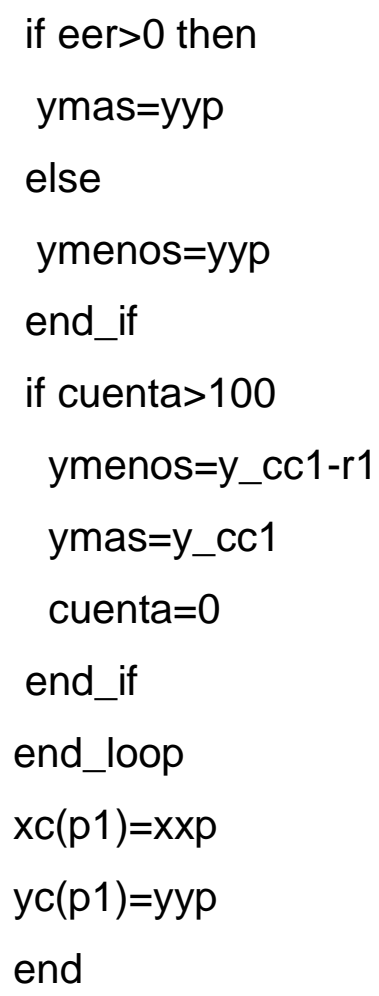

def genera_prim

poi0=poi0

poi1=poi1

poi2=poi2

poi3=poi3

poi4=poi4

poi5=poi5

poi6=poi6

poi7=poi7

$\mathrm{n} 1=\mathrm{n} 1$

$\mathrm{n} 2=\mathrm{n} 2$

n3=n3

rat1=rat1

rat2=rat2

rat3=rat3

gname=gname 
gname2=gname2

if tipo $=0$ then

command

gen zon brick p0 gp poi0 p1 gp poi1 p2 gp poi2 p3 gp poi3 p4 gp poi4 \&

p5 gp poi5 p6 gp poi6 $\mathrm{p} 7 \mathrm{gp}$ poi7 size $\mathrm{n} 1 \mathrm{n} 2 \mathrm{n} 3$ ratio rat1 rat2 rat3 group gname slot 1

end_command

else

if tipo $=1$ then

command

gen zon wedge p0 gp poi0 p1 gp poi1 p2 gp poi2 p3 gp poi3 p4 gp poi4 \&

p5 gp poi5 size $n 1 \mathrm{n} 2 \mathrm{n} 3$ ratio rat1 rat2 rat3 group gname slot 1

end_command

else

end_if

end_if

poi0 $=$ poi0 +1

poi $1=$ poi $1+1$

poi2=poi2 +1

poi3=poi3 +1

poi4=poi4+1

poi5=poi5+1

poi6=poi6 +1

poi $7=$ poi7 +1

end

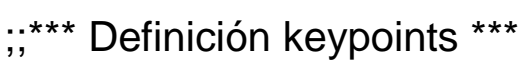

def parm_geom

;CCentro del pilote central

;;-------------------- 


$$
\begin{aligned}
& x \_c e n=0.0 \\
& y \_c e n=0.0 \\
& z z \_c e n=z \text { _viga }
\end{aligned}
$$

;Contornos del modelo

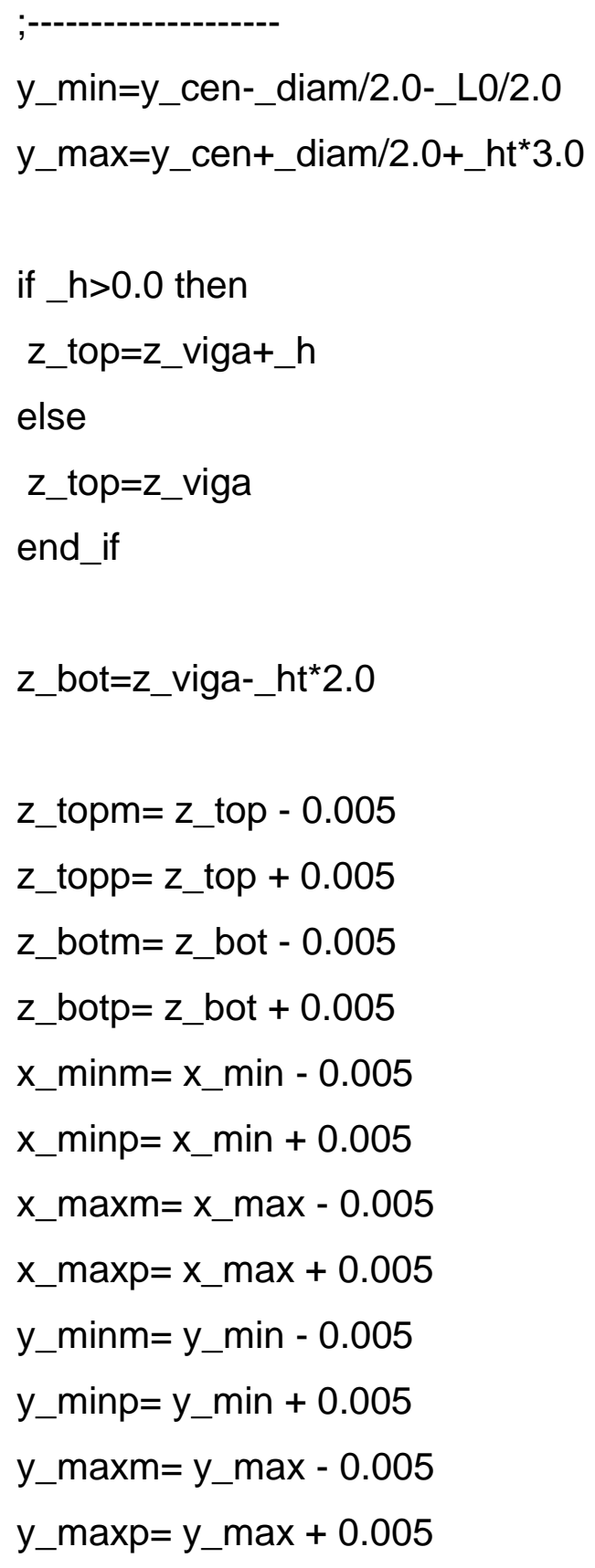




$$
\begin{aligned}
& \text { y_1=y_cen+_B/2.0 } \\
& \text { y_3=y_cen-_B/2.0 } \\
& \text { z_9=z_viga-_A } \\
& \text { z_8=z_viga-_dnf } \\
& \text { z_7=z_viga-_e1 } \\
& \text { z_6=z_viga-_hl } \\
& \text { z_5=z_7-_e2 } \\
& \text { z_4=z_6-_el } \\
& \text { z_3=z_4-_eR } \\
& \text { z_2=z_viga-_dnft } \\
& \text { z_1 =z_viga-_ht }
\end{aligned}
$$

if $z \_2>z$ _ 3 then

z_2=z_3-1.0

end_if

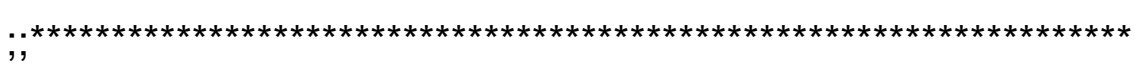

$$
\begin{aligned}
& \text { nx1 }=4 \\
& n \times 2=2 \\
& n \times 3=n x 1 \\
& \operatorname{ratx} 1=1.0 \\
& \operatorname{ratx} 2=1.0 \\
& \text { ratx3=1.0 } \\
& \text { ny2 }=2 \\
& \text { ny3 }=4 \\
& \text { ny4 }=\text { ny3 } \\
& \text { ny5 }=\text { ny2 } \\
& \text { ny6 }=\text { int }\left(\left(y \_2-y \_1\right) / 0.5\right) \\
& \text { ny7 }=10 \\
& \text { ny1 }=\text { int }\left(\left(y \_3-y \_m i n\right) / 0.75\right)
\end{aligned}
$$




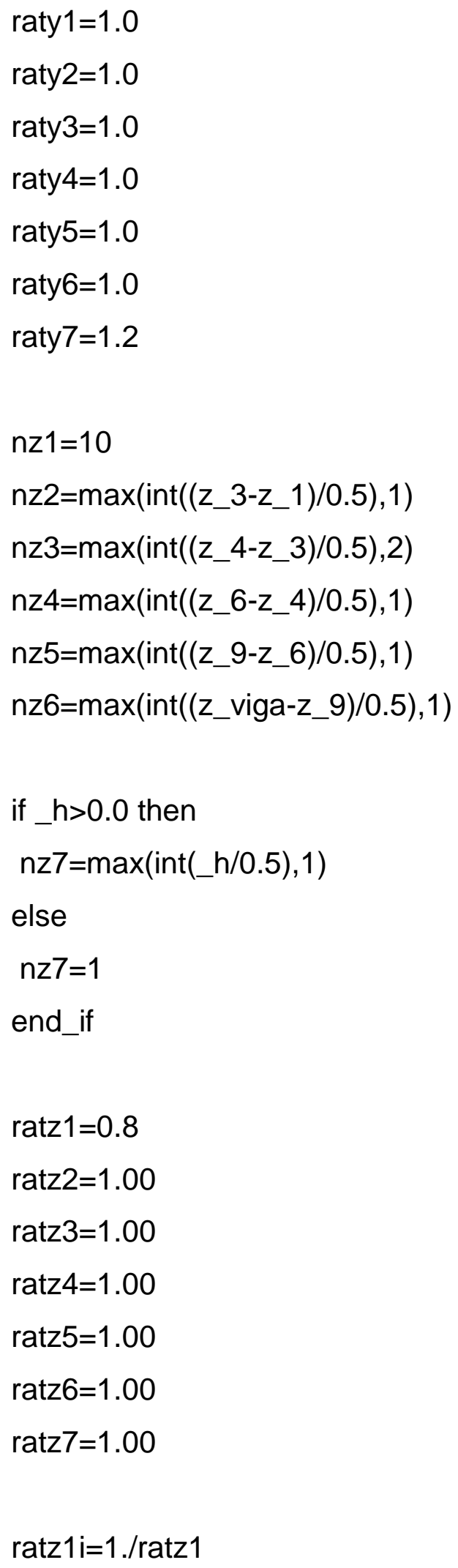


;;keypoints

$$
\begin{aligned}
& \text { loop } \mathrm{k}(18,25) \\
& x c(1000+k)=x \_c e n \\
& \text { end_loop } \\
& \text { yc }(1018)=y \_ \text {min } \\
& \text { yc (1019) }=y \_3 \\
& \text { yc }(1021)=y \_c e n \\
& \text { yc }(1020)=y \text { _cen-_diam } / 2.0 \\
& \text { yc }(1022)=y \_c e n+\text { diam } / 2.0 \\
& \mathrm{yc}(1023)=\mathrm{y} \_1 \\
& \text { yc (1024)=y_2 } \\
& \mathrm{yc}(1025)=\mathrm{y} \_\max
\end{aligned}
$$

$$
\text { loop } \mathrm{k}(1,8)
$$

$$
\begin{aligned}
& x c(1000+k)=x \_ \text {min } \\
& y c(1000+k)=y c(1000+k+17) \\
& \text { end_loop }
\end{aligned}
$$

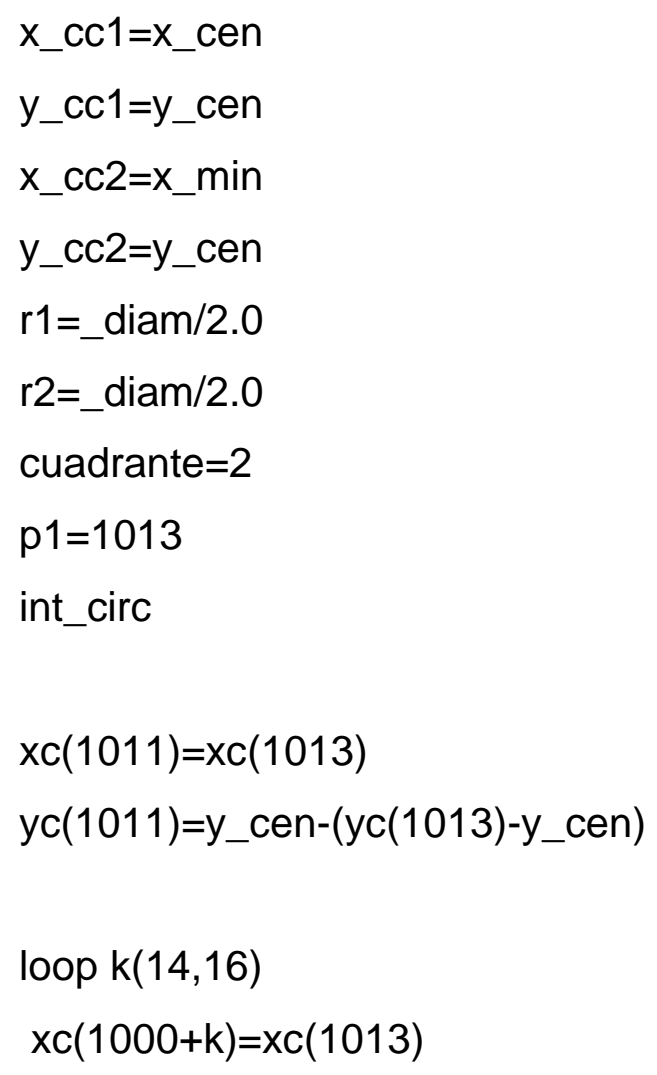




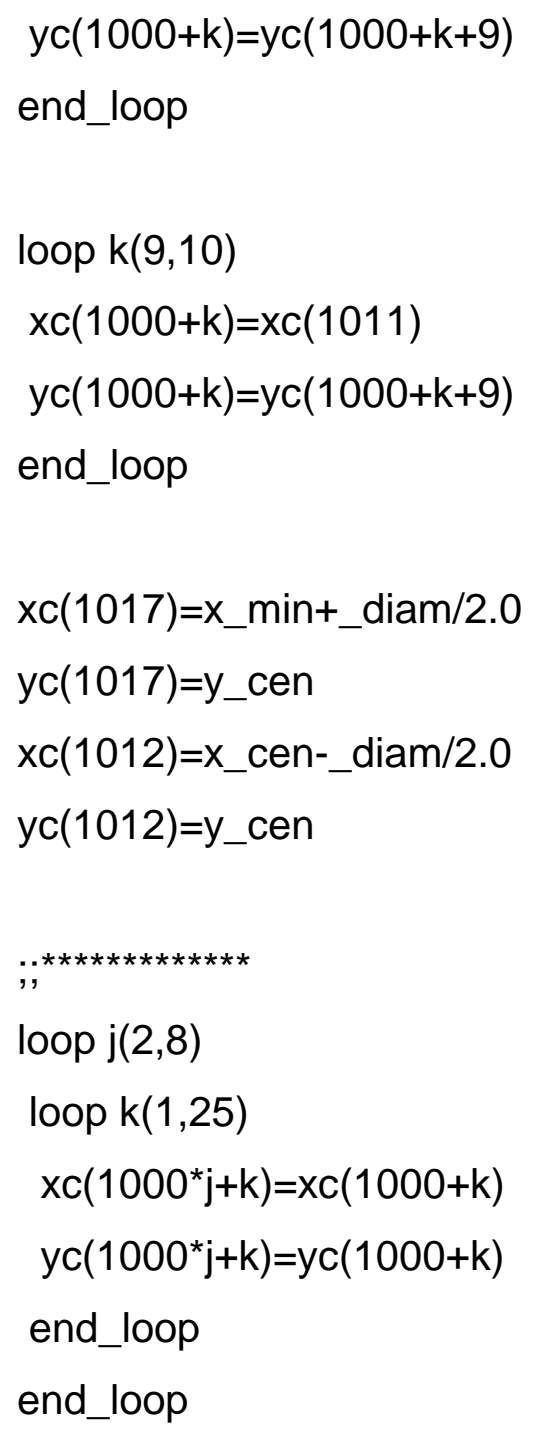




$$
\begin{aligned}
& \mathrm{yc}(8014)=\mathrm{yc}(7014)+(\mathrm{zc}(8014)-\mathrm{zc}(7014))^{*} \text { _alfa } \\
& \mathrm{yc}(8023)=\mathrm{yc}(7023)+(\mathrm{zc}(8023)-\mathrm{zc}(7023))^{*} \text { _alfa }
\end{aligned}
$$

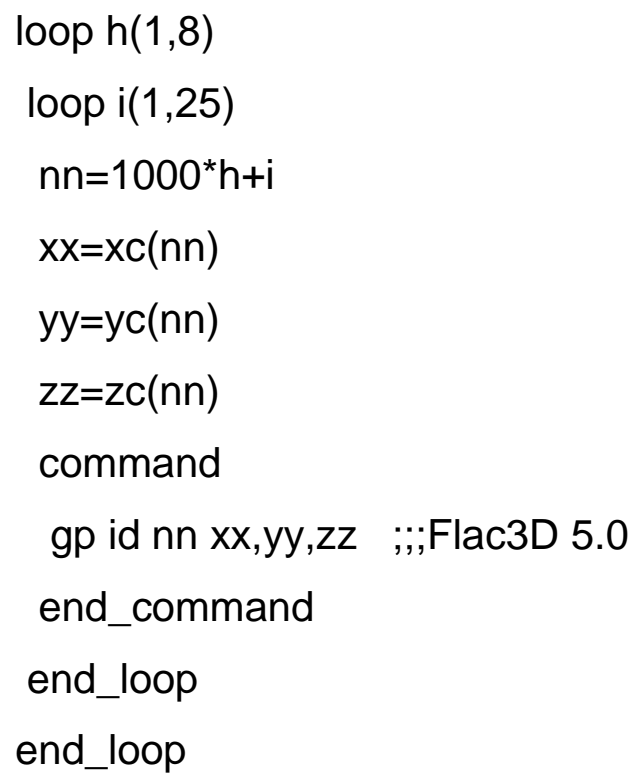

end

parm_geom

\section{;;GENERACIÓN DE PRIMITIVAS}

$;$

;,tipo=0 brick, tipo=1 wedge

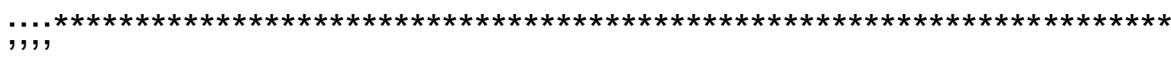

;; 1000-2000

set tipo=0 poi $=1001$ poi $1=1009$ poi $2=1002$ poi $3=2001$ poi $4=1010$ poi5=2002 poi6=2009 poi $7=2010$

set gname='terreno' $n 1=n \times 1$ n2=ny $1 \mathrm{n} 3=\mathrm{nz} 1$ rat $1=$ ratx 1 rat2=raty 1 rat $3=$ ratz 1 genera_prim

set gname='terreno' $n 1=n x 1 \mathrm{n} 2=\mathrm{ny} 2 \mathrm{n} 3=\mathrm{nz} 1$ rat $1=$ ratx1 rat2=raty 2 rat3=ratz1 genera_prim

set gname='terreno' $n 1=n x 1 \mathrm{n} 2=\mathrm{ny} 3 \mathrm{n} 3=\mathrm{nz} 1$ rat $1=$ ratx 1 rat2=raty3 rat3=ratz1 
genera_prim

set gname='terreno' $n 1=n x 1 \mathrm{n} 2=\mathrm{ny} 4 \mathrm{n} 3=\mathrm{nz} 1$ rat $1=$ ratx 1 rat2=raty 4 rat3=ratz 1 genera_prim

set gname='terreno' $\mathrm{n} 1=\mathrm{nx} 1 \mathrm{n} 2=\mathrm{ny} 5 \mathrm{n} 3=\mathrm{nz} 1$ rat1=ratx1 rat2=raty 5 rat3=ratz1 genera_prim

set gname='terreno' $\mathrm{n} 1=\mathrm{nx} 1 \mathrm{n} 2=\mathrm{ny} 6 \mathrm{n} 3=\mathrm{nz} 1$ rat $1=$ ratx 1 rat2=raty 6 rat $3=$ ratz 1 genera_prim

set gname='terreno' $n 1=n x 1 \mathrm{n} 2=\mathrm{ny} 7 \mathrm{n} 3=\mathrm{nz} 1$ rat1=ratx1 rat2=raty 7 rat3=ratz1 genera_prim

set tipo=1 poi0=2017 poi $1=2012$ poi2=1017 poi3=2011 poi4=1012 poi5=1011 set gname='terreno' n1=nx2 n2=nz1 n3=ny3 rat1=ratx2 rat2=ratz1i rat3=raty3 genera_prim

set tipo=1 poi0=2012 poi $1=2017$ poi2=1012 poi3=2013 poi4=1017 poi5=1013 set gname='terreno' n1=nx2 n2=nz1 n3=ny4 rat1=ratx2 rat2=ratz1i rat3=raty4 genera_prim

set tipo=0 poi0=1009 poi $1=1018$ poi $2=1010$ poi3=2009 poi $4=1019$ poi5=2010 poi6=2018 poi7=2019

set gname='terreno' n1=nx3 n2=ny1 n3=nz1 rat1=ratx3 rat2=raty 1 rat3=ratz1 genera_prim

set gname='terreno' $n 1=n \times 3$ n2=ny2 n3=nz1 rat1=ratx3 rat2=raty2 rat3=ratz1 genera_prim

set tipo $=0$ poi $=1011$ poi $=1020$ poi $=1017$ poi3=2011 poi $4=1021$ poi5=2017 poi6=2020 poi7=2021

set gname='terreno' $n 1=n \times 3$ n2=ny3 n3=nz1 rat1=ratx3 rat2=raty3 rat3=ratz1 genera_prim

set tipo $=0$ poi $0=1017$ poi $1=1021$ poi $2=1013$ poi $3=2017$ poi $4=1022$ poi $5=2013$ poi6=2021 poi7=2022

set gname='terreno' $n 1=n \times 3$ n2=ny4 n3=nz1 rat1=ratx3 rat2=raty4 rat3=ratz1 genera_prim 
set tipo=0 poi $=1013$ poi $1=1022$ poi $2=1014$ poi $3=2013$ poi $4=1023$ poi $5=2014$ poi6=2022 poi $7=2023$

set gname='terreno' n1=nx3 n2=ny5 n3=nz1 rat1=ratx3 rat2=raty5 rat3=ratz1 genera_prim

set gname='terreno' $n 1=n \times 3 n 2=n y 6 n 3=n z 1$ rat $1=$ ratx3 rat2=raty 6 rat $3=$ ratz 1 genera_prim

set gname='terreno' $n 1=n \times 3$ n2=ny $7 \mathrm{n} 3=\mathrm{nz} 1$ rat $1=$ ratx3 rat2=raty 7 rat3=ratz1 genera_prim

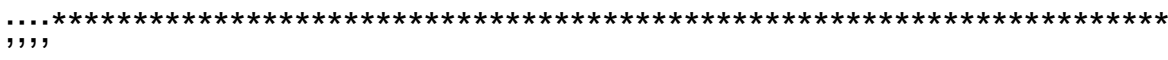

;; 2000-3000

set tipo=0 poi0=2001 poi $1=2009$ poi2=2002 poi3=3001 poi $4=2010$ poi5=3002 poi6=3009 poi7=3010

set gname='terreno' $n 1=n x 1 \mathrm{n} 2=\mathrm{ny} 1 \mathrm{n} 3=\mathrm{nz} 2$ rat1=ratx1 rat2=raty1 rat3=ratz2 genera_prim

set gname='terreno' $n 1=n x 1 \mathrm{n} 2=\mathrm{ny} 2 \mathrm{n} 3=\mathrm{nz} 2$ rat1=ratx1 rat2=raty2 rat3=ratz2 genera_prim

set gname='pilote1' $\mathrm{n} 1=\mathrm{nx} 1 \mathrm{n} 2=\mathrm{ny} 3 \mathrm{n} 3=\mathrm{nz} 2$ rat1=ratx1 rat2=raty3 rat3=ratz2 genera_prim

set gname='pilote1' $n 1=n x 1$ n2=ny4 n3=nz2 rat1=ratx1 rat2=raty4 rat3=ratz2 genera_prim

set gname='terreno' $\mathrm{n} 1=\mathrm{nx} 1 \mathrm{n} 2=\mathrm{ny} 5 \mathrm{n} 3=\mathrm{nz} 2$ rat $1=$ ratx 1 rat2=raty 5 rat3=ratz2 genera_prim

set gname='terreno' $\mathrm{n} 1=\mathrm{nx} 1 \mathrm{n} 2=\mathrm{ny} 6 \mathrm{n} 3=\mathrm{nz} 2$ rat $1=$ ratx 1 rat2=raty 6 rat $3=$ ratz2 genera_prim

set gname='terreno' $n 1=n x 1 \mathrm{n} 2=\mathrm{ny} 7 \mathrm{n} 3=\mathrm{nz} 2$ rat $1=$ ratx 1 rat2=raty 7 rat3=ratz2 genera_prim

set tipo=1 poi0=3017 poi $1=3012$ poi2=2017 poi3=3011 poi4=2012 poi5=2011 set gname='pilote $1 l^{\prime} \mathrm{n} 1=\mathrm{nx} 2 \mathrm{n} 2=\mathrm{nz} 2 \mathrm{n} 3=\mathrm{ny} 3$ rat1=ratx2 rat2=ratz2 rat3=raty3 genera_prim

set tipo $=1$ poi $0=3012$ poi $1=3017$ poi $2=2012$ poi3=3013 poi $4=2017$ poi5=2013 
set gname='pilote1l' n1=nx2 n2=nz2 n3=ny4 rat1=ratx2 rat2=ratz2 rat3=raty4 genera_prim

set tipo=0 poi $=2009$ poi $1=2018$ poi $2=2010$ poi3=3009 poi $4=2019$ poi5=3010 poi6=3018 poi7 $=3019$

set gname='terreno' $n 1=n \times 3$ n2=ny $1 \mathrm{n} 3=\mathrm{nz} 2$ rat $1=$ ratx3 rat2=raty 1 rat3=ratz2 genera_prim

set gname='terreno' $n 1=n \times 3$ n2=ny2 n3=nz2 rat1=ratx3 rat2=raty2 rat3=ratz2 genera_prim

set tipo $=0$ poi $0=2011$ poi $1=2020$ poi $2=2017$ poi3=3011 poi $4=2021$ poi5=3017 poi6=3020 poi7 $=3021$

set gname='pilote 2 ' n1=nx3 n2=ny3 n3=nz2 rat1=ratx3 rat2=raty3 rat3=ratz2 genera_prim

set tipo $=0$ poi $0=2017$ poi $1=2021$ poi $2=2013$ poi3 $=3017$ poi $4=2022$ poi5 $=3013$ poi6=3021 poi7 $=3022$

set gname='pilote2' n1=nx3 n2=ny4 n3=nz2 rat1=ratx3 rat2=raty4 rat3=ratz2 genera_prim

set tipo $=0$ poi $=2013$ poi $1=2022$ poi $=2014$ poi3=3013 poi4=2023 poi5=3014 poi6=3022 poi7 $=3023$

set gname='terreno' $\mathrm{n} 1=\mathrm{nx} 3 \mathrm{n} 2=\mathrm{ny} 5 \mathrm{n} 3=\mathrm{nz} 2$ rat $1=$ ratx3 rat2=raty5 rat3=ratz2 genera_prim

set gname='terreno' $n 1=n \times 3$ n2=ny 6 n3=nz2 rat1=ratx3 rat2=raty 6 rat3=ratz2 genera_prim

set gname='terreno' $n 1=n \times 3$ n2=ny $7 \mathrm{n} 3=\mathrm{nz} 2$ rat $1=$ ratx3 rat2=raty 7 rat3=ratz2 genera_prim

$;, ; ;$

;; 3000-4000

set tipo $=0$ poi $0=3001$ poi $1=3009$ poi $2=3002$ poi $3=4001$ poi $4=3010$ poi5=4002 poi6=4009 poi $7=4010$

set gname='relleno' $\mathrm{n} 1=\mathrm{nx} 1 \mathrm{n} 2=\mathrm{ny} 1 \mathrm{n} 3=\mathrm{nz} 3$ rat1=ratx1 rat2=raty 1 rat3=ratz3 genera_prim 
set gname='relleno' $n 1=n x 1 \mathrm{n} 2=\mathrm{ny} 2 \mathrm{n} 3=\mathrm{nz} 3$ rat1=ratx1 rat2=raty 2 rat3=ratz3 genera_prim

set gname='pilote 1 ' n1=nx1 n2=ny3 n3=nz3 rat1=ratx1 rat2=raty3 rat3=ratz3 genera_prim

set gname='pilote 1 ' $\mathrm{n} 1=\mathrm{nx} 1 \mathrm{n} 2=\mathrm{ny} 4 \mathrm{n} 3=\mathrm{nz} 3$ rat1=ratx1 rat2=raty4 rat3=ratz3 genera_prim

set gname='terreno' $n 1=n x 1$ n2=ny5 n3=nz3 rat1=ratx1 rat2=raty5 rat3=ratz3 genera_prim

set gname='terreno' $n 1=n x 1 \mathrm{n} 2=\mathrm{ny} 6 \mathrm{n} 3=\mathrm{nz} 3$ rat $1=$ ratx 1 rat2=raty 6 rat3=ratz3 genera_prim

set gname='terreno' $n 1=n \times 1$ n2=ny 7 n3=nz3 rat $1=$ ratx 1 rat2=raty 7 rat3=ratz3 genera_prim

set tipo=1 poi $=4017$ poi $1=4012$ poi $2=3017$ poi3=4011 poi4=3012 poi5=3011 set gname='pilote 1 I' $n 1=n \times 2$ n2=nz3 n3=ny3 rat1=ratx2 rat2=ratz3 rat3=raty3 genera_prim

set tipo $=1$ poi $0=4012$ poi $1=4017$ poi2=3012 poi3=4013 poi4=3017 poi5=3013 set gname='pilote1l' $n 1=n \times 2$ n2=nz3 n3=ny4 rat1=ratx2 rat2=ratz3 rat3=raty4 genera_prim

set tipo=0 poi $=3009$ poi $1=3018$ poi $2=3010$ poi $3=4009$ poi $4=3019$ poi $5=4010$ poi6 $=4018$ poi $=4019$

set gname='relleno' n1=nx3 n2=ny1 n3=nz3 rat1=ratx3 rat2=raty 1 rat3=ratz3 genera_prim

set gname='relleno' n1=nx3 n2=ny2 n3=nz3 rat1=ratx3 rat2=raty 2 rat3=ratz3 genera_prim

set tipo=0 poi $=3011$ poi $1=3020$ poi $2=3017$ poi $3=4011$ poi $4=3021$ poi $5=4017$ poi6 $=4020$ poi $7=4021$

set gname='pilote2' n1=nx3 n2=ny3 n3=nz3 rat1=ratx3 rat2=raty3 rat3=ratz3 genera_prim

set tipo=0 poi $0=3017$ poi $1=3021$ poi $2=3013$ poi3=4017 poi $4=3022$ poi5=4013 poi6 $=4021$ poi $7=4022$ 
set gname='pilote2' $n 1=n \times 3$ n2=ny4 n3=nz3 rat1=ratx3 rat2=raty4 rat3=ratz3 genera_prim

set tipo $=0$ poi $0=3013$ poi $1=3022$ poi $2=3014$ poi $3=4013$ poi $4=3023$ poi5=4014 poi6=4022 poi $=4023$

set gname='terreno' $\mathrm{n} 1=\mathrm{nx} 3 \mathrm{n} 2=\mathrm{ny} 5 \mathrm{n} 3=\mathrm{nz} 3$ rat1=ratx3 rat2=raty5 rat3=ratz3 genera_prim

set gname='terreno' $\mathrm{n} 1=\mathrm{nx} 3 \mathrm{n} 2=\mathrm{ny} 6 \mathrm{n} 3=\mathrm{nz} 3$ rat1=ratx3 rat2=raty 6 rat3=ratz3 genera_prim

set gname='terreno' $n 1=n \times 3$ n2=ny $7 \mathrm{n} 3=\mathrm{nz} 3$ rat $1=$ ratx3 rat2=raty 7 rat3=ratz3 genera_prim

$; ; ; ;$

;; 4000-5000

set tipo $=0$ poi $0=4001$ poi $1=4009$ poi $2=4002$ poi3 $=5001$ poi $4=4010$ poi $5=5002$ poi6 $=5009$ poi $7=5010$

set gname='solera' $\mathrm{n} 1=\mathrm{nx} 1 \mathrm{n} 2=\mathrm{ny} 1 \mathrm{n} 3=\mathrm{nz} 4$ rat1=ratx1 rat2=raty1 rat3=ratz4 genera_prim

set gname='solera' n1=nx1 n2=ny2 n3=nz4 rat1=ratx1 rat2=raty2 rat3=ratz4 genera_prim

set gname='pilote 1 ' $\mathrm{n} 1=\mathrm{nx} 1 \mathrm{n} 2=\mathrm{ny} 3 \mathrm{n} 3=\mathrm{nz} 4$ rat1=ratx1 rat2=raty3 rat3=ratz4 genera_prim

set gname='pilote 1 ' $\mathrm{n} 1=\mathrm{nx} 1 \mathrm{n} 2=\mathrm{ny} 4 \mathrm{n} 3=\mathrm{nz} 4$ rat1=ratx1 rat2=raty4 rat3=ratz4 genera_prim

set gname='terreno' $n 1=n x 1$ n2=ny5 n3=nz4 rat1=ratx1 rat2=raty5 rat3=ratz4 genera_prim

set gname='terreno' $n 1=n x 1 \mathrm{n} 2=\mathrm{ny} 6 \mathrm{n} 3=\mathrm{nz} 4$ rat $1=$ ratx 1 rat2=raty 6 rat3=ratz4 genera_prim

set gname='terreno' $n 1=n x 1 \mathrm{n} 2=\mathrm{ny} 7 \mathrm{n} 3=\mathrm{nz} 4$ rat1=ratx1 rat2=raty7 rat3=ratz4 genera_prim

set tipo $=1$ poi0 $=5017$ poi $1=5012$ poi2 $=4017$ poi3=5011 poi4=4012 poi5=4011 set gname='pilote1l' n1=nx2 n2=nz4 n3=ny3 rat1=ratx2 rat2=ratz4 rat3=raty3 
genera_prim

set tipo $=1$ poi $0=5012$ poi $1=5017$ poi $2=4012$ poi $3=5013$ poi $4=4017$ poi $5=4013$ set gname='pilote1l' n1=nx2 n2=nz4 n3=ny4 rat1=ratx2 rat2=ratz4 rat3=raty4 genera_prim

set tipo $=0$ poi $0=4009$ poi $1=4018$ poi $2=4010$ poi $3=5009$ poi $4=4019$ poi $5=5010$ poi6 $=5018$ poi $7=5019$

set gname='solera' $\mathrm{n} 1=\mathrm{nx} 3 \mathrm{n} 2=\mathrm{ny} 1 \mathrm{n} 3=\mathrm{nz} 4$ rat1 $=$ ratx3 rat2=raty1 rat3=ratz4 genera_prim

set gname='solera' $\mathrm{n} 1=\mathrm{nx} 3 \mathrm{n} 2=\mathrm{ny} 2 \mathrm{n} 3=\mathrm{nz} 4$ rat1=ratx3 rat2=raty2 rat3=ratz4 genera_prim

set tipo $=0$ poi $=4011$ poi $1=4020$ poi $2=4017$ poi $3=5011$ poi $4=4021$ poi $5=5017$ poi6 $=5020$ poi $7=5021$

set gname='pilote 2 ' n1=nx3 n2=ny3 n3=nz4 rat1=ratx3 rat2=raty3 rat3=ratz4 genera_prim

set tipo $=0$ poi $0=4017$ poi $1=4021$ poi $2=4013$ poi $3=5017$ poi $4=4022$ poi $5=5013$ poi6=5021 poi7 $=5022$

set gname='pilote2' n1=nx3 n2=ny4 n3=nz4 rat1=ratx3 rat2=raty4 rat3=ratz4 genera_prim

set tipo $=0$ poi $0=4013$ poi $1=4022$ poi $2=4014$ poi $3=5013$ poi $4=4023$ poi $5=5014$ poi6 $=5022$ poi $7=5023$

set gname='terreno' $n 1=n \times 3$ n2=ny5 n3=nz4 rat1=ratx3 rat2=raty5 rat3=ratz4 genera_prim

set gname='terreno' $n 1=n \times 3$ n2=ny 6 n3=nz4 rat1=ratx3 rat2=raty 6 rat3=ratz4 genera_prim

set gname='terreno' $n 1=n \times 3 n 2=n y 7 n 3=n z 4$ rat1=ratx3 rat2=raty 7 rat3=ratz4 genera_prim

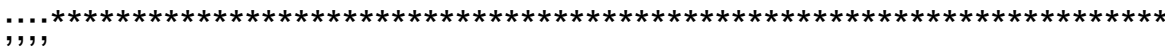

;; 5000-6000 
set tipo $=0$ poi $=5001$ poi $1=5009$ poi $=5002$ poi $3=6001$ poi $4=5010$ poi $5=6002$ poi6=6009 poi $7=6010$

set gname='excava' $n 1=n x 1$ n2=ny $1 \mathrm{n} 3=\mathrm{nz} 5$ rat1=ratx1 rat2=raty 1 rat3=ratz5 genera_prim

set gname='excava' $n 1=n x 1$ n2=ny2 n3=nz5 rat1=ratx1 rat2=raty 2 rat3=ratz5 genera_prim

set gname='pilote 1 ' $\mathrm{n} 1=\mathrm{nx} 1 \mathrm{n} 2=\mathrm{ny} 3 \mathrm{n} 3=\mathrm{nz} 5$ rat1=ratx1 rat2=raty3 rat3=ratz5 genera_prim

set gname='pilote1' $\mathrm{n} 1=\mathrm{nx} 1 \mathrm{n} 2=\mathrm{ny} 4 \mathrm{n} 3=\mathrm{nz} 5$ rat1=ratx1 rat2=raty4 rat3=ratz5 genera_prim

set gname='terreno' $\mathrm{n} 1=\mathrm{nx} 1 \mathrm{n} 2=\mathrm{ny} 5 \mathrm{n} 3=\mathrm{nz} 5$ rat $1=$ ratx 1 rat2=raty 5 rat3=ratz5 genera_prim

set gname='terreno' $n 1=n x 1$ n2=ny6 n3=nz5 rat1=ratx1 rat2=raty 6 rat3=ratz5 genera_prim

set gname='terreno' $n 1=n \times 1$ n2=ny $7 \mathrm{n} 3=\mathrm{nz} 5$ rat $1=$ ratx 1 rat2=raty 7 rat3=ratz5 genera_prim

set tipo=1 poi0=6017 poi $1=6012$ poi2=5017 poi3=6011 poi $4=5012$ poi5=5011 set gname='pilote 1 I' n1=nx2 n2=nz5 n3=ny3 rat1=ratx2 rat2=ratz5 rat3=raty3 genera_prim

set tipo $=1$ poi $0=6012$ poi $1=6017$ poi $=5012$ poi3=6013 poi $4=5017$ poi5=5013 set gname='pilote1l' n1=nx2 n2=nz5 n3=ny4 rat1=ratx2 rat2=ratz5 rat3=raty4 genera_prim

set tipo $=0$ poi $0=5009$ poi $1=5018$ poi $2=5010$ poi3 $=6009$ poi $4=5019$ poi $5=6010$ poi6=6018 poi $=6019$

set gname='excava' $n 1=n x 3$ n2=ny 1 n3=nz5 rat1=ratx3 rat2=raty 1 rat3=ratz5 genera_prim

set gname='excava' $n 1=n \times 3$ n2=ny2 n3=nz5 rat1=ratx3 rat2=raty 2 rat3=ratz5 genera_prim

set tipo $=0$ poi $0=5011$ poi $1=5020$ poi $2=5017$ poi $3=6011$ poi $4=5021$ poi $5=6017$ poi6 $=6020$ poi $=6021$ 
set gname='pilote 2 ' $n 1=n \times 3$ n2=ny3 n3=nz5 rat1=ratx3 rat2=raty3 rat3=ratz5 genera_prim

set tipo $=0$ poi $0=5017$ poi $1=5021$ poi $2=5013$ poi $3=6017$ poi $4=5022$ poi5=6013 poi6=6021 poi7 $=6022$

set gname='pilote $2^{\prime}$ n1=nx3 n2=ny4 n3=nz5 rat1=ratx3 rat2=raty4 rat3=ratz5 genera_prim

set tipo $=0$ poi $0=5013$ poi $1=5022$ poi $2=5014$ poi $3=6013$ poi $4=5023$ poi $5=6014$ poi6=6022 poi $7=6023$

set gname='terreno' $\mathrm{n} 1=\mathrm{nx} 3 \mathrm{n} 2=\mathrm{ny} 5 \mathrm{n} 3=\mathrm{nz} 5$ rat $1=$ ratx3 rat2=raty 5 rat3=ratz5 genera_prim

set gname='terreno' $\mathrm{n} 1=\mathrm{nx} 3 \mathrm{n} 2=\mathrm{ny} 6 \mathrm{n} 3=\mathrm{nz} 5$ rat $1=$ ratx 3 rat2=raty 6 rat $3=$ ratz 5 genera_prim

set gname='terreno' $\mathrm{n} 1=\mathrm{n} \times 3 \mathrm{n} 2=\mathrm{ny} 7 \mathrm{n} 3=\mathrm{nz} 5$ rat $1=$ ratx3 rat2=raty 7 rat3=ratz5 genera_prim

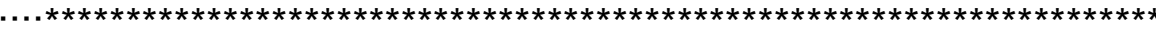

;; 6000-7000

set tipo $=0$ poi $=6001$ poi $1=6009$ poi $=6002$ poi3 $=7001$ poi $4=6010$ poi5=7002 poi6=7009 poi7=7010

set gname='excava' $n 1=n x 1 \mathrm{n} 2=\mathrm{ny} 1 \mathrm{n} 3=\mathrm{nz} 6$ rat $1=$ ratx 1 rat2=raty 1 rat3=ratz6 genera_prim

set gname='viga' $n 1=n \times 1$ n2=ny2 n3=nz6 rat1=ratx1 rat2=raty2 rat3=ratz6 genera_prim

set gname='pilote 1 ' $\mathrm{n} 1=\mathrm{nx} 1 \mathrm{n} 2=\mathrm{ny} 3 \mathrm{n} 3=\mathrm{nz} 6$ rat1=ratx1 rat2=raty3 rat3=ratz6 genera_prim

set gname='pilote1' $\mathrm{n} 1=\mathrm{nx} 1 \mathrm{n} 2=\mathrm{ny} 4 \mathrm{n} 3=\mathrm{nz} 6$ rat1=ratx1 rat2=raty4 rat3=ratz6 genera_prim

set gname='viga' $\mathrm{n} 1=\mathrm{nx} 1 \mathrm{n} 2=\mathrm{ny} 5 \mathrm{n} 3=\mathrm{nz} 6$ rat1=ratx1 rat2=raty5 rat3=ratz6 genera_prim

set gname='terreno' $\mathrm{n} 1=\mathrm{nx} 1 \mathrm{n} 2=\mathrm{ny} 6 \mathrm{n} 3=\mathrm{nz} 6$ rat1=ratx1 rat2=raty 6 rat3=ratz6 genera_prim

set gname='terreno' $\mathrm{n} 1=\mathrm{nx} 1 \mathrm{n} 2=\mathrm{ny} 7 \mathrm{n} 3=\mathrm{nz} 6$ rat $1=$ ratx 1 rat2=raty 7 rat3=ratz6 genera_prim 
set tipo $=1$ poi $0=7017$ poi $1=7012$ poi $2=6017$ poi $3=7011$ poi $4=6012$ poi $5=6011$ set gname='pilote 1 l' $n 1=n \times 2$ n2=nz6 n3=ny3 rat1=ratx2 rat2=ratz6 rat3=raty3 genera_prim

set tipo=1 poi0=7012 poi $1=7017$ poi2 $=6012$ poi3=7013 poi4=6017 poi5=6013 set gname='pilote1l' $n 1=n \times 2$ n2=nz6 n3=ny4 rat1=ratx2 rat2=ratz6 rat3=raty4 genera_prim

set tipo $=0$ poi $0=6009$ poi $1=6018$ poi $2=6010$ poi $3=7009$ poi $4=6019$ poi5 $=7010$ poi6=7018 poi7 $=7019$

set gname='excava' $n 1=n \times 3$ n2=ny $1 \mathrm{n} 3=\mathrm{nz} 6$ rat $1=$ ratx3 rat2=raty 1 rat3=ratz6 genera_prim

set gname='viga' n1=nx3 n2=ny2 n3=nz6 rat1=ratx3 rat2=raty2 rat3=ratz6 genera_prim

set tipo $=0$ poi $0=6011$ poi $1=6020$ poi $2=6017$ poi $3=7011$ poi $4=6021$ poi5=7017 poi6=7020 poi7 $=7021$

set gname='pilote2' $\mathrm{n} 1=\mathrm{n} \times 3 \mathrm{n} 2=\mathrm{ny} 3 \mathrm{n} 3=\mathrm{nz} 6$ rat1=ratx3 rat2=raty3 rat3=ratz6 genera_prim

set tipo $=0$ poi $0=6017$ poi $1=6021$ poi $2=6013$ poi $3=7017$ poi $4=6022$ poi $5=7013$ poi6=7021 poi7 $=7022$

set gname='pilote 2 ' n1=nx3 n2=ny4 n3=nz6 rat1=ratx3 rat2=raty4 rat3=ratz6 genera_prim

set tipo $=0$ poi $0=6013$ poi $1=6022$ poi $2=6014$ poi $3=7013$ poi $4=6023$ poi5=7014 poi6=7022 poi $=7023$

set gname='viga' n1=nx3 n2=ny5 n3=nz6 rat1=ratx3 rat2=raty5 rat3=ratz6 genera_prim

set gname='terreno' $n 1=n \times 3$ n2=ny 6 n3=nz6 rat $1=$ ratx3 rat2=raty 6 rat3=ratz6 genera_prim

set gname='terreno' $\mathrm{n} 1=\mathrm{nx} 3 \mathrm{n} 2=\mathrm{ny} 7 \mathrm{n} 3=\mathrm{nz} 6$ rat $1=$ ratx3 rat2=raty 7 rat3=ratz6 genera_prim

def recrece 
if $\_h>0$ then

command

set tipo $=0$ poi $0=7001$ poi $1=7009$ poi $2=7002$ poi3 $=8001$ poi $4=7010$ poi5=8002 poi6=8009 poi7 $=8010$

set gname='excava' $n 1=n x 1 \mathrm{n} 2=\mathrm{ny} 1 \mathrm{n} 3=\mathrm{nz} 7$ rat $1=$ ratx 1 rat2=raty 1 rat $3=$ ratz7 genera_prim

set gname='excava' $n 1=n x 1 \mathrm{n} 2=\mathrm{ny} 2 \mathrm{n} 3=\mathrm{nz} 7$ rat $1=$ ratx 1 rat2=raty 2 rat $3=$ ratz 7 genera_prim

set gname='excava' $n 1=n x 1 \mathrm{n} 2=\mathrm{ny} 3 \mathrm{n} 3=\mathrm{nz} 7$ rat1=ratx1 rat2=raty3 rat3=ratz7 genera_prim

set gname='excava' $n 1=n x 1 \mathrm{n} 2=\mathrm{ny} 4 \mathrm{n} 3=\mathrm{nz} 7$ rat1=ratx1 rat2=raty 4 rat3=ratz7 genera_prim

set gname='excava' $\mathrm{n} 1=\mathrm{nx} 1 \mathrm{n} 2=\mathrm{ny} 5 \mathrm{n} 3=\mathrm{nz} 7$ rat1=ratx1 rat2=raty 5 rat3=ratz 7 genera_prim

set gname='terreno' $\mathrm{n} 1=\mathrm{nx} 1 \mathrm{n} 2=\mathrm{ny} 6 \mathrm{n} 3=\mathrm{nz} 7$ rat1=ratx1 rat2=raty6 rat3=ratz7 genera_prim

set gname='terreno' $\mathrm{n} 1=\mathrm{nx} 1 \mathrm{n} 2=\mathrm{ny} 7 \mathrm{n} 3=\mathrm{nz} 7$ rat $1=$ ratx 1 rat2=raty 7 rat3=ratz7 genera_prim

set tipo $=1$ poi $=8017$ poi $1=8012$ poi $2=7017$ poi3 $=8011$ poi $4=7012$ poi5 $=7011$ set gname='excava' $n 1=n \times 2$ n2=nz7 n3=ny3 rat1=ratx2 rat2=ratz7 rat3=raty3 genera_prim

set tipo $=1$ poi $0=8012$ poi $1=8017$ poi $2=7012$ poi3 $=8013$ poi $4=7017$ poi5=7013 set gname='excava' $n 1=n \times 2$ n2=nz7 n3=ny4 rat1=ratx2 rat2=ratz7 rat3=raty4 genera_prim

set tipo $=0$ poi0 $=7009$ poi $1=7018$ poi $2=7010$ poi3 $=8009$ poi $4=7019$ poi $5=8010$ poi6=8018 poi7 $=8019$

set gname='excava' $n 1=n \times 3$ n2=ny $1 \mathrm{n} 3=\mathrm{nz} 7$ rat $1=$ ratx3 rat2=raty 1 rat3=ratz7 genera_prim set gname='excava' n1=nx3 n2=ny2 n3=nz7 rat1=ratx3 rat2=raty 2 rat3=ratz7 genera_prim 
set tipo=0 poi0=7011 poi $1=7020$ poi $2=7017$ poi3 $=8011$ poi $4=7021$ poi5=8017 poi6=8020 poi $7=8021$

set gname='excava' n1=nx3 n2=ny3 n3=nz7 rat1=ratx3 rat2=raty3 rat3=ratz7 genera_prim

set tipo $=0$ poi $0=7017$ poi $1=7021$ poi $2=7013$ poi3 $=8017$ poi $4=7022$ poi5=8013 poi6=8021 poi7 $=8022$

set gname='excava' $n 1=n \times 3$ n2=ny4 n3=nz7 rat1=ratx3 rat2=raty4 rat3=ratz7 genera_prim

set tipo $=0$ poi $0=7013$ poi $1=7022$ poi $2=7014$ poi3 $=8013$ poi $4=7023$ poi5=8014 poi6=8022 poi7 $=8023$

set gname='excava' $\mathrm{n} 1=\mathrm{nx} 3 \mathrm{n} 2=\mathrm{ny} 5 \mathrm{n} 3=\mathrm{nz} 7$ rat1=ratx3 rat2=raty5 rat3=ratz 7 genera_prim

set gname='terreno' $n 1=n \times 3$ n2=ny 6 n3=nz7 rat1=ratx3 rat2=raty 6 rat3=ratz7 genera_prim

set gname='terreno' $n 1=n \times 3$ n2=ny 7 n3=nz7 rat1=ratx3 rat2=raty7 rat3=ratz7 genera_prim

end_command

end_if

end

recrece

def morf1

$x \times 1=x c(p 1)$

$\mathrm{yy} 1=\mathrm{yc}(\mathrm{p} 1)$

$x \times 2=x c(p 2)$

yy2=yc(p2)

$x \times 3=x c(p 3)$

yy3=yc(p3)

$\mathrm{xx} 4=\mathrm{xc}(\mathrm{p} 4)$

yy4=yc(p4)

loop $\mathrm{i}(1, \mathrm{n} 1+1)$ 


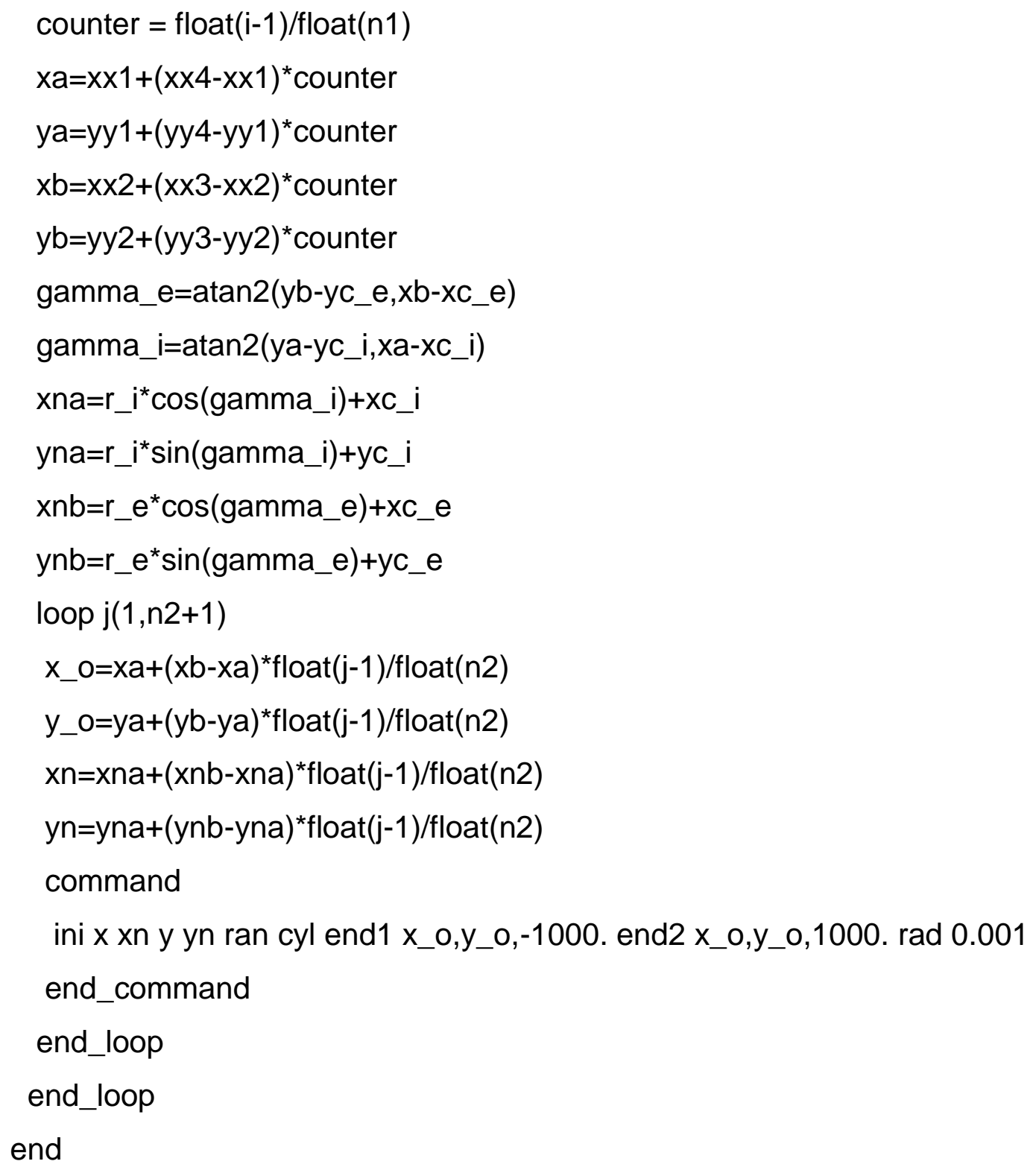


set $n 1=n y 3 n 2=n x 2$

set $x c \_e=x \_c 2$ yc_e=y_c2 xc_i=x_c1 yc_i=y_c1 r_e=r_pil r_i=r_pil

morf1

set $\mathrm{p} 1=1013 \mathrm{p} 2=1013 \mathrm{p} 3=1017 \mathrm{p} 4=1012$

set $\mathrm{n} 1=\mathrm{ny} 3 \mathrm{n} 2=\mathrm{nx} 2$

set $x c \_e=x \_c 2 y c \_e=y \_c 2 x c \_i=x \_c 1$ yc_i$=y \_c 1 r$ _e=r_pil r_i=r_pil

morf1

def morf2

$x \times 1=x c(p 1)$

$\mathrm{yy} 1=\mathrm{yc}(\mathrm{p} 1)$

$x \times 2=x c(p 2)$

yy2=yc(p2)

$x \times 3=x c(p 3)$

yy3=yc(p3)

$\mathrm{xx} 4=\mathrm{xc}(\mathrm{p} 4)$

$\mathrm{yy} 4=\mathrm{yc}(\mathrm{p} 4)$

loop $\mathrm{i}(1, \mathrm{n} 1+1)$

counter $=$ float(i-1)/float(n1)

$x a=x x 1+(x x 4-x x 1)^{*}$ counter

ya $=y y 1+(y y 4-y y 1)^{*}$ counter

$x b=x \times 2+(x \times 3-x \times 2) *$ counter

yb=yy2+(yy3-yy2)* counter

gamma_e=atan2(yb-yc_e,xb-xc_e)

gamma_i=atan2(ya-yc_i,xa-xc_i)

$x n a=r \_i^{*} \cos \left(g a m m a \_i\right)+x c \_i$

yna $=r \_i^{*} \sin ($ gamma_i)+yc_i

loop $\mathrm{j}(1, \mathrm{n} 2+1)$

if razon=1 then

$x \_0=x a+(x b-x a)^{\star}$ float $(j-1) /$ float(n2)

y_o=ya+(yb-ya) ${ }^{\star}$ float(j-1)/float(n2) 


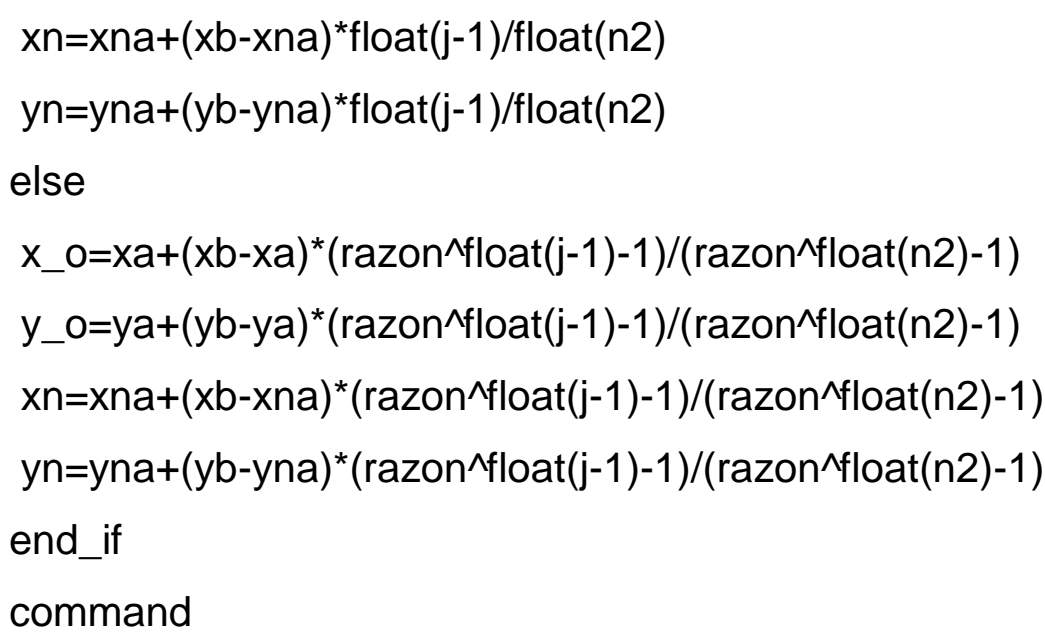




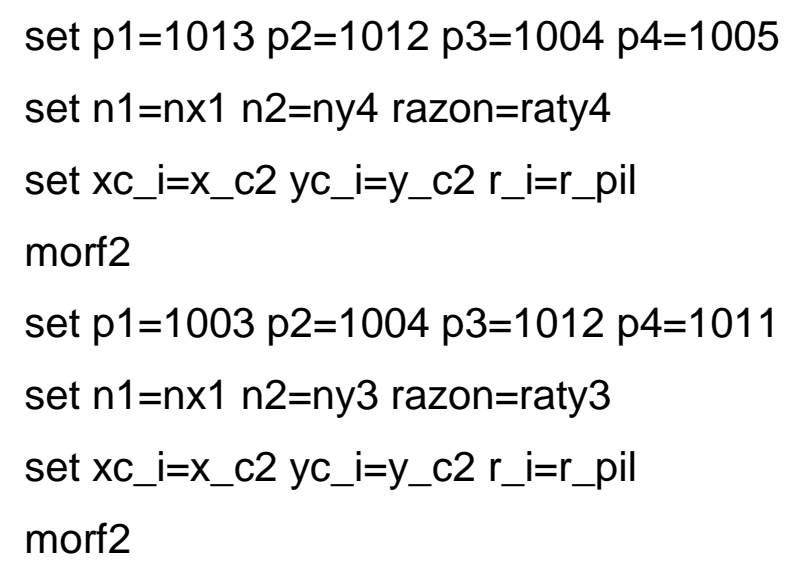

set $p 1=1013 p 2=1017$ p3=1021 p4=1022

set $\mathrm{n} 1=\mathrm{n} \times 3 \mathrm{n} 2=\mathrm{ny} 4$ razon=raty 4

set $x c \_i=x \_c 1$ yc_i=y_c1 $r \_i=r \_p i l$

morf2

set $p 1=1011 p 2=1017$ p3=1021 p4=1020

set $\mathrm{n} 1=\mathrm{n} \times 3 \mathrm{n} 2=\mathrm{ny} 3$ razon=raty3

set $x c \_i=x \_c 1$ yc_i $=y \_c 1 r \_i=r \_p i l$

morf2

gen merge 1e-3

gen zon ref dip 90. dd 90. ori x_cen,0.,0.

save pantalla_gr0.sav

gen zon copy 0.,0.,100. ran group pilote2 slot 1 gen zon copy $0 ., 0 ., 100$. ran group pilote 1 I slot 1 gen merge 1e-3

gen zon copy $0 ., 0 .,-100$. ran group pilote 1 slot 1 gen zon copy 0.,0.,-100. ran group pilote1I slot 1 z z_bot,z_top gen merge 1e-3

gen zon densify global nseg 1,1,2 ran z z_top,1000. group pilote2 slot 1 
gen zon densify local nseg 1,2,1 ran z z_top,1000. group pilote1l slot 1 ;cuñas gen zon densify global nseg 1,1,2 ran z-1000.,z_bot group pilote1 slot 1 gen zon densify local nseg 1,2,1 ran z -1000.,z_bot group pilote1/ slot 1 ;cuñas

group pilote2 slot 3 ran z z_top, 1000 .

group pilote1 slot 3 ran $z-1000 ., z$ bot

gen zon copy $0 ., 0 ., 150$. ran group solera slot 1

gen zon densify global nseg 1,1,2 ran z z_top,1000. group solera slot 1

gen zon copy $0 ., 0 ., 150$. ran group viga slot 1

gen zon densify global nseg 1,1,2 ran z z_top,1000. group viga slot 1

save pantalla_gr1.sav

ca pantalla_calculo.f3dat

RET 


\section{Archivo pantalla_cálculo.f3dat}

config creep

fix $x$ ran $x x \_$minm, $x \_$minp

fix $x$ ran $x$ x_maxm, $x \_$maxp

fix y ran y y_minm,y_minp

fix y ran y y_maxm,y_maxp

fix $x$ y z ran z z_botm,z_botp

set $g 10$.

mo mech mo ran z z_bot,z_top

mo mech null ran $z-1000 ., z \_$bot

mo mech null ran z z_top, 1000 .

;;***Geología

group terreno1 slot 2 ran z z_7,z_top

group terreno2 slot 2 ran z z_5,z_7

group terreno3 slot 2 ran z z_bot,z_5

mo mech cvisc ran group terreno1 slot 2

mo mech cvisc ran group terreno2 slot 2

;*** Propiedades de cálculo

pro bulk=_bulk1 coh=_cc1 fric=_ff1 tens=_tens 1 dens=_gamma1 ran group terreno1 slot 2

pro kshear=_kshear1 $\mathrm{kvis}=\_\mathrm{kvisc} 1$ mshear=_mshear1 mvis=_mvisc1 ran group terreno1 slot 2

pro bulk=_bulk2 coh=_cc2 fric=_ff2 tens=_tens2 dens=_gamma2 ran group terreno2 slot 2

pro kshear=_kshear2 $\mathrm{kvis}=\_\mathrm{kvisc} 2$ mshear=_mshear2 $\mathrm{mvis}=\_$mvisc2 ran group terreno2 slot 2 
pro young=_ee3 pois=_pois3 $\mathrm{coh}=\_\mathrm{cc} 3$ fric=_ff3 tens=_tens3 dens=_gamma3 ran group terreno3 slot 2

; Condiciones iniciales

def cotas_agua

$$
\text { z_fre }=\text { z_8 }
$$$$
\text { z_frem }=z \text { fre }-0.001
$$$$
\text { z_frep }=z \text { fre }+0.001
$$

$\mathrm{pp} 0=\mathrm{z} \_$fre ${ }^{*} 1 \mathrm{e} 4$

$\mathrm{ppOm}=-\mathrm{pp} 0$

end

cotas_agua

ini pp pp0 grad 0.0. -1e4 ran z z_botm z_frep

def ini_tens

pnt=zone_head

loop while pnt \# null

if $z \_$model(pnt)\#'null' then

z_el=z_zcen(pnt)

if z_el>z_7 then

z_szz(pnt) $=-(\text { z_top-z_el })^{*}$ _gamma1*10.

else

if z_el>z_5 then

z_szz $($ pnt $)=-\left(z \_t o p-z \_7\right)^{*}$ _gamma1 ${ }^{*} 10 .-\left(z \_7-z \_e l\right)^{*}$ _gamma2 ${ }^{*} 10$.

else

z_szz(pnt)=-(z_top-z_7 $)^{*}$ _gamma1*10.-(z_7-z_5)*_gamma2*10.-(z_5-

z_el) ${ }^{*}$ gamma3*10.

end_if

end_if 


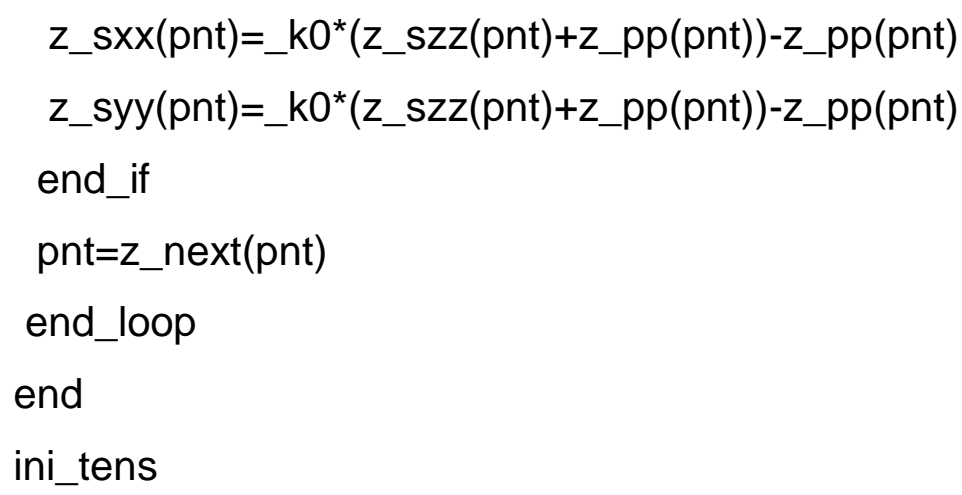


solve ratio $1 \mathrm{e}-5$

save @fname

end_command

end_if

end

excava_banco

hist gp zdisp x_cen,y_min,z_viga

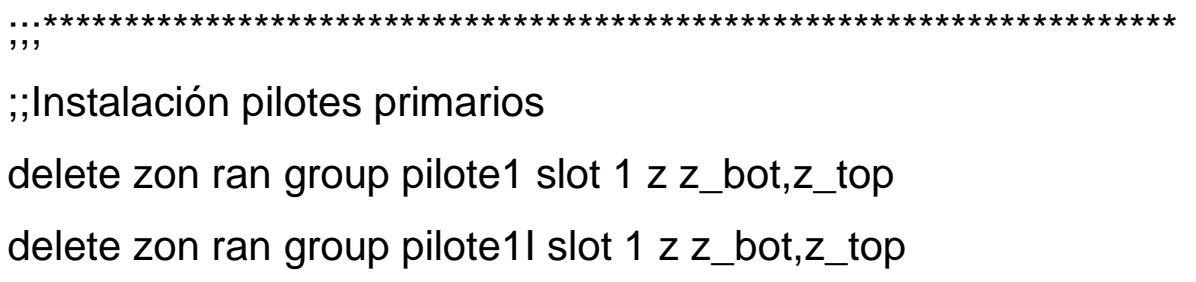

mo el ran group pilote1 slot 3

pro young=_youngP1 pois=_poisP1 dens=_densP1 ran group pilote1 slot $1 \mathrm{z}$ 1000.,z_bot

pro young=_youngP1i pois=_poisP1i dens=_densP1i ran group pilote $1 \mathrm{l}$ slot $1 \mathrm{z}$ $-1000 ., z$ bot

$$
\begin{aligned}
& \text { def_cotas_int1 } \\
& z \text { _int1=z_1-100. } \\
& z \text { _int2=z_viga-100. } \\
& \text { z_int1m=z_int1-0.001 } \\
& \text { z_int1p=z_int1+0.001 } \\
& \text { z_int2m=z_int2-0.001 } \\
& \text { z_int2p=z_int2+0.001 } \\
& \text { y_top1=y_1+1.5 } \\
& \text { y_top2=y_1 +5.0 } \\
& \text { r_pilp=r_pil+0.01 }
\end{aligned}
$$




$$
\begin{aligned}
& \text { z_vigam =z_viga-0.001 } \\
& z \_1 m=z \_1-0.001 \\
& z \_1 p=z \_1+0.001 \\
& x \_c 2 b=-x \_c 2 \\
& \text { end } \\
& \text { _cotas_int1 }
\end{aligned}
$$

hist gp zdisp x_cen,y_top1,z_top

hist gp zdisp $\mathrm{x} \_$cen,y_top2,z_top

ini $x s 0$ ys 0 zs 0

int 1 face ran $x x \_$minp,x_maxm $z z \_$int1p,z_int2m

int 2 face ran $x x \_$minp, $x \_$maxm $z z$ int1 $m, z$ int $1 p$

ini $z$ add 100. ran group pilote 1 slot 3

def prop_int

$$
\begin{gathered}
\text { _ff1 int=_ff } 1{ }^{*} 2 . / 3 . \\
\text { fff2int=_ff } 2 * 2 . / 3 . \\
\text { fff3int=_ff } 3^{\star} 2 . / 3 . \\
\text { fffRint=_ffR }{ }^{\star} 2 . / 3 .
\end{gathered}
$$

end

prop_int

int 1 prop kn 1 e9 ks 1 e9 coh=_cc1 fric=_ff 1 int ran z z_7,z_topp

int 1 prop kn 1 e9 ks 1 e9 $\mathrm{coh}=\_c c 2$ fric $=$ ffeint ran $z$ z_5,z_7

int 1 prop kn 1 e9 ks 1 e9 coh=_cc3 fric=_ff3int ran z z_bot,z_5

int 2 prop kn 1 e9 ks 1 e9 coh=_cc3 fric=_ff3int

int 1 perm on

int 2 perm on

;;,carga agua 
apply nstr pp0m grad 0.,0.,1e4 ran cyl end1 x_c2,y_c2,z_fre end2 $x \_c 2, y \_c 2, z \_1 m$ rad $r \_p i l p \&$

$x$ x_minp,x_maxm

apply nstr pp0m grad 0.,0.,1e4 ran cyl end1 $x \_c 2 b, y \_c 2, z \_f r e$ end2 $x \_c 2 b, y \_c 2, z \_1 m$ rad r_pilp \&

x x_minp,x_maxm

def_archivo

fname ='pant_'+string(_caso)+'_01.sav'

end

_archivo

set creep off

solve ratio $1 \mathrm{e}-5$

save@fname

$; ;$

;;Instalación pilotes secundarios

delete zon ran group pilote2 slot $1 \mathrm{z} z$ zbot,z_top

delete zon ran group pilote1I slot $1 \mathrm{z} z$ z_bot,z_top

mo el ran group pilote2 slot 3

pro young=_youngP2 pois=_poisP2 dens=_densP2 ran group pilote2 slot $3 \mathrm{z}$ z_top, 1000.

def_cotas_int2

z_int3=z_1+100.

z_int4=z_viga +100 .

z_int3m=z_int3-0.001

$z$ int3p $=z$ int3 +0.001

z_int4m=z_int4-0.001 
z_int $4 p=z \_$int $4+0.001$

end

_cotas_int2

ini xs 0 ys 0 zs 0 ran cyl end 1 x_cen,y_cen,-100. end2 x_cen,y_cen,50. rad r_pilp

int 3 face ran $x$ x_minp,x_maxm z z_int3p,z_int4m group pilote2 slot 1

int 5 face ran $x x \_$minp, $x \_$maxm $z z \_$int3p,z_int $4 m$ group pilote2 slot 1 not

int 4 face ran $x x \_$minp, $x \_$maxm $z z$ int3m,z_int3p

ini $z$ add -100 . ran group pilote 2 slot 3

int 3 prop kn 1 e9 ks 1 e9 $\mathrm{coh}=\_c c 1$ fric $=$ ff 1 int ran z z_ 7,z_topp

int 3 prop kn 1 e9 ks 1 e9 $\mathrm{coh}=\_c c 2$ fric $=$ ff 2int ran $z \mathrm{z} \_5, z \_7$

int 3 prop kn 1 e9 ks 1 e9 coh=_cc3 fric=_ff3int ran z z_bot,z_5

int 4 prop kn 1 e9 $\mathrm{ks} 1$ e9 $\mathrm{coh}=\_\mathrm{cc} 3 \mathrm{fric}=$ ff $3 i n t$

int 5 prop kn 1 e9 ks 1 e9 $\mathrm{coh}=0.0$ fric $=45$.

int 3 perm on

int 4 perm on

int 5 perm on

apply nstr pp0m grad 0.,0.,1e4 ran cyl end1 $x \_c 1, y \_c 1, z \_f r e$ end2 $x \_c 1, y \_c 1, z \_1 m$ rad r_pilp \&

x x_minp,x_maxm group pilote1i slot 1 not group pilote1 slot 1 not

def_archivo

fname ='pant_'+string(_caso)+'_02.sav'

end

_archivo

set creep off

solve ratio $1 \mathrm{e}-5$ 
save@fname

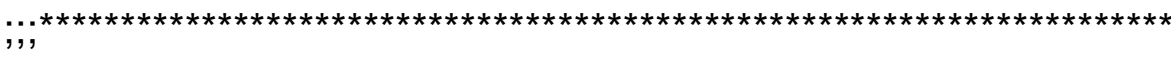

;:Excavación hasta viga de atado y anclajes

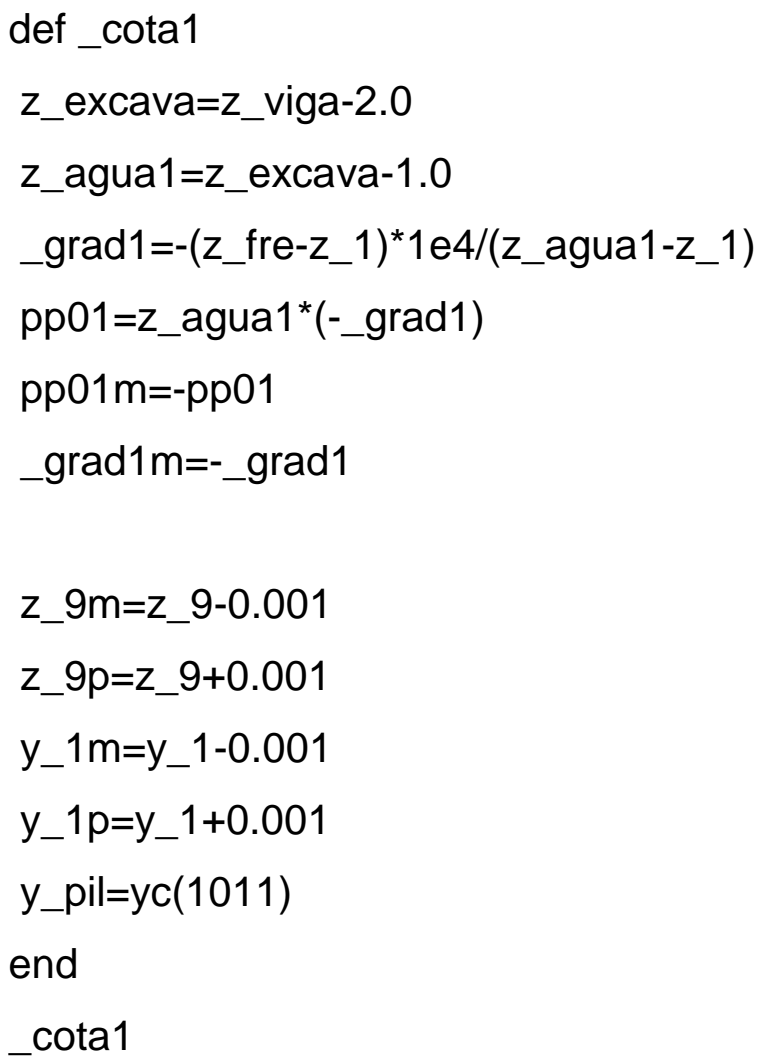

mo null ran group excava slot 1 z z_excava,z_viga delete zon ran group viga slot 1 z z_excava,z_viga

ini zex 50

def lee_presiones_ini

pnt=zone_head

loop while pnt\#null

z_extra (pnt,5)=z_pp(pnt)

pnt=z_next(pnt)

end_loop

end 
lee_presiones_ini

def baja_agua

if z_8>z_agua1 then

command

ini pp pp01 grad 0. 0._grad1 ran z z_1,z_agua1 y y_minm,y_cen

ini pp 0 ran z z_agua1,z_topp y y_minm,y_cen

ini pp 0 ran mo el

apply remove nstre ran cyl end1 x_c1,y_c1,z_vigam end2 x_c1,y_c1,z_1p rad r_pilp y y_minm,y_pil

apply remove nstre ran cyl end1 $x \_c 2, y \_c 1, z \_v i g a m$ end2 $x \_c 2, y \_c 1, z \_1 p$ rad r_pilp y y_minm,y_pil

apply remove nstre ran cyl end1 $x \_c 2 b, y \_c 1, z \_v i g a m$ end2 $x \_c 2 b, y \_c 1, z \_1 p$ rad r_pilp y y_minm,y_pil

apply nstr pp01m grad 0.,0.,_grad1m ran cyl end1 x_c1,y_c1,z_agua1 end2 $x \_c 1, y \_c 1, z \_1 p$ rad r_pilp \&

y y_minm,y_pil $x$ x_minp,x_maxm

apply nstr pp01m grad 0.,0.,_grad1m ran cyl end1 $\mathrm{x} \_c 2, y \_c 1, z \_a g u a 1$ end2 $x \_c 2, y \_c 1, z \_1 p$ rad r_pilp \&

y y_minm,y_pil $x$ x_minp,x_maxm

apply nstr pp01m grad 0.,0.,_grad1m ran cyl end1 x_c2b,y_c1,z_agua1 end2 $x \_c 2 b, y \_c 1, z \_1 p$ rad $r \_p i l p \&$

y y_minm,y_pil $x$ x_minp,x_maxm

end_command

end_if

end

baja_agua

def cambia_tens

pnt=zone_head

loop while pnt\#null

pp_actual=z_pp(pnt) 


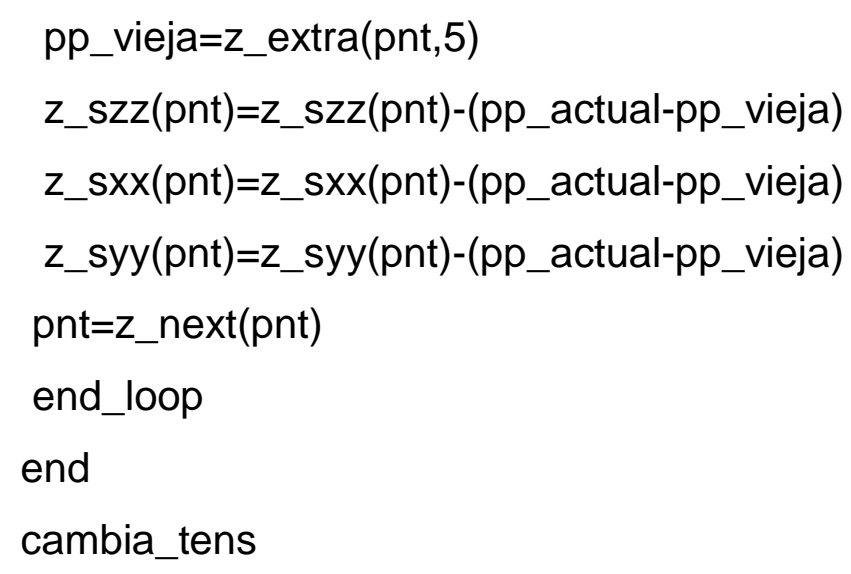

mo el ran group viga slot 1

pro young=_young $V$ pois=_pois $V$ dens=_dens $V$ ran group viga slot $1 \mathrm{z}$ z_top, 1000 .

ini xs 0 ys 0 zs 0 ran z z_9m,z_topp

int 6 face ran $z$ z_9m,z_9p

int 7 face ran z z_topp,1000. y y_1m,y_1p

int 6 prop kn 1 e9 $\mathrm{ks} 1 \mathrm{e} 9 \mathrm{coh}=\_\mathrm{cc} 1 \mathrm{fric}=$ ff 1 int

int 7 prop kn 1 e9 $\mathrm{ks} 1 \mathrm{e} 9 \mathrm{coh}=\_\mathrm{cc} 1 \mathrm{fric}=$ fffint

ini $z$ add -150 . ran group viga slot 1

int 1 prop kn 1 e9 ks 1 e9 coh=1e10 tens=1e10 ran z z_9,z_viga

int 3 prop kn 1 e9 ks 1 e9 coh=1e10 tens=1e10 ran z z_9,z_viga

;;Anclaje
def pone_cable
idc=idc
zini=z_viga-_A2
yini=y_3+0.00001
xini=x_cen
ang=_beta*degrad

Modelos de comportamiento para el control de pantallas ancladas en terrenos con fluencia 


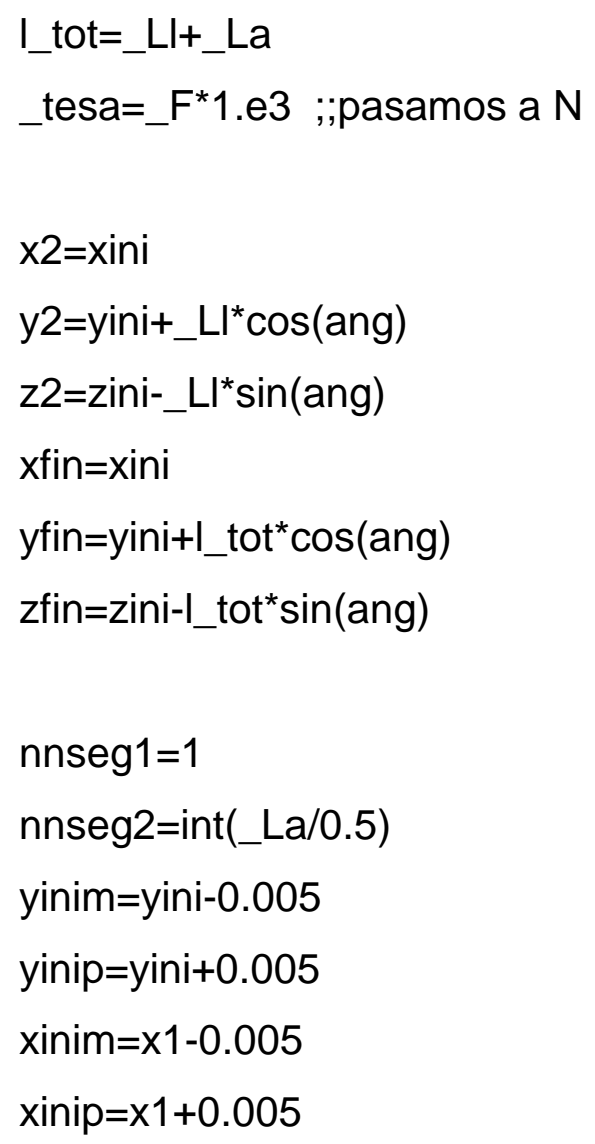

command

sel cable id idc beg xini,yini,zini end x2,y2,z2 nseg nnseg1

sel cable id idc beg x2,y2,z2 end xfin,yfin,zfin nseg nnseg2

sel link attach $x d$ rigid $y d$ rigid $z d$ rigid ran y yinim,yinip

sel cable id idc pro e 1 e7 $x c a=$ area gr_k 1 e9 gr_c 0 yten=_carga ran y yini,y2 ;parte libre

sel cable id idc pro e=e_cable $\mathrm{xca}=\_$area gr_k $1 \mathrm{e} 9 \mathrm{gr} \_\mathrm{c}=\_$grout $y$ ten=_carga ran y y2,yfin ;parte anclada

sel cable id idc pretension_tesa ran y yini,y2

end_command

end

set idc $=101$

pone_cable

def_archivo

fname ='pant_'+string(_caso)+'_03.sav' 
end

_archivo

set creep off

solve ratio $1 e-5$

save@fname

;;Tesado

sel cable id 101 pro e=e_cable

$;, ; *$

;;Excavación hasta NME

def_cota2

z_excava $=$ z_3

z_agua2=z_excava-1.0

-grad2=-(z_fre-z_1)*1e4/(z_agua2-z_1)

pp02=z_agua2* ${ }^{*}-$ grad2)

pp02m=-pp02

_grad2m=-_grad2

y_his $1=\left(y \_3+y \_\min \right)^{\star} 0.5$

end

_cota2

mo null ran group excava slot 1 z z_3,z_viga

delete zon ran group solera slot $1 \mathrm{z} z$ zexcava,z_viga

mo null ran group relleno slot 1 z z_excava,z_viga

ini zex 50

lee_presiones_ini

ini pp pp02 grad 0.0._grad2 ran z z_1,z_agua2 y y_minm,y_cen 
ini pp 0 ran z z_agua2,z_top y y_minm,y_cen

ini pp 0 ran mo el

def lee_inc

pnt=zone_head

loop while pnt\#null

pp_actual=z_pp(pnt)

pp_vieja=z_extra $(p n t, 5)$

z_extra(pnt,6)=pp_actual-pp_vieja

pnt=z_next(pnt)

end_loop

end

lee_inc

cambia_tens

apply remove nstre ran cyl end1 $\mathrm{x} \_c 1, y \_c 1, z \_v i g a m$ end2 $x \_c 1, y \_c 1, z \_1 p$ rad r_pilp y y_minm,y_pil

apply remove nstre ran cyl end1 $\mathrm{x} \_c 2, y \_c 1, z \_v i g a m$ end2 x_c2,y_c1,z_1p rad r_pilp y y_minm,y_pil

apply remove nstre ran cyl end1 $x \_c 2 b, y \_c 1, z \_v i g a m ~ e n d 2 x \_c 2 b, y \_c 1, z \_1 p$ rad r_pilp y y_minm,y_pil

apply nstr pp02m grad 0.,0.,_grad2m ran cyl end1 x_c1,y_c1,z_agua2 end2 $x \_c 1, y \_c 1, z \_1 p$ rad r_pilp \&

y y_minm,y_pil $x$ x_minp,x_maxm

apply nstr pp02m grad 0.,0.,_grad2m ran cyl end1 x_c2,y_c1,z_agua2 end2 x_c2,y_c1,z_1prad r_pilp \&

y y_minm,y_pil $x$ x_minp,x_maxm

apply nstr pp02m grad 0.,0.,_grad2m ran cyl end1 x_c2b,y_c1,z_agua2 end2 $x \_c 2 b, y \_c 1, z \_1 p$ rad $r \_p i l p \&$

y y_minm,y_pil $x$ x_minp,x_maxm 
def_archivo

fname ='pant_'+string(_caso)+'_04.sav'

end

_archivo

hist gp zdisp $\mathrm{x} \_c e n, y \_\min , z \_3$

hist gp zdisp $\mathrm{x} \_$cen,y_his1,z_3

hist gp zdisp $\mathrm{x} \_c e n, y \_3, z \_3$

set creep off

solve ratio $1 \mathrm{e}-5$

save@fname

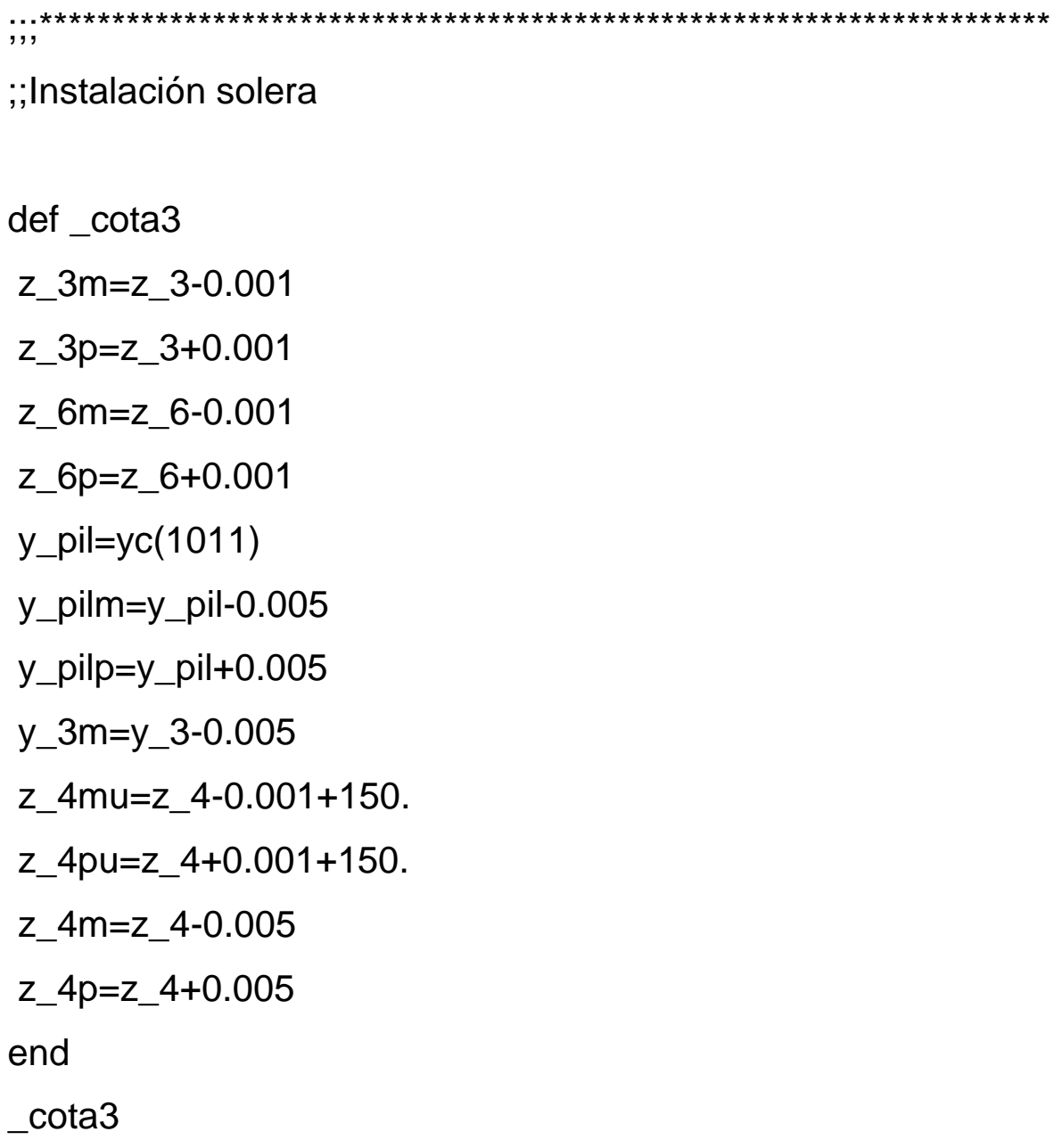


mo mo ran group relleno slot 1

pro young=_eeR pois=_poisR coh=_ccR fric=_ffR tens=_tens $R$ dens=_gammaR ran group relleno slot 1

ini pp 0 ran group relleno slot 1

ini xs 0 ys 0 zs 0 ran z z_3p,z_6p y y_3m,y_pilp

ini xs 0 ys 0 zs 0 ran z z_4m,z_4p y y_min,y_pilp

mo el ran group solera slot 1

pro young=_youngS pois=_poisS dens=_densS ran group solera slot $1 \mathrm{z}$ z_top, 1000 .

int 8 face ran z z_4mu,z_4pu

int 8 prop kn 1 e9 $\mathrm{ks} 1$ e9 $\mathrm{coh}=\_\mathrm{ccR}$ fric=_ffRint

ini xs 0 ys 0 zs 0 ran group solera slot 1

ini $x s 0$ ys 0 zs 0 ran group relleno slot $1 z z \_3 p, z \_4 p$

ini $z$ add -150 . ran group solera slot 1

;,empotramiento de la pantalla

int 1 pro coh 1 e10 tens 1 e10 ran z z_4,z_6

int 3 pro coh 1 e10 tens 1 e10 ran z z_4,z_6

def_archivo

fname ='pant_'+string(_caso)+'_05.sav'

end

_archivo

hist gp zdisp $\mathrm{x} \_$cen,y_min,z_6

hist gp zdisp x_cen,y_his1,z_6

hist gp zdisp $\mathrm{x} \_$cen,y_3,z_6 
set creep off

solve ratio 1 e-5

save@fname

;;recuperación freático

ini zex 50

lee_presiones_ini

ini pp pp0 grad 0. 0. -1e4 ran z z_1,z_4 y y_minm,y_cen

ini pp 0 ran mo el

cambia_tens

apply remove nstre ran cyl end1 $\mathrm{x} \_c 1, \mathrm{y} \_\mathrm{c} 1, \mathrm{z}$ _vigam end2 $\mathrm{x} \_\mathrm{c} 1, \mathrm{y} \_\mathrm{c} 1, \mathrm{z} \_1 \mathrm{p}$ rad r_pilp y y_minm,y_pil

apply remove nstre ran cyl end1 x_c2,y_c1,z_vigam end2 x_c2,y_c1,z_1p rad r_pilp y y_minm,y_pil

apply remove nstre ran cyl end1 $\mathrm{x} \_\mathrm{c} 2 \mathrm{~b}, \mathrm{y} \_c 1, \mathrm{z} \_$vigam end2 $\mathrm{x} \_c 2 b, y \_c 1, z \_1 p$ rad r_pilp y y_minm,y_pil

apply nstr pp0m grad 0.,0.,1e4 ran cyl end1 $x \_c 1, y \_c 1, z \_4$ end2 $x \_c 1, y \_c 1, z \_1 p$ rad r_pilp \&

y y_minm,y_pil x x_minp,x_maxm

apply nstr pp0m grad 0.,0.,1e4 ran cyl end1 x_c2,y_c1,z_4 end2 $x \_c 2, y \_c 1, z \_1 p$ rad r_pilp \&

y y_minm,y_pil x x_minp,x_maxm

apply nstr pp0m grad 0.,0.,1e4 ran cyl end1 $x \_c 2 b, y \_c 1, z \_4$ end2 x_c2b,y_c1,z_1p rad r_pilp \&

y y_minm,y_pil $x$ x_minp,x_maxm

apply nstr pp0m grad 0.,0.,1e4 ran z z_4m,z_4p y y_minm,y_pil $x$ x_minp,x_maxm 
def_archivo

fname ='pant_'+string(_caso)+'_06.sav'

end

_archivo

set creep off

solve ratio $9 \mathrm{e}-6$

save@fname

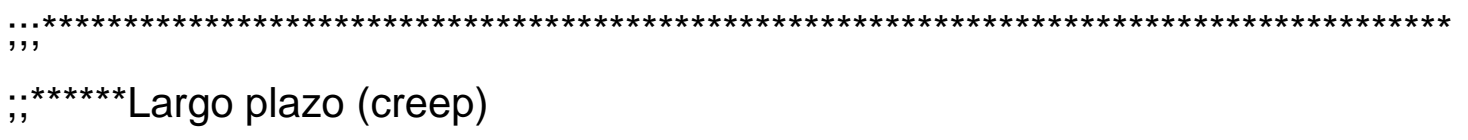

ini gpex 50

ini gpex 60

ini gpex 70

def lee_disp_ini

pnt=gp_head

loop while pnt\#null

gp_extra $(p n t, 5)=g p \_x d i s p(p n t)$

gp_extra $(p n t, 6)=g p \_y d i s p(p n t)$

gp_extra $(p n t, 7)=g p \_z d i s p(p n t)$

pnt=gp_next(pnt)

end_loop

end

lee_disp_ini

ini xd 0 yd 0 zd 0 ;;inicializamos desplazamientos de la fase instántanea ini $x v 0$ yv 0 zv 0

sel node init $x d 0$ yd 0 zd 0 xr 0 yr 0 zr 0

sel node init xvel 0 yvel 0 zvel 0 
set creep on

set cr mindt 1 maxdt 1000.

set $\mathrm{cr}$ dt 10

set $\mathrm{cr}$ dt auto on

set cr lfob 1e-3 ufob 5e-3

set cr Imul $1.01 \mathrm{cr}$ umul .9

def $t$ dias

t_dias= crtime/86400. ;;tiempo en días

end

hist t_dias

;*** Instrumentación

def lee_disp_pil

;punto control en losa

$x 0 \_$losa $=x c(1019)$

y0_losa=yc(1019)

command

hist gp zdisp x0_losa,y0_losa,z_6

hist gp ydisp x0_losa,y0_losa,z_6

hist gp xdisp x0_losa,y0_losa,z_6

end_command

;puntos en pilotes

x0_pil=xc(1020)

y0_pil=yc(1020)

z0_pil=z_6+0.55

n_punto $=0$

loop while z0_pil<z_9

command

hist gp zdisp x0_pil,y0_pil,z0_pil

hist gp ydisp $x 0$ pil,y0_pil,z0_pil

hist gp xdisp x0_pil,y0_pil,z0_pil 


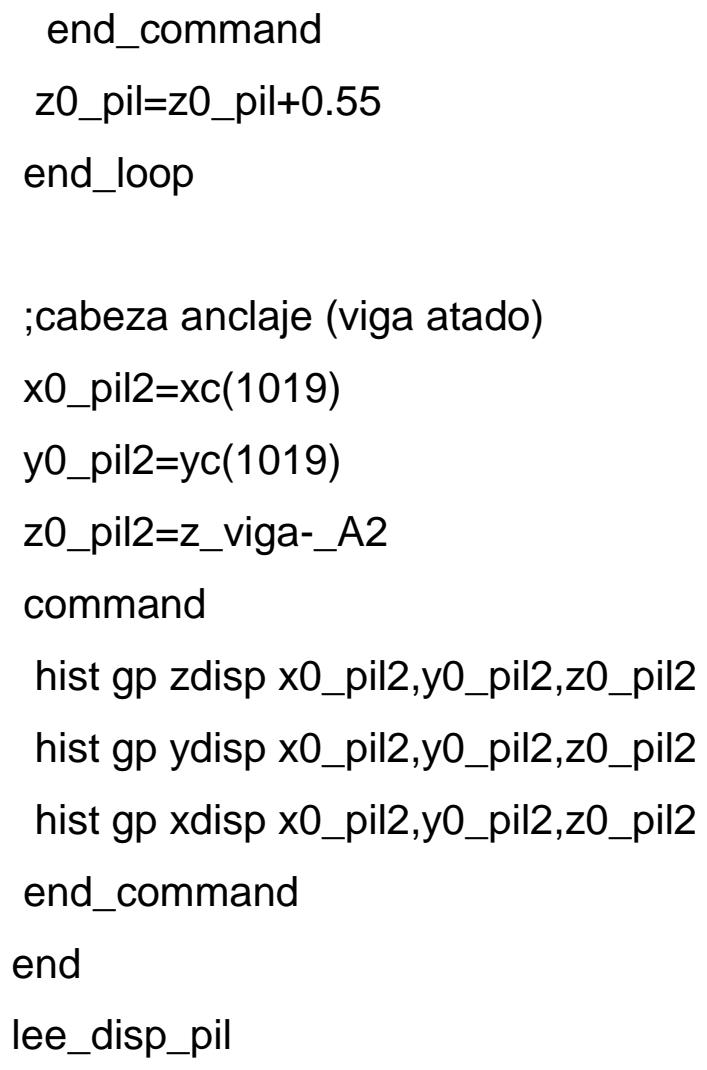




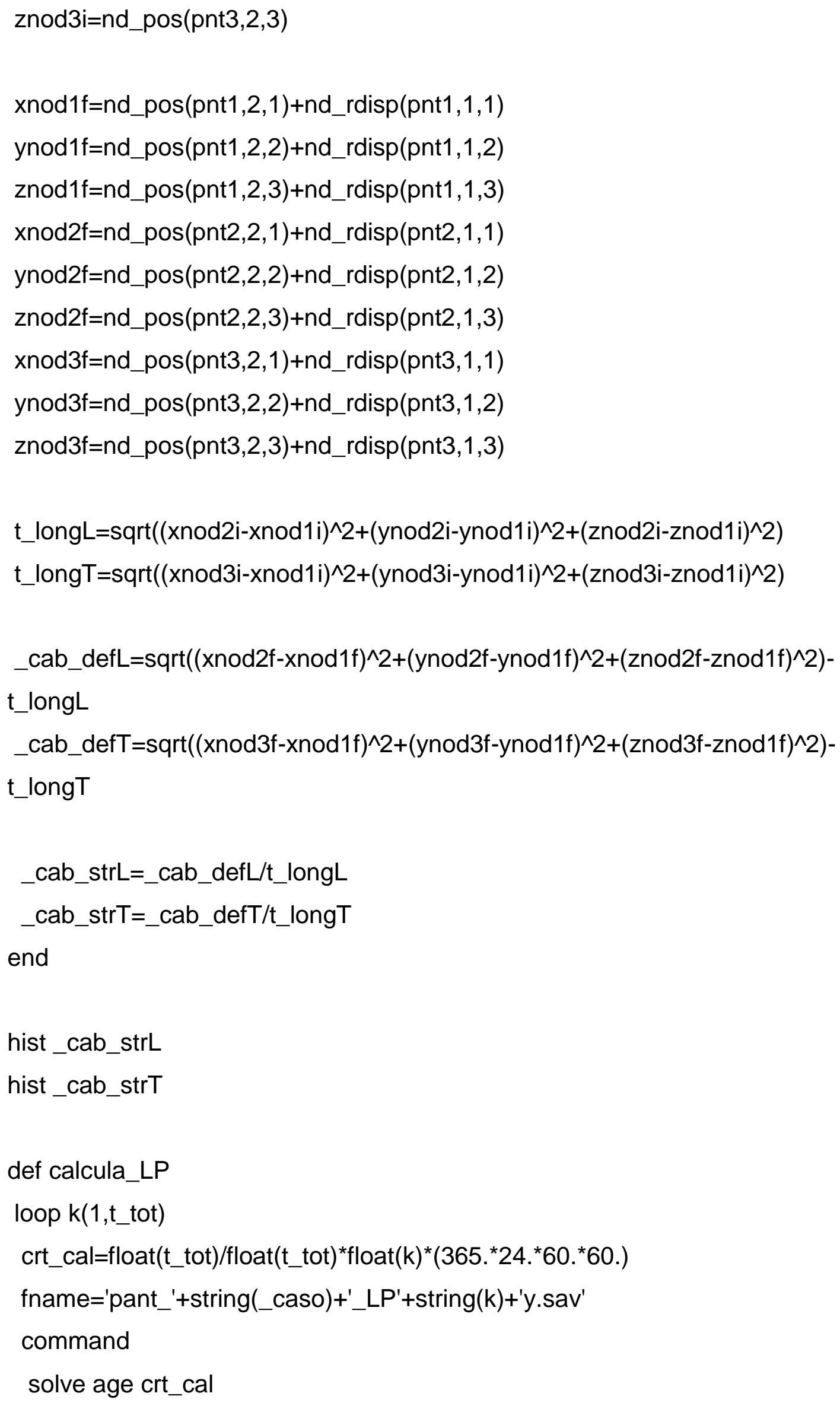


save @fname

end_command

end_loop

end

set t_tot=20 ;;tiempo total en años

calcula_LP

RET 